University of New Hampshire

University of New Hampshire Scholars' Repository

Spring 2020

\title{
Element-Based Multi-Objective Optimization Methodology Supporting a Transportation Asset Management Framework for Bridge Planning and Programming
}

Karim Naji

University of New Hampshire, Durham

Follow this and additional works at: https://scholars.unh.edu/dissertation

\section{Recommended Citation}

Naji, Karim, "Element-Based Multi-Objective Optimization Methodology Supporting a Transportation Asset Management Framework for Bridge Planning and Programming" (2020). Doctoral Dissertations. 2513.

https://scholars.unh.edu/dissertation/2513

This Dissertation is brought to you for free and open access by the Student Scholarship at University of New Hampshire Scholars' Repository. It has been accepted for inclusion in Doctoral Dissertations by an authorized administrator of University of New Hampshire Scholars' Repository. For more information, please contact Scholarly.Communication@unh.edu. 
ELEMENT-BASED MULTI-OBJECTIVE OPTIMIZATION METHODOLOGY SUPPORTING A TRANSPORTATION ASSET MANAGEMENT FRAMEWORK FOR BRIDGE PLANNING AND PROGRAMMING

BY

\section{KARIM NAJI}

\section{DISSERTATION}

Submitted to the University of New Hampshire in Partial Fulfillment of the Requirements for the Degree of

Doctor of Philosophy

in

Civil and Environmental Engineering

May, 2020 
This dissertation was examined and approved in partial fulfillment of the requirements for the degree of Doctor of Philosophy in Civil and Environmental Engineering by:

Dissertation Director, Dr. Erin Santini-Bell, Chair and Professor

Department of Civil and Environmental Engineering University of New Hampshire

Dr. James Malley, Professor

Department of Civil and Environmental Engineering University of New Hampshire

Dr. Robert Henry, Associate Professor

Department of Civil and Environmental Engineering

University of New Hampshire

Dr. Kyle Kwiatkowski, Assistant Professor

Department of Civil and Environmental Engineering

University of New Hampshire

Dr. Robert Zobel, Program Coordinator

Long-Term Bridge Performance Program

Office of Infrastructure Research and Development

Federal Highway Administration

On April 3, 2020

Original approval signatures are on file with the University of New Hampshire Graduate School. 


\section{DEDICATION}

To my parents

To the memory of my uncle, Naser Neji

To the memory of my mentor, Abderrazak Hamam 


\section{ACKNOWLEDGEMENTS}

I would like to express my sincere appreciation to my advisor, Dr. Erin Santini-Bell, for her constant guidance, immense support, and encouragement during every phase of this research.

I would like to acknowledge all members of my Ph.D. advisory committee, Dr. James Malley, Dr.

Robert Henry, Dr. Kyle Kwiatkowski, and Dr. Robert Zobel, for their feedback, valuable discussions, and assistance.

I would like to gratefully thank all my colleagues at work and my friends for their friendship, encouragement in all my pursuits, and moral support that helped me go through the stressful times. 


\section{TABLE OF CONTENTS}

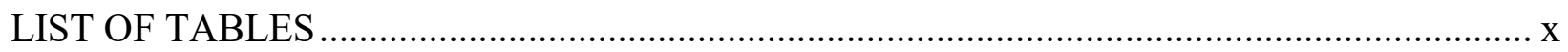

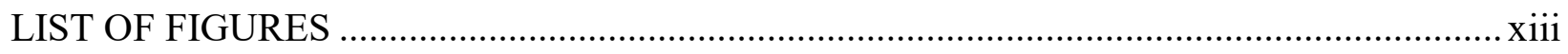

LIST OF ABBREVIATIONS ...................................................................................... xvii

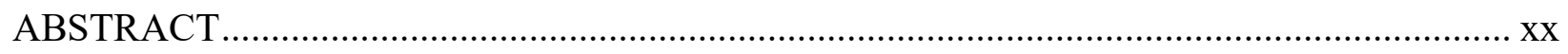

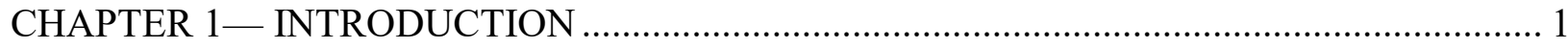

1.1 Path Leading to the Proposed Research.................................................................... 1

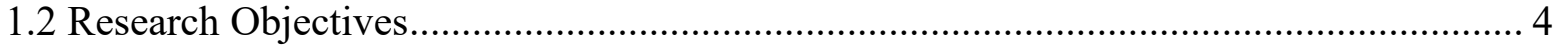

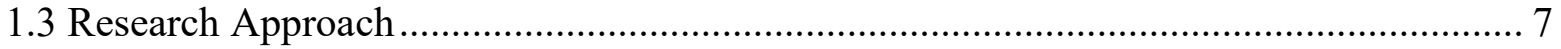

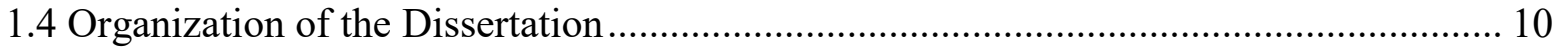

CHAPTER 2 - LITERATURE REVIEW .................................................................... 14

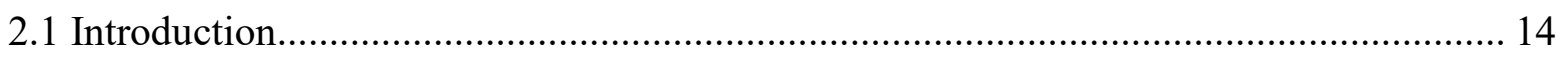

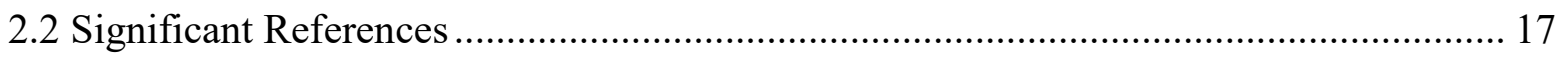

2.3 Transportation Planning Process ...................................................................... 21

2.4 Bridge Programming Process ............................................................................... 23

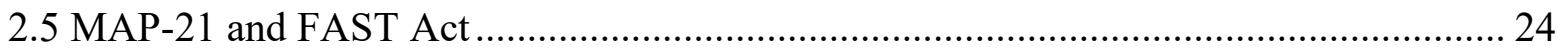

2.5.1 Performance-Based Planning Requirements.................................................... 26

2.5.2 Transportation Asset Management Plan Requirements ....................................... 28

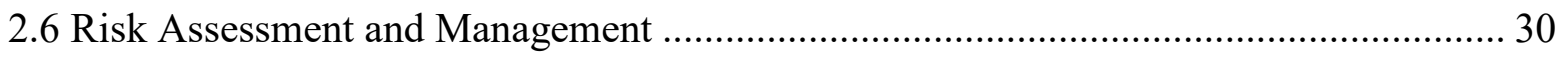

2.7 Optimization in Bridge Management ................................................................... 32

2.8 Multi-Objective Optimization Approaches .............................................................. 36

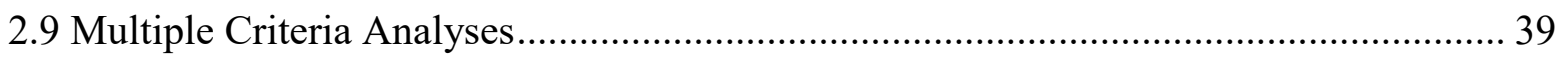

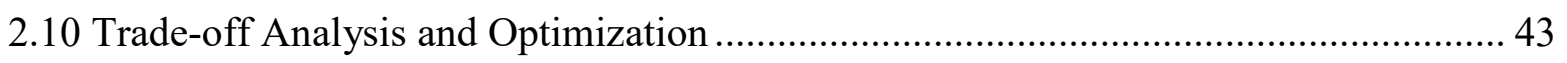

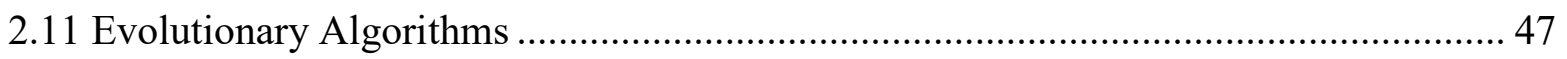

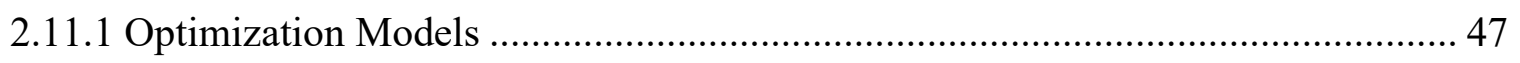

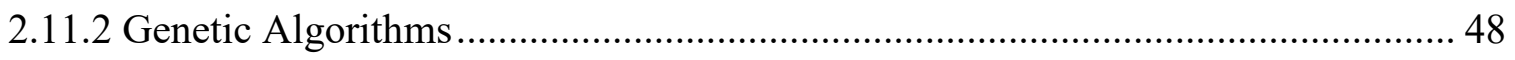

2.11.3 Non-Dominated Sorting Genetic Algorithm-II............................................ 52

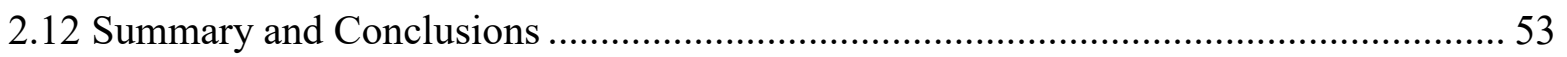


CHAPTER 3 - TRANSPORTATION ASSET MANAGEMENT FRAMEWORK \& ELEMENT-BASED MULTI-OBJECTIVE OPTIMIZATION METHODOLOGY .................. 55

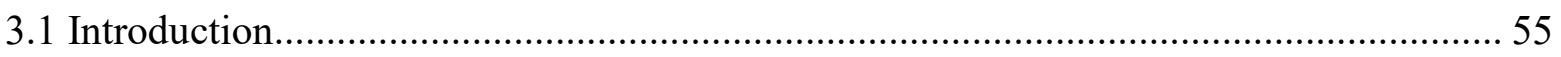

3.2 Proposed Transportation Asset Management Framework........................................... 57

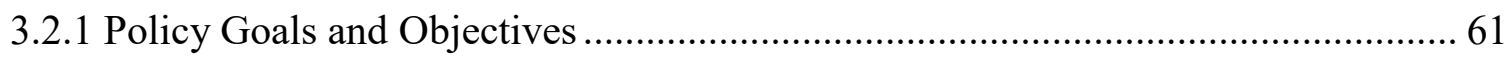

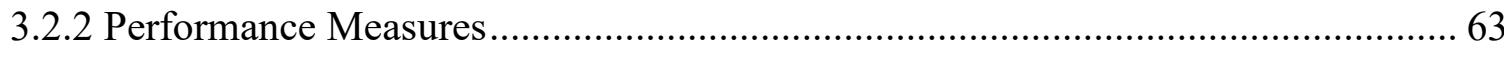

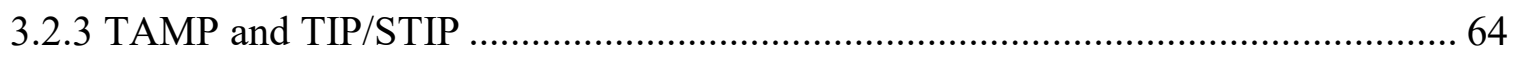

3.3 Proposed Element-Based Multi-Objective Methodology .......................................... 66

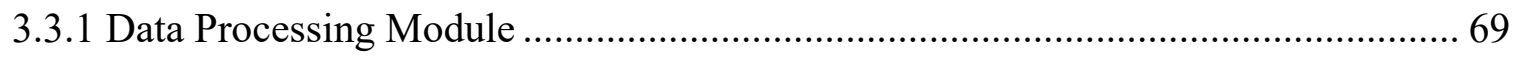

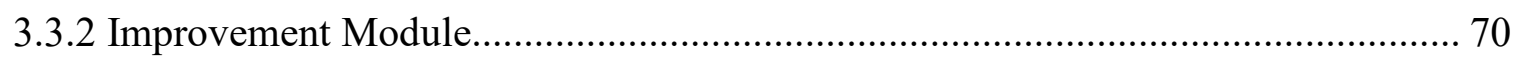

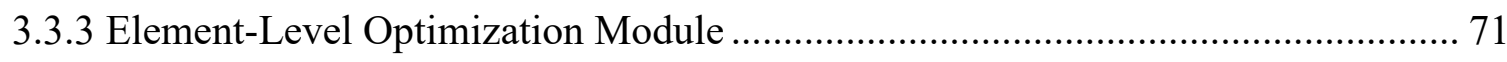

3.3.4 Bridge-Level Optimization Module............................................................... 72

3.3.5 Network-Level Optimization Module.............................................................. 73

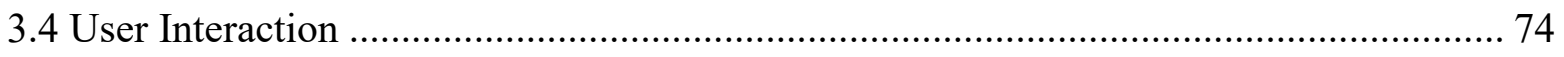

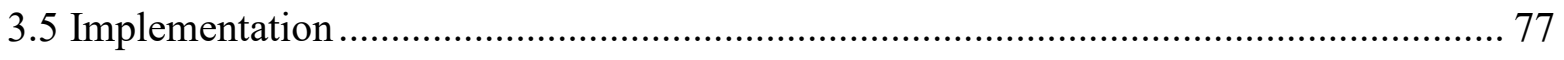

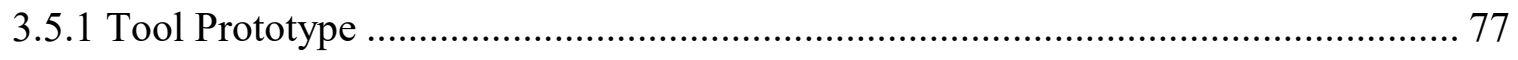

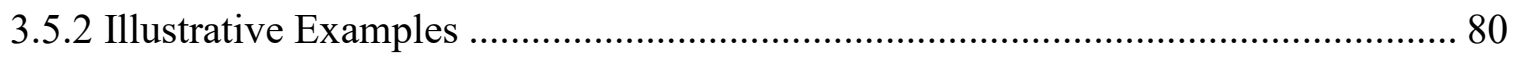

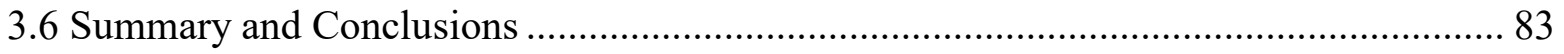

CHAPTER 4 — DATA PROCESSING MODULE........................................................ 85

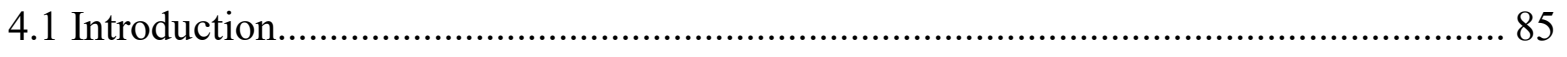

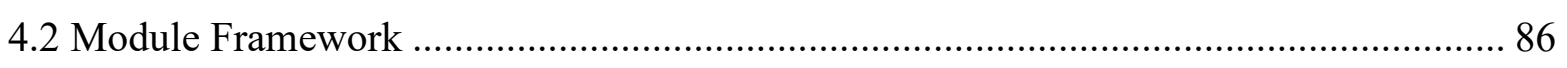

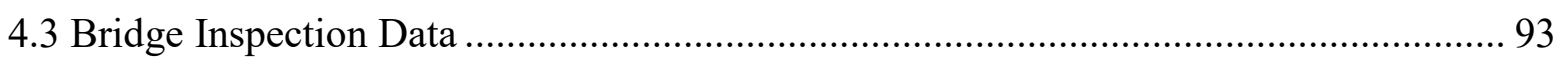

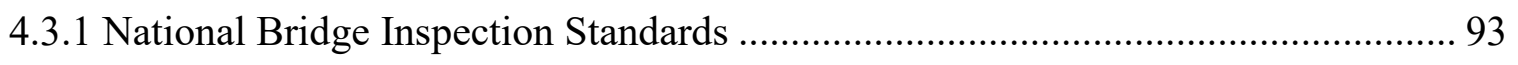

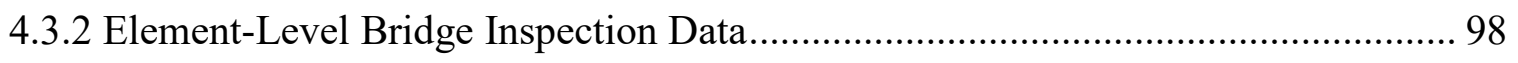

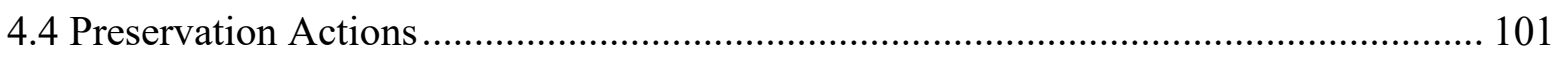

4.5 Element Preservation Action Costs ....................................................................... 102

4.6 Functional Improvement Action .......................................................................... 102

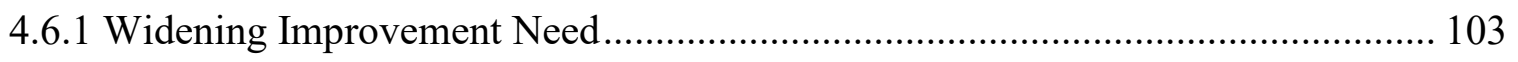

4.6.2 Raising Improvement Need ....................................................................... 104

4.6.3 Strengthening Improvement Need ................................................................ 104

4.6.4 Functional Improvement Cost........................................................................ 105

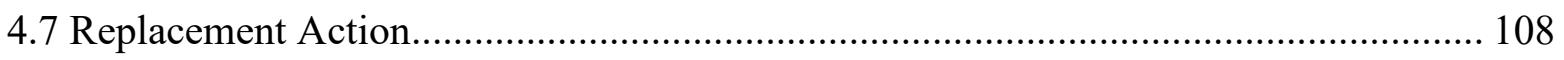

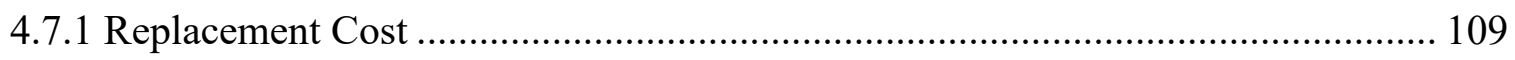

4.8 Bridge User Cost............................................................................................... 110 


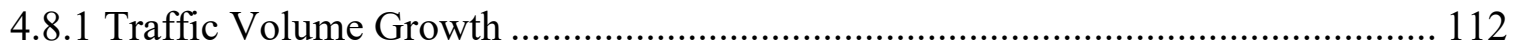

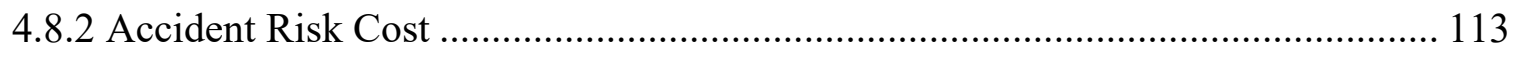

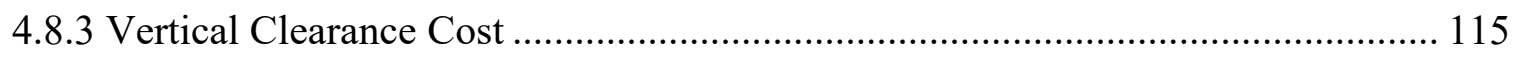

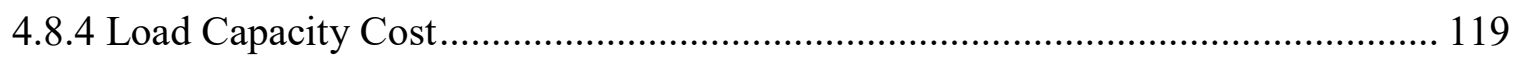

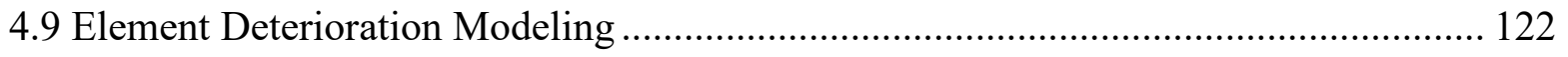

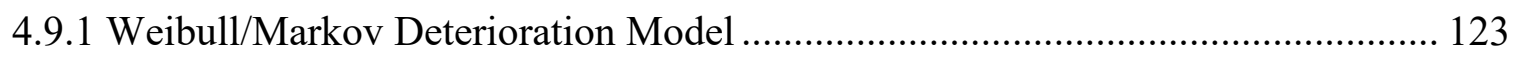

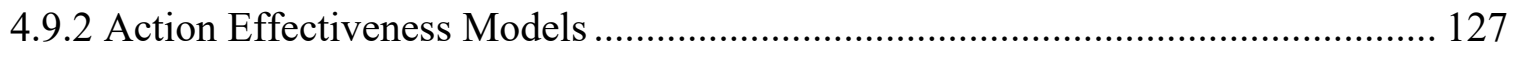

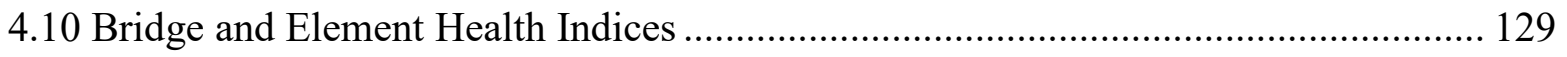

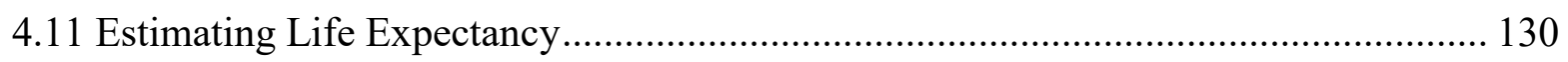

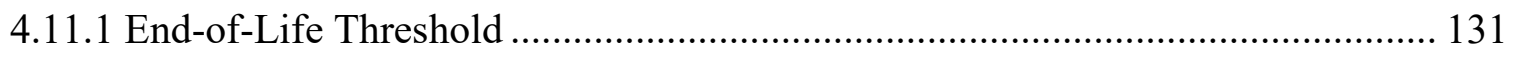

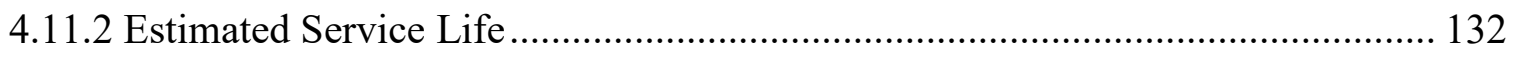

4.11.3 Estimated Remaining Service Life ............................................................ 133

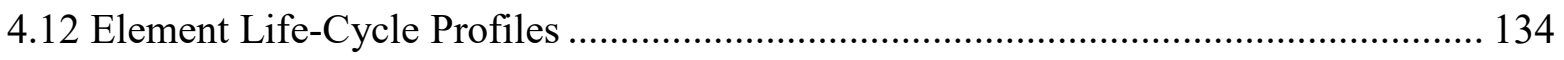

4.12.1 Do-Nothing Life-Cycle Profile ...................................................................... 134

4.12.2 Replacement Only Life-Cycle Profile ………………...................................... 136

4.12.3 Action Effectiveness Life-Cycle Profile.............................................................. 138

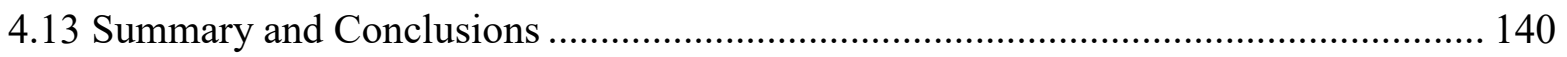

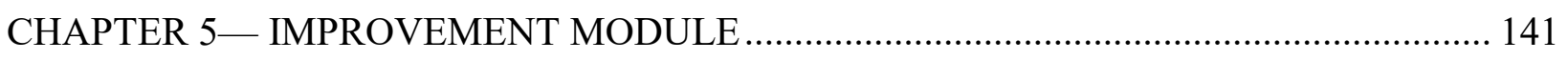

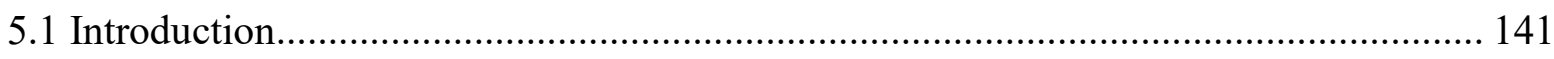

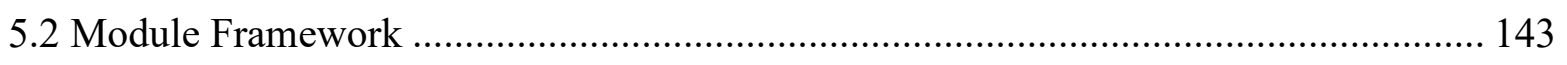

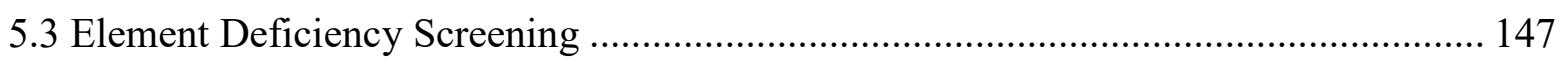

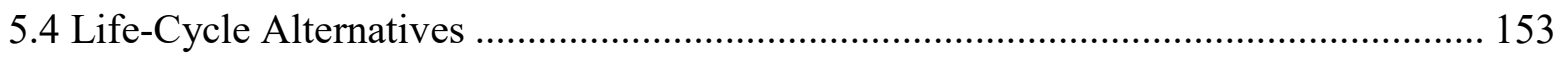

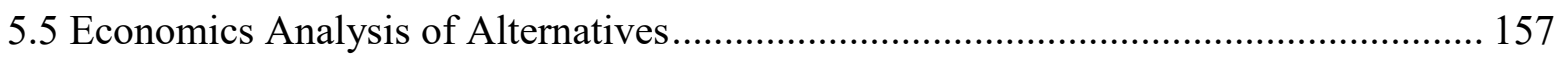

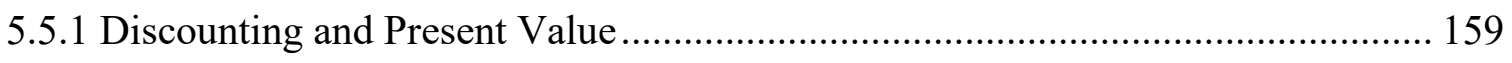

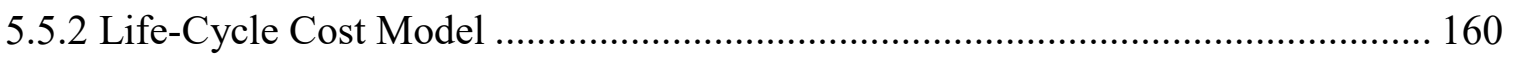

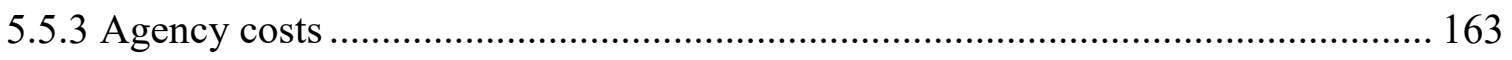

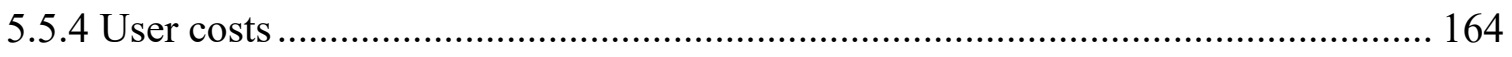

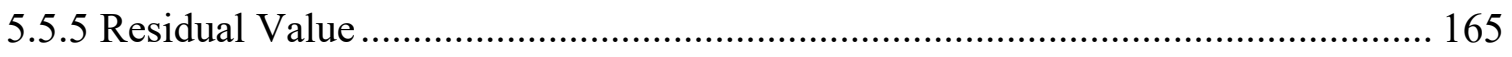

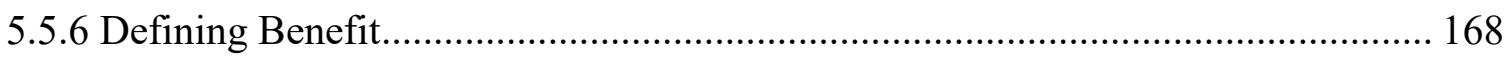

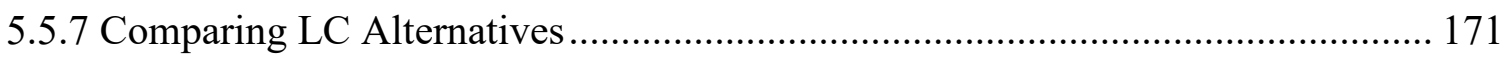

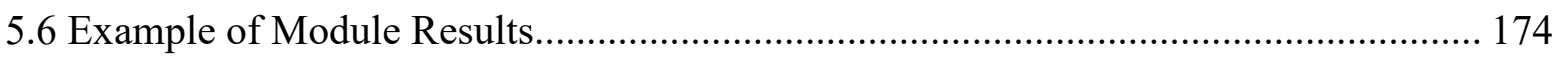

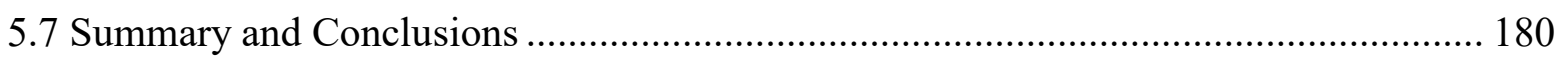




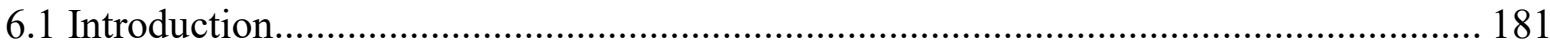

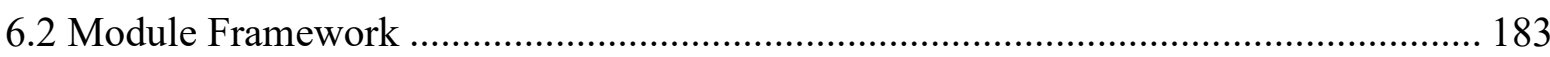

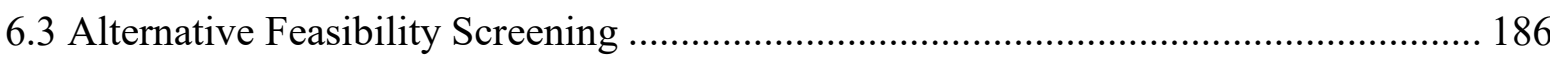

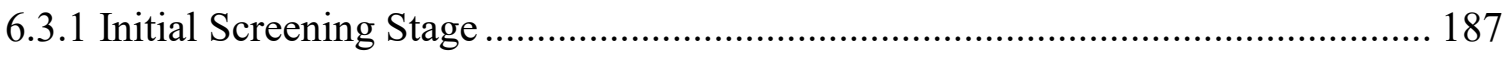

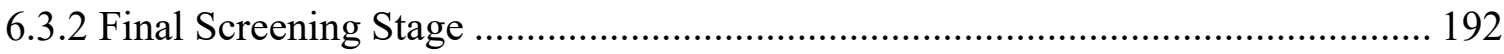

6.4 Element-Level Optimization Problem Formulation ...................................................... 197

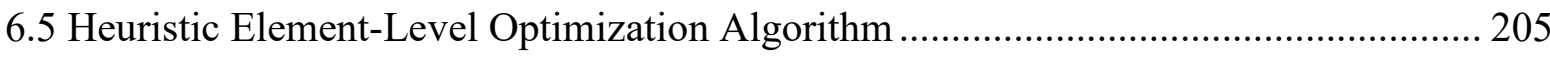

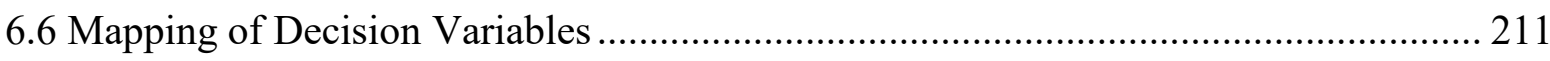

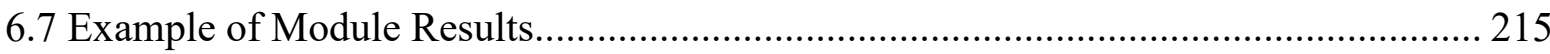

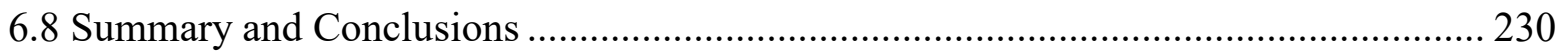

CHAPTER 7-BRIDGE-LEVEL OPTIMIZATION MODULE ........................................... 232

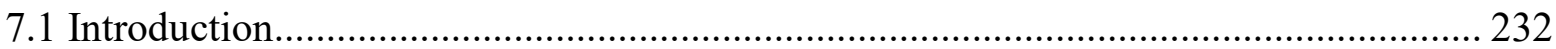

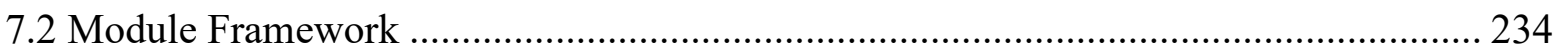

7.3 Bridge-Level Optimization Problem Formulation........................................................ 237

7.4 Heuristic Bridge-Level Optimization Algorithm...................................................... 244

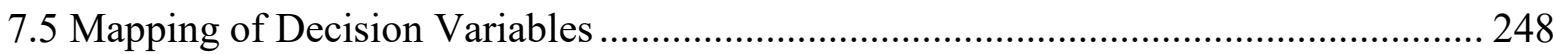

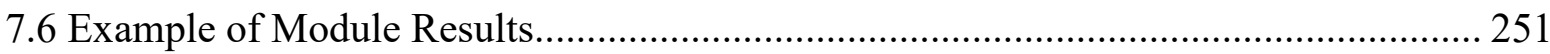

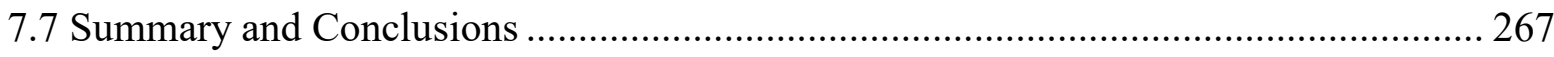

CHAPTER 8 - NETWORK-LEVEL OPTIMIZATION MODULE ………………………....... 269

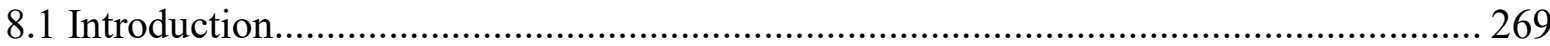

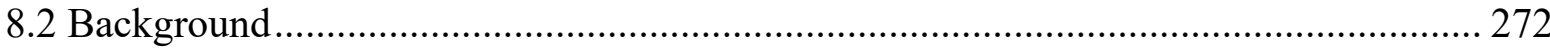

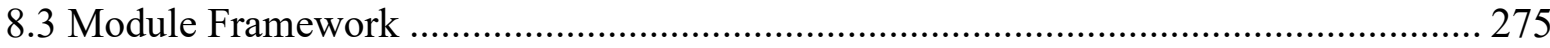

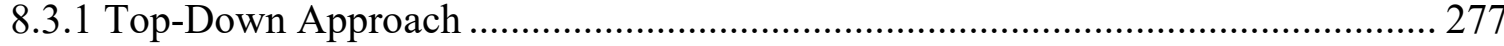

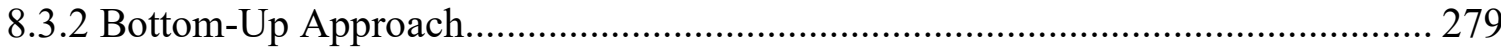

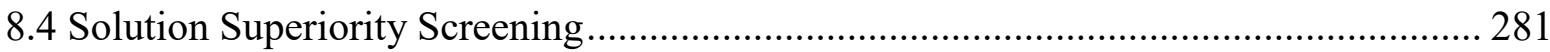

8.5 Network-Level Optimization Problem Formulations .................................................... 284

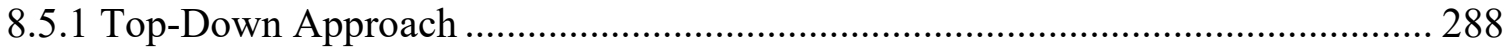

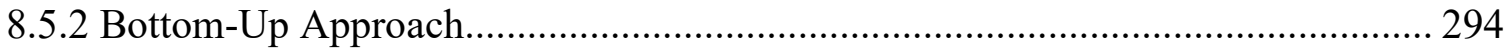

8.6 Heuristic Network-Level Optimization Algorithms .................................................... 298

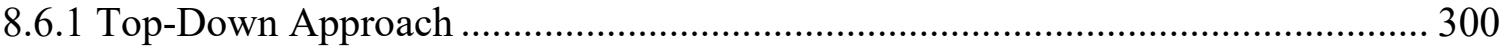

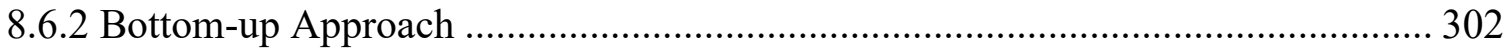

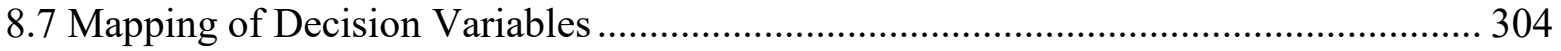




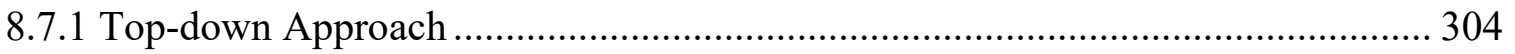

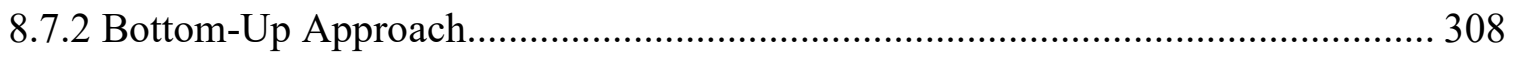

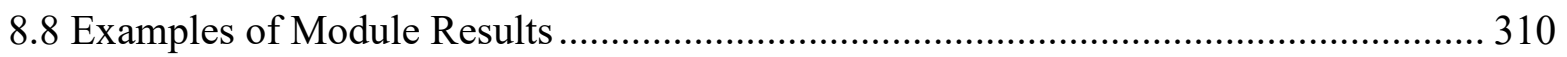

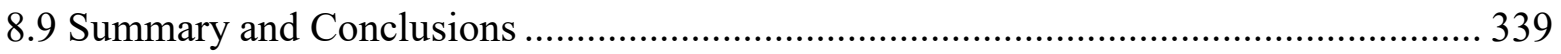

CHAPTER 9-CONCLUSIONS, CONTRIBUTIONS AND RECOMMENDATIONS ......... 342

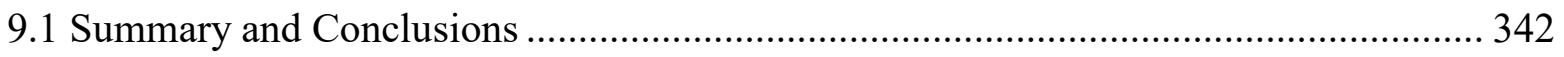

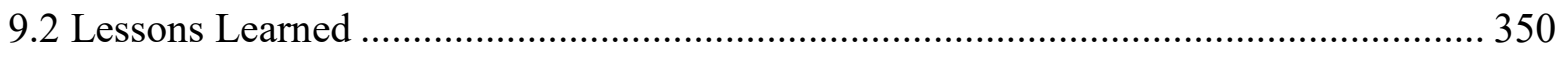

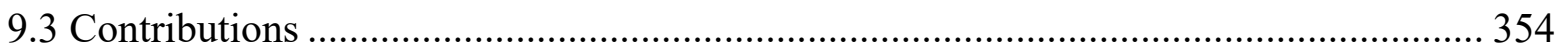

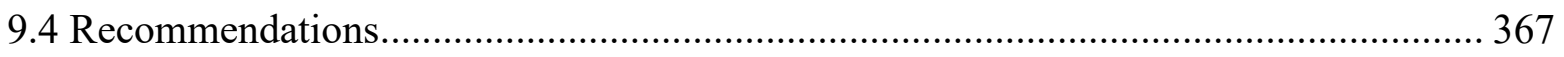

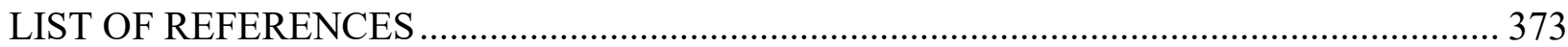




\section{LIST OF TABLES}

Table 1.1 Activities associated with the research tasks ............................................................ 8

Table 2.1 Significant references identified in the literature review........................................... 17

Table 2.2 Example of relative weights: individual performance measures ................................... 41

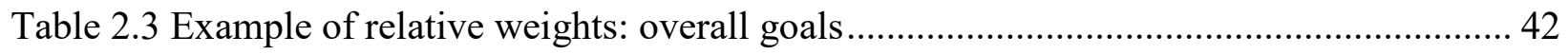

Table 2.4 Advantages and disadvantages of well-known GAs.................................................. 51

Table 3.1 NYSDOT's pavement and bridge performance targets and gaps................................... 63

Table 3.2 Inspection data, major attributes and characteristics of each sample bridge in the

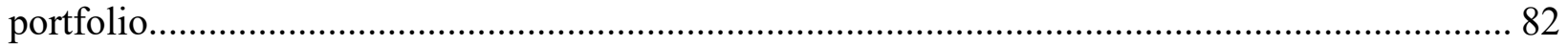

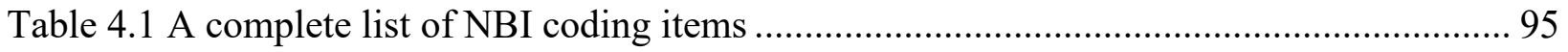

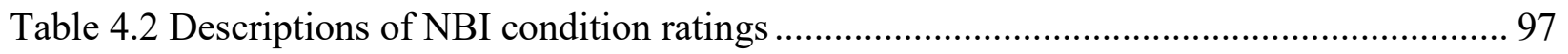

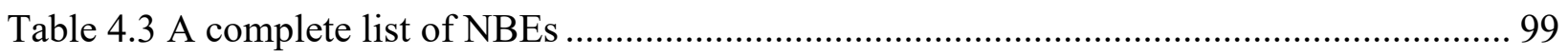

Table 4.4 Condition state definitions for Element 12-Deck Reinforced Concrete .................. 100

Table 4.5 Example of condition state quantities assigned to Element 107-Steel Open

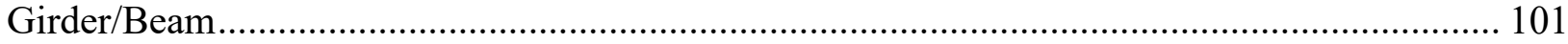

Table 4.6 Example of the feasible treatments assigned to Element 207-Steel Column Tower

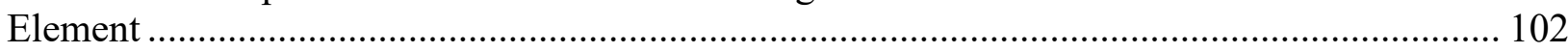

Table 4.7 Example of a matrix of transition probabilities ..................................................... 125

Table 4.8 Example of AE models for Element 207-Steel Column Tower............................... 128

Table 4.9 Example of ESL calculations for Element 110 (ESL=75 years) ............................... 133

Table 5.1 Preservation action triggers used by MnDOT …………………………………..... 148

Table 5.2 Example of preservation only screening results for all program years....................... 151

Table 5.3 Example of post-major screening results for program year 4 .................................... 151

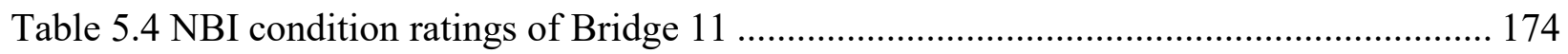

Table 5.5 Condition states of the 12 elements of Bridge 11 .................................................. 175

Table 5.6 Module LC alternative results produced using the tool prototype for Bridge 11 ....... 176

Table 6.1 Representation of the 3125 MRR LC alternatives (with an initial intervention in program year 4) generated by the improvement module for Element $226 \ldots \ldots \ldots \ldots \ldots \ldots \ldots \ldots \ldots \ldots \ldots . . . . . . . . . . . . . .190$

Table 6.2 Representation of the 644 feasible MRR LC alternatives (with an initial intervention in program year 4) identified through the initial screening stage for Element 226. 191

Table 6.3 Representation of the best feasible 86 MRR LC alternatives (with an initial intervention in program year 4) identified through the final screening stage for Element 226.. 194 Table 6.4 Example of input matrices for use in the ELO process ............................................. 196 


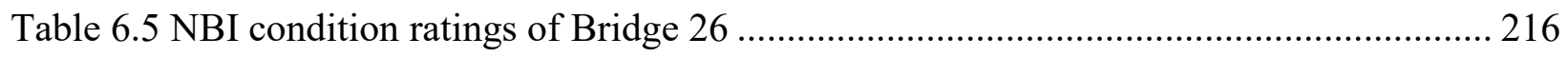

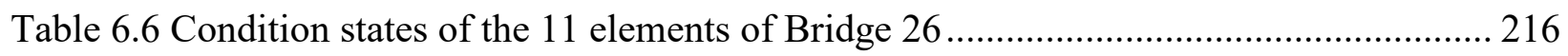

Table 6.7 Defining the different ELO problems of the example .............................................. 217

Table 6.8 Results associated with the 41 solutions obtained for the 2-objective ELO problem,

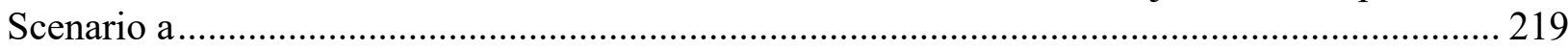

Table 6.9 Results associated with the 2-objective ELO solutions producing the least bridge LCC values for the three improvement types

Table 6.10 Element improvement actions associated with the 2-objective ELO solutions producing the least bridge LCC values for the three improvement types. 228

Table 6.11 Feasible preservation treatments associated with the improvement actions recommended by the 2-objective ELO solutions producing the least bridge LCC values for the three improvement types. 229

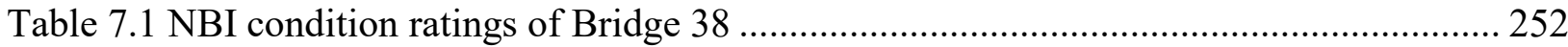

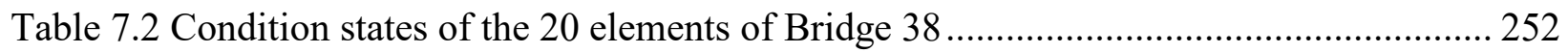

Table 7.3 Defining the different BLO problems of the example .............................................. 253

Table 7.4 Results associated with the 35 solutions obtained for the 2-objective BLO problem,

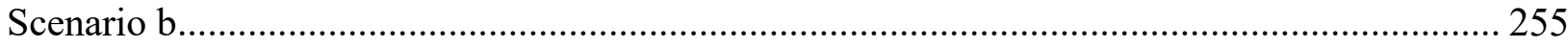

Table 7.5 Results associated with the solutions producing the least bridge LCC values for the

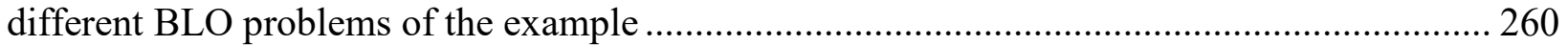

Table 7.6 Element improvement actions associated with the solutions producing the least bridge LCC values for the 2-objective BLO problems ..................................................................... 264

Table 7.7 Feasible preservation treatments associated with the improvement actions recommended by the solutions producing the least bridge LCC values for the 2-objective BLO

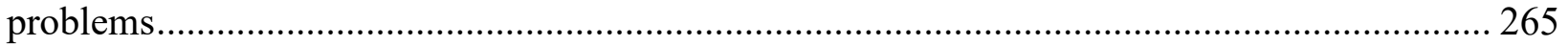

Table 8.1 Example of MKP with 10 objects and two knapsacks............................................. 287

Table 8.2 Obtained Pareto solution set for the MKP example defined in Table 8.1 .................. 287

Table 8.3 Defining the different NLO problems of the examples under Case A ........................ 313

Table 8.4 Defining the different NLO problems of the examples under Case B........................ 314

Table 8.5 Results produced by the solutions with the maximum network LCC benefits for the 3objective NLO problems ................................................................................................. 321

Table 8.6 Initial investment distributions over the 10 -year program period for the 3 -objective

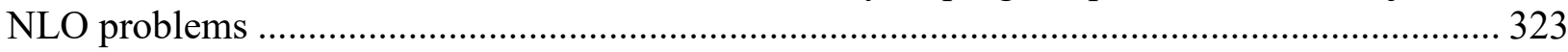

Table 8.7 Results produced by the NLO solutions recommended for Example 3, Scenario 2a,

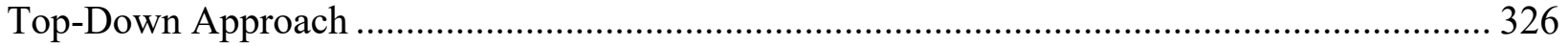

Table 8.8 Bridge-level results of the NLO solution with the maximum network LCC benefit for Example 3, Scenario 2a, Top-Down Approach 329 
Table 8.9 LC alternatives recommended by NLO Solution 3 with the maximum network LCC benefit for Example 3, Scenario 2a, Top-Down Approach ………………………………...... 333

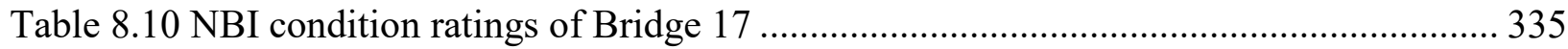

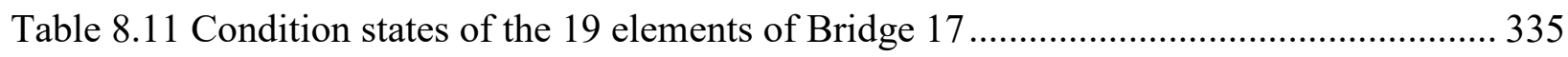

Table 8.12 LC alternatives recommended by NLO Solution 3 for Bridge 17 .......................... 336

Table 8.13 Feasible preservation treatments associated with the improvement actions recommended by NLO Solution 3 for Bridge 17 ................................................................. 337 


\section{LIST OF FIGURES}

Figure 2.1 Performance-based planning framework. Adapted from Performance-Based Planning and Programming Guidebook (Grant, D’Ignazio, Bond, and McKeeman, 2013).................... 28

Figure 2.2 MnDOT's bridge risk assessment quantifying risks, impacts, and consequences. Adapted from the NCHRP Report 706: Uses of Risk Management and Data Management to Support Target-setting for Performance-based Resource Allocation by Transportation Agencies (Cambridge Systematics, Inc., 2011).

Figure 2.3 Classification of MOO methods. Redrawn from the original in Trade-Off Analysis in Multi-Objective Optimization for Transportation Asset Management (Bai, 2012). .................. 36

Figure 2.4 Schematic of a preference-based MOO approach. Redrawn from the original in MultiObjective Optimization Using Evolutionary Algorithms (Deb, 2001).................................... 38

Figure 2.5 Schematic of an ideal MOO approach. Redrawn from the original in Multi-Objective Optimization Using Evolutionary Algorithms (Deb, 2001).

Figure 2.6 Example of a trade-off analysis. Adapted from the NCHRP Report 806: Cross-Asset Resource Allocation and the Impact on Transportation System Performance (Maggiore \& Ford, 2016).

Figure 2.7. Front-end interface of the MOOS network-level model. Adapted from the NCHRP Report 590: Multi-Objective Optimization for Bridge Management Systems (Patidar et al., 2007).

Figure 2.8. Example of program results as displayed in BrM version 5.2.3. Adapted from AASHTOWare Bridge Management 5.2.3.: Conducting Trade-off Analysis (Boyle, 2017)...... 46

Figure 2.9 The different families of optimization models. Redrawn from the original in Metaheuristics: From Design to Implementation (Talbi, 2009).

Figure 2.10 The different classifications of optimization algorithms. Redrawn from the original in Metaheuristics: From Design to Implementation (Talbi, 2009).

Figure 2.11 A flowchart of the working principle of a GA. Redrawn form the original in Multi-

Objective Optimization Using Evolutionary Algorithms (Deb, 2009).

Figure 3.1 Asset management framework recommended by the Guide. Redrawn from the original in the Transportation Asset Management Guide (Cambridge Systematics, Inc. et al., 2002).

Figure 3.2 The proposed goal-driven TAM framework

Figure 3.3 Hierarchy of Caltrans' objectives. Redrawn from the original in the SHOPP Report Phase 2: Application of a Project Prioritization Framework to the 2016 SHOPP (Caltrans, 2016).

Figure 3.4 CDOT's historical budget levels by asset class. Adapted from the 2013 CDOT's RiskBased Asset Management Plan (Cambridge Systematics, Inc., 2013). .................................. 65

Figure 3.5 The proposed EB-MOO methodology flow diagram .......................................... 67

Figure 3.6 Hierarchical structure of the MATLAB-based tool prototype ................................ 78 
Figure 3.7 Example of results as displayed in the MATLAB-based tool prototype. 79

Figure 4.1 Data processing module framework.

Figure 4.2 Truck height histogram for Florida's Interstate roadways. Adapted from Project Planning Models for Florida's Bridge Management System (Sobanjo \& Thompson, 2004).... 118

Figure 4.3 Truck height histogram for Florida's Non-Interstate roadways. Adapted from Project Planning Models for Florida's Bridge Management System (Sobanjo \& Thompson, 2004). ... 118

Figure 4.4 Truck weight histogram for Florida's Interstate roadways. Adapted from Project Planning Models for Florida's Bridge Management System (Sobanjo \& Thompson, 2004).... 121

Figure 4.5 Truck weight histogram for Florida's Non-Interstate roadways. Adapted from Project Planning Models for Florida's Bridge Management System (Sobanjo \& Thompson, 2004).... 121

Figure 4.6 Example of DN LC profile for Element 110_-Open Girder/Beam, Reinforced Concrete 135

Figure 4.7 Example of RO LC profile for Element 202-Column, Steel 137

Figure 4.8 Example of AE LC profile for Element 215-Abutment, Reinforced Concrete ...... 139

Figure 5.1 Improvement module framework..... 146

Figure 5.2 Bridge condition over time. Redrawn from the original in the FHWA's Bridge Preservation Guide (FHWA, 2018).

Figure 5.3 Example of element deficiency screening for (a) preservation only; (b) post-major improvement 150

Figure 5.4 Example of predicted health indices for (a) preservation needs; (b) preservation needs succeeding a major bridge improvement in program year 4 152

Figure 5.5 Simulation of (a) MRR LC Alternative 2593 (action path 4-0-3-3-2); (b) FCI LC Alternative 357 (action path 5-2-4-1-1). 156

Figure 5.6 Example of LC profile showing different LCCs and residual values for (a) MRR LC Alternative 2215; (b) FCI LC Alternative 28; (c) REP LC Alternative 54 ............................. 162

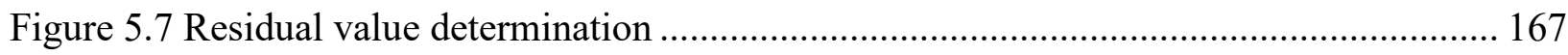

Figure 5.8 Example of baseline LC alternative profile of (a) DN; (b) RO ............................ 169

Figure 5.9 Health indices predicted for Bridge 11 under (a) DN LC Alternative; (b) Combination of MRR LC Alternatives; (c) RO LC Alternative (d); Combination of FCI LC Alternatives; (e) FCI/REP LC Alternative 1; (f) Combination of REP LC Alternatives ................................... 179

Figure 6.1 Element-level optimization module framework ........................................... 185

Figure 6.2 Illustration of the initial stage of the alternative feasibility screening process ......... 189

Figure 6.3 Illustration of the final stage of the alternative feasibility screening process .......... 193

Figure 6.4 Heuristic element-level optimization algorithm .............................................. 210

Figure 6.5 Illustration of the chromosome encoding of an ELO solution ............................... 214 
Figure 6.6 Obtained solutions for the 2-objective ELO problems under (a) Scenario a, (b)

Scenario b, (c) Scenario c; the 3-objective ELO problems under (d) Scenario a, (e) Scenario b, (f) Scenario c; the 7-objective ELO problems under (g) Scenario a, (h) Scenario b, (k) Scenario c

Figure 6.7 Predicted bridge health indices for the 2-objective ELO problems under (a) Scenario a, (b) Scenario b, (c) Scenario c; the 3-objective ELO problems under (d) Scenario a, (e) Scenario b, (f) Scenario c; the 7-objective ELO problems under (g) Scenario a, (h) Scenario b, (k) Scenario c. 223

Figure 6.8 Predicted health indices associated with the solutions producing the least bridge LCC values for the 2-objective ELO problems under (a) Scenario a, (b) Scenario b, (c) Scenario c; the 3-objective ELO problems under (d) Scenario a, (e) Scenario b, (f) Scenario c; the 7-objective ELO problems under (g) Scenario a, (h) Scenario b, (k) Scenario c. Predicted health indices under (l) DN LC Alternative; (m) RO LC Alternative; (n) FCI/REP LC Alternative 1............ 226

Figure 7.1 Bridge-level optimization module framework ................................................ 236

Figure 7.2 Heuristic bridge-level optimization algorithm .............................................. 247

Figure 7.3 Illustration of the chromosome encoding of a BLO solution ................................ 250

Figure 7.4 Obtained solutions for the 2-objective BLO problems under (a) Scenario a, (b) Scenario b; the 3-objective BLO problems under (c) Scenario a, (d) Scenario b; the 7-objective BLO problems under (e) Scenario a, (f) Scenario b 257

Figure 7.5 Predicted bridge health indices for the 2-objective BLO problems under (a) Scenario a, (b) Scenario b; the 3-objective BLO problems under (c) Scenario a, (d) Scenario b; the 7objective BLO problems under (e) Scenario a, (f) Scenario b..... 259

Figure 7.6 Predicted health indices associated with the solutions producing the least bridge LCC values for the 2-objective BLO problems under (a) Scenario a, (b) Scenario b; the 3-objective BLO problems under (c) Scenario a, (d) Scenario b; the 7-objective BLO problems under (e) Scenario a, (f) Scenario b. Predicted health indices under (g) DN LC Alternative; (h) RO LC Alternative 262

Figure 8.1 Network-level optimization module framework for the top-down approach........... 278

Figure 8.2 Network-level optimization module framework for the bottom-up approach ......... 280

Figure 8.3 Illustration of the solution superiority screening process .................................... 283

Figure 8.4 Heuristic network-level optimization algorithm for the top-down approach ........... 301

Figure 8.5 Heuristic network-level optimization algorithm for the bottom-up approach.......... 303

Figure 8.6 Illustration of the chromosome encoding of a top-down approach NLO solution.... 307

Figure 8.7 Illustration of the chromosome encoding of a bottom-up approach NLO solution .. 309

Figure 8.8 Obtained Pareto frontiers for the 2-objective NLO problems of (a) Example 1, (c) Example 5, (e) Example 6; the 3-objective NLO problems of (b) Example 3, (d) Example 7, (f)

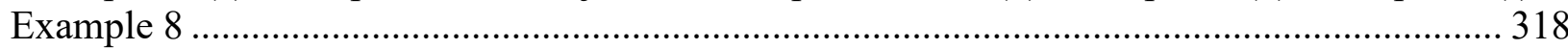

Figure 8.9 Network costs produced by the solutions with the maximum network LCC benefits for the 3-objective NLO problems (Table 8.5).... 322 
Figure 8.10 Network health indices produced by the solutions with the maximum network LCC benefits for the 3-objective NLO problems (Table 8.5) ................................................. 322

Figure 8.11 (a) Selected bridges; (b) Initial agency spending distribution over the 10-year program period for the 3-objective NLO problems (Table 8.6) 324

Figure 8.12 Annual initial agency costs per improvement type produced by the NLO solution with the maximum network LCC benefit for Example 3, Scenario 2a, Top-Down Approach .. 331

Figure 8.13 Network health indices produced by the NLO solutions recommended for Example 3, Scenario 2a, Top-Down Approach 331

Figure 8.14 Predicted health indices for Bridge 17 and all its elements under (a) LC alternatives recommended by NLO Solution 3; (b) DN LC Alternative; (c) RO LC Alternative 338 


\section{LIST OF ABBREVIATIONS}

Abbreviation

AASHTO

$\mathrm{AE}$

ASTM

BMS

$\mathrm{BrM}$

BLO

$\mathrm{BNT}_{\text {elm }}$

$\mathrm{BNT}_{\text {brg }}$

BCA

BLCCA

CFR

CDOT

Caltrans

CS

DOT

DN

EB-MOO

EA

ESL

ELO

FCI

FAST Act

Florida DOT

FTA

FHWA

GA

HPMS

HI
Description

American Association of State Highway and Transportation Officials Action Effectiveness

American Society for Testing and Materials

Bridge Management System

AASHTOWare Bridge Management Software

Bridge-Level Optimization

Element Life-Cycle Cost Benefit

Bridge Life-Cycle Cost Benefit

Benefit Cost Analysis

Bridge Life-Cycle Cost Analysis

Code of Federal Regulations

Colorado Department of Transportation

California Department of Transportation

Condition State

Department of Transportation

Do-Nothing

Element-Based Multi-Objective Optimization Methodology

Evolutionary Algorithm

Estimated Service Life

Element-Level Optimization

Functional Improvement

Fixing America's Surface Transportation Act

Florida Department of Transportation

Federal Transit Administration

Federal Highway Administration

Genetic Algorithm

Highway Performance Monitoring System

Health Index 


\begin{tabular}{|c|c|}
\hline IBC & Incremental Benefit-Cost \\
\hline IBCA & Incremental Benefit-Cost Analysis \\
\hline IAC & Initial Agency Cost \\
\hline $\mathrm{IAC}_{\mathrm{elm}}$ & Element Initial Agency Cost \\
\hline IAC $C_{b r g}$ & Bridge Initial Agency Cost \\
\hline IT & Information Technology \\
\hline LC & Life-Cycle \\
\hline $\mathrm{LCC}$ & Life-Cycle Cost \\
\hline LCCA & Life-Cycle Cost Analysis \\
\hline $\mathrm{LCC}_{\mathrm{brg}}$ & Bridge Life-Cycle Cost \\
\hline LCP & Life-Cycle Planning \\
\hline LRTP & Long-Range Transportation Plan \\
\hline LOS & Level of Service \\
\hline LTBP & Long-Term Bridge Performance \\
\hline MAP-21 & Moving Ahead for Progress in the 21 st Century Act \\
\hline MRR & Maintenance, Repair, and Rehabilitation \\
\hline MATLAB & Matrix Laboratory \\
\hline MCDM & Multi-Criteria Decision-Making \\
\hline $\mathrm{MOO}$ & Multi-Objective Optimization \\
\hline MODA & Multi-Objective Decision Analysis \\
\hline MPO & Metropolitan Planning Organization \\
\hline MOEA & Multi-Objective Evolutionary Algorithm \\
\hline MTP & Metropolitan Transportation Plan \\
\hline MnDOT & Minnesota Department of Transportation \\
\hline MOOS & Multi-Objective Optimization System \\
\hline $\mathrm{MIC}_{\mathrm{brg}}$ & Bridge Major Improvement Cost \\
\hline MOGA & Multi-Objectives Genetic Algorithm \\
\hline NSGA-II & Non-dominated Sorting Genetic Algorithm-II \\
\hline MCKP & Multi-Choice Knapsack Problem \\
\hline MCMDKP & Multi-Choice Multi-Dimensional Knapsack Problem \\
\hline MKP & Multiple Knapsack Problem \\
\hline
\end{tabular}


NCHRP National Cooperative Highway Research Program

NHS National Highway System

NBIAS National Bridge Investment Analysis System

NAT Network Analysis Tool

NYSDOT New York State Department of Transportation

NBIS National Bridge Inspection Standards

NLO Network-Level Optimization

NBI National Bridge Inventory

NHS National Highway System

NBE National Bridge Element

NP-hard Non-Deterministic Polynomial-Time Hardness

PLAT Project Level Analysis Tool

$\mathrm{PAC}_{\mathrm{elm}} \quad$ Element Preservation Action Cost

PLCC $_{\text {elm }} \quad$ Element Preservation Life-Cycle Cost

SPEA-2 Strength Pareto Evolutionary Algorithm 2

PESA Pareto Envelope-based Selection Algorithm

REP Replacement

RO Replacement Only

RSL Remaining Service Life

$\mathrm{RV}_{\text {elm }} \quad$ Element Residual Value

RV Residual Value

State DOT State Department of Transportation

SHOPP State Highway Operation and Protection Program

TIP/STIP Transportation Improvement Program/Statewide Transportation

Improvement Program

TAMP Transportation Asset Management Plan

TAM Transportation Asset Management

TRB Transportation Research Board

USC $_{\text {brg }} \quad$ Bridge User Cost

USC United States Code 


\begin{abstract}
The Moving Ahead for Progress in the 21st Century Act (MAP-21) mandates the development of a risk-based transportation asset management plan and use of a performance-based approach in transportation planning and programming. This research introduces a systematic element-based multi-objective optimization (EB-MOO) methodology integrated into a goal-driven transportation asset management framework to

(1) improve bridge management,

(2) support state departments of transportation with their transition efforts to comply with the MAP-21 requirements,

(3) determine short- and long-term intervention strategies and funding requirements, and

(4) facilitate trade-offs between funding levels and performance.
\end{abstract}

The proposed methodology focuses on one transportation asset class (i.e., bridge) and is structured around the following five modules:

1. Data Processing Module,

2. Improvement Module,

3. Element-level Optimization Module,

4. Bridge-level Optimization Module, and

5. Network-level Optimization Module.

To overcome computer memory and processing time limitations, the methodology relies on the following three distinct screening processes:

1. Element Deficiency Process, 
2. Alternative Feasibility Process, and

3. Solution Superiority Screening Process.

The methodology deploys an independent deterioration model (i.e., Weibull/Markov model), to predict performance, and a life-cycle cost model, to estimate life-cycle costs and benefits. Lifecycle (LC) alternatives (series of element improvement actions) are generated based on a new simulation arrangement for three distinct improvement types:

1. maintenance, repair and rehabilitation (preservation);

2. functional improvement; and

3. replacement.

A LC activity profile is constructed separately for each LC alternative action path. The methodology consists of three levels of optimization assessment based on the Pareto optimality concept:

(1) an element-level optimization, to identify optimal or near-optimal element intervention actions for each deficient element (in a poor condition state) of a candidate bridge;

(2) a bridge-level optimization, to identify combinations of optimal or near-optimal element intervention actions for a candidate bridge; and

(3) a network-level optimization, following either a top-down or bottom-up approach, to identify sets of optimal or near-optimal element intervention actions for a network of bridges.

A robust metaheuristic genetic algorithm (i.e., Non-dominated Sorting Genetic Algorithm II, [NSGA-II]) is deployed to handle the large-sized multi-objective optimization problems. A MATLAB-based tool prototype was developed to test concepts, demonstrate effectiveness, and 
communicate benefits. Several examples of unconstrained and constrained scenarios were established for implementing the methodology using the tool prototype.

Results reveal the capability of the proposed EB-MOO methodology to generate a high quality of Pareto optimal or near-optimal solutions, predict performance, and determine appropriate intervention actions and funding requirements. The five modules collectively provide a systematic process for the development and evaluation of improvement programs and transportation plans. Trade-offs between Pareto optimal or near-optimal solutions facilitate identifying best investment strategies that address short- and long-term goals and objective priorities. 


\section{CHAPTER 1-INTRODUCTION}

\subsection{Path Leading to the Proposed Research}

Managing and maintaining the nation's transportation infrastructure has always been a challenge for transportation agencies as funding resources continue to diminish with increased public demands and expectations. Ensuring safety, serviceability and reliability of highway bridges in the United States has always been a priority. Bridges are one of the most visible and essential components of the transportation system. For instance, collapse of a bridge due to lack of maintenance could cause loss of lives and impact the regional transportation network and economy.

State departments of transportation (state DOTs) select projects from their transportation improvement program/statewide transportation improvement program (TIP/STIP) documentsdrawn from and consistent with their long-range transportation plans (LRTPs)—based on specified prioritizations and resource allocations. Usually, allocations and prioritizations in the TIP/STIP documents are driven by historical precedents, funding restrictions, and investment needs determined by either state asset management systems, leadership discretions, political considerations, or priorities relative to state DOTs (Maggiore \& Ford, 2016).

Many state DOTs limit application of their bridge management systems (BMSs) to just monitoring conditions, identifying maintenance activities, and programming potential projects on a "worstfirst" basis. Advanced capabilities of BMSs (such as optimization, deterioration modeling, tradeoff analysis, life-cycle cost analysis, and cost-benefit analysis) are not generally being utilized to 
support setting policies and program priorities, and recommending candidate projects and preservation treatments that support achievement of goals and objectives established in the LRTP (Cambridge Systematic 2009). The link between the LRTP and actual programmed projects and resource allocations in the TIP/STIP will remain elusive if existing decision-making environment and business practices persist (Federal Highway Administration [FHWA], 1999).

The Moving Ahead for Progress in the 21st Century Act (MAP-21) was enacted in 2012 to fortify this linkage, address needs, improve existing practices, and provide a new way of management and doing business. MAP-21 introduced a new decision-making environment by increasing emphasis on performance management, including new national performance measures, mandating the development of a risk-based transportation asset management plan (TAMP), and requiring the use of a performance-based approach in transportation planning and programming to support national goals and improve accountability and transparency. MAP-21 established new national goals to address the many challenges facing the nation transportation system. Challenges include improving safety, reliability and efficiency of the system and freight movement while protecting the environment, maintaining infrastructure condition in a "state of good repair," reducing traffic congestion, and streamlining project development and delivery process. The Fixing America's Surface Transportation Act (FAST Act) was enacted in 2015 and continued MAP-21's overall performance management and performance-based planning and programming.

To implement the new performance provisions of MAP-21 and FAST Act, several rulemakings were developed in multiple phases by FHWA and finalized within the past years establishing regulations for compliance. The final rules established national performance measures within specific areas and mandated use of measures in the performance-based planning and programming process. A performance-based LRTP provides enough details, criteria and strategies to support 
investment decision making and identify TIP/STIP projects — contributing to the established goals, objectives and targets. The final rules structured the mandated TAMP to include objectives and measures, pavement and bridge conditions, performance gaps, investment strategies leading to achievement of performance targets, risk management analysis, life-cycle planning process, and financial plan. In addition, states DOTs are required to integrate their TAMPs into their statewide transportation planning process.

To satisfy these performance measurement final rules, state DOTs started developing goals, objectives, performance measures, and targets as guiding criteria to drive the overall new decisionmaking environment throughout all stages of project and program development—-from the longrange planning to implementation and delivery. Efforts are increasing to support the transition, especially after the mandate TAMP development: (1) developing frameworks allowing the integration of these criteria into existing transportation asset management (TAM) practices, and (2) providing decision makers with useful procedures, methodologies, set of data, techniques, and analytical tools are also increasing.

As part of such frameworks, methodologies based on multi-criteria decision-making (MCDM) or multi-objective optimization (MOO) became apparent to support the prioritization and resource allocation efforts. For instance, in 2014, the California DOT (Caltrans) developed a multi-objective decision analysis (MODA) tool (Caltrans, 2016) using a compensatory technique (based on multiattribute value theory). The MODA tool applies a value function to identify an optimized portfolio of projects from a pool of candidate projects that align with the agency's goals and objectives. The tool was utilized in the development process of Caltrans' TAMP. 
This research introduces a systematic bridge element-based multi-objective optimization (EBMOO) methodology integrated into a goal-driven TAM framework to

(1) improve bridge management,

(2) support state DOTs with their transition efforts to comply with the MAP-21 requirements,

(3) determine short- and long-term intervention strategies and funding requirements, and

(4) facilitate trade-offs between funding levels and performance.

The proposed methodology focuses on one transportation asset class (i.e., bridge). It is structured around the following five modules:

1. Data Processing Module

2. Improvement Module

3. Element-level Optimization Module

4. Bridge-level Optimization Module

5. Network-level Optimization Module

\subsection{Research Objectives}

The main objectives of this research can be summarized as follows:

\section{Conducting a Literature Review}

- Conduct a comprehensive literature review to identify relevant studies and best practices to support the research objectives, refine the focus, and ensure no duplication of efforts

- Explore different investment decision-making and project prioritization methods used by state DOTs and other transportation agencies and recognize their capabilities and limitations 


\section{Proposing a Goal-Driven TAM Framework}

- Transfer previous work on TAM and best practices to refine the conceptual TAM framework introduced in the research proposal

- Discuss the different steps of the proposed framework including its benefits and practical challenges

- Identify future research aiming to expand the proposed framework capabilities to accommodate other transportation asset classes or modes

Proposing an EB-MOO Methodology

- Examine various optimization techniques, analytical and decision support tools, forecasting and cost models, and MCDM methods used by state DOTs and other transportation agencies

- Incorporate previous work on bridge decision making involving multiple criteria or objectives into the proposed MOO methodology_permitting decision makers to transfer preferred preferences and decision criteria or objectives

- Develop a systematic EB-MOO methodology based on a quantitative process able to identify optimal or near-optimal intervention actions and funding needs - supporting the development of short- and long-term investment strategies, and trade-offs between investment levels and performance

- Develop a flexible EB-MOO methodology able to support the TIP/STIP development and amendment process - by identifying candidate bridge projects, setting program and project priorities, and assessing impacts of programmed types of work (i.e., preservation, rehabilitation and replacement) or funding allocations 
- Develop an EB-MOO methodology that consists of three levels of optimization assessment:

(1) an element-level optimization (ELO), to identify optimal or near-optimal element intervention actions for each deficient element (in a poor condition state) of a candidate bridge;

(2) a bridge-level optimization (BLO), to identify combinations of optimal or nearoptimal element intervention actions for a candidate bridge; and

(3) a network-level optimization (NLO), following either a top-down or bottom-up approach, to identify sets of optimal or near-optimal element intervention actions for a network of bridges.

\section{Implementation of the Proposed EB-MOO Methodology using a Tool Prototype}

- Develop a MATLAB-based tool prototype structured around the proposed EB-MOO methodology

- Develop a tool prototype that considers decision makers' preferences, predicts performance, identifies optimal or near-optimal element intervention actions, and determines funding requirements

- Develop a tool prototype able to accommodate user-specified measures (objectives), inputs and preferences, and commonly collected data from widely-used state and national management systems

- Implement the different concepts of the proposed EB-MOO methodology through examples of unconstrained and constrained (budget and performance) scenarios using the developed tool prototype to test/validate, prove effectiveness and demonstrate and communicate potential benefits 
- Assess the ability of the tool prototype to recognize short- and long-term investment needs by examining the recommended intervention strategies, determined funding requirements, and predicted performance.

\subsection{Research Approach}

The research approach consists of five main tasks to accomplish. Table 1.1 lists the activities associated with each task. The following are brief discussions of these five tasks:

\section{Task 1: Conducting a Literature Review}

Task 1 of this research consists of conducting a comprehensive review of literature across multiple resources (such as search engines, databases, specific procedures and publications by transportation agencies, and library catalogs) to fully explore the availability of work related to the research areas and objectives, and identify state-of-the-art studies and best practices.

\section{Task 2: Proposing a Goal-Driven Transportation Asset Management Framework}

Task 2 further explores and refines the conceptual TAM framework introduced in the research proposal-transferring previous work on TAM and best practices identified throughout the literature review under Task 1.

\section{Task 3: Proposing an Element-Based Multi-Objective Methodology}

The intent of Task 3 is to transfer previous work (recognized throughout the literature review) on bridge decision making involving multiple criteria or objectives to develop a systematic EB-MOO methodology able to identify optimal or near-optimal intervention actions and funding needs, support the development of short- and long-term investment strategies, and facilitate trade-offs between investment levels and performance. 


\section{Task 4: Development of a Tool Prototype}

The development of a tool prototype falls under Task 4; it's primarily for ease of use and to implement the proposed EB-MOO methodology. The objective is to design a tool prototype that consists of the five proposed EB-MOO methodology modules (detailed in the subsequent chapters), considers decision makers' preferences, predicts performance, identifies optimal or near-optimal element intervention actions, and determines funding requirements. The designed tool prototype should complement common decision support tools and BMSs, and builds upon their existing capabilities.

\section{Task 5: Implementation of the Methodology through Examples of Scenarios}

The main objective of Task 5 is to implement the proposed EB-MOO concepts through several examples of constrained and unconstrained (budget and performance) scenarios using the tool prototype to test/validate, prove effectiveness, and demonstrate and communicate potential benefits of the methodology. The tool prototype produced under Task 4 will be used primarily for the implementation efforts.

Table 1.1 Activities associated with the research tasks

\begin{tabular}{|l|}
\hline Research Activities \\
\hline Task 1: Conducting a Literature Review-Chapter $\mathbf{2}$ \\
\hline $\begin{array}{l}\text { Identify resources (e.g., search engines, databases, specific procedures and publications by } \\
\text { transportation agencies, and library catalogs) needed to conduct a comprehensive literature review }\end{array}$ \\
\hline $\begin{array}{l}\text { Identify various practices of state DOTs and other transportation agencies, and relevant studies that } \\
\text { could support the research objectives }\end{array}$ \\
\hline $\begin{array}{l}\text { Explore the identified work and best practices related to the research areas and objectives, and } \\
\text { recognize capabilities and limitations }\end{array}$ \\
\hline $\begin{array}{l}\text { Group literature review results based on the research areas and provide a high-level summary of } \\
\text { findings }\end{array}$ \\
\hline Task 2: Proposing a Goal-Driven Transportation Asset Management Framework-Chapters $\mathbf{3} \& \mathbf{9}$ \\
\hline $\begin{array}{l}\text { Provide an overview of TAM principles, and how they are being applied based on the literature review } \\
\text { findings }\end{array}$ \\
\hline
\end{tabular}


Transfer previous work on TAM and best practices to refine the conceptual TAM framework (introduced in the research proposal)

Discuss the different steps of the proposed framework, including its benefits and practical challenges

Discuss how can existing data and tools be used to support the proposed framework

Recommend future research/studies aiming to advance the capabilities of the proposed EB-MOO

methodology, and expand the TAM framework to accommodate other transportation asset classes or modes

Task 3: Proposing an Element-Based Multi-Objective Methodology-Chapters 3,4,5,6,7, \&8 Identify various optimization techniques, analytical and decision support tools, forecasting and cost models, MCDM methods, and utility functions used by state DOTs and other transportation agencies Transfer the most appropriate work and best practices related to bridge decision making involving MOO and MCDM to develop a flexible EB-MOO methodology — structured around the different modules and supported by a MOO process

Examine various deterioration and cost models used by state DOTs and other transportation agencies, and incorporate the most suitable ones into the EB-MOO methodology

Examine various MOO techniques and approaches used by BMSs, discuss advantages and limitations, and adapt the most appropriate for each assessment level (i.e., element-, bridge-, and network-level optimization)

Finalize the conceptual flow diagram of the EB-MOO methodology (introduced in the research proposal) and develop a framework for each of its modules

Task 4: Development of a Tool Prototype-Chapters 4,5,6,7, \&8

Explore various investment decision-making analyses, project prioritization methods and optimization tools used by state DOTs and other transportation agencies, and discuss their capabilities (e.g., lifecycle cost analysis, sensitivity analysis, and trade-off analysis) and limitations

Develop a MATLAB-based tool prototype structured around the proposed EB-MOO modulestransferring appropriate features found in the explored tools under Task 1

Describe the processes and features included in the tool prototype, discuss their interactions and capabilities, and provide instructions on how to effectively use them

Task 5: Implementation of the Methodology through Examples of Scenarios-Chapters 5,6,7, \&8 Implement the proposed EB-MOO concepts through examples of unconstrained and constrained (budget and performance) scenarios using the developed tool prototype

Define a portfolio of sample bridges based on availability of relevant data, decision-making entity, geographical area, vicinity, or other characteristics

Post-process obtained optimization solutions by verifying the recommended priorities and infographic depictions produced by the tool prototype

Assess the capability of the tool to recognize short- and long-term investment needs by examining the recommended improvement strategies, determined funding requirements, and predicted performance 


\subsection{Organization of the Dissertation}

The reminder of the dissertation is organized as follows:

Chapter 2 summarizes the literature search on previous work and best practices in the areas of TAM, risk assessment and management, MCDM, and MOO. The chapter includes brief descriptions of the identified significant references, and discusses the transportation planning and programming process and the performance-based planning and TAMP requirements of MAP 21 . This chapter also covered various MOO approaches and methods, risk assessment, trade-off and multiple criteria analyses, and evolutionary algorithms.

Chapter 3 introduces the refined goal-driven TAM framework. The chapter presents the TAM principles used to structure the framework and discusses the different steps of the framework and interactions with the long-range planning and programming process. An overview of the proposed EB-MOO methodology is included in this chapter. Each of the five methodology modules is discussed in a separate section. The examples of scenarios, the portfolio of sample bridges, and the developed MATLAB-based tool porotype used for the methodology implementation are introduced in this chapter.

Chapter 4 describes the data processing module and its different underlying concepts. A framework of the module is presented. The chapter defines the improvement actions (i.e., maintenance, repair and rehabilitation [MRR], functional improvement [FCI], and replacement [REP] actions) and functional improvement needs (i.e., widening, raising, and strengthening needs), and discusses the two common types of bridge inspection data. It presents the independent deterioration model (i.e., Weibull/Markov model) used to estimate the life expectancy of an element and predict its performance over an analysis period. The chapter further lays out the 
process of assessing improvement needs, and the integrated user cost models to estimate incurred user costs. The chapter also presents the correlation/regression formulas used to estimate major improvement costs to eliminate incurred user costs, relieve the bridge from restrictions, and/or provide the required level of service (LOS) and design standards. The three types of element lifecycle (LC) profiles (i.e., "do-nothing," "replacement only," and "action effectiveness" LC profiles) are discussed in this chapter with illustrative examples.

Chapter 5 presents a basic framework to visualize the modeling approach followed to generate LC alternatives (series of element improvement actions) and predict performance and estimate life-cycle costs (LCCs) and LCC benefits. It describes the novel screening process, to focus on potential deficient (in a poor condition state) elements, and the new simulation arrangement, to generate realistic ("real-life") LC alternatives, for three distinct improvement types (i.e., MRR, FCI and REP) based on agency's preservation policies and/or practices. An illustrative example using the tool prototype is presented in this paper to demonstrate the capability of the module in producing reliable LC alternative results - to be transferred to the optimization modules to serve as the optimization input parameters.

Chapter 6 presents a basic framework of the ELO module illustrating the different concepts and processes. The chapter introduces the heuristic algorithm designed to solve the ELO problems. It describes the alternative feasibility screening process developed to reduce the ELO problem size to a manageable size and improve computational time. The ELO problem types and formulations, and the mapping approach of the problem decision variables are also discussed. The chapter includes an illustrative example using the developed tool prototype. The example consists of different ELO problems under unconstrained scenarios. One sample bridge is used in this example to demonstrate the capability of this module in producing ELO solutions (optimal or near-optimal), 
recommending set of element intervention actions, predicting element performance, and determining funding requirements for the specified improvement type and program year.

Chapter 7 introduces the BLO module and the heuristic algorithm designed to solve the BLO problems. The chapter presents the module framework and discusses the interaction between the ELO and BLO modules. The BLO problem types and formulations, and the mapping approach of the problem decision variables are also discussed. The chapter includes an illustrative example using the developed tool prototype. The example consists of different BLO problems under constrained (by budget and/or performance) and unconstrained scenarios. One sample bridge is used in this example to demonstrate the capability of the module in producing BLO solutions (optimal or near optimal), recommending set of element intervention actions and timings, predicting bridge performance, and determining funding requirements for the entire program period.

Chapter 8 introduces the NLO module. It discusses the solution superiority screening process used to guide the optimization search toward global optimality within a reasonable computational time. The chapter defines the top-down and bottom-up approaches followed by the NLO. For each approach, the chapter lays out the optimization framework, the optimization problem types and formulations, the mapping approach of decision variables, and the heuristic algorithm. Two cases of budget- and performance-constrained scenarios can be analyzed throughout this module: Case A — all bridge in the portfolio must be selected, and Case B - not necessarily all bridges in the portfolio must be selected. Several examples of constrained (by budget and/or performance) and unconstrained scenarios were established for the module implementation using the tool prototype. The examples are defined in this chapter based on optimization goals and problem types. A small portfolio of 40 sample bridges (introduced in Chapter 3) is used in these examples. Results of these 
examples are visually presented in this chapter to demonstrate the capability of the module in generating Pareto frontiers (fronts hosting optimal solutions), predicting network performance, determining investment needs, and facilitating trade-off analyses. Additionally, the diversity and quality of obtained NLO solutions (by either the top-down approach or the bottom-up approach), and the recommended intervention strategies (maintaining the desired network performance within the available budget) are also examined and discussed in this chapter.

Chapter 9 summarizes the research work, highlights the contributions and lessons learned, and provides overall conclusions and several recommendations for future research or studies. 


\section{CHAPTER 2-LITERATURE REVIEW}

- Task 1: Conducting a Literature Review

\subsection{Introduction}

Task 1 of this research consists of conducting a comprehensive review of literature across multiple resources, such as search engines, databases, specific procedures and publications by transportation agencies, and library catalogs, in accordance with the Transportation Research Circular (E-C194) (Avni et al., 2015) to fully explore the availability of research work and findings related to the research areas and objectives, identify best practices, and gain answers to the following eleven questions:

1) How do state DOTs, metropolitan planning organizations (MPOs) and other transportation agencies establish goals and objectives to guide their TAM processes?

2) How do performance measures and targets get selected by state DOTs, MPOs and other transportation agencies?

3) How are TAM frameworks structured, and what types of measures are driving the process?

4) How are TAM frameworks being implemented and assessed for effectiveness?

5) How can TAM frameworks support transparency, investment decisions, resource allocations, planning and programming, program delivery, and cost-effectiveness?

6) What are the different types of decision-making methodologies/techniques/tools involving multiple criteria/objectives used by state DOTs and other transportation agencies to support 
bridge management, economical spending decisions, and trade-offs between criteria/objectives or investment scenarios?

7) What are the different risk assessment analyses/approaches/frameworks/methods/tools used by state DOTs and other transportation agencies?

8) How could a quantitative risk-based decision-making process be used to support bridge programming (scheduling bridge intervention actions) and planning (identifying long-term investment strategies and supporting the TIP/STIP and TAMP development)?

9) What are the different optimization techniques, methods, and tools and utility/value functions used by state DOTs and other transportation agencies to address multiple criteria/objectives transportation problems?

10) What are the different performance forecasting and cost models, and economic LC analyses used by BMSs, state DOTs, and other transportation agencies to prioritize bridge intervention actions, and identify funding needs?

11) How are inputs and preferences (from decision makers and experts) and commonly collected data (from state and national asset management systems) being used to support a MCDM process?

The intent of the literature review is to support the research objectives and refine the focus by exploring various practices of transportation agencies, answering the above questions, and identifying relevant research — to ensure no duplication of efforts but rather build on previous research. The literature review focused on the following areas:

- Bridge performance measures

- Bridge management practice

- Bridge maintenance and improvement activities 
- Bridge deterioration models

- Data collection and application tools

- Life-cycle cost analysis

- Cost-benefit analysis

- Incremental-benefit cost analysis

- Cost models

- MCMD methods/techniques

- MOO methods/techniques

- Transportation asset management

- Transportation planning and programming

- Transportation investment strategies and allocation of resources

- Risk assessment and management

- Bridge- and network-level optimization methods

- Evolutionary algorithms

- Trade-off, sensitivity, and "What-if" scenario analyses

This effort involved the collection of relevant domestic and international research and studies (journals, research reports, dissertations, conference papers or presentations, etc.) and references (i.e., agency's policies and publications, procedures, manuals, specifications, standards, and technical reports). The result is a list of resources related to the research areas. Findings relevant to the research objectives from this thorough literature review were summarized and grouped based on the listed areas. The gathered information and practices provided the background to refine the conceptional TAM framework (introduced in the research proposal) and develop the EB-MOO methodology. 


\subsection{Significant References}

The literature review identified significant references relevant to the research objectivesreflecting the current state-of-the-art in TAM, risk assessment, MCDM and MOO. These significant references were used to refine the conceptional TAM framework, and design the EBMOO methodology. Table 2.1 lists these significant references. Each of them is accompanied with a brief description.

Table 2.1 Significant references identified in the literature review

\begin{tabular}{|c|c|c|c|}
\hline Name & Author & Year & Brief Description* \\
\hline $\begin{array}{l}\text { Transportation Asset } \\
\text { Management Guide: A } \\
\text { Focus on Implementation }\end{array}$ & $\begin{array}{l}\text { American } \\
\text { Association of } \\
\text { State Highway } \\
\text { and } \\
\text { Transportation } \\
\text { Officials } \\
\text { (AASHTO) }\end{array}$ & $2013 b$ & $\begin{array}{l}\text { The document provides state DOTs and } \\
\text { other transportation agencies guidance on } \\
\text { implementing asset management concepts } \\
\text { and principles within their business } \\
\text { processes. }\end{array}$ \\
\hline Asset Management Primer & FHWA & 1999 & $\begin{array}{l}\text { This document explains the basics of asset } \\
\text { management: What is asset management? } \\
\text { Why do we need asset management? An } \\
\text { overview of current practices in asset } \\
\text { management and a vision into the future } \\
\text { for improving the process are presented. }\end{array}$ \\
\hline $\begin{array}{l}\text { Performance-based Planning } \\
\text { and Programming } \\
\text { Guidebook }\end{array}$ & $\begin{array}{l}\text { Grant, M., } \\
\text { D’Ignazio, J., } \\
\text { Bond, A., \& } \\
\text { McKeeman, A. }\end{array}$ & 2013 & $\begin{array}{l}\text { The guidebook is designed to highlight } \\
\text { effective practices to help transportation } \\
\text { agencies in moving toward a performance- } \\
\text { based approach for planning and } \\
\text { programming. }\end{array}$ \\
\hline $\begin{array}{l}\text { Transportation Asset } \\
\text { Management Guide: } \\
\text { Prepared for National } \\
\text { Cooperative Highway } \\
\text { Research Program (NCHRP) } \\
\text { Project, 20-24(11) }\end{array}$ & $\begin{array}{l}\text { Cambridge } \\
\text { Systematics, } \\
\text { Inc., Parsons } \\
\text { Brinckerhoff } \\
\text { Quade \& } \\
\text { Douglas, Inc., } \\
\text { Ray Jorgenson } \\
\text { Associates, Inc., } \\
\& \text { Thompson, P. } \\
\text { D. }\end{array}$ & 2002 & $\begin{array}{l}\text { The guide is designed to help } \\
\text { transportation agencies develop and apply } \\
\text { asset management principles, techniques, } \\
\text { and tools that can advance the management } \\
\text { of their transportation assets. }\end{array}$ \\
\hline
\end{tabular}




\begin{tabular}{|c|c|c|c|}
\hline $\begin{array}{l}\text { NCHRP Report 590: Multi- } \\
\text { Objective Optimization for } \\
\text { Bridge Management } \\
\text { Systems }\end{array}$ & $\begin{array}{l}\text { Patidar, V., } \\
\text { Labi, S., Sinha, } \\
\text { K. C., \& } \\
\text { Thompson, P. D }\end{array}$ & 2007 & $\begin{array}{l}\text { The report describes a MOO methodology } \\
\text { driven by user-specified preferences and } \\
\text { criteria proposed for the network level and } \\
\text { the project level }\end{array}$ \\
\hline $\begin{array}{l}\text { NCHRP Report 806: Cross- } \\
\text { Asset Resource Allocation } \\
\text { and the Impact on } \\
\text { Transportation System } \\
\text { Performance }\end{array}$ & $\begin{array}{l}\text { Maggiore, M., } \\
\text { \& Ford, K. }\end{array}$ & 2016 & $\begin{array}{l}\text { The report provides transportation agencies } \\
\text { guidance on allocating limited resources } \\
\text { among asset classes and organizational } \\
\text { units to provide optimal system } \\
\text { performance. }\end{array}$ \\
\hline $\begin{array}{l}\text { NCHRP Report 483: Bridge } \\
\text { Life-Cycle Cost Analysis } \\
\text { (BLCCA) }\end{array}$ & Hugh, $\mathrm{H}$. & 2003 & $\begin{array}{l}\text { The report contains findings of a study to } \\
\text { develop a BLCCA methodology for use by } \\
\text { transportation agencies. }\end{array}$ \\
\hline $\begin{array}{l}\text { NCHRP Report 706: Uses of } \\
\text { Risk Management and Data } \\
\text { Management to Support } \\
\text { Target-Setting for } \\
\text { Performance-based Resource } \\
\text { Allocation by Transportation } \\
\text { Agencies }\end{array}$ & $\begin{array}{l}\text { Cambridge } \\
\text { Systematics, } \\
\text { Inc. }\end{array}$ & $2011 a$ & $\begin{array}{l}\text { The report describes how risk management } \\
\text { and data management may be used by } \\
\text { transportation agencies to support target- } \\
\text { setting for performance-based resource } \\
\text { allocation. }\end{array}$ \\
\hline $\begin{array}{l}\text { NCHRP Report 545: } \\
\text { Analytical Tools for Asset } \\
\text { Management }\end{array}$ & $\begin{array}{l}\text { Cambridge } \\
\text { Systematics, } \\
\text { Inc., PB } \\
\text { Consult, \& } \\
\text { System Metrics } \\
\text { Group, Inc. }\end{array}$ & 2005 & $\begin{array}{l}\text { This report presents new analytical tools to } \\
\text { support asset management. Emphasis is } \\
\text { given to the tools needed to assist } \\
\text { transportation agencies in trade-off } \\
\text { decisions for resource allocation. }\end{array}$ \\
\hline $\begin{array}{l}\text { NCHRP Synthesis 397: } \\
\text { Bridge Management } \\
\text { Systems for Transportation } \\
\text { Agency Decision Making. }\end{array}$ & $\begin{array}{l}\text { Markow, M. J., } \\
\text { \& Hyman, W. } \\
\text { A. }\end{array}$ & 2009 & $\begin{array}{l}\text { This study gathers information on current } \\
\text { practices in the network-level resource } \\
\text { allocation for bridge programs. The study } \\
\text { explores how BMSs of transportation } \\
\text { agencies are deployed in this process. }\end{array}$ \\
\hline $\begin{array}{l}\text { NCHRP Report 666: Target- } \\
\text { Setting Methods and Data } \\
\text { Management to Support } \\
\text { Performance-based Resource } \\
\text { Allocation by Transportation } \\
\text { Agencies }\end{array}$ & $\begin{array}{l}\text { Cambridge } \\
\text { Systematics, } \\
\text { Inc. }\end{array}$ & 2010 & $\begin{array}{l}\text { The report describes methods that state } \\
\text { DOTs and other transportation agencies } \\
\text { can use for setting performance targets to } \\
\text { achieve multiple objectives. The report } \\
\text { also discusses how data management } \\
\text { systems can support performance-based } \\
\text { decision making. }\end{array}$ \\
\hline $\begin{array}{l}\text { NCHRP Report 551: } \\
\text { Performance Measures and } \\
\text { Targets for Transportation } \\
\text { Asset Management }\end{array}$ & $\begin{array}{l}\text { Cambridge } \\
\text { Systematics, } \\
\text { Inc., PB } \\
\text { Consult, \& } \\
\text { Texas } \\
\text { Transportation } \\
\text { Institute }\end{array}$ & 2006 & $\begin{array}{l}\text { The report describes the research effort and } \\
\text { provides the current state-of-the practice } \\
\text { on the use of performance measures, } \\
\text { principally in the context of TAM. }\end{array}$ \\
\hline
\end{tabular}




\begin{tabular}{|c|c|c|c|}
\hline $\begin{array}{l}\text { Trade-off Analysis in Multi- } \\
\text { Objective Optimization for } \\
\text { Transportation Asset } \\
\text { Management }\end{array}$ & Bai, Q. & 2012 & $\begin{array}{l}\text { The dissertation introduces } \\
\text { a hybrid Non-dominated Sorting Genetic } \\
\text { Algorithm-II (NSGA-II) method to } \\
\text { generate Pareto frontiers to conduct trade- } \\
\text { off analyses between investment and } \\
\text { performance. }\end{array}$ \\
\hline $\begin{array}{l}\text { Explicit Building Block } \\
\text { Multi-Objective } \\
\text { Evolutionary Computation: } \\
\text { methods and Applications }\end{array}$ & Day, R. O. & 2005 & $\begin{array}{l}\text { The dissertation presents principles, } \\
\text { techniques and performance of various } \\
\text { evolutionary optimization algorithms and } \\
\text { proposes a robust algorithm based on } \\
\text { common evolutionary optimization } \\
\text { concepts. }\end{array}$ \\
\hline $\begin{array}{l}\text { Project Planning Models for } \\
\text { Florida's Bridge } \\
\text { Management System }\end{array}$ & $\begin{array}{l}\text { Sobanjo, J. O., } \\
\text { \& Thompson, P. } \\
\text { D. }\end{array}$ & 2004 & $\begin{array}{l}\text { This study updated Florida's user cost } \\
\text { models in terms of truck weight and height } \\
\text { characteristics, and moveable bridge } \\
\text { openings on Florida's roadways. }\end{array}$ \\
\hline $\begin{array}{l}\text { Implementation of the } 2013 \\
\text { AASHTO Manual for } \\
\text { Bridge Element Inspection }\end{array}$ & $\begin{array}{l}\text { Sobanjo, J. O., } \\
\text { \& Thompson, P. } \\
\text { D. }\end{array}$ & $2016 a$ & $\begin{array}{l}\text { This study developed new transition times } \\
\text { for deterioration between condition states } \\
\text { and revised the action effectiveness models } \\
\text { based on the new AASHTO bridge } \\
\text { element inspection manual. Preservation } \\
\text { unit costs and other cost parameters were } \\
\text { also revised for Florida's BMS. }\end{array}$ \\
\hline $\begin{array}{l}\text { Decision Support for Bridge } \\
\text { Programming and Budgeting }\end{array}$ & $\begin{array}{l}\text { Sobanjo, J. O., } \\
\text { \& Thompson, P. } \\
\text { D. }\end{array}$ & 2007 & $\begin{array}{l}\text { The report discusses a bridge decision } \\
\text { support tool developed for Florida DOT to } \\
\text { determine performance at any given level } \\
\text { of funding over 10-year planning horizon. }\end{array}$ \\
\hline $\begin{array}{l}\text { National Bridge Investment } \\
\text { Analysis System (NBIAS) } \\
\text { Technical Manual }\end{array}$ & $\begin{array}{l}\text { Cambridge } \\
\text { Systematics, } \\
\text { Inc. }\end{array}$ & 2011 & $\begin{array}{l}\text { The technical manual supplements the user } \\
\text { manual and provides key technical features } \\
\text { of version } 4.0 \text { of NBIAS. }\end{array}$ \\
\hline $\begin{array}{l}\text { Enhancement of the FDOT's } \\
\text { Project Level and Network } \\
\text { Level Bridge Management } \\
\text { Analysis Tools }\end{array}$ & $\begin{array}{l}\text { Sobanjo, J. O., } \\
\text { \& Thompson, P. } \\
\text { D }\end{array}$ & 2011 & $\begin{array}{l}\text { The research improved the deterioration, } \\
\text { action effectiveness, and cost model for } \\
\text { Florida DOT's bridge management } \\
\text { analysis tools. New accident models were } \\
\text { formulated. }\end{array}$ \\
\hline $\begin{array}{l}\text { Florida DOT's Project Level } \\
\text { Analysis Tool (PLAT) User } \\
\text { Manual }\end{array}$ & $\begin{array}{l}\text { Thompson, P. } \\
\text { D.\& Sobanjo, J. } \\
\text { O. }\end{array}$ & 2016 & $\begin{array}{l}\text { This manual is designed to help learn about } \\
\text { the different features of the Florida DOT's } \\
\text { PLAT and how to perform a project-level } \\
\text { analysis. }\end{array}$ \\
\hline $\begin{array}{l}\text { Metaheuristics: From Design } \\
\text { to Implementation. }\end{array}$ & Talbi, E. G. & 2009 & $\begin{array}{l}\text { The book covers metaheuristics applicable } \\
\text { to MOO problems. It presents the main } \\
\text { design questions and search components } \\
\text { for all families of metaheuristics. }\end{array}$ \\
\hline
\end{tabular}




\begin{tabular}{|c|c|c|c|}
\hline $\begin{array}{l}\text { NCHRP Report 713: } \\
\text { Estimating Life } \\
\text { Expectancies of Highway } \\
\text { Assets. }\end{array}$ & $\begin{array}{l}\text { Thompson, P. } \\
\text { D., Ford, K. M., } \\
\text { Armin, M. H.R., } \\
\text { Labi, S., Sinha, } \\
\text { K. C., \& } \\
\text { Shirole, A. }\end{array}$ & 2012 & $\begin{array}{l}\text { The guide describes current methods for } \\
\text { estimating life expectancies of highway } \\
\text { assets and how to apply life expectancy } \\
\text { models. }\end{array}$ \\
\hline $\begin{array}{l}\text { Multi-Objective } \\
\text { Optimization using } \\
\text { Evolutionary Algorithms }\end{array}$ & Deb, K., & 2001 & $\begin{array}{l}\text { The book discusses in-depth each } \\
\text { evolutionary algorithm with examples of } \\
\text { real-world problems. }\end{array}$ \\
\hline $\begin{array}{l}\text { A Large-Scale Optimization } \\
\text { Algorithm to Support Cross- } \\
\text { Assets Long-Term Planning } \\
\text { in Transportation Asset } \\
\text { Management }\end{array}$ & Kachua, S. G. & 2012 & $\begin{array}{l}\text { The research develops and implements a } \\
\text { large-scale linear programming } \\
\text { formulation to support the long-term } \\
\text { rehabilitation and maintenance resource } \\
\text { allocation for road networks. }\end{array}$ \\
\hline $\begin{array}{l}\text { Multi-Criteria Optimization } \\
\text { in Bridge Management }\end{array}$ & Patidar V. & 2006 & $\begin{array}{l}\text { The dissertation discusses a multi-criteria } \\
\text { optimization methodology developed for } \\
\text { the bridge decision-making problem based } \\
\text { on the utility theory concepts. }\end{array}$ \\
\hline $\begin{array}{l}\text { Multi-objective } \\
\text { Optimization Algorithms } \\
\text { Considering Objective } \\
\text { Preferences and Solution } \\
\text { Clusters }\end{array}$ & Taboada, H. & 2007 & $\begin{array}{l}\text { The thesis presents new approaches that } \\
\text { provide a balance between the } \\
\text { determination of single solutions and a set } \\
\text { of Pareto optimal solutions. }\end{array}$ \\
\hline $\begin{array}{l}\text { A Summary and Comparison } \\
\text { of MOEA Algorithms. }\end{array}$ & Kunkle, D. & 2005 & $\begin{array}{l}\text { The document briefly summarizes and } \\
\text { compares various well-known multi- } \\
\text { objective evolutionary algorithms } \\
\text { (MOEAs). }\end{array}$ \\
\hline $\begin{array}{l}\text { Feasibility of Incremental } \\
\text { Benefit-Cost Analysis for } \\
\text { Optimal Allocation of } \\
\text { Limited Budgets to } \\
\text { Maintenance, Rehabilitation } \\
\text { and Replacement of Bridges }\end{array}$ & $\begin{array}{l}\text { Farid, F., } \\
\text { Johnston, D. W., } \\
\text { Laverde, M.A., } \\
\text { Chen, C. J. \& } \\
\text { Rihani, B. S. }\end{array}$ & 1988 & $\begin{array}{l}\text { The study investigates the applicability of } \\
\text { the Incremental Benefit-Cost program } \\
\text { (called INCBEN) in allocating limited } \\
\text { bridge improvement budgets and compares } \\
\text { results with different sufficiency rating } \\
\text { methods. }\end{array}$ \\
\hline $\begin{array}{l}\text { Synthesis of National and } \\
\text { International Methodologies } \\
\text { Used for Bridge Health } \\
\text { Indices }\end{array}$ & $\begin{array}{l}\text { Chase, S., Adu- } \\
\text { Gyamfi, Y., Akt } \\
\text { an, A., } \\
\text { \& Minaie, E. }\end{array}$ & 2016 & $\begin{array}{l}\text { The study reviews the state-of-the-art with } \\
\text { respect to bridge condition indices used to } \\
\text { assess performance of bridges in the } \\
\text { United States and other countries. }\end{array}$ \\
\hline $\begin{array}{l}\text { Development of Optimal } \\
\text { Strategies for Bridge } \\
\text { Management Systems. }\end{array}$ & $\begin{array}{l}\text { Mohamed, H. A. } \\
\text { H. }\end{array}$ & 1995 & $\begin{array}{l}\text { The dissertation discussed a } \\
\text { comprehensive framework developed to } \\
\text { efficiency utilize available resources and } \\
\text { minimize the loss of benefits for bridge } \\
\text { management systems. }\end{array}$ \\
\hline
\end{tabular}




\begin{tabular}{|c|c|c|c|}
\hline $\begin{array}{l}\text { The Development of } \\
\text { Optimal Strategies for } \\
\text { Maintenance Rehabilitation } \\
\text { and Replacement of } \\
\text { Highway Bridges, Final } \\
\text { Report Vol. 6: Bridge } \\
\text { Performance and }\end{array}$ & $\begin{array}{l}\text { Jiang, Y., \& } \\
\text { Sinha, K. C. }\end{array}$ & 1989 & $\begin{array}{l}\text { This volume provides the results of the } \\
\text { research conducted on the development of } \\
\text { an optimization model for bridge project } \\
\text { selection. It also includes a discussion on } \\
\text { bridge condition deterioration } \\
\text { curves and appropriate performance } \\
\text { prediction models. }\end{array}$ \\
\hline
\end{tabular}

* Descriptions are from the documents

\subsection{Transportation Planning Process}

The MPOs represent the multi-modal transportation interests of a specific urbanized region and usually made up of representatives from local governments and transportation agencies. The MPOs develop Metropolitan Transportation Plans (MTPs) for their regions. The state DOT develops a LRTP for the entire state, including both metropolitan and nonmetropolitan areas. The MTPs and the LRTP are the fundamental products of the initial planning process. To ensure consistency, these long-range plans are developed in cooperation between the state DOT and the MPOs. The 23 United States Code (USC) 134(h) provision established the following ten factors to define the scope of this initial planning process:

- $\quad$ Support Economic Vitality

- Increase Safety

- Increase Security

- Increase Accessibility

- Protect and Enhance the Environment

- Enhance Integration and Connectivity

- Promote System Efficiency

- Emphasize System Preservation 
- Improve Resiliency and Reliability

- Enhance Travel and Tourism

These plans consider existing and future transportation system needs and resources for the next 20-year horizon—provide directions and supporting information for subsequent planning activities. Generally, the LRTP does not list individual projects but describes management and investment strategies - required to ensure preservation and improvement of the multi-modal transportation system statewide. The MTP specifies resources expected and recommends strategies for projects and programs needed in the metropolitan area. The next step in the planning process consists of identifying specific transportation projects for implementation in the near term, including funding requirements. The TIP and STIP are both short-range programs to identify priority transportation projects to be funded in the upcoming four years and drawn from and consistent with the MTPs and LRTP. These short-range programs are fiscally constrained and usually combined in one TIP/STIP document, capturing all transportation-related projects for the entire state, and approved by the Federal Transit Administration (FTA) and FHWA.

The NCHRP Report 806: Cross-Asset Resource Allocation and the Impact on Transportation System Performance (Maggiore \& Ford, 2016) studied various practices of resource allocation decision making. The study recognized four common approaches among state DOTs for making investment decisions and setting program/project priorities:

(1) decisions are determined based on historical precedents and funding restrictions (past allocations and priorities) for a program;

(2) decisions are driven largely by asset management systems that determine investment needs; 
(3) decisions are driven mostly by leadership discretions, political considerations, and other nontechnical inputs to align available resources with priorities relative to the state DOT; and

(4) decisions are based on performance determined by condition data, forecasting models, and analytical tools.

\subsection{Bridge Programming Process}

One of the main objectives of a BMS is to manage bridges under constrained budgets and resources. Many BMSs address three aspects of bridge management: (1) assessing existing bridge conditions, (2) forecasting deteriorations, and (3) identifying improvement needs (Elbehairy, 2007). Twenty-six state DOTs were surveyed as part of the NCHRP Synthesis 397: Bridge Management Systems for Transportation Agency Decision Making (Markow \& Hyman, 2009). The survey revealed that most of the bridge projects listed in the TIP/STIP were being developed using specific prioritization criteria/procedures and/or professional judgments. Few state DOTs use BMSs for project programming and TIP/STIP development to recommend candidate projects and preservation treatments. At the project programming level, asset managers prioritize bridge work activities based on projected budgets in the TIP/STIP—for many state DOTs, the TIP/STIP defines their annual bridge programs (Markow \& Hyman, 2009). Saito and Sinha (1989) discussed common methods used by state DOTs to select bridge projects for funding. Some select projects based on "worst-first" basis while others rely on ranking methods or computer-based systematic analyses. To identify candidate projects, state DOTs generally use the bridge sufficiency ratings, component ratings, health indices, or other factors (such as the type of bridge, location, volume of traffic, level of service, functional deficiencies, detour length, number of injuries, and fatalities). 
Most state DOTs use BMSs for collecting and managing inspection data and identifying potential projects or maintenance activities. BMSs are being used as repositories of bridge data without deploying the advanced functionalities such as prediction of future trends, optimization, trade-off analysis, life-cycle cost analysis (LCCA), and cost-benefit analysis (BCA) (Cambridge Systematics, Inc., 2009). The NCHRP Synthesis of Highway Practice 243: Methods for Capital Programming and Project Selection (Neumann, 1997) highlights common reasons behind not fully utilizing the capabilities of BMSs to support setting program priorities and establishing investment levels. Some of the reasons include the following:

- Concerns that outcomes from BMSs will overtake engineering judgments and executive policy directions

- Problems with system integration, limited historical data, and lack of expertise

- Sufficiency rating, deficiency status, and other condition assessment methods continue to serve as the primary instruments for setting priorities

\subsection{MAP-21 and FAST Act}

On December 4, 2015, FAST Act was signed into law succeeding MAP-21, which was enacted in 2012. The 23 USC 150(a) provision of MAP-21 created a performance-based program to strengthen existing transportation systems by focusing on national transportation goals, increasing accountability and transparency, and improving decision making through better informed planning and programming. FAST Act continued MAP-21's overall performance management approach, which requires state DOTs to invest resources in transportation projects to collectively progress toward the national performance goals. 
The following are the seven areas of the national performance goals, as established in MAP-21, 23 USC 150(b):

- Safety-To achieve a significant reduction in traffic fatalities and serious injuries on all public roads.

- Infrastructure Condition-To maintain the highway infrastructure asset system in a stateof-good repair

- Congestion Reduction-To achieve a significant reduction in congestion on the National Highway System

- System Reliability -To improve the efficiency of the surface transportation system

- Freight Movement and Economic Vitality-To improve the national freight network, strengthen the ability of rural communities to access national and international trade markets, and support regional economic development.

- Environmental Sustainability-To enhance the performance of the transportation system while protecting and enhancing the natural environment.

- Reduced Project Delivery Delays - To reduce project costs, promote jobs and the economy, and expedite the movement of people and goods by accelerating project completion through eliminating delays in the project development and delivery process, including reducing regulatory burdens and improving agencies' work practices 
To implement the new performance provisions of MAP-21 and FAST Act, several rulemakings were developed by FHWA in multiple phases. The rules were finalized in 2016 and published in the Federal Register, establishing requirements for compliance. The following are the finale rules:

- Pavement and Bridge Condition Performance Measures Final Rule

- System Performance/Freight/Congestion Mitigation and Air Quality Performance Measures Final Rule

- Highway Safety Improvement Program Final Rule

- Safety Performance Measures Final Rule

- Planning Final Rule

- Asset Management Plan Final Rule

- Transit Asset Management Final rule

- Public Transportation Safety Program Final Rule

\subsubsection{Performance-Based Planning Requirements}

Performance measures within the areas listed below were established through several final rules to monitor transportation system performance and conditions, inform investment decisions, and increase accountability and transparency. The planning final rule requires state DOTs and MPOs to set aspirational targets as part of their MTPs and LRTPs. State DOTs and MPOs may elect to use additional performance measures; however, it's highly recommended they be aligned with the following national goals, 23 USC 150(c), where applicable.

- Pavement condition on the Interstate System and on remainder of the National Highway System (NHS)

- Performance of the Interstate System and the remainder of the NHS 
- Bridge condition on the NHS

- Fatalities and serious injuries_-both number and rate per vehicle mile traveled on all public roads

- Traffic congestion

- On-road mobile source emissions

- Freight movement on the Interstate System

MAP-21 mandates the use of measures to evaluate performance. These measures will be used in the performance-based planning and programming process. The process links the LRTP to the TIP/STIP. A performance-based LRTP provides enough details, criteria and strategies toward achieving goals, objectives and performance targets, and supports prioritization and selection (programming) of projects for the TIP/STIP. Figure 2.1 illustrates the performance-based planning and programming framework as discussed in the FHWA's Performance-Based Planning and Programming Guidebook (Grant et al., 2013). The figure shows the different steps of a performance-based planning and programming process. Performance-based planning is led by goals and objectives supported by performance measures-linked to the national goals described in MAP-21. The LRTP is guided by goals, objectives, and performance measures to identify trends and targets, define strategies, and develop investment priorities. Performance-based programming takes place next, where specific projects and programs are identified and prioritized to be included in the TIP/STIP — based on contribution to attainment of targets. The entire process is monitored for adjustments and future planning efforts. 


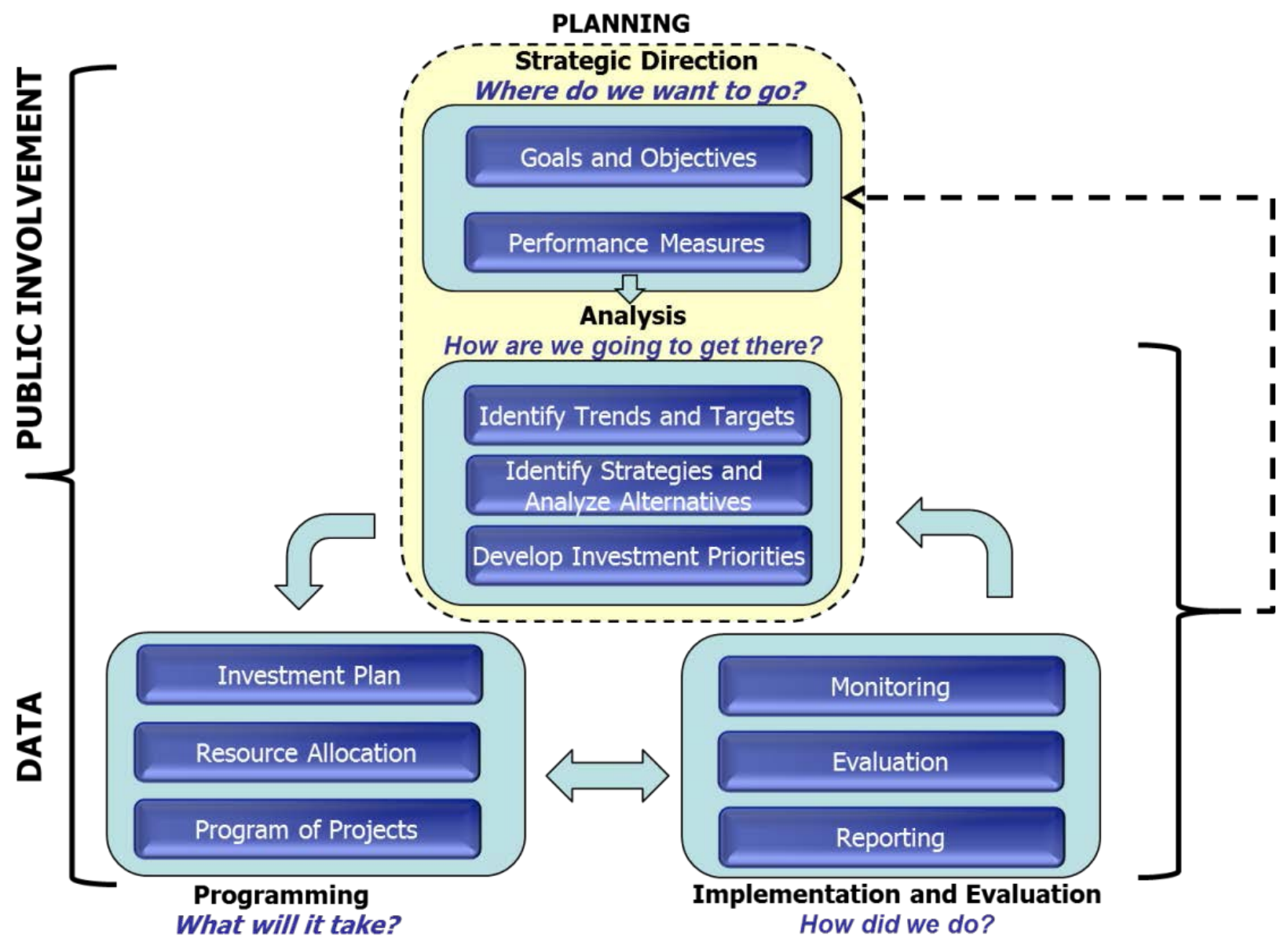

Figure 2.1 Performance-based planning framework. Adapted from Performance-Based Planning and Programming Guidebook (Grant, D’Ignazio, Bond, and McKeeman, 2013).

\subsubsection{Transportation Asset Management Plan Requirements}

TAM, in its broadest sense, is defined by AASHTO Subcommittee on Asset Management (AASHTO, 2013b) as "a strategic and systematic process of operating, maintaining, upgrading, and expanding physical assets effectively through their life cycles. It focuses on business and engineering practices for resource allocation and utilization, with the objective of better decision making based on quality information and well-defined objectives." Through an effective data-driven asset management approach, state DOTs can improve investment decisions, asset performance, resource allocations, and accountability and transparency. To embrace such approach as the main business practice for state DOTs, MAP-21 and FAST mandated the development of a risk-based TAMP for the 
NHS to improve or preserve asset conditions and system performance. The following seven elements, 23 Code of Federal Regulations (CFR) 515.9, must be included in the TAMP:

- Conditions of pavement and bridge

- Asset management objectives and measures

- Identification of any performance gap

- Life-cycle planning

- Risk management analysis

- Financial plan

- Investment strategies

The TAMP is framed to include investment strategies leading to make progress toward achievement of performance targets. The plan should address both short- and long-term needs - to improve or preserve the condition and performance of the NHS. A risk management analysis is required as part of the TAMP development. State DOTs are required to consider various risks associated with current and future environmental conditions (risks due to failure, extreme weather, seismic, financing, etc.). In addition, the TAMP final rule directs state DOTs to coordinate with the MPOs and other stakeholders when establishing performance measures and targets. The planning final rule requires states DOTs to integrate their TAMPs into their statewide transportation planning process. State DOTs are required to submit to FHWA their TAMPs for approval, meeting all requirements, along with documentations to demonstrate implementation. On a 4-year basis, the plan is revised and resubmitted for approval. 


\subsection{Risk Assessment and Management}

The NCHRP Report 632: An Asset-Management Framework for the Interstate Highway System (Cambridge Systematics, Inc., 2009) classifies risks into two possible categories: internal programmatic and external non-programmatic. Internal programmatic risks involve state DOT's internal operations (planning, budgeting, program delivery, maintenance, managing, etc.), whereas risks beyond the state DOT's control such as natural-related hazards (flooding, earthquakes, hurricanes, sea level rising, etc.) or human actions (collisions, terrorist attacks, etc.) are classified as external non-programmatic.

The NCHRP Report 632 states that "significant work has been performed on risk assessment for transportation in recent years." Efforts have been made to assess the threat of natural and manmade hazards in BMSs. Vulnerability or risk cost models quantify consequences resulting from natural hazards (such as earthquake, scour, and flooding). The NCHRP Project 20-07, Task 378, (Thompson, 2018) developed a risk assessment guideline for the LCCA in BMSs. The guideline is based on likelihood probability models for sixteen different hazards. The project also developed a process for monetizing risk. This type of LCCs can be considered in the LCCA to account for risk.

As stated earlier, a TAMP must identify risks that can affect the NHS condition and performance, including risks associated with current and future environmental conditions (risks due to failure, extreme weather, seismic, financing, etc.). The FHWA publication, Incorporating Risk Management into Transportation Asset Management Plans (FHWA, 2017b), defines risk and provides guidance on how to incorporate risk into a TAMP to meet the risk-based TAMP requirements. The intent of the guidance is to assist state DOTs with the development of their TAMPs. However, the concepts 
are applicable to any transportation agency responsible for managing pavements, bridges or other assets.

Risks that could impact the ability to deliver planned investments or effectively manage assets, the performance of a network of assets or a single asset, or the achievement of performance targets are generally identified, prioritized and added to a risk register. Experts adjust these predefined risks or add new noteworthy risks for consideration. A risk assessment analysis relies on collected data and inputs provided by experts. Each identified risk is quantified with an overall score equals to the product of its likelihood of occurrence and its associated consequences. Criticality or vulnerability levels are also assigned to each bridge or network of bridges to determine importance factors as part of the analysis. The risk assessment results (qualitative or quantitative) can be used to inform decisions, evaluate investment strategies, or develop a risk management plan.

Figure 2.2 illustrates an example of Minnesota DOT's (MnDOT) risk assessment matrix as presented in the NCHRP Report 706: Uses of Risk Management and Data Management to Support Target-setting for Performance-based Resource Allocation by Transportation Agencies (Cambridge Systematics, Inc., 2011). Separate scaling tables and relative weights for different types of risk that could jeopardize the serviceability of a bridge are used to estimate its resilience indicator. Importance factor is also determined for each bridge based on its provided service to a specific network (impacts on the public). At the end, a function of combined weighted importance factors and resilience indicators is utilized to determine a single network resilience indicator (Cambridge Systematics, Inc., 2011). 


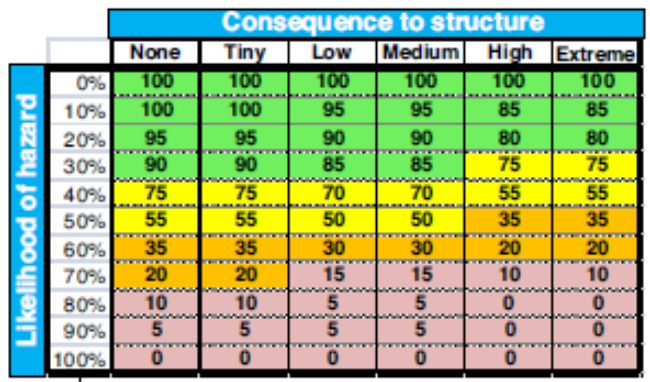

(separate scaling table or formula for each type of risk

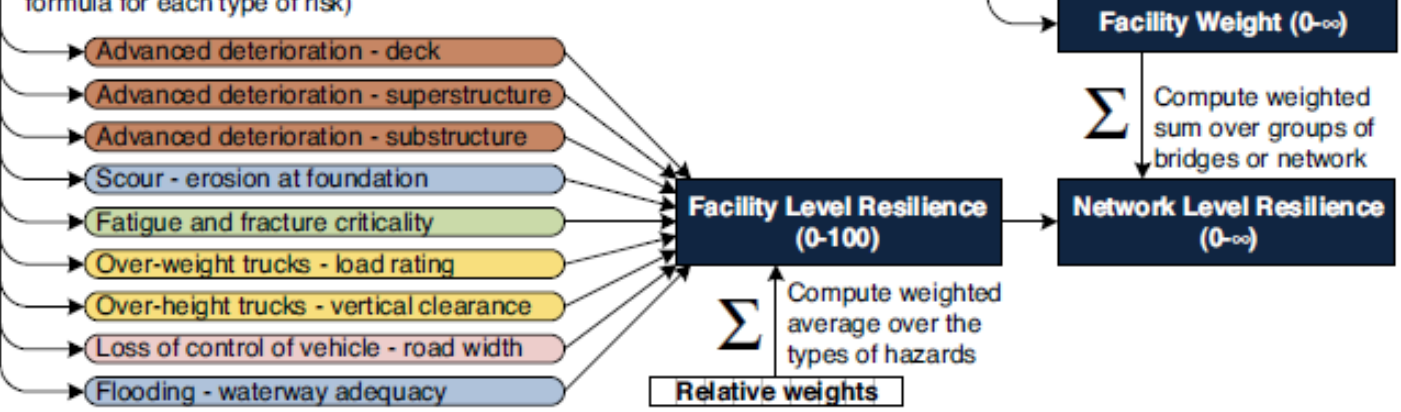

Figure 2.2 MnDOT's bridge risk assessment quantifying risks, impacts, and consequences. Adapted from the NCHRP Report 706: Uses of Risk Management and Data Management to Support Target-setting for

Performance-based Resource Allocation by Transportation Agencies (Cambridge Systematics, Inc., 2011).

\subsection{Optimization in Bridge Management}

The literature review revealed that empirical and non-empirical ranking methods, incremental benefit-cost analysis (IBCA), multiple criteria analysis (discussed later), and optimization techniques are widely applied for selecting projects. Ranking methods evaluate several projectrelated factors and produce a ranking value corresponding to a relative order of importance to be used by decision makers. Though, the ranking results do not necessarily lead to optimal projects. Farid et al. (1988) investigated the feasibility of utilizing the IBCA for optimal allocation of limited budgets to maintenance, rehabilitation, and replacement of 25 bridges in North Carolina. The study proved that the IBCA rankings are superior to those produced by other priority rating methods and can be used to identify bridge improvement alternatives. However, the IBCA cannot produce optimal sets of bridge improvement alternatives under limited budgets. On the other hand, 
optimization techniques produce optimal solutions by either maximizing or minimizing specified objectives (simultaneously or combining them into a single objective) and subject to a set of constraints (Saito \& Sinha, 1989).

A large variety of optimization techniques, such as mathematical programming (e.g., dynamic programming, linear or non-linear programming, integer or continuous programming, and goal programming) and metaheuristics (e.g., evolutionary algorithms, tabu search, ant colony, particle swarm, and shuffled frog-leading) have been applied to solve bridge optimization problems. The use of one technique versus another depends on the characteristics of the optimization problem. Krugler et al. (2007) highlighted that optimization techniques have been used for solving practical problems successfully in different disciplines. Resource allocation problems are treated in most times using different optimization techniques. However, the study acknowledged that the complexity associated with resource allocation problems - optimizing benefits (objectives) while satisfying constraints-limits the applicability of such techniques in transportation areas. The authors further pointed out to several studies that describe various mathematical optimization models for resource allocation problems; though, most of them cannot be applied directly to transportation decision problems.

Krugler et al. (2007) in the same study discussed the two common approaches used for solving multiple criteria/objectives decision problems. One of these approaches consists of combining multiple criteria (or objectives) into a single criterion (or a single objective) function, after being ranked based on decision makers' preferences and assigned appropriate weights. In most cases when this approach is considered, heuristic solution techniques are usually used, since the resulting function is nonlinear. The other one considers simultaneously all criteria (or objectives) in the mathematical formulation by applying "true" MOO techniques - permitting decision makers to 
examine competing decision criteria/objectives - to identify optimal solutions. However, this approach is less common due to the extra efforts involving system integration and collection of extensive data, and reliance on specific expertise.

Separate investment analysis tools or systems using optimization techniques also have been developed for bridge investment decision making either by state DOTs, the FHWA, or researchers. The most popular system is NBIAS, which was designed for modeling national bridge investment needs and trade-offs between funding and performance. The system is mainly used in the development process of the Status of the Nation's Highways, Bridges, and Transit: Conditions \& Performance Report to Congress (U.S. Department of Transportation, 2015) - to provide the current condition state of bridges and projected national system performance under a set of different investment scenarios. NBIAS relies on a linear programming optimization and an analytical framework like the one in the AASHTOWare Bridge Management software (BrM).

The NCHRP Report 590: Multi-Objective Optimization for Bridge Management Systems (Patidar et al., 2007) (product of the NCHRP Project 12-67) reveals that LCCAs had been integrated into most optimization techniques used by BMSs, including the incremental benefit-cost method to produce near-optimal solutions, to speed-up optimization by varying constraints without resolving the entire problem. The report indicated that the annual budget and performance measure (expressed as structural health index, sufficiency rating, or other condition-based indices) are typically the criteria that drive the BMS optimization process.

(Saito \& Sinha, 1989; Patidar, 2006) pointed out that the integer programming and dynamic programming techniques have been widely used to support decision making. In the integer programming technique, the decision variables have a value of either 0 or 1 . A project is selected 
if a decision variable is corresponding to 1 , and it is not selected if the variable is 0 . The dynamic programming technique transforms an "n-variable" problem into a series of "n 1-variable" problems. The optimization usually requires a less computational effort and produces global optimal solutions. Saito and Sinha (1989) developed an optimization procedure for Indiana DOT, using a combination of integer programming and dynamic programming techniques to identify optimal bridge projects. Integer programming is used to maximize the statewide bridge system effectiveness under a limited budget and other established constraints for each program period. Dynamic programming uses the results of integer programming to select the overall optimal strategy, maximizing the system effectiveness over the entire planning horizon. However, the computational time increases substantially when dealing with an increased number of decision variables.

The Multi-Objective Optimization System (MOOS) is a standalone optimization tool developed by researchers (as part of the NCHRP Project 12-67) for bridge investment decision making. MOOS is a spreadsheet-based tool to help bridge program managers to visualize the performance of a network of bridges by showing projected investment needs, performance, and trade-offs between funding levels and targets. Florida DOT, back in 2007, developed a standalone application (to supplement its BMS) called Network Analysis Tool using MOO and an incremental benefitcost heuristic to predict network performance at any given level of funding for a 10-year planning horizon (Sobanjo \& Thompson, 2016c). The application was developed in Microsoft Excel spreadsheet incorporating several of the MOOS concepts detailed in the NCHRP Report 590 (Patidar et al., 2007). 


\subsection{Multi-Objective Optimization Approaches}

MOO approaches are classified based on whether the decision makers' preferences are utilized in the optimization process: preferences and non-preferences approaches. In the work of Bai (2012), the following Figure 2.3 is presented to illustrate the classification introduced by Hwang and Masud (1979) and Miettinen (1999).

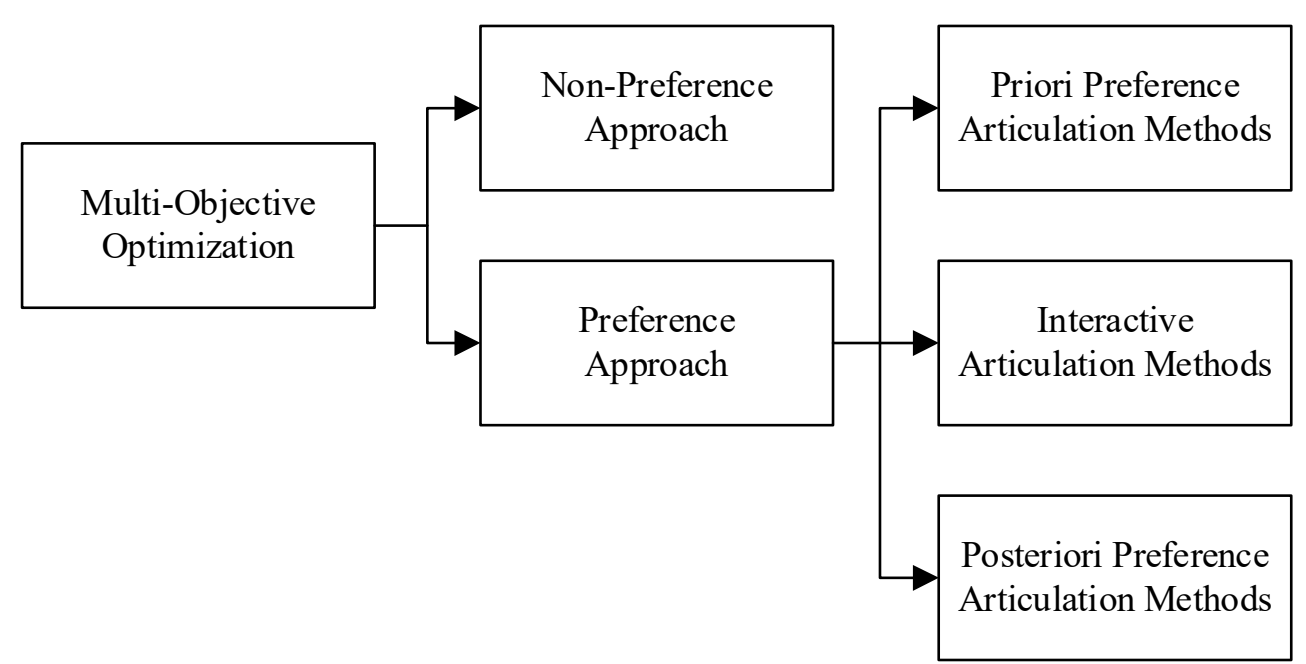

Figure 2.3 Classification of MOO methods. Redrawn from the original in Trade-Off Analysis in MultiObjective Optimization for Transportation Asset Management (Bai, 2012).

The non-preference approach doesn't rely on the decision makers' preferences or assume any information about the importance of objective in the optimization process. A heuristic is generally used to find a single optimal solution. However, in the preference approach, decision makers' preferences are incorporated in the optimization process (Miettinen, 1999; Bai, 2012). The preference approach is divided into the following three categories based on the stage where the preference is articulated (Bai, 2012).

- Priori preference articulation - prior to the optimization, transforming the MOO problem into a single-objective optimization problem by incorporating the preferences. 
- Interactive preference articulation - the preferences are incorporated simultaneously with the optimization process; the decision maker interactively participates in the optimization process.

- Posteriori preference articulation-Pareto (subsequently discussed) solutions are first determined, then the decision maker chooses one of the Pareto solutions as the best solution based on preferences.

Deb (2001) defined the MOO methods that convert multiple objectives into a single objective as "classical" methods. These methods form a composite objective function, weighted sum of the objectives in accordance to the assigned preferences. The following methods are considered classical MOO methods:

- Weighted Sum Method

- $E$-Constraint Method

- Weighted Metric Methods

- Rotated Weighted Metric Method

- Benson's Method

- Value Function Method

- Goal Programming Methods

The author named this type of approach (followed by these classical methods) a preference-based MOO approach. The author stressed out that this approach is highly subjective, not straightforward, and largely sensitive to the relative preferences. A schematic of this approach is shown in Figure 2.4. 


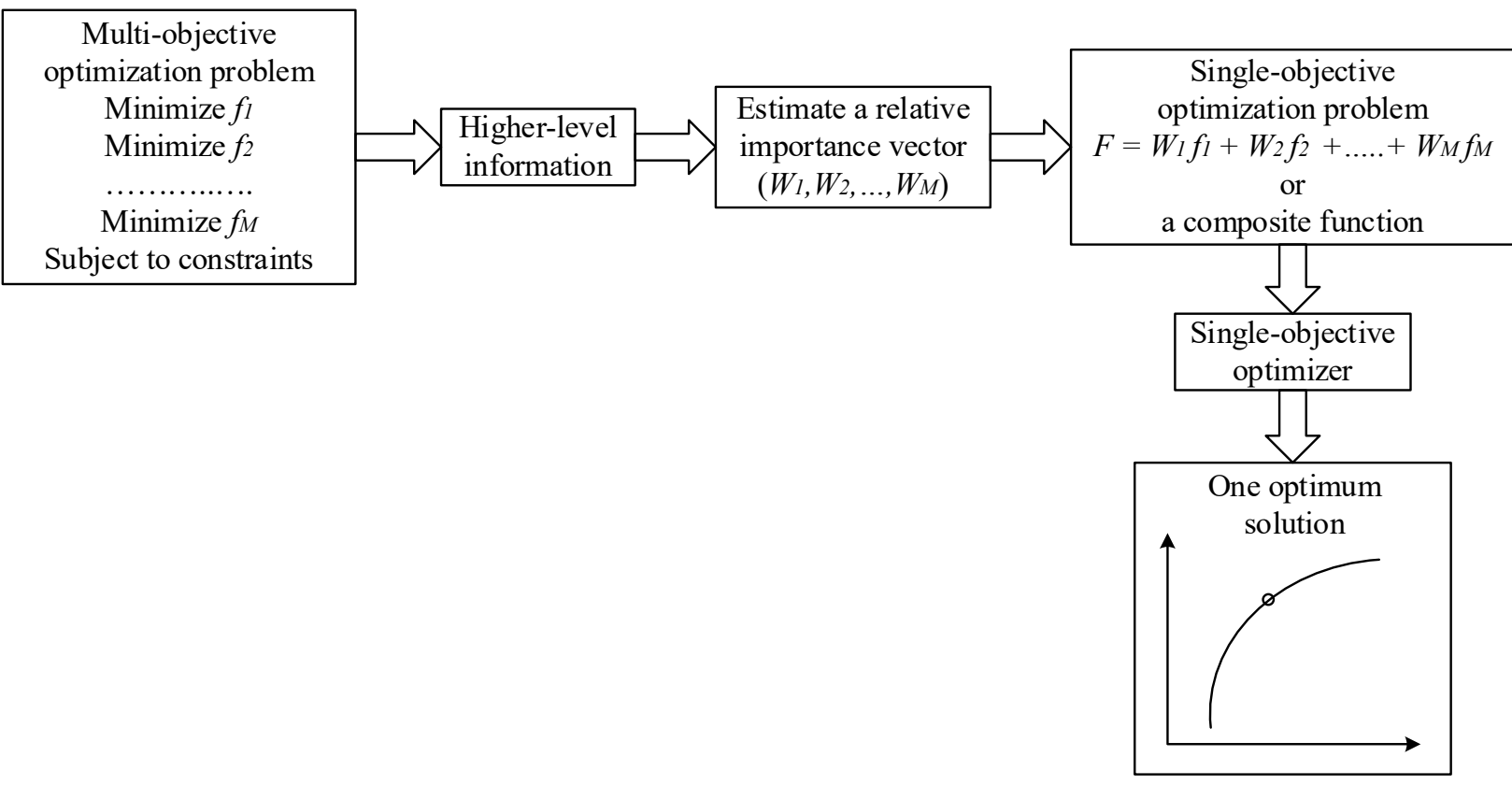

Figure 2.4 Schematic of a preference-based MOO approach. Redrawn from the original in MultiObjective Optimization Using Evolutionary Algorithms (Deb, 2001).

Deb (2001) suggested the following two steps for an ideal MOO approach:

- Step1 - find a multiple trade-off optimal solutions by equally considering all objectives to be important

- Step2-choose one of the obtained solution using higher-level information.

Figure 2.5 illustrates a recommended ideal MOO approach. The author considered this approach is more methodical, more practical, and less subjective — it doesn't require any relative preferences and provides a complete knowledge of the problem. User-supplied information is used only to evaluate and compare obtained trade-off solutions. The proposed EB-MOO methodology is structured around this ideal MOO approach. 


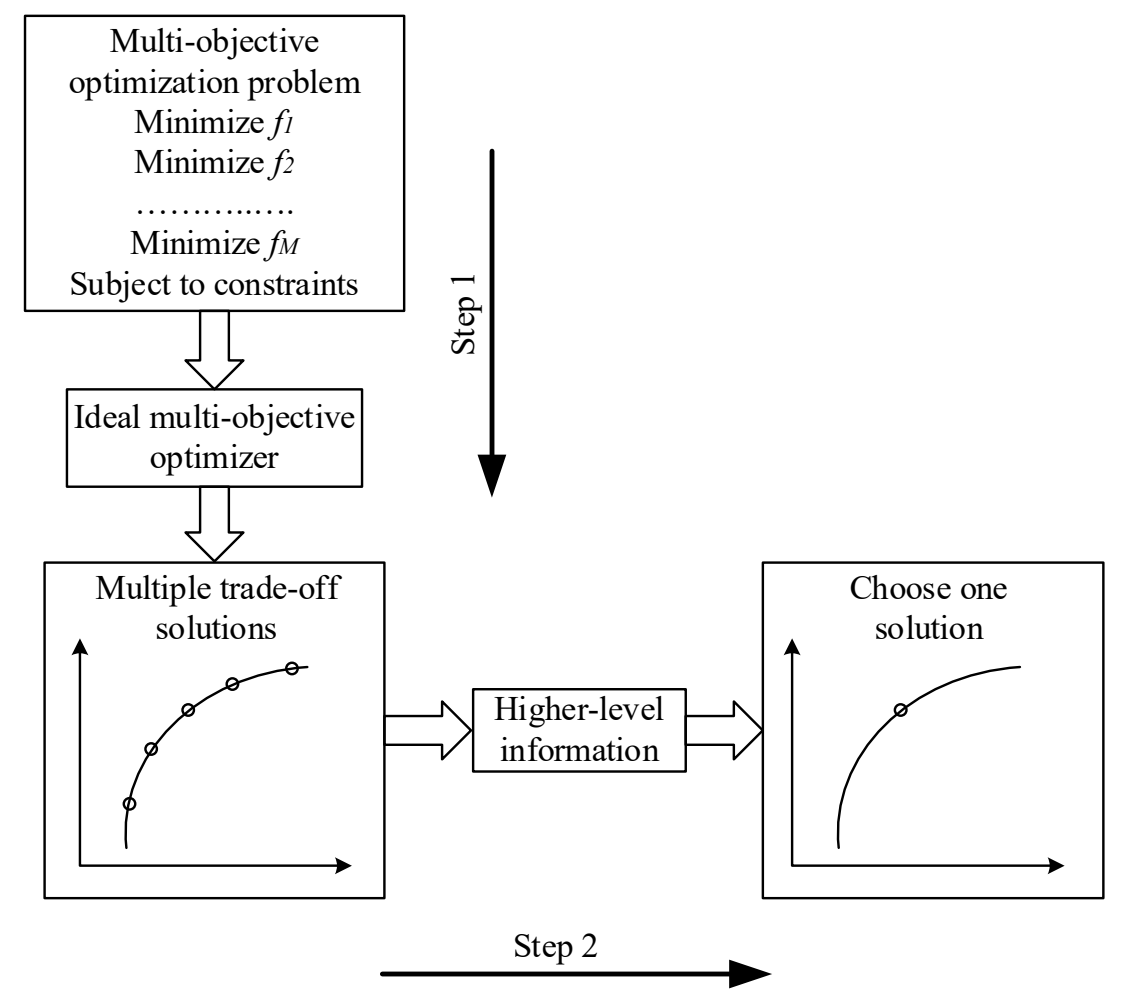

Figure 2.5 Schematic of an ideal MOO approach. Redrawn from the original in Multi-Objective Optimization Using Evolutionary Algorithms (Deb, 2001).

\subsection{Multiple Criteria Analyses}

Multiple criteria (or multiple objectives) analysis is widely used to support a decision making involving multiple criteria (monetary and non-monetary). It consists of using preferences to guide project selection through weighting and scoring procedures (Maggiore \& Ford, 2016). A representative score combining agency weighting and scaling preferences is assigned to be used in the prioritization or optimization process. Various methods and techniques are used in the assessment, including the weighted sum method (WMS), weighted product method (WPM), utility function method, simple multi-attribute rating technique (SMART), analytic hierarchy process (AHP), preference ranking organization method for enrichment evaluation (PROMETHEE), elimination and choice translating reality (ELECTRE), and hierarchical decision trees (Shoghli, 2014). These methods and techniques capture and quantify decision makers' preferences and 
account for inherent risks and uncertainties associated with the criteria. However, predicted decision outcomes may vary considerably based on the chosen technique or method.

Krugler et al. (2007) demonstrated that a decision-making problem involving multiple decision criteria can easily be implemented if all criteria are combined into a single criterion utility through the process of weighting, scaling and amalgamation - consistent with the multi-attribute utility theory (Keeney \& Raiffa, 1993) to account for the inherent risk and uncertainty associated with the different criteria. Criteria can be formulated using linear or nonlinear functions; thought, a sensitivity analysis is generally needed to identify the most suitable models (Krugler et al., 2007). Labi (2014) explicitly described these three steps of the process in his book Introduction to Civil Engineering Systems: A Systems Perspective to the Development of Civil Engineering Facilities. The weighing process consists of assigning weights (importance factors) to criteria and plays a very influential role in the selection of treatments. Direct weighting method, Delphi method, regression techniques, observed-derived weighting method, AHP, etc. are used to set relative weights. To apply the weights, the decision criteria of different units need to be scaled. The scaling process provides a common scale of measurement. Scaling is a leveling technique used to convert criteria with different dimensions (monetary and non-monetary) or units of measurement into a normalized scale to be compared. Different methods and techniques have been used for scaling such as linear scaling functions, monetization, direct rating, mid-value splitting method, gamble method, and others. The amalgamation process involves combining single-criterion utility functions to an overall utility function, considering the established weights. Different methods and techniques have been used for amalgamation such as WMS, WPM, AHP of amalgamation, ELECTRE, goal programming method, and step method (STEM). 
In the NCHRP Report 590 (Patidar et al., 2007), a multi-criterion (i.e. bridge condition, safety, traffic flow disruption, and vulnerability) approach to evaluate bridge improvement alternatives using an optimization heuristic process is introduced for BMSs. The report proposes a rational process for constructing a total utility function by

(1) developing a single-criterion utility function for each performance measure or goal;

(2) scaling each single-criterion utility function to a common scale, capturing decision makers' preferences; and

(3) combining the scaled single-criterion utility functions using relative weights, capturing decision makers' preferences.

The determination of relative weights requires an initial feedback from each decision maker to capture preferences; different weighing methods and aggregations techniques are then deployed to produce the best group representation. Tables 2.2 and 2.3 summarize the relative weights recommended for the studied goals and performance measures.

Table 2.2 Example of relative weights: individual performance measures

\begin{tabular}{|c|c|c|c|}
\hline Overall Goal & $\begin{array}{c}\text { Performance } \\
\text { Measure }\end{array}$ & Relative Weight & Total \\
\hline \multirow{3}{*}{$\begin{array}{l}\text { Condition } \\
\text { Preservation }\end{array}$} & NBI Ratings & 0.271 & \multirow{3}{*}{1.000} \\
\hline & Health Index & 0.507 & \\
\hline & Sufficiency Rating & 0.222 & \\
\hline \multirow{3}{*}{ NBI Ratings } & Deck & 0.330 & \multirow{3}{*}{1.000} \\
\hline & Superstructure & 0.340 & \\
\hline & Substructure & 0.330 & \\
\hline \multirow{2}{*}{ Traffic Safety } & Geometric Rating & 0.570 & \multirow{2}{*}{1.000} \\
\hline & Inventory Rating & 0.430 & \\
\hline \multirow{4}{*}{$\begin{array}{l}\text { Protection } \\
\text { from Extreme } \\
\text { Events }\end{array}$} & Scour & 0.385 & \multirow{4}{*}{1.000} \\
\hline & Fatigue & 0.265 & \\
\hline & Earthquake & 0.205 & \\
\hline & Other & 0.145 & \\
\hline
\end{tabular}

Note. Adapted from the NCHRP Report 590: Multi-Objective Optimization for Bridge Management Systems (Patidar et al., 2007). 
Table 2.3 Example of relative weights: overall goals

\begin{tabular}{lc}
\hline \multicolumn{1}{c}{ Overall Goal } & Relative Weight \\
\hline Bridge Preservation & 0.360 \\
\hline Safety & 0.205 \\
\hline Protection from Extreme Events & 0.150 \\
\hline Agency Cost & 0.175 \\
\hline User Cost & 0.110 \\
\hline Total & 1.000 \\
\hline
\end{tabular}

Note. Adapted from the NCHRP Report 590: Multi-Objective Optimization for Bridge Management Systems (Patidar et al., 2007).

As emphasized in Chapter 1, efforts to incorporate MODA into bridge management practice by state DOTs and MPOs are expected to increase, especially with the transportation performance measurement requirements of MAP 21 (discussed earlier). Caltrans is considered one of the earliest state DOTs that moved toward this transition. The State Highway Operation and Protection Program (SHOPP) Pilot Project Report, Phase 1: A Framework for Project Prioritization (Caltrans, 2015) documents Caltrans' project prioritization process which is based on a customized MODA. A tool prototype that applies a value function and uses MODA was produced in Microsoft Excel to identify an optimized portfolio of projects from a pool of SHOPP projects. The tool supports the TAMP development process and increases transparency of the project selection process.

AssetManager NT, discussed in the NCHRP Report 545: Analytical tools for asset management (Cambridge Systematics, Inc., 2005), is another spreadsheet-based tool developed by researchers as an investment analysis tool to integrate data from other investment analysis and management systems such as NBIAS and BrM. The tool explores the impacts of different levels of investment categories over the long term; investment categories could be defined based on asset classes (pavement, bridge, etc.), geographical areas (districts, regions, etc.), or sub-networks (NHS, primary corridors, local network of roads, etc.). 


\subsection{Trade-off Analysis and Optimization}

Kachua (2011) defined trade-off analysis as a simulation of "what if" scenarios to examine the long-term impacts of different investment strategies for decision making. The use of optimization and trade-off analysis in TAM permits decision makers to identify appropriate strategies/scenarios, understand relationships between different strategies/scenarios, and communicate any impacts. A practical approach to perform a trade-off analysis is to investigate optimal solutions, representing a Pareto solution set. A solution in a Pareto solution set (or Pareto solution) is superior to the rest of the solutions in the search space, and no Pareto solution is superior than the other Pareto solutions. The improvement of an objective associated with a Pareto solution results in worsening of another one, at least. (Konak et al., 2006; Zitzler et al., 2000; Shoghli, 2014) pointed out to the importance of

(1) the best-known Pareto frontier to be as close as possible to the true Pareto frontier,

(2) the best-known Pareto solutions to be uniformly distributed and diverse to provide the decision makers a true understanding of trade-offs, and

(3) the best-known Pareto frontier to capture the whole spectrum of the true Pareto frontier.

A diverse set of Pareto solutions cannot be obtained by transferring a MOO problem into a singleobjective optimization problem — constructing a Pareto frontier (or trade-off frontier) requires several independent runs by varying certain parameters; and yet, optimal solutions are not guaranteed. MOO techniques, consisting of a simultaneous optimization of multiple competing objectives subject to constraints, attempt to provide a diverse set of optimal or near-optimal solutions - constituting a frontier of trade-offs between objectives. They help providing a complete knowledge of the problem (Talbi, 2009). 
The NCHRP Report 806: Cross-Asset Resource Allocation and the Impact on Transportation System Performance (Maggiore \& Ford, 2016) presents Figure 2.6 as an example of trade-off analysis between two performance measures to inform decision makers of the impacts of moving from one area of the Pareto frontier to another. Performance targets are also plotted to assist in the decision-making process.

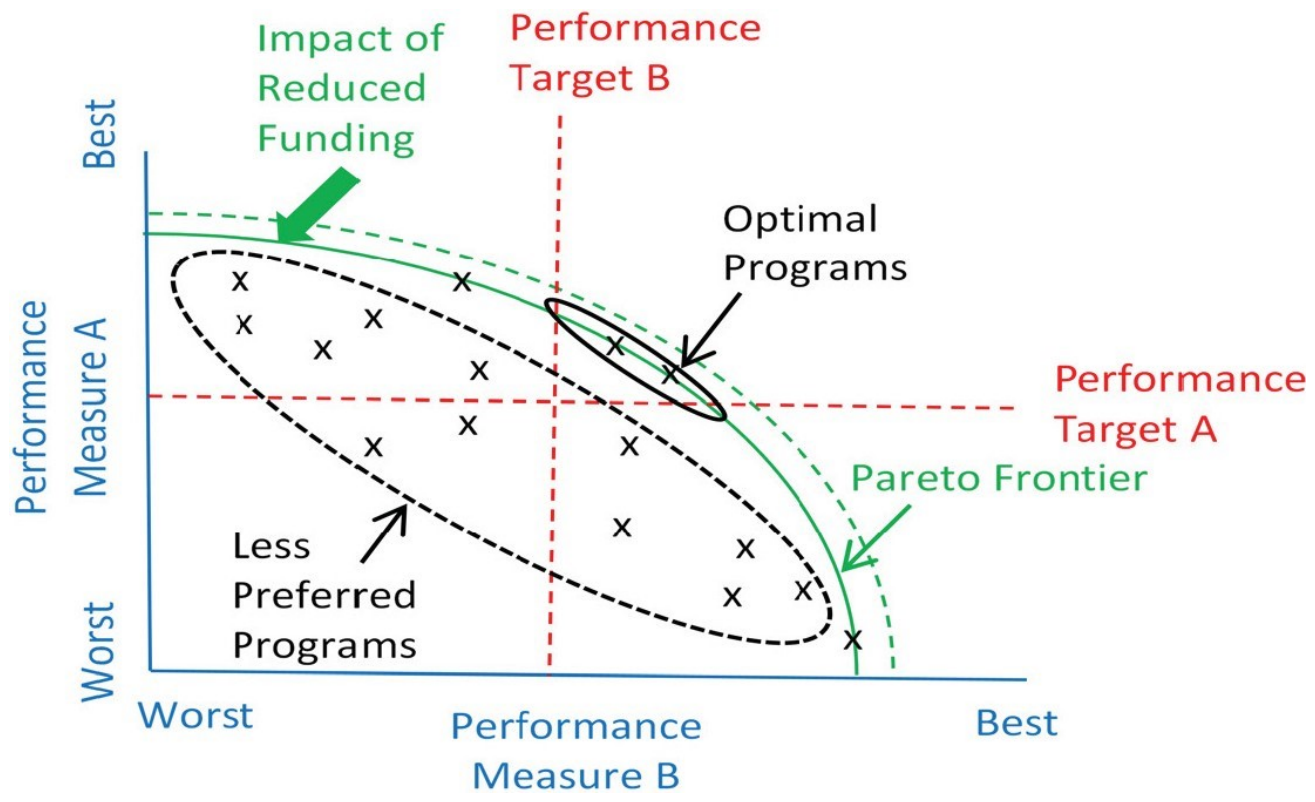

Figure 2.6 Example of a trade-off analysis. Adapted from the NCHRP Report 806: Cross-Asset Resource Allocation and the Impact on Transportation System Performance (Maggiore \& Ford, 2016).

Bai (2012) proposed a trade-off-based MOO framework for TAM. A hybrid algorithm based on NSGA-II was developed to generate Pareto frontiers for conducting trade-off analyses between costs and performance measures. The proposed algorithm converges faster and generates betterdistributed Pareto frontiers when compared to traditional algorithms. The trade-off relationships between performance measures do vary at different budget levels. The chance-constrained programming was applied to incorporate performance measure uncertainties into the proposed MOO framework. Trade-off relationships under uncertainties do vary at different confidence levels - a high confidence requirement results in lower performance at the same cost level. 
Kachua (2011) developed a large-scale linear programming algorithm to support long-term investment planning for transportation and municipal infrastructure. Through a case study, the developed linear programming algorithm demonstrated that it is feasible to implement cross-asset optimization and trade-off analysis to support long-term planning. The linear programming algorithm considers "what if" scenarios and effectiveness of individual treatments for a large-sized network. It also examines all possible treatment options for a given asset type and provides information on how to select the right treatment, at the right place, and at the right time.

Figure 2.7 shows the front-end interface of the MOOS network-level model (discussed in the previous section) to help bridge program managers to visualize the performance of a network of bridges - showing projected investment needs and performance, and trade-offs between funding levels and performance. This front-end interface provides most of the controls and outputs in one convenient layout while reserving all complex mathematical calculations for back-end processing.

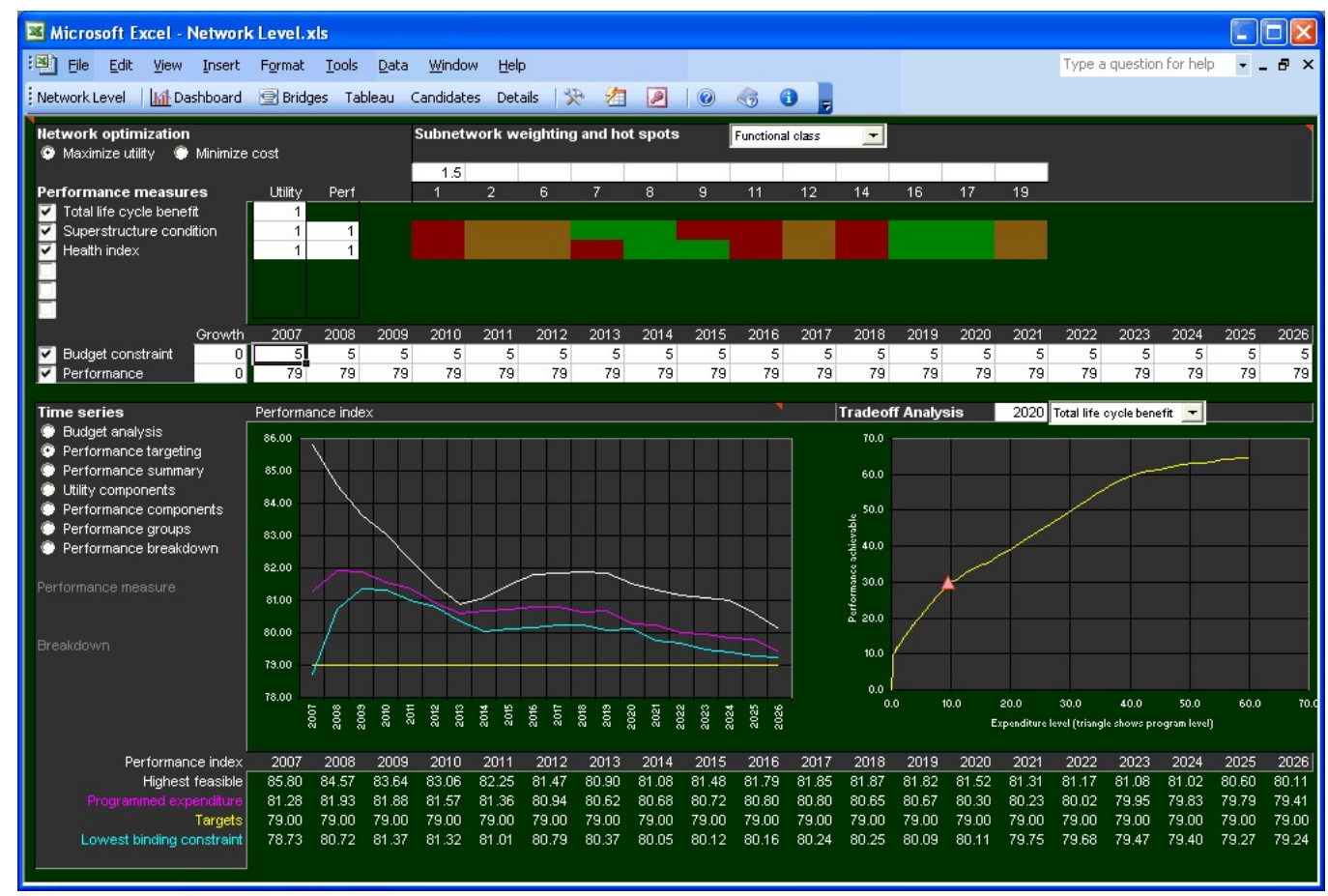

Figure 2.7. Front-end interface of the MOOS network-level model. Adapted from the NCHRP Report 590: Multi-Objective Optimization for Bridge Management Systems (Patidar et al., 2007). 
BrM version 5.2.3 facilitates trade-off analyses for prioritizing preservation and maintenance work through its scenario explorer module. Users can create multiple scenarios to evaluate impacts of different funding levels. They can also create scenarios for several items that may impact the results of the optimization (for example, scenarios for different performance targets such as the percent of deck area of structurally deficient bridges). Figure 2.8 is an example of program results displayed in BrM version 5.2.3. The green triangle on the benefit-cost frontier, which is constructed based on a well-known incremental benefit-cost (IBC) heuristic (discussed in Chapter 5), shown in the upper-right graph, is selected by the user as the preferred program strategy. The graph also shows other program strategies with greater utility benefits (measuring the benefit of combined objectives) but involving higher costs (Boyle, 2017).

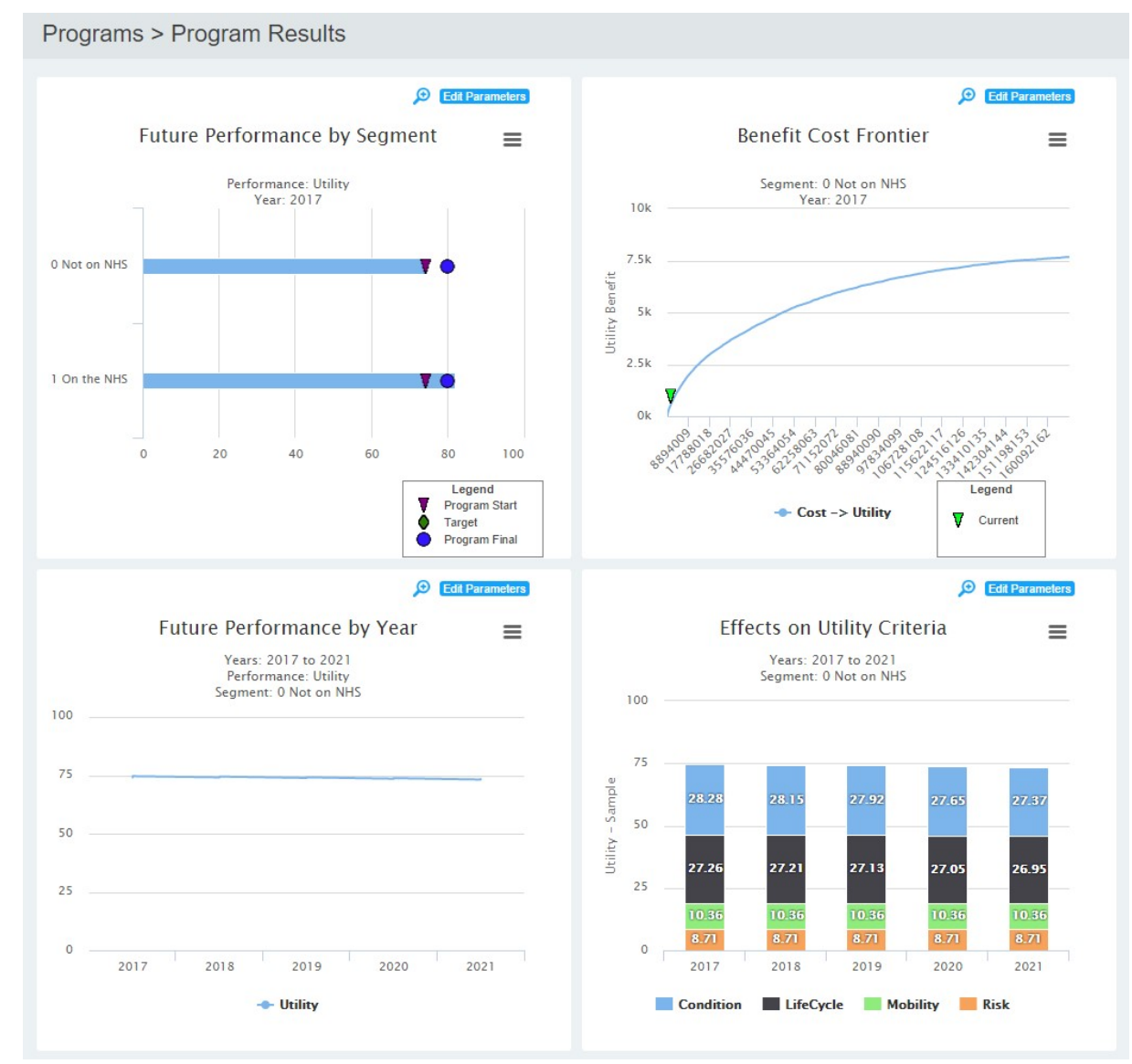

Figure 2.8. Example of program results as displayed in BrM version 5.2.3. Adapted from AASHTOWare Bridge Management 5.2.3.: Conducting Trade-off Analysis (Boyle, 2017). 


\subsection{Evolutionary Algorithms}

\subsubsection{Optimization Models}

Figure 2.9 was developed by Talbi (2009) to illustrate the different families of optimization models used to solve decision-making problems. The most common models belong to the mathematical programming family such as integer programming, dynamic programming, linear programming, and goal programming.

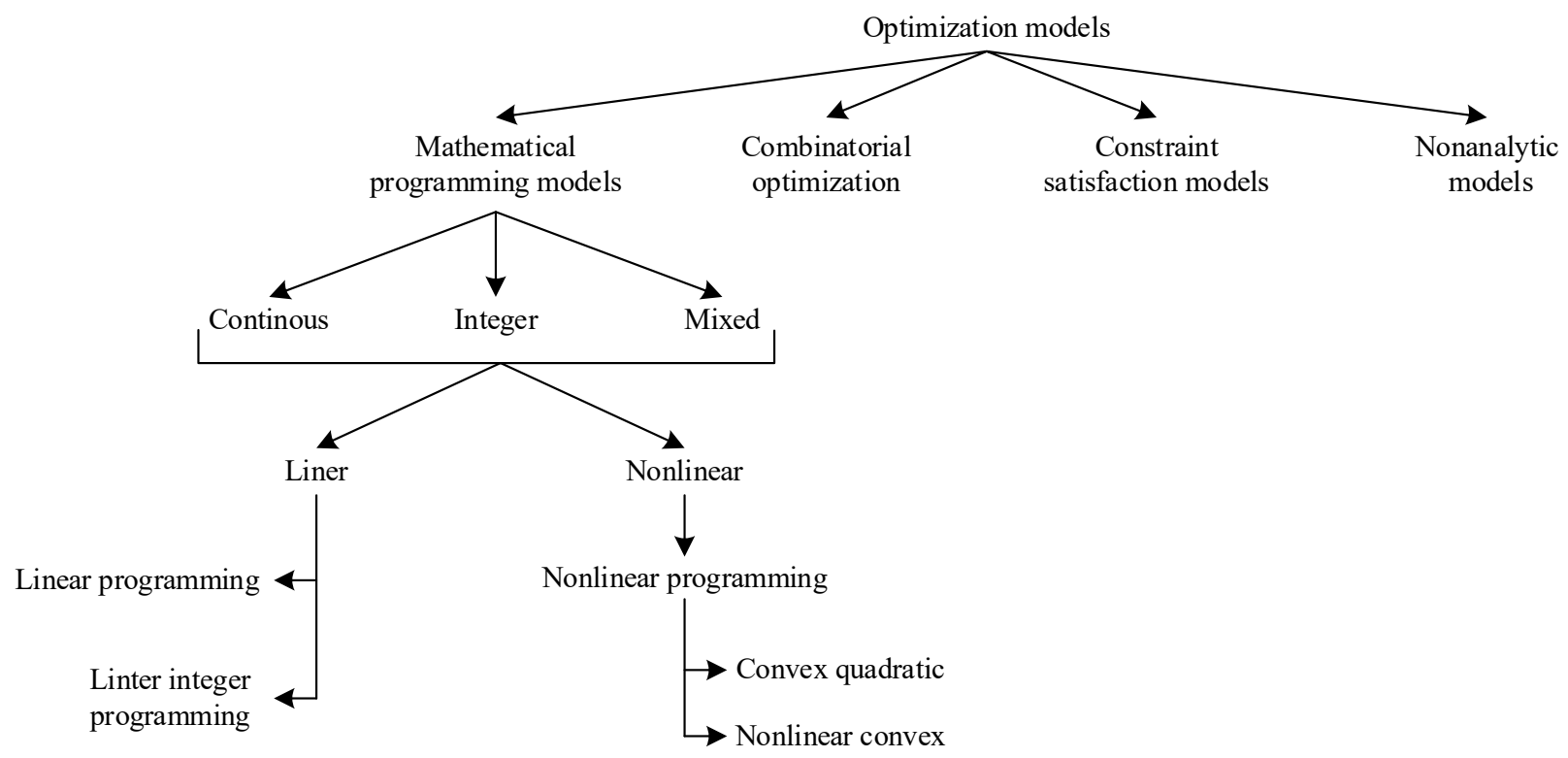

Figure 2.9 The different families of optimization models. Redrawn from the original in Metaheuristics: From Design to Implementation (Talbi, 2009).

The author further classified the different optimization algorithms as shown in Figure 2.10 into two main categories: Exact algorithms and heuristics. Exact algorithms guarantee finding a single optimal solution but applicable to only small size optimization problems. On the contrary, approximate algorithms can handle large-scale optimization problems. The approximate algorithms are decomposed into two classes: specific heuristics and metaheuristics. Specific heuristics are problem-dependent, whereas metaheuristics are applicable to a large variety of optimization problems. Most of the metaheuristics mimic natural metaphors (based on biological 
and animal behaviors) such as genetic algorithms (GAs) and ant colony optimization (ACO) to solve complex optimization problems (Talbi, 2009). Both heuristics and metaheuristics cannot guarantee optimal solutions but very close to the real Pareto optimal solutions (Bai, 2012). Different performance criteria, such as quality of solutions, computational effort (search time), robustness, ease of use, simplicity, flexibility, development cost, are used to evaluate metaheuristics (Talbi, 2009).

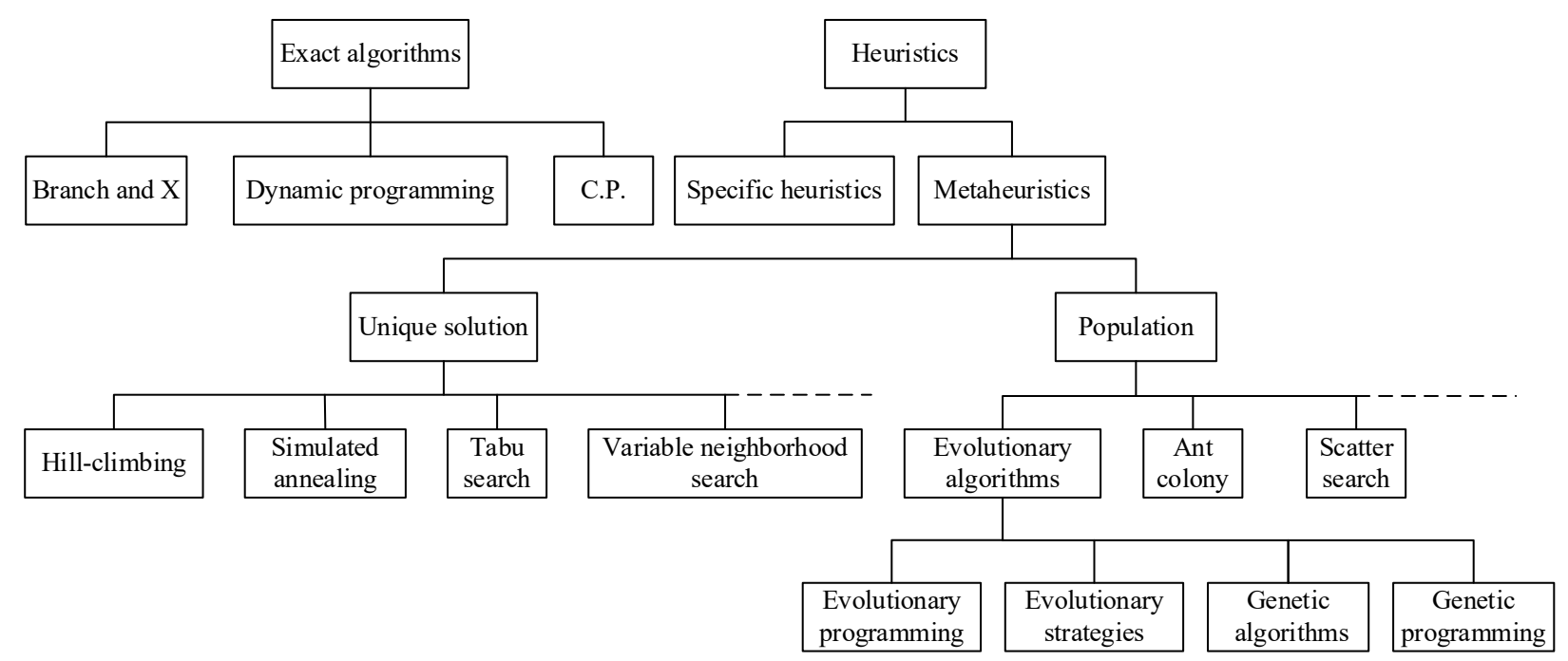

Figure 2.10 The different classifications of optimization algorithms. Redrawn from the original in Metaheuristics: From Design to Implementation (Talbi, 2009).

\subsubsection{Genetic Algorithms}

Evolutionary algorithms (EAs) are population-based search metaheuristics inspired by Darwinian evolutionary theory. EAs produce a high quality of solutions (optimal or near-optimal) in a reasonable time for non-linear large-sized problems. EAs are divided into several branches such as GA, shuffled frog leaping (SFL), ACO, particle swarm optimization (PSO), and others. The literature review was devoted to study GAs and identify the most appropriate for integration-it's believed that GAs are well-suited for the different complex optimization problems of this research (i.e., non-linear and combinatorial optimization problems). 
GAs (Holland, 1975) are stochastic search heuristics that rely on the "survival of the fittest" principle from the biological sciences, and the use of evolution operators makes them very effective in performing global search. They are widely used (in system modeling and identification, planning and control, resource allocation, data mining, engineering design, machine learning, and other domains) and capable of handling discrete and combinatorial optimization problems with many decision variables (Kachua, 2011). Deb (2009) described the different involved genetic operators in greater detail in his book Multi-Objective Optimization Using Evolutionary Algorithms.

Figure 2.11 shows a flowchart of the working principle of a GA. The cycle starts by generating a random population of solutions (a random set of chromosomes; each is composed of a string of genes). The objective function values and constraint violations are calculated for each solution. A performance function or relative merit using the calculated values is assigned (refer to as the fitness). The fitness of the population is then evaluated. If the termination condition is not satisfied, the population is modified through three main genetic operators (i.e., reproduction, crossover and mutation operators), and a better fitted population is generated. This first cycle represents the first generation (or first iteration). The algorithm exits the loop when the termination criterion is satisfied. 


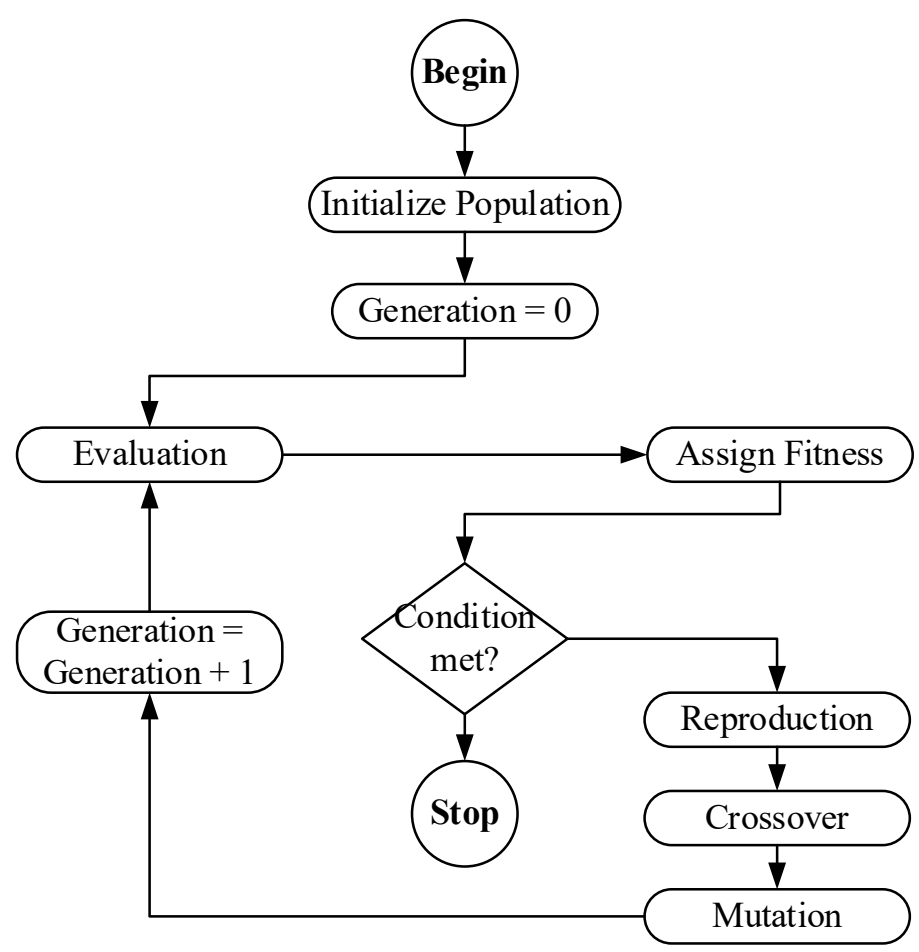

Figure 2.11 A flowchart of the working principle of a GA. Redrawn form the original in Multi-Objective Optimization Using Evolutionary Algorithms (Deb, 2009).

Schaffer (1985) developed the first genetic algorithm Vector Evaluation Genetic Algorithm (VEGA). The second wave of genetic algorithms includes Pareto Archived Evolution Strategy (PAES) (Knowles \& Corne, 2000), Pareto Envelope-based Selection Algorithm (PESA) (Corne et al., 2000), Niched Pareto Genetic Algorithm 2 (NPGA 2) (Erickson et al., 2001), Strength Pareto Evolutionary Algorithm 2 (SPEA-2) (Zitzler et al., 2001), NSGA-II (Deb et al., 2002), and others. Table 2.4 lists the advantages and disadvantages of well-known GAs as highlighted by Konak (2006) in the study Multi-Objective Optimization Using Genetic Algorithms: A Tutorial. Reliability Engineering \& System Safety. 
Table 2.4 Advantages and disadvantages of well-known GAs

\begin{tabular}{|c|c|c|}
\hline Algorithm & Advantages & Disadvantages \\
\hline $\begin{array}{l}\text { Vector Evaluated } \\
\text { Genetic Algorithm } \\
\text { (VEGA) }\end{array}$ & $\begin{array}{l}\text { First multi-objective GA Straightforward } \\
\text { implementation }\end{array}$ & $\begin{array}{l}\text { Converges to the extreme of } \\
\text { each objective }\end{array}$ \\
\hline $\begin{array}{l}\text { Multi-Objectives } \\
\text { Genetic Algorithm } \\
\text { (MOGA) }\end{array}$ & Simple extension of single-objective GA & $\begin{array}{l}\text { Usually slow convergence } \\
\text { Problems related to niche size } \\
\text { parameter }\end{array}$ \\
\hline $\begin{array}{l}\text { Weight-Based Genetic } \\
\text { Algorithm } \\
\text { (WBGA) }\end{array}$ & $\begin{array}{l}\text { Simple extension of single } \\
\text { objective GA }\end{array}$ & $\begin{array}{l}\text { Difficulties in nonconvex } \\
\text { objective function space }\end{array}$ \\
\hline $\begin{array}{l}\text { Niched Pareto Genetic } \\
\text { Algorithm } \\
\text { (NPGA) }\end{array}$ & $\begin{array}{l}\text { Very simple selection process with } \\
\text { tournament selection }\end{array}$ & $\begin{array}{l}\text { Difficulties in nonconvex } \\
\text { objective function space }\end{array}$ \\
\hline $\begin{array}{l}\text { Random Weight Genetic } \\
\text { Algorithm } \\
\text { (RWGA) }\end{array}$ & Efficient and easy to implement & $\begin{array}{l}\text { Performance depends on cell } \\
\text { sizes } \\
\text { Prior information needed about } \\
\text { objective space }\end{array}$ \\
\hline $\begin{array}{l}\text { Pareto Envelope-based } \\
\text { Selection Algorithm } \\
\text { (PESA) }\end{array}$ & $\begin{array}{l}\text { Easy to implement } \\
\text { Computationally efficient }\end{array}$ & $\begin{array}{l}\text { Not a population based } \\
\text { approach }\end{array}$ \\
\hline $\begin{array}{l}\text { Pareto Archived } \\
\text { Evolution Strategy } \\
\text { (PAES) }\end{array}$ & $\begin{array}{l}\text { Random mutation hill-climbing strategy } \\
\text { Easy to implement } \\
\text { Computationally efficient }\end{array}$ & $\begin{array}{l}\text { Performance depends on cell } \\
\text { sizes }\end{array}$ \\
\hline $\begin{array}{l}\text { Non-dominated Sorting } \\
\text { Genetic Algorithm } \\
\text { (NSGA) }\end{array}$ & Fast convergence & $\begin{array}{l}\text { Problems related to niche size } \\
\text { parameter }\end{array}$ \\
\hline $\begin{array}{l}\text { Non-dominated Sorting } \\
\text { Genetic Algorithm II } \\
\text { (NSGA-II) }\end{array}$ & $\begin{array}{l}\text { Single parameter }(\mathrm{N}) \\
\text { Well tested } \\
\text { Efficient }\end{array}$ & $\begin{array}{l}\text { Crowding distance works in } \\
\text { objective space only }\end{array}$ \\
\hline $\begin{array}{l}\text { Strength Pareto } \\
\text { Evolutionary Algorithm } \\
\text { (SPEA) }\end{array}$ & $\begin{array}{l}\text { Well tested } \\
\text { No parameter for clustering }\end{array}$ & Complex clustering algorithm \\
\hline $\begin{array}{l}\text { Strength Pareto } \\
\text { Evolutionary Algorithm } \\
2 \text { (SPEA-2) }\end{array}$ & $\begin{array}{l}\text { Improved SPEA } \\
\text { Makes sure extreme points are preserved }\end{array}$ & $\begin{array}{l}\text { Computationally expensive } \\
\text { fitness and density calculation }\end{array}$ \\
\hline $\begin{array}{l}\text { Rank-Density based } \\
\text { Genetic Algorithm } \\
\text { (RDGA) }\end{array}$ & $\begin{array}{l}\text { Dynamic cell update } \\
\text { Robust with respect to the number of } \\
\text { objectives }\end{array}$ & $\begin{array}{l}\text { More difficult to implement } \\
\text { than others }\end{array}$ \\
\hline $\begin{array}{l}\text { Dynamic Multi- } \\
\text { Objective Evolutionary } \\
\text { Algorithm (DMOEA) }\end{array}$ & $\begin{array}{l}\text { Includes efficient techniques to update } \\
\text { cell densities } \\
\text { Adaptive approaches to set GA } \\
\text { parameters }\end{array}$ & $\begin{array}{l}\text { More difficult to implement } \\
\text { than others }\end{array}$ \\
\hline
\end{tabular}




\subsubsection{Non-Dominated Sorting Genetic Algorithm-II}

NSGA-II is arguably the most popular for solving MOO problems. It's a well-known nondomination based algorithm among the GAs. The algorithm relies on a non-dominated sorting process. The population is sorted based on non-domination, and only the best " $\mathrm{N}$ " individuals are kept, where "N" is the population size. It is highly referenced in the literature. NSGA-II is capable of handling large-sized NP-hard combinatorial optimization problems (discussed later in the dissertation). It has the following three distinct features (Deb, 2009):

(1) deploys an elitist principle,

(2) deploys an explicit diversity preserving mechanism, and

(3) emphasizes non-dominated solutions.

NSGA-II is robust and reliable for solving MOO problems with less computational efforts. It ensures solution diversity and convergence to a near true Pareto frontier. NSGA-II is deployed as the main optimizer for the three optimization modules proposed in this research. It's easy to substitute this chosen optimizer by any other metaheuristic algorithm with similar capabilities such as SPEA-2, PESA, and MOGA. However, the quality of obtained solution sets should be evaluated using experimental tests or performance metrics (e.g., hypervolume, generational distance, epsilon indicator, and inverted generational distance metrics). Performance metrics generally consider the following three aspects of a solution set, discussed in the study of Okabe and Sendhoff (as cited in Riquelme et al., 2015):

(1) convergence, i.e., closeness to the theoretical Pareto frontier;

(2) diversity, i.e., distribution as well as spread; and

(3) number of solutions. 


\subsection{Summary and Conclusions}

In this chapter, a review of the previous work, studies, and best practices related to the research areas and objectives is presented. The chapter summarized the literature search on TAM, risk assessment and management, MCDM, and MOO. The chapter included brief descriptions of the identified significant references. The gathered information and best practices from these references provided the background to refine the conceptual TAM framework and shape the EB-MOO methodology. The chapter discussed the transportation planning and programming process and the new performance-based planning and TAMP requirements of MAP 21. Risk could impact the delivery of investment strategies or the performance of a network of assets or a single asset. Several best practices and studies on risk assessment and management were also highlighted in this chapter. However, the integration of a risk assessment model into the proposed EB-MOO methodology is beyond the research scope.

The literature review revealed that empirical and non-empirical ranking methods, IBCA, multiple criteria analyses are widely applied for selecting bridge projects. A large variety of optimization techniques such as mathematical programming, metaheuristics, and optimization algorithms have been applied to solve bridge optimization problems. The use of one technique versus another depends on the characteristics of the optimization problem. Optimization techniques generally produce optimal or near-optimal solutions by either maximizing or minimizing preferred objectives subject to a set of constraints. Various investment analysis tools and systems using optimization techniques for bridge investment decision making either by state DOTs, FHWA, or researchers were discoursed in this chapter.

The chapter discussed the different MOO approaches, methods and techniques, and analytical tools commonly used to support investment decision making involving multiple criteria (or objectives). 
The importance of carrying out a trade-off analysis in TAM has been emphasized in this chapter. Obtained set of optimal solutions (representing a Pareto solution set) can be further examined through trade-off analyses to identify appropriate strategies/scenarios, understand relationships between them, and communicate any impacts. EAs produce a high quality of solutions (optimal or near-optimal) in a reasonable time for non-linear large-sized optimization problems. The literature review was devoted to investigate this family of population-based search metaheuristics, specifically focusing on GAs to identify the most appropriate for integration. It was determined that the metaheuristic NSGA-II is well-suited for solving the different complex optimization problems of this research (i.e., non-linear and combinatorial optimization problems) in less computational efforts - it guarantees solution diversity and convergence to a near true Pareto frontier. NSGA-II is deployed as the main optimizer for the three optimization modules proposed in this research. 


\section{CHAPTER 3-TRANSPORTATION ASSET MANAGEMENT FRAMEWORK \& ELEMENT-BASED MULTI-OBJECTIVE OPTIMIZATION METHODOLOGY}

- Task 2: Proposing a Goal-Driven Transportation Asset Management Framework

- Task 3: Proposing an Element-Based Multi-Objective Optimization Methodology

\subsection{Introduction}

This chapter introduces the proposed goal-driven TAM framework applying the principles of the Transportation Asset Management Guide: Prepared for NCHRP Project, 20-24(11) (Cambridge Systematics, Inc. et al., 2002) (hereinafter referred to as "Guide") to state DOTs with their transition efforts to performance management and performance-based planning and programming. The framework focuses on one transportation asset class (i.e., bridge) and relies on quality data and agency established policies, goals, performance measures and targets, anticipated funding levels, and customer expectations to guide the management process of assets. The framework can be expanded to accommodate other asset classes or modes. The framework is designed to be integrated into the long-range planning and programming process - to provide more transparency, address public needs, and support the development and evaluation of the LRTP, TIP/STIP, and TAMP. Previous work on TAM and best practices were identified throughout a comprehensive literature review (refer to Chapter 2). The framework is structured around a continuing performance monitoring to assess effectiveness, identify gaps, and adjust as needed.

Little research work has been focused on systematic element-based optimization methodologies for bridge project selection. This research was also undertaken to develop a novel MOO 
methodology to assess element improvement needs of existing bridges and recommend nearoptimal or optimal investment strategies for an effective management of bridge activities in both short- and long-term planning horizons. This chapter provides an overview of the proposed methodology and its five modules (i.e., data processing, improvement, ELO, BLO, and NLO modules). The methodology relies on three distinct screening processes (i.e., element deficiency, alternative feasibility, and solution superiority screening processes) to overcome computer memory and processing time limitations. It's designed to support the development of short- and long-term investment strategies, TIP/STIP development/amendment process, setting of performance targets and program/project priorities, and trade-offs between investment scenarios and performance. The methodology is integrated into the framework, serving as a decision support tool, to support identifying candidate bridge projects, setting project priorities, revaluating funding allocations, or assessing impacts of programmed types of bridge work (i.e., preservation, rehabilitation, and replacement) in the TIP/STIP on system performance.

A MATLAB-based tool prototype structured around the proposed five EB-MOO modules was developed to be utilized for the implementation of the methodology. Several examples of unconstraint and constraint (budget and performance) scenarios were established to test concepts, prove effectiveness, and demonstrate and communicate potential benefits. Results reveal the capability of the methodology to recognize short- and long-term investment needs for bridge programming and planning process. The EB-MOO methodology produces reliable $\mathrm{LC}$ alternative results, generates optimal or near-optimal solutions, predicts performance, and determines longterm intervention strategies and funding requirements. The methodology facilitates trade-offs between funding scenarios and performance. It can be expanded to accommodate other asset classes or modes. 


\subsection{Proposed Transportation Asset Management Framework}

The intent of Task 2 of this research is to transfer previous work on TAM and best practices identified throughout the comprehensive literature review (under Task 1) into a relevant TAM framework. A goal-driven TAM framework is introduced as part of this research to support state DOTs with their transition efforts to performance management and performance-based planning and programming. The framework is founded based on the following principles of TAM as defined in the Guide:

- Policy goals and objectives, including the role of policy formulation in asset management and ways in which policy guidance can benefit from improved asset management;

- Planning and programming, focusing on best practices in reaching decisions on resource allocation for investments in transportation infrastructure;

- Program delivery, looking at options in resource utilization and management methods to deliver programs and services; and

- Information and analysis, including use of information technology (IT) at each stage of asset management; monitoring of asset performance and feedback of this information to improve decision processes in the future, and reporting and communication of key information and results (Cambridge Systematics, Inc. et al., 2002).

The Guide envisions these principles to be applied throughout key business processes using the IT support as shown in Figure 3.1. 


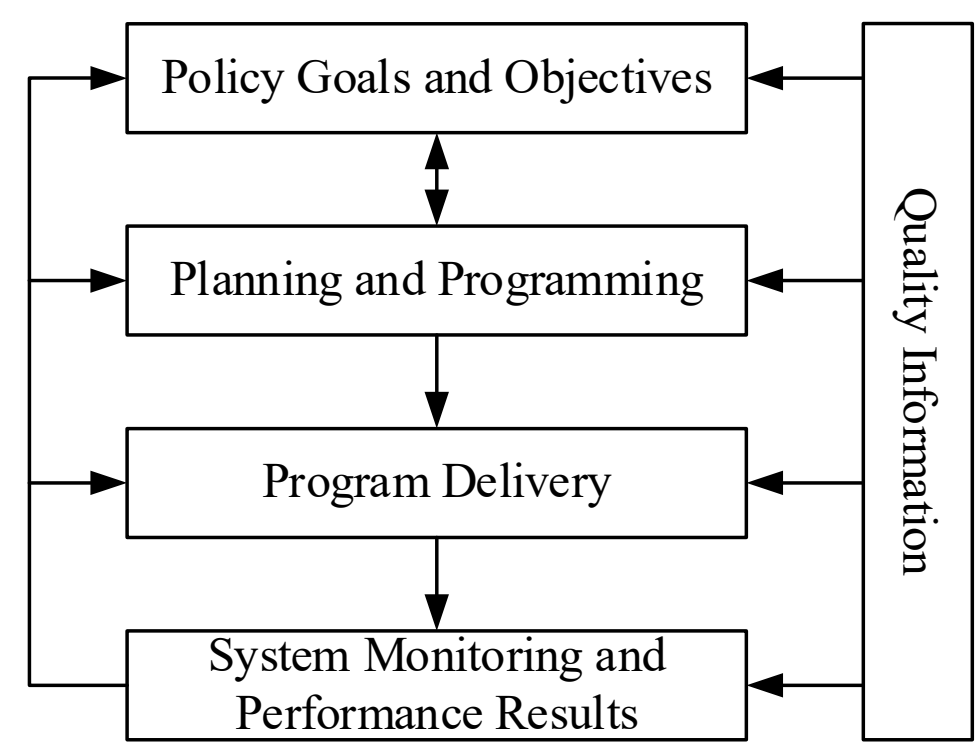

Figure 3.1 Asset management framework recommended by the Guide. Redrawn from the original in the Transportation Asset Management Guide (Cambridge Systematics, Inc. et al., 2002).

The proposed framework focuses mainly on one major transportation asset class (i.e., bridge) state DOTs have been collecting bridge data for years. Though, the framework can expand to accommodate other asset classes (e.g., pavement, sign structures, retaining walls, state DOT's facilities, and intelligent transportation system installations) or even other modes. The framework is driven by policy goals and objectives for better planning and programming, and program delivery. The integrated EB-MOO methodology relies on quality data information, wellestablished performance measures and targets to produce reliable investment strategies progressing toward the defined goals and objectives - trade-offs among asset classes, programs, types of investments, and funding levels to produce informed funding allocations and project prioritizations.

The framework is also structured around a continuing performance monitoring and comparing to performance targets - to assess effectiveness of different programs and determine the necessary adjustments to program areas, goals and objectives, or performance targets. The framework is 
integrated into the long-range planning process to assist in the development and evaluation of the TIP/STIP; for instance, screening projects or setting project priorities in the TIP/STIP based on long-term goals and feedback from public outreach. As stated in Chapter 2, MAP-21 includes a requirement for state DOTs to develop "a risk-based transportation asset management plan for the National Highway System to improve or preserve the condition of the assets and the performance of the system." This risk-based TAMP documents current asset conditions, decision-making practices about allocating resources and managing assets, and progress toward performance targets and national goals. The proposed framework supports the development of the six elements (discussed in Chapter 2) required to be included in the plan. The proposed framework is illustrated in Figure 3.2. 


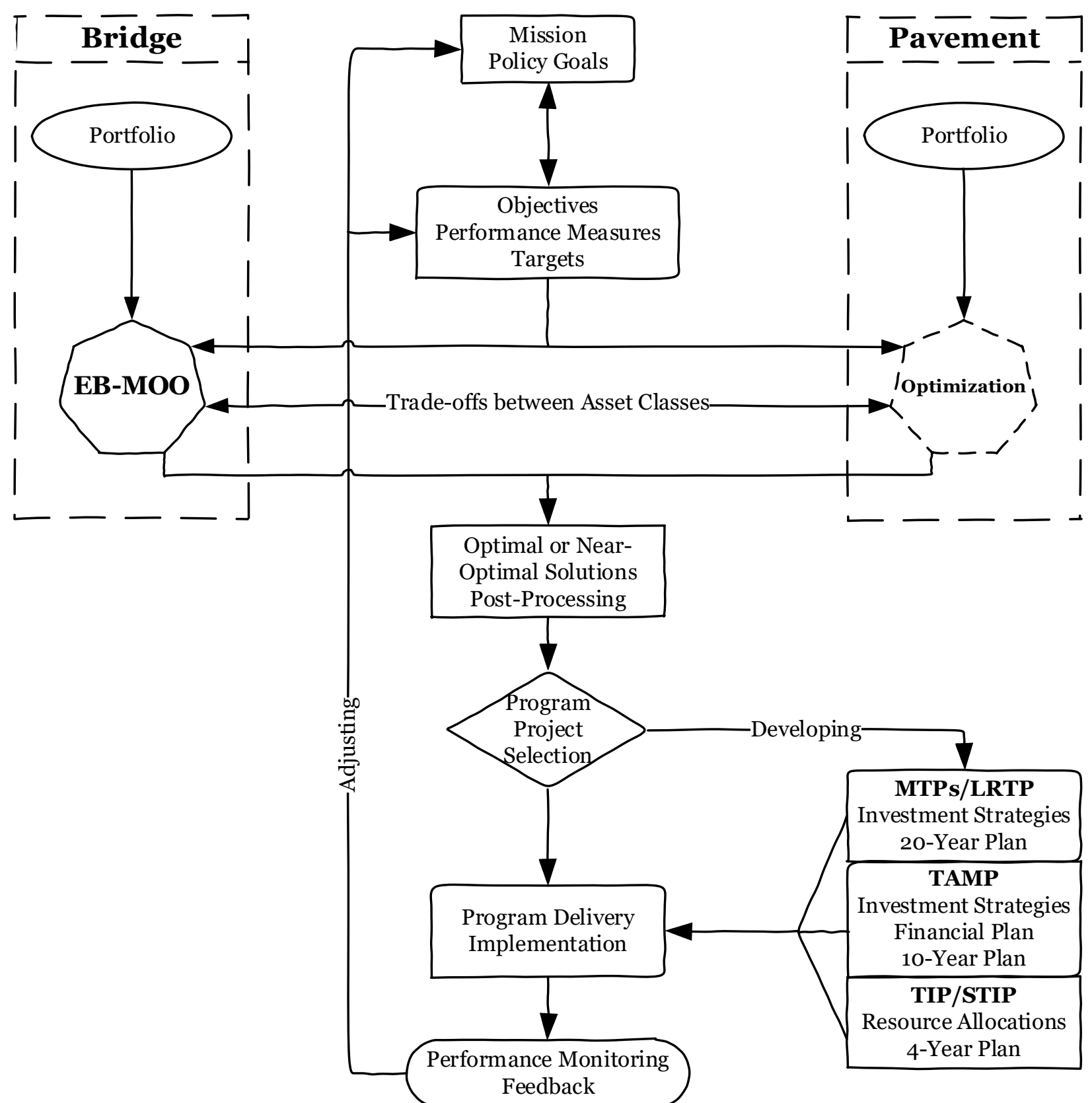

Figure 3.2 The proposed goal-driven TAM framework 


\subsubsection{Policy Goals and Objectives}

As discussed previously, the framework is driven by policy goals and objectives guiding the state DOT's asset management process for investment decisions, resource allocations, and program delivery, considering anticipated funding levels and customers' needs as determined during public outreach in the planning process. State DOTs share a high level of consistency in their choice of policy goals and objectives, despite the great differences in their asset management practices (FHWA, 1999). Goals and objectives are often established to address safety, infrastructure preservation, mobility and congestion, economic development, environmental stewardship, and organizational effectiveness; however, priorities among these goals vary between state DOTs (FHWA, 1999). Most state DOTs had established goals and objectives linked to the national performance goals. MAP-21 requires state DOTs to invest resources in transportation projects, progressing toward the national goals - the current FAST Act continues MAP-21's performance management approach (discussed in Chapter 2). The following Figure 3.3 illustrates the hierarchy of objectives used in the SHOPP Report Phase 2: Application of a Project Prioritization Framework to the 2016 SHOPP (Caltrans, 2016) to represent the Caltrans' fundamental objectives, sub-objectives, and the relationships to the department's mission, vision, and goals. A similar objectives hierarchy should also be developed to support the framework. 


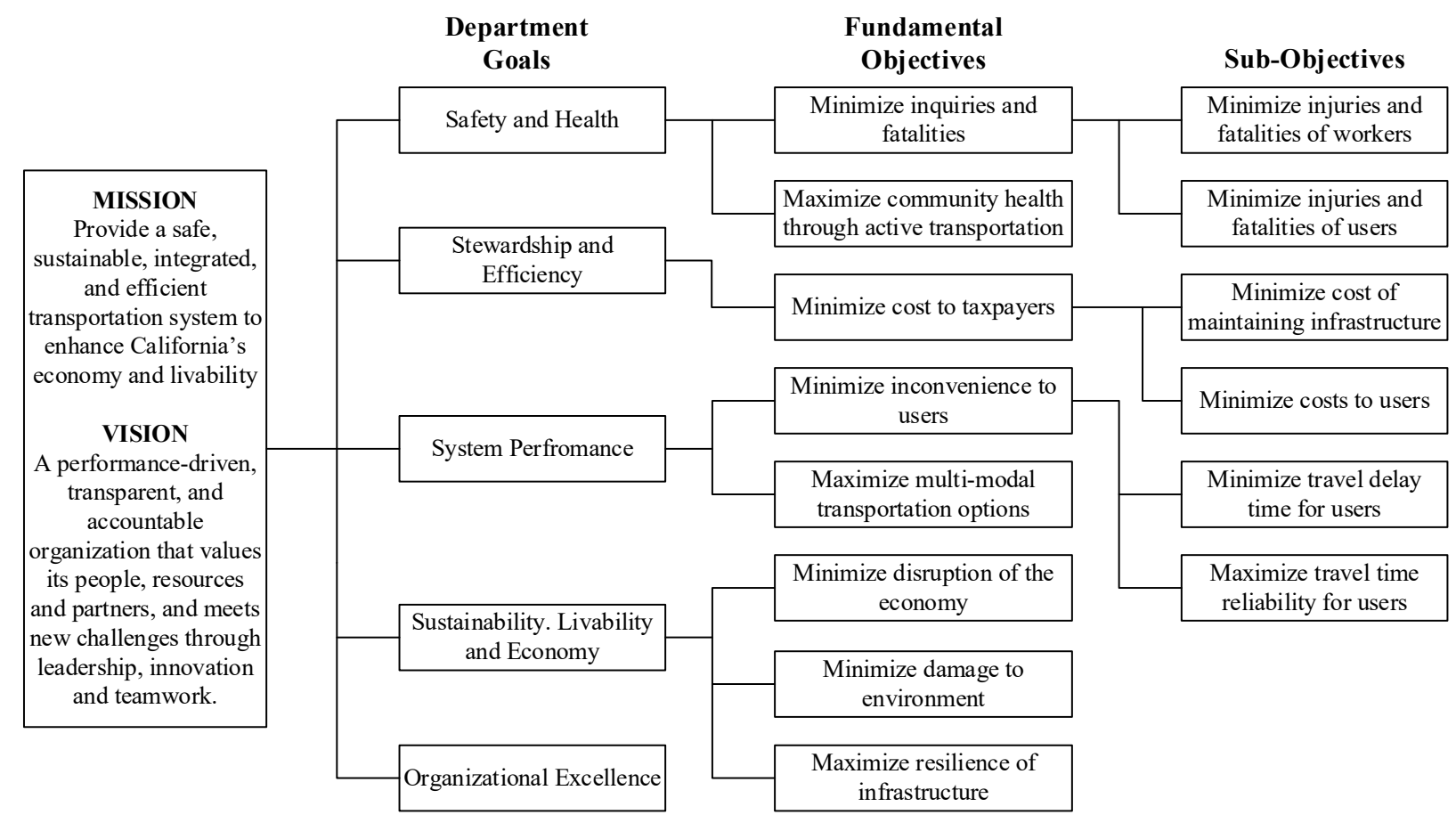

Figure 3.3 Hierarchy of Caltrans' objectives. Redrawn from the original in the SHOPP Report Phase 2: Application of a Project Prioritization Framework to the 2016 SHOPP (Caltrans, 2016).

Policy goals and objectives are usually included in statutes, internal regulations, or policy directives of state DOTs defining the overall priorities for its asset management process (Cambridge Systematics, Inc. et al., 2002). Asset management is a customer-focused and goaldriven decision-making process. Information on funding levels and customer (traveling public, freight carriers, etc.) expectations, and considering feedback from stakeholders (i.e., MPOs and other planning partners, federal agencies, and resource agencies) are crucial in defining priority goals and objectives - that can be translated to specific performance measures and targets, providing transparency and addressing public needs. 


\subsubsection{Performance Measures}

Establishing practices of performance measures and targets vary considerably among state DOTs. Most state DOTs have similar capabilities for tracking performance measures and targets related to safety and conditions of pavements and bridges (Cambridge Systematics, Inc., 2006); Table 3.1, as an example, shows the New York State DOT's (NYSDOT) established condition performance targets for pavements and bridges. NYSDOT demonstrates that both pavement and bridge conditions are expected to worsen over the next ten years if existing investment strategies are kept the same.

Table 3.1 NYSDOT's pavement and bridge performance targets and gaps

\begin{tabular}{|c|c|c|c|c|c|}
\hline & Performance Measure & $\begin{array}{l}\text { Baseline } \\
2013\end{array}$ & $\begin{array}{c}\text { State of } \\
\text { Good } \\
\text { Repair }\end{array}$ & $\begin{array}{l}\text { Target } \\
2023\end{array}$ & $\begin{array}{l}\text { Performance } \\
\text { Gap }\end{array}$ \\
\hline \multirow{2}{*}{ 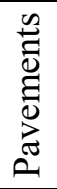 } & $\%$ of VMT on Good and Excellent & $71 \%$ & $88 \%$ & $59 \%$ & $29 \%$ \\
\hline & $\%$ of Poor & $10 \%$ & $10 \%$ & $36 \%$ & $26 \%$ \\
\hline \multirow{4}{*}{$\frac{\infty}{0}$} & $\%$ of Deficient & $49 \%$ & $25 \%$ & $50.5 \%$ & $25.5 \%$ \\
\hline & $\%$ of Poor & $23 \%$ & $10 \%$ & $29 \%$ & $19 \%$ \\
\hline & $\begin{array}{l}\% \text { of Preservation } \\
\text { (Good and Fair Protective) }\end{array}$ & $56 \%$ & $75 \%$ & $53 \%$ & $22 \%$ \\
\hline & $\%$ of Correctable (Fair Corrective) & $21 \%$ & $15 \%$ & $18 \%$ & $3 \%$ \\
\hline
\end{tabular}

Performance measures and targets for goal areas related to economic development, congestion, mobility, system operations, livability, energy efficiency, climate change, environment, and sustainability have not been widely considered in the decision-making process or are still immature (lack of well-developed measures with quantifiable targets). Performance measures are not used only for investment decision making but also for funding advocacy and transparency — to convey current system performance and what has been accomplished with existing tax dollars. There is a wide body of literature on transportation performance measures — used by state DOTs, MPOs, 
county and municipal governments, transit agencies, and international transportation agencies. The framework relies on well-defined performance measures to track progress toward established goals and objectives.

\subsubsection{TAMP and TIP/STIP}

As explained in Chapter 2, state DOTs are responsible for the development of the LRTP to identify improvement needs of their multi-modal transportation system in the next 20-year planning horizon. This step of the planning process is so essential for resource allocation decisions. The LRTP sets the stage for the development of a financially constrained TIP/STIP that identifies priority improvement projects for the next 4 years. The TAMP is designed to be a living document. The described processes and estimated funding levels in the TAMP are revised and improved on a biennial cycle to reflect current asset conditions, TIP/STIP updates, or new policy goals, objectives, performance measures and targets, or identified risks. The TAMP, like the LRTP, can be considered as a business plan that reveals the level of investments needed to meet the state DOT's policy goals, objectives, and performance targets related to the transportation system. The proposed goal-driven TAM framework supports the TIP/STIP development or amendment process - or other methods of optimizations, prioritizations, and trade-offs. The integrated EBMOO methodology serves as a decision support tool to

(1) identify candidate bridge projects for inclusion in the LRTP or TIP/STIP;

(2) set project/program priorities, revaluate funding allocations, or assess impacts of programmed types of bridge work (i.e., preservation, rehabilitation, and replacement) in the TIP/STIP on system performance; and

(3) evaluate different investment strategies and set targets through scenario analyses. 
Established objectives, performance measures and targets, budget allocations and constraints, and identified risks are presented in these plans and need to be transferred to the integrated EB-MOO methodology. For instance, specified budget constraints should be aligned with the financial plan included in the TAMP — historical and current bridge budgets, anticipated revenues, and long-term preservation investment strategies (identified funding allocations in the TIP/STIP over the next 4 years for the bridge projects). Figure 3.4 shows historical budget levels of the Colorado DOT (CDOT) by asset class from 2008 to 2014. At a minimum, objectives and bridge performance measures and targets identified in the TAMP should be the basis for driving the framework. Setting additional measures and targets must be well-defined, linked to the state DOT's goals and objectives, and trackable with quantifiable and reachable targets.

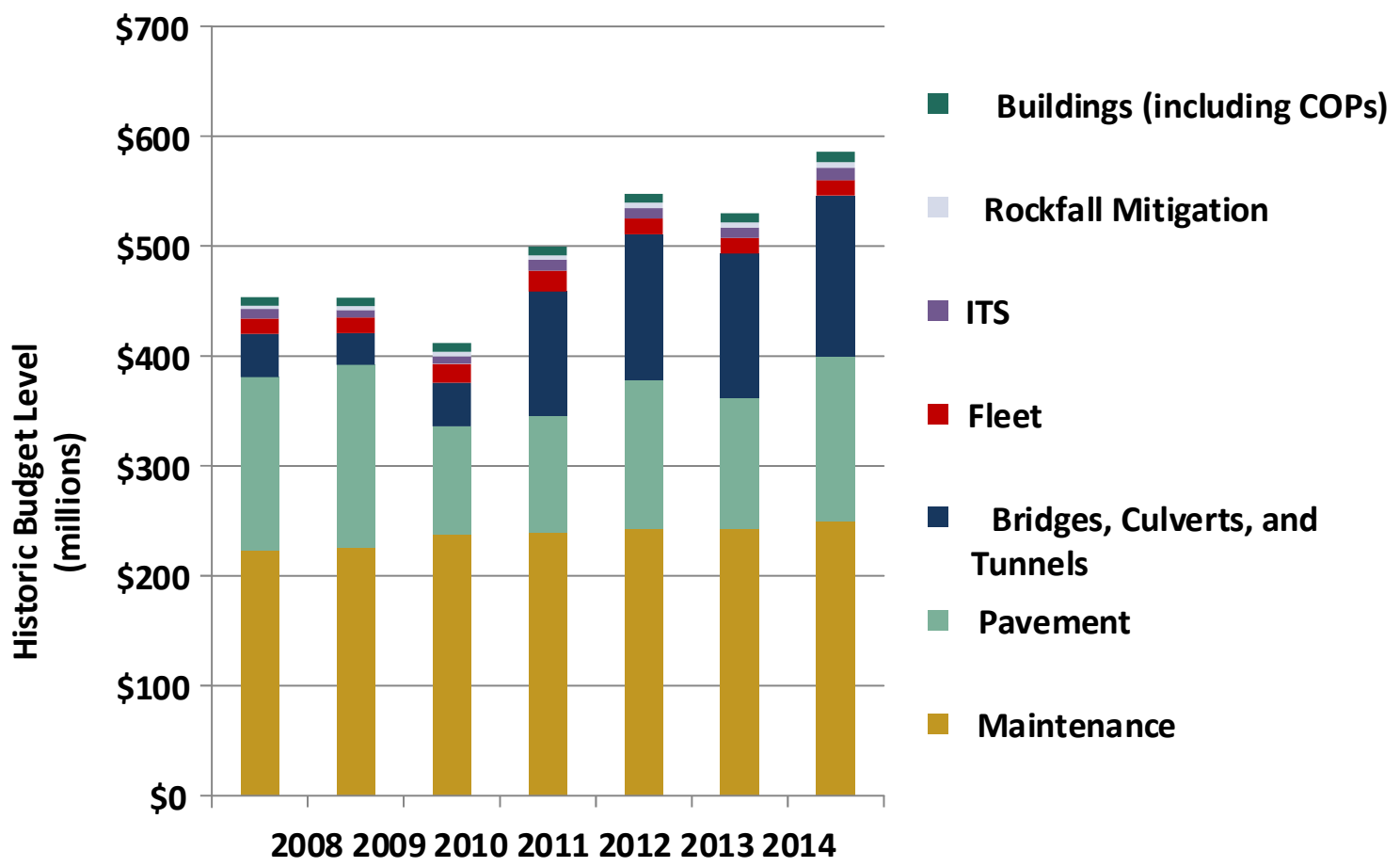

Figure 3.4 CDOT's historical budget levels by asset class. Adapted from the 2013 CDOT's Risk-Based Asset Management Plan (Cambridge Systematics, Inc., 2013). 


\subsection{Proposed Element-Based Multi-Objective Methodology}

The intent of Task 3 of this research is (1) to transfer previous work on bridge decision making involving multiple criteria/objectives (identified throughout the literature review of Task 1); and (2) to develop a flexible decision-making methodology, accompanying the proposed goal-driven TAM framework, relying on a "true" MOO technique - permitting decision makers to examine competing decision criteria/objectives (performance measures). The methodology improves bridge management and supports the development of the 20-year LRTP-identifying improvement needs for their multi-modal transportation system, and a financially constrained 4year TIP/STIP (listing high-priority improvement projects). The proposed EB-MOO methodology identifies optimal or near-optimal element intervention actions and determines anticipated funding needs through a robust $\mathrm{MOO}$ algorithm and several quantitative processes driven by decision makers' preferences and inputs. The methodology focuses on one transportation asset class (i.e., bridge). It is structured around five modules (i.e., data processing, improvement, ELO, BLO, and NLO modules). A flow diagram illustrating the interactions between these different modules is shown in Figure 3.5. 


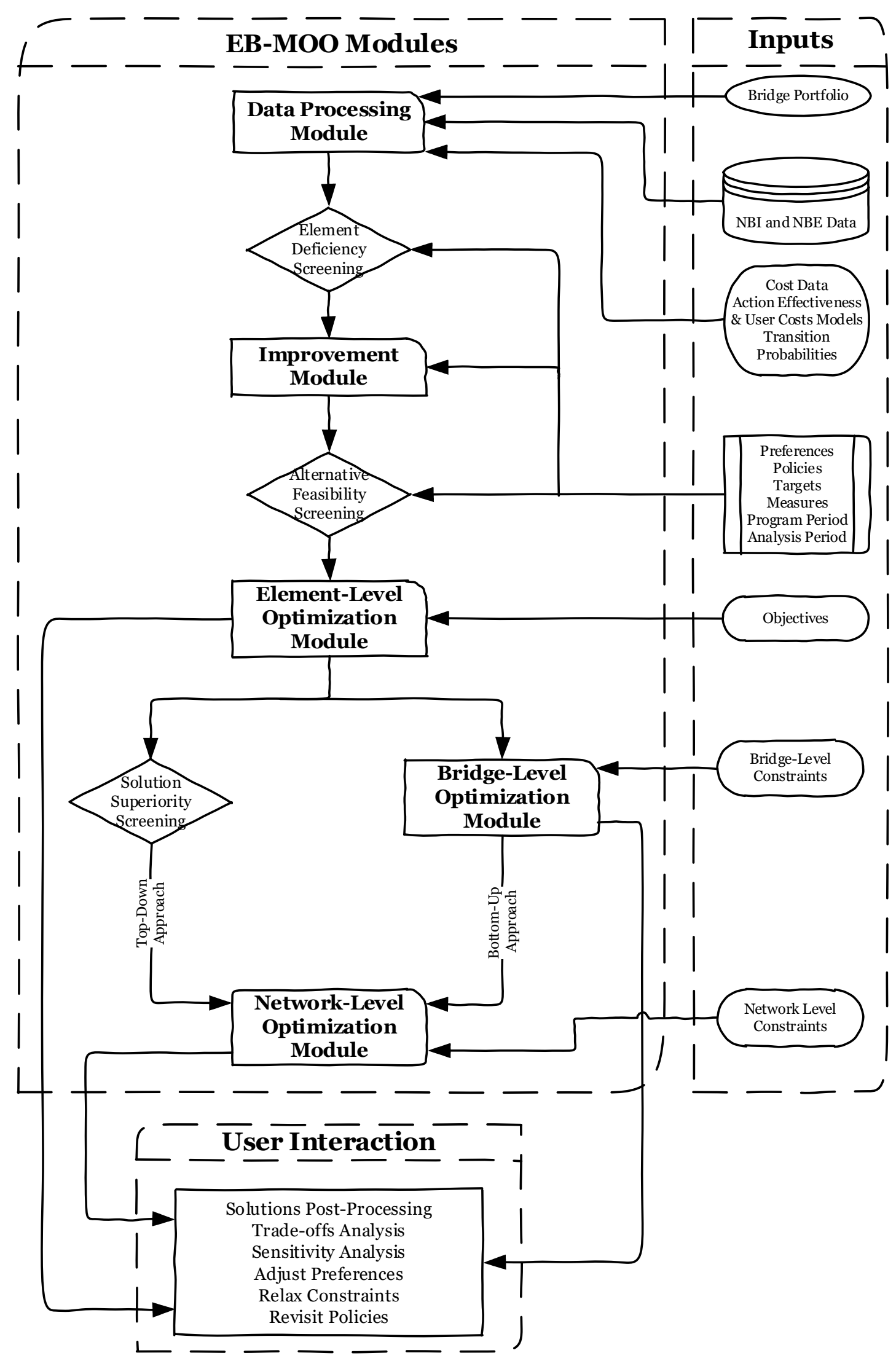

Figure 3.5 The proposed EB-MOO methodology flow diagram 
The proposed EB-MOO methodology relies on three distinct screening processes (i.e., element deficiency, alternative feasibility, and solution superiority screening processes) to overcome computer memory and processing time limitations. Leading-edge forecasting and up-to-date cost models were integrated. An independent deterioration model is incorporated to predict performance and a LCC model to estimate LCCs and LCC benefits. LC alternatives (series of element improvement actions) are generated based on a new simulation arrangement for three distinct improvement types: MRR, FCI, and REP. These improvement types consist with the defined work types of the TAMP. The requirement of 23 CFR 515.5 specifies the following five work types to be considered in the TAMP life-cycle planning (LCP), financial planning, and investment strategy analysis processes.

1. Initial Construction (i.e. new construction)

2. Maintenance

3. Preservation

4. Rehabilitation

5. Reconstruction (i.e. replacement)

A LC activity profile is constructed separately for each LC alternative action path. $\mathrm{LC}$ alternative results are produced for further evaluation. The methodology consists of three levels of optimization assessment based on the Pareto optimality concept:

(1) ELO, to identify optimal or near-optimal element intervention actions for each deficient element (in a poor condition state) of a candidate bridge;

(2) BLO, to identify combinations of optimal or near-optimal element intervention actions for a candidate bridge; and 
(3) NLO, following either a top-down or bottom-up approach, to identify sets of optimal or near-optimal element intervention actions for a network of bridges.

The methodology depends on a quantitative process driven by decision makers' preferences (derived from agency polices, objectives and constraints) and bridge-related data. Various optimization techniques, decision support tools, forecasting and cost models, and MCDM methods used by state DOTs and other transportation agencies were examined; the appropriate processes and concepts in bridge management and decision making involving multiple objectives were incorporated in the methodology. A genetic $\mathrm{MOO}$ algorithm is adapted due to the stochastic nature of the optimization problems and the large number of variables involved in the selection of intervention actions. A robust metaheuristic algorithm (i.e., NSGA-II; discussed in Chapter 2) is deployed to handle the large-sized MOO problems. The subsequent chapters are devoted to these modules. The proposed five modules are overviewed in the following subsections.

\subsubsection{Data Processing Module}

A portfolio of candidate bridges or programmed bridges in the TIP/STIP is introduced in the data processing module to be considered throughout the different processes and examined at the element, bridge, and network levels. The module relies on decision makers' preferences and inputs, quality data from state and national data management systems or external sources, and information provided in the TAMP and TIP/STIP. Deterioration modeling is the main driver of the element LC condition required in an optimization analysis. Leading-edge forecasting and up-to-date cost models were integrated in this module. The adapted independent deterioration model (i.e., Weibull/Markov model) is used to estimate the life expectancy of an element and predict its performance over an extended LC period. Improvement needs based on functional deficiencies are assessed at this stage of analysis. An integrated cost models are used to estimate incurred user 
costs due to existing functional deficiencies. Proven correlation/regression formulas are used to estimate major improvement costs required to eliminate incurred user costs or relieve the bridge from all its restrictions, and provide the required LOS and design standards. A "do-nothing” (DN) LC profile is constructed for each element as part of this module.

\subsubsection{Improvement Module}

The module can be deployed independently to support the development of LRTP, TIP/STIP and TAMP. Improvement model results can be used with common economic analyses (i.e., LCCA, BCA and IBCA) and optimization techniques to identify short- and long-term bridge investment needs, and recommend bridge programs and implementation schedules. The FHWA LCP Guidance Using a Life-Cycle Planning Process to Support Asset Management (FHWA, 2017a) recommends the following five steps:

1. Select asset classes and networks to be analyzed

2. Define LCP strategies

3. Set LCP scenario inputs

4. Develop and run the LCP scenarios

5. Provide input to financial planning

The module supports the implementation of these five steps. It generates LC alternatives, estimates LCCs and LCC benefits, and predicts performance (health indices). A program period is specified based on existing programming practice. Throughout the modeling approach, each bridge in the portfolio is evaluated separately for each program year. Results are organized per program year for further processing. The LC economic analysis extends beyond the programming phase. The 
total LC period (including the program period) is referred to as "analysis period." The length of this period depends on the agency's preservation policies and preferences.

Each bridge in the portfolio is screened for candidacy through a screening process referred to as "element deficiency screening process." A bridge identified with at least a deficient element is considered a candidate for improvement. A "replacement only" (RO) LC profile is constructed for each identified deficient element. The DN and RO LC profiles represent the "baseline" against which the LC alternative economic benefits (i.e., LCC benefits) are compared. DN and base agency costs are computed and discounted to present value for each deficient element. An "action effectiveness" (AE) LC profiles is constructed separately for each deficient element. An AE LC profile of an element is allied with one of the three improvement types (i.e., MRR, FCI and REP). It's represented by a series of actions following a unique LC alternative action path. A LC alternative is defined by a program year, a path of improvement actions, and an improvement type. Different types of LCCs (incurred during the entire analysis period) and residual values (applied at the end of the analysis period) are estimated for each LC alternative. Also, element health indices at different points in time are determined.

\subsubsection{Element-Level Optimization Module}

For each program year, as stated earlier, the improvement module identifies the potential deficient elements and estimates the bridge initial agency costs, user costs, and major improvement costs. These LC results are transferred to this module for further processing and use in the optimization phase. A screening process referred to as "alternative feasibility screening" is deployed to identify a set of LC alternatives for each identified deficient element per improvement type and per program year. Using this process, the optimization problem size is reduced by eliminating economically unattractive LC alternatives. Best feasible LC alternatives are recognized and set 
aside to be used in the optimization phase. Results associated with the identified best LC alternatives are grouped and reorganized per improvement type and per program year in single matrices. Each matrix includes one type of results per improvement type and per program year, encompassing all deficient elements. The optimization process relies on these preset matrices as input parameters, including the bridge user and initial agency costs, to direct the search of Pareto optimal or near-optimal LC alternatives. The optimization solution results per improvement type are produced for each program year. The optimization results contain the recommended LC alternatives for all deficient elements, initial agency costs, bridge health indices at different points in the analysis period, and optimized element-level objective values.

\subsubsection{Bridge-Level Optimization Module}

The module has two main purposes: (1) supporting the development of bridge improvement or preservation programs, and (2) producing bridge-level input parameters for the bottom-up approach NLO. The module identifies best (optimal or near-optimal) sets of improvement actions and timings for future work on a candidate bridge. The BLO module addresses one bridge at a time, recommending a set of optimal or near-optimal element improvement strategies. BLO results are stored to serve the NLO process.

ELO results from the preceding module are grouped and expressed in summation formulas over the entire program period and all deficient elements. The grouped ELO solutions compete for minimum bridge LCCs, maximum bridge LCC benefits, and/or maximum bridge health indices (bridge-level objectives). As discussed earlier, the ELO module focuses on finding optimal or nearoptimal solutions (Pareto solutions) per program year and per improvement type for each deficient element of a given bridge in the portfolio. These ELO results per improvement type and per program year for each element are transferred to this module as input parameters. They are grouped 
and reorganized in single matrices - covering the entire program period and the three improvement types. This arrangement allows to unite all recommended ELO solutions to compete at the BLO. The BLO results contain the recommend LC alternatives associated with the BLO solutions (each element is assigned to an optimal or near-optimal LC alternative, an improvement type and a program year), initial agency costs, bridge health indices at different points in the analysis period, and the optimized bridge-level objective values.

\subsubsection{Network-Level Optimization Module}

The module is designed to identify funding needs and short- and long-term investment strategies for each deficient element of a bridge in the portfolio (network) and facilitate trade-offs between funding scenarios and performance. There are two NLO approaches among BMSs: (1) top-down approach, optimization determines network-level goals, and then improvement needs for individual bridges; and (2) bottom-up approach, where bridge improvement needs are determined first. The top-down and bottom-up approaches are defined differently in this research. Basically, a top-down approach is followed when ELO solutions are used in determining network-level investment needs. ELO solutions, associated with the three improvement types and all program years, for each bridge in the portfolio compete at this level. Only one ELO solution per bridge is selected. A screening process referred to as "solution superiority screening" is deployed to reduce the computational time for the top-down-approach optimization. The screening also guarantees the inclusion of superior ELO solutions. Improvement rules or triggers can be established at this higher

level to substitute the adapted screening process or complement it. The bottom-up approach is followed when BLO solutions are chosen as the optimization input parameters instead. No more than one BLO solution per bridge is selected. 
The optimization problem is either constrained (by budget and/or performance) or unconstrained (involving only the selection criterion). The unconstrained problem can be used to estimate the budget required to address identified improvement needs, or determine whether a program is under- or over-funded. Usually, this information is used to justify budget requests through the legislative process. The budget-constrained problem can be used to anticipate short- and long-term network improvement strategies under limited funds - supporting the development of bridge programs or TIPs/STIPs. The module outcomes involve the recommended LC alternatives associated with the NLO solutions (each bridge element is assigned to an optimal or near-optimal LC alternative, an improvement type, and a program year), initial agency costs, network health indices at different points in the analysis period, and optimized network-level objective values.

\subsection{User Interaction}

The methodology relies on inputs and preferences from experts and decision makers to support the implemented processes and different analyses (i.e., LCCA, optimization process, sensitivity analysis, trade-off analysis, and adjustment of measures and targets). Due to the large variation among bridge management and maintenance practices and preservation policies adapted by state DOTs, inputs should come from experts familiar with the state DOT's internal procedures and practices and with experience and knowledge of design features, different damage modes and deterioration mechanisms, and performance history of bridges within their operational environments. Preferences should be provided by asset managers or program planners involved in asset management, planning and programming, development of policy objectives and performance measures, or resource allocations. Asset managers can validate optimized priorities, funding requirements, and produced infographic depictions. They can also run sensitivity/scenario analysis for funding uncertainty by manipulating budget constraints, discount rates, or other factors. 
A priori articulation of preference approach (discussed in Chapter 2) is often followed when multiple objectives are aggregated into one, as in the weighted sum or utility function method. Decision makers provide preferences (relative weights) prior the optimization process. The methodology follows a different approach referred to as posteriori articulation of preference approach-Pareto solutions are first determined, and then presented to the decision makers to select the best one based on preferences. This approach helps providing a complete knowledge of the problem and exploring the whole set of Pareto solutions (Talbi, 2009). The multiple criteria analyses discussed in Chapter 2 facilitate the selection of the best solution.

Optimization results can be further explored by a trade-off or "what-if" scenario analysis (covered in Chapter 2) between obtained Pareto solutions. The analysis allows decision makers to trade-off between sets of LC alternatives or optimization objectives. This type of analysis is essential for identifying the appropriate course of actions, adjusting preferences and funding levels, and communicating resulting impacts. Pareto solutions are indispensable for this type of analysis. Pareto solutions from different scenarios, in alignment with the long-term goals, are evaluated for possible implications on resource allocations and performance.

The NCHRP Report 666: Target-Setting Methods and Data Management to Support PerformanceBased Resource Allocation by Transportation Agencies identifies targets as "a quantifiable point in time at which an organization achieves all or a portion of its goals," and emphasizes "the importance of tracking and assessing the impacts of measures and targets on actual investments" (Cambridge Systematics, Inc., 2010). It's important to periodically revisit measures and targets to ensure their effectiveness in the actual decision making and the development of long-term investment strategies. Asset managers through the proposed EB-MOO modules will be able to perform this kind of assessments and recommend adjustments to targets or measures over time- 
for example, based on trends of actual investments and separate analyses, measures that were proven to be irrelevant or ineffective, or targets believed to be unachievable (set too high).

A broad range of risk events could impact achievement of bridge-related performance targets and the ability to deliver planned investments or manage assets effectively, and the performance of a network of assets or a single asset. Considering risk in the decision-making process supports achieving a reasonable informed decision— by assessing likelihoods of occurrence and associated consequence. Risk events can have impacts on system levels in various terms-impacting performance and ability to deliver recommended investments or effectively manage assets. At a minimum, experts are encouraged to identify risks that could impact the serviceability and achievement of performance targets. Bridge-related risk events addressed by the TAMP's risk management element can be included for consistency and completeness. The optimized timing of interventions over the analysis period should be explored considering any identified relevant risks - usually based on bridge or network attributes such as location, environmental, traffic volume, etc.

Considering the risk aspect is beyond the scope of this research. Nevertheless, the EB-MOO methodology is well-suited to accommodate any risk assessment models. Incorporating a risk assessment model into the proposed EB-MOO methodology is recommended for future research. The inclusion of risk assessment models allows to factor risk into the LCC calculations. As mentioned in Chapter 2, the risk assessment guideline developed as part of the NCHRP Project 20-07, Task 378, (Thompson et al., 2018) includes likelihood probability models for sixteen different hazards and a process for monetizing consequences of service distribution (risk costs) well-suited for the LCCA in BMSs. These guideline models can be incorporated to add the risk aspect in the recommended investment strategies. 


\subsection{Implementation}

\subsubsection{Tool Prototype}

As part of Task 1, various available BMSs and investment decision-making and project prioritization tools used by state DOTs, MPOs and other transportation agencies were explored in terms of their capabilities and limitations. A standalone tool complementing common decision support tools and builds upon their existing capabilities was designed under Task 4. The tool prototype accommodates user-specified performance measures, inputs and preferences, and commonly collected data from widely-used state and national data management systems (such as National Bridge Inventory [NBI] System, Highway Performance Monitoring System [HPMS], BrM, Highway Safety Information System [HSIS], Fatality Analysis Reporting System [FARS], and Long-Term Bridge Performance [ LTBP] InfoBridge).

The tool prototype was produced in MATLAB, primarily for ease of use and to implement the EB-MOO methodology through several examples of scenarios. The tool prototype should be considered as a "proof of concept" rather than a complete rigorous software ready for operational implementation. The tool is structured around the five EB-MOO methodology modules discussed in earlier. Each module is executed independently. Module output files are saved in one location and accessible for use by the different processes and the main optimizer. The tool consists of multiple MATLAB-coded scripts that provide most of the controls while reserving all complex mathematical calculations for back-end processing. Figure 3.6 shows the tool's hierarchical structure in the form of directories (folders) and their subdirectories (subfolders), hosting most of the MATLAB-coded scripts and functions. An illustration of the tool produced results is presented in Figure 3.7. 
4 MATLAB R2018b - academic use
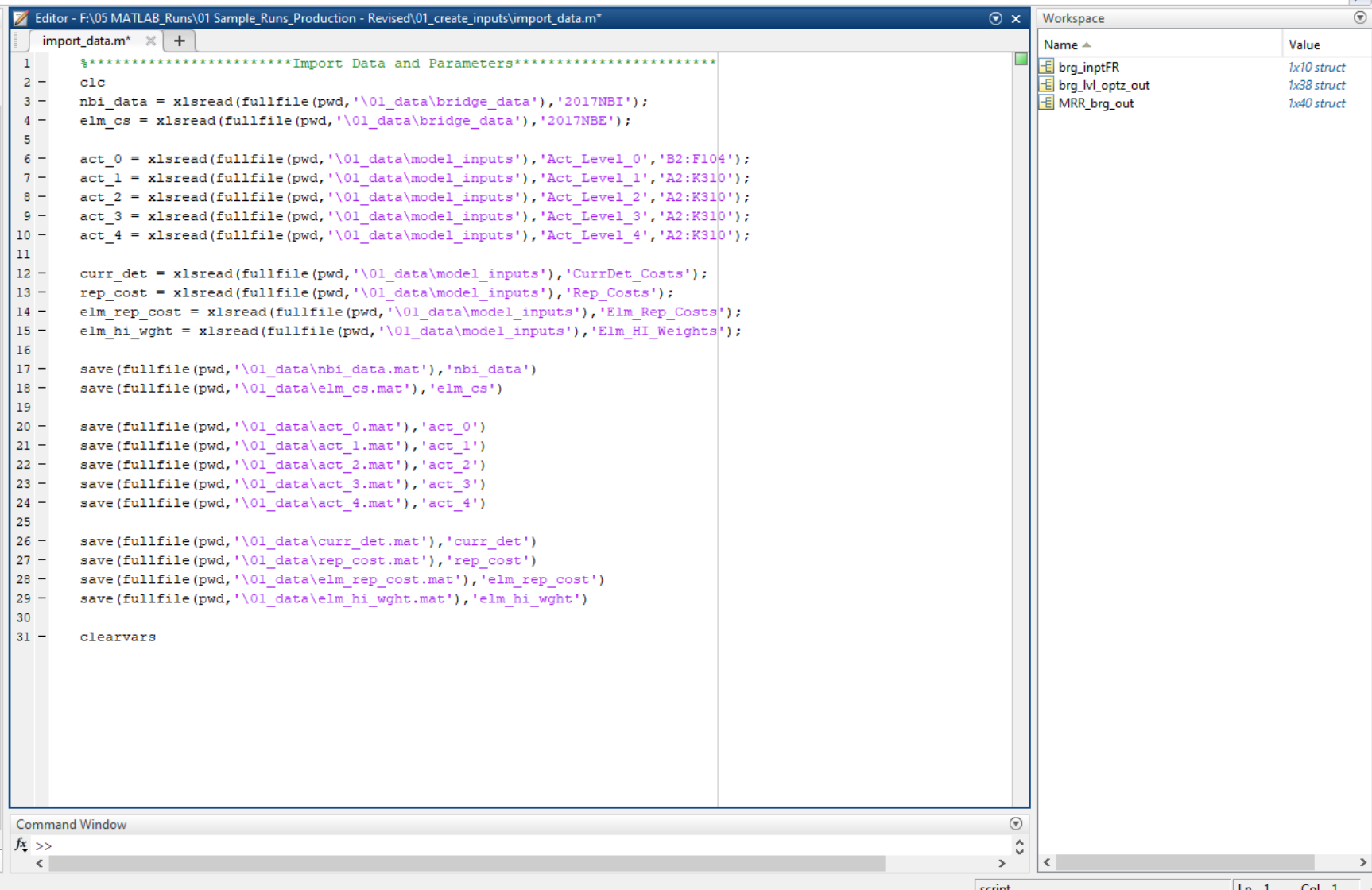

Figure 3.6 Hierarchical structure of the MATLAB-based tool prototype 
brg_inptFR(1).brg

\begin{tabular}{|c|c|c|c|c|c|c|c|c|c|c|c|c|c|c|c|c|c|c|}
\hline Fields & 国 elm & Ђ brg_elm_id & {$[1]$ nbi_data } & {$[1]$ nbi_data_under } & 啗 fct_imp & 臽 deck_area & 乌 PVacc_all & Ђ PVcl_all & 乌. PVopr_all & 固 MRR & 国 FCI_REP & 帠 elm_pckd_ref & $\boxplus$ elm_pckd_nn $\boxplus$ elm_non_pckd_ref & ef 点 HLelm & 写 HI_brg & 阽DN_HI_brg & 吅 elm_pckd_id & \\
\hline 1 & $7 \times 10$ struct & ]$[12,107,215,22 \ldots$ & $1 \times 137$ cell & $1 \times 137$ cell & {$[0,0,0]$} & {$[640.7000,1.0 \ldots$} & 1754 double & $1 \times 54$ double & 1754 double & $1 \times 1$ struct & $1 \times 1$ struct & {$[1,2,3,4,5,6,7,8,9, \ldots$} & 10 & [] 10x7 double & {$[100,78.835 \ldots$} & {$[85.1156,68.17 \ldots \ldots$} & {$[12,107,215,227, \ldots$} & 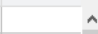 \\
\hline 2 & $1 \times 21$ struct & $1 \times 21$ double & $1 \times 137$ cell & $1 \times 137$ cell & {$[0,0,0]$} & {$[4.9152 \mathrm{e}+03, \ldots$} & 1754 double & 1754 double & 1,54 double & $1 \times 1$ struct & $1 \times 1$ struct & $1 \times 21$ double & 21 & [] 21177 double & {$[100,79.095 \ldots$} & {$[83.2221,69.62 \ldots$} & 1 1x21 double & \\
\hline 3 & $1 \times 21$ struct & 1x21 double & $7 \times 137$ cell & $1 \times 137$ cell & {$[0,0,0]$} & {$[4.9152 \mathrm{e}+03, \ldots$} & 1754 double & $1 \times 54$ double & $1 \times 54$ double & $1 \times 1$ struct & $7 \times 1$ struct & 1×21 double & 21 & [] 21x7 double & {$[100,78.637 \ldots$} & {$[83.3248,69.27 \ldots$} & $1 \times 21$ double & \\
\hline 4 & $1 \times 11$ struct & $1 \times 11$ double & $1 \times 137$ cell & [] & {$[0,0,1]$} & {$[2.0511 \mathrm{e}+03, \ldots$} & 1554 double & $1 \times 54$ double & 1554 double & $1 \times 1$ struct & $1 \times 1$ struct & 1x11 double & 11 & [] 11x7 double & {$[100,76.776 \ldots$} & {$[83.1543,69.38 \ldots$} & . 1x11 double & \\
\hline 5 & $1 \times 18$ struct & 1x18 double & $1 \times 137$ cell & [] & {$[1,0,0]$} & {$[641.5600,80 \ldots$} & 1554 double & $1 \times 54$ double & 1554 double & $1 \times 1$ struct & $1 \times 1$ struct & 1x18 double & 18 & [] 18x7 double & {$[100,77.700 \ldots$} & {$[77.0011,61.90 \ldots$} & $1 \times 18$ double & \\
\hline 6 & $1 \times 10$ struct & {$[12,104,215,22 \ldots$.} & $1 \times 137$ cell & $1 \times 137$ cell & {$[0,0,1]$} & {$[1.8726 \mathrm{e}+03, \ldots$} & 1754 double & $1 \times 54$ double & $1 \times 54$ double & $1 \times 1$ struct & $1 \times 1$ struct & {$[1,2,3,4,5,6,7,7,9, \ldots, \ldots$} & 10 & [] 10x7 double & {$[100,77.104 \ldots \ldots$} & {$[86.5556,69.83 \ldots \ldots$} & {$[12,104,215,227, \ldots$} & \\
\hline 7 & $1 \times 9$ struct & {$[12,102,215,22 \ldots$} & $1 \times 137$ cell & $1 \times 137$ cell & {$[0,0,1]$} & {$[3.3171 \mathrm{e}+03, \ldots$} & 1754 double & 1754 double & $1 \times 54$ double & $1 \times 1$ struct & $1 \times 1$ struct & {$[1,2,3,4,5,6,7,8,9]$} & 9 & [] $9 \times 7$ double & {$[100,76.313 \ldots$} & {$[83.6012,66.95 \ldots$} & {$[12,102,215,227, \ldots$} & \\
\hline 8 & $1 \times 13$ struct & $1 \times 13$ double & $1 \times 137$ cell & $1 \times 137$ cell & {$[0,0,0]$} & {$[4.2699 \mathrm{e}+03, \ldots$} & 1754 double & $1 \times 54$ double & 1754 double & $1 \times 1$ struct & $1 \times 1$ struct & $1 \times 13$ double & 13 & [] $13 \times 7$ double & {$[100,76.122 \ldots]$} & {$[89.1607,69.23 \ldots$} & $1 \times 13$ double & \\
\hline 9 & $1 \times 10$ struct & {$[12,109,215,22 \ldots$} & $.1 \times 137$ cell & $1 \times 137$ cell & {$[0,1,0]$} & {$[2.3608 \mathrm{e}+03, \ldots$} & 1754 double & $1 \times 54$ double & 1754 double & $1 \times 1$ struct & $1 \times 1$ struct & {$[1,2,3,4,5,6,7,8,9, \ldots$} & 10 & [] 1007 double & {$[100,80.553 \ldots$} & {$[72.0622,68.47 \ldots$} & {$[12,109,215,227, \ldots$} & \\
\hline 10 & $1 \times 10$ struct & {$[12,105,109,21 \ldots$} & $1 \times 137$ cell & $1 \times 137$ cell & {$[0,1,0]$} & {$[1.2732 \mathrm{e}+03, \ldots$} & 1754 double & 1754 double & 1754 double & $1 \times 1$ struct & $1 \times 1$ struct & {$[1,2,3,4,5,6,7,8,9, \ldots$} & 10 & [] 10x7 double & {$[100,79.328 . \ldots$} & {$[69.9121,66.07 \ldots$} & {$[12,105,109,215, \ldots$} & \\
\hline 11 & $1 \times 12$ struct & 1x12 double & $1 \times 137$ cell & [] & {$[1,0,0]$} & {$[1.0010 \mathrm{e}+03, \ldots$} & 1754 double & 1754 double & $1 \times 54$ double & $1 \times 1$ struct & $1 \times 1$ struct & 1x12 double & 12 & [] $12 \times 7$ double & {$[100,77.560 \ldots$} & {$[78.1442,66.14 \ldots$} & 1712 double & \\
\hline 12 & $1 \times 12$ struct & 1x12 double & $1 \times 137$ cell & [] & {$[1,0,0]$} & {$[1.0010 \mathrm{e}+03, \ldots$} & 1754 double & $1 \times 54$ double & 1.54 double & $1 \times 1$ struct & $1 \times 1$ struct & 1x12 double & 12 & [] $12 \times 7$ double & {$[100,77.563 \ldots$} & {$[73.0928,64.54 \ldots$} & $1 \times 12$ double & \\
\hline 13 & $1 \times 19$ struct & 1x19 double & $1 \times 137$ cell & [] & {$[1,0,0]$} & {$[1.4749 \mathrm{e}+03, \ldots$} & 1754 double & 1754 double & $1 \times 54$ double & $1 \times 1$ struct & $1 \times 1$ struct & 1x19 double & 19 & [] 19x7 double & {$[100,77.827 \ldots$} & {$[80,3309,63.83 \ldots$} & $1 \times 19$ double & \\
\hline 14 & $7 \times 13$ struct & 1x13 double & $1 \times 137$ cell & $1 \times 137$ cell & {$[0,0,1]$} & {$[6.7144 \mathrm{e}+03, \ldots$} & $7 \times 54$ double & $1 \times 54$ double & $1 \times 54$ double & $1 \times 1$ struct & $1 \times 1$ struct & 1x13 double & 13 & [] $13 \times 7$ double & {$[100,77.125 \ldots \ldots$} & {$[80.3016,66.47 \ldots$} & $1 \times 13$ double & \\
\hline 15 & $1 \times 18$ struct & Tx18 double & $7 \times 137$ cell & $7 \times 137$ cell & {$[0,0,1]$} & {$[1.8044 \mathrm{e}+04, \ldots$} & 1754 double & 1754 double & 1,54 double & $1 \times 1$ struct & $1 \times 1$ struct & Tx18 double & 18 & [] 18x7 double & {$[100,76.941 \ldots$} & {$[82.8708,67.09 . \ldots$} & $1 \times 18$ double & \\
\hline 16 & $1 \times 10$ struct & {$[12,107,215,22 \ldots$} & . $1 \times 137$ cell & $1 \times 137$ cell & {$[0,1,0]$} & {$[657.5800,90 \ldots$} & 1754 double & 1754 double & 1754 double & $1 \times 1$ struct & $1 \times 1$ struct & {$[1,2,3,4,5,6,7,8,9, \ldots$} & 10 & [] 10x7 double & {$[100,77.139 . \ldots$} & {$[81.3262,65.45 \ldots$} & {$[12,107,215,227, \ldots$} & \\
\hline 17 & $1 \times 19$ struct & $1 \times 19$ double & $1 \times 137$ cell & $1 \times 137$ cell & {$[1,0,1]$} & {$[3.4621 \mathrm{e}+04, \ldots$} & 1754 double & $1 \times 54$ double & 1754 double & $1 \times 1$ struct & $1 \times 1$ struct & 1x19 double & 19 & [] 19x7 double & {$[100,76.218 \ldots]$} & {$[74.6651,58.87 \ldots$} & $1 \times 19$ double & \\
\hline 18 & $1 \times 22$ struct & 1722 double & $1 \times 137$ cell & [] & {$[1,0,1]$} & {$[7,8863 \mathrm{e}+03, \ldots$} & 1754 double & $1 \times 54$ double & 1754 double & $1 \times 1$ struct & $1 \times 1$ struct & 1x22 double & 22 & [] $22 \times 7$ double & {$[100,76.696 \ldots \ldots$} & {$[81.5918,64.04 \ldots$} & $1 \times 22$ double & \\
\hline 19 & $1 \times 20$ struct & 1720 double & $1 \times 137$ cell & $1 \times 137$ cell & {$[1,0,0]$} & {$[822.1600,1.0 \ldots$} & 1754 double & $1 \times 54$ double & 1754 double & $1 \times 1$ struct & $1 \times 1$ struct & $1 \times 20$ double & 20 & [] 20x7 double & {$[100,76.103 \ldots$} & {$[74.1815,58.18 \ldots$} & $1 \times 20$ double & \\
\hline 20 & $1 \times 10$ struct & {$[12,107,215,22 \ldots$} & $1 \times 137$ cell & $1 \times 137$ cell & {$[1,1,0]$} & {$[380.1200,47 \ldots$} & 1754 double & $1 \times 54$ double & 1754 double & $1 \times 1$ struct & $1 \times 1$ struct & {$[1,2,3,4,5,6,7,8,9, \ldots$} & 10 & [] 10x7 double & {$[100,78.087 \ldots$} & {$[83.2202,67.12 \ldots$} & {$[12,107,215,227, \ldots$} & \\
\hline 21 & $1 \times 10$ struct & {$[12,107,215,22 \ldots$} & $1 \times 137$ cell & $1 \times 137$ cell & {$[1,1,0]$} & {$[380.1200,47 \ldots$} & 1754 double & $1 \times 54$ double & 1754 double & $1 \times 1$ struct & $1 \times 1$ struct & {$[1,2,3,4,5,6,7,8,9, \ldots$} & 10 & [] 10x7 double & {$[100,78.087 \ldots$} & {$[75.3858,63.45 \ldots$} & {$[12,107,215,227, \ldots$} & \\
\hline 22 & $1 \times 6$ struct & {$[30,102,113,15 \ldots$} & $.1 \times 137$ cell & [] & {$[1,0,1]$} & {$[109.7200,63 \ldots$} & 1754 double & $1 \times 54$ double & 1,54 double & $1 \times 1$ struct & $1 \times 1$ struct & {$[1,2,3,4,4,5,6]$} & 6 & [] $6 x 7$ double & {$[100,73.707 \ldots \ldots$} & {$[62,4406,46.70 \ldots$} & {$[30,102,113,152, \ldots$} & \\
\hline 23 & $1 \times 16$ struct & $1 \times 16$ double & $1 \times 137$ cell & [] & {$[1,0,1]$} & {$[2.2011 \mathrm{e}+04, \ldots$} & 1,54 double & $1 \times 54$ double & 1754 double & $1 \times 1$ struct & $1 \times 1$ struct & $1 \times 16$ double & 16 & [] $16 \times 7$ double & {$[100,77.595 \ldots$} & {$[79.2582,65.89 . \ldots$} & $1 \times 16$ double & \\
\hline 24 & $7 \times 15$ struct & $1 \times 15$ double & $1 \times 137$ cell & $1 \times 137 \mathrm{cell}$ & {$[0,0,0]$} & {$[7.0959 e+03, \ldots$} & 1754 double & $1 \times 54$ double & 1754 double & $1 \times 1$ struct & $1 \times 1$ struct & $1 \times 15$ double & 15 & [] $15 \times 7$ double & {$[100,78.510 \ldots$} & {$[83.6649,69.97 \ldots$} & 1715 double & \\
\hline 25 & $1 \times 10$ struct & {$[12,109,215,22 \ldots$} & $.1 \times 137$ cell & [] & {$[0,0,1]$} & {$[1.2899 e+04, \ldots$} & 1754 double & 1.54 double & 1754 double & $1 \times 1$ struct & $1 \times 1$ struct & {$[1,2,3,4,5,6,7,8, \ldots \ldots$} & 10 & [1 1007 double & {$[100,80.412 \ldots]$} & {$[77.6360,62.09 \ldots]$} &.$[12,109,215,220, \ldots$ & \\
\hline 26 & $1 \times 11$ struct & $1 \times 11$ double & $1 \times 137$ cell & [] & {$[1,0,0]$} & {$[550.4000,67 \ldots$} & 1754 double & $1 \times 54$ double & 1.54 double & $1 \times 1$ struct & $1 \times 1$ struct & $1 \times 11$ double & 11 & [] 11x7 double & {$[100,78.919 \ldots$} & {$[84.2914,67.51 \ldots$} & 17x11 double & \\
\hline 27 & $1 \times 8$ struct & {$[38,215,225,23 \ldots$} & $1 \times 137$ cell & [] & {$[1,0,0]$} & {$[196.9400,22 \ldots$} & 1754 double & $1 \times 54$ double & 1754 double & $1 \times 1$ struct & $1 \times 1$ struct & {$[1,2,3,4,5,6,7,8]$} & 8 & [] $8 \times 7$ double & {$[100,74.994 \ldots$} & {$[82.8730,66.69 \ldots$} & {$[38,215,225,234, \ldots$} & \\
\hline 28 & 177 struct & {$[38,215,226,23 \ldots$} & $1 \times 137$ cell & $1 \times 137$ cell & {$[0,1,0]$} & {$[460.4600,63 \ldots$} & 1754 double & $1 \times 54$ double & $1 \times 54$ double & $1 \times 1$ struct & $1 \times 1$ struct & {$[1,2,3,4,5,6,7]$} & 7 & [] $7 \times 7$ double & {$[100,71.631 \ldots$} & {$[77.9524,63.87 \ldots$} & {$[38,215,226,234, \ldots$} & \\
\hline 29 & $1 \times 18$ struct & $1 \times 18$ double & $7 \times 137$ cell & $1 \times 137$ cell & {$[0,0,0]$} & {$[2.8311 \mathrm{e}+03, \ldots$} & 1754 double & 1754 double & 1,54 double & $1 \times 1$ struct & $1 \times 1$ struct & $1 \times 18$ double & 18 & [] 18x7 double & {$[100,71.487 \ldots$} & {$[74.5234,60.48 \ldots$} & $1 \times 18$ double & \\
\hline 30 & $1 \times 18$ struct & $1 \times 18$ double & $7 \times 137$ cell & $7 \times 137$ cell & {$[0,0,0]$} & {$[2.8311 \mathrm{e}+03, \ldots$} & 1754 double & 1754 double & 1754 double & $1 \times 1$ struct & $1 \times 1$ struct & $1 \times 18$ double & 18 & [] $18 \times 7$ double & {$[100,71.491 \ldots$} & {$[74.5223,60.46 \ldots$} & 1 1x18 double & \\
\hline 31 & $1 \times 8$ struct & {$[38,210,215,22 \ldots$} & $1 \times 137$ cell & [] & {$[1,0,0]$} & {$[117.8200,13 \ldots$} & 1754 double & $1 \times 54$ double & 1754 double & $1 \times 1$ struct & $1 \times 1$ struct & {$[1,2,3,4,5,6,7,8]$} & 8 & [] $8 \times 7$ double & {$[100,75.292 \ldots$} & {$[81.5832,67.29 \ldots$} & {$[38,210,215,225, \ldots$} & \\
\hline 32 & $1 \times 10$ struct & {$[12,102,210,21 \ldots$} & $1 \times 137$ cell & $1 \times 137$ cell & {$[0,0,0]$} & {$[2646,3.7265 \ldots$} & 1754 double & $1 \times 54$ double & 1754 double & $1 \times 1$ struct & $1 \times 1$ struct & {$[1,2,3,4,5,6,7,8,9, \ldots$} & 10 & [] $10 \times 7$ double & {$[100,76.077 \ldots$} & {$[86.1846,68.00 \ldots$} & {$[12,102,210,215, \ldots$} & \\
\hline 33 & $1 \times 10$ struct & {$[12,102,210,21 \ldots$} & $1 \times 137$ cell & $1 \times 137$ cell & {$[0,0,0]$} & {$[3174,4,4700 \ldots$} & 1,554 double & 1054 double & $1 \times 54$ double & $1 \times 1$ struct & $1 \times 1$ struct & {$[1,2,3,4,4,6,6,7,8,9, \ldots$} & 10 & [] 10x7 double & {$[100,75.984 \ldots$} & {$[86.1020,67.96 \ldots]$} & {$[12,102,210,215, \ldots$} & \\
\hline 34 & $1 \times 14$ struct & 1x14 double & $1 \times 137$ cell & [] & {$[0,0,0]$} & {$[304,4400,44 \ldots$} & 1754 double & $1 \times 54$ double & $1 \times 54$ double & $1 \times 1$ struct & $1 \times 1$ struct & 1x14 double & 14 & [] $14 \times 7$ double & {$[100,76.251 \ldots$} & {$[77.3560,60.52 \ldots$} & $1 \times 14$ double & \\
\hline 35 & $1 \times 13$ struct & $1 \times 13$ double & $1 \times 137$ cell & [] & {$[1,0,0]$} & {$[1.8462 \mathrm{e}+03, \ldots$} & 1054 double & $7 \times 54$ double & 1.554 double & $1 \times 1$ struct & $1 \times 1$ struct & $1 \times 13$ double & 13 & [] $13 \times 7$ double & {$[100,75.479 \ldots$} & {$[78.4886,61.60 \ldots$} & $1 \times 13$ double & \\
\hline 36 & $1 \times 17$ struct & 1x17 double & $1 \times 137$ cell & [] & {$[1,0,1]$} & {$[1.7019 \mathrm{e}+03, \ldots$} & 1,54 double & $1 \times 54$ double & 1754 double & $1 \times 1$ struct & $1 \times 1$ struct & 1x17 double & 17 & [] $17 \times 7$ double & {$[100,77.005 \ldots \ldots$} & {$[65.6164,59.56 \ldots$} & Tx17 double & \\
\hline 37 & $1 \times 20$ struct & $1 \times 20$ double & $7 \times 137$ cell & $1 \times 137$ cell & {$[1,0,1]$} & {$[1.3149 \mathrm{e}+03, \ldots$} & 1754 double & 1754 double & 1754 double & $1 \times 1$ struct & $1 \times 1$ struct & $1 \times 20$ double & 20 & [] $20 \times 7$ double & {$[100,78.700 \ldots$} & {$[80.5919,66.08 . .$.} & $17 \times 20$ double & \\
\hline 38 & $1 \times 20$ struct & $1 \times 20$ double & $1 \times 137$ cell & $1 \times 137$ cell & {$[1,0,1]$} & {$[1.3149 e+03, \ldots$} & 1754 double & $1 \times 54$ double & 1.554 double & $1 \times 1$ struct & $1 \times 1$ struct & 1x20 double & 20 & [] $20 \times 7$ double & {$[100,78.700 \ldots$} & {$[80.5919,66.08 \ldots$} & $17 \times 20$ double & \\
\hline in & 150 ctmint & 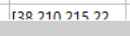 & 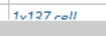 & & inn 11 & 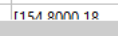 & & & & & & - & 。 & mourtanithe & $\operatorname{rim} 7510 n$ & re2 19276500 & 128210215320 & \\
\hline
\end{tabular}

Figure 3.7 Example of results as displayed in the MATLAB-based tool prototype 


\subsubsection{Illustrative Examples}

The main objective of Task 5 is to implement the proposed EB-MOO methodology through examples of different scenarios. Task 5 provides an opportunity to evaluate the effectiveness of the proposed methodology. The tool prototype produced under Task 4 is primarily used for this purpose. Several examples of unconstraint and constraint (budget and performance) scenarios were established to test/validate concepts, prove effectiveness, and demonstrate and communicate potential benefits—-by predicting investment needs and performance.

The data needed for the tool prototype vary considerably based on the optimization technique and approach (bottom-up or top-down approach) being applied, and the demanded level of assessment and analysis. A sample set of existing bridges (portfolio) was carefully chosen for all these examples based on relevant data availability/completeness, common features, attributes, and other factors - required to carry out the tool prototype's optimization, deterioration forecasting, LC economic analysis, and other processes of the different modules - to ensure a high reliability of outcomes. The tool prototype can handle a portfolio of many bridges. The selected portfolio consists of 40 bridges from the same network-sharing the same decision-making entity, geographical area, vicinity, or other characteristics. Table 3.2 includes the inspection data, major attributes and characteristics of each bridge in the portfolio. The portfolio provided an excellent opportunity to implement all stages of the EB-MOO methodology.

Example results are validated by verifying optimized priorities, funding requirements, and infographic depictions produced by the tool prototype. Results proved the capability of the methodology to recognize short- and long-term investment needs for bridge programming and planning. Sample bridges were examined at different assessment levels. The tool produced reliable LC alternative results, generated Pareto optimal or near-optimal solutions, predicted performance, 
and determined long-term intervention strategies and funding requirements. The implementation proved that the tool can facilitate performing trade-off analyses between funding scenarios and performance — where specific criteria and constraints are imposed to determine the required levels of investment to meet performance targets - or to evaluate the impact of resource allocation decisions. As stated earlier, the tool can serve as a framework to carry out a sensitivity analysis on obtained results for funding uncertainty or other uncertainties to make any necessary adjustments. Throughout this dissertation, results from the EB-MOO methodology implementation using the tool prototype are summarized and displayed in tabulations and graphs to help visualize/communicate assessment outcomes and demonstrate effectiveness and benefits. 
Table 3.2 Inspection data, major attributes and characteristics of each sample bridge in the portfolio

\begin{tabular}{|c|c|c|c|c|c|c|c|c|c|c|c|c|c|c|c|c|c|}
\hline 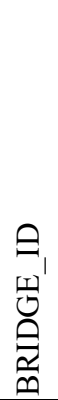 & 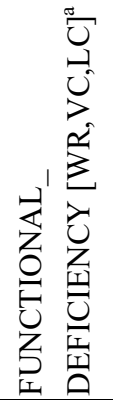 & 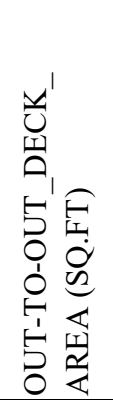 & 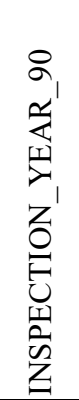 & 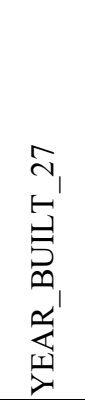 & 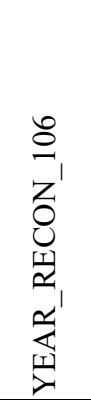 & 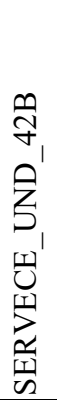 & 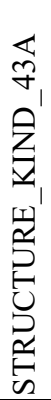 & 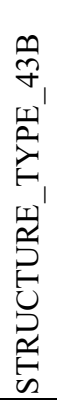 & 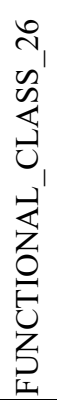 & 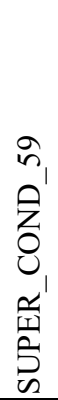 & 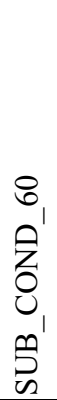 & 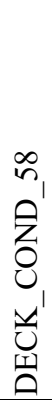 & 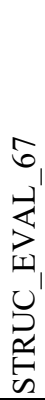 & 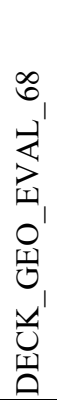 & 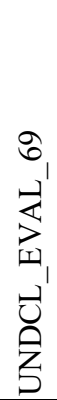 & 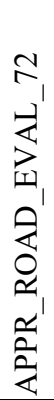 & 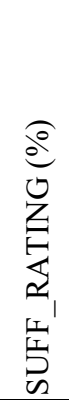 \\
\hline 1 & {$[0,0,0]$} & 1035.6 & 2015 & 1956 & 1992 & 4 & 3 & 2 & 12 & 7 & 7 & 7 & 7 & 9 & 9 & 8 & 92.1 \\
\hline 2 & {$[0,0,0]$} & 7168 & 2015 & 1999 & 0 & 6 & 3 & 16 & 14 & 5 & 7 & 8 & 5 & 6 & 5 & 6 & 82.2 \\
\hline 3 & {$[0,0,0]$} & 7168 & 2015 & 1999 & 0 & 6 & 3 & 16 & 14 & 5 & 7 & 8 & 5 & 6 & 6 & 8 & 83.2 \\
\hline 4 & {$[0,0,1]$} & 2858.4 & 2015 & 1987 & 2011 & 5 & 5 & 2 & 11 & 8 & 7 & 8 & 7 & 7 & 78 & 9 & 93.1 \\
\hline 5 & {$[1,0,0]$} & 805.68 & 2015 & 1948 & 0 & 5 & 3 & 16 & 14 & 6 & 7 & 7 & 6 & 4 & 78 & 8 & 52.5 \\
\hline 6 & {$[0,0,1]$} & 2492.8 & 2016 & 1986 & 0 & 1 & 6 & 21 & 11 & 7 & 7 & 7 & 7 & 6 & 5 & 8 & 91.7 \\
\hline 7 & {$[0,0,1]$} & 7016.9 & 2015 & 2013 & 0 & 1 & 4 & 6 & 11 & 8 & 8 & 8 & 8 & 9 & 3 & 9 & 96 \\
\hline 8 & {$[0,0,0]$} & 6901.4 & 2017 & 2006 & 0 & 1 & 4 & 6 & 14 & 8 & 8 & 7 & 8 & 9 & 9 & 7 & 98.9 \\
\hline 9 & {$[0,1,0]$} & 2759.4 & 2016 & 1964 & 2004 & 1 & 5 & 2 & 11 & 7 & 7 & 6 & 7 & 4 & 3 & 9 & 83.9 \\
\hline 10 & {$[0,1,0]$} & 2259.9 & 2016 & 1964 & 2004 & 1 & 5 & 2 & 11 & 5 & 7 & 5 & 5 & 6 & 3 & 8 & 72.4 \\
\hline 11 & {$[1,0,0]$} & 1210.6 & 2017 & 1957 & 0 & 5 & 3 & 2 & 2 & 7 & 7 & 6 & 6 & 3 & 78 & 8 & 74.3 \\
\hline 12 & {$[1,0,0]$} & 1210.6 & 2017 & 1957 & 0 & 5 & 3 & 2 & 2 & 7 & 7 & 6 & 6 & 3 & 78 & 8 & 74.3 \\
\hline 13 & {$[1,0,0]$} & 1149.1 & 2016 & 1927 & 2005 & 5 & 3 & 17 & 2 & 6 & 5 & 7 & 5 & 2 & 78 & 3 & 59.9 \\
\hline 14 & {$[0,0,1]$} & 11598 & 2016 & 1955 & 0 & 4 & 3 & 12 & 11 & 6 & 7 & 5 & 6 & 9 & 3 & 8 & 66 \\
\hline 15 & {$[0,0,1]$} & 23317 & 2015 & 1967 & 0 & 6 & 4 & 10 & 12 & 5 & 7 & 5 & & 4 & 9 & 8 & 43.8 \\
\hline 16 & {$[0,1,0]$} & 909.51 & 2015 & 1953 & 0 & 1 & 3 & 2 & 12 & 7 & 6 & 6 & 6 & 2 & 3 & 8 & 61 \\
\hline 17 & {$[1,0,1]$} & 39791 & 2016 & 1953 & 0 & 6 & 4 & 10 & 12 & 5 & 6 & 5 & 5 & 2 & 3 & 8 & 44.1 \\
\hline 18 & {$[1,0,1]$} & 6913.4 & 2016 & 1941 & 0 & 5 & 3 & 15 & 14 & 5 & 5 & 6 & 5 & 2 & 78 & 6 & 46.5 \\
\hline 19 & {$[1,0,0]$} & 1051.6 & 2016 & 1963 & 2011 & 6 & 3 & 16 & 14 & 6 & 6 & 6 & 6 & 3 & 3 & 9 & 68.5 \\
\hline 20 & {$[1,1,0]$} & 472.94 & 2015 & 1958 & 0 & 1 & 3 & 2 & 14 & 7 & 7 & 7 & 7 & 2 & 3 & 8 & 74.9 \\
\hline 21 & {$[1,1,0]$} & 472.94 & 2015 & 1958 & 0 & 1 & 3 & 2 & 14 & 7 & 7 & 6 & 7 & 2 & 3 & 8 & 74.9 \\
\hline 22 & {$[1,0,1]$} & 63.3 & 2017 & 2005 & 0 & 5 & 3 & 0 & 9 & 6 & 6 & 6 & 5 & 2 & 78 & 6 & 62.8 \\
\hline 23 & {$[1,0,1]$} & 20731 & 2015 & 1938 & 1998 & 5 & 4 & 10 & 2 & 5 & 6 & 5 & 5 & 2 & 78 & 8 & 57.7 \\
\hline 24 & {$[0,0,0]$} & 13202 & 2015 & 1993 & 0 & 6 & 4 & 2 & 6 & 6 & 7 & 7 & 6 & 9 & 78 & 8 & 99.7 \\
\hline 25 & {$[0,0,1]$} & 17003 & 2016 & 1965 & 0 & 5 & 5 & 2 & 14 & 5 & 6 & 6 & 5 & 5 & 78 & 8 & 65.5 \\
\hline 26 & {$[1,0,0]$} & 678.4 & 2015 & 1952 & 0 & 5 & 3 & 2 & 2 & 7 & 6 & 7 & 6 & 5 & 78 & 8 & 77.2 \\
\hline 27 & {$[1,0,0]$} & 229 & 2016 & 1948 & 0 & 5 & 1 & 1 & 2 & 7 & 7 & 7 & 7 & 3 & 78 & 8 & 80 \\
\hline 28 & {$[0,1,0]$} & 630.89 & 2015 & 1958 & 0 & 1 & 1 & 1 & 14 & 7 & 7 & 7 & 7 & 5 & 3 & 8 & 77.9 \\
\hline 29 & {$[0,0,0]$} & 4312.5 & 2015 & 2003 & 0 & 6 & 3 & 16 & 14 & 7 & 7 & 7 & 7 & 4 & 3 & 8 & 83.6 \\
\hline 30 & {$[0,0,0]$} & 4312.5 & 2015 & 2004 & 0 & 6 & 3 & 16 & 14 & 7 & 7 & 7 & 7 & 4 & 3 & 8 & 83.6 \\
\hline 31 & {$[1,0,0]$} & 138.37 & 2015 & 1927 & 1950 & 5 & 1 & 1 & 14 & 6 & 6 & 6 & 6 & 3 & 78 & 9 & 79.7 \\
\hline 32 & {$[0,0,0]$} & 3726.5 & 2016 & 2006 & 0 & 1 & 4 & 6 & 14 & 8 & 8 & 8 & 8 & 7 & 6 & 9 & 98.1 \\
\hline 33 & {$[0,0,0]$} & 4470.1 & 2016 & 2007 & 0 & 1 & 4 & 6 & 14 & 8 & 8 & 8 & 8 & 7 & 6 & 9 & 96.2 \\
\hline 34 & {$[0,0,0]$} & 446.04 & 2015 & 1941 & 0 & 5 & 3 & 15 & 14 & 5 & 7 & 6 & 5 & 4 & 78 & 9 & 56.4 \\
\hline 35 & {$[1,0,0]$} & 2337.2 & 2016 & 1913 & 1995 & 5 & 3 & 16 & 14 & 6 & 6 & 7 & 5 & 2 & 78 & 8 & 55 \\
\hline 36 & {$[1,0,1]$} & 1979 & 2015 & 1953 & 1966 & 5 & 3 & 2 & 2 & 7 & 5 & 6 & 5 & 4 & 78 & 7 & 60.1 \\
\hline 37 & {$[1,0,1]$} & 1727.8 & 2015 & 1959 & 0 & 6 & 3 & 16 & 14 & 5 & 7 & 6 & 5 & 4 & 5 & 8 & 42.5 \\
\hline 38 & {$[1,0,1]$} & 1727.8 & 2015 & 1959 & 0 & 6 & 3 & 16 & 14 & 5 & 7 & 6 & 5 & 4 & 5 & 8 & 42.5 \\
\hline 39 & {$[0,0,1]$} & 181.8 & 2016 & 1949 & 1957 & 5 & 1 & 1 & 2 & 7 & 6 & 7 & 5 & 4 & 78 & 9 & 69.5 \\
\hline 40 & {$[1,0,1]$} & 119.21 & 2016 & 1941 & 1954 & 5 & 1 & 1 & 2 & 7 & 7 & 7 & 5 & 3 & 78 & 9 & 59.6 \\
\hline
\end{tabular}

Note. $\mathrm{WR}=$ width required; $\mathrm{VC}=$ vertical clearance; $\mathrm{LC}=$ load capacity.

${ }^{\text {a }}$ The value of 1 represents the existence of the functional deficiency, otherwise the value of 0 . 


\subsection{Summary and Conclusions}

The intent of Task 2 is to transfer previous work on TAM and best practices, identified throughout the literature review under Task 1, to refine the conceptual TAM framework introduced in the research proposal. This chapter presented the refined goal-driven TAM framework, applying the principles of the Guide and discussed the different framework steps and interactions with the TAMP and the long-range planning and programming process. A comprehensive overview of the proposed EB-MOO methodology integrated into this refined TAM framework is included in this chapter. Each of the five modules of the methodology is presented separately. The methodology serves as a decision support tool for identifying optimal or near-optimal intervention actions and funding needs, and aiding the development of short- and long-term investment strategies, and trade-offs between investment levels and performance.

The chapter elaborated on the posteriori articulation of preference approach followed by methodology_-Pareto solutions are first determined, and then presented to the decision makers to select the best based on preferences. The chapter touched on the different types of analyses (i.e., sensitivity, "what-if" scenario, and trade-off analyses) that can be performed to explore the whole set of Pareto solutions and communicate resulting impacts of limited resources and needs to achieve performance goals. Although the risk aspect is beyond the scope of this research, the chapter emphasized on the importance of considering it in the decision-making process to attain a reasonable informed decision. The proposed EB-MOO methodology is well-suited to incorporate a risk assessment model in the future.

The development of a tool prototype structured around the five modules of the EB-MOO methodology falls under Task 4-a tool prototype that complements common decision support tools and BMSs, and builds upon their existing capabilities. This chapter discussed the developed 
MATLAB-based tool prototype used to implement the EB-MOO methodology. Several examples of unconstrained and constrained scenarios were established as part of Task 5 to test/validate concepts, prove effectiveness, and demonstrate and communicate potential benefits using the tool prototype. A sample set of existing bridges (portfolio) chosen for all these examples is introduced in this chapter. The tool was capable to produce reliable LC alternative results, generate Pareto optimal or near-optimal solutions, predict performance, and determine long-term intervention strategies and funding requirements. 


\section{CHAPTER 4-DATA PROCESSING MODULE}

- Task 3: Proposing an Element-Based Multi-Objective Optimization Methodology

- Task 4: Development of a Tool Prototype

\subsection{Introduction}

The main objective of the proposed EB-MOO methodology is to determine short- and long-term investment needs and support recommending programs and implementation schedules. The methodology is structured around five modules (i.e., data processing, improvement, ELO, BLO, and NLO modules). It consists of three levels of optimization assessment based on the Pareto optimality concept: element level, bridge level, and network level (following either a top-down or bottom-up approach). It relies on three distinct screening processes (i.e., element deficiency, alternative feasibility, and solution superiority screening processes). A separate chapter is dedicated to discuss each of these modules and their processes in greater detail.

This chapter discusses the modeling approach used for data processing. A portfolio of candidate bridges or programmed bridges in the TIP/STIP is introduced in the data processing module to be considered throughout the different processes of the different modules and examined at the element, bridge and network levels. The module relies on decision makers' preferences and inputs, quality data, and information provided in the TAMP and TIP/STIP. Leading-edge forecasting and up-to-date cost models are integrated into this module. Deterioration modeling is the main driver of the element LC condition required in the optimization analysis. The chapter discusses the independent deterioration model used to estimate the life expectancy of an element and predict its 
performance over an analysis period. The chapter also lays out the process of assessing improvement needs based on functional deficiencies. It discusses the cost models adapted to estimate incurred user costs due to existing functional deficiencies. The chapter presents the different correlation/regression formulas used to estimate major improvement costs - to eliminate incurred user costs or relieve the bridge from all restrictions, and provide the required LOS and design standards. The three types of element LC profiles (i.e., DN, RO, and AE LC profiles) are introduced in this chapter with illustrative examples.

\subsection{Module Framework}

Effectively implementing a decision support tool based on multiple criteria/objectives is a challenge for most state DOTs: many of the stumbling blocks are associated with the data quality and availability, data collection methods and costs, outdated databases, and lack of comprehensive inventories and trained data analysts. A sound $\mathrm{MOO}$ methodology requires a complete high quality of data to support its involved analyses and processes (e.g., deterioration and cost modeling, screening processes, BCA, LCCA, and trade-off analysis). The proposed methodology is a datadriven process that relies on accurate "good" data to produce reliable results. Quality data from state and national data management systems or external sources, user's preferences and inputs, and information retrieved from the TAMP and TIP/STIP are processed in this module.

Data are being collected by state DOTs and MPOs—data related to location-related information, attributes, inspection, maintenance, costs and funding, average daily traffic, truck traffic, congestion, mobility and safety, environment, etc. The data processing module is designed to accommodate user-specified performance measures and commonly collected data from widelyused state and national data management systems (e.g., BrM, NBI system, HPMS, and LTBP InfoBridge) to ensure a high reliability of outcomes. The needed data vary considerably based on 
the optimization technique and approach (e.g., bottom-up or top-down approach) being applied, and the demanded levels of assessment and analyses. Established objectives, performance measures and targets, budget allocations, and any constraints need to be transferred into this module in a consistent way.

The TAMP, like the LRTP, can be considered as a business plan that reveals the level of investments needed to meet the state DOT's policy goals, objectives, and performance targets related to its transportation system. For example, the specified budget constraints in the optimization module should be aligned with the financial plan included in the TAMP — historical and current bridge budgets, anticipated revenues, and long-term preservation investment strategies (identified funding allocations in the TIP/STIP over the next 4 years for the bridge projects).

Deterioration modeling is the main driver of the element LC condition required in the optimization process. The data processing and improvement modules rely on an independent deterioration model to predict bridge improvement needs. Different deterioration forecasting models were compared in previous research, and the Weibull/Markov (hybrid) model is overwhelming recommended for modern BMSs (Thompson et al., 2012). This hybrid model is used to forecast conditions of individual elements (e.g., deck, girders, floor beams, pins, hangers, and bearings). The model is used in BrM and Florida DOT's PLAT (Sobanjo \& Thompson, 2016b). This same hybrid model is adapted for element deterioration prediction. Florida DOT's transition probabilities and Weibull parameters (Sobanjo \& Thompson, 2016a) are used by default.

Figure 4.1 represents a flow diagram to visualize the data processing approach for each bridge in the portfolio. A set of bridges (portfolio) is introduced in this module to be considered throughout the subsequent modules, and examined at the element, bridge, and network levels. In this module, 
each bridge in the portfolio is evaluated separately. The stored data are located and processed at this initial stage to determine the estimated service life (ESL), DN condition states, and health indices for each bridge element.

For a comprehensive LCCA, state DOTs use different cost models based on operational, maintenance, preservation and rehabilitation, and replacement costs and other influential costs such as user, detour, or crash costs (Hugh, 2003). Improvement needs based on existing functional deficiencies are assessed, and major improvement costs and user costs are also determined in this module for each bridge in the portfolio. A set of feasible treatments are defined for each element condition state. Treatments classified as MRR or preservation treatments (these two terms "MRR" and "preservation" are used interchangeably throughout this dissertation) are committed mainly to preserve the element or restore it to a better condition. Each element condition state is associated with up to five treatments. One of these treatments is always the DN option to represent taking no action. These treatments are grouped into five main actions (i.e., MRR Actions 0, 1, 2, 3, and 4). Unit costs are assigned to each of these defined treatments. Each of these treatment is discussed in the subsequent section. Only MRR Action 0, which represents the DN option, is used in this module. Florida DOT's AE models (Sobanjo \& Thompson, 2016a) are adapted after being slightly modified to agree with these definitions.

The module distinguishes functional improvement needs from preservation needs. Preservation needs (as assessed in the improvement module) are identified for each element condition state, while functional improvement needs are resulting from functional deficiencies associated with the entire bridge. All bridges listed in the portfolio are assessed for functional improvement needs. The module follows a common approach used by BrM (licensed to numerous state DOTs), NBIAS 
(used by FHWA), and other state DOT's BMSs to assess the economic consequences in terms of user costs due to functional deficiencies - possibly affecting the LOS provided to the users.

The major cost of improvement needed to eliminate the functional deficiencies is estimated. Widening, raising, and/or strengthening (symbolized by FCI Action 5) improvement needs are assessed for each bridge based on several mathematical models. Each of these models rely on proven correlation/regression formulas, LOS and design standards, and unit costs. A functional improvement relieves the bridge from certain restrictions and reduces or eliminates any incurred user costs; however, a replacement (symbolized by REP Action 6) is assumed to relieve the bridge from all its restrictions, provide the required LOS and design standards, and eliminate all incurred user costs. The module assesses the replacement option of the entire bridge regardless of its element condition states or restrictions - assuming the replacement option is always feasible; and therefore, it's eligible to compete. These estimated bridge costs are transferred to the improvement module for consideration in the LCCA. NBIAS's and Florida DOT's user cost models (Cambridge Systematics, 2011b; Sobanjo \& Thompson, 2004) are adapted for assessing functional improvement and replacement needs. These default models can be substituted or customized as appropriate.

The total LC period is referred to as "analysis period." The length of this period depends on the agency's preservation policies and preferences. A fixed long analysis period (usually around 50 years) produces realistic LC alternatives (discussed in Chapter 5) covering most of the anticipated element service life. An analysis period of 54 years is used by default. Three types of LC profiles (i.e., DN, RO, and AE LC profiles) are constructed for each element to predict bridge and element health indices at different points in time, and estimate remaining service lives and LCCs. In this module, only the DN LC profiles are constructed to determine the element ESL, DN condition 
states, and DN health indices at different points in time. The DN LC profile represents the "base" scenario of predicting condition of an untreated element (no actions are performed to extend the element ESL). This scenario simulates the element declining condition when no action (i.e., MRR Action 0) is ever taken until reaching its end-of-life threshold. The element deficiency screening process of the improvement module (discussed in the following chapter) relies on the DN LC profile produced results to evaluate the extent of element deterioration. Some elements (classified as "deficient elements") may have deteriorated to a level where major repairs or proactive preservation efforts are necessary, and others may show no sign of deterioration. The subsequent sections are devoted to overview these underlying concepts. The module approach is based on the following concepts:

1. Retrieve the different types of stored data associated with each bridge in the portfolio

2. Construct the DN LC profile for each element of each bridge in the portfolio

3. Predict the ESL, DN condition states, and DN health indices for each element at different points in the analysis period

4. Predict the $\mathrm{DN}$ health indices for each bridge at different points in the analysis period

5. Organize the produced DN LC profile results by element for each bridge in the portfolio

6. Assess the functional improvement needs based on existing functional deficiencies for each bridge in the portfolio

7. Estimate the economic consequences in terms of user costs due to existing functional deficiencies for each bridge in the portfolio

8. Estimate the major improvement cost (i.e., FCI Action 5) required to relieve existing functional deficiencies for each bridge in the portfolio 
9. Estimate the major improvement cost (i.e., REP Action 6) required to relieve each bridge in the portfolio from all its existing restrictions, provide the required LOS and design standards, and eliminate all incurred user costs

10. Store the estimated major improvement and user costs for each bridge in the portfolio 


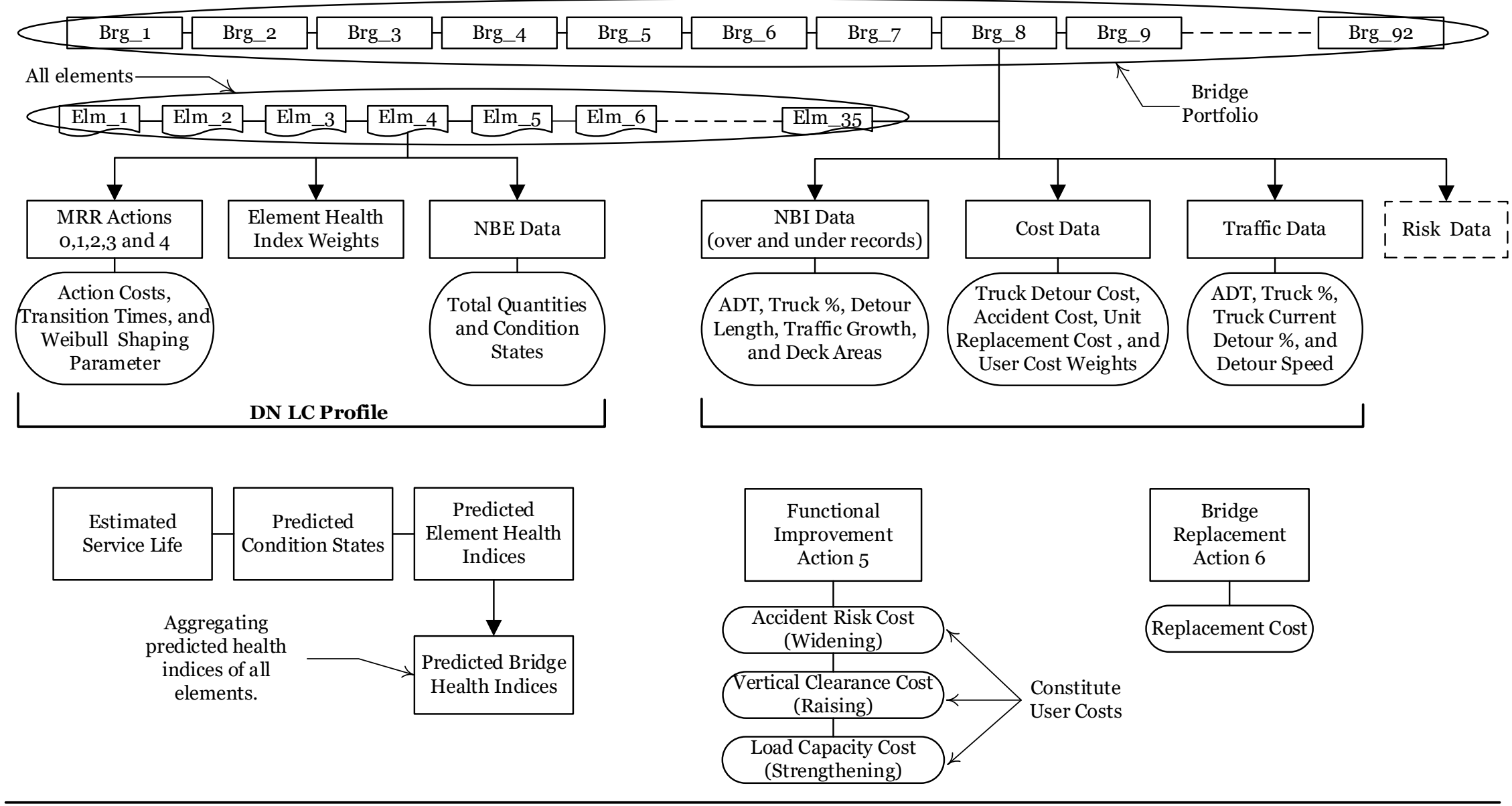

Figure 4.1 Data processing module framework 


\subsection{Bridge Inspection Data}

As stated earlier, the proposed EB-MOO methodology is a data-driven process that relies on accurate "good" data from state and national data management systems or external sources to produce reliable results. Most state DOTs use BMSs to host NBI data (i.e., inventory, appraisal and condition data) and element-level inspection data, as well as maintenance management systems for maintenance-related data. These systems are used to inventory new structures, update conditions, track work accomplishments and costs, measure performance, predict deterioration trends, and recommend maintenance activities, preservation treatments and candidate bridge improvement (preservation, rehabilitation or replacement) projects. Other systems are also widely used by state DOTs to collect different types of data such as pavement condition data, mobility data, safety data, and environmental data. Along with these different state systems, a handful of national systems (i.e., NBI system, HPMS, NBIAS, HSIS, FARS, and LTBP InfoBridge) exist to provide national data in a consistent format. State DOTs are required to annually collect and submit most of these national data to comply with the federal data collection requirements and support the development of the Status of the Nation's Highways, Bridges, and Transit: Conditions \& Performance Report to Congress (U.S. Department of Transportation, 2015), the national performance measurement, and the federal resource allocation (Cambridge Systematics, Inc., 2009).

\subsubsection{National Bridge Inspection Standards}

The National Bridge Inspection Standards (NBIS) (23 USC 151) established in 1971. The core requirement of the NBIS is the periodic inspection of bridges carrying public traffic and greater than $20 \mathrm{ft}$. in length. Approximately, bridge inspection data from over 600,000 of the nation's bridges carrying public roads are annually being reported and maintained in the NBI system, since 
1983. State DOTs annually report their inspection data to FHWA to be stored in the NBI system. To ensure consistent reported NBI data, state DOTs collect the data in accordance with the inspection procedures and format outlined in the FHWA's Recording and Coding Guide for the Structure Inventory and Appraisal of the Nation's Bridges (FHWA, 1995). The recording and coding guide specifies $116 \mathrm{NBI}$ coding items (structure inventory and appraisal items), describing a bridge location, geometrics, age, traffic, load capacity, structural condition, and other relevant features. Table 4.1 represents a complete list of these items. 
Table 4.1 A complete list of NBI coding items

\begin{tabular}{|c|c|c|c|c|c|c|c|c|c|}
\hline \multicolumn{10}{|c|}{ NBI Coding Items } \\
\hline 1 & State Code & 22 & Owner & $43 \mathrm{~A}$ & $\begin{array}{l}\text { Kind of } \\
\text { Material/Design }\end{array}$ & 62 & Culverts & 96 & Total Project Cost \\
\hline 8 & Structure Number & 26 & $\begin{array}{l}\text { Functional Class Of } \\
\text { Inventory Rte. }\end{array}$ & $43 \mathrm{~B}$ & $\begin{array}{l}\text { Type of } \\
\text { Design/Construction }\end{array}$ & 63 & $\begin{array}{l}\text { Method Used To Determine } \\
\text { Operating Rating }\end{array}$ & 97 & $\begin{array}{l}\text { Year Of Improvement } \\
\text { Cost Estimate }\end{array}$ \\
\hline 5 & Inventory Route & 27 & Year Built & 44 & $\begin{array}{l}\text { Structure Type, } \\
\text { Approach Spans }\end{array}$ & 64 & Operating Rating & 98 & Border Bridge \\
\hline $5 \mathrm{~A}$ & Record Type & 28 & $\begin{array}{l}\text { Lanes On/Under } \\
\text { Structure }\end{array}$ & $44 \mathrm{~A}$ & $\begin{array}{l}\text { Kind of } \\
\text { Material/Design }\end{array}$ & 65 & $\begin{array}{l}\text { Method Used To Determine } \\
\text { Inventory Rating }\end{array}$ & $98 \mathrm{~A}$ & Neighboring State Code \\
\hline $5 \mathrm{~B}$ & Route Signing Prefix & $28 \mathrm{~A}$ & Lanes On Structure & 44B & $\begin{array}{l}\text { Type of } \\
\text { Design/Construction }\end{array}$ & 66 & Inventory Rating & $98 \mathrm{~B}$ & Percent Responsibility \\
\hline $5 \mathrm{C}$ & $\begin{array}{l}\text { Designated Level of } \\
\text { Service }\end{array}$ & $28 \mathrm{~B}$ & Lanes Under Structure & 45 & $\begin{array}{l}\text { Number Of Spans In } \\
\text { Main Unit }\end{array}$ & 67 & Structural Evaluation & 99 & $\begin{array}{l}\text { Border Bridge Structure } \\
\text { Number }\end{array}$ \\
\hline $5 \mathrm{D}$ & Route Number & 29 & Average Daily Traffic & 46 & $\begin{array}{l}\text { Number Of Approach } \\
\text { Spans }\end{array}$ & 68 & Deck Geometry & 100 & $\begin{array}{l}\text { STRAHNET Highway } \\
\text { Designation }\end{array}$ \\
\hline $5 \mathrm{E}$ & Directional Suffix & 30 & $\begin{array}{l}\text { Year Of Average Daily } \\
\text { Traffic }\end{array}$ & 47 & $\begin{array}{l}\text { Inventory Rte Total } \\
\text { Horz Clearance }\end{array}$ & 69 & $\begin{array}{l}\text { Underclear, Vertical \& } \\
\text { Horizontal }\end{array}$ & 101 & $\begin{array}{l}\text { Parallel Structure } \\
\text { Designation }\end{array}$ \\
\hline 2 & $\begin{array}{l}\text { Highway Agency } \\
\text { District }\end{array}$ & 31 & Design Load & 48 & $\begin{array}{l}\text { Length Of Maximum } \\
\text { Span }\end{array}$ & 70 & Bridge Posting & 102 & Direction Of Traffic \\
\hline 3 & $\begin{array}{l}\text { County (Parish) } \\
\text { Code }\end{array}$ & 32 & $\begin{array}{l}\text { Approach Roadway } \\
\text { Width }\end{array}$ & 49 & Structure Length & 71 & Waterway Adequacy & 103 & $\begin{array}{l}\text { Temporary Structure } \\
\text { Designation }\end{array}$ \\
\hline 4 & Place Code & 33 & Bridge Median & 50 & Curb/Sidewalk Widths & 72 & $\begin{array}{l}\text { Approach Roadway } \\
\text { Alignment }\end{array}$ & 104 & $\begin{array}{l}\text { Highway System Of } \\
\text { Inventory Route }\end{array}$ \\
\hline 6 & Features Intersected & 34 & Skew & $50 \mathrm{~A}$ & $\begin{array}{l}\text { Left Curb/Sidewalk } \\
\text { Width }\end{array}$ & 75 & Type of Work & 105 & Federal Lands Highways \\
\hline $6 \mathrm{~A}$ & Features Intersected & 35 & Structure Flared & $50 \mathrm{~B}$ & $\begin{array}{l}\text { Right Curb/Sidewalk } \\
\text { Width }\end{array}$ & $75 \mathrm{~A}$ & Type of Work Proposed & 106 & Year Reconstructed \\
\hline $6 \mathrm{~B}$ & $\begin{array}{l}\text { Critical Facility } \\
\text { Indicator }\end{array}$ & 36 & Traffic Safety Features & 51 & $\begin{array}{l}\text { Bridge Roadway } \\
\text { Width Curb-To-Curb }\end{array}$ & $75 \mathrm{~B}$ & Work Done By & 107 & Deck Structure Type \\
\hline 7 & $\begin{array}{l}\text { Facility Carried By } \\
\text { Structure }\end{array}$ & $36 \mathrm{~A}$ & Bridge Railings & 52 & $\begin{array}{l}\text { Deck Width, Out-To- } \\
\text { Out }\end{array}$ & 76 & $\begin{array}{l}\text { Length Of Structure } \\
\text { Improvement }\end{array}$ & 108 & $\begin{array}{l}\text { Wearing Surface/ } \\
\text { Protective System }\end{array}$ \\
\hline 9 & Location & $36 \mathrm{~B}$ & Transitions & 53 & $\begin{array}{l}\text { Min Vert Clear Over } \\
\text { Bridge Roadway }\end{array}$ & 90 & Inspection Date & $108 \mathrm{~A}$ & Type of Wearing Surface \\
\hline 10 & $\begin{array}{l}\text { Inventory Rte, Min } \\
\text { Vert Clearance }\end{array}$ & $36 \mathrm{C}$ & Approach Guardrail & 54 & $\begin{array}{l}\text { Minimum Vertical } \\
\text { Underclearance }\end{array}$ & 91 & $\begin{array}{l}\text { Designated Inspection } \\
\text { Frequency }\end{array}$ & 108B & Type of Membrane \\
\hline 11 & Kilometerpoint & $36 \mathrm{D}$ & $\begin{array}{l}\text { Approach Guardrail } \\
\text { Ends }\end{array}$ & $54 \mathrm{~A}$ & Reference Feature & 92 & Critical Feature Inspection & $108 \mathrm{C}$ & Deck Protection \\
\hline 12 & $\begin{array}{l}\text { Base Highway } \\
\text { Network }\end{array}$ & 37 & Historical significance & $54 \mathrm{~B}$ & $\begin{array}{l}\text { Minimum Vertical } \\
\text { Underclearance }\end{array}$ & $92 \mathrm{~A}$ & Fracture Critical Details & 109 & $\begin{array}{l}\text { Average Daily Truck } \\
\text { Traffic }\end{array}$ \\
\hline 13 & $\begin{array}{l}\text { Inventory Route, } \\
\text { Subroute Number }\end{array}$ & 38 & Navigation Control & 55 & $\begin{array}{l}\text { Min Lateral Underclear } \\
\text { On Right }\end{array}$ & $92 \mathrm{~B}$ & Underwater Inspection & 110 & $\begin{array}{l}\text { Designated National } \\
\text { Network }\end{array}$ \\
\hline $13 \mathrm{~A}$ & $\begin{array}{l}\text { LRS Inventory } \\
\text { Route }\end{array}$ & 39 & $\begin{array}{l}\text { Navigation Vertical } \\
\text { Clearance }\end{array}$ & $55 \mathrm{~A}$ & Reference Feature & $92 \mathrm{C}$ & Other Special Inspection & 111 & $\begin{array}{l}\text { Pier or Abutment } \\
\text { Protection }\end{array}$ \\
\hline $13 \mathrm{~B}$ & Subroute Number & 40 & $\begin{array}{l}\text { Navigation Horizontal } \\
\text { Clearance }\end{array}$ & $55 \mathrm{~B}$ & $\begin{array}{l}\text { Minimum Lateral } \\
\text { Underclearance }\end{array}$ & 93 & $\begin{array}{l}\text { Critical Feature Inspection } \\
\text { Dates }\end{array}$ & 112 & NBIS Bridge Length \\
\hline 16 & Latitude & 41 & $\begin{array}{l}\text { Structure } \\
\text { Open/Posted/Closed }\end{array}$ & 56 & $\begin{array}{l}\text { Min Lateral Underclear } \\
\text { On Left }\end{array}$ & $93 \mathrm{~A}$ & Fracture Critical Details Date & 113 & Scour Critical Bridges \\
\hline 17 & Longitude & 42 & Type Of Service & 58 & Deck & $93 \mathrm{~B}$ & Underwater Inspection Date & 114 & $\begin{array}{l}\text { Future Average Daily } \\
\text { Traffic }\end{array}$ \\
\hline 19 & $\begin{array}{l}\text { Bypass/Detour } \\
\text { Length }\end{array}$ & $42 \mathrm{~A}$ & $\begin{array}{l}\text { Type of Service On } \\
\text { Bridge }\end{array}$ & 59 & Superstructure & $93 \mathrm{C}$ & Other Special Inspection Date & 115 & $\begin{array}{l}\text { Year of Future Average } \\
\text { Daily Traffic }\end{array}$ \\
\hline 20 & Toll & $42 \mathrm{~B}$ & $\begin{array}{l}\text { Type of Service Under } \\
\text { Bridge }\end{array}$ & 60 & Substructure & 94 & Bridge Improvement Cost & 116 & $\begin{array}{l}\text { Minimum Navigation } \\
\text { Vertical Clearance, Vertical } \\
\text { Lift Bridge }\end{array}$ \\
\hline 21 & $\begin{array}{l}\text { Maintenance } \\
\text { Responsibility }\end{array}$ & 43 & Structure Type, Main & 61 & $\begin{array}{l}\text { Channel/Channel } \\
\text { Protection }\end{array}$ & 95 & Roadway Improvement Cost & & \\
\hline
\end{tabular}

Note. Adapted from the FHWA's Recording and Coding Guide for the Structure Inventory and Appraisal of the Nation's Bridges (FHWA, 1995). 
The NBI condition rating reflects the range of the physical condition of the major bridge structural components: deck, superstructure, substructure, and culvert. The recording and coding guide defines condition ratings on a scale of 0 to 9 . Turner and Richardson stated that this scale indicates the overall structural integrity but a little information about the type and location of the defect and/or damage (as cited in Elbehairy, 2007). For example, an NBI condition rating of 3 (serious condition) is used to describe a bridge component failure. This low condition rating signals to a serious deterioration that could compromise the structural capacity, safety, and serviceability of the bridge; however, in most cases, the extent of the deterioration is limited to a local element and not the entire component of the bridge. Table 4.2 provides a description of each of these NBI condition ratings. 
Table 4.2 Descriptions of NBI condition ratings

\begin{tabular}{|c|c|c|}
\hline Code & Condition & Description \\
\hline $\mathrm{N}$ & Not Applicable & \\
\hline 9 & Excellent Condition & \\
\hline 8 & $\begin{array}{l}\text { Very Good } \\
\text { Condition }\end{array}$ & No problems noted. \\
\hline 7 & Good Condition & Some minor problems. \\
\hline 6 & $\begin{array}{l}\text { Satisfactory } \\
\text { Condition }\end{array}$ & Structural elements show some minor deterioration. \\
\hline 5 & Fair Condition & $\begin{array}{l}\text { All primary structural elements are sound but may have } \\
\text { minor section loss, cracking, spalling or scour. }\end{array}$ \\
\hline 4 & Poor Condition & Advanced section loss, deterioration, spalling or scour. \\
\hline 3 & Serious Condition & $\begin{array}{l}\text { Loss of section, deterioration, spalling or scour have } \\
\text { seriously affected primary structural components. Local } \\
\text { failures are possible. Fatigue cracks in steel or shear cracks } \\
\text { in concrete may be present. }\end{array}$ \\
\hline 2 & Critical Condition & $\begin{array}{l}\text { Advanced deterioration of primary structural elements. } \\
\text { Fatigue cracks in steel or shear cracks in concrete may be } \\
\text { present or scour may have removed substructure support. } \\
\text { Unless closely monitored it may be necessary to close the } \\
\text { bridge until corrective action is taken. }\end{array}$ \\
\hline 1 & $\begin{array}{l}\text { Imminent Failure } \\
\text { Condition }\end{array}$ & $\begin{array}{l}\text { Major deterioration or section loss present in critical } \\
\text { structural components or obvious vertical or horizontal } \\
\text { movement affecting structure stability. Bridge is closed to } \\
\text { traffic but corrective action may put back in light service. }\end{array}$ \\
\hline 0 & Failed Condition & Out of service - beyond corrective action. \\
\hline
\end{tabular}

The recording and coding guide classifies a bridge as "structurally deficient" if at least one of its NBI condition ratings (deck, superstructure, and substructure) is rated 4 or less, or at least one of its NBI appraisal ratings (structural condition or waterway adequacy) is rated 2 or less. FHWA (23 CFR 490.409-Calculation of National Performance Management Measures for Assessing Bridge Condition) classifies these condition ratings as follows: 7, 8, or 9 represents "Good" condition; 5 or 6 means "Fair" condition; and 4 or less indicates "Poor" condition. The intent of this classification is to develop condition targets for NHS bridges in these defined conditions, expressed as the percentage of bridge deck area. 


\subsubsection{Element-Level Bridge Inspection Data}

The NBI condition ratings describe the overall bridge component conditions but not individual elements as the element-level bridge inspection data. The ratings are susceptible to subjective interpretation by inspectors. Moreover, the ratings provide information on the levels of severity but lack to quantify the extent of the deterioration and/or damage (Adams et al., 2009). On the other hand, for example, instead of rating the condition of the entire superstructure component, an element-level bridge inspection looks at the condition of the individual elements, such as girders, floor beams, pins and hangers, and bearings, and captures the severity and extent (percentage) of the defects.

Several state DOTs have been collecting this type of element-level data since the '90s. MAP-21, 23 USC 144(d)(2), mandated to begin collecting the element-level bridge inspection data starting from October 1, 2014, for each NHS bridge in accordance with 23 CFR 650 Subpart C. Since this requirement, all state DOTs have been collecting the element-level data. The use of this type of element-level data provides a thorough and objective assessment of the bridge condition. The element-level data permit to adequately assess the damage modes experienced in different elements. Table 4.3 lists the national bridge elements (NBEs) specified in the AASHTO Manual for Bridge Element Inspection (AASHTO, 2013a). NBEs are intended to facilitate and standardize the collection of bridge element conditions across the country. NBEs represent the primary structural components of bridges needed to determine the overall condition and safety of the primary load carrying members (i.e., material and construction types of decks/slabs, bridge railings, superstructures, substructures, bearings, joints, and culverts). 
Table 4.3 A complete list of NBEs

\begin{tabular}{|c|c|c|}
\hline \multicolumn{3}{|c|}{ Decks / Slabs (NBEs) } \\
\hline El. No. & Element Name & Units \\
\hline 12 & Reinforced Concrete Deck & AREA (sq. ft.) \\
\hline 13 & Prestressed Concrete Deck & AREA (sq. ft.) \\
\hline 15 & Prestressed Concrete Top Flange & AREA (sq. ft.) \\
\hline 16 & Reinforced Concrete Top Flange & AREA (sq. ft.) \\
\hline 28 & Steel Deck-Open Grid & AREA (sq. ft.) \\
\hline 29 & Steel Deck-Concrete Filled & AREA (sq. ft.) \\
\hline 30 & Steel Deck-Corrugated/Orthotropic/Etc. & AREA (sq. ft.) \\
\hline 31 & Timber Deck & AREA (sq. ft.) \\
\hline 38 & Reinforced Concrete Slab & AREA (sq. ft.) \\
\hline 54 & Timber Slab & AREA (sq. ft.) \\
\hline 60 & Other Material Deck & AREA (sq. ft.) \\
\hline 65 & Other Material Slab & AREA (sq. ft.) \\
\hline
\end{tabular}

\begin{tabular}{|c|c|c|}
\hline \multicolumn{3}{|c|}{ Superstructures (NBEs) } \\
\hline El. No. & Element Name & Units \\
\hline 102 & Closed Web/Box Girder, Steel & LENGTH (ft.) \\
\hline 104 & Closed Web/Box Girder, Prestressed Concrete & LENGTH (ft.) \\
\hline 105 & Closed Web/Box Girder, Reinforced Concrete & LENGTH (ft.) \\
\hline 106 & Closed Web/Box Girder, Other & LENGTH (ft.) \\
\hline 107 & Open Girder/Beam, Steel & LENGTH (ft.) \\
\hline 109 & Open Girder/Beam, Prestressed Concrete & LENGTH (ft.) \\
\hline 110 & Open Girder/Beam, Reinforced Concrete & LENGTH (ft.) \\
\hline 111 & Open Girder/Beam, Timber & LENGTH (ft.) \\
\hline 112 & Open Girder/Beam, Other & LENGTH (ft.) \\
\hline 113 & Stringer, Steel & LENGTH (ft.) \\
\hline 115 & Stringer, Prestressed Concrete & LENGTH (ft.) \\
\hline 116 & Stringer, Reinforced Concrete & LENGTH (ft.) \\
\hline 117 & Stringer, Timber & LENGTH (ft.) \\
\hline 118 & Stringer, Other & LENGTH (ft.) \\
\hline 120 & Truss, Steel & LENGTH (ft.) \\
\hline 135 & Truss, Timber & LENGTH (ft.) \\
\hline 136 & Truss, Other & LENGTH (ft.) \\
\hline 141 & Arch, Steel & LENGTH (ft.) \\
\hline 142 & Arch, Other & LENGTH (ft.) \\
\hline 143 & Arch, Prestressed Concrete & LENGTH (ft.) \\
\hline 144 & Arch, Reinforced Concrete & LENGTH (ft.) \\
\hline 145 & Arch, Masonry & LENGTH (ft.) \\
\hline 146 & Arch, Timber & LENGTH (ft.) \\
\hline 147 & Cable - Main, Steel & LENGTH (ft.) \\
\hline 148 & Cable - Secondary, Steel & EACH \\
\hline 149 & Cable - Secondary, Other & EACH \\
\hline 152 & Floor Beam, Steel & LENGTH (ft.) \\
\hline 154 & Floor Beam, Prestressed Concrete & LENGTH (ft.) \\
\hline 155 & Floor Beam, Reinforced Concrete & LENGTH (ft.) \\
\hline 156 & Floor Beam, Timber & LENGTH (ft.) \\
\hline 157 & Floor Beam, Other & LENGTH (ft.) \\
\hline 161 & Pin, Pin and Hanger Assembly, or both & EACH \\
\hline 162 & Gusset Plate & EACH \\
\hline
\end{tabular}

\begin{tabular}{|c|l|c|}
\hline \multicolumn{3}{|c|}{ Substructures (NBEs) } \\
\hline El. No. & \multicolumn{1}{|c|}{ Element Name } & Units \\
\hline 202 & Columns, Steel & EACH \\
\hline 203 & Columns, Other & EACH \\
\hline 204 & Columns, Prestressed Concrete & EACH \\
\hline 205 & Columns, Reinforced Concrete & EACH \\
\hline 206 & Columns, Timber & EACH \\
\hline 207 & Column Tower (Trestle), Steel & LENGTH (ft.) \\
\hline 208 & Column Tower (Trestle), Timber & LENGTH (ft.) \\
\hline 210 & Pier Wall, Reinforced Concrete & LENGTH (ft.) \\
\hline 211 & Pier Wall, Other & LENGTH (ft.) \\
\hline 212 & Pier Wall, Timber & LENGTH (ft.) \\
\hline 213 & Pier Wall, Masonry & LENGTH (ft.) \\
\hline 215 & Abutment, Reinforced Concrete & LENGTH (ft.) \\
\hline 216 & Abutment, Timber & LENGTH (ft.) \\
\hline 217 & Abutment, Masonry & LENGTH (ft.) \\
\hline 218 & Abutment, Other & LENGTH (ft.) \\
\hline 219 & Abutment, Steel & LENGTH (ft.) \\
\hline 220 & Pile Cap/Footing, Reinforced Concrete & LENGTH (ft.) \\
\hline 225 & Pile, Steel & EACH \\
\hline 226 & Pile, Prestressed Concrete & EACH \\
\hline 227 & Pile, Reinforced Concrete & EACH \\
\hline 228 & Pile, Timber & EACH \\
\hline 229 & Pile, Other & EACH \\
\hline 231 & Pier Cap, Steel & LENGTH (ft.) \\
\hline 233 & Pier Cap, Prestressed Concrete & LENGTH (ft.) \\
\hline 234 & Pier Cap, Reinforced Concrete & LENGTH (ft.) \\
\hline 235 & Pier Cap, Timber & LENGTH (ft.) \\
\hline 236 & Pier Cap, Other & LENGTH (ft.) \\
\hline & & \\
\hline
\end{tabular}

\begin{tabular}{|c|l|c|}
\hline \multicolumn{3}{|c|}{ Bridge Rails (NBEs) } \\
\hline El. No. & \multicolumn{1}{|c|}{ Element Name } & Units \\
\hline 330 & Metal Bridge Railing & LENGTH (ft.) \\
\hline 331 & Reinforced Concrete Bridge Railing & LENGTH (ft.) \\
\hline 332 & Timber Bridge Railing & LENGTH (ft.) \\
\hline 333 & Other Bridge Railing & LENGTH (ft.) \\
\hline 334 & Masonry Bridge Railing & LENGTH (ft.) \\
\hline
\end{tabular}

\begin{tabular}{|c|l|c|}
\hline \multicolumn{3}{|c|}{ Joints (BMEs) } \\
\hline El. No. & \multicolumn{1}{|c|}{ Element Name } & Units \\
\hline 300 & Strip Seal Expansion Joint & LENGTH (ft.) \\
\hline 301 & Pourable Joint Seal & LENGTH (ft.) \\
\hline 302 & Compression Joint Seal & LENGTH (ft.) \\
\hline 303 & Assembly Joint/Seal (Modular) & LENGTH (ft.) \\
\hline 304 & Open Expansion Joint & LENGTH (ft.) \\
\hline 305 & Assembly Joint without Seal & LENGTH (ft.) \\
\hline 306 & Other Joint & LENGTH (ft.) \\
\hline
\end{tabular}

\begin{tabular}{|c|l|c|}
\hline \multicolumn{3}{|c|}{ Bearings (NBEs) } \\
\hline El. No. & \multicolumn{1}{|c|}{ Element Name } & Units \\
\hline 310 & Elastomeric Bearing & EACH \\
\hline 311 & Movable Bearing (roller, sliding, etc.) & EACH \\
\hline 312 & Enclosed/Concealed Bearing & EACH \\
\hline 313 & Fixed Bearing & EACH \\
\hline 314 & Pot Bearing & EACH \\
\hline 315 & Disk Bearing & EACH \\
\hline 316 & Other Bearing & EACH \\
\hline
\end{tabular}

\begin{tabular}{|c|l|c|}
\hline \multicolumn{3}{|c|}{ Culverts (NBEs) } \\
\hline El. No. & \multicolumn{1}{|c|}{ Element Name } & Units \\
\hline 240 & Culvert, Steel & LENGTH (ft.) \\
\hline 241 & Culvert, Reinforced Concrete & LENGTH (ft.) \\
\hline 242 & Culvert, Timber & LENGTH (ft.) \\
\hline 243 & Culvert, Other & LENGTH (ft.) \\
\hline 244 & Culvert, Masonry & LENGTH (ft.) \\
\hline 245 & Culvert, Prestressed Concrete & LENGTH (ft.) \\
\hline
\end{tabular}

\begin{tabular}{|c|c|c|}
\hline \multicolumn{3}{|c|}{ Approach Slabs (BMEs) } \\
\hline El. No. & Element Name & Units \\
\hline 320 & Prestressed Concrete Approach Slab & AREA (sq. ft.) \\
\hline 321 & Reinforced Concrete Approach Slab & AREA (sq. ft.) \\
\hline
\end{tabular}

Note. Adapted from the FHWA Training Course: Introduction to Element-Level Bridge Inspection (FHWA, 2015).

\begin{tabular}{|c|l|c|}
\hline \multicolumn{3}{|c|}{ Wearing Surface and Protective Systems (BMEs) } \\
\hline El. No. & \multicolumn{1}{|c|}{ Element Name } & Units \\
\hline 510 & Wearing Surface & AREA (sq. ft.) \\
\hline 515 & Steel Protective Coating & AREA (sq. ft.) \\
\hline 520 & Concrete Reinforcing Steel Protective System & AREA (sq. ft.) \\
\hline 521 & Concrete Protective Coating & AREA (sq. ft.) \\
\hline
\end{tabular}


Each element has a unit of measure and four defined condition states (denoted as CS1, CS2, CS3, and CS4). A higher condition state indicates a higher severity of the damage and/or deterioration of the element. The general definitions of the condition states are as follows: CS1-Good, CS2Fair, CS3-Poor, and CS4-Severe. Table 4.4 shows the specific condition state definitions for Element 12-Deck Reinforced Concrete. The bridge inspector records the quantity or percentage of each element found in each condition state. Table 4.5 presents an example of quantities assigned to the four condition states for Element 107 (Steel Open Girder/Beam) and its steel protective coating (Element 515).

Table 4.4 Condition state definitions for Element 12-Deck Reinforced Concrete

\begin{tabular}{|c|c|c|c|c|}
\hline \multicolumn{5}{|c|}{ Condition State Definitions: Element 12 - RC Deck } \\
\hline Defect & $\mathrm{CS} 1$ & $\mathrm{CS} 2$ & $\mathrm{CS} 3$ & CS4 \\
\hline $\begin{array}{l}\text { Delamination / Spall / } \\
\text { Patched Area } \\
(1080)\end{array}$ & None & $\begin{array}{l}\text { Delaminated. } \\
\text { Spall } 1 \text { in. or less } \\
\text { deep or } 6 \text { in. or } \\
\text { less in diameter. } \\
\text { Patched area that } \\
\text { is sound. }\end{array}$ & $\begin{array}{l}\text { Spall greater than } 1 \text { in. } \\
\text { deep or greater than } 6 \\
\text { in. diameter. Patched } \\
\text { area that is unsound or } \\
\text { showing distress. Does } \\
\text { not warrant structural } \\
\text { review. }\end{array}$ & $\begin{array}{l}\text { The condition } \\
\text { warrants a } \\
\text { structural } \\
\text { review to } \\
\text { determine the } \\
\text { effect on } \\
\text { strength or }\end{array}$ \\
\hline $\begin{array}{l}\text { Exposed Rebar } \\
(1090)\end{array}$ & None & $\begin{array}{l}\text { Present without } \\
\text { measurable } \\
\text { section loss. }\end{array}$ & $\begin{array}{l}\text { Present with } \\
\text { measurable section } \\
\text { loss, but does not } \\
\text { warrant structural } \\
\text { review. }\end{array}$ & $\begin{array}{l}\text { serviceability } \\
\text { of the element } \\
\text { or bridge; OR a } \\
\text { structural } \\
\text { review has }\end{array}$ \\
\hline $\begin{array}{l}\text { Efflorescence / Rust } \\
\text { Staining (1120) }\end{array}$ & None & $\begin{array}{l}\text { Surface white } \\
\text { without build-up } \\
\text { or leaching } \\
\text { without rust } \\
\text { staining. }\end{array}$ & $\begin{array}{l}\text { Heavy build-up with } \\
\text { rust staining. }\end{array}$ & $\begin{array}{l}\text { been completed } \\
\text { and the defects } \\
\text { impact strength } \\
\text { or } \\
\text { serviceability }\end{array}$ \\
\hline $\begin{array}{l}\text { Cracking } \\
(1130)\end{array}$ & $\begin{array}{l}\text { Width less than } \\
0.012 \text { in. or } \\
\text { spacing greater } \\
\text { than } 3.0 \mathrm{ft} \text {. }\end{array}$ & $\begin{array}{l}\text { Width } 0.012-0.05 \\
\text { in. or spacing of } \\
1.0-3.0 \mathrm{ft} .\end{array}$ & $\begin{array}{l}\text { Width greater than } 0.05 \\
\text { in. or spacing of less } \\
\text { than } 1 \mathrm{ft} .\end{array}$ & $\begin{array}{l}\text { of the element } \\
\text { or bridge. }\end{array}$ \\
\hline
\end{tabular}

Note. Adapted from the FHWA Training Course: Introduction to Element-Level Bridge Inspection (FHWA, 2015). 
Table 4.5 Example of condition state quantities assigned to Element 107-Steel Open Girder/Beam

\begin{tabular}{lcccccc}
\hline Element & $\begin{array}{c}\text { Total } \\
\text { Qty }\end{array}$ & Units & $\begin{array}{c}\text { CS1 } \\
\text { Qty }\end{array}$ & $\begin{array}{c}\text { CS2 } \\
\text { Qty }\end{array}$ & $\begin{array}{c}\text { CS3 } \\
\text { Qty }\end{array}$ & $\begin{array}{c}\text { CS4 } \\
\text { Qty }\end{array}$ \\
\hline 107 - Steel Open Girder/Beam & 1098 & $\mathrm{ft}$. & 1018 & 80 & 0 & 0 \\
\hline 515 - Steel Protective Coating & 13931 & sq. ft. & 0 & 13851 & 0 & 80 \\
\hline $\begin{array}{l}\text { Note. Adapted from the FHWA Training Course: Introduction to Element-Level } \\
\text { Bridge Inspection (FHWA, 2015). }\end{array}$
\end{tabular}

\subsection{Preservation Actions}

A set of feasible treatments is defined for each element condition state. Treatments classified as MRR (or preservation) treatments are committed mainly to preserve the element or restore it to a better condition. Each element condition state is associated with up to five treatments. One of these treatments is always the DN option to represent taking no action. These treatments are grouped into five main actions: MRR Action 0, MRR Action 1, MRR Action 2, MRR Action 3 and MRR Action 4. MRR Action 0 includes only the DN option. The other actions involve special treatments, minor and/or major repairs, and/or entire or partial element replacement.

Unit costs are assigned to each of these treatments. The total unit cost includes the direct and indirect costs. Table 4.6 shows an example of the different feasible treatments assigned to Element 207 (Steel Column Tower Element). The table is organized by MRR action, element condition state, treatment name, treatment key, total unit cost, and direct unit cost. In this example, MRR Action 4 can only be performed when the element is in CS3 or CS4. Only one feasible treatment under MRR Action 4 is assigned to each of these condition states:

- For CS3, MRR Action 4-Mitigate settlement or scour

- $\quad$ For CS4, MRR Action 4-Replace unit 
Table 4.6 Example of the feasible treatments assigned to Element 207-Steel Column Tower Element

\begin{tabular}{cccccc}
\hline $\begin{array}{c}\text { MRR } \\
\text { Action }\end{array}$ & $\begin{array}{c}\text { Element } \\
\text { CS }\end{array}$ & Treatment Name & $\begin{array}{c}\text { Treatment } \\
\text { Key }\end{array}$ & $\begin{array}{c}\text { Total Unit } \\
\text { Cost }(\$)\end{array}$ & $\begin{array}{c}\text { Direct Unit } \\
\text { Cost (\$) }\end{array}$ \\
\hline 1 & 2 & Spot blast & 319 & 102.74 & 11.42 \\
1 & 3 & Spot blast and minor repair & 302 & 102.74 & 11.42 \\
1 & 4 & Spot blast and major repair & 202 & 2054.63 & 228.29 \\
2 & 2 & Spot blast and minor repair & 302 & 102.74 & 11.42 \\
2 & 3 & Spot blast and major repair & 202 & 2054.63 & 228.29 \\
2 & 4 & Repair distortion & 248 & 2054.63 & 228.29 \\
3 & 3 & Repair distortion & 248 & 2054.63 & 228.29 \\
3 & 4 & Mitigate settlement or scour & 247 & 9702.47 & 1078.05 \\
4 & 3 & Mitigate settlement or scour & 247 & 9702.47 & 1078.05 \\
4 & 4 & Replace unit & 144 & 38809.88 & 4312.21 \\
\hline
\end{tabular}

Note. Retrieved from Implementation of the 2013 AASHTO Manual for Bridge Element Inspection (Sobanjo \& Thompson, 2016a).

\subsection{Element Preservation Action Costs}

An element preservation action costs (symbolized by $\mathrm{PAC}_{\mathrm{elm}}$ ) is the cost of implementing a preservation action. The cost is made up of the direct and indirect costs and applied at the end of the implementation year. The direct cost is the sum of all costs involved to preserve, repair, or replace the element. The other component is the sum of all costs indirectly incurred (e.g., costs of maintaining traffic, and engineering design). Florida DOT's AE models (Sobanjo \& Thompson, 2016a) are incorporated in the improvement module (discussed in this Chapter 5) to estimate these preservation action costs - slightly modified to agree with the defined actions for this research.

\subsection{Functional Improvement Action}

The proposed EB-MOO methodology distinguishes functional improvement needs from preservation needs. Preservation needs are identified for each element condition state, while functional improvement needs are resulting from functional deficiencies associated with the entire bridge. All bridges in the portfolio are assessed for functional improvement needs. A functional improvement (symbolized by FCI Action 5) relieves the bridge from certain restrictions and 
reduces or eliminates any incurred user costs. Widening, raising, and/or strengthening improvement needs are assessed for each bridge based on several mathematical models. Each of these models rely on proven correlation/regression formulas, LOS and design standards, and unit costs. NBIAS's and Florida DOT's user cost models (Cambridge Systematics, 2011b; Sobanjo \& Thompson, 2004) are adapted for assessing functional improvement needs.

\subsubsection{Widening Improvement Need}

\section{Bridge Roadway Width Deficiency}

Widening improvement need is assessed when the bridge roadway width is less than the required width. The bridge roadway width is specified by NBI coding item 51 for the roadways on the structure, and NBI coding item 47 for roadways under the structure. However, only NBI coding item 51 is accepted-it's assumed that it is infeasible to widen the roadway under a bridge. To determine if the bridge carries a highway traffic, the module examines the coded value of NBI coding item $42 \mathrm{~A}$. If NBI coding item $42 \mathrm{~A}$ is coded as either $1,4,5,6,7$, or 8 , then the bridge is deemed to be carrying a highway traffic, and the recorded value of NBI coding item 51 is accepted for the assessment. Required width is calculated by Equation (4.1) as follows:

(Cambridge Systematics, 2011b)

Required Width $=2 \times$ LOS Shoulder Width + Number of Lanes $\times$ LOS Lane Width

where

Required Width $\quad=$ roadway width required for the bridge;

Number of Lanes $\quad=$ number of lanes of the roadway, specified by NBI coding item 28;

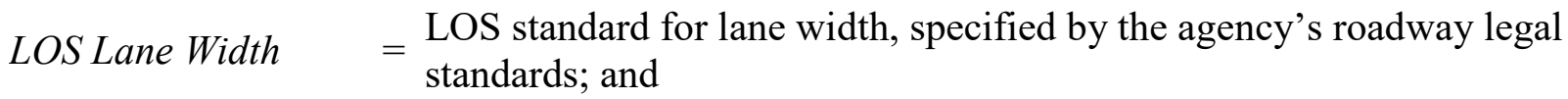
LOS Shoulder Width = LOS standard for shoulder width, specified by the agency's roadway
legal standards. 


\subsubsection{Raising Improvement Need}

\section{Vertical Clearance Deficiency}

Raising improvement need is assessed when the recorded minimum vertical clearance over the roadway (NBI coding item 10) fails to meet the agency's LOS standard for vertical clearance. Hence, the bridge vertical clearance is presumed deficient and forcing a percentage of truck traffic (based on truck height) to use alternative routes. The module uses the following Equation (4.2) to examine the recorded minimum vertical clearance over the roadway.

(Cambridge Systematics, 2011b)

Vertical Clearance $<$ LOS Vertical Clearance

where

minimum vertical clearance over the roadway, specified by NBI coding

Vertical Clearance $=$ item 28 (if the value is missing or invalid, then NBI coding item 53 is used for the roadway on the bridge, and NBI coding item 54 is used for roadways under the bridge); and

LOS Vertical
Clearance $\quad$ LOS standard for vertical clearance, specified by the agency's roadway Clearance legal standards.

\subsubsection{Strengthening Improvement Need}

\section{Load Capacity deficiency}

Strengthening improvement is assessed when the recorded bridge operating load rating (NBI coding item 46) fails to meet the agency's LOS standard for bridge operating load rating. Hence, the bridge load rating is presumed deficient and creating certain truck traffic (based on truck height) to use longer detour routes. The module uses the following Equation (4.3) to examine the recorded operating load rating. 
(Sobanjo \& Thompson, 2004).

Operating Load Rating < LOS Operating Load Rating

where

Operating Load $=$ current bridge operating load rating, specified by NBI coding item 46; and
Rating LOS Operating = LOS standard for bridge operating load rating, specified by the agency's Load Rating bridge load rating and posting legal standards.

\subsubsection{Functional Improvement Cost}

Two types of bridge-level major improvement costs (denoted by $\mathrm{MIC}_{\mathrm{brg}}$ ) are determined by the module: functional improvement and replacement costs. Functional improvement cost is the cost of improvement needed to eliminate incurred user costs. Functional improvement cost includes widening cost (required to eliminate the bridge width deficiency), raising cost (required to eliminate the bridge vertical clearance deficiency), and/or strengthening cost (required to eliminate the bridge load capacity deficiency). Replacement cost is the cost incurred to relieve the bridge from all its restrictions (discussed in the subsequent section). Major improvement cost needed to eliminate any existing functional deficiencies is estimated. Bridges identified with multiple functional improvements needs, the highest of the calculated improvement costs is used. The module determines the major cost of a functional improvement action (FCI Action 5) performed in a program year by Equation (4.4) as follows:

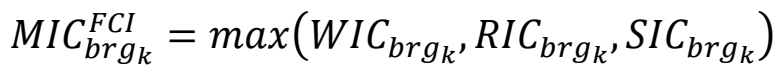

where

$M I C_{b r g_{k}}^{F C I}=\begin{aligned} & \text { major cost of a functional improvement action (FCI Action 5) performed on bridge } \\ & k \text { in a program year; }\end{aligned}$ $W I C_{\text {brg }_{k}}=$ cost of a widening improvement performed on bridge $k$ in a program year; 
$R I C_{\text {brg }_{k}}=$ cost of a raising improvement performed on bridge $k$ in a program year; and $S I C_{\text {brg }_{k}}=$ cost of a strengthening improvement performed on bridge $k$ in a program year.

\subsubsection{Widening Improvement Cost}

The cost of widening improvement is always incurred within the program period to eliminate the bridge roadway width deficiency. It is possible a widening improvement won't eliminate all accident costs, but it substantially reduces them. For simplicity and consistency, the module assumes a functional improvement action (FCI Action 5) eliminates all incurred user costs. The cost of a widening improvement performed on a bridge in a program year is calculated by Equation (4.5) as follows:

$W I C_{\text {brg }_{k}}=R W_{\text {brg }_{k}} \times L_{b r g_{k}} \times W U C$

where

$W C_{\text {brg }_{k}}=$ cost of a widening improvement performed on bridge $k$ in a program year;

$R W_{\text {brg }_{k}}=$ roadway width required for bridge $k$, determined by Equation (4.1);

$L_{b r g_{k}}=$ length of bridge $k$, specified by NBI coding item 49; and

WUC = $\begin{aligned} & \text { unit cost of widening per square meter of bridge deck area, specified by the agency } \\ & \text { (Florida DOT's unit costs are used by default). }\end{aligned}$ 


\subsubsection{Raising Improvement Cost}

The cost of raising improvement is the incurred cost to eliminate the bridge vertical clearance deficiency. It's possible a raising improvement won't eliminate all user costs due to the vertical clearance deficiency, but it substantially reduces them. For simplicity and consistency, the module assumes a functional improvement eliminates all incurred user costs. The cost of a raising improvement performed on a bridge in a program year is calculated by Equation (4.6) as follows:

$R I C_{\operatorname{brg}_{k}}=R W_{b r g_{k}} \times L_{b r g_{k}} \times R U C$

where

$R I C_{b r g_{k}}=$ cost of a raising improvement performed on bridge $k$ in a program year;

$R W_{\operatorname{brg}_{k}}=$ roadway width required for bridge $k$, determined by Equation (4.1);

$L_{\text {brg }_{k}}=$ length of bridge $k$, specified by NBI coding item 49; and

$R U C=\begin{aligned} & \text { unit cost of raising per square meter of bridge deck area, specified by the agency } \\ & \text { (Florida DOT's unit costs are used by default). }\end{aligned}$ 


\subsubsection{Strengthening Improvement Cost}

The cost of strengthening improvement is the incurred cost to eliminate the bridge operating load rating deficiency. It's possible a strengthening improvement won't eliminate all user costs due to this load rating deficiency, but it substantially reduces them. For simplicity and consistency, the module assumes a functional improvement eliminates all incurred user costs. The cost of a strengthening improvement performed on a bridge in a program year is calculated by Equation (4.7) as follows:

$S I C_{\operatorname{brg}_{k}}=R W_{\operatorname{brg}_{k}} \times L_{\operatorname{brg}_{k}} \times S U C$

where

$R I C_{\text {brg }_{k}}=$ cost of a strengthening improvement performed on bridge $k$ in a program year;

$R W_{\text {rrg }_{k}}=$ roadway width required for bridge $k$, determined by Equation (4.1);

$L_{\text {brg }_{k}}=$ length of bridge $k$, specified by NBI coding item 49; and

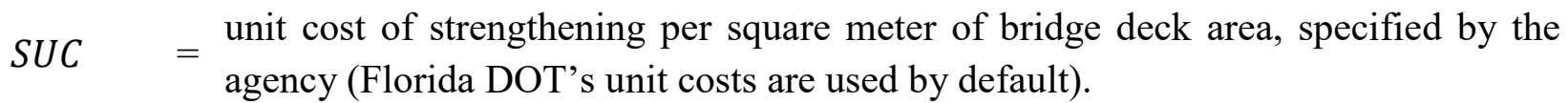

\subsection{Replacement Action}

A functional improvement relieves the bridge from certain restrictions and reduces or eliminates any incurred user costs; however, a replacement action (REP Action 6) is assumed to relieve the bridge from all its restrictions, provide the required LOS and design standards, and eliminate all incurred user costs. The module assesses the replacement option for a candidate bridge regardless of its element condition states or restrictions - assuming the replacement option is always feasible; and therefore, it's eligible to compete. NBIAS's and Florida DOT's user cost models Cambridge Systematics, 2011b; Sobanjo \& Thompson, 2004) are adapted for the assessment of replacement needs. 


\subsubsection{Replacement Cost}

The module calculates the bridge replacement cost same as BrM, NBIAS and Florida DOT's PLAT. The cost of replacing a bridge is determined by Equation (4.8) as follows:

$M I C_{b r g_{k}}^{R E P}=D D A_{\text {rrg }_{k}} \times R C_{b r g_{k}} \times S F$

where

$M I C_{\text {brg }_{k}}^{R E P}=$ major cost of replacing bridge $k$ in a program year;

$D D A_{\operatorname{brg}_{k}}=\begin{aligned} & \text { new deck area of bridge } k \text {, meeting design standards for lane and shoulder widths, } \\ & \text { determined by Equation (4.9); }\end{aligned}$ unit cost of reconstructing bridge $k$ per square meter of bridge deck area, meeting

$R C_{\text {brg }_{k}}=$ design standards, specified by the agency (Florida DOT's unit costs are used by default); and

$S F \quad=$ cost increase coefficient, specified by the agency (1 is the default).

$D D A_{\operatorname{brg}_{k}}=R D W_{\operatorname{brg}_{k}} \times L_{b r g_{k}}$

where

$D D A_{\operatorname{brg}_{k}}=$ new deck area of bridge $k$, meeting design standards for lane and shoulder widths;

$R D W_{\text {brg }_{k}}=$ design width required for bridge $k$, determined by Equation (4.10); and

$L_{\text {rrg }_{k}}=$ length of bridge $\mathrm{k}$, specified by NBI coding item 49. 
(Sobanjo \& Thompson, 2004).

Required Design Width $=2 \times$ Design Shoulder Width + Number of Lanes $\times$ Design Lane (4.10)

where

Required Design Width = design width required for the bridge;

Number of Lanes $\quad=$ number of lanes of the roadway, specified by NBI coding item 28;

Design Lane Width = $\begin{aligned} & \text { design standard for lane width, specified by the agency's roadway } \\ & \text { legal standards; and }\end{aligned}$

$\begin{aligned} \text { Design Shoulder Width }= & \begin{array}{l}\text { design standard for shoulder width, specified by the agency's } \\ \text { roadway legal standards. }\end{array}\end{aligned}$

\subsection{Bridge User Cost}

The bridge user cost is the sum of all costs incurred by users over the bridge LC. It is important to use the same type of user costs and most significant in the LCC calculation to ensure consistency and no bias. User costs associated with work zones include delays, vehicle operating costs, and costs related to vehicle crashes. While these costs are important to consider in a LCCA to truly reflect the overall LCC, they are often omitted due to their similarity (FHWA, 2002). The nonwork zone-related user costs are generally associated with the bridge functional deficienciesvehicles must take detours due to a narrow bridge width, or restricted vertical clearance or load capacity.

These functional deficiencies may increase vehicle operating costs (due to detours) or reduce safety (increase in accident rates). Several models exist in estimating these user costs. Generally, these models are based on regression formulas taking into consideration the traffic growth over time. The NCHRP Synthesis of Highway Practice 494: Life-cycle Cost Analysis for Management of Highway Assets (Flannery et al., 2016) discuses a study conducted for South Carolina DOT in 2008. The study revealed that $60 \%$ of the 33 surveyed state DOTs don't include user costs in their 
LCCAs. The data processing module provides an avenue to incorporates these substantial costs into a LCCA of the improvement module.

As stated earlier, the module assesses each bridge independently for functional improvement needs. When improvement needs are warranted, the economic consequences, in terms of user costs, are estimated for each analysis year. For the identified deficiency, a user cost for each analysis year is calculated, including action implementation years. Accident risk, vertical clearance, and load capacity costs (each of them is subsequently defined) are the only user costs computed by the module. The sum of these three types of user costs constitutes the total bridge user cost. The following Equation (4.11) is applied to compute the total user cost for an analysis year.

$U S C_{b r g_{k}}^{y}=A R C_{b r g_{k}}^{y}+V C C_{b r g_{k}}^{y}+L O C_{b r g_{k}}^{y}$

where

$U S C_{b^{\prime} g_{k}}^{y} \quad=$ bridge $k$ total user cost for analysis year $y$;

$A R C_{b r g_{k}}^{y} \quad=$ bridge $k$ accident risk cost for analysis year $y$;

$V C C_{b r g_{k}}^{y} \quad=$ bridge $k$ vertical clearance cost for analysis year $y$; and

$L_{O C} C_{b r g_{k}}^{y} \quad=$ bridge $k$ load capacity cost for analysis year $y$.

Total user costs for all analysis years are discounted to present values and added to the LCC. To provide a uniform basis for assessing alternatives, no user costs are recognized prior to analysis year 1. Element deterioration and traffic growth are assumed to contribute to the increase of user costs over time. No additional user costs are considered beyond the end of the analysis periodpass this point, only the residual value (covered in Chapter 5) is added to LC profile. The module 
follows a common approach used by BrM, NBIAS, and other state DOT's BMSs to assess the economic consequences in term of user costs due to functional deficiencies - possibly affecting LOS provided to the users.

\subsubsection{Traffic Volume Growth}

The traffic volume growth is considered in the user cost calculations. The module assumes traffic volumes change over the analysis period. For each roadway on and under the bridge, the traffic volume is estimated for each analysis year. Traffic volume is forecasted using an exponential interpolation between two average daily traffic (ADT) points: observed and future. The same interpolation approach as Florida DOT's PLAT, NBIAS and BrM is used. However, if any required variable to compute the traffic volume growth is missing, the module relies on the latest available traffic volume and ignores the proportional effect. The forecast ADT of a given analysis year is evaluated by Equation (4.12) as follows:

(Cambridge Systematics, 2011b)

$A D T_{y}=A D T_{y_{0}}\left(\frac{A D T_{y_{1}}}{A D T_{y_{0}}}\right)^{\frac{y-y_{0}}{y_{1}-y_{0}}}$

where

$A D T_{y}=$ the forecast $\mathrm{ADT}$ for analysis year $y$

$A D T_{y_{0}}=$ the recent $\mathrm{ADT}(\mathrm{NBI}$ coding item 29);

$y_{0}=$ the year of recent ADT (NBI coding item 30);

$A D T_{y_{1}}=$ the future ADT (NBI coding item 114);

$y_{1}=$ the year of future ADT (NBI coding item 115); and

$y=$ the analysis year of forecast ADT. 


\subsubsection{Accident Risk Cost}

NBIAS assumes the accident risk rate is affected by the bridge roadway width deficiency. NBIAS calculates the annual reduction in accident risk costs using the following Equation (4.13) (Cambridge Systematics, 2011b).

$A R A C=W_{a c c} \times 365 \times A D T \times C_{a c c} \times\left(A R^{c u r r}-A R^{r e q}\right)$

where

$A R A C=$ annual reduction in accident risk costs;

$W_{a c c}=$ accident risk cost weight, specified by the agency;

$A D T=$ average daily traffic on the bridge;

$C_{a c c}=$ average cost per accident, specified by the agency;

$A R^{\text {curr }}=$ current accident rate based on the bridge width, determined by Equation (4.14); and $A R^{r e q}=$ required accident rate based on design standards;

$A R^{c u r r}=K \times W^{-D} \times\left(1+0.5 \frac{B-R_{a}}{B-C}\right)$

where

$K=$ roadway width in feet;

$W \quad=$ improvement model scaling coefficient (default value is 200);

$D \quad=$ improvement model parameter (default value is 6.5 );

$B=\begin{aligned} & \text { improvement model parameter for the maximum approach alignment rating (default } \\ & \text { value is 9); }\end{aligned}$

$C=\begin{aligned} & \text { improvement model parameter for the minimum approach alignment rating (default } \\ & \text { value is } 2 \text { ); and }\end{aligned}$

$R_{a} \quad=$ approach alignment rating on the bridge (NBI coding item 72). 
Equation (4.13) is used to estimate the accident risk cost incurred by the bridge roadway width deficiency for a given analysis year. The equation is slightly modified by eliminating the term of $A R^{r e q}$ and considering an accident risk cost weight factor, $W_{a c c}$. Agencies often assign weight factors, less than 1 , to different user costs to reduce their effect on the analysis - by default, a constant weight factor equals to 1 is chosen. The module assumes a widening improvement restores the required bridge roadway width (by design standards), and the required accident rate equals to 0 - to some extent, results are more conservative than Equation (4.13). The following Equation (4.15) is the derived equation applied to calculate the bridge accident risk cost for an analysis year.

$A R C_{b r g_{k}}^{y}=W_{a c c} \times 365 \times A D T_{y} \times C_{a c c} \times A R_{b r g_{k}}^{c u r r}$

where

$A R C_{\mathrm{brg}_{k}}^{y}=$ bridge $k$ accident risk cost for analysis year $y$;

$W_{a c c} \quad=$ accident risk cost weight, specified by the agency;

$A D T_{y}=$ forecast $\mathrm{ADT}$ for analysis year $y$, determined by Equation (4.12);

$C_{a c c}=$ average cost per accident, specified by the agency; and

$A R_{\text {brg }_{k}}^{\text {curr }}=$ current accident rate based on bridge $k$ width, determined by Equation (4.14); 


\subsubsection{Vertical Clearance Cost}

Florida DOT's user cost model assumes that user costs due to vertical clearance restrictions occur when the bridge vertical clearance is determined deficient. For simplicity and consistency, the module eliminates all user costs after performing a functional improvement action (FCI Action 5). The annual reduction in vertical clearance costs is calculated using the following Equation (4.16) (Sobanjo \& Thompson, 2016b).

$A V C C=W_{v t c} \times 365 \times A D T \times C_{d e t} \times T R \times\left(T D_{c u r r}-T D_{\text {imp }}\right)$

where

$A V C C=$ annual reduction in vertical clearance costs;

$A D T=$ average daily traffic on the bridge;

$C_{\text {det }} \quad=$ detour cost per truck, determined by Equation (4.18);

$T R=$ fraction of trucks in the ADT, specified by NBI coding item 109;

$T D_{\text {curr }}=$ percent of trucks detoured by the current bridge (Figures 4.2 and 4.3 );

$T D_{i m p}=$ percent of trucks detoured by the improved bridge; and

$W_{v t c} \quad=$ vertical clearance cost weight, specified by the agency;

Equation (4.16) is used to estimate the cost incurred by the bridge vertical clearance deficiency for an analysis year. The equation is slightly modified by eliminating the term of $T D_{i m p}$. Agencies often assign weight factors, less than 1 , to different user costs to reduce their effect on the analysis - by default, a constant vertical clearance cost weight factor, $W_{v t c}$, equals to 1 is chosen. The module assumes a raising improvement restores the bridge vertical clearance required by design standards, and the percentage of trucks detoured by the improved bridge equals to 0 - to 
some extent, results are more conservative than Equation (4.16). The following Equation (4.17) is the derived equation applied to calculate the bridge vertical clearance cost for an analysis year.

$V C C_{b r g_{k}}^{y}=W_{v t c} \times 365 \times A D T_{y} \times C_{d e t} \times T R \times T D_{b r g_{k}}$

where

$V C C_{b r g_{k}}^{y}=$ bridge $k$ vertical clearance cost for analysis year $y$;

$W_{v t c} \quad=$ vertical clearance cost weight, specified by the agency;

$A D T_{y} \quad=$ forecast $\mathrm{ADT}$ for analysis year $y$, determined by Equation (4.12);

$C_{d e t} \quad=$ detour cost per truck, determined by Equation (4.18);

$T R=$ fraction of trucks in the ADT, specified by NBI coding item 109; and

$T D_{\text {brg }_{k}}=$ percent of trucks detoured by bridge $k$ (Figures 4.2 and 4.3). 


\section{$\underline{\text { Detour Cost per truck }}$}

Florida DOT's user cost model is adapted to calculate the detour cost per truck. The cost is a combination of vehicle operating costs (due to the detour distance) and travel time costs (due to the detour time). Similar approach is used by BrM and NBIAS to determine the detour cost per truck. The following Equation (4.18) is used for bridges with either vertical clearance or load capacity deficiency (Sobanjo \& Thompson, 2016b).

Detour Cost per Truck $=$ VOC $\times$ Bypass Length $+T T \times \frac{\text { Bypass Length }}{\text { Bypass Speed }}$

where

Detour Cost per $=$ detour cos per truck for the bridge;
Truck

VOC = unit vehicle operating cost per km of detour, specified by the agency;

Bypass Length $=$ detour distance for the bridge, specified by NBI coding item 19;

TT = unit travel time cost per hour of detour, specified by the agency; and

Bypass Speed $=\begin{aligned} & \text { speed on the detour route, product of default speed value (based on functional } \\ & \text { class specified by the agency) and bypass speed factor (default set as } 0.9) .\end{aligned}$

\section{Percent of Trucks Detoured}

Sobanjo and Thompson (2004) developed the truck height histograms in Figures 4.2 and 4.3 to estimate the percent of trucks detoured by any given vertical clearance restriction for Interstate (top) and Non-Interstate roadways. The percentage of trucks detoured by a bridge, $T D_{\operatorname{brg}_{k}}$, is determined by one of these two histograms. 


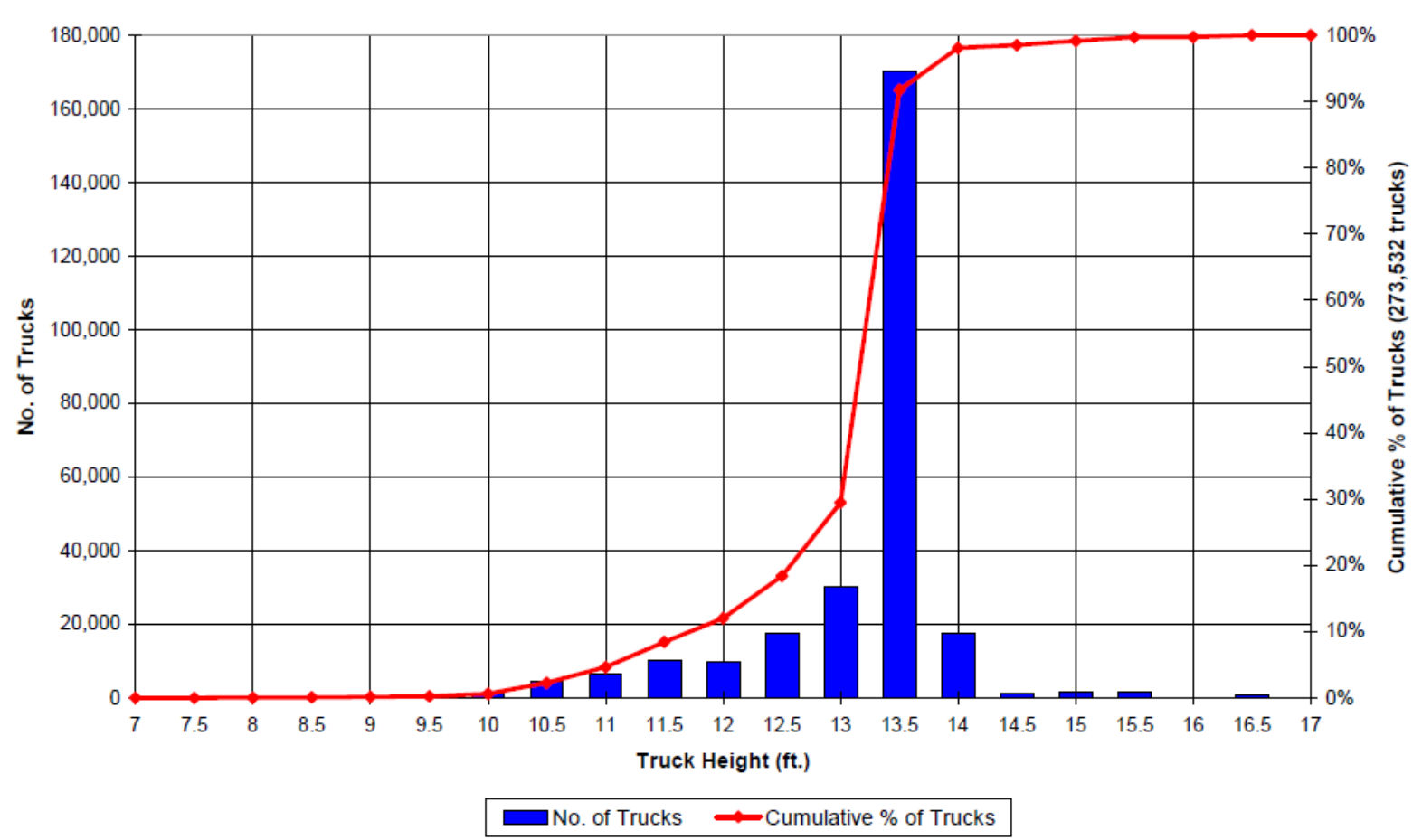

Figure 4.2 Truck height histogram for Florida's Interstate roadways. Adapted from Project Planning Models for Florida's Bridge Management System (Sobanjo \& Thompson, 2004).

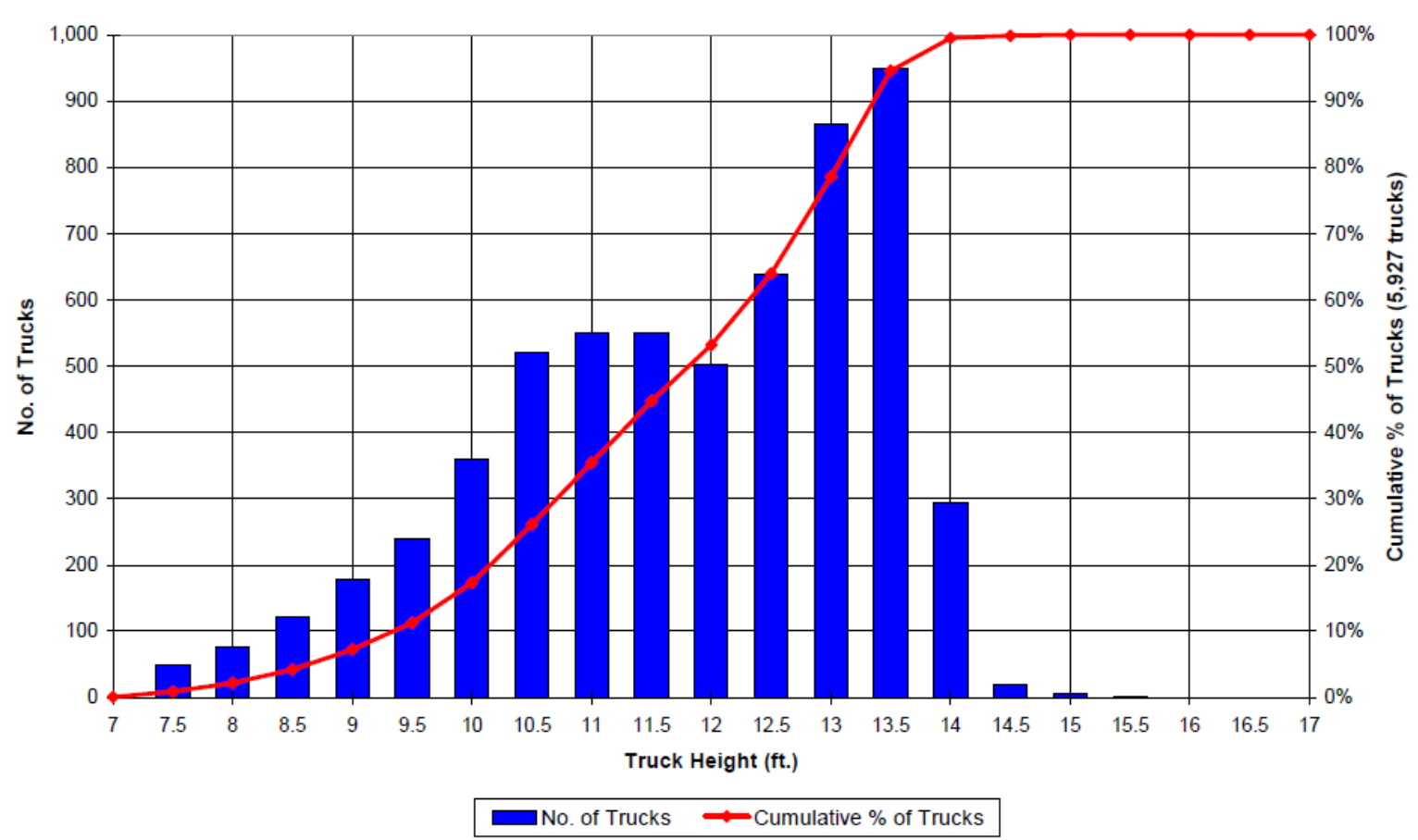

Figure 4.3 Truck height histogram for Florida's Non-Interstate roadways. Adapted from Project Planning Models for Florida's Bridge Management System (Sobanjo \& Thompson, 2004). 


\subsubsection{Load Capacity Cost}

Florida DOT's user cost model assumes that user costs due to load capacity restrictions occur when the bridge operating load rating is determined inadequate. For simplicity and consistency, the module eliminates all user costs after performing a functional improvement action (FCI Action 5). The annual reduction in load capacity costs is calculated using the following Equation (4.19) (Sobanjo \& Thompson, 2016b).

$A L C C=W_{l c} \times 365 \times A D T \times C_{d e t} \times T R \times\left(T D_{\text {curr }}-T D_{\text {imp }}\right)$

where

$A L C C=$ annual reduction in load capacity costs;

$A D T=$ average daily traffic on the bridge;

$C_{d e t}=$ detour cost per truck, determined by Equation (4.18);

$T R=$ fraction of trucks in the ADT, specified by NBI coding item 109;

$T D_{\text {curr }}=$ percent of trucks detoured by the current bridge (Figures 4.4 and 4.5 );

$T D_{i m p}=$ percent of trucks detoured by the improved bridge; and

$W_{l c} \quad=$ load capacity cost weight, specified by the agency.

Equation (4.19) is used to estimate the cost incurred by the bridge load capacity deficiency for an analysis year. The equation is slightly modified by eliminating the term of $T D_{-} i m p$. Agencies often assign weight factors, less than 1 , to different user costs to reduce their effect on the analysis - by default, a constant load capacity cost weight factor, $W_{l c}$, equals to 1 is chosen. The module assumes a strengthening improvement restores the bridge load capacity required by design standards, and the percentage of trucks detoured by the improved bridge equals to 0 - to some 
extent, results are more conservative than Equation (4.19). The following Equation (4.20) is the derived equation applied to calculate the bridge load capacity cost for an analysis year.

$\operatorname{LOC}_{\mathrm{brg}_{k}}^{y}=W_{l c} \times 365 \times A D T_{y} \times C_{d e t} \times T R \times T D_{b r g_{k}}$

where

$\operatorname{LOC}_{\mathrm{brg}_{k}}^{y}=$ bridge $k$ load capacity cost for analysis year $y$;

$W_{l c} \quad=$ load capacity cost weight, specified by the agency;

$A D T_{y} \quad=$ forecast $\mathrm{ADT}$ for analysis year $y$, determined by Equation (4.12);

$C_{\text {det }} \quad=$ detour cost per truck, determined by Equation (4.18);

$T R=$ fraction of trucks in the ADT, specified by NBI coding item 109; and

$\mathrm{TD}_{\mathrm{brg}_{k}}=$ percent of trucks detoured by bridge $k$ (Figures 4.4 and 4.5).

\section{$\underline{\text { Percent of Trucks Detoured }}$}

Truck weight histograms were developed by Sobanjo and Thompson (2004) to determine the percent of trucks detoured by any given operating load restriction for Interstate and Non-Interstate roadways. The percentage of trucks detoured by a bridge, $T D_{b r g}$, is determined by one of the following histograms (Figures 4.4 and 4.5) based on the bridge roadway classification. 


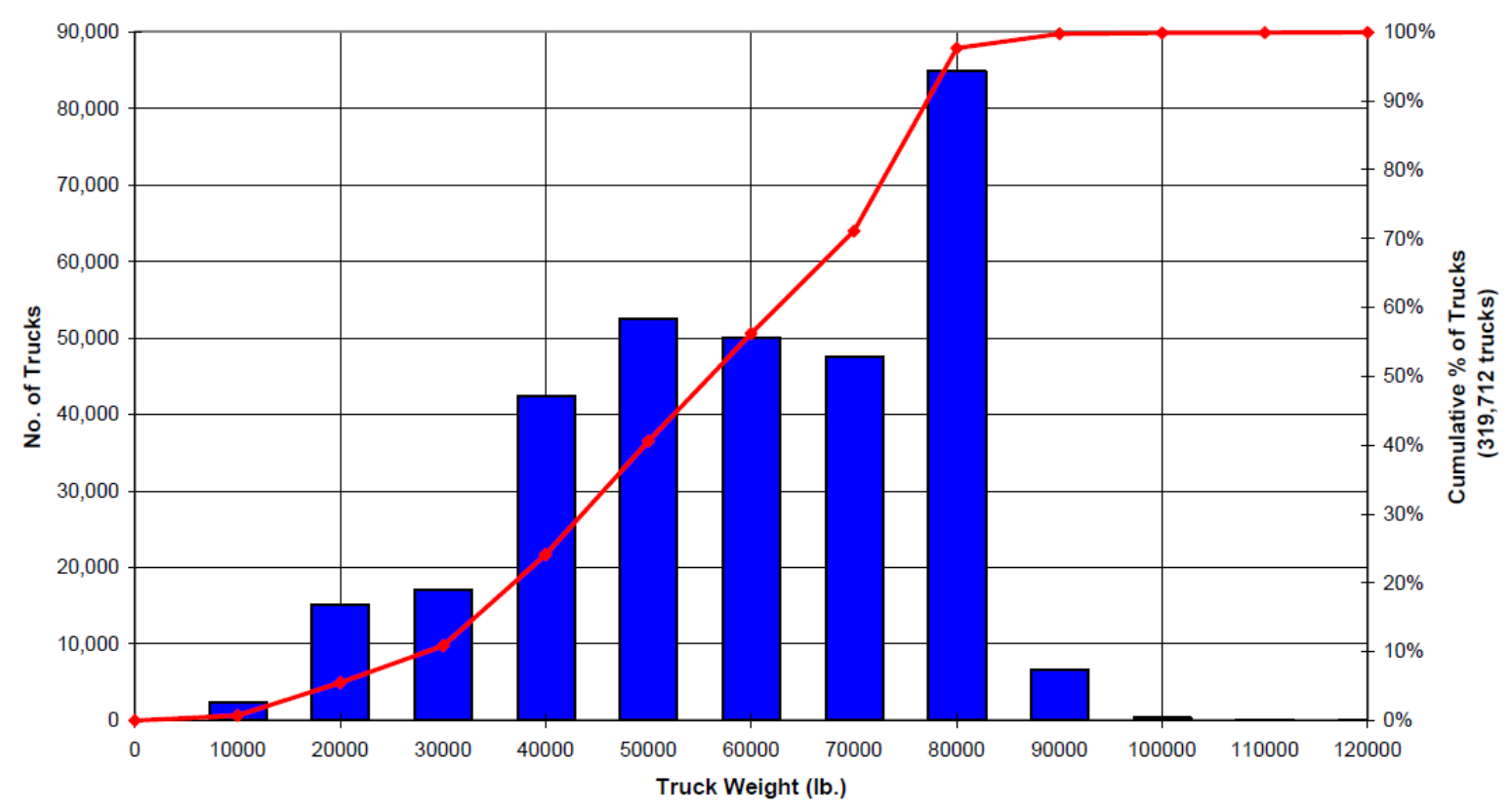

$\square$ No. of Trucks $\quad$ Cumulative $\%$ of Trucks

Figure 4.4 Truck weight histogram for Florida's Interstate roadways. Adapted from Project Planning Models for Florida's Bridge Management System (Sobanjo \& Thompson, 2004).

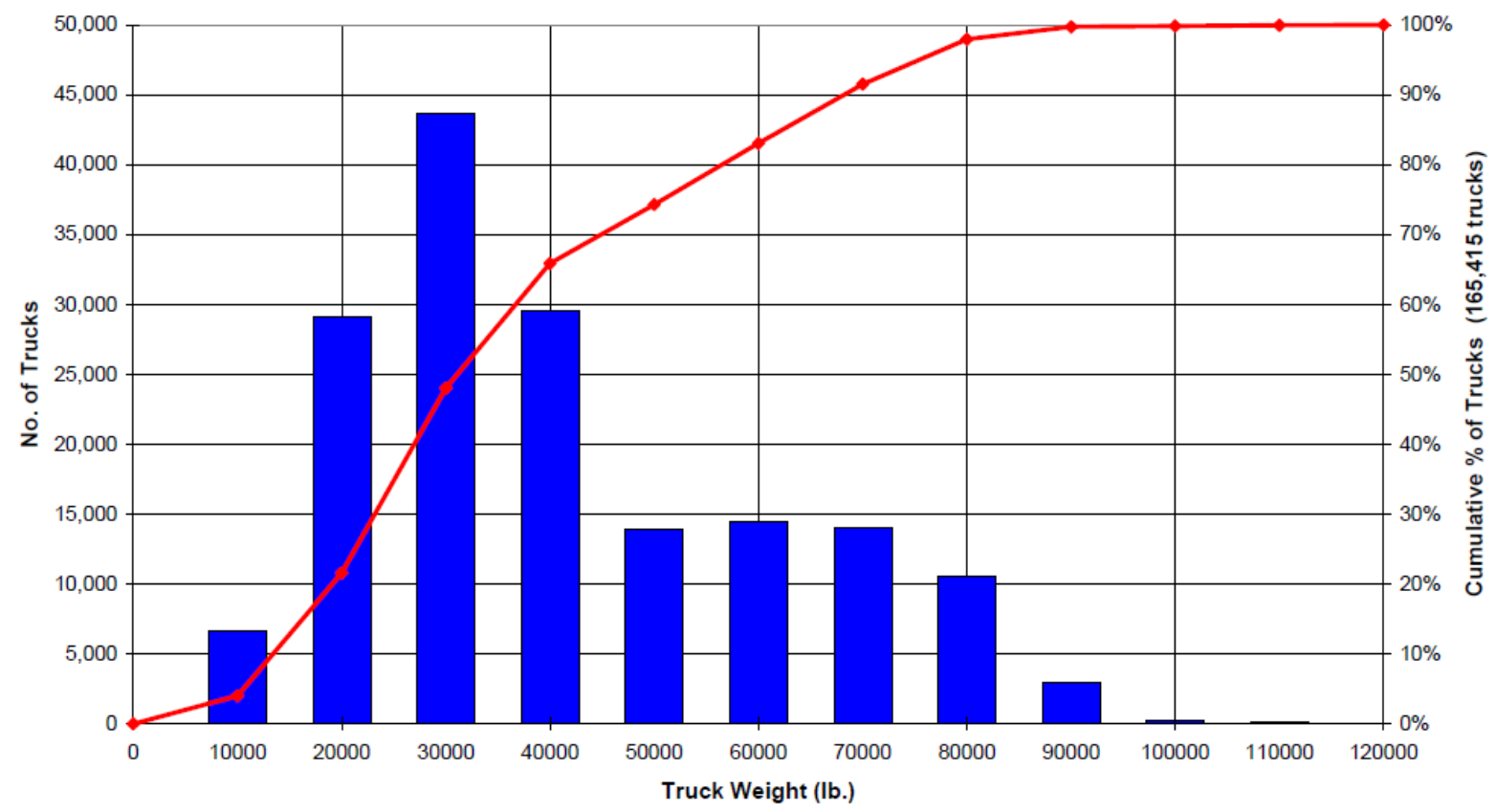

No. of Trucks $\leadsto$ Cumulative $\%$ of Trucks

Figure 4.5 Truck weight histogram for Florida's Non-Interstate roadways. Adapted from Project Planning Models for Florida's Bridge Management System (Sobanjo \& Thompson, 2004). 


\subsection{Element Deterioration Modeling}

Deterioration modeling is the main driver of the element LC condition required in the optimization process. Deterioration models are used to forecast conditions of key bridge components (i.e., deck, superstructure, and substructure) or their individual elements (e.g., decks, girders, floor beams, pins, hangers, and bearings) based on current and historical NBI and element-level bridge inspection data. These data are sampled and analyzed based on deterministic or probabilistic models to develop deterioration curves.

Over the past several decades, deterioration models have matured from purely deterministic models to probabilistic models using Markov decision process assumptions in estimating transition probabilities - assuming transition probabilities of future state depends only on the present state. Markov-based deterioration models improved the accuracy of prediction and allowed to capture the stochastic nature of the deterioration; however, they can result in incorrect decision making due to the independency between future and past state histories. This limitation is well-recognized by state DOTs; it is expected that the FHWA's LTBP Program will ultimately lead to the development of improved deterioration models. In the meantime, several state DOTs have taken the initiative to develop more accurate deterioration models based on parametric models that follow theoretical distribution defined by set of parameters or a combined parametric and Markov model. The hybrid model provides the most accurate modeling of deterioration that is possible (Thompson et al., 2012).

Elements deteriorate at different rates in different natural environments. The concept of bridge environment is incorporated in many deterioration models. A bridge is assigned to an environment based on the climate zone definitions of HPMS. Likewise, the different effects of protective elements (such as coatings, wearing surfaces, cathodic protections, joints, and drainage systems) 
are considered in deterioration models by introducing the concept of deterioration refinements. BrM and Florida DOT's PLAT include features to account for these refinements. These concepts are ignored in the module deterioration modeling. The inclusion of any deterioration refinements is beyond the scope of this research. Though, the module is well-suited to accommodate any deterioration refinements that quantify effects of different protections or environments. The module assumes the predicted condition occurs at the end of the analyzed year, and the recorded inspection date reflects the most recent inspection date.

\subsubsection{Weibull/Markov Deterioration Model}

Various deterioration models used by state DOTs were examined in the literature under Task 1. Their advantages and limitations were studied. Different deterioration forecasting models were compared in previous research, and the hybrid (Weibull/Markov) model is overwhelming recommended for use in modern BMSs. The Weibull model estimates the time of a bridge element remains in "good" condition — tracking progression through time without lowering the condition state because much of the element's life is spent in the "good" condition state. Once the element moves to the "fair" condition state, the Markov model predicts deterioration based on history.

Markov models have rapid decline in condition from CS1 to CS2 - in reality, this effect is not observed. Sobanjo and Thompson (2010) provided a solution by using a Weibull age-dependent function to model the probability of remaining in condition state. In Markov models, probabilities of transition from $\mathrm{CS} 1$ to $\mathrm{CS} 2$ are constant; therefore, this enhancement will make them agedependent variables. The Weibull survival function can slow the initial deterioration, providing a more realistic prediction. Subsequent condition states are still modeled using Markov models. It is possible to develop a completely age-dependent Weibull survival probability deterioration model if all the individual state transitions are analyzed independently-each asset is in only one 
condition state at a time and can move to only one other state between inspections (Thompson et al., 2012).

Florida DOT's PLAT and BrM use this hybrid (Weibull/Markov) model. The module relies on this same independent hybrid model for predicting element conditions. The Weibull model is used only to model the transition of an element from CS1 to CS2. The other transitions (i.e., CS2 to CS3 and CS3 to CS4) are modeled using the Markov model. Florida DOT's transition probabilities and Weibull parameters (Sobanjo \& Thompson, 2016a) are used by default. The Weibull/Markov model is expressed by the following Equation (4.21).

(Thompson et al., 2012).

$Y_{k}=Y_{k-1} \times P_{k-1, k}+Y_{k} \times P_{k, k}$

where

$Y_{k} \quad=$ forecasted percentage of the element in condition state $k$;

$Y_{k-1}=$ current percentage of the element in condition state $k-1$;

$Y_{k} \quad=$ current percentage of the element in condition state $k$;

$P_{k-1, k}=\begin{aligned} & \text { probability of the element changes from condition state } k \text { - } 1 \text { to } k \text { in the next year } \\ & \text { determined by Equation (4.22); and }\end{aligned}$

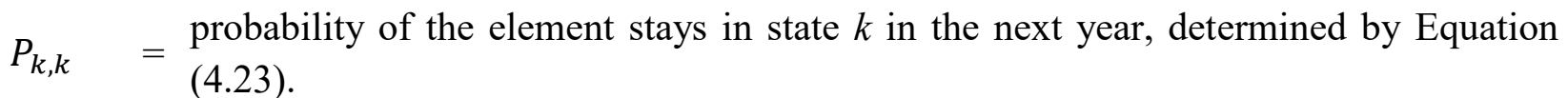
The deterioration model can also be expressed by a matrix of transition probabilities. This expression is convenient to predict condition for any future point in time by just matrix multiplication. Each element has a transition probability matrix. Each matrix cell represents the probability of making a transition from one condition state to another, depending only on the initial condition state. Each cell of the matrix is the probability that, in one year, an element in the row's 
condition state (i.e., CS1, CS2, CS3, or CS4) will deteriorate into the column's condition state (i.e., CS1, CS2, CS3, or CS4). All rows must sum to $100 \%$. Therefore, the deterioration of an element can be represented by a vector, with one value for each condition state. Table 4.7 represents an example of such matrix.

Table 4.7 Example of a matrix of transition probabilities

\begin{tabular}{|c|c|c|c|c|}
\cline { 2 - 5 } \multicolumn{1}{c|}{} & CS1 & CS2 & CS3 & CS4 \\
\hline CS1 & $92 \%$ & $8 \%$ & $0 \%$ & $0 \%$ \\
\hline CS2 & $0 \%$ & $89 \%$ & $11 \%$ & $0 \%$ \\
\hline CS3 & $0 \%$ & $0 \%$ & $85 \%$ & $15 \%$ \\
\hline CS4 & $0 \%$ & $0 \%$ & $0 \%$ & $100 \%$ \\
\hline
\end{tabular}

The example includes DN (no improvement action is performed) transition probabilities. If the element is in CS1, it has $8 \%$ chance of moving to CS2 in one year and $92 \%$ chance of staying in CS1. If the element is in CS3, it has $85 \%$ chance to remain in the same condition sate and $15 \%$ chance to move to CS4 in one year.

As stated earlier, Markov model doesn't depend on past condition information and is used to predict transition probabilities for $\mathrm{CS} 2, \mathrm{CS} 3$, and CS4 of an element in the next year. Transition probabilities can be derived from the median number of years between known transitions. It is an appropriate way to develop a deterioration model from expert judgments and mining historical inspection data (Thompson et al., 2012). The Markov model uses the following formulas (4.22) and (4.23) to estimate the transition probabilities. 
(Thompson et al., 2012).

$P_{k, k}=0.5^{\frac{1}{T_{k}}}$

$P_{k-1, k}=1-P_{k-1, k-1}$

where

$P_{k, k}=$ probability of the element changes from condition state $k-1$ to $k$ in the next year;

$P_{k-1, k}=$ probability of the element stays in state $k$ in the next year; and

$T_{k}=\begin{aligned} & \text { median number of years that the element stays in condition state } k \text {, before transition to } \\ & \text { the next condition state. }\end{aligned}$

The Weibull model applies the following formulas (4.24) and (4.25) to estimate the transition probabilities for CS1 in the next year.

(Thompson et al., 2012).

$P_{1,1}=\exp \left[-\left(\frac{g}{\alpha}\right)^{\beta}+\left(\frac{g-1}{\alpha}\right)^{\beta}\right]$

$P_{1,2}=1-P_{1,1}$

where

$P_{1,1}=$ probability of the element stays in CS1 in the next year;

$P_{1,2}=$ probability of the element changes from CS1 to CS2 in the next year;

$\alpha=$ scaling factor, estimated by Equation (4.26);

$\beta=\begin{aligned} & \text { shaping parameter that controls the initial slowing effect on deterioration, specified by } \\ & \text { the agency; and }\end{aligned}$

$\mathrm{g} \quad=$ age of the element, estimated by Equation (4.27) 
The scaling factor is estimated using the following Equation (4.26).

$\alpha=\frac{T_{1}}{\ln 2^{\frac{1}{\beta}}}$

$\alpha \quad=$ scaling factor;

$\beta \quad=$ shaping parameter; and

$T_{1}=$ median number of years the element stays in CS1, before transition to CS2.

The age of the element is initially (year 0) estimated using the following Equation (4.27). For the consecutive years, the age of the element is incremented by 1 .

$$
\begin{aligned}
& g=10 \alpha \frac{\log \left(-\ln \left(Y_{1}\right)\right)}{\beta} \\
& g=\text { estimated age of the element; } \\
& \alpha=\text { scaling factor; } \\
& \beta=\text { shaping parameter; and } \\
& Y_{1}=\text { initial percentage of the element in CS1. }
\end{aligned}
$$

\subsubsection{Action Effectiveness Models}

The effect of an action on condition can be expressed by a matrix of probabilities. Each condition state has zero to multiple feasible actions. Each action results in a distribution of condition states immediately following its implementation. The distribution is expressed in transition probabilities. A study for Florida DOT (Sobanjo \& Thompson, 2011) estimated transition probabilities when a preservation action is taken for different groups of bridge elements based on an inventory of more than 19,000 structures and inspection data history of 14 years. These transition probabilities institute the AE models used in Florida DOT's PLAT. These models were refined in 2016 to align 
with the new AASHTO elements (AASHTO, 2013; Sobanjo \& Thompson, 2016a). Similar AE models were developed for use in BrM, NBIAS, and several state DOT's BMSs. The module relies on the Florida DOT's AE models to predict conditions of the different elements when an action is taken. The following Table 4.8 presents the AE models assigned to Element 207—Steel Column Tower.

Table 4.8 Example of AE models for Element 207—Steel Column Tower

\begin{tabular}{|c|c|c|c|c|c|c|c|}
\hline \multirow[t]{2}{*}{ Action } & \multirow{2}{*}{$\begin{array}{c}\text { Initial } \\
\text { Condition } \\
\text { State }\end{array}$} & \multirow[t]{2}{*}{ Treatment Name } & \multirow{2}{*}{$\begin{array}{l}\text { Treatment } \\
\text { Key }\end{array}$} & \multicolumn{4}{|c|}{$\begin{array}{c}\text { Probability after Performing Action } \\
(\%)\end{array}$} \\
\hline & & & & $\mathrm{CS} 1$ & $\mathrm{CS} 2$ & CS3 & CS4 \\
\hline 1 & 2 & Spot blast & 319 & 70.84 & 29.16 & 0 & 0 \\
\hline 1 & 3 & Spot blast and minor repair & 302 & 70.84 & 29.16 & 0 & 0 \\
\hline 1 & 4 & Spot blast and major repair & 202 & 76.89 & 23.10 & 0 & 0 \\
\hline 2 & 2 & Spot blast and minor repair & 302 & 70.84 & 29.16 & 0 & 0 \\
\hline 2 & 3 & Spot blast and major repair & 202 & 76.89 & 23.11 & 0 & 0 \\
\hline 2 & 4 & Repair distortion & 248 & 76.89 & 23.11 & 0 & 0 \\
\hline 3 & 3 & Repair distortion & 248 & 76.89 & 23.11 & 0 & 0 \\
\hline 3 & 4 & $\begin{array}{l}\text { Mitigate settlement or } \\
\text { scour }\end{array}$ & 247 & 68.62 & 31.34 & 0.04 & 0 \\
\hline 4 & 3 & $\begin{array}{l}\text { Mitigate settlement or } \\
\text { scour }\end{array}$ & 247 & 68.62 & 31.34 & 0.04 & 0 \\
\hline 4 & 4 & Replace unit & 144 & 100 & 0 & 0 & 0 \\
\hline
\end{tabular}

Note. Retrieved from Implementation of the 2013 AASHTO Manual for Bridge Element Inspection (Sobanjo \& Thompson, 2016a).

Table 4.8 is organized by action, condition state, treatment name, treatment key, and probability into a condition state. To explain the table, for instance, if the element is initially in CS3, and Action 2 (Treatment Key 202-Spot blast and major repair) is performed, then $76.89 \%$ of the element will be in CS1 and $23.10 \%$ in CS2 immediately after the action. All rows must sum to $100 \%$. 


\subsection{Bridge and Element Health Indices}

Bridges are essential parts of the nation transportation infrastructure. Collapse of a critical bridge due to its deteriorating condition can be devastating - it could cause loss of lives, disrupt the regional transportation network, and impact the regional economy. State DOTs use a variety of performance measures and indices to assess the structural or functional health of a bridge, the performance of a network, or support their decision-making process. The structurally deficient status, NBI condition rating, and sufficiency rating are commonly used performance measures to support the bridge programming process. Bridge health indices are likewise useful for structural health comparisons and resource allocation for a network of bridges. They are generally applied at the network level and used as performance measures to track conditions over time and evaluate the LC performance (Adams et al., 2009). BMSs rely on element-level inspection data to compute bridge and element health indices (Chase et al., 2016).

Caltrans' bridge and element health indices are used by BrM, Florida DOT's PLAT, and several state DOT's BMSs. The presented module uses these same condition measures to assess the extent of deterioration and effectiveness of actions. Element and bridge health indices are computed by the following Equations (4.28) and (4.29), respectively. Element health indices are first determined by Equation (4.28), and then weighted, aggregated and divided by the sum of all their weighs in Equation (4.29), constituting an overall bridge health index. 
$H I_{e l m_{i}}=\left(P 1_{e l m_{i}}\right)+\frac{2}{3}\left(P 2_{e l m_{i}}\right)+\frac{1}{3}\left(P 3_{e l m_{i}}\right)$

$H I_{b r g_{k}}=\frac{\sum_{i \in E_{k}}\left(W_{\text {elm }_{i}} \times Q_{\text {elm }_{i}} \times H I_{\text {elm }}\right)}{\sum_{i \in E_{k}} W_{\text {elm }}}$

where

$H I_{e l m_{i}}=$ health index of element $i$

$H_{\text {brg }_{k}}=$ health index of bridge $k$;

$P 1_{\text {elm }}$ = percentage of element $i$ in CS1;

$P 2_{\text {elm }}$ = percentage of element $i$ in CS2;

$P 3_{\text {elm }}=$ percentage of element $i$ in CS3;

$W_{\text {elm }}=$ health index weight for element $i$;

$Q_{e l m_{i}}=$ quantity of element $i$; and

$E_{k} \quad=$ set of bridge $k$ elements.

A change in the overall bridge health index is generally minuscule. Element health indices are weighted, aggregated and divided by the sum of all their weighs to constitute this overall percentage. Improving few element health indices (after factoring their weights) won't dramatically change the overall percentage. It takes substantial improvement efforts to alter the overall bridge health index. Despite this drawback, these adapted health indices still provide an appropriate measure to assess the performance of a single element and the overall bridge; however, they are not a complete measure of the value of the agency's investment (Chase et al., 2016).

\subsection{Estimating Life Expectancy}

Asset life expectancy is defined as the length of time until the asset is replaced or removed from service. Estimating life expectancies of an asset provides the ability to realistically assess 
performance and investment strategies. Incorporating asset life expectancy into a LCC model and/or decision-making process allows agencies to develop bridge improvement programs with greater confidence. Methods for estimating life expectancy can be either empirical or mechanistic. Physical-based methods are considered mechanistic because they generally involve field or laboratory testing using destructive or non-destructive techniques to measure certain asset physical properties. However, methods developed based on Makov chains, machine learning algorithms, Weibull distributions, or duration and regression models are classified as empirical (Thompson et al., 2012). These methods can be simple or more involving. In this research, one of the empirical methods recommended in the NCHRP Report 713 (Thompson et al., 2012) is considered.

\subsubsection{End-of-Life Threshold}

A replacement alternative is generally triggered when the condition reaches an unacceptable level of performance. A minimum performance threshold (or trigger) reflects the point at which intermediate maintenance actions are no longer cost-effective (Thompson et al., 2012). The definition of end of service life depends on agency's LOS standards, maintenance practices and program policies-The AASHTO Manual for Bridge Element Inspection (AASHTO, 2013a) provides useful "end-of-life" definitions. There may be more than one performance measure or trigger defining the end of life of asset or its components. NBI condition ratings serve as simplistic triggers to set preservation priorities. For instance, a bridge end of service life can be defined when its superstructure condition rating drops to 4 or less-a preservation is no longer a viable option, and only a major rehabilitation or replacement can be considered. The worst-defined condition state of an element is generally presumed as the optimal level for replacement. It's convenient to define an element end of service life when there is a $50 \%$ chance the element is in its worst-defined condition state. A bridge end of service life (or end-of-life) could be defined when $50 \%$ of all its 
elements reach their worst-defined condition states (Thompson et al., 2012). In this research, the element end-of-life threshold is defined as the worst-defined condition equals to $50 \%$. The worstdefined condition state of an element is taken as CS4.

\subsubsection{Estimated Service Life}

The NCHRP Report 713 (Thompson et al., 2012) states that no published sources of accurately estimating life expectancies of bridges or elements exist; Markov transition probabilities are commonly used to estimate element life expectancies. It further lays out a simplified approach to determine an element life expectancy from a matrix of Markov transition probabilities. The Markov prediction formula is used iteratively, extending the forecast until the end-of-life threshold is reached. The module follows this same approach to estimate the service life of an element (ESL). The computation is based on the hybrid (Weibull/Markov) prediction formula, Equation (4.21). The calculation begins with an element in perfect condition $(\mathrm{CS} 1=100 \%)$ and iterates for each year until reaching the end-of-life threshold $(\mathrm{CS} 4=50 \%)$. Table 4.9 illustrates a computational example of ESL for Element 110-Open Girder/Beam, Reinforced Concrete. In this example, the end-oflife threshold (CS4=50.95\%) is reached in year 75. Accordingly, the module assigns an ESL of 75 years to Element 110 . 
Table 4.9 Example of ESL calculations for Element 110 (ESL=75 years)

\begin{tabular}{|c|c|c|c|c|}
\hline & \multicolumn{4}{|c|}{ Condition Sates (\%) } \\
\hline Year & CS1 & $\mathrm{CS} 2$ & CS3 & CS4 \\
\hline 0 & 100.00 & 0.00 & 0.00 & 0.00 \\
\hline 1 & 99.93 & 0.07 & 0.00 & 0.00 \\
\hline 2 & 99.74 & 0.26 & 0.00 & 0.00 \\
\hline 3 & 99.41 & 0.58 & 0.01 & 0.00 \\
\hline 4 & 98.95 & 1.01 & 0.04 & 0.00 \\
\hline 5 & 98.36 & 1.55 & 0.08 & 0.00 \\
\hline 6 & 97.65 & 2.20 & 0.14 & 0.01 \\
\hline 7 & 96.82 & 2.94 & 0.23 & 0.01 \\
\hline 8 & 95.86 & 3.77 & 0.35 & 0.02 \\
\hline 9 & 94.79 & 4.67 & 0.50 & 0.04 \\
\hline 10 & 93.61 & 5.65 & 0.68 & 0.06 \\
\hline- & - & - & - & - \\
\hline- & - & - & - & - \\
\hline- & - & - & - & - \\
\hline 70 & 3.93 & 24.18 & 26.96 & 44.93 \\
\hline 71 & 3.58 & 23.49 & 26.78 & 46.15 \\
\hline 72 & 3.26 & 22.79 & 26.58 & 47.37 \\
\hline 73 & 2.96 & 22.11 & 26.36 & 48.57 \\
\hline 74 & 2.69 & 21.43 & 26.12 & 49.77 \\
\hline 75 & 2.44 & 20.75 & 25.86 & 50.95 \\
\hline 76 & 2.21 & 20.09 & 25.58 & 52.12 \\
\hline 77 & 1.99 & 19.43 & 25.29 & 53.29 \\
\hline 72 & 2.69 & 19.50 & 24.01 & 53.80 \\
\hline \multicolumn{5}{|c|}{ Hybrid Deterioration Model } \\
\hline \multicolumn{2}{|c|}{ Shaping } & \multicolumn{3}{|c|}{ Transition Times (years) } \\
\hline & $\beta$ & $T_{1-2}$ & $\mathrm{~T}_{2-3}$ & $\mathrm{~T}_{3-4}$ \\
\hline & .00 & 32.40 & 15.69 & 14.93 \\
\hline
\end{tabular}

Note. $\beta=$ Weibull shaping parameter;

$\mathrm{T}_{1-2}=$ Transition time from CS1 to CS2;

$\mathrm{T}_{2-3}=$ Transition time from CS2 to CS3;

$\mathrm{T}_{3-4}=$ Transition time from CS3 to CS4.

\subsubsection{Estimated Remaining Service Life}

When no actions are considered in the entire LC of an element, the remaining service life (RSL) is easily computed by subtracting the element current age from its ESL. However, if an action takes place at any point in time, then it is more accurate to use a condition-based approach—-taking 
advantage of the deterioration and equivalent age models (Thompson et al., 2012). Alike the computational approach of the ESL, the hybrid prediction formula, Equation (4.21), is used iteratively until a known condition state is reached as long as the element did not reach its end-oflife threshold. The known condition can be converted to an equivalent age from the deterioration curve. This equivalent age is then subtracted from the element ESL to determine its RSL (refer to Figure 5.7 in Chapter 5).

\subsection{Element Life-Cycle Profiles}

Three types of LC profiles (i.e., DN, RO, and AE LC profiles) are constructed for each element to predict bridge and element health indices at different points in time, and estimate RSLs and LCCs.

\subsubsection{Do-Nothing Life-Cycle Profile}

Constructing a DN LC profile for each element as part of the data processing phase permits to predict the bridge and element DN health indices at the end of each analysis year and estimate the element RSLs. The DN transition times, Weibull model parameters, and recent condition states associated with each element are organized and stored in this module to be used by the hybrid deterioration model. An example of DN LC profile is illustrated in Figure 4.6. The schematic represents a "base" scenario of predicting condition of an untreated element (no actions performed to extend its ESL). This scenario simulates the element declining condition when no action is ever taken until reaching its end-of-life threshold. Element deficiency screening process (discussed in Chapter 5) relies on the DN LC profile. The element doesn't experience any improvement actions at any point in time. 


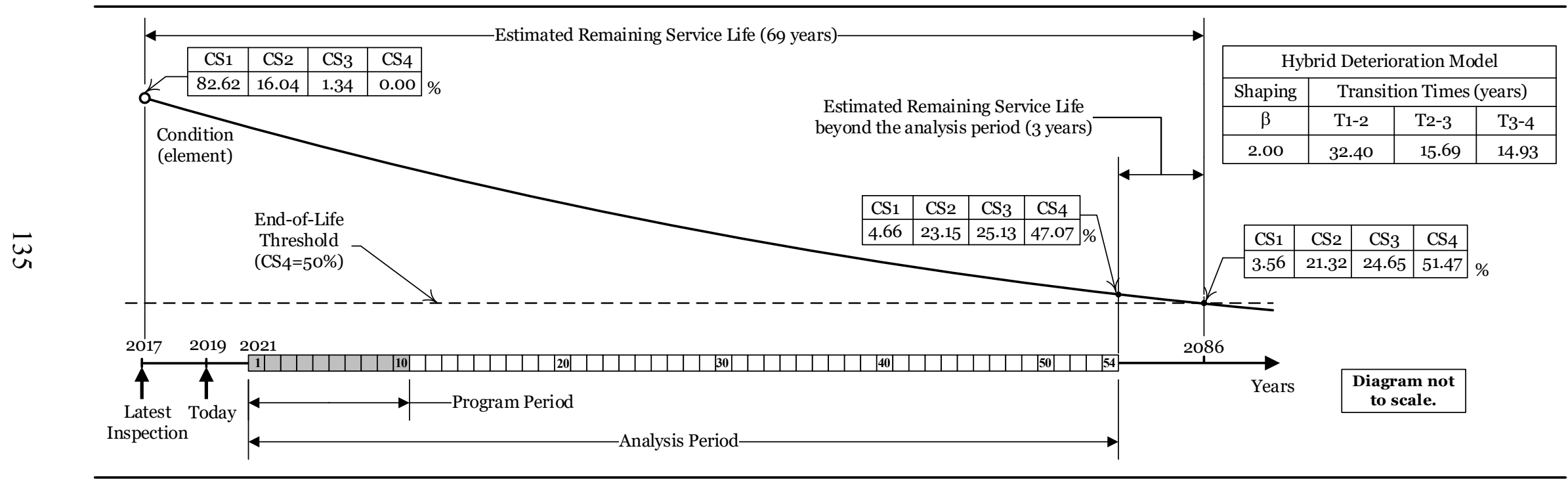

Figure 4.6 Example of DN LC profile for Element 110 — Open Girder/Beam, Reinforced Concrete 


\subsubsection{Replacement Only Life-Cycle Profile}

Constructing a RO LC profile for each element as part of the data processing phase permits to predict the bridge and element health indices at the end of each analysis year, estimate the element RSLs beyond the analysis period and ESLs for the RO scenario. The DN transition times, Weibull model parameters, and recent condition states associated with each element are organized and stored in this module to be used by the hybrid deterioration model. Figure 4.7 illustrates an example of RO LC profile. The schematic represents another "base" scenario of predicting condition of an element that experiences only replacement actions. The schematic mimics the "worst-first" strategy: the element gets entirely replaced as it deteriorates to a poor condition without experiencing any treatments (no actions). The element is replaced whenever its worst-defined condition (i.e., CS4) reaches the end-of-life threshold (CS4=50\%). Every time the element is replaced to extend its ESL, CS1 is restored to $100 \%(\mathrm{CS} 1=100 \%)$. 


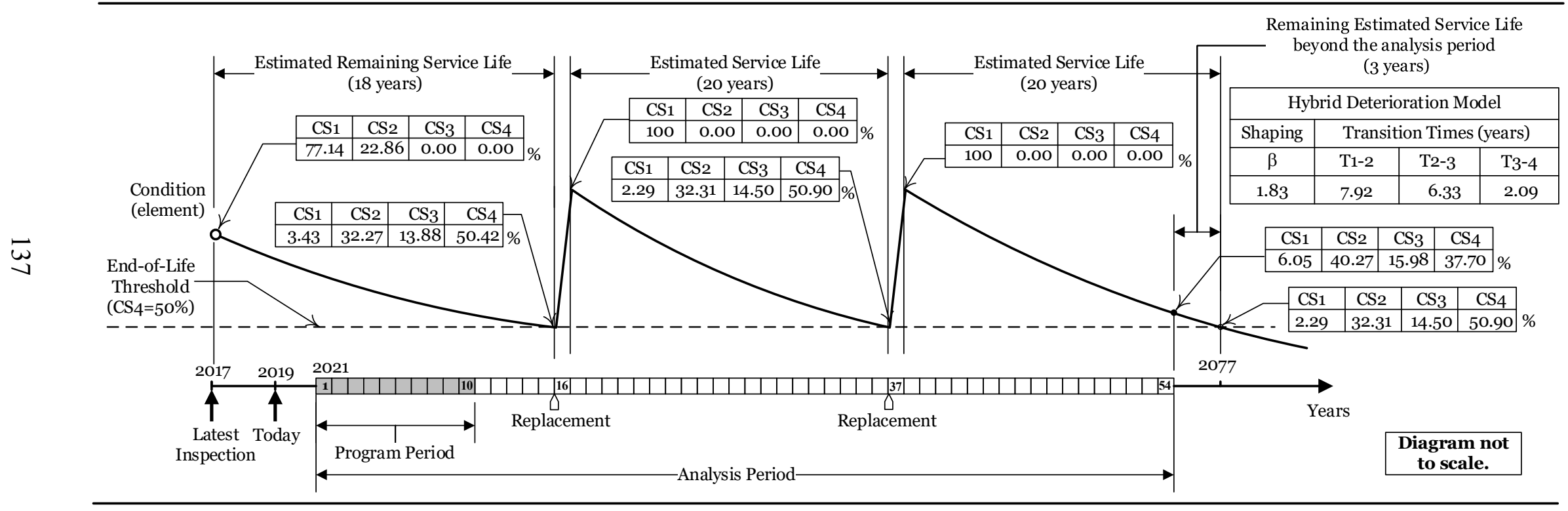

Figure 4.7 Example of RO LC profile for Element 202 - Column, Steel 


\subsubsection{Action Effectiveness Life-Cycle Profile}

An AE LC of an element is represented by a series of actions separated by an inaction period. The module assumes that a preservation policy is followed throughout the LC of the bridge and a major improvement work on the bridge fully restores all its elements. Preservation actions are considered the most cost-effective actions for the long term. Preservation actions extend the service life of an element by slowing down deterioration. Thus, they always subsequent a major improvement work on the bridge for the remaining analysis period. Preservation actions account for the large portion of the AE LC profile.

An AE LC profile for each possible combination of actions for each element is constructed by the improvement module to predict the AE conditions, determine the bridge and element health indices at the end of specified analysis year, and estimate the element RSLs beyond the analysis period. The DN transition times, Weibull model parameters, and recent condition states associated with each element are organized and stored in this module to be used by the improvement module - the hybrid deterioration model is used to forecast conditions within the inaction periods; whereas, the AE models, within the action implementation years. An example of AE LC profile is illustrated in Figure 4.8. represents an "improvement" scenario of predicting condition of a treated element (experiencing various actions separated by a waiting period). When an action is taken, an immediate change in condition happens according to the AE model, while subsequent forecasting, up to the next action, relies on the hybrid deterioration model-using the improved condition as the starting point. This scenario simulates a preservation strategy approach. The element experiences preservation actions that extend its service life-reaching the end-of-life threshold within the analysis period is deferred. 


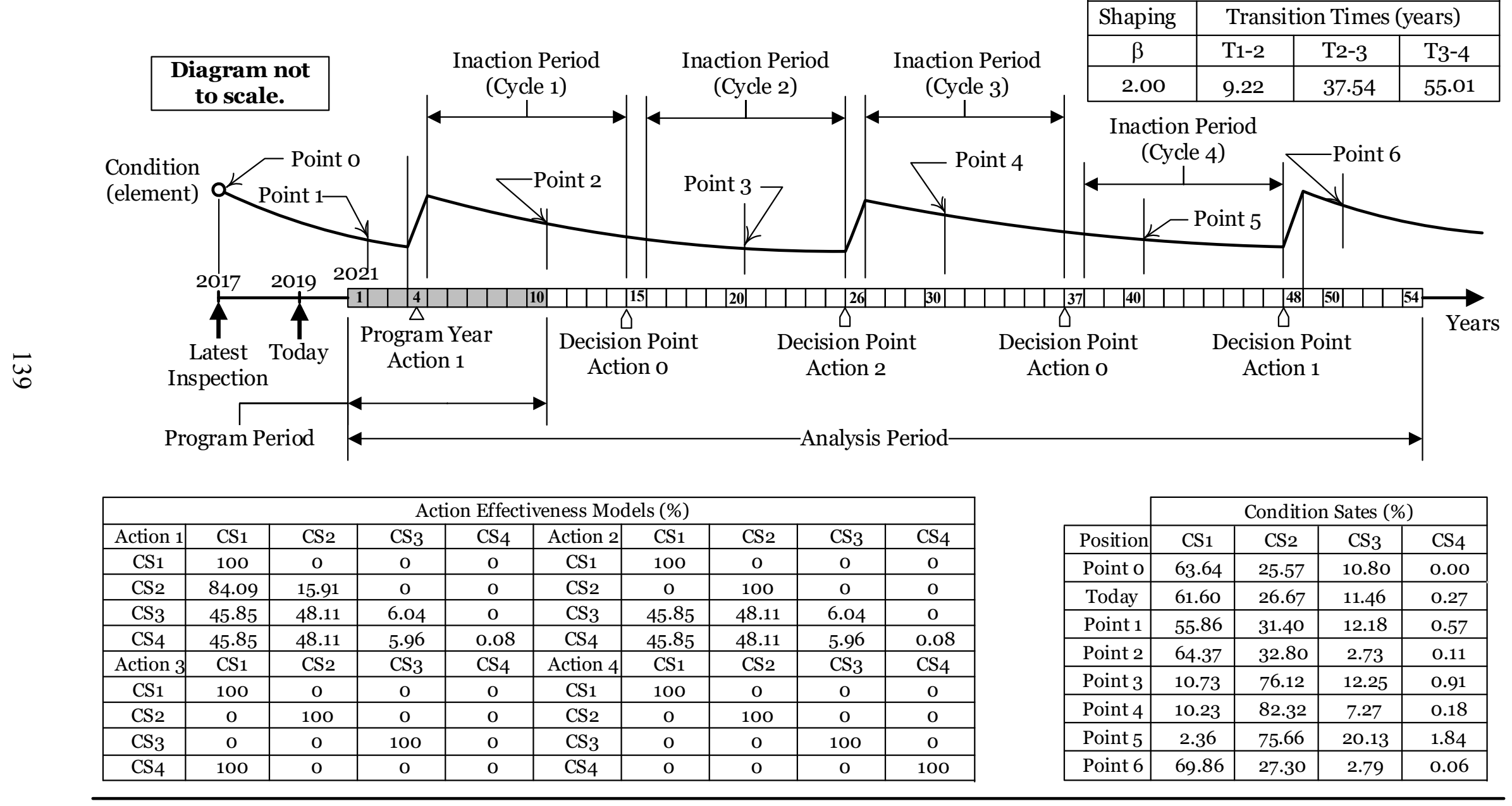

Figure 4.8 Example of AE LC profile for Element 215-Abutment, Reinforced Concrete 


\subsection{Summary and Conclusions}

This chapter introduced the data processing module framework and described its different underlying concepts. The module relies on decision makers' preferences and inputs, quality data, and information provided in the TAMP and TIP/STIP, and leading-edge forecasting and up-todate user cost models. The chapter discussed the two common types of bridge inspection data (i.e., NBI and NBE inspection data), the Weibull/Markov deterioration model used to predict the performance of an element over an analysis period and determine its ESL and RSL, the adapted AE models to forecast element conditions when actions are taken, and the performance measures (i.e., Caltrans' bridge and element health indices) used to assess the extent of deterioration and effectiveness of actions.

The chapter defined the preservation, functional improvement and replacement actions (i.e., MRR Actions 0 to 4, FCI Action 5, and REP Action 6). It laid out the process of assessing function improvement needs (i.e., widening, raising, and strengthening improvement needs) and revealed the user cost models used to estimate incurred user costs. The chapter presented the different equations used to estimate major improvement costs to eliminate any incurred user costs, relieve the bridge from any restrictions, and/or provide the required LOS and design standards. The three types of element LC profiles representing the "base" and "improvement" scenarios (i.e., DN, RO, and AE LC profiles) were introduced in this chapter. The module assumes that a preservation policy is followed throughout the LC of the bridge, and a major improvement work on the bridge fully restores all its elements. 


\section{CHAPTER 5-IMPROVEMENT MODULE}

- Task 3: Proposing an Element-Based Multi-Objective Optimization Methodology

- Task 4: Development of a Tool Prototype

- Task 5: Implementation of the Methodology through Examples of Scenarios

\subsection{Introduction}

State DOTs are responsible for the LRTP development to identify improvement needs of their multi-modal transportation system in the next 20-year planning horizon. As stated in Chapter 2, the LRTP sets the stage for the development of a financially constrained transportation TIP/STIP that identifies high-priority improvement projects for the next 4 years. State DOTs are required to include a financial plan in their risk-based TAMPs that identifies the required annual costs to implement their asset improvement strategies over a minimum of 10-year period. All these plans are essential for resource allocation decisions. The improvement module was developed taking into consideration this planning and programming process and TAMP's financial plan requirements.

As funding resources continue to diminish, state DOTs continue to face the challenge of effectively prioritizing bridge interventions and making informed and economical investment decisions as well as anticipating future funding needs. BMSs can be utilized to help objectively make such decisions at the bridge and network levels. The primary objective of a BMS is to assist agencies in managing bridge data, performing economic analysis at the bridge and network levels, and recommending work programs. At the bridge level, LCCA is performed, and interventions over 
the bridge remaining life are selected; whereas at the network level, bridges that yield the maximum network benefits are identified from a list of candidate bridges.

A comprehensive BMS provides a systemic approach for prioritizing and selecting bridge improvement alternatives (O' Connor \& Hyman, 1989). Not all possible combinations of improvement actions and timings over an extended analysis period get considered in the LC economic analyses - capturing incurred costs due to each possible alternative and any effect on performance leads to tremendous computational efforts and processing time. Thus, the recommended alternatives do not necessarily guarantee the optimal allocation of resources (Kachua, 2012). The improvement module is designed to overcome most of these limitations.

This chapter presents a basic framework to visualize the modeling approach followed to generate LC alternatives (series of element improvement actions). It summarizes the underlying processes and concepts. The module relies on a novel screening process to focus on potential deficient elements, and a simulation arrangement to generate realistic ("real-life") LC alternatives for MRR, FCI and REP improvement types based on agency's preservation policies and/or practices. The module relies on an independent deterioration model (covered in Chapter 4) to predict performance, and a LCC model to estimate LCCs and LCC benefits. A LC activity profile is constructed separately for each LC alternative action path and each program year. The module was implemented through a sample of bridges to test concepts, and demonstrate effectiveness and potential benefits. An illustrative example using the tool prototype is presented in this chapter. 


\subsection{Module Framework}

As mentioned in the introduction, the improvement module was designed taking into consideration the planning and programming process and TAMP's financial plan requirements. The module can be deployed independently to support the development of LRTP, TIP/STIP, and TAMP. Improvement module results can be used with common economic analyses and optimization techniques to identify short- and long-term bridge investment needs, and recommend bridge programs and implementation schedules.

Figure 5.1 introduces a basic flow diagram to visualize the modeling approach for generating LC alternatives, estimating LCCs and LCC benefits, and predicting performance (health indices). A program period of 10 years is considered by default. The period could be shortened to improve the processing time or agree with existing programming practice. A program period should at least cover the time horizon of a fiscally constrained TIP/STIP (i.e., 4 years). Though, a longer period extended to the TAMP financial plan time horizon allows to support monitoring of performance targets and resource allocations. Throughout the improvement module, each bridge in the portfolio is evaluated separately for each program year. Results are organized per program year for further processing. Although the initial agency intervention always performed within the program timeframe, the LC economic analysis extends beyond this short-term of programming phase. As mentioned in Chapter 3, the length of the analysis period depends on the agency's preservation policies and preferences. A consistent period of analysis allows to fairly compare the generated LC alternatives. A fixed long analysis period (usually around 50 years) produces realistic LC alternatives covering most of the anticipated element service life. An analysis period of 54 years is used by default. 
Each bridge in the portfolio is screened for candidacy through the element deficiency screening process. The screening process uses the forecasting results of the DN LC profiles constructed in the data processing module (discussed in Chapter 4). A bridge identified with at least a deficient element is considered a candidate for improvement. A RO LC profile is constructed for each identified deficient element. The DN and RO LC profiles represent the "baseline" against which the LC alternative economic benefits (i.e., LCC benefits) are compared. For each candidate bridge, $\mathrm{DN}$ and base user costs covering the entire analysis period are computed and discounted to present value. DN and base agency costs are computed and discounted to present value for each deficient element. Similarly, relying on the hybrid deterioration model discussed in Chapter 4, DN and base bridge and element health indices at different points in time are determined.

As illustrated in Figure 5.1, each program year (initial agency intervention) is associated with various LC profiles. Each LC profile is constructed separately and allied with one of the three improvement types: MRR, FCI, and REP. A LC alternative is defined by a program year, a path of actions, and an improvement type. AE LC profiles (discussed in Chapter 4) are constructed separately for each deficient element. An AE LC profile (or LC activity profile) of an element is represented by a series of actions following a unique LC alternative action path. Different types of LCCs (incurred during the entire analysis period) and residual values (applied at the end of the analysis period) are estimated for each LC alternative. Element health indices at different points in time are determined. The module framework exemplified in Figure 5.1 is based on the following concepts:

1. Separate LC profiles by improvement type: MRR, FCI, and REP

2. Locate the DN LC profile results (from the data processing module) associated with each element of a bridge in the portfolio 
3. Construct the RO LC profiles (base LC profiles) for each element of a bridge in the portfolio

4. Compute and discount to present value all base costs and residual values for each element of a bridge in the portfolio

5. Determine base health indices at different points in the analysis period for each element of a bridge in the portfolio

6. Identify the candidate bridges and their potential deficient elements through the element deficiency screening process

7. Construct all possible LC alternative profiles per improvement type and per program year for each identified deficient element

8. Join LC profiles of each identified deficient element with their corresponding LC alternatives

9. Compute and discount to present value all LCCs and residual values relevant to each LC alternative

10. Use LCCs, residual values, and base LCCs to compute LCC benefits

11. Predict element health indices at different points in the analysis period relevant to each LC alternative

12. Organize LC alternative results by element, improvement type, and program year for each candidate bridge 


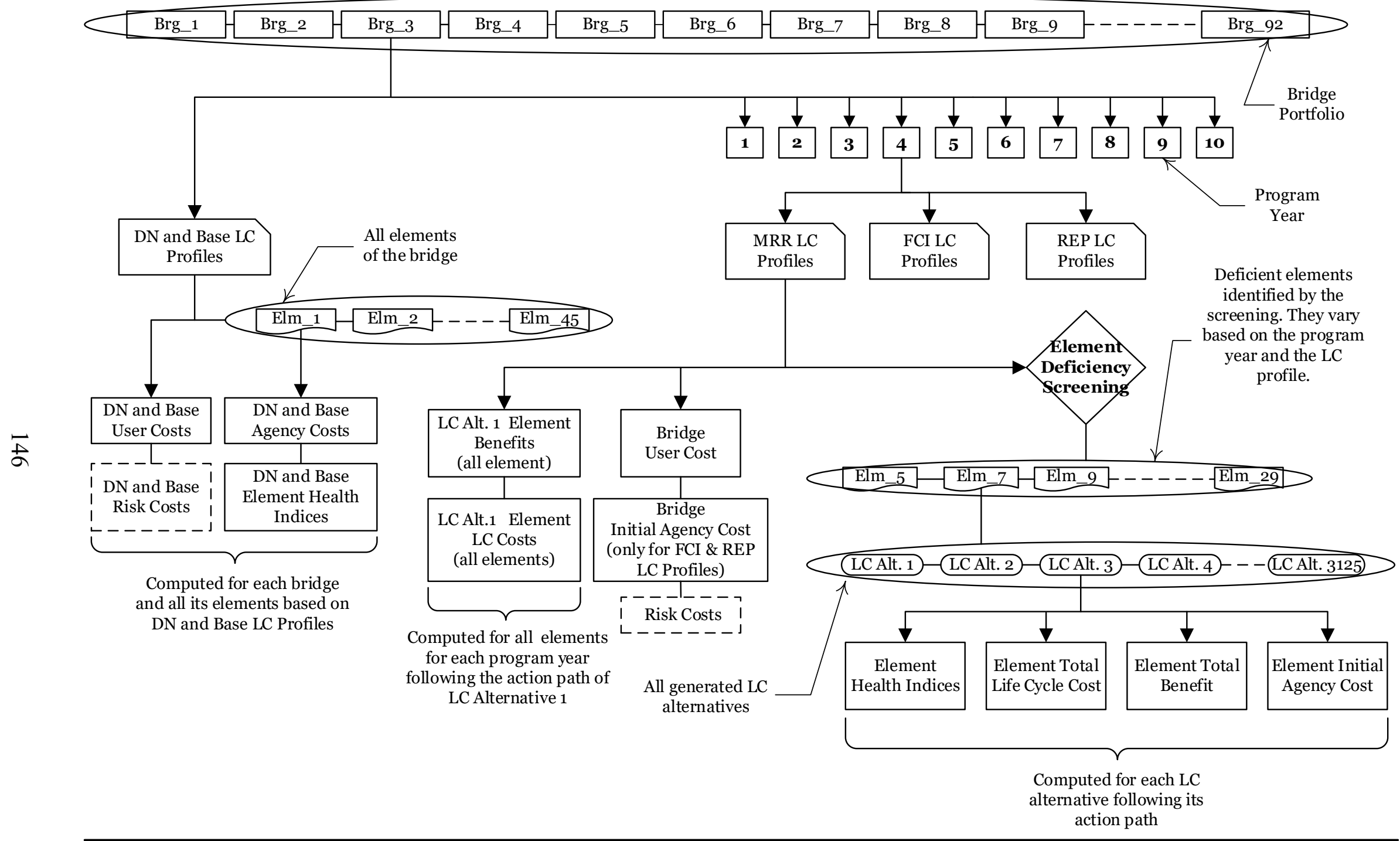

Figure 5.1 Improvement module framework 


\subsection{Element Deficiency Screening}

The proposed module depends on a screening process that evaluates the extent of element deterioration. Some elements may have deteriorated to a level where major repairs or proactive preservation efforts are necessary, and others may show no sign of deterioration. The FHWA's Bridge Preservation Guide (FHWA, 2018) provides examples of established agency's rules for preservation programs. Agency's rules or criteria for bridge preservation strategies prevent or delay deterioration, extend serviceability, and maintain a "state of good repair." The following Figure 5.2 is borrowed from the guide, representing the three common bridge improvement programs, and how they interact over time. A preservation program can extend the service life of a bridge in "good" or "fair" condition. Preservation actions are no longer effective when the bridge enters the "poor" condition zone. The bridge becomes a candidate for rehabilitation or replacement to restore its "good" or "fair" condition.

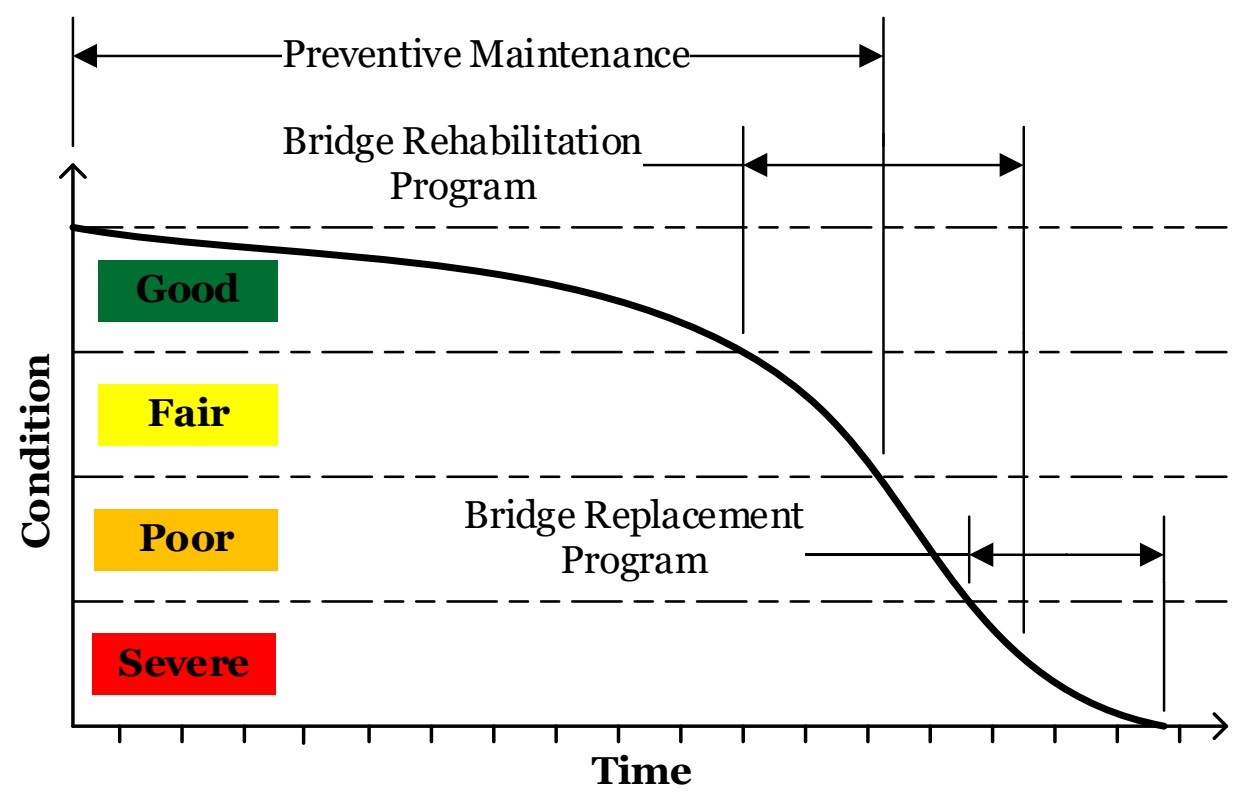

Figure 5.2 Bridge condition over time. Redrawn from the original in the FHWA's Bridge Preservation Guide (FHWA, 2018). 
A preservation policy contains information on the element deterioration rates, and costs and benefits of feasible preservation improvement actions. Simulation rules or triggers are driven from these preservation policies. Improvement interventions are recommended based on these triggers (e.g., condition state level or health index). A higher percentage in CS3 or CS4 indicates the severity of the damage or advanced deterioration of the element. Table 5.1 shows the preservation action triggers used by MnDOT for the different groups of elements.

Table 5.1 Preservation action triggers used by MnDOT

\begin{tabular}{ll}
\hline \multicolumn{1}{c}{ Elements } & \multicolumn{1}{c}{ Preservation Action Triggers } \\
\hline Steel elements & More than $15 \%$ in Condition State 3 or 4 \\
Steel protective coatings & More than $20 \%$ in Condition State 3 or 4 \\
Reinforced concrete elements & More than $10 \%$ in Condition State 3 or 4 \\
Prestressed concrete elements & More than $10 \%$ in Condition State 3 or 4 \\
Timber elements & More than $10 \%$ in Condition State 3 or 4 \\
Concrete deck or slab elements & More than $15 \%$ in Condition State 3 or 4 \\
Wearing surface elements & More than $15 \%$ in Condition State 3 or 4 \\
Joints elements & More than $10 \%$ in Condition State 3 or 4 \\
Bearing elements & More than $10 \%$ in Condition State 3 or 4 \\
\hline
\end{tabular}

Note. Adapted from the MnDOT Bridge Preservation and Improvement Guidelines (MnDOT, 2015).

To support this concept, the module identifies preservation needs for each bridge included in the portfolio. Each bridge is screened for candidacy. This process is referred to as "element deficiency screening." A bridge with or expected to acquire deficient elements is considered a candidate for improvement. The focus is to ensure vulnerable bridges are being elevated in the programming process. A bridge identified with no potential deficient elements is excluded from considerationthe "state of good repair" is assumed to be maintained in the entire analysis period. As mentioned earlier, bridge and element health indices are common performance measures used by agencies for prioritizing preservation and improvement projects. NBI condition and appraisal ratings, sufficiency ratings, load capacities, etc. are also among common performance measures. Element health index is the only performance measure used to assess preservation needs in this module. 
Users set minimum element health index limits at different points in the analysis period. Element health indices at the end of analysis years 10,20,30,40, and 50 are predicted for each element. A health index lower-frontier is constructed for each element (joining minimum limits). Elements with health indices falling under this lower-frontier are classified as "deficient."

Figure 5.3(a) illustrates a screening example for potential preservation needs. The same set of identified potential deficient elements is considered for all program years. The DN health index curve (denoted by MRR LC Alternative 1) starting from the last inspection date is considered in the determination. Figure 5.3(b) illustrates another screening example of identifying potential deficient elements. In this example, preservation needs are assessed for the period succeeding the element replacement year. All elements are replaced (restoring CS1 to 100\%) in program year 4 because of a major improvement (i.e., FCI Action 5 or REP Action 6) performed on the entire bridge. For each element, the DN health index curve (denoted by FCI or REP LC Alternative 1) starting from the end of program year 4 (initial intervention; $\mathrm{CS} 1=100 \%$ ) is considered in this example. A separate screening is performed for each of the other program years. 


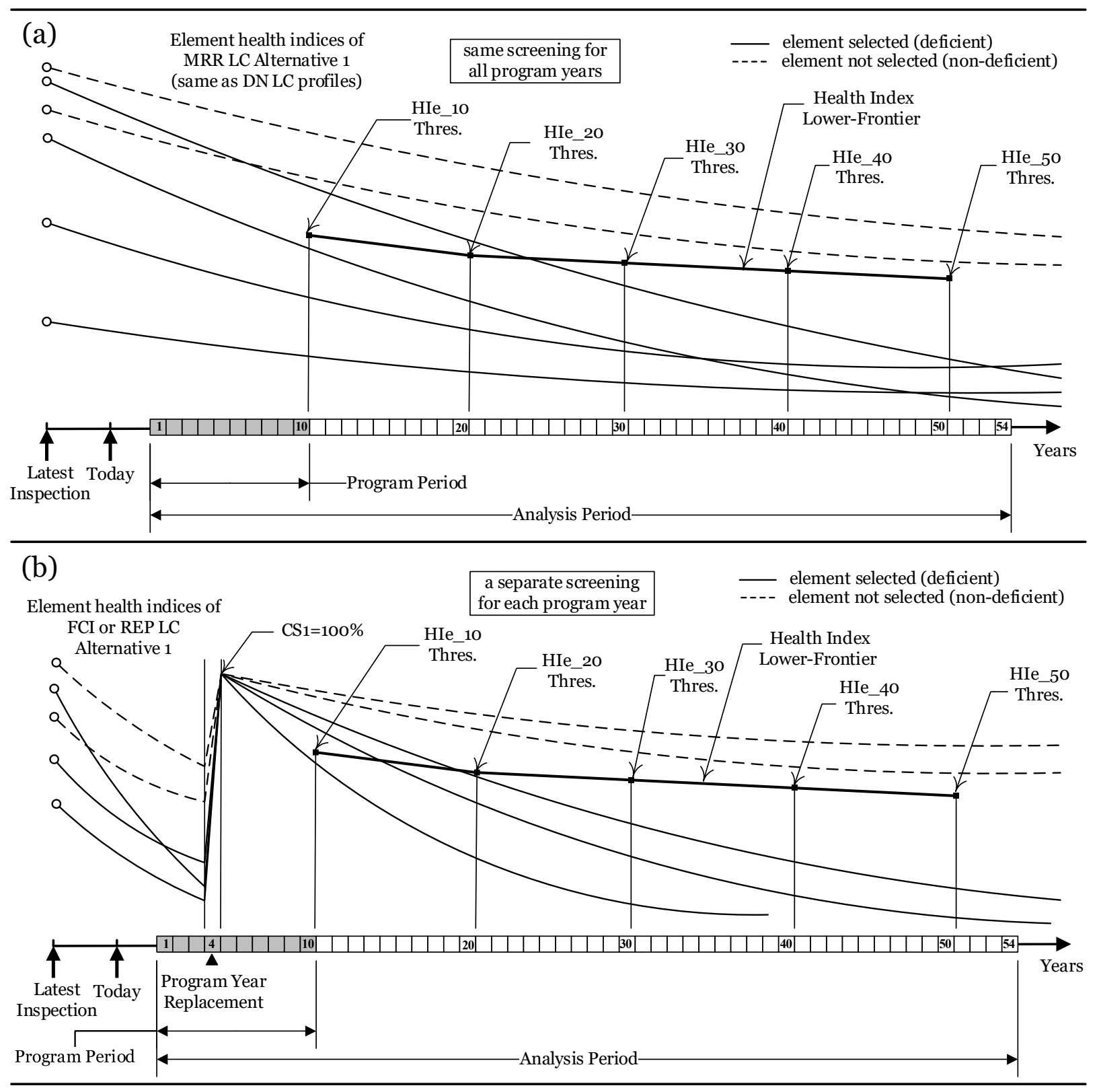

Figure 5.3 Example of element deficiency screening for (a) preservation only; (b) post-major improvement

Tables 5.2 and 5.3 presents examples of screened results (i.e., ESLs, RSLs, and DN health indices)

for a bridge with 12 elements. Table 5.2 values relate to the preservation only screening, and Table

5.3, to the post-major improvement screening. 
Table 5.2 Example of preservation only screening results for all program years

\begin{tabular}{|c|c|c|c|c|c|c|c|c|c|}
\hline Element & $\begin{array}{l}\text { ESLelm }_{\text {elm }} \\
\text { (years) }\end{array}$ & $\begin{array}{l}\mathrm{RSL}_{\mathrm{elm}} \\
\text { (years) }\end{array}$ & $\begin{array}{l}\mathrm{HI} 1_{\text {elm }} \\
(\%)\end{array}$ & $\begin{array}{c}\text { HI10 }{ }_{\text {elm }} \\
(\%)\end{array}$ & $\begin{array}{c}\text { HI20 elm } \\
(\%)\end{array}$ & $\begin{array}{c}\mathrm{HI} 30_{\mathrm{elm}} \\
(\%)\end{array}$ & $\begin{array}{c}\mathrm{HI}^{\mathrm{H}} 0_{\mathrm{elm}} \\
(\%)\end{array}$ & $\begin{array}{c}\mathrm{HI} 50_{\mathrm{elm}} \\
(\%)\end{array}$ & $\begin{array}{l}\mathrm{HI}^{\mathrm{H} 54_{\mathrm{elm}}} \\
(\%)\end{array}$ \\
\hline 12 & 129 & 66 & 71.70 & 62.73 & 57.07 & 52.52 & 48.26 & 44.22 & 42.67 \\
\hline 107 & 77 & 20 & 95.18 & 73.12 & 57.88 & 49.52 & 42.45 & 36.06 & 33.71 \\
\hline 205 & 200 & 143 & 99.77 & 97.58 & 92.64 & 85.76 & 77.96 & 70.18 & 67.22 \\
\hline $215^{\mathrm{a}}$ & 75 & 18 & 99.95 & 99.48 & 98.37 & 96.69 & 94.49 & 91.86 & 90.71 \\
\hline 220 & 123 & 57 & 80.19 & 64.64 & 54.84 & 49.47 & 44.72 & 40.35 & 38.70 \\
\hline 226 & 141 & 63 & 63.03 & 58.20 & 53.16 & 48.47 & 44.13 & 40.12 & 38.61 \\
\hline $234^{\mathrm{a}}$ & 408 & 351 & 99.98 & 99.81 & 99.38 & 98.70 & 97.76 & 96.56 & 96.00 \\
\hline 301 & 24 & 0 & 86.90 & 58.64 & 33.16 & 17.49 & 8.92 & 4.48 & 3.40 \\
\hline 311 & 58 & 0 & 62.10 & 47.87 & 36.22 & 28.15 & 21.74 & 16.64 & 14.92 \\
\hline 313 & 58 & 0 & 63.51 & 48.26 & 36.14 & 28.08 & 21.69 & 16.60 & 14.89 \\
\hline 330 & 28 & 0 & 96.52 & 71.70 & 38.84 & 17.63 & 7.27 & 2.85 & 1.94 \\
\hline $331^{\mathrm{a}}$ & 151 & 91 & 98.37 & 97.25 & 94.88 & 91.31 & 86.60 & 80.92 & 78.44 \\
\hline Bridge & & & $\mathrm{HI} 1_{\text {brg }}$ & HI10 brg & HI20 brg & $\mathrm{HI} 30_{\mathrm{brg}}$ & HI40 brg & $\mathrm{HI} 50_{\mathrm{brg}}$ & $\mathrm{HI}_{54} 4_{\mathrm{brg}}$ \\
\hline 11 & & & 78.14 & 66.14 & 57.73 & 51.91 & 46.88 & 42.31 & 40.59 \\
\hline
\end{tabular}

Note. A health index lower-frontier of $80 \%$ (deficiency screening thresholds, a minimum health index of $80 \%$ every 10 years).

${ }^{a}$ Non-deficient element.

Table 5.3 Example of post-major screening results for program year 4

\begin{tabular}{|c|c|c|c|c|c|c|c|c|c|}
\hline Element & $\begin{array}{l}\text { ESL }_{\text {elm }} \\
\text { (years) }\end{array}$ & $\begin{array}{l}\mathrm{RSL}_{\mathrm{elm}} \\
\text { (years) }\end{array}$ & $\begin{array}{l}\mathrm{HI} 1_{\text {elm }} \\
(\%)\end{array}$ & $\begin{array}{c}\text { HI10 }{ }_{\text {elm }} \\
(\%)\end{array}$ & $\begin{array}{c}\text { HI20 elm } \\
(\%)\end{array}$ & $\begin{array}{c}\mathrm{HI} 30_{\mathrm{elm}} \\
(\%)\end{array}$ & $\begin{array}{c}\text { HI40 elm } \\
(\%)\end{array}$ & $\begin{array}{c}\text { HI50 } 0_{\text {elm }} \\
(\%)\end{array}$ & $\begin{array}{c}\text { HI54 } 4_{\text {elm }} \\
(\%)\end{array}$ \\
\hline 12 & 129 & 79 & 71.70 & 82.20 & 64.69 & 58.19 & 53.55 & 49.24 & 47.58 \\
\hline 107 & 77 & 27 & 95.18 & 90.91 & 67.29 & 55.16 & 47.40 & 40.52 & 37.97 \\
\hline 205 & 200 & 162 & 99.77 & 99.49 & 96.35 & 90.73 & 83.47 & 75.59 & 72.47 \\
\hline $215^{\mathrm{a}}$ & 75 & 69 & 99.95 & 99.89 & 99.21 & 97.92 & 96.08 & 93.74 & 92.69 \\
\hline 220 & 123 & 73 & 80.19 & 91.27 & 67.15 & 57.70 & 52.23 & 47.27 & 45.39 \\
\hline 226 & 141 & 91 & 63.03 & 96.88 & 82.00 & 67.03 & 57.82 & 52.03 & 50.08 \\
\hline $234^{\mathrm{a}}$ & 408 & 393 & 99.98 & 99.96 & 99.71 & 99.20 & 98.45 & 97.43 & 96.94 \\
\hline 301 & 24 & 0 & 86.90 & 83.91 & 52.80 & 29.32 & 15.33 & 7.79 & 5.92 \\
\hline 311 & 58 & 8 & 62.10 & 90.75 & 63.57 & 48.50 & 38.35 & 29.99 & 27.10 \\
\hline 313 & 58 & 8 & 63.51 & 90.75 & 63.57 & 48.50 & 38.35 & 29.99 & 27.10 \\
\hline 330 & 28 & 0 & 96.52 & 92.78 & 61.29 & 31.11 & 13.63 & 5.52 & 3.79 \\
\hline $331^{\mathrm{a}}$ & 151 & 126 & 98.37 & 99.81 & 98.56 & 96.03 & 92.21 & 87.21 & 84.93 \\
\hline Bridge & & & $\mathrm{HI} 1_{\text {brg }}$ & HI10 $0_{\text {brg }}$ & $\mathrm{HI} 20_{\mathrm{brg}}$ & $\mathrm{HI} 30_{\mathrm{brg}}$ & HI40 brg & $\mathrm{HI} 50_{\mathrm{brg}}$ & $\mathrm{HI} 4_{\mathrm{brg}}$ \\
\hline 11 & & & 78.14 & 84.89 & 66.12 & 57.81 & 52.14 & 47.17 & 45.30 \\
\hline
\end{tabular}

Note. A health index lower-frontier of $80 \%$ (deficiency screening thresholds, a minimum health index of $80 \%$ every 10 years).

${ }^{a}$ Non-deficient element. 
Figure 5.4(a) displays the predicted health indices (at different analysis years) for each element of a bridge screened for preservation needs within a 54-year analysis period. The horizontal dashed line represents a health index lower-frontier (deficiency screening thresholds, a minimum health index of $80 \%$ every 10 years). The dashed curves above this frontier distinguish the non-deficient elements from the deficient ones. For MRR improvement type and all program years, the three identified non-deficient elements (i.e., Elements 215, 234, and 331) won't be processed by the module, only the deficient ones. The thick connected lines in black represent the overall DN bridge health indices. Figure 5.4(b) shows the predicted health indices for each element of the same bridge screened for preservation needs succeeding a major bridge improvement in program year 4 . The same three elements were identified non-deficient for FCI and REP improvement types with a major improvement in program year 4.

(a)

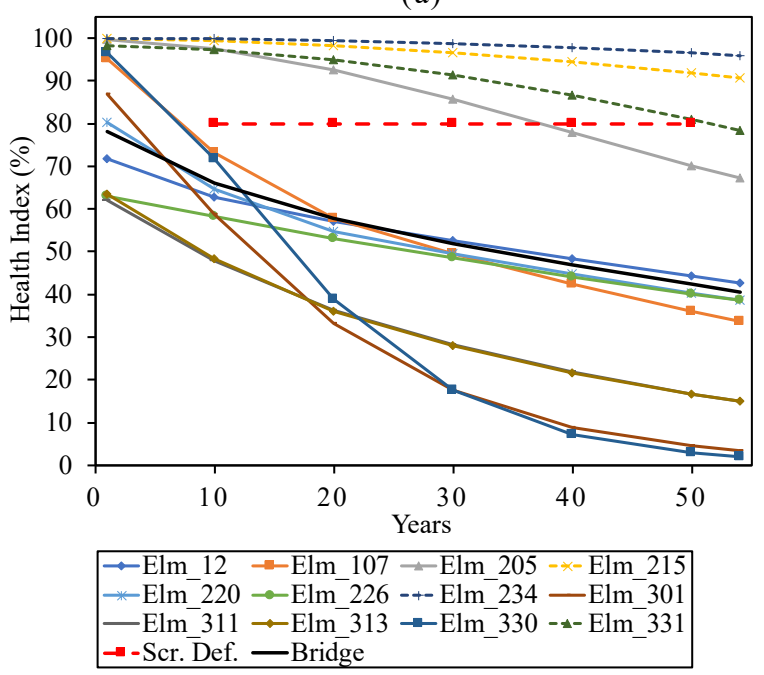

(b)

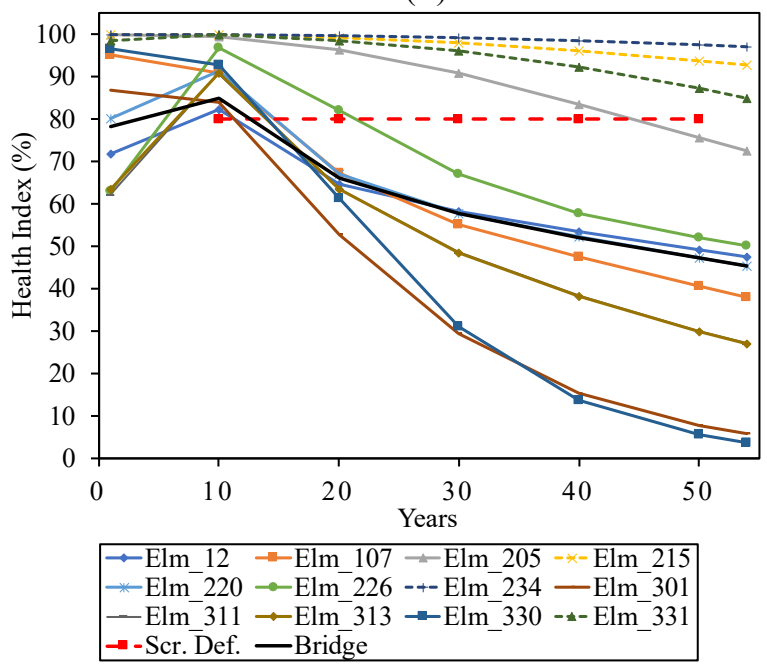

Figure 5.4 Example of predicted health indices for (a) preservation needs; (b) preservation needs succeeding a major bridge improvement in program year 4 


\subsection{Life-Cycle Alternatives}

Three types of LC profiles (i.e., DN, RO, and AE LC profiles) are constructed for each element to predict bridge and element health indices at different points in time, and estimate RSLs and LCCs. Chapter 4 discusses these three types of LC profiles in more detail-Examples of the three LC profiles are illustrated in Figures 4.7, 4.8, and 4.9. An AE LC profile represents an "improvement" scenario of predicting condition of a treated element. An AE profile of an element is represented by a series of improvement actions. When an action is taken, an immediate change in condition happens per the $\mathrm{AE}$ model, while subsequent forecasting up to the next action is based on the hybrid model. The module assumes that a preservation policy is followed throughout the LC of the bridge, and a major improvement work on the bridge fully restores all elements. Preservation actions (discussed in Chapter 4) are considered the most cost-effective actions for the long term. Thus, they always subsequent a major improvement work on the bridge for the remaining analysis period. Preservation actions account for the large portion of the AE LC profile.

To generate LC alternatives for each element, all possible AE LC profiles must first be constructed. A LC alternative is defined by a path of actions and an improvement type. Each AE LC profile is laid out in a cash-flow diagram following a LC alternative action path. A waiting period separates these actions. Each program year (initial intervention) is associated with multiple LC profiles. Each LC profile is allied with an improvement type (i.e., MRR, FCI or REP). Each MRR LC alternative calls for a specific path of MRR actions covering the entire analysis period. Each FCI LC alternative calls for a specific path of MRR actions covering the remainder period succeeding the initial intervention action (i.e., FCI Action 5)-which is assigned separately to the entire bridge. The same concept does apply for defining a REP LC alternative except the initial intervention action is a complete bridge replacement (i.e., REP Action 6). 
All possible alternatives can be generated; however, the number will be unmanageable. Given there are five groups of MRR actions (MRR Actions $0,1,2,3$, and 4) that can be performed in any year, for a 54-year analysis period, approximately 5.55 E73 $\left(5^{54}\right) \mathrm{LC}$ alternatives for each element. It's impractical to manage this huge number of LC alternatives due to the problem complexitysolving a problem with this size through an optimization algorithm would be beyond the capability of most computers due to memory limitation and processing time. Thus, a realistic ("real-life") arrangement providing a dramatic reduction in number of LC alternatives is vital.

A waiting period (referred to as inaction or deferment period) is introduced to significantly reduce the number of LC alternatives. The longer the waiting period, the less LC alternatives can be generated, but deterioration will likely increase, and preservation actions become less feasible. This inaction period should reflect maintenance and preservation practices. The period should be defined as part of the agency's preservation policies. Deferment rules can also be developed based these preservation policies. Variable inaction periods can be specified for different actions when applied. However, inconsistent inaction periods will complicate the LC modeling. The following rules are considered when generating LC alternatives:

- Action effectiveness profile consists of 5 cycles

- First cycle falls always after a program year

- Preservation action selection is made at the end of each inaction period (referred to as decision point)

- Ten years of inaction period between decision points (based on common practice)

- Action is implemented in one year 
Based on these rules, $3125 \mathrm{MRR}, 625 \mathrm{FCI}$, and $625 \mathrm{REP}$ LC alternatives per program year are generated for each deficient element. Thus, for a 10-year program period, a total of 31,250 of unique MRR LC profiles, 6,250 FCI LC profiles, and 6,250 REP LC profiles are constructed for each deficient element. Figure 5.5(a) illustrates an example of an action path defining a specific MRR LC alternative. The initial intervention (for this example, MRR Action 4) takes place in program year 4, followed by MRR Action 0, MRR Action 3, MRR Action 3, and MRR Action 2 spaced by 10-year of inaction period. This action path 4-0-3-3-2 is unique to MRR LC Alternative 2593. Figure 5.5(b) shows a path of actions for an FCI LC alternative. The initial intervention (for this example, FCI Actions 5) takes place in program year 4 and performed on the bridge followed by a series of preservation actions (MRR Actions 2, 4, 1, and 1, in that order, spaced by 10-year of inaction period) performed on a deficient element. As well, this action path 5-2-4-1-1 is unique to FCI LC Alternative 357. 


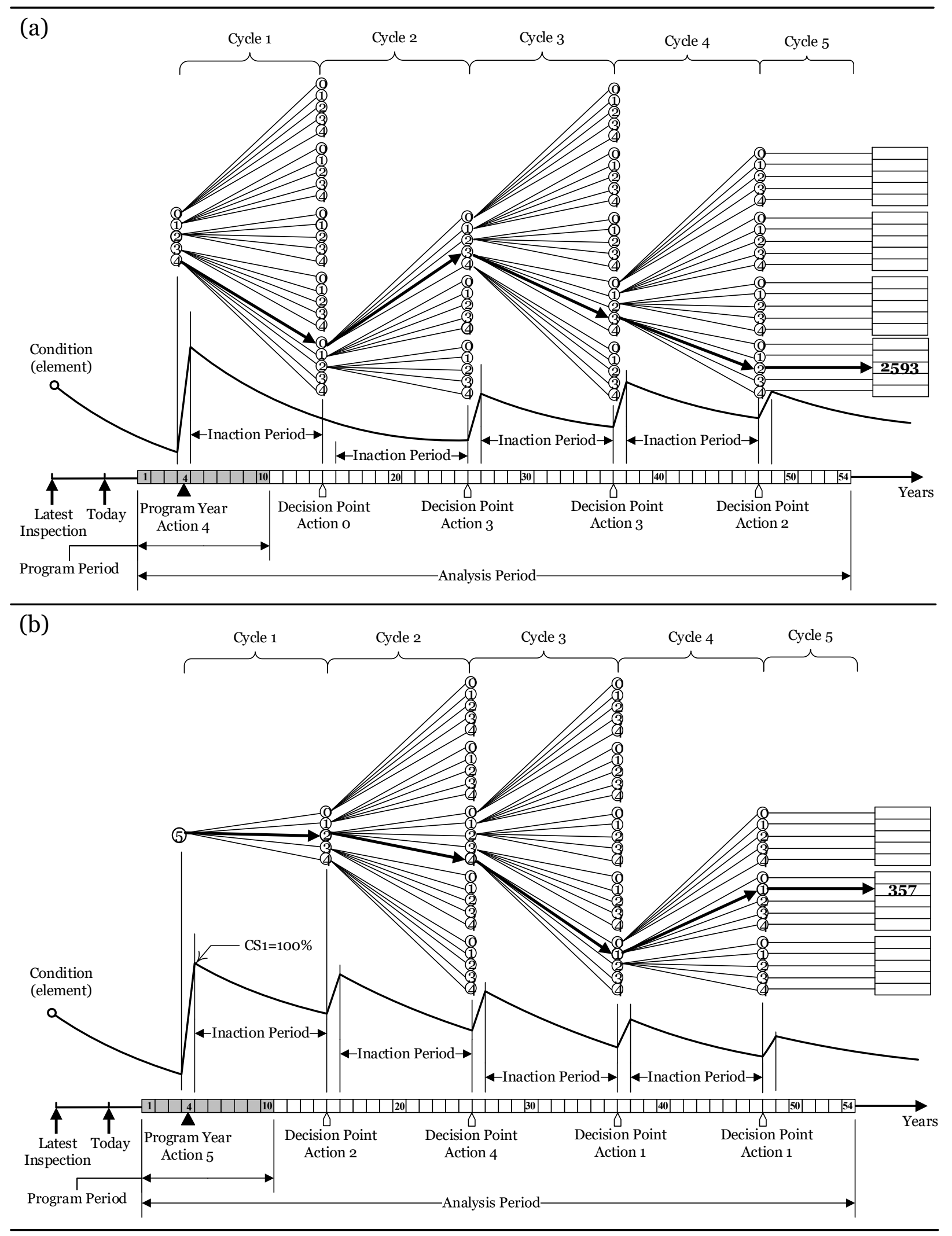

Figure 5.5 Simulation of (a) MRR LC Alternative 2593 (action path 4-0-3-3-2); (b) FCI LC Alternative 357 (action path 5-2-4-1-1) 


\subsection{Economics Analysis of Alternatives}

The LC economic analysis is one of the most common decision support tools used by transportation agencies for planning and programming. The analysis assesses the total economic worth of an asset by evaluating the initial agency costs and all costs anticipated over its service life. MAP-21 (23 CFR 515.9) requires state DOTs to incorporate a LCP process into their TAMPs, at a minimum, for pavements and bridges on the NHS and recommends a similar process for other transportation assets. To support transportation agencies with this requirement, FHWA (2017a) published a guidance on how to develop a LCP process. The guidance discusses the following to be included in a LCP process and focusses mainly on the LC economic analysis aspect covered in item 4.

1. The state DOT's asset condition targets for each asset class or asset sub-group;

2. Identification of deterioration models for each asset class or asset sub-group, assets other than NHS pavements and bridges are optional;

3. Potential work types across the whole life of each asset class or asset sub-group with their relative unit costs; and

4. A strategy for managing each asset class or asset sub-group by minimizing its LCCs, while achieving the state DOT's asset condition targets for NHS pavements and bridges under 23 USC 150(d).55 (FHWA, 2017a).

LCCA is a technique founded on the principles of economic analysis to help in the evaluation of the overall long-term economic efficiency between competing alternatives of investment options (AASHTO, 1986). LCCA allows agencies to assess the total economic worth of an asset by analyzing discounted initial agency costs and all expected costs over the life of the asset. The literature (under Task 1) revealed that multiple LCCA methods are being used to support the 
decision making. The NCHRP Report 483: Bridge Life-Cycle Cost Analysis (Hugh, 2003) provides a commonly accepted bridge LCCA approach to support agencies in selecting appropriate bridge improvement alternatives. The approach is incorporated in the LCC model of this module. The NCHRP Synthesis of Highway Practice 494: Life-Cycle Cost Analysis for Management of Highway Assets (Flannery et al., 2016) discusses the results of a survey on LCCA tools used by agencies, completed for Caltrans in 2011. Among the seventeen states participated in the survey, five reported using the FHWA's Real Cost Tool, three developed their own LCCA tools, three use custom spreadsheets, one uses both AASHTOWare products and custom software, and the remaining did not specify the tool being used. The survey identified two common tools for bridge LCCA: Bridge LCC and BLCCA. Bridge LCC developed in 2003 based on the American Society for Testing and Materials (ASTM) practice for measuring the LCCs of buildings and building systems and is primarily used to compare project alternatives. BLCCA (mentioned in Chapter 2) was developed under the NCHRP Project 12-43 (Hugh, 2003), including agency, user and vulnerability cost models.

As emphasized in Chapter 2, efforts have been made to assess the threat of natural and man-made hazards in BMSs. Vulnerability or risk cost models quantify consequences resulting from natural hazards (such as earthquake, scour, and flooding). The NCHRP Project 20-07, Task 378, (Thompson, 2018) developed a risk assessment guideline for the LCCA in BMSs based on likelihood probability models for sixteen different hazards and a process for monetizing risk. The guideline can be considered in the LCCA to account for risk. The module LCC model doesn't consider risk costs. Only agency and user costs are considered in the LCC model. The risk aspect is beyond the scope of this research. Nevertheless, the module is well-suited to admit the recommended guideline concepts or other risk models. 
The FHWA's Life-Cycle Cost Analysis Primer (FHWA, 2002) distinguishes between LCCA and BCA. The latter compares benefits as well as costs in selecting the superior alternatives. It can be applied to alternatives with different level of services because it considers all types of costs and benefits. However, LCCA can be used to compare only alternatives providing the same LOS, and the most cost-effective is selected. The proposed EB-MOO methodology deviates from both these common approaches for selecting alternatives. In most cases, other non-economic preferences (objectives), targets, and restrictions (constraints) contribute in the decision making. Generated LC alternatives won't be compared in this module, only LCCs and LCC benefits are determined and discounted to the current year (present time). The recommendation of optimal or near-optimal alternatives, accounting for all these conflicting objectives, takes place in the optimization modules.

\subsubsection{Discounting and Present Value}

Comparing costs incurred at different times in a LC profile is achieved by a net present value analysis. All future cash flows are discounted to present values. This conversion provides a common ground for comparison. No standard value for discount rate exists. The rate is usually specified based on experience or current practice. It is important that the same discount rate is used when comparing LCCs or benefits. Only the real interest rate without inflation is considered in the module LCC model. Including inflated unit costs at every point in the LC profile complicate the analysis. Inflation is less predictable and does not affect results unless costs are modeled to inflate at different rates (Sobanjo \& Thompson, 2016b). 
After omitting inflation, the discount factor is determined by Equation (5.1) as follows:

$d r=\frac{1}{1+r i}$

where

$d r \quad=$ discount rate; and

$r i=\begin{aligned} & \text { real interest rate, usually it's specified by the agency as part of the asset management } \\ & \text { policy. }\end{aligned}$ The present value of a future cost/benefit is calculated by Equation (5.2) as follows:

$P V=\frac{F V_{N}}{(1+d r)^{N}}$

where

$P V \quad=$ present value of a one-time future cost/benefit;

$F V_{N}=$ future value of the cost/benefit estimated at time $N$;

$d r \quad=$ discount rate, determined by Equation (5.1); and

$N=$ time interval between the base year (usually the current year or the first year of the analysis period) and the analysis year.

\subsubsection{Life-Cycle Cost Model}

At the bridge level, most BMSs estimate the LCCs associated with different intervention strategies for a given bridge. Thus, the most feasible alternative is recommended-suggesting an intervention scope at the appropriate timing with the expected performance and condition. The LCC model integrated in this module assumes that a preservation policy is followed throughout the bridge LC, and a major improvement work on the bridge fully restores all its elements $(\mathrm{CS} 1=100 \%)$. Preservation actions account for a large portion of the element LC profile. They are considered the most cost-effective actions for the long-term, extending the element service life by 
slowing down deterioration. Accordingly, as defined in Chapter 4, preservation actions always follow a major improvement work on the bridge for the remainder of the analysis period. For each element, a LC profile is constructed and present values are determined for relevant agency and user costs, residual values, and benefits. Figures 5.6(a), (b), and (c) illustrate three examples of MRR, FCI, and REP LC alternative profiles. Diagrams include the typical types of incurred LCCs and residual values. LCC model is expressed by Equations (5.3) and (5.4) as follows:

$$
\begin{aligned}
& P L C C_{e l m_{i}}^{v_{p j r}}=P A C_{e l m_{i}}^{v_{p j r}}-R V_{e l m_{i}}^{v_{p j r}} \\
& L C C_{b r g_{k}}^{C_{p j k}}=U S C_{b r g_{k}}^{p j}+M I C_{b r g_{k}}^{p_{j}}+\sum_{i \in E_{k}} P L C C_{e l m_{i}}^{v_{p j r}}
\end{aligned}
$$

where

$v_{p j r}=$ improvement type $p$ LC alternative $r$ with an initial intervention in program year $j$;

$\operatorname{PLCC}_{e l m_{i}}^{v_{p j r}}=$ total preservation LCCs incurred in element $i \mathrm{LC}$ alternative $v_{p j r}$ profile;

$P A C_{e l m_{i}}^{v_{p j r}}=\begin{aligned} & \text { sum of all discounted preservation action costs incurred in element } i \mathrm{LC} \text { alternative } \\ & v_{p j r} \text { profile; }\end{aligned}$ $R V_{e l m_{i}}^{v_{p j r}}=\begin{aligned} & \text { discounted residual value applied at the end of element } i \mathrm{LC} \text { alternative } v_{p j r} \text { profile, } \\ & \text { refer to Equation (5.5); }\end{aligned}$ $C_{p j k}=\begin{aligned} & \text { bridge } k \text { combination of improvement type } p \text { LC alternatives with an initial } \\ & \text { intervention in program year } j ;\end{aligned}$ $L_{C C} C_{\text {brg }_{k}}^{C_{p j k}}=$ total LCC of combination $C_{p j k}$ for bridge $k$;

sum of all discounted user costs incurred due to bridge $k$ (user cost for an analysis $U S C_{\text {brg }_{k}}^{p j}=\begin{aligned} & \text { year is determined by Equation (4.11) in Chapter 4; for FCI and REP improvement } \\ & \text { types, only user costs between beginning of program year } 1 \text { and end of program year }\end{aligned}$ $j$ are considered, otherwise from the entire analysis period); 
discounted major cost of improvement type $p$ performed on bridge $k$ in program year $M I C_{\text {brg }_{k}}^{p j}=\begin{aligned} & j \text { (for FCI and REP improvement types, the cost is determined by Equations (4.4) } \\ & \text { and (4.8) in Chapter 4, respectively; for MRR improvement type, the cost is always }\end{aligned}$ equals to zero); and

$E_{k} \quad=$ set of bridge $k$ elements.
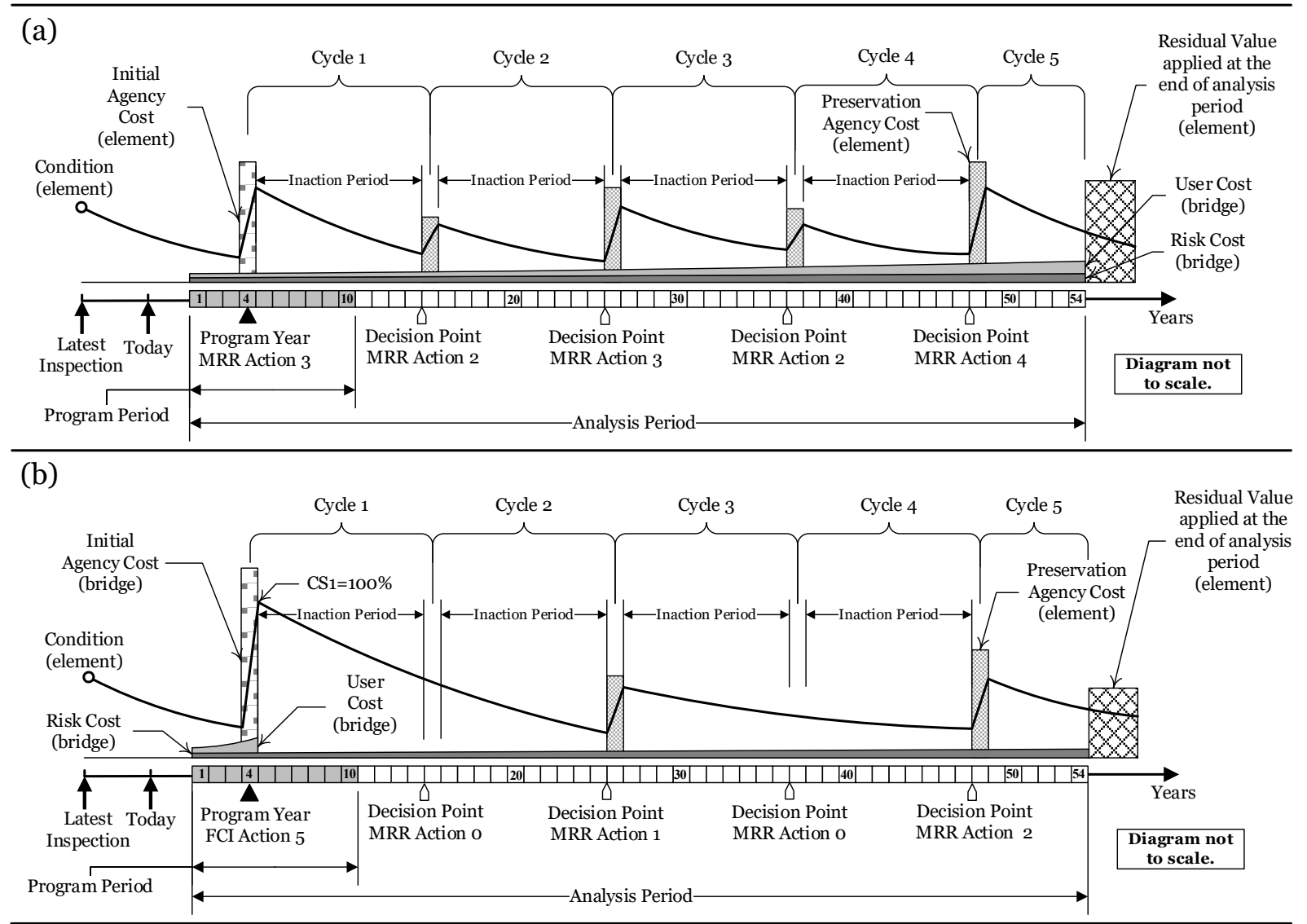

(c)

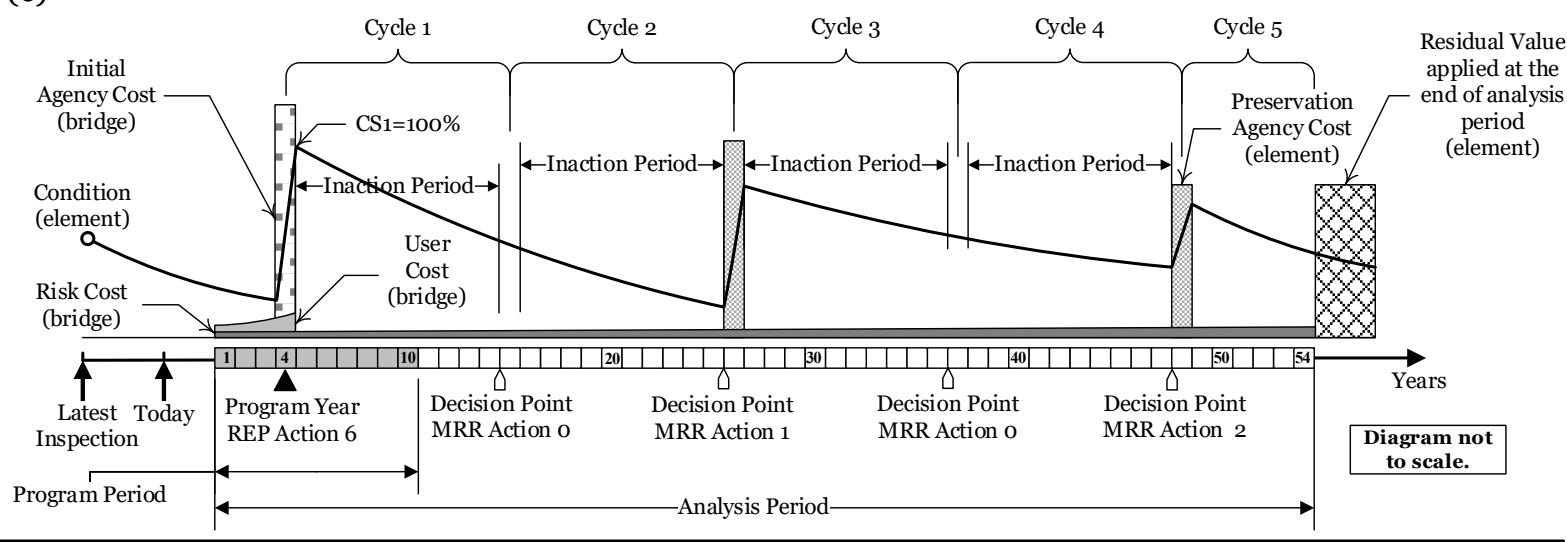

Figure 5.6 Example of LC profile showing different LCCs and residual values for (a) MRR LC Alternative 2215; (b) FCI LC Alternative 28; (c) REP LC Alternative 54 


\subsubsection{Agency costs}

Agency costs represent the combination of all costs incurred by the agency over time. These costs are incurred to implement preservation and improvement actions. Agency costs are classified into three cost categories: element preservation, bridge major improvement, and initial agency costs.

\section{Element Preservation Action Costs}

Preservation cost (discussed in Chapter 4) is an element-level cost (symbolized by PACelm $_{\text {elm }}$ in the LCC model) of implementing a preservation action. The cost is made up of direct and indirect costs and applied at the end of the implementation year. The direct cost is the sum of all costs involved to preserve, repair, or replace the element. The other component is the sum of all costs indirectly incurred such as costs of maintaining traffic and engineering design.

\section{Bridge Major Improvement Costs}

Two types of bridge-level major improvement costs (symbolized by $\mathrm{MIC}_{\text {brg }}$ in the $\mathrm{LCC}$ model) are considered as major improvement costs: functional improvement and replacement costs, defined in Chapter 4 by Equations (4.4) and (4.8), respectively. Functional improvement cost includes widening cost (required to eliminate the bridge width deficiency), raising cost (required to eliminate the bridge vertical clearance deficiency), and/or strengthening cost (required to eliminate the bridge load capacity deficiency). Replacement cost is the cost incurred to relieve the bridge from all its restrictions, provide required LOS and design standards, and eliminate all incurred user costs. These costs depend on different factors such as the bridge size, type and material, location, feature being crossed, and other attributes. 


\section{Initial Agency costs}

The initial intervention cost is referred to as initial agency cost (or IAC) in a LC profile. The cost is always incurred in a program year. The element- and bridge-level IACs are defined as follows:

- Element-level IAC (denoted by $\mathrm{IAC}_{\text {elm}}$ ) is the cost of implementing the first preservation action on an element. The cost appears only in MRR LC profiles-FCI or REP LC profiles do not assume any perseveration actions in the program period.

- Bridge-level IAC (denoted by $\mathrm{IAC}_{\mathrm{brg}}$ ) is an initial cost incurred for the entire bridge. For MRR LC profiles, IAC $\mathrm{brg}_{\mathrm{brg}}$ is the sum of all deficient element IACs; whereas for FCI or REP LC profiles, $\mathrm{IAC}_{\mathrm{brg}}$ is the combination of the bridge major improvement costs (i.e., $\mathrm{MIC}_{\mathrm{brg}}$ ) and all its deficient element IACs.

\subsubsection{User costs}

Chapter 4 discusses the different types of user costs being modeled. Equation (4.11) in Chapter 4 defines the total bridge user cost for an analysis year. User cost (USC $\mathrm{brg}_{\mathrm{rg}}$ in the LCC model) is the sum of all costs incurred by users over the bridge LC (analysis period). It is important to use the same type of user costs and most significant in the LCC calculation to ensure consistency and no bias. The LCC model computes three types of user costs: accident risk, vertical clearance, and load capacity costs, defined in Chapter 4 by Equations (4.15), (4.17), and (4.20), respectively. A user cost due to a functional deficiency is calculated for each analysis year, including action implementation years. All user costs are discounted to present values and added to the bridge LCC (denoted by $\mathrm{LCC}_{\mathrm{brg}}$ in the LCC model). To provide a uniform basis for assessing alternatives, no user costs are recognized prior to year 1. Element deterioration and traffic growth are assumed to contribute to the increase of user costs over time. No further user costs are considered beyond the end of the analysis period. 


\subsubsection{Residual Value}

LC alternatives produce different RSLs (defined in Chapter 4) or none for the same analysis period. Residual value (RV) is the economic value of RSL applied at the end of an analysis period. For a fair comparison, each element RV for each LC alternative is estimated, discounted, and subtracted from the $\mathrm{LCC}_{\mathrm{brg}}$. A few methods exist to estimate RVs. The Life-Cycle Cost Analysis in Pavement Design-Interim Technical Bulletin (Walls \& Smith, 1998) considers the RV as a product between the asset cost and the percentage design life remaining at the end of the analysis period. A different approach based on bridge condition ratings for estimating RVs is presented in the NHCRP Report 483 (Hugh, 2003). The RV of an element (denoted by $\mathrm{RV}_{\text {elm }}$ in the LCC model) is estimated using the proportional approach.

Figure 5.7 assists in visualizing the RV modeling approach. A straight line is constructed joining the element replacement cost and the end-of-life threshold (dashed line) over its projected ESL (defined in Chapter 4). The RSL beyond the analysis period is determined. The RV is then identified as the proportional side to the element replacement cost side. No other costs are considered beyond the end of the analysis period. It's assumed that operation continues beyond the analysis period in the indefinite future, and no salvage values are realized. The RV modeling approach is expressed by Equation (5.5) as follows:

$R V_{e l m_{i}}^{v_{p j r}}=\frac{E S L_{e l m_{i}}}{R S L_{e l m_{i}}^{v_{p r r}}} \times R U C_{e l m_{i}} \times Q_{e l m_{i}}$

where

$v_{p j r} \quad=$ improvement type $p$ LC alternative $r$ with an initial intervention in program year $j$;

$R V_{e l m_{i}}^{v_{p j r}}=$ discounted residual value applied at the end of element $i \mathrm{LC}$ alternative $v_{p j r}$ profile; 


$$
\begin{aligned}
& E S L_{e l m_{i}}=\text { ESL of element } i \\
& R S L_{e l m_{i}}^{v_{p j r}}=\text { RSL of element } i \text { as a result of LC alternative } v_{p j r} ; \\
& R U C_{e l m_{i}}=\text { replacement unit cost of element } i \text {, and } \\
& Q_{e l m_{i}}=\text { quantity of element } i .
\end{aligned}
$$




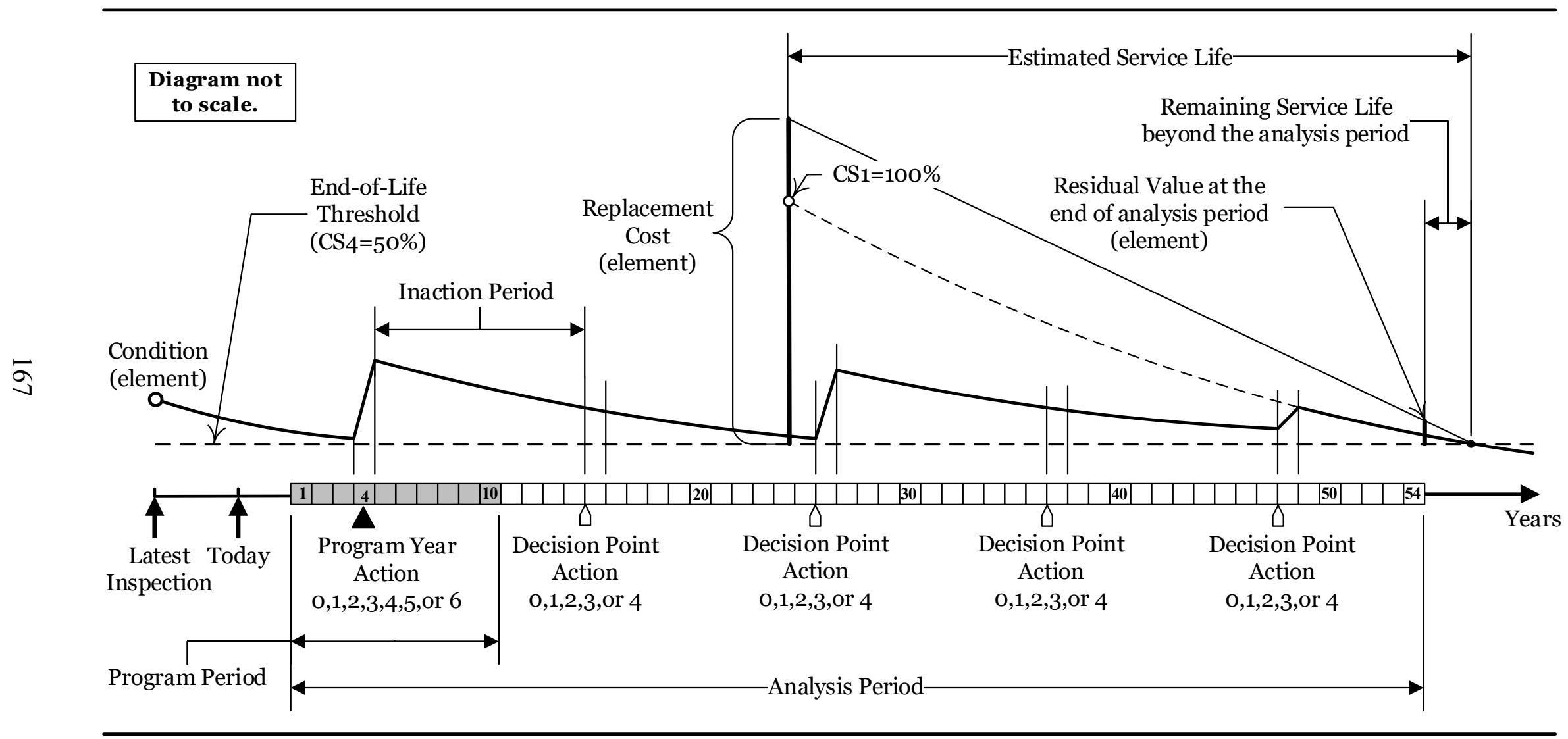

Figure 5.7 Residual value determination 


\subsubsection{Defining Benefit}

Benefit should be carefully defined to capture the effect of an alternative on strategic objectives and performance targets. The NCHRP Report 590 (Patidar et al., 2007) defines benefit as the savings in LCC of "doing something" relative to the "do-nothing" alternative, or the increase in utility of "doing something" rather than "doing nothing." Benefits can be either positive or negative depending on whether the discounted "doing something" LCC exceeds the discounted “do-nothing” LCC or not. Positive benefit is generally desirable. The benefit is calculated for each element LC alternative and transferred to the ELO module. The total benefit of a set of element LC alternatives for a bridge constitutes one of the objectives to be optimized.

The economic benefit of an element LC alternative is calculated by subtracting the alternative LCC from its base LCC. Both costs are discounted to present values. For each bridge, a base user cost covering the entire analysis period is computed and discounted to present value. The DN and RO LC profiles are constructed for each element (covered in Chapter 4). These two base LC profiles define the "baseline" alternative against which the economic benefits (LCC benefits) of improvement alternatives are compared. The base LCC (sum of all user costs plus any element replacement costs) may be zero or negative if the element RSL extends beyond the analysis period. Figures 5.8(a) and (b) illustrate two examples of DN, and RO LC profiles. The diagrams include the typical types of incurred LCCs and RVs. 

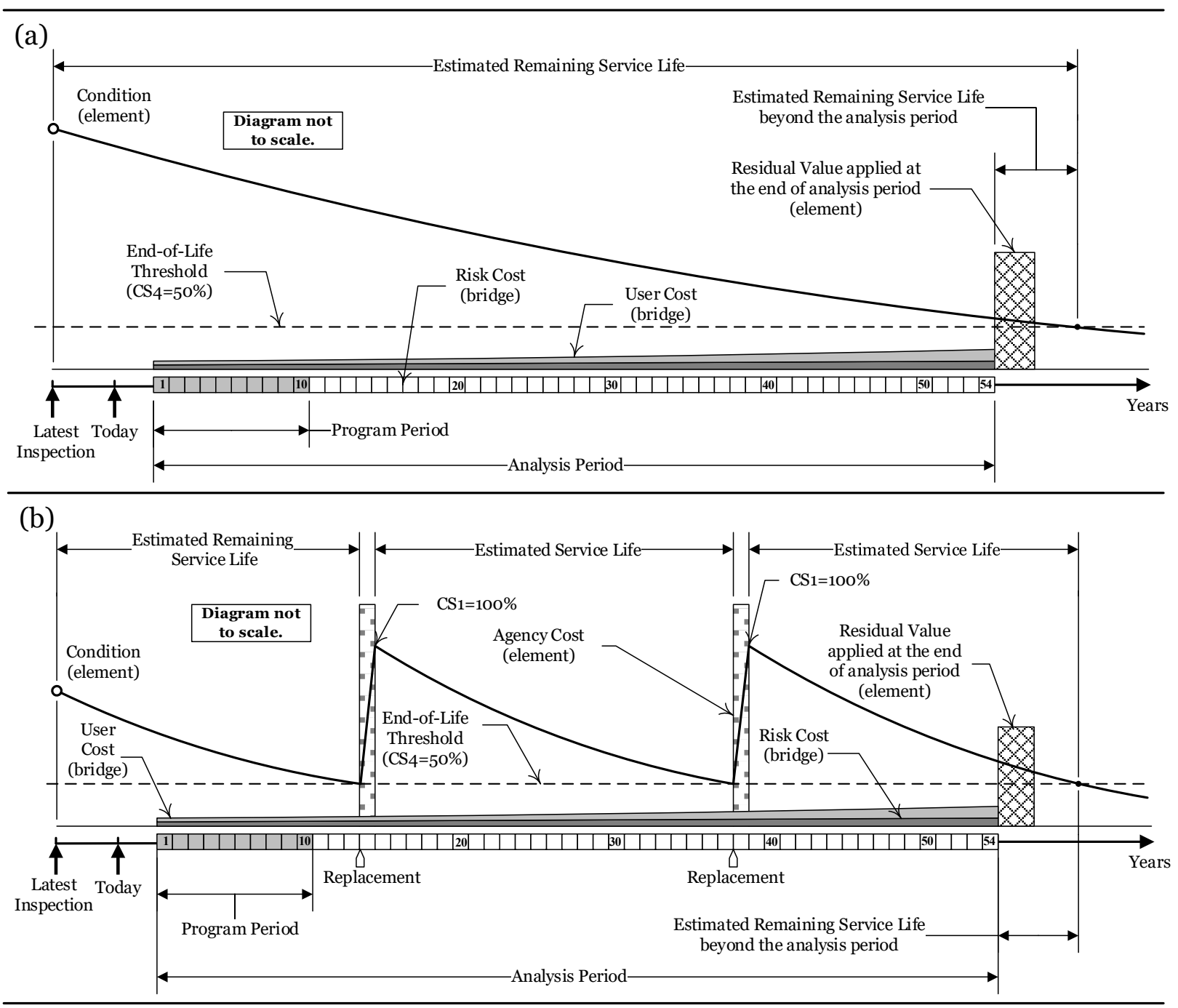

Figure 5.8 Example of baseline LC alternative profile of (a) DN; (b) RO

As discussed previously and illustrated in Figures 5.6 (a), (b), and (c), three types of AE LC profiles (i.e., MRR, FCI, and REP LC profiles) are constructed for each element. User costs cannot be eliminated by preservation actions; therefore, they have no effect on "preservation" LCC benefits. Element-level LCC benefits (symbolized by BNT $_{\text {elm }}$ in the LCC model) are calculated separately (element LCC of minus its base LCC). The sum of all these element-level LCC benefits constitutes the bridge-level LCC benefit (symbolized by BNT $\mathrm{brg}_{\text {) }}$. 
The following Equations (5.6) through (5.9) are developed around these definitions to compute the base LCCs and LCC benefits.

$$
\begin{aligned}
& L C C_{e l m_{i}}^{b}=A C_{e l m_{i}}^{b}-R V_{e l m_{i}}^{b} \\
& L C C_{b r g_{k}}^{b}=U S C_{b r g_{k}}^{b}+\sum_{i \in E_{k}} L C C_{e l m_{i}}^{b} \\
& B N T_{e l m_{i}}^{v_{p j r}}=L C C_{e l m_{i}}^{b}-P L C C_{e l m_{i}}^{v_{p j r}} \\
& B N T_{b^{2} g_{k}}^{C_{p j k}}=L C C_{b r g_{k}}^{b}-L C C_{b r g_{k}}^{C_{p j k}}
\end{aligned}
$$

where

$v_{p j r} \quad=$ improvement type $p$ LC alternative $r$ with an initial intervention in program year $j$;

$L C C_{\text {elm }}^{b}=$ total base $\mathrm{LCC}$ for element $i$

$A C_{e l m_{i}}^{b} \quad=$ sum of all discounted agency costs incurred in element $i$ base LC profile;

$R V_{e l m_{i}}^{b} \quad=$ discounted residual value applied at the end of element $i$ base LC profile;

$L_{C C} C_{b r g_{k}}^{b}=$ total base LCC for bridge $k$;

$U S C_{b r g_{k}}^{b}=\begin{aligned} & \text { sum of all discounted base user costs incurred over the entire analysis period for } \\ & \text { bridge } k ;\end{aligned}$

$B N T_{e l m_{i}}^{v_{p j r}}=$ LCC benefit of LC alternative $v_{p j r}$ for element $i$

$P L C C_{e l m_{i}}^{v_{p j r}}=\begin{aligned} & \text { discounted preservation LCCs incurred in element } i \mathrm{LC} \text { alternative } v_{p j r} \text { profile, refer } \\ & \text { to Equation (5.3); }\end{aligned}$ $C_{p j k}=\begin{aligned} & \text { combination of improvement type } p \mathrm{LC} \text { alternatives with an initial intervention in } \\ & \text { program year } j \text { for bridge } k ;\end{aligned}$ $B N T_{b r g_{k}}^{C_{p j k}}=$ total LCC benefit of combination $C_{p j k}$ for bridge $k$; and $L C C_{b r g_{k}}^{C_{p j k}}=$ total LCC for bridge $k$ combination $C_{p j k}$, refer to Equation (5.4). 


\subsubsection{Comparing LC Alternatives}

Three common economic decision analyses are used to compare alternatives in BMSs: LCCA, BCA, and IBCA. The FHWA's Life-Cycle Cost Analysis Primer (FHWA, 2002) distinguishes between BCA and LCCA. Not all types of costs need to be explored in a LCCA, only costs that significantly differ among alternatives. LCCA identifies the most cost-effective alternative among alternatives providing the same LOS. BCA compares benefits as well as costs to identify the best alternative; it can be applied to alternatives with different LOSs. Alternatives are prioritized by sorting their ratios of benefit to cost—ratios equal to or greater than 1 are considered acceptable. When the budget is constrained, alternatives with the highest benefit-cost ratios are selected following a priority. BCA is desirable for comparing independent alternatives. However, ranking alternatives based on their benefit-cost ratios usually yields misleading results. The IBCA analysis is considered a superior to BCA (Farid et al., 1988).

IBCA is based on the well-known economic law of Diminishing Marginal Return-incremental increase of investment produces smaller benefits. IBC ratio is defined as "the ratio of the extra benefits of advancing from one improvement level to the next, divided by the corresponding extra cost" (O' Connor, 1989). The alternative IBC ratio is determined by calculating the difference in benefit divided by the difference in cost of the alternative compared to the next less costly alternative. The ratio is calculated for each alternative, and the one that produces the maximum net of benefit (i.e., the highest IBC ratio) is selected.

Robert (2017) describes how IBCA is used in NBIAS to provide a near-optimal solution for a budget-constrained problem rather than exact optimization methods - due to their limitations for large-sized problems. NBIAS relies on an IBC heuristic called "MINBEN," described in (Robert et al., 2009), for sorting alternatives. The heuristic provides a near-optimal solution within 
acceptable running time. Alternatives that do not follow the Law of Diminishing Marginal Return are eliminated from consideration. Under this law, more expensive alternatives produce smaller IBC ratios - an incremental cost produces a less proportionate increase in benefit. Accordingly, the alternative that satisfies the available budget and produces the highest benefit per additional cost is selected (Patidar et al., 2007).

BrM uses a multi-criteria decision analysis based on the utility theory. Each criterion for a bridge is associated with a utility value determined through weighting, scaling and amalgamation techniques (discussed in Chapter 2). Four criteria are involved in the analysis: condition, LCC, mobility, and risk. BrM defines benefit as the change to a bridge as a result of work. BrM generates bridge and network actions based on user-specified preservation and network policies, respectively. The benefit of an action is calculated from the incremental increase in the overall utility of the bridge (referred to as $\Delta$ Utility). A $\Delta$ Utility ratio is determined for each action. The system selects actions with the highest $\Delta$ Utility ratios. A cutoff is reached when the performance and budget constraints are met (Johnson \& Boyle, 2017).

Any of these discussed analyses can be utilized to compare the module produced LC alternatives. A given bridge with just few elements can be associated with an enormous number of possible combinations of LC alternatives. For example, taking a bridge with only 9 elements, the 3125 MRR LC alternatives per program year for each element generates $2.84 \mathrm{E} 31\left(3125^{9}\right)$ of possible combinations of MRR LC alternatives per program year. At the element level, superior LC alternatives per program year and per improvement type can be identified for each element using either one of the discussed analyses. These superior alternatives constitute feasible combinations of $\mathrm{LC}$ alternatives for the entire program period and the three improvement types. 
The bridge LCCs and LCC benefits, Equations (5.4) and (5.9), are determined for each of these feasible combinations to be compared through an economic analysis. Again, either BCA or IBCA can be applied to find the ultimate combination. In most cases, other non-economic preferences (objectives), targets and restrictions (constraints) contribute in the decision making. Optimization methodologies are effective in optimizing multiple competing objectives (such as minimizing the LCC, and maximizing the benefit and performance) subject to constraints (e.g., limited available budget and minimum level of acceptable performance). As explained in Chapter 2, optimization methodologies guarantee a diverse set of optimal or near-optimal solutions — constituting a frontier of trade-offs (i.e., Pareto frontier). The optimization problem size can be reduced by eliminating economically unattractive LC alternatives (generally, alternatives with negative LCC benefits or other specified criteria) associated with the deficient elements. The remainder alternatives represent the feasible $\mathrm{LC}$ alternatives to be transferred to the optimization.

The proposed EB-MOO methodology deviates from these common approaches (i.e., BCA and IBCA) used by most BMSs for selecting alternatives. LC alternatives won't be compared at this stage, only the LCCs and LCC benefits are determined and discounted to present time. The module LC alternative results are transferred to the optimization modules. The results will be processed at the ELO to obtain optimal or near-optimal combinations of LC alternatives per program year for each analyzed bridge. 


\subsection{Example of Module Results}

The module was implemented through a sample set of bridges to prove effectiveness and demonstrate potential benefits. The MATLAB-based tool prototype was used for the implementation. Bridges were selected based on common features, attributes, and data completeness. For each sample bridge, the tool successfully identified potential deficient elements, predicted performance, generated LC alternatives, constructed LC profiles, and determined all incurred LCCs and LCC benefits. Table 5.4 presents the NBI condition ratings of one of the sample bridges (i.e., Bridge 11; Table 3.2 in Chapter 3 lists all sample bridges used for the different illustrative examples). Table 5.5 shows the latest condition states of the 12 elements of this sample bridge. Three of the 12 elements (i.e., Elements 215, 234 and 331) were identified as non-deficient by the element deficiency screening process.

Table 5.4 NBI condition ratings of Bridge 11

\begin{tabular}{|c|c|c|c|c|c|c|c|c|c|c|c|c|c|c|c|c|}
\hline \multicolumn{17}{|c|}{ Bridge 11} \\
\hline 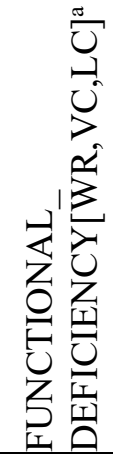 & 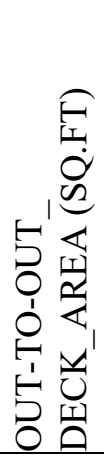 & 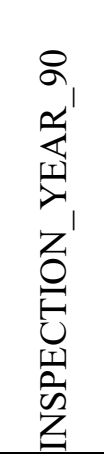 & 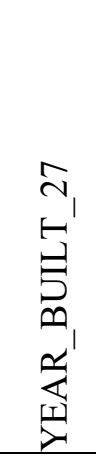 & 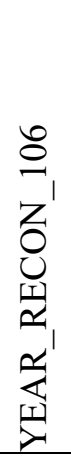 & 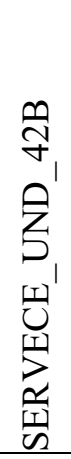 & 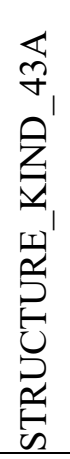 & 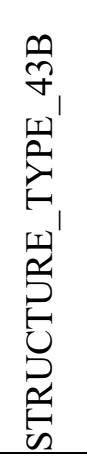 & 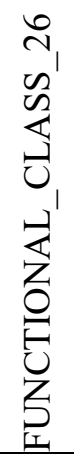 & 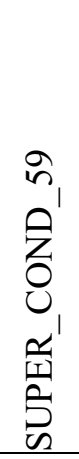 & 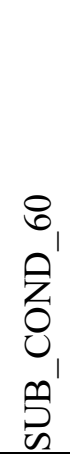 & 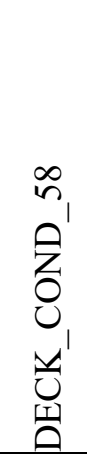 & 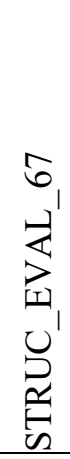 & 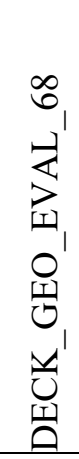 & 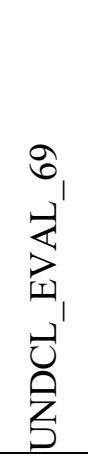 & 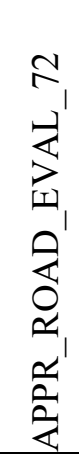 & 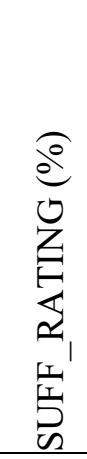 \\
\hline 0 & 1211 & 2017 & 1957 & 0 & 5 & 3 & 2 & 2 & 7 & 7 & 6 & 6 & 3 & 78 & 8 & 74.3 \\
\hline
\end{tabular}

Note. $\mathrm{WR}=$ width required; $\mathrm{VC}=$ vertical clearance; $\mathrm{LC}=$ load capacity.

${ }^{a}$ The value of 1 represents to the existence of the functional deficiency, otherwise the value of 0 . 
Table 5.5 Condition states of the 12 elements of Bridge 11

\begin{tabular}{lcccccccccccc}
\hline & \multicolumn{11}{c}{ Elements of Bridge 11 } \\
\cline { 2 - 13 } & 12 & 107 & 205 & 215 & 220 & 226 & 234 & 301 & 311 & 313 & 330 & 331 \\
\hline CS1 (\%) & 29.4 & 99.3 & 100 & 100 & 58.78 & 0 & 100 & 93.1 & 32 & 40 & 99.8 & 95.9 \\
CS2 (\%) & 70.4 & 0.53 & 0 & 0 & 33.11 & 95.8 & 0 & 6.86 & 36 & 24 & 0 & 3.93 \\
CS3 (\%) & 0.07 & 0.11 & 0 & 0 & 8.11 & 4.17 & 0 & 0 & 32 & 36 & 0.13 & 0.13 \\
CS4 (\%) & 0 & 0 & 0 & 0 & 0 & 0 & 0 & 0 & 0 & 0 & 0 & 0 \\
Qt (sq.ft) & 13,0 & 1,91 & 8 & 79 & 148 & 24 & 112 & 204 & 25 & 25 & 764 & 764 \\
W $_{\text {elm (\%) }}$ & 25 & 49 & 40 & 13 & 10 & 17 & 13 & 12 & 12 & 12 & 16 & 14 \\
ESL (years) & 129 & 77 & 200 & 75 & 123 & 141 & 408 & 24 & 58 & 58 & 28 & 151 \\
RSL (years) & 66 & 20 & 143 & 18 & 57 & 63 & 351 & 0 & 0 & 0 & 0 & 91 \\
RU (\$) & 54 & 2,06 & 38,8 & 1,5 & 194,0 & 38,8 & 1,1 & 72 & 9,0 & 9,0 & 296 & 212 \\
\hline
\end{tabular}

${ }^{\mathrm{a}} \mathrm{RSL}$ is estimated from program year 1 (2020).

Table 5.6 includes the module LC alternative results obtained using the tool prototype for this sample bridge. The analysis period is set for 54 years, including a 10 -year program period. The discount rate is $4 \%$ over 56 years (analysis period plus the period from current year, 2018, to program year 1, 2020). The table section under "Elements" header includes the LCCs incurred by the two base LC alternatives (i.e., DN and RO LC Alternatives) and other improvement type LC alternatives sharing the same program year 4 . MRR, FCI and REP LC alternatives are assigned to each element. FCI LC Alternative 1 and REP LC Alternative 1 simulate the scenario of performing a major bridge intervention with no subsequent preservation actions. The section under "Bridge" header includes the overall bridge LCCs for each improvement type combination-combining all element LC alternatives of the same improvement type. Each of these combinations represents a unique bridge alternative for program year 4 . This unique combination is one of the many possible combinations for a bridge with 9 deficient elements. As explained earlier, the number of possible combinations is overwhelming; the best combination can be efficiently identified using either BCA, IBCA, or optimization heuristics. 
Table 5.6 Module LC alternative results produced using the tool prototype for Bridge 11

\begin{tabular}{|c|c|c|c|c|c|c|c|c|c|c|c|c|c|c|c|}
\hline & \multicolumn{13}{|c|}{ Elements } & \multirow{5}{*}{\multicolumn{2}{|c|}{ Bridge }} \\
\hline & Ek & 12 & 107 & 205 & 215 & 220 & 226 & 234 & 301 & 311 & 313 & 330 & 331 & & \\
\hline & $\overline{\mathrm{Qelm}_{\mathrm{el}}}$ & 13030 (sq.ft) & $1903(\mathrm{ft})$ & 8 (each) & $79(\mathrm{ft})$ & $148(\mathrm{ft})$ & 24 (each) & $112(\mathrm{ft})$ & 204 (ft) & 25 (each) & 25 (each) & $764(\mathrm{ft})$ & 764 (ft) & & \\
\hline & RUC (\$) & 54 & 2,062 & 38,810 & 1,591 & 194,049 & 38,810 & 1,186 & 72 & 9,009 & 9,009 & 296 & 212 & & \\
\hline & $\mathrm{ESL}_{\text {elm }}$ (years) & 129 & 77 & 200 & 75 & 123 & 141 & 408 & 24 & 58 & 58 & 28 & 151 & & \\
\hline \multirow{4}{*}{ 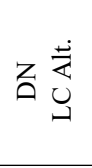 } & $\mathrm{RSL}_{\text {elm }}$ (years) & 66 & 20 & 143 & 18 & 57 & 63 & 351 & 0 & 0 & 0 & 0 & 91 & $\mathrm{USC}_{\mathrm{brg}}(\$)$ & 753,711 \\
\hline & $\mathrm{RV}_{\mathrm{elm}}(\$)$ & 39,924 & 113,334 & 24,687 & 3,355 & $1,480,051$ & 46,282 & 12,703 & 0 & 0 & 0 & 0 & 10,835 & $\mathrm{LCC}_{\mathrm{brg}}(\$)$ & $-977,460$ \\
\hline & $\mathrm{AC}_{\mathrm{elm}}(\$)$ & 0 & 0 & 0 & 0 & 0 & 0 & 0 & 0 & 0 & 0 & 0 & 0 & & \\
\hline & $\mathrm{LCC}_{\mathrm{elm}}(\$)$ & $-39,924$ & $-113,334$ & $-24,687$ & $-3,355$ & $-1,480,051$ & $-46,282$ & $-12,703$ & 0 & 0 & 0 & 0 & $-10,835$ & & \\
\hline \multirow{4}{*}{ ○这 } & $\mathrm{RSL}_{\text {elm }}$ (years) & 66 & 20 & 143 & 18 & 57 & 63 & 351 & 16 & 45 & 44 & 0 & 91 & $\mathrm{USC}_{\mathrm{brg}}(\$)$ & 753,711 \\
\hline & $\mathrm{RV}_{\mathrm{elm}}(\$)$ & 39,924 & 113,334 & 24,687 & 3,355 & $1,480,051$ & 46,282 & 12,703 & 1,082 & 19,434 & 19,002 & 0 & 10,835 & $\mathrm{LCC}_{\mathrm{brg}}(\$)$ & $-848,273$ \\
\hline & $\mathrm{AC}_{\text {elm }}(\$)$ & 0 & 0 & 0 & 0 & 0 & 0 & 0 & 8,146 & 41,707 & 43,375 & 75,478 & 0 & & \\
\hline & $\mathrm{LCC}_{\mathrm{elm}}(\$)$ & $-39,924$ & $-113,334$ & $-24,687$ & $-3,355$ & $-1,480,051$ & $-46,282$ & $-12,703$ & 7,063 & 22,273 & 24,373 & 75,478 & $-10,835$ & & \\
\hline \multirow{5}{*}{ 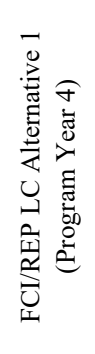 } & Alt. Reference & 1 & 1 & 1 & 1 & 1 & 1 & 1 & 1 & 1 & 1 & 1 & 1 & $\mathrm{USC}_{\mathrm{brg}}(\$)$ & 77,673 \\
\hline & $\mathrm{RSL}_{\text {elm }}$ (years) & 79 & 27 & 162 & 69 & 73 & 91 & 393 & 0 & 8 & 8 & 0 & 126 & $\begin{array}{l}\mathrm{MIC}_{\text {brg }}(\$) \\
(\mathrm{FCI} / \mathrm{REP})\end{array}$ & $\begin{array}{l}2,089,162 \\
3,769,103\end{array}$ \\
\hline & $\mathrm{RV}_{\mathrm{elm}}(\$)$ & 47,787 & 153,002 & 27,967 & 12,862 & $1,895,504$ & 66,851 & 14,223 & 0 & 3,455 & 3,455 & 0 & 15,002 & $\begin{array}{l}\mathrm{LCC}_{\mathrm{brg}}(\$) \\
(\mathrm{FCI} / \mathrm{REP})\end{array}$ & $\begin{array}{r}-73,272 \\
1,606,668\end{array}$ \\
\hline & $\mathrm{AC}_{\mathrm{elm}}(\$)$ & 0 & 0 & 0 & 0 & 0 & 0 & 0 & 0 & 0 & 0 & 0 & 0 & $\begin{array}{l}\mathrm{BNT}_{\text {brg }}(\mathrm{DN})(\$) \\
(\mathrm{FCI} / \mathrm{REP})\end{array}$ & $\begin{array}{r}-904,188 \\
-2,584,128\end{array}$ \\
\hline & $\mathrm{LCC}_{\mathrm{elm}}(\$)$ & $-47,787$ & $-153,002$ & $-27,967$ & $-12,862$ & $-1,895,504$ & $-66,851$ & $-14,223$ & 0 & $-3,455$ & $-3,455$ & 0 & $-15,002$ & $\begin{array}{l}\mathrm{BNT}_{\text {brg }}(\mathrm{RO})(\$) \\
(\mathrm{FCI} / \mathrm{REP})\end{array}$ & $\begin{array}{r}-775,000 \\
-2,454,941 \\
\end{array}$ \\
\hline
\end{tabular}


Table 5.6 Module LC alternative results produced using the tool prototype for Bridge 11 (continued)

\begin{tabular}{|c|c|c|c|c|c|c|c|c|c|c|c|c|c|c|c|}
\hline & \multicolumn{13}{|c|}{ Elements } & \multirow{2}{*}{\multicolumn{2}{|c|}{ Bridge }} \\
\hline & $\mathrm{Ek}$ & 12 & 107 & 205 & 215 & 220 & 226 & 234 & 301 & 311 & 313 & 330 & 331 & & \\
\hline \multirow{9}{*}{ 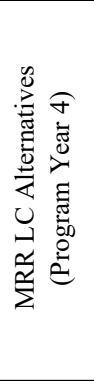 } & Alt. Reference & 392 & 2172 & 3071 & 1 & 1132 & 895 & 1 & 2001 & 481 & 3120 & 471 & 1 & $\mathrm{USC}_{\mathrm{brg}}(\$)$ & 753,711 \\
\hline & Action Path & $0-3-0-3-1$ & $3-2-1-4-1$ & $4-4-2-4-1$ & $0-0-0-0-0$ & $1-4-0-1-1$ & $1-2-0-3-4$ & $0-0-0-0-0$ & $3-1-0-0-0$ & $0-3-4-1-0$ & $4-4-4-3-4$ & $0-3-3-4-0$ & $0-0-0-0-0$ & $\mathrm{MIC}_{\mathrm{brg}}(\$)$ & 0 \\
\hline & $\mathrm{RSL}_{\mathrm{elm}}$ (years) & 117 & 69 & 185 & 18 & 115 & 103 & 351 & 0 & 36 & 0 & 0 & 91 & $\mathrm{IAC}_{\mathrm{brg}}(\$)$ & 501,406 \\
\hline & $\mathrm{RV}_{\mathrm{elm}}(\$)$ & 70,774 & 391,004 & 31,938 & 3,355 & $2,986,068$ & 75,667 & 12,703 & 0 & 15,547 & 0 & 0 & 10,835 & $\mathrm{LCC}_{\mathrm{brg}}(\$)$ & $-866,476$ \\
\hline & $\mathrm{PAC}_{\mathrm{elm}}(\$)$ & 187,965 & $1,428,367$ & 336 & 0 & 301,668 & 44,623 & 0 & 6,394 & 8,352 & 0 & 0 & 0 & $\mathrm{BNT}_{\text {brg }}(\mathrm{DN})(\$)$ & $-110,984$ \\
\hline & $\operatorname{IAC}_{\text {elm }}(\$)$ & 0 & 243,917 & 0 & 0 & 212,905 & 44,584 & 0 & 0 & 0 & 0 & 0 & 0 & $\mathrm{BNT}_{\mathrm{brg}}(\mathrm{RO})(\$)$ & 18,203 \\
\hline & $\mathrm{PLCC}_{\text {elm }}(\$)$ & 117,191 & $1,037,363$ & $-31,602$ & $-3,355$ & $-2,684,400$ & $-31,044$ & $-12,703$ & 6,394 & $-7,195$ & 0 & 0 & $-10,835$ & & \\
\hline & $\mathrm{BNT}_{\text {elm }}(\mathrm{DN})(\$)$ & $-157,115$ & $-1,150,697$ & 6,915 & 0 & $1,204,349$ & $-15,238$ & 0 & $-6,394$ & 7,195 & 0 & 0 & 0 & & \\
\hline & $\mathrm{BNT}_{\mathrm{elm}}(\mathrm{RO})(\$)$ & $-157,115$ & $-1,150,697$ & 6,915 & 0 & $1,204,349$ & $-15,238$ & 0 & 669 & 29,468 & 24,373 & 75,478 & 0 & & \\
\hline \multirow{8}{*}{ 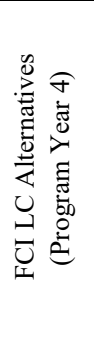 } & Alt. Reference & 68 & 365 & 509 & 1 & 146 & 279 & 1 & 622 & 601 & 381 & 444 & 1 & $\mathrm{USC}_{\mathrm{brg}}(\$)$ & 77,673 \\
\hline & Action Path & $5-0-2-3-2$ & $5-2-4-2-4$ & $5-4-0-1-3$ & $5-0-0-0-0$ & $5-1-0-4-0$ & $5-2-1-0-3$ & $5-0-0-0-0$ & $5-4-4-4-1$ & $5-4-4-0-0$ & $5-3-0-1-0$ & $5-3-2-3-3$ & $5-0-0-0-0$ & $\mathrm{MIC}_{\mathrm{brg}}(\$)$ & $2,089,162$ \\
\hline & $\mathrm{RSL}_{\text {elm }}$ (years) & 94 & 61 & 190 & 18 & 84 & 116 & 351 & 16 & 14 & 36 & 2 & 91 & $\mathrm{IAC}_{\mathrm{brg}}(\$)$ & $2,089,162$ \\
\hline & $R V_{\text {elm }}(\$)$ & 56,861 & 345,670 & 32,801 & 3,355 & $2,181,128$ & 85,217 & 12,703 & 1,082 & 6,046 & 15,547 & 1,798 & 10,835 & $\mathrm{LCC}_{\mathrm{brg}}(\$)$ & 976,447 \\
\hline & $\mathrm{PAC}_{\mathrm{elm}}(\$)$ & 10,109 & $1,452,933$ & 672 & 0 & 49,101 & 7,824 & 0 & 2,141 & 0 & 9,195 & 30,681 & 0 & $\mathrm{BNT}_{\text {brg }}(\mathrm{DN})(\$)$ & $-1,953,907$ \\
\hline & $\operatorname{PLCC}_{\text {elm }}(\$)$ & $-46,753$ & $1,107,262$ & $-32,129$ & $-3,355$ & $-2,132,026$ & $-77,393$ & $-12,703$ & 1,059 & $-6,046$ & $-6,352$ & 28,883 & $-10,835$ & $\mathrm{BNT}_{\text {brg }}(\mathrm{RO})(\$)$ & $-1,824,720$ \\
\hline & $\mathrm{BNT}_{\text {elm }}(\mathrm{DN})(\$)$ & 6,829 & $-1,220,597$ & 7,442 & 9,506 & 651,975 & 31,112 & 1,520 & $-1,059$ & 6,046 & 6,352 & $-28,883$ & 4,167 & & \\
\hline & $\mathrm{BNT}_{\mathrm{elm}}(\mathrm{RO})(\$)$ & 6,829 & $-1,220,597$ & 7,442 & 0 & 651,975 & 31,112 & 0 & 6,004 & 28,319 & 30,725 & 46,595 & 0 & & \\
\hline \multirow{8}{*}{ 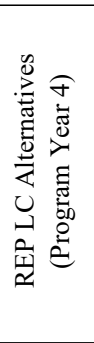 } & Alt. Reference & 3 & 18 & 398 & 1 & 327 & 224 & 1 & 459 & 549 & 624 & 112 & 1 & $\mathrm{USC}_{\mathrm{brg}}(\$)$ & 77,673 \\
\hline & Action Path & $6-0-0-0-2$ & $6-0-0-3-2$ & $6-3-0-4-2$ & $6-0-0-0-0$ & $6-2-3-0-1$ & $6-1-3-4-3$ & $6-0-0-0-0$ & $6-3-3-1-3$ & $6-4-1-4-3$ & $6-4-4-4-3$ & $6-0-4-2-1$ & $6-0-0-0-0$ & $\mathrm{MIC}_{\mathrm{brg}}(\$)$ & $3,769,103$ \\
\hline & $\mathrm{RSL}_{\text {elm }}$ (years) & 93 & 69 & 185 & 18 & 112 & 118 & 351 & 6 & 29 & 17 & 18 & 91 & $\mathrm{IAC}_{\mathrm{brg}}(\$)$ & $3,769,103$ \\
\hline & $\mathrm{RV}_{\mathrm{elm}}(\$)$ & 56,256 & 391,004 & 31,938 & 3,355 & $2,908,170$ & 86,686 & 12,703 & 406 & 12,524 & 7,342 & 16,181 & 10,835 & $\mathrm{LCC}_{\text {brg }}(\$)$ & $2,382,381$ \\
\hline & $\mathrm{PAC}_{\mathrm{elm}}(\$)$ & 8,670 & $1,841,285$ & 592 & 0 & 117,995 & 5,100 & 0 & 3,189 & 10,388 & 0 & 43,034 & 0 & $\mathrm{BNT}_{\text {brg }}(\mathrm{DN})(\$)$ & $-3,359,841$ \\
\hline & $\operatorname{PLCC}_{\mathrm{elm}}(\$)$ & $-47,587$ & $1,450,281$ & $-31,346$ & $-3,355$ & $-2,747,424$ & $-81,586$ & $-12,703$ & 2,783 & $-2,136$ & $-7,342$ & 26,854 & $-10,835$ & $\mathrm{BNT}_{\text {brg }}(\mathrm{RO})(\$)$ & $-3,230,653$ \\
\hline & $\mathrm{BNT}_{\mathrm{elm}}(\mathrm{DN})(\$)$ & 7,663 & $-1,563,616$ & 6,659 & 9,506 & $1,267,373$ & 35,305 & 1,520 & $-2,783$ & 2,136 & 7,342 & $-26,854$ & 4,167 & & \\
\hline & $\mathrm{BNT}_{\mathrm{elm}}(\mathrm{RO})(\$)$ & 7,663 & $-1,563,616$ & 6,659 & 0 & $1,267,373$ & 35,305 & 0 & 4,280 & 24,409 & 31,715 & 48,625 & 0 & & \\
\hline
\end{tabular}


Figures 5.10(a) through (f) illustrate the element health indices at different analysis years produced by the LC alternatives in Table 5.6. The horizontal dashed line represents a health index lowerfrontier (deficiency screening thresholds, a minimum health index of $80 \%$ every 10 years). The dashed curves above this frontier distinguish the non-deficient elements from the deficient ones. The thick connected lines in black represent the overall bridge health indices for the different combinations of LC alternatives in Table 5.6. 
(a)

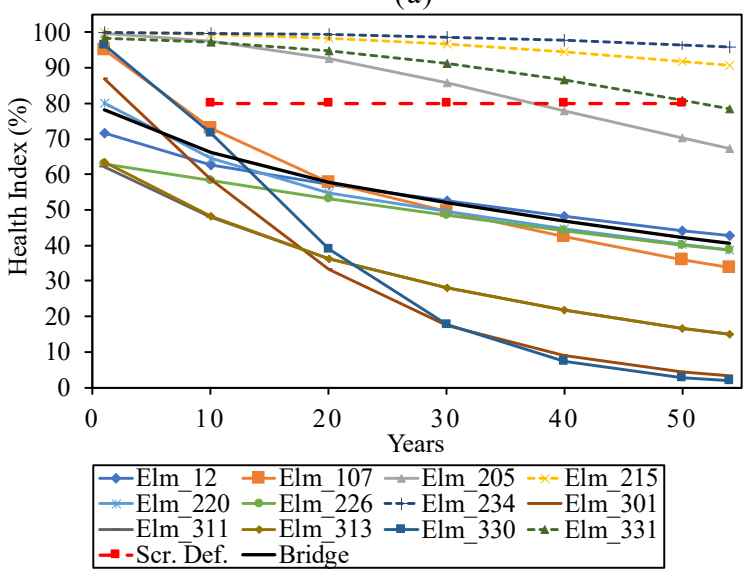

(c)

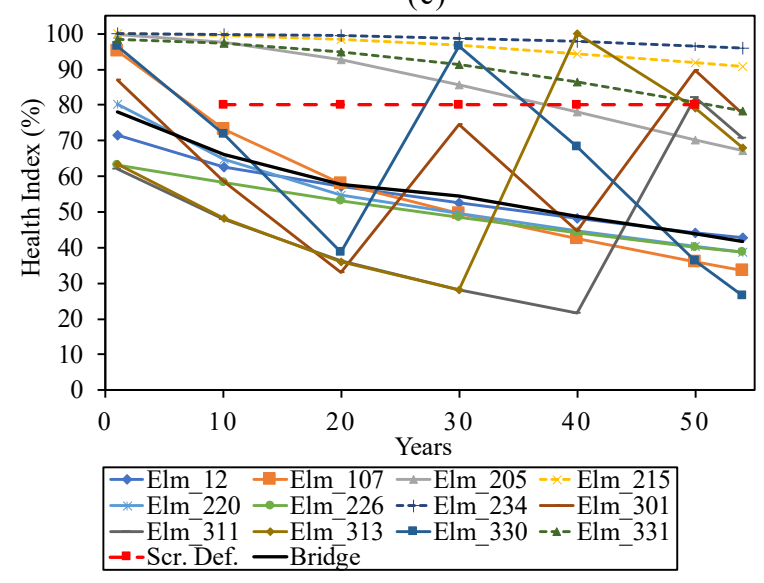

(e)

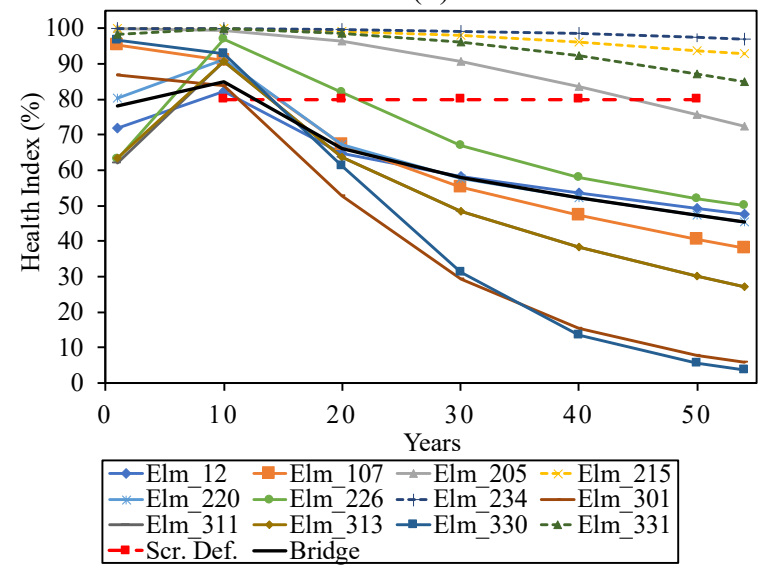

(b)

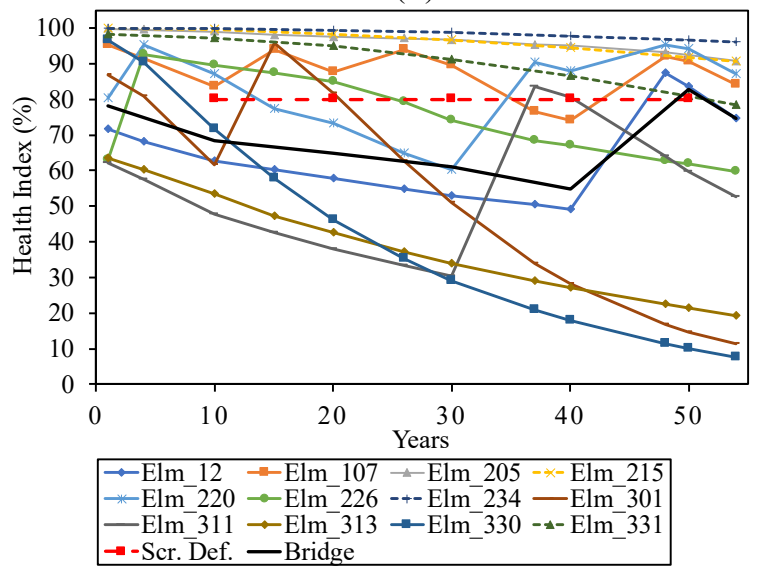

(d)

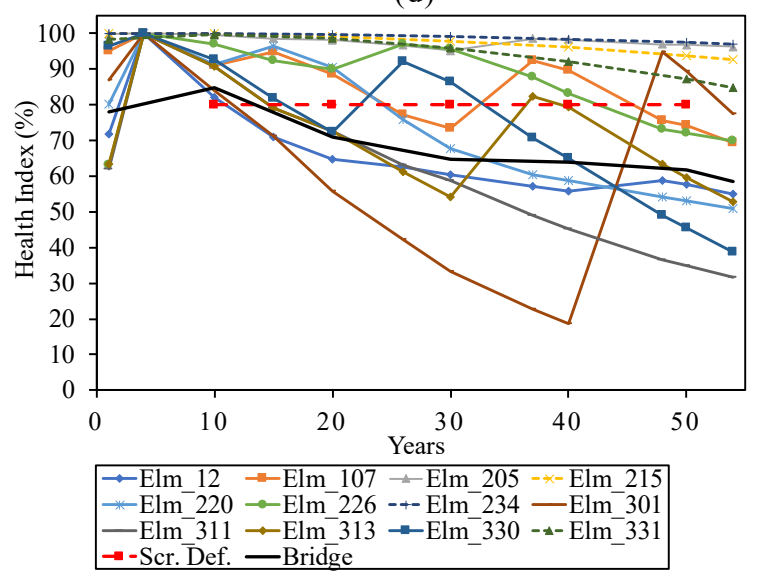

(f)

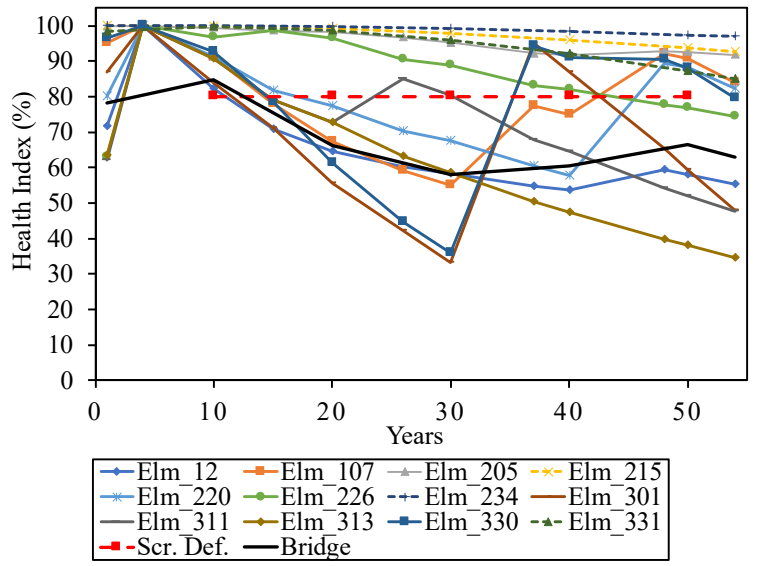

Figure 5.9 Health indices predicted for Bridge 11 under (a) DN LC Alternative; (b) Combination of MRR

LC Alternatives; (c) RO LC Alternative (d); Combination of FCI LC Alternatives; (e) FCI/REP LC Alternative 1; (f) Combination of REP LC Alternatives 


\subsection{Summary and Conclusions}

This chapter presented a basic framework to visualize the modeling approach followed to generate LC alternatives (series of element improvement actions) and estimate LCCs and LCC benefits. The chapter introduced a novel screening process, to focus on potential deficient elements, and a new simulation arrangement, to generate realistic ("real-life") LC alternatives, for the three improvement types (i.e., MRR, FCI, and REP). The module relies on two independent models (i.e., deterioration and LCC models) to predict conditions and estimate LCCs and LCC benefits. The LCC and benefit modeling approaches are expressed in this chapter. An illustrative example of module results using the MATLAB-based tool prototype is also included. The implementation proved the capability of the module in producing reliable LC alternative results. The tool successfully identified potential deficient elements, predicted performance, generated LC alternatives, constructed LC profiles, and determined incurred LCCs and LCC benefits.

The module results can be used independently to determine bridge investment needs for bridge programming and planning. $\mathrm{BCA}$, IBCA, or optimization heuristics can be deployed to identify bridge combinations of LC alternatives representing the proper intervention strategies. The proposed EB-MOO methodology deviates from common economic decision analyses (i.e., BCA and IBCA) used by BMSs for selecting alternatives. LC alternatives won't be compared at this stage, only LCCs and LCC benefits are determined and discounted to present time. The module LC alternative results are transferred to the ELO module (discussed in the subsequent chapter) to be used in the optimization process. 


\section{CHAPTER 6-ELEMENT-LEVEL OPTIMIZATION MODULE}

- Task 3: Proposing an Element-Based Multi-Objective Optimization Methodology

- Task 4: Development of a Tool Prototype

- Task 5: Implementation of the Methodology through Examples of Scenarios

\subsection{Introduction}

The main objective of the EB-MOO methodology is to determine short- and long-term investment needs and support recommending programs and implementation schedules. The methodology consists of five modules (i.e., data processing, improvement, ELO, BLO, and NLO modules). The discussion up to this point has largely focused on the data processing and improvement modules. Chapters 4 and 5 introduced the different processes and models incorporated in these two modules: a novel screening process to identify potential deficient bridge elements, an independent deterioration model to predict performance, a LCC model to estimate LCCs and LCC benefits, and a simulation arrangement to generate realistic LC alternatives for MRR, FCI and REP improvement types. Chapters 6,7 , and 8 are devoted to the three optimization modules. This chapter discusses the ELO module framework and its different processes, introduces the proposed heuristic optimization algorithm, and includes an illustrative example using the developed tool prototype.

For each program year, LC alternatives of a deficient element-generated as part of the improvement module - are independently screened. The module screening process recognizes the best feasible LC alternatives for each program year based on the specified criteria and optimization 
goals. Sorting outcomes are rearranged and grouped to capture all deficient elements. Module results associated with these best feasible LC alternatives drive the optimization process.

The literature review revealed that MOO methodologies, aiming to produce optimal or nearoptimal sets of long-term intervention strategies, are less common. Most of the MOO methods used in modern BMSs reduce the problem to a single-objective optimization problem by scaling, weighting, and aggregating all competing objectives. The recommended long-term needs are determined only at the higher levels (i.e., bridge level or network level). This chapter introduces a "true" MOO methodology that relies on results from the data processing and improvement modules. The module optimization requires a simultaneous optimization of multiple competing objectives (e.g., minimize the LCC and maximize the LCC benefit and/or health indices at different points in time for a candidate bridge) and seeks to arrive at the best trade-off between them. The aim is to obtain a diverse set of ELO solutions per improvement type and per program year as close as possible to the true Pareto frontier.

This module focuses on finding a set of Pareto optimal or near-optimal ELO solutions per program year for each improvement type. A set of LC alternatives is derived from each solution. Each recommended LC alternative represents a set of best actions for a deficient element over the analysis period — associated budgets, LCCs, and LCC benefits, and conditions are subsequently retrieved. Results associated with these element-level LC alternatives (or solutions) serve as the fundamental inputs for the subsequent optimization modules.

The optimization problem is formulated as a "Multi-Choice Knapsack Problem" (MCKP) in terms of discrete decision variables (binary values), involving only the selection criterion. A LC alternative is selected for an element when the corresponding decision variable is 1 , and not 
selected, when the variable is 0 . This type of optimization problem is referred to as combinatorial optimization problem. It's considered difficult to solve combinatorial problems in a reasonable time - generally near-optimal rather than optimal solutions are obtained. ELO objectives and constraints are expressed in terms of these decision variables. Because of the stochastic nature of the optimization problem and the large number of variables involved in the selection of intervention strategies; a genetic algorithm was chosen for the optimization. A robust metaheuristic algorithm (i.e., NSGA-II, covered in Chapter 2) is deployed as the main optimizer to solve the computational complexity of the module optimization problems.

\subsection{Module Framework}

A framework of the ELO module is illustrated in Figure 6.1. As discussed in Chapter 5, each bridge in the portfolio is evaluated separately for each improvement type and each program year: the LCC model estimates bridge initial agency, user, and major improvement costs; the screening process identifies bridge deficient elements; and the simulation arrangement generates all possible LC alternatives for each identified deficient element-their corresponding preservation LCCs ( $\left.\mathrm{PLCC}_{\text {elms }}\right)$ and $\mathrm{LCC}$ benefits $\left(\mathrm{BNT}_{\text {elms }}\right)$, and health indices are determined. These output data are transferred to this module for further processing at this optimization level. The module framework exemplified in Figure 6.1 is based on the following concepts:

1. A screening process referred to as "alternative feasibility screening," discussed in the subsequent section, is deployed to identify a set of best feasible LC alternatives for each identified deficient element per improvement type and per program year. For illustration, Figure 6.1 shows an alternative feasibility screening linked to MRR improvement type and program year 4. The screening is specific to Element 107 of Bridge 47 . Using this screening process, the optimization problem size is reduced by eliminating the economically 
unattractive LC alternatives - associated results are sorted based on the preferred screening criteria and/or optimization goals.

2. Best feasible LC alternatives are recognized and set aside to be used in the optimization process. Results (i.e., PLCC $_{\text {elms, }}$ IAC $_{\text {elms, }}, \mathrm{BNT}_{\text {elms, }}$, and element health indices) associated with these identified LC alternatives are grouped and reorganized per improvement type and per program year in single matrices as shown in Figure 6.1.

3. Each matrix includes one type of LC alternative results per improvement type and per program year, encompassing all deficient elements. The optimization process relies on these preset matrices as input parameters, including bridge user and initial agency costs, to direct the search toward optimal or near-optimal LC alternatives.

4. Three optimization solution output matrices are produced for each improvement type and each program year. As shown in Figure 6.1, the first matrix (bottom, far-left) contains the recommended LC alternatives for all deficient elements, the second matrix, the resulted initial agency costs and health indices, and the third matrix, the optimized element-level objective values. 


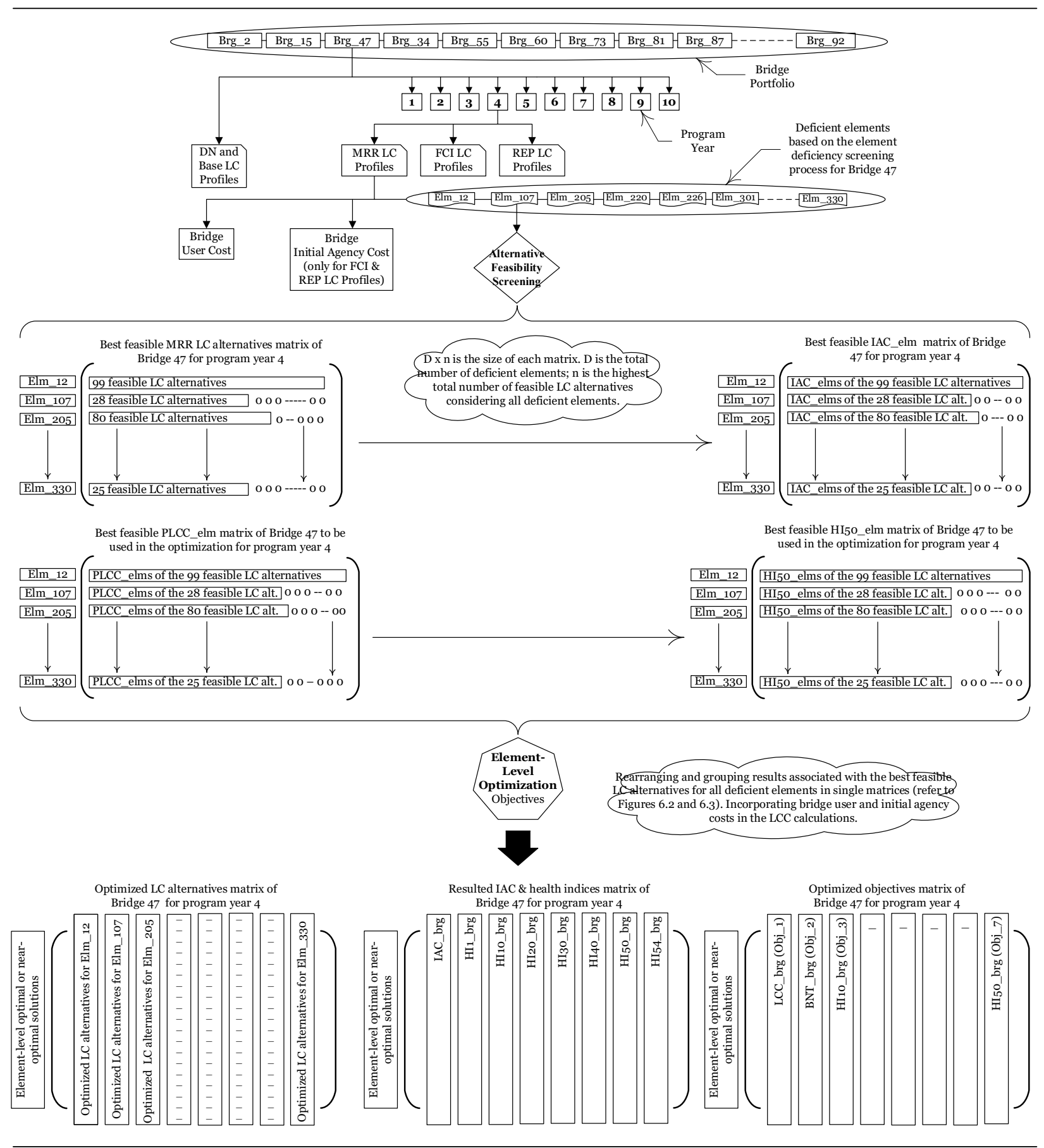

Figure 6.1 Element-level optimization module framework 


\subsection{Alternative Feasibility Screening}

Chapter 5 discusses the three common economic decision analyses used in BMSs to compare alternatives: LCCA, BCA, and IBCA. Any of these analyses can be utilized to compare the produced LC alternatives. However, prioritizing LC alternatives based on their economic preferences usually yields misleading results. Other non-economic preferences (objectives), targets and restrictions (constraints) are not being considered in the search of best sets of LC alternatives. The module optimization problem deals with multiple competing objectives (e.g., minimizing the bridge LCC, and maximizing the bridge LCC benefit and health indices at different points in time) subject to constraints (e.g., available budget and/or minimum acceptable performance). MOO techniques are considered the most effective for these types of optimization problems. They guarantee a diverse set of optimal or near-optimal solutions-constituting a frontier of trade-offs (i.e., Pareto frontier). The module relies on a robust MOO technique to obtain these optimal or near-optimal solutions.

A given bridge can be associated with a large number of possible combinations of LC alternatives. For example, taking a bridge with only 9 elements, the 3125 MRR LC alternatives per program year for each element generate $2.84 \mathrm{E} 31\left(3125^{9}\right)$ of possible combinations of MRR LC alternatives per program year. This huge number makes the optimization problem very challenging and costly to solve in terms of computational time and computer memory. This large-scale optimization problem becomes extremely difficult to manage. Achieving heuristic solutions as close as to the Pareto frontier requires a tremendous computational effort. The need for a strategy that guides the optimization search toward global optimality within a reasonable computational time became indispensable. 
A screening process referred to as "alternative feasibility screening" is introduced to make the problem more tractable without affecting the quality of solutions - attaining a manageable problem size dramatically improves the optimization computational time. To achieve a reasonable problem size and guarantee inclusion of most suitable LC alternatives, the process relies on two independent stages of screening:

(1) an initial screening stage-feasible LC alternatives are identified after eliminating the economically unattractive ones, and

(2) a final screening stage - feasible LC alternatives producing results in alignment with the optimization goal are further identified and classified as the "best" feasible LC alternatives for consideration.

The following subsections discuss both stages in more detail.

\subsubsection{Initial Screening Stage}

Figure 6.2 illustrates the initial stage of the screening process. To identify the preliminary set of feasible LC alternatives for each deficient element, all possible LC alternatives, generated as part of the improvement module, are sorted through this initial stage. Each of them is screened for feasibility. A LC alternative is classified as feasible if the following two screening criteria are satisfied.

- Criteria 1-Element health indices produced by the LC alternative outdo the element health index thresholds. Users set element health index thresholds (minimum acceptable limits) at the end of analysis years 10,20,30,40, and 50. For each improvement type and each program year, predicted element health indices at these specified analysis points in time are compared to their corresponding thresholds. A health index lower-frontier is 
constructed for each deficient element (joining the specified thresholds as indicated in Figure 6.2). Any produced health indices falling below this lower-frontier is classified as "unfeasible" and eliminated from further screening or consideration.

- Criteria 2-Element LCC benefit (BNT $\left.\mathrm{Blm}_{\mathrm{lm}}\right)$ of the $\mathrm{LC}$ alternative is positive. If LC alternatives produce only negative $\mathrm{BNT}_{\text {elms, }}$, then the sorting follows an ascending order. LC alternatives producing the largest $\mathrm{BNT}_{\text {elms }}$ are considered - starting from the top (the largest LCC benefit) to a specified cutoff in ascending order (refer to Figure 6.2 for illustration). The remaining LC alternatives beyond the cutoff are deemed unfeasible. 


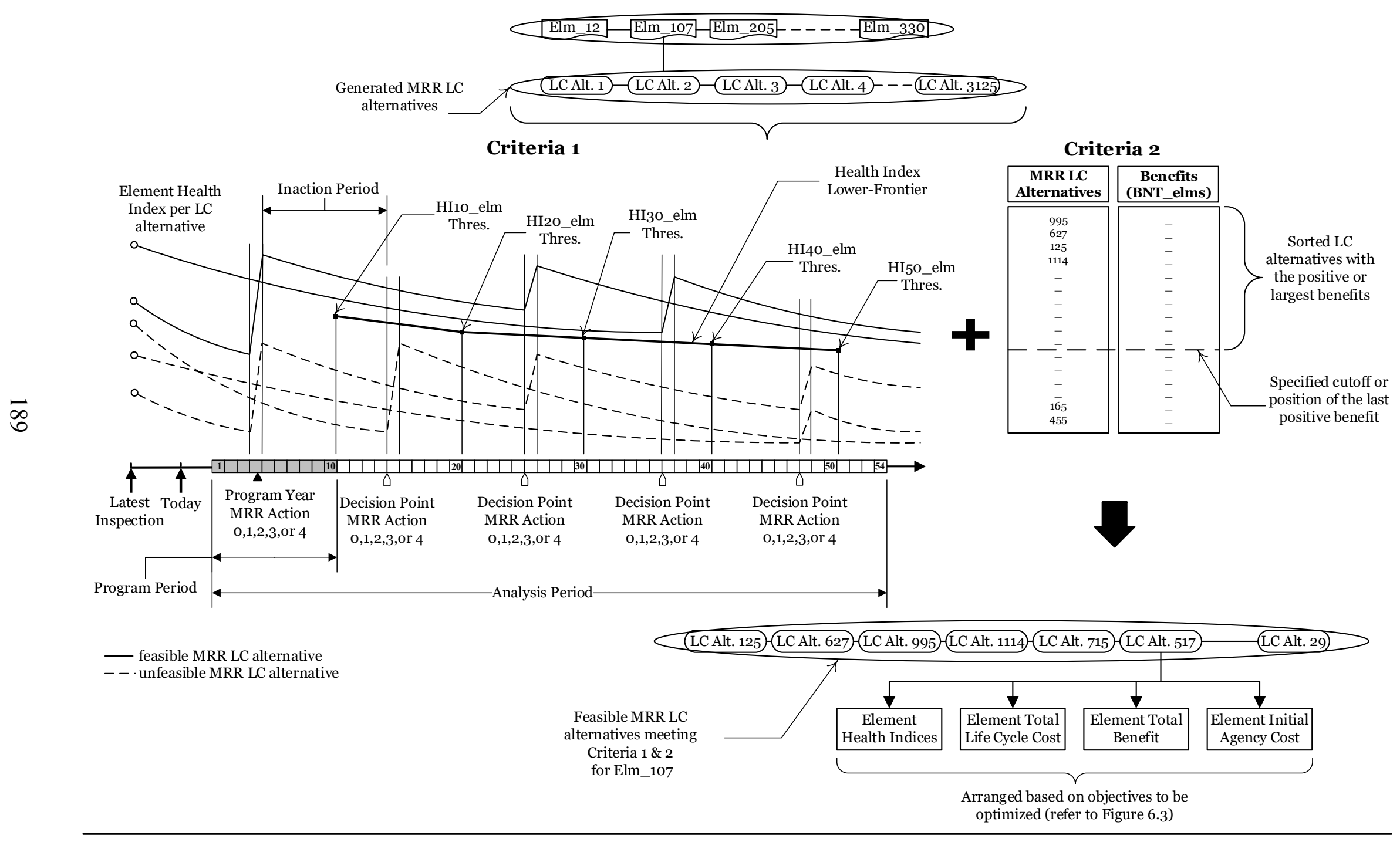

Figure 6.2 Illustration of the initial stage of the alternative feasibility screening process 
Results associated with all possible LC alternatives for each deficient element are transferred to this module and arranged by improvement type and program year. The results are sorted through this initial stage based on the established screening criteria. Table 6.1 is a representation of the 3125 MRR LC alternatives generated by the improvement module for a deficient element (i.e., Element 226). The table includes the associated results which are unique for Element 226, MRR improvement type, and program year 4 .

Table 6.1 Representation of the 3125 MRR LC alternatives (with an initial intervention in program year 4) generated by the improvement module for Element 226

\begin{tabular}{|c|c|c|c|c|c|c|c|c|c|}
\hline & $\begin{array}{l}\text { Alt. } \\
\text { Ref. }\end{array}$ & $\begin{array}{c}\text { HI10 elm } \\
(\%)\end{array}$ & $\begin{array}{c}\mathrm{HI}^{2} 0_{\mathrm{elm}} \\
(\%)\end{array}$ & $\begin{array}{c}\text { HI30 } 0_{\text {elm }} \\
(\%)\end{array}$ & $\begin{array}{c}\mathrm{HI}^{4} 0_{\text {elm }} \\
(\%)\end{array}$ & $\begin{array}{c}\mathrm{HI}^{50 \mathrm{elm}} \\
(\%)\end{array}$ & $\begin{array}{c}\mathrm{BNT}_{\text {elm }} \\
(\$)\end{array}$ & $\begin{array}{c}\text { PLCC }_{\text {elm }} \\
(\$)\end{array}$ & $\begin{array}{c}\mathrm{IAC}_{\text {elm }} \\
(\$)\end{array}$ \\
\hline \multirow{28}{*}{ 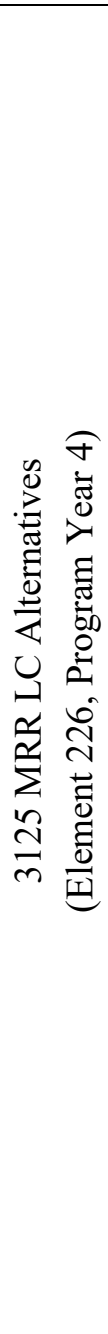 } & 1 & 58.20 & 53.16 & 48.47 & 44.13 & 40.12 & $-46,282$ & $-46,282$ & $-46,282$ \\
\hline & 2 & 58.20 & 53.16 & 48.47 & 44.13 & 85.05 & $-92,563$ & $-46,282$ & $-46,282$ \\
\hline & 3 & 58.20 & 53.16 & 48.47 & 44.13 & 73.52 & $-67,718$ & $-46,282$ & $-46,282$ \\
\hline & 4 & 58.20 & 53.16 & 48.47 & 44.13 & 56.76 & $-48,719$ & $-46,282$ & $-46,282$ \\
\hline & 5 & 58.20 & 53.16 & 48.47 & 44.13 & 40.51 & $-25,641$ & $-46,282$ & $-46,282$ \\
\hline & 6 & 58.20 & 53.16 & 48.47 & 85.87 & 76.17 & $-87,421$ & $-46,282$ & $-46,282$ \\
\hline & 7 & 58.20 & 53.16 & 48.47 & 85.87 & 95.46 & $-96,971$ & $-46,282$ & $-46,282$ \\
\hline & 8 & 58.20 & 53.16 & 48.47 & 85.87 & 83.64 & $-88,624$ & $-46,282$ & $-46,282$ \\
\hline & - & - & - & & - & - & - & - & - \\
\hline & - & - & - & - & - & - & - & - & - \\
\hline & - & - & - & - & - & - & - & - & - \\
\hline & - & - & - & - & - & - & - & - & - \\
\hline & 864 & 89.47 & 94.90 & 87.53 & 84.47 & 78.23 & $-80,740$ & $-46,282$ & $-46,282$ \\
\hline & 865 & 89.47 & 94.90 & 87.53 & 84.47 & 77.83 & $-82,062$ & $-46,282$ & $-46,282$ \\
\hline & 866 & 89.47 & 94.90 & 87.53 & 81.29 & 72.32 & $-80,151$ & $-46,282$ & $-46,282$ \\
\hline & 867 & 89.47 & 94.90 & 87.53 & 81.29 & 94.35 & $-92,640$ & $-46,282$ & $-46,282$ \\
\hline & 868 & 89.47 & 94.90 & 87.53 & 81.29 & 81.66 & $-82,009$ & $-46,282$ & $-46,282$ \\
\hline & - & - & - & - & - & - & - & - & - \\
\hline & - & - & - & - & - & - & - & - & - \\
\hline & - & - & - & - & - & - & - & - & - \\
\hline & - & - & - & - & - & - & - & - & - \\
\hline & - & - & - & - & - & - & - & - & - \\
\hline & 3120 & 58.72 & 54.14 & 49.84 & 55.60 & 51.08 & 2,010 & $-46,282$ & 5,007 \\
\hline & 3121 & 58.72 & 54.14 & 49.84 & 45.83 & 41.69 & 5,301 & $-46,282$ & 5,007 \\
\hline & 3122 & 58.72 & 54.14 & 49.84 & 45.83 & 85.49 & $-38,042$ & $-46,282$ & 5,007 \\
\hline & 3123 & 58.72 & 54.14 & 49.84 & 45.83 & 73.13 & $-13,350$ & $-46,282$ & 5,007 \\
\hline & 3124 & 58.72 & 54.14 & 49.84 & 45.83 & 56.43 & 4,119 & $-46,282$ & 5,007 \\
\hline & 3125 & 58.72 & 54.14 & 49.84 & 45.83 & 42.09 & 23,431 & $-46,282$ & 5,007 \\
\hline
\end{tabular}


Table 6.2 presents an example of sorting results. LC alternative results from Table 6.1 were processed through the initial screening stage. For program year 4, the initial screening stage identified 644 out of the 3125 MRR LC alternatives meeting both Criteria 1 and 2. 644 MRR LC alternatives with positive $\mathrm{BNT}_{\text {elms }}$ and element health indices above the specified thresholds $(60 \%$ as the minimum acceptable health index every 10 years) were identified. These 644 MRR LC alternatives are classified as feasible for program year 4. The remaining 2481 MRR LC alternatives are disregarded.

Table 6.2 Representation of the 644 feasible MRR LC alternatives (with an initial intervention in program year 4) identified through the initial screening stage for Element 226

\begin{tabular}{|c|c|c|c|c|c|c|c|c|c|}
\hline & \multirow{2}{*}{$\begin{array}{l}\text { Alt. } \\
\text { Ref. }\end{array}$} & \multicolumn{5}{|c|}{$\begin{array}{r}\text { Criteria } 1 \\
\text { (Element Health Inde }\end{array}$} & \multirow{2}{*}{$\begin{array}{c}\text { Criteria } 2 \\
\left(\mathrm{BNT}_{\mathrm{elm}} \geq 0\right) \\
\mathrm{BNT}_{\mathrm{elm}} \\
(\$)\end{array}$} & \multirow[b]{2}{*}{$\begin{array}{l}\text { PLCC }_{\text {elm }} \\
(\$)\end{array}$} & \multirow[b]{2}{*}{$\begin{array}{l}\mathrm{IAC}_{\text {elm }} \\
(\$)\end{array}$} \\
\hline & & $\begin{array}{l}\text { HI10 elm } \\
(\%)\end{array}$ & $\begin{array}{l}\mathrm{HI} 20_{\mathrm{elm}} \\
(\%)\end{array}$ & $\begin{array}{l}\mathrm{HI} 30_{\mathrm{elm}} \\
(\%)\end{array}$ & $\begin{array}{l}\mathrm{HI} 40_{\mathrm{elm}} \\
(\%)\end{array}$ & $\begin{array}{l}\text { HI50 elm } \\
(\%)\end{array}$ & & & \\
\hline \multirow{24}{*}{ 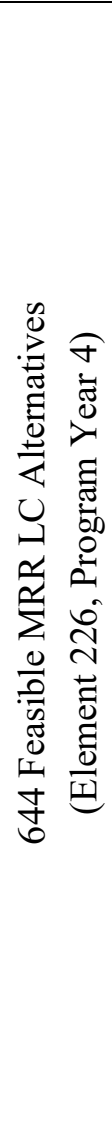 } & 631 & 89.47 & 76.62 & 63.77 & 89.94 & 79.53 & 44,078 & $-90,359$ & $-46,282$ \\
\hline & 632 & 89.47 & 76.62 & 63.77 & 89.94 & 96.34 & 51,424 & $-97,706$ & $-46,282$ \\
\hline & 633 & 89.47 & 76.62 & 63.77 & 89.94 & 85.93 & 44,878 & $-91,160$ & $-46,282$ \\
\hline & 634 & 89.47 & 76.62 & 63.77 & 89.94 & 83.21 & 42,695 & $-88,976$ & $-46,282$ \\
\hline & 635 & 89.47 & 76.62 & 63.77 & 89.94 & 82.80 & 43,547 & $-89,828$ & $-46,282$ \\
\hline & - & - & - & - & - & - & - & - & - \\
\hline & - & - & - & - & - & - & - & - & - \\
\hline & - & - & - & - & - & - & - & - & - \\
\hline & - & - & - & - & - & - & - & - & - \\
\hline & 878 & 89.47 & 85.05 & 74.19 & 62.62 & 70.91 & 21,317 & $-67,598$ & $-46,282$ \\
\hline & 881 & 89.47 & 85.05 & 74.19 & 92.17 & 81.36 & 36,108 & $-82,389$ & $-46,282$ \\
\hline & 882 & 89.47 & 85.05 & 74.19 & 92.17 & 96.80 & 42,719 & $-89,001$ & $-46,282$ \\
\hline & 883 & 89.47 & 85.05 & 74.19 & 92.17 & 87.28 & 36,704 & $-82,985$ & $-46,282$ \\
\hline & 884 & 89.47 & 85.05 & 74.19 & 92.17 & 85.14 & 35,925 & $-82,207$ & $-46,282$ \\
\hline & - & - & - & - & - & - & - & - & - \\
\hline & - & - & - & - & - & - & - & - & - \\
\hline & - & - & - & - & - & - & - & - & - \\
\hline & - & - & - & - & - & - & - & - & - \\
\hline & - & - & - & - & - & - & - & - & - \\
\hline & 1785 & 65.07 & 60.08 & 88.98 & 95.81 & 88.18 & 8,278 & $-54,559$ & $-46,282$ \\
\hline & 1787 & 65.07 & 60.08 & 88.98 & 85.22 & 95.54 & 6,844 & $-53,125$ & $-46,282$ \\
\hline & 1792 & 65.07 & 60.08 & 88.98 & 82.41 & 94.62 & 8,021 & $-54,303$ & $-46,282$ \\
\hline & 1797 & 65.07 & 60.08 & 88.98 & 81.99 & 94.54 & 10,549 & $-56,831$ & $-46,282$ \\
\hline & 1798 & 65.07 & 60.08 & 88.98 & 81.99 & 82.08 & 165 & $-46,446$ & $-46,282$ \\
\hline
\end{tabular}




\subsubsection{Final Screening Stage}

The initial stage of the screening process focuses on recognizing the feasible LC alternatives for each deficient element. This final stage improves the computational time by imposing additional screening criteria. The aim is to further reduce the optimization problem size without affecting the solution quality. However, this step can be omitted if the computational time is not a concern. This stage seeks to identify the feasible LC alternatives producing results in alignment with the optimization goal. For a given program year, feasible LC alternative results pertaining to each deficient element are sorted and arranged in accordance to each objective to be optimized. This sorting approach intents to direct the optimization search toward global optimality within a reasonable computational time. The approach reduces the dimensionality of the optimization search space (i.e., the space of all feasible solutions) by emphasizing on the best feasible LC alternatives. It allows to efficiently explore the search space toward the optimal frontier.

For each program year, feasible LC alternatives producing the best objective values are distinguished. This additional screening assures inclusion of the best feasible LC alternative results to serve as input parameters in the optimization process. These best LC alternatives are compatible with the ELO goal. Thus, solutions producing maximum or minimum objective values, depending on the optimization goal, are guaranteed. Obtained solutions are considered superior to all other solutions in the search space. A screening example for a bridge with 9 deficient elements is used for illustration in Figure 6.3. A cutoff value is established to limit the total number of best feasible LC alternatives to be considered. The example optimization goal is to simultaneously minimize the bridge LCC and maximize the bridge LCC benefit and health index in year 20. In this example, feasible element $\mathrm{LC}$ alternatives results (i.e., PLCC elms, $\mathrm{BNT}_{\text {elms, }}$, and $\mathrm{HI} 20_{\mathrm{elms}}$ ) are sorted

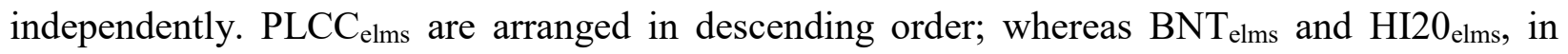


ascending order. A cutoff value is set as " 50 " for this example - the 50 smallest PLCC elms, and the 50 highest $\mathrm{BNT}_{\text {elms }}$ and $\mathrm{HI} 20_{\text {elms }}$ are chosen. Feasible LC alternatives associated with these selected 150 values are clustered, and any duplicate is eliminated. The final set includes the best feasible LC alternatives for consideration.

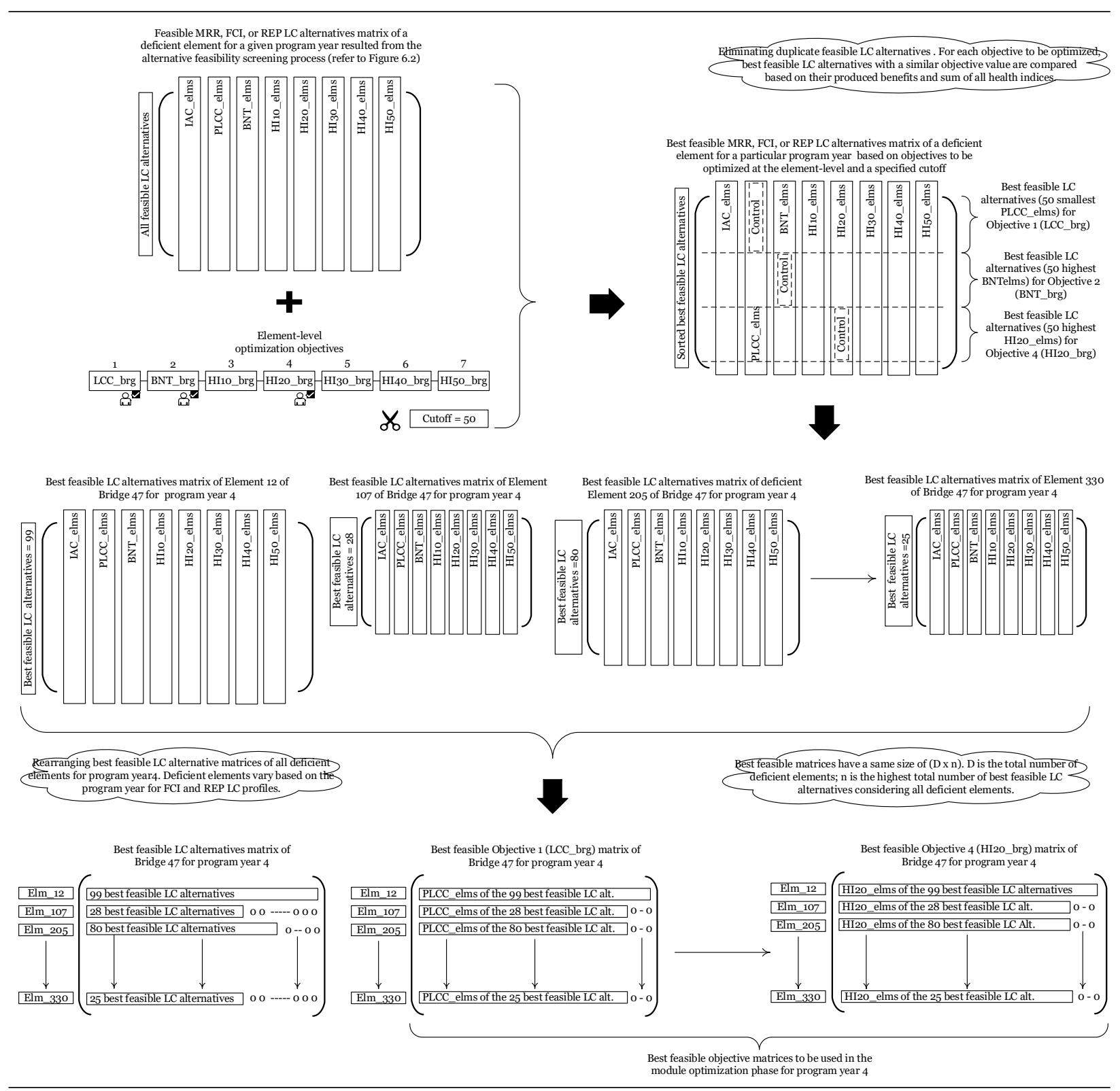

Figure 6.3 Illustration of the final stage of the alternative feasibility screening process 
Table 6.3 shows an example of final stage screening results. The same 644 feasible MRR LC alternatives of Table 6.2 are used in this example. A cutoff value of 50 was considered. Three objectives to be optimized-minimizing $\mathrm{LCC}_{\mathrm{brg}}$, and maximizing $\mathrm{BNT}_{\mathrm{brg}}$ and $\mathrm{HI} 2 \mathrm{O}_{\mathrm{brg}}$. By following the sorting steps described earlier, the number was reduced to 86 feasible MRR LC alternatives. They are all regarded as the best feasible MRR LC alternatives with an initial intervention in program year 4 for Element 226.

Table 6.3 Representation of the best feasible 86 MRR LC alternatives (with an initial intervention in program year 4) identified through the final screening stage for Element 226

\begin{tabular}{|c|c|c|c|c|c|c|c|c|c|}
\hline & \multirow{2}{*}{$\begin{array}{l}\text { Alt. } \\
\text { Ref. }\end{array}$} & \multicolumn{5}{|c|}{$\begin{array}{c}\text { Criteria } 1 \\
\text { (Element Health Index } \geq 60 \% \text { ) }\end{array}$} & \multirow{2}{*}{$\begin{array}{c}\text { Criteria } 2 \\
\left(\mathrm{BNT}_{\text {elm }} \geq 0\right) \\
\mathrm{BNT}_{\text {elm }}{ }^{\mathrm{a}} \\
(\$)\end{array}$} & \multirow[b]{2}{*}{$\begin{array}{c}\mathrm{PLCC}_{\text {elm }}{ }^{\mathrm{a}} \\
\text { (\$) }\end{array}$} & \multirow[b]{2}{*}{$\begin{array}{c}\mathrm{IAC}_{\text {elm }} \\
(\$)\end{array}$} \\
\hline & & $\begin{array}{c}\mathrm{HI}^{\mathrm{H}} 0_{\mathrm{elm}} \\
(\%)\end{array}$ & $\begin{array}{c}\mathrm{HI} 20_{\mathrm{elm}}{ }^{\mathrm{a}} \\
(\%)\end{array}$ & $\begin{array}{c}\text { HI30 } 0_{\text {elm }} \\
(\%)\end{array}$ & $\begin{array}{c}\mathrm{HI}^{4} 40_{\mathrm{elm}} \\
(\%)\end{array}$ & $\begin{array}{c}\mathrm{HI} 50_{\mathrm{elm}} \\
(\%)\end{array}$ & & & \\
\hline \multirow{20}{*}{ 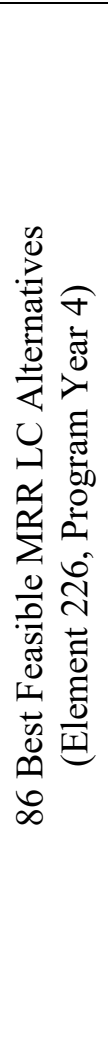 } & 632 & 89.47 & $76.62^{b}$ & 63.77 & 89.94 & 96.34 & 51,424 & $-97,706$ & $-46,282$ \\
\hline & 652 & 89.47 & $76.62^{b}$ & 92.31 & 80.49 & 93.56 & 49,955 & $-96,236$ & $-46,282$ \\
\hline & 656 & 89.47 & $76.62^{b}$ & 92.31 & 96.50 & 84.88 & 47,016 & $-93,298$ & $-46,282$ \\
\hline & 657 & 89.47 & $76.62^{b}$ & 92.31 & 96.50 & 97.67 & 52,159 & $-98,440$ & $-46,282$ \\
\hline & - & - & - & - & - & - & - & - & - \\
\hline & - & - & - & - & - & - & - & - & - \\
\hline & - & - & - & - & - & - & - & - & - \\
\hline & - & - & - & - & - & - & - & - & - \\
\hline & 792 & 89.47 & 94.90 & 96.56 & 89.05 & 96.15 & $50,323^{b}$ & $-96,604$ & $-46,282$ \\
\hline & 793 & 89.47 & 94.90 & 96.56 & 89.05 & 85.38 & $42,832^{\mathrm{b}}$ & $-89,113$ & $-46,282$ \\
\hline & 794 & 89.47 & 94.90 & 96.56 & 89.05 & 82.43 & $40,674^{b}$ & $-86,956$ & $-46,282$ \\
\hline & 795 & 89.47 & 94.90 & 96.56 & 89.05 & 81.99 & $42,392^{b}$ & $-88,674$ & $-46,282$ \\
\hline & - & - & - & - & - & - & - & - & - \\
\hline & - & - & - & - & - & - & - & - & - \\
\hline & - & - & - & - & - & - & - & - & - \\
\hline & - & - & - & - & - & - & - & - & - \\
\hline & 1162 & 89.47 & 82.64 & 93.94 & 88.34 & 96.14 & 47,246 & $-93,527^{c}$ & $-46,282$ \\
\hline & 1167 & 89.47 & 82.64 & 93.94 & 86.75 & 95.62 & 47,456 & $-93,737^{c}$ & $-46,282$ \\
\hline & 1172 & 89.47 & 82.64 & 93.94 & 86.55 & 95.58 & 48,930 & $-95,211^{\mathrm{c}}$ & $-46,282$ \\
\hline & 1232 & 89.47 & 82.64 & 76.22 & 92.83 & 96.92 & 46,703 & $-92,985^{\mathrm{c}}$ & $-46,282$ \\
\hline
\end{tabular}

${ }^{\mathrm{a}}$ ELO objective. ${ }^{\mathrm{b}}$ Highest values. ${ }^{\mathrm{c}}$ Smallest values. 
Table 6.4 presents an example of input matrices for use in the ELO process. Four input matrices are displayed in this table. Each of them captures a specific type of results allied with the best feasible MRR LC alternatives with an initial intervention in program year 4. The matrices have the same size or dimension. Each row is assigned to a deficient element (a total of 9 deficient elements for this example). Columns are dedicated to the corresponding best feasible LC alternative results. The total number of columns is the highest number of best feasible LC alternatives, comparing all deficient elements (96 for this example). The zeros represent no associated LC alternatives or results. 
Table 6.4 Example of input matrices for use in the ELO process

\begin{tabular}{|c|c|c|c|c|c|c|c|c|c|c|c|c|c|c|c|}
\hline \multirow{2}{*}{$\begin{array}{l}\text { Deficient } \\
\text { Element }\end{array}$} & \multicolumn{15}{|c|}{ Best Feasible LC MRR Alternatives ${ }^{a}$} \\
\hline & 1 & 2 & 3 & 4 & - & 44 & 45 & 46 & - & 91 & 92 & 93 & 94 & 95 & 96 \\
\hline 12 & 132 & 152 & 157 & 160 & - & 1047 & 1152 & 1157 & - & 0 & 0 & 0 & 0 & 0 & 0 \\
\hline 107 & 131 & 132 & 133 & 152 & - & 907 & 908 & 911 & - & 0 & 0 & 0 & 0 & 0 & 0 \\
\hline 205 & 7 & 32 & 132 & 152 & - & 782 & 783 & 784 & - & 2672 & 2682 & 2707 & 2732 & 2907 & 3032 \\
\hline 220 & 132 & 137 & 152 & 157 & - & 791 & 792 & 793 & - & 0 & 0 & 0 & 0 & 0 & 0 \\
\hline 226 & 632 & 652 & 656 & 657 & - & 785 & 786 & 787 & - & 0 & 0 & 0 & 0 & 0 & 0 \\
\hline 301 & 782 & 2032 & 2657 & 0 & - & 0 & 0 & 0 & - & 0 & 0 & 0 & 0 & 0 & 0 \\
\hline 311 & 777 & 781 & 782 & 783 & - & 1042 & 1043 & 1047 & - & 0 & 0 & 0 & 0 & 0 & 0 \\
\hline 313 & 777 & 781 & 782 & 783 & - & 1037 & 1038 & 1042 & - & 0 & 0 & 0 & 0 & 0 & 0 \\
\hline \multirow[t]{2}{*}{330} & 157 & 158 & 160 & 162 & - & 0 & 0 & 0 & - & 0 & 0 & 0 & 0 & 0 & 0 \\
\hline & \multicolumn{15}{|c|}{ HI20 $0_{\text {elms }}$ Produced by the Best Feasible MRR LC Alternatives ${ }^{a}(\%)$} \\
\hline 12 & 80.52 & 80.52 & 80.52 & 80.52 & - & 66.32 & 66.32 & 66.32 & - & 0 & 0 & 0 & 0 & 0 & 0 \\
\hline 107 & 85.56 & 85.56 & 85.56 & 85.56 & - & 88.27 & 88.27 & 88.27 & - & 0 & 0 & 0 & 0 & 0 & 0 \\
\hline 205 & 92.64 & 92.64 & 98.87 & 98.87 & - & 99.37 & 99.37 & 99.37 & - & 99.27 & 99.27 & 99.27 & 99.27 & 97.55 & 97.54 \\
\hline 220 & 85.97 & 85.97 & 85.97 & 85.97 & - & 89.99 & 89.99 & 89.99 & - & 0 & 0 & 0 & 0 & 0 & 0 \\
\hline 226 & 76.62 & 76.62 & 76.62 & 76.62 & - & 94.90 & 94.90 & 94.90 & - & 0 & 0 & 0 & 0 & 0 & 0 \\
\hline 301 & 82.04 & 81.66 & 81.66 & 0 & - & 0 & 0 & 0 & - & 0 & 0 & 0 & 0 & 0 & 0 \\
\hline 311 & 80.18 & 80.18 & 80.18 & 80.18 & - & 62.70 & 62.70 & 62.70 & - & 0 & 0 & 0 & 0 & 0 & 0 \\
\hline 313 & 80.50 & 80.50 & 80.50 & 80.50 & - & 63.58 & 63.58 & 63.58 & - & 0 & 0 & 0 & 0 & 0 & 0 \\
\hline \multirow[t]{2}{*}{330} & 76.22 & 76.22 & 76.22 & 76.22 & - & 0 & 0 & 0 & - & 0 & 0 & 0 & 0 & 0 & 0 \\
\hline & \multicolumn{15}{|c|}{$\mathrm{BNT}_{\text {elms }}$ Produced by the Best Feasible MRR LC Alternatives ${ }^{\mathrm{a}}(\$)$} \\
\hline 12 & 33,270 & 32,665 & 33,270 & 27,826 & - & 31,440 & 32,665 & 33,270 & - & 0 & 0 & 0 & 0 & 0 & 0 \\
\hline 107 & 221,002 & 277,669 & 226,445 & 272,003 & - & 152,207 & 109,139 & 35,156 & - & 0 & 0 & 0 & 0 & 0 & 0 \\
\hline 205 & 8,632 & 8,805 & 8,632 & 8,632 & - & 8,805 & 8,392 & 8,425 & - & 8,625 & 8,638 & 8,716 & 8,794 & 8,640 & 8,783 \\
\hline 220 & $1,506,017$ & $1,396,110$ & $1,480,051$ & $1,506,017$ & - & $1,048,483$ & $1,463,936$ & $1,267,894$ & - & 0 & 0 & 0 & 0 & 0 & 0 \\
\hline 226 & 51,424 & 49,955 & 47,016 & 52,159 & - & 48,379 & 42,157 & 49,503 & - & 0 & 0 & 0 & 0 & 0 & 0 \\
\hline 301 & 1,150 & 710 & 1,150 & 0 & - & 0 & 0 & 0 & - & 0 & 0 & 0 & 0 & 0 & 0 \\
\hline 311 & 19,866 & 16,411 & 20,729 & 13,744 & - & 14,611 & 5,464 & 16,375 & - & 0 & 0 & 0 & 0 & 0 & 0 \\
\hline 313 & 19,866 & 16,411 & 20,729 & 14,010 & - & 10,554 & 792 & 14,757 & - & 0 & 0 & 0 & 0 & 0 & 0 \\
\hline \multirow[t]{2}{*}{330} & 16,181 & 9,584 & 8,090 & 4,362 & - & 0 & 0 & 0 & - & 0 & 0 & 0 & 0 & 0 & 0 \\
\hline & \multicolumn{15}{|c|}{ PLCC $_{\text {elms }}$ Produced by the Best Feasible MRR LC Alternatives ${ }^{a}(\$)$} \\
\hline 12 & $-73,194$ & $-72,589$ & $-73,194$ & $-67,749$ & - & $-71,364$ & $-72,589$ & $-73,194$ & - & 0 & 0 & 0 & 0 & 0 & 0 \\
\hline 107 & $-334,337$ & $-391,004$ & $-339,780$ & $-385,337$ & - & $-265,541$ & $-222,473$ & $-148,490$ & - & 0 & 0 & 0 & 0 & 0 & 0 \\
\hline 205 & $-33,319$ & $-33,492$ & $-33,319$ & $-33,319$ & - & $-33,492$ & $-33,080$ & $-33,112$ & - & $-33,312$ & $-33,325$ & $-33,403$ & $-33,481$ & $-33,327$ & $-33,470$ \\
\hline 220 & $-2,986,068$ & $-2,876,161$ & $-2,960,102$ & $-2,986,068$ & - & $-2,528,534$ & $-2,943,987$ & $-2,747,944$ & - & 0 & 0 & 0 & 0 & 0 & 0 \\
\hline 226 & $-97,706$ & $-96,236$ & $-93,298$ & $-98,440$ & - & $-94,661$ & $-88,439$ & $-95,785$ & - & 0 & 0 & 0 & 0 & 0 & 0 \\
\hline 301 & $-1,150$ & -710 & $-1,150$ & 0 & - & 0 & 0 & 0 & - & 0 & 0 & 0 & 0 & 0 & 0 \\
\hline 311 & $-19,866$ & $-16,411$ & $-20,729$ & $-13,744$ & - & $-14,611$ & $-5,464$ & $-16,375$ & - & 0 & 0 & 0 & 0 & 0 & 0 \\
\hline 313 & $-19,866$ & $-16,411$ & $-20,729$ & $-14,010$ & - & $-10,554$ & -792 & $-14,757$ & - & 0 & 0 & 0 & 0 & 0 & 0 \\
\hline \multirow[t]{2}{*}{330} & $-16,181$ & $-9,584$ & $-8,090$ & $-4,362$ & - & 0 & 0 & 0 & - & 0 & 0 & 0 & 0 & 0 & 0 \\
\hline & \multicolumn{15}{|c|}{ IAC $_{\text {elms }}$ Produced by the Best Feasible MRR LC Alternatives ${ }^{\mathrm{a}}(\$)$} \\
\hline 12 & 0 & 0 & 0 & 0 & - & 0 & 0 & 0 & - & 0 & 0 & 0 & 0 & 0 & 0 \\
\hline 107 & 0 & 0 & 0 & 0 & - & 0 & 0 & 0 & - & 0 & 0 & 0 & 0 & 0 & 0 \\
\hline 205 & 0 & 0 & 0 & 0 & - & 0 & 0 & 0 & - & 0.79 & 0.79 & 0.79 & 0.79 & 0.79 & 0.79 \\
\hline 220 & 0 & 0 & 0 & 0 & - & 0 & 0 & 0 & - & 0 & 0 & 0 & 0 & 0 & 0 \\
\hline 226 & 0 & 0 & 0 & 0 & - & 0 & 0 & 0 & - & 0 & 0 & 0 & 0 & 0 & 0 \\
\hline 301 & 0 & 556.35 & 0 & 0 & - & 0 & 0 & 0 & - & 0 & 0 & 0 & 0 & 0 & 0 \\
\hline 311 & 0 & 0 & 0 & 0 & - & 0 & 0 & 0 & - & 0 & 0 & 0 & 0 & 0 & 0 \\
\hline 313 & 0 & 0 & 0 & 0 & - & 0 & 0 & 0 & - & 0 & 0 & 0 & 0 & 0 & 0 \\
\hline 330 & 0 & 0 & 0 & 0 & - & 0 & 0 & 0 & - & 0 & 0 & 0 & 0 & 0 & 0 \\
\hline
\end{tabular}

a Matrices have one same size of $(D \times n)$, where $D$ is the total number of deficient elements, and $n$ is the highest total number of best feasible LC alternatives considering all deficient elements. 


\subsection{Element-Level Optimization Problem Formulation}

A MOO attempts to provide a set of solutions that defines the best trade-off between multiple objectives. Each solution is represented by a vector of decision variables that satisfies constraints and optimizes various competing objectives. An optimal solution for one of the objectives is usually non-optimal for the remaining objectives. In a single-objective optimization problem, the superiority of a solution over other solutions is easily determined by comparing their objective values. In a MOO problem, the goodness of a solution is determined by the dominance. The product of a MOO is generally a set of non-dominated solutions. The solutions are described as Pareto optimal solutions (or non-dominated solutions), shaping a boundary referred to as Pareto frontier. Each solution on the Pareto frontier is not dominated by any other feasible solution (refer to Chapter 2 for more discussion). A typical MOO problem consists of several objectives subject to inequality and equality constraints. The problem can be formulated as follows:

Objectives

Minimize/maximize $f_{m}(x)$

$\forall m=1,2, \ldots M$

Subject to

$g_{j}(x) \geq 0$

$\forall j=1,2, \ldots J$

$h_{k}(x)=0$

$\forall k=1,2, \ldots K$

$x_{i} L \leq x_{i} \leq x_{i} U$

$\forall i=1,2, \ldots n$

where

$f_{m}(x) \quad=$ objective function $m$

$g_{j}(x) \quad=$ inequality constraint $j$

$h_{k}(x) \quad=$ equality constraint $k$;

$x \quad=\quad$ vector of $n$ decision variables, $x=\left(x_{1}, x_{2}, \ldots . x_{n}\right)$; 


$$
\begin{array}{ll}
x_{i} & =\text { decision variable } i \\
x_{i} L & =\text { lower } x_{i} \text { bound; and } \\
x_{i} U & =\text { upper } x_{i} \text { bound. }
\end{array}
$$

Objective functions $\left\{f_{1}(x), f_{2}(x), \ldots f_{M}(x)\right\}$ constitute a multi-dimensional objective space. Decision variable space is constituted by variable bounds that restrict each variable $x_{i}$ to take a value within a lower $x_{i} L$ bound and an upper $x_{i} U$ bound. A solution $x$ that satisfies all constraints and variable bounds is a feasible solution; otherwise, it is called an infeasible solution. A feasible solution space hosts all feasible solutions and is defined by the constraints. The solution space size is generally linked to the number of decision variables $(n)$.

Optimization problems are divided into two categories: those with continuous decision variables and those with discrete decision variables (Taboada, 2007). The latter are referred to as combinatorial optimization problems - they are difficult to solve in a reasonable time and require excessive computer memory (Chinneck, 2006). The module optimization problem falls under this combinatorial category. It's defined in terms of discrete decision variables. ELO objectives are functions of these discrete decision variables. The binary variables were found to be suitable for this type of combinatorial optimization problem. A LC alternative is selected for an element if the corresponding decision variable is 1 , and not selected if the variable is 0 .

The module focuses on finding a set of Pareto solutions per improvement type and per program year. In this research, Pareto solutions encompass the optimal or near-optimal (very close to optimal) solutions. The solutions serve as the fundamental inputs for the subsequent optimization modules. The module optimization problem requires a simultaneous optimization of multiple competing objectives and seeks to arrive at the best trade-off between them. The aim is to obtain 
a diverse set of ELO solutions as close as possible to the Pareto frontier. For a giving candidate bridge, the optimization problem goal is to simultaneously minimize the bridge LCC and maximize the bridge LCC benefit and/or health indices at different points in time subject to a restricted decision variable space; only one LC alternative (one choice) must be picked from a set of best feasible LC alternatives (multiple choices) for each deficient element. The alternative feasibility screening process discussed in the previous section identifies the best feasible LC alternatives per improvement type and per program year for each deficient element. Results associated with these best feasible LC alternatives are grouped and rearranged to capture all deficient elements and cover the entire program period. These arranged results are used as the optimization input parameters.

One common application of the knapsack problem is in capital budgeting (Patidar, 2006). A simple knapsack problem can be explained using this analogous situation. For instance, a problem involves a set of items and a specified knapsack of capacity, where each item is associated with a certain profit and weight. The objective is to find a subset of items such that the sum of these weighted items does not exceed the specified knapsack capacity and yields a maximum combined profit. This simple knapsack problem has many variations in the operations research literature. The problem is considered a MCKP due to the set of choices for each class (Patidar, 2006). Generally, a MCKP involves a set of different classes, each class contains multiple items, and the objective is to pick exactly one (restricting the decision variable space) and maximize the overall profit without exceeding the specified knapsack capacity.

A MCKP is considered as an NP-hard (non-deterministic polynomial-time hardness) optimization problem - required time to achieve optimal solutions grows exponentially with the size of decision variable space. Typically, MCKPs are solved using heuristics or metaheuristics to achieve an approximate (near-optimal rather than optimal) solutions within a reasonable computational effort 
(Thompson et al., 2008). The next section covers the heuristic optimization algorithm developed for this purpose. The module MCKP can be mathematically expressed as follows:

\section{Objectives}

Minimize $L C C_{b r g_{k}}^{p j}$

Maximize $B N T_{b r g_{k}}^{p j}$

$\forall p \in I$

Maximize $H I_{b r g_{k}}^{y p j}$

$\forall j \in T$

$\forall y \in H$

Subject to

$\sum_{r \in A_{k l}^{p j}} X_{l r}=1$

$\forall l \in D_{k}^{p j}$

where

$L C C_{b r g_{k}}^{p j}=U S C_{b r g_{k}}^{p j}+M I C_{b r g_{k}}^{p j}+\sum_{i \in E_{k}} P L C C_{e l m_{i}}^{v_{p j 1}}+\sum_{l \in D_{k}^{p j}} \sum_{r \in A_{k l}^{p j}}\left[X_{l r}\left(P L C C_{e l m_{l}}^{v_{p j r}}-P L C C_{e l m_{l}}^{v_{p j 1}}\right)\right]$
$B N T_{b^{p j} g_{k}}^{p j}=U S C_{b^{\prime} g_{k}}^{b}-U S C_{b r g_{k}}^{p j}-M I C_{\text {brg }_{k}}^{p j}+\sum_{i \in E_{k}} B N T_{e l m_{i}}^{v_{p j 1}}+\sum_{l \in D_{k}^{p j}} \sum_{r \in A_{k l}^{p j}}\left[X_{l r}\left(B N T_{e l m_{l}}^{v_{p j r}}-B N T_{e l m_{l}}^{v_{p j 1}}\right)\right]$

$H I_{\text {brg }_{k}}^{\text {ypj }}=\frac{\sum_{i \in E_{k}}\left[H I_{e l m_{i}}^{v_{p j 1}}\left(W_{e l m_{i}} Q_{e l m_{i}}\right)\right]+\sum_{l \in D_{k}^{p j}} \sum_{r \in A_{k l}^{p j}}\left[X_{l r}\left(W_{e l m_{l}} Q_{e l m_{l}}\right)\left(H I_{e l m_{l}}^{y v_{p j r}}-H I_{e l m_{l}}^{y v_{p j 1}}\right)\right]}{\sum_{i \in E_{k}}\left(W_{e l m_{i}} Q_{e l m_{i}}\right)}$

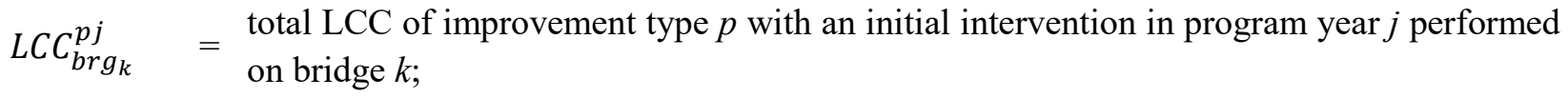
$B N T_{b r g_{k}}^{p j}=\begin{aligned} & \text { total LCC benefit of improvement type } p \text { with an initial intervention in program year } j \\ & \text { performed on bridge } k ;\end{aligned}$

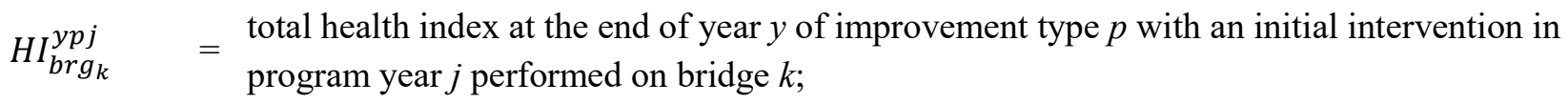




$$
\begin{aligned}
& X_{l r} \quad=\begin{array}{l}
\text { binary decision variable }\left(X_{l r}=1 \text { if a LC alternative } r \text { of a deficient element } l\right. \text { is selected, } \\
\text { otherwise } \left.X_{l r}=0\right) ;
\end{array} \\
& v_{p j r} \quad=\text { improvement type } p \text { LC alternative } r \text { with an initial intervention in program year } j \text {; } \\
& \text { PLCC } v_{\text {elm }}^{v_{i}}=\text { total preservation LCCs incurred in element } i \text { LC alternative } v_{p j r} \text { profile; } \\
& B N T_{e l m_{i}}^{v_{p j r}}=\begin{array}{l}
\text { sum of all discounted preservation action costs incurred in element } i \text { LC alternative } v_{p j r} \\
\text { profile; }
\end{array} \\
& H I_{\text {elm }}^{y v_{p j}}=\text { element } l \text { health index at the end of year } y \text { in LC alternative } v_{p j r} \text { profile; } \\
& W_{\text {elm }} \quad=\text { health index weight for element } i \\
& Q_{\text {elm }}=\text { quantity of element } i \\
& \text { sum of all discounted user costs incurred due to bridge } k \text { (for FCI and REP improvement } \\
& \begin{aligned}
U S C_{b r g_{k}}^{p j}= & \text { types, only user costs between beginning of program year } 1 \text { and end of program year } j \text { are } \\
& \text { considered, otherwise from the entire analysis period); }
\end{aligned} \\
& U S C_{b r g_{k}}^{b} \quad=\text { sum of all discounted base user costs incurred over the entire analysis period for bridge } k \text {; } \\
& M_{I C_{\text {brg }}}^{p j}=\begin{array}{l}
\text { discounted major cost of improvement type } p \text { performed on bridge } k \text { in program year } j \text { (for } \\
\text { MRR improvement type, the cost is equals to zero); }
\end{array} \\
& E_{k} \quad=\text { set of bridge } k \text { elements; } \\
& D_{k}^{p j} \quad=\begin{array}{l}
\text { set of bridge } k \text { deficient elements for improvement } p \text { with an initial intervention in program } \\
\text { year } j ;
\end{array} \\
& A_{k l}^{p j}=\begin{array}{l}
\text { set of best feasible LC alternatives of deficient element } l \text { of bridge } k \text { for improvement } p \\
\text { with an initial intervention in program year } j ;
\end{array} \\
& I \quad=\text { set of the three improvement types (i.e., MRR, FCI, and REP); } \\
& T \quad=\text { set of all program years (i.e., the program period); and } \\
& H \quad=\text { set of specified years in the analysis period. }
\end{aligned}
$$

Formulating the problem correctly is so essential to achieve high-quality solutions. Different formulations for overcoming the size of the problem were investigated. The proposed formulation is flexible enough to accommodate various ELO goals with one common constraint (i.e., picking 
only one LC alternative for each deficient element) for an improvement type and a program year. The formulation is suitable for the three improvement types (i.e., MRR, FCI, and REP) and any analyzed program year. For example, an ELO goal may involve minimizing the bridge LCC, Equation (6.2), maximizing the bridge LCC benefit, Equation (6.3), and maximizing the bridge health indices at the end of years 20 and 40, Equation (6.4). In this example, four objectives subject to one picking criterion will be optimized for the specified improvement type and program year.

This generalized formulation calls for an individual optimization for each program year. This yearby-year optimization strategy decomposes the optimization problem and further reduces the number of decision variables. A multi-year strategy generates a large number of decision variables. The computational time to reach convergence is significant, especially when dealing with a large network of bridges. To explain, deploying a multi-year optimization strategy generates 20,000 decision variables over a program period of 10 years for a bridge with 20 deficient elements, where each of them is associated with $100 \mathrm{LC}$ alternatives. However, the year-by-year strategy generates only 2,000 decision variables. This reduced number of decision variables is considered practicable even for a large network of bridges.

In this module, the different ELO objectives (bridge LCC, LCC benefit, and health index) are mathematically formulated. Only one constraint (i.e., picking criterion) is incorporated. Constraints that guarantee performance and satisfy the budget limitation are not being considered at this level. At the bridge level and network level, these constraints are integrated in the problem formulation. A set of Pareto solutions (each solution is a combination of best feasible LC alternatives identified for all deficient elements) per improvement type and per program year is recommended for each bridge in the portfolio. Making the optimization problem as an unconstrained problem (except for the picking criterion) at this level increases the search effort 
and generates unwanted element-level Pareto solutions - that can't satisfy either the budget or performance constraint at the bridge level or network level. The elimination of unwanted ELO solution is basically delayed at this level. Nevertheless, this approach permits to explore enough areas of the search space, and therefore increasing diversity of ELO solutions.

As explain earlier, the decision in the module optimization problem is a binary choice — one of the best feasible LC alternatives is either selected or rejected for each deficient element. A unique feasible solution in the solution space is represented by a vector of binary decision variables called "chromosome." The encoding of decision variables is illustrated and discussed later in this chapter. The non-dominated solutions of the entire feasible solution space constitute the Pareto solution set. Equation (6.1) restricts the decision variable space. This constraint equation guarantees the selection of only one best feasible LC alternative for each deficient element. LC Alternative 1 is always assigned to the non-deficient elements. For MRR improvement type, the non-deficient elements don't experience preservation actions; MRR LC Alternative 1 (representing the DN LC Alternative) is assigned to each of them. For FCI and REP improvement types, the non-deficient elements still get replaced, but no preservation actions will follow; FCI/REP LC alternative 1 is assigned to mimic this scenario. In contrasts, the deficient elements undertake the recommended LC alternatives.

Each element regardless of its deficiency status always contributes to the bridge LCC, LCC benefit, and health indices calculations. The element-level objectives, Equations (6.2), (6.3), and (6.4), are interdependent on the decision variable, $X_{l r}$. The overall bridge LCC, benefit, or health index consists of three distinct contributors: deficient elements, non-deficient elements, and bridge itself. The contributions of deficient elements are summed over all deficient elements, $D_{k}^{p j}$, and all best LC alternatives, $A_{k l}^{p j}$, after applying their corresponding decision variables. However, the 
bridge and its non-deficient elements do not associate with any decision variables; their contributions are configured separately.

Equation (6.2) defines the bridge LCC per improvement type and per program year. The equation is derived from Equation (5.4) in Chapter 5. The different components of this equation are explicitly defined in Chapter 5. The equation factors the different types of LCCs associated with the deficient and non-deficient elements - combining preservation LCCs incurred by the best feasible LC alternatives of all deficient elements. $A_{k l}^{p j}$ is the set of best feasible LC alternatives of deficient element $l$ of bridge $k$ for improvement $p$ (MRR, FCI, or REP) with an initial intervention in program year $j$. The set is determined by the alternative feasibility screening process (as discussed earlier in this chapter). LC Alternative 1 is always assigned to the non-deficient elements. Thus, only the LCCs incurred by these LC alternatives are factored in the overall bridge LCC expression. The equation incorporates any incurred total user cost and major improvement cost performed on the entire bridge. These costs per improvement type and per program year are separately computed by the LCC model of the improvement module (refer to Chapter 5 for the LCC model).

Equation (6.3) defines the bridge LCC benefit per improvement type and per program year. The equation is originated from Equation (5.8) in Chapter 5. Basically, the bridge LCC benefit per improvement type and per program year is the LCC savings - the difference between its total base LCC, Equation (5.7) in Chapter 5, and its LCC defined by Equation (6.2). Equation (6.4) defines the overall bridge health index per improvement type and per program year. It's expressed in element health indices. Equation (4.29) in Chapter 4 was used to arrive to this expression of Equation (6.4). In a similar way, weighted element health indices at the end of the analysis year specified in set $T$ for deficient and non-deficient elements are combined and divided by the sum of 
the element health index weights. Again, the deficient element health indices are associated with the best feasible LC alternatives of $A_{k l}^{p j}$, and the non-efficient elements with the LC Alternative 1.

\subsection{Heuristic Element-Level Optimization Algorithm}

The simplest approach to solve this large size of optimization problem is through

(1) the utility function, constructing a single-objective utility function representing all objectives by deploying a process of weighting, scaling, and amalgamation; or

(2) the weighted sum method, computing the sum of weighted objectives (where each objective is multiplied by a weight representing its relative importance).

The approach transfers the MOO problem to a single-objective problem that could be easily solved by mathematical programming algorithms/methods such as Branch and Bound algorithm, Simplex method, and Lagrange-Multiplier method. Although the approach is straightforward and guarantees global optimality, it's sensitive to the selected weights, requires advance knowledge of relative importance of each objective, and limits solution diversity. It requires several independent runs by varying weights to achieve the desired diversity. Thus, the computational cost will be more significant. This approach is deemed not effective for a "true" MOO problem (Talbi, 2009) (see Chapter 2 for more discussion). Deb (2009) specified two main goals of a "true" MOO algorithm:

(1) Able to obtain a set of solutions close to the optimal solutions

(2) The obtained set of solutions should be diverse to represent the spread of a true set of optimal solutions

In the literature review as part of Task 1, several MOO algorithms were evaluated based on these two goals, and in terms of computational speed, accuracy and robustness. The literature review revealed that EAs are well-suited for this type of problems. EAs are population-based search 
metaheuristics inspired by Darwinian evolutionary theory. The ability of a population to evolve into the Pareto frontier in a single run makes EAs attractive for solving MOO problems. As stated earlier, mathematical programming algorithms/methods require repetitive runs to find a set of Pareto solutions; and yet, these repetitive runs do not necessarily guarantee convergence to optimality. However, EAs permit to obtain a high quality of Pareto solutions in a single run (Taboada, 2007). EAs are divided in several branches such as GA, SFL, ACO, and PSO (refer to Chapter 2 for more discussion on EAs). GAs are stochastic search that rely on the "survival of the fittest" principle from the biological sciences, and the use of evolution operators makes them very effective in performing global search. They are widely used and capable of handling problems with many decision variables (Kachua, 2011). NSGA-II is a well-known metaheuristic algorithm among the GAs. An overview of GAs and the metaheuristic NSGA-II is provided in Chapter 2. NSGA-II is capable of handling large-sized NP-hard combinatorial optimization problems. It includes the following three features (Deb et al., 2002):

(1) deploys an elitist principle,

(2) deploys an explicit diversity preserving mechanism, and

(3) emphasizes non-dominated solutions.

NSGA-II is robust and reliable for solving MOO problems with less computational efforts. For optimization problems with a manageable size, it guarantees diversity of obtained solutions and convergence to a near true Pareto frontier. Because of all these advantages, NSGA-II is used as the main optimizer for the three optimization modules. NSGA-II was customized to accommodate the optimization problem characteristics for each these modules. Though, the optimizer algorithm can be substituted by any other metaheuristic algorithm with similar capabilities such as SPEA-2, PESA and MOGA. 
Figure 6.4 presents the flowchart of the heuristic algorithm designed to solve the module optimization problem. NSGA-II is integrated within the presented algorithm to handle optimization tasks. The brute-force (or exhaustive) search technique is deployed to enumerate and examine obtained solutions. For each program year, the brute-force search technique systematically assesses every single solution after several evaluations (for NSGA-II, the product of population size and total number of generations equals to the total number of evaluations). If all solutions of this total number of evaluations satisfy the constraints, they are then considered feasible, and the algorithm moves on to the next program year. Otherwise, if any solution violates a constraint, the whole set of solutions is rejected, the total number of evaluations is increased by an increment, and new solutions are obtained to be assessed for the same program year. This simple search technique controls the number of runs per program year, efficiently manages the computational time, and guarantees the feasibility of every single produced solution.

Users set the population size, initial evaluations, evaluation increment, maximum evaluations, number of bridges to be evaluated, program period, and improvement type. The initial evaluations, population size, including other optimizer default parameters (such as crossover and mutation probabilities) must be carefully chosen. These genetic algorithm parameters and inputs are essential to ensure a high quality of obtained solutions. The quality of obtained solution sets should be evaluated using performance metrics or through experimental tests. Performance metrics can be used to examine the performance of the genetic optimizer. As mentioned in Chapter 2, Okabe and Sendhoff (as cited in Riquelme et al., 2015) stated that performance metrics (such as hypervolume, generational distance, epsilon indicator, and inverted generational distance metrics) generally consider the following three aspects of a solution set:

(1) the convergence, i.e., closeness to the theoretical Pareto optimal frontier; 
(2) the diversity, i.e., distribution as well as spread; and

(3) the number of solutions.

The convergence, optimality of the solutions, diversity along the Pareto frontier, computational time, computer memory use, etc. can also be assessed by conducting different experiments. The best combination of parameters, initial evaluations, and population size can be identified by trial and error. For instance, a consistent Pareto frontier is an indication of convergence or a high quality of solutions. Any observed difference in the Pareto frontier shape when increasing the total number of initial evaluations or population size requires adjustment of certain inputs or parameters. The key steps of the designed heuristic algorithm are as follows:

1. The algorithm starts with the first bridge $(i=1)$, and a total number of evaluations (Eval) equals to the total number of initial evaluations (Eval_inc).

2. For each program year $j$, best feasible matrices associated with bridge $i$ and improvement type $p$ are located from the improvement module output data (refer to Chapter 5).

3. A random initial population of size $N 1$ is generated following the chromosome structure (discussed in the subsequent section).

4. While the total number of evaluations $($ Eval + Eval_inc $)$ is less than the specified maximum value (Eval_max), the brute-force search technique is deployed.

5. The optimizer NSGA-II is called to solve the optimization problem for these evaluations.

6. The obtained ELO solution results of these evaluations are saved.

7. Feasibility of the obtained solution set is verified.

8. If all constraints are met, the algorithm exits the while loop of brute-force search technique; otherwise, the total number of evaluations (Eval) is increased by an increment (Eval_inc), 
the population is taken as the population of this number of evaluations, and steps 5,6 and 7 get repeated.

9. The algorithm advances to the next program year $(j=j+1)$ and repeats the whole process again (the above steps).

10. Once the entire program period is covered, the algorithm progresses to the next bridge $(i=i+1)$, and so on.

11. The algorithm terminates after all $n b$ bridges being considered for each program year. 


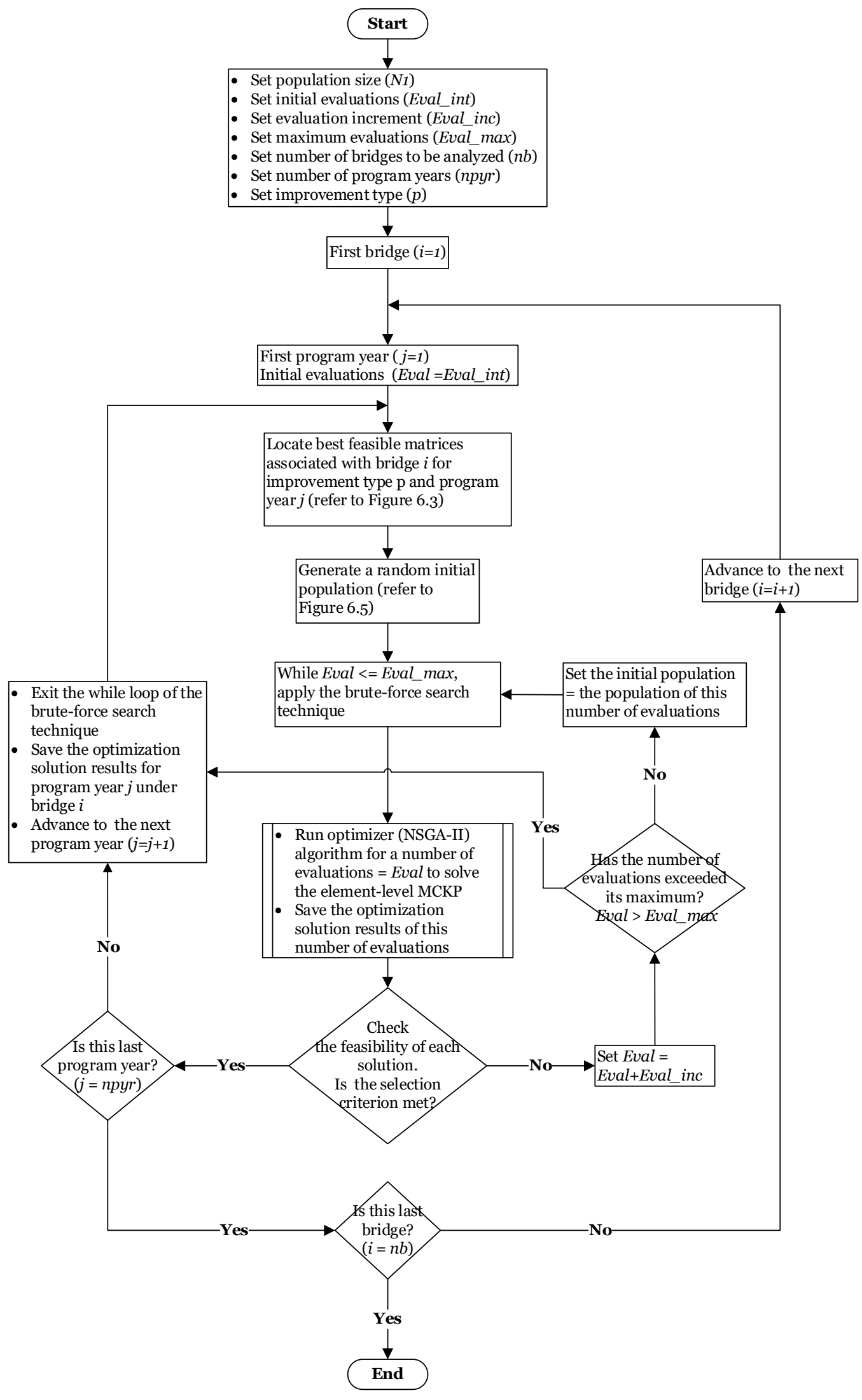

Figure 6.4 Heuristic element-level optimization algorithm 


\subsection{Mapping of Decision Variables}

There is a considerable literature on GAs. Chapter 2 overviews the basics in greater detail. GAs generate a set of chromosomes (a population of solutions). Each of them is made of a series of genes. Each of these genes represents a decision variable (controlling features of the chromosome). The entire chromosome represents a solution to the problem. A selection/fitness operator is commonly used to evaluated the "fitness" of a chromosome. It is intended to improve the population quality by mimicking the "survival of the fittest." The less fitted ones are eliminated. There are many methods in selecting the best chromosomes, such as roulette wheel selection, stochastic universal sampling selection, rank selection, tournament selection, and steady-state selection.

The module genetic optimizer (i.e., NSGA-II) relies on an elitism operator for chromosome selection. The best (elite) chromosomes are copied to a new population. The elitism operator increases the performance of GA; it ensures the best chromosomes remain in the population. For each generation, new chromosomes are created by two mating operators: crossover and mutation. Crossover operator mimics the biological reproduction. The fittest chromosomes are mixed to form new chromosomes. The process is repeated until the entire offspring population is formed. Since these chromosomes survived the selection, the crossover operator preserves features of the two best chromosomes - crossover between chromosomes is implemented by a single/multi-point crossover or a uniform binary crossover. Mutation operator adds randomness to the chromosomes

of the offspring population. The randomness introduces diversity in the population; and therefore, it improves the search space exploration for superior solutions.

Once a new generation is produced using these three genetic operators, the process of selection 
and mating is repeated until finding the optimal or near-optimal solutions to the problem. The iteration continues until the predetermined number of generations or a certain termination criterion is reached. However, the process requires an effective mapping mechanism (refer to as "encoding") of chromosomes. Binary, permutation, value, and tree encoding are among the most popular encoding schemes in GAs. The key of achieving better performance and truthful representation of the problem lies in the chromosome structure-ability to encode a solution to a series of genes to form a chromosome. Frangopol and Liu (as cited in cited in Kachua, 2012) emphasized that the problem encoding demands most of the effort, comparing to the problem itself.

GAs can process a large number of chromosomes per iteration (representing one population). Although a very large population size increases the search space, the large number of chromosomes adversely impact the computer memory and computational time (Taboada, 2007). The larger the population size, the more computational efforts will take. Hence, it's essential to control the population size in terms of number of chromosomes. Similarly, the number of genes (i.e., decision variables) per chromosome affects the GA performance. The alternative screening process and the year-by-year optimization strategy (described earlier) both combined make the problem tractable. As demonstrated previously, considering only the best feasible LC alternatives and calling for an individual optimization under each program year considerably reduces the number of decision variables per chromosome.

Figure 6.4 illustrates the chromosome encoding used to represent an ELO solution. A chromosome encompasses a total number of $D . n$ genes, where $D$ is the total number of deficient elements, and $n$ is the largest total number of feasible $\mathrm{LC}$ alternatives considering all deficient elements. A binary encoding scheme is adapted because of its simplicity and the allocation characteristics of the problem. Each chromosome has one binary string of genes (composed of either 0 or 1), and each 
gene represents a characteristic of the ELO solution. The figure illustrates the process of assigning decision variables to a feasible MRR LC alternative matrix of size $(D \times n)$ of a sample bridge (Bridge 47) for a program year (program year 4). In this illustrative example, the first $n$ genes represent the first deficient element (Elm_12), the next $n$ genes represent the next element (Elm_107), and so on until the last element (Elm_330). A gene with a value of 1 signifies a LC alternative is selected for the element; in contrast, a value of 0 signifies no selection. This encoding ensures the representation of all deficient elements and their corresponding feasible LC alternatives. A population of size N1 simply includes $N 1$ chromosomes with different random binary values. For any program year, each chromosome is structured in the same manner. 


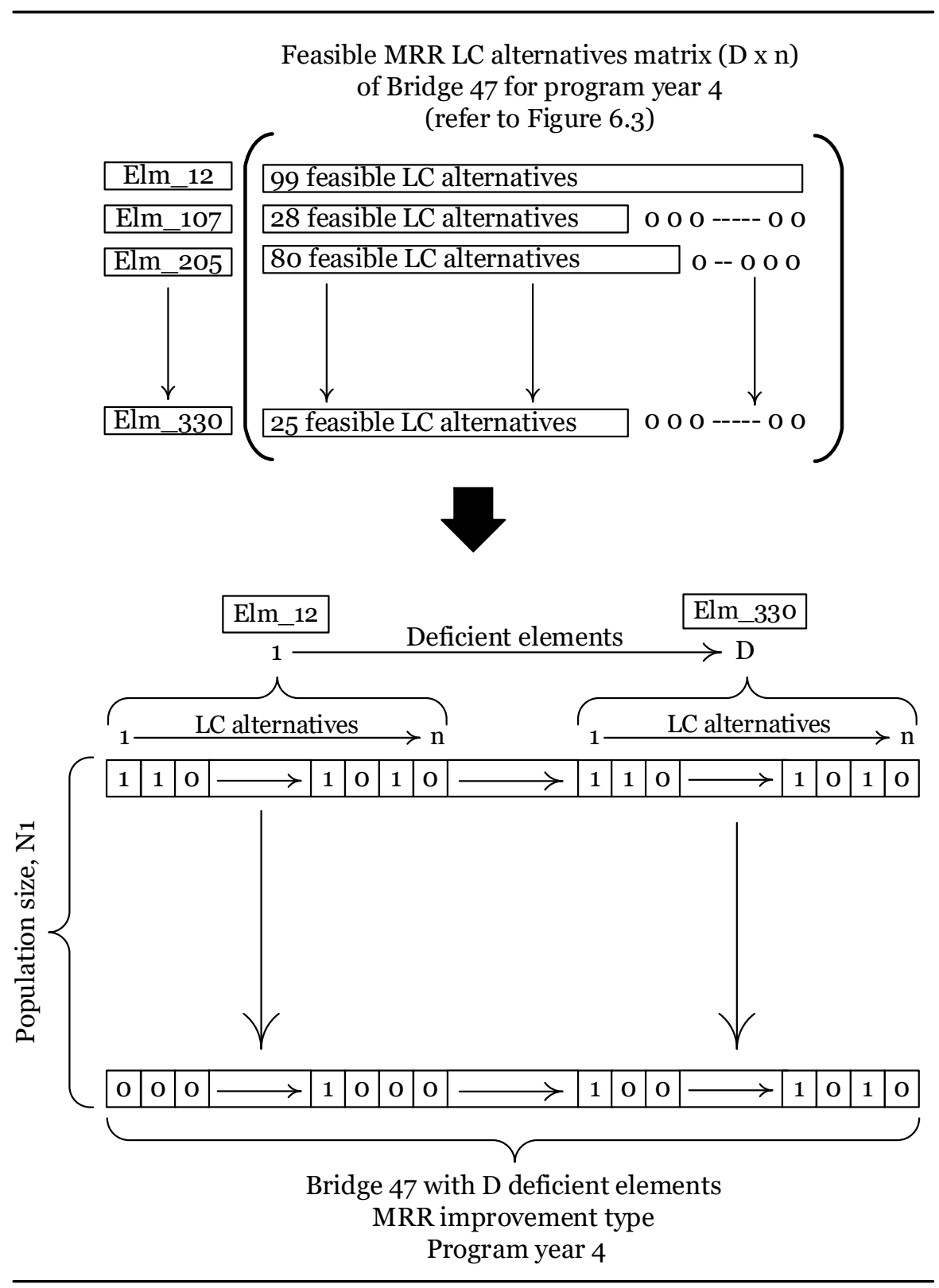

Figure 6.5 Illustration of the chromosome encoding of an ELO solution 


\subsection{Example of Module Results}

The methodology was implemented through different examples to test concepts, prove effectiveness, and demonstrate potential benefits. These examples provided an excellent opportunity to apply the EB-MOO methodology. The examples include detailed tables and charts to communicate outcomes from the different modules. A portfolio of 40 sample bridges (introduced in Chapter 3) is used to implement the different stages of the EB-MOO methodology. These sample bridges were selected based on common features, attributes, and data completeness. Table 3.2 in Chapter 3 provides more information related to the characteristics of these sample bridges.

As discussed in Chapter 3, the MATLAB-based tool prototype, structured around the five EBMOO modules, was mainly developed for the implementation. The tool prototype is considered as a "proof of concept" rather than a complete rigorous software ready for operational implementation. Using the tool prototype, the preliminary outcomes associated with these sample bridges produced by the improvement module were transferred to this ELO module. For each improvement type and each program year, the tool produced ELO solutions, recommended set of intervention actions, predicted performance, and determined budget requirements.

As stated earlier, the year-by-year optimization strategy is adapted to decompose the ELO problem and further reduce the number decision variables. An ELO run is independently performed for each program year. Through the example presented herein, only one program year (i.e., program year 6) is examined for illustration. One of the sample bridges in the portfolio (i.e., Bridge 26) is used in this example. Table 6.5 presents the NBI condition ratings of this sample bridge and other related attributes. Table 6.6 shows the latest condition states of the 11 elements of this sample 
bridge. The bridge was built in 1952. It's a steel multi-girder bridge and has a sufficiency rating of $77.2 \%$. The bridge was identified with one functional deficiency: bridge roadway width.

Table 6.5 NBI condition ratings of Bridge 26

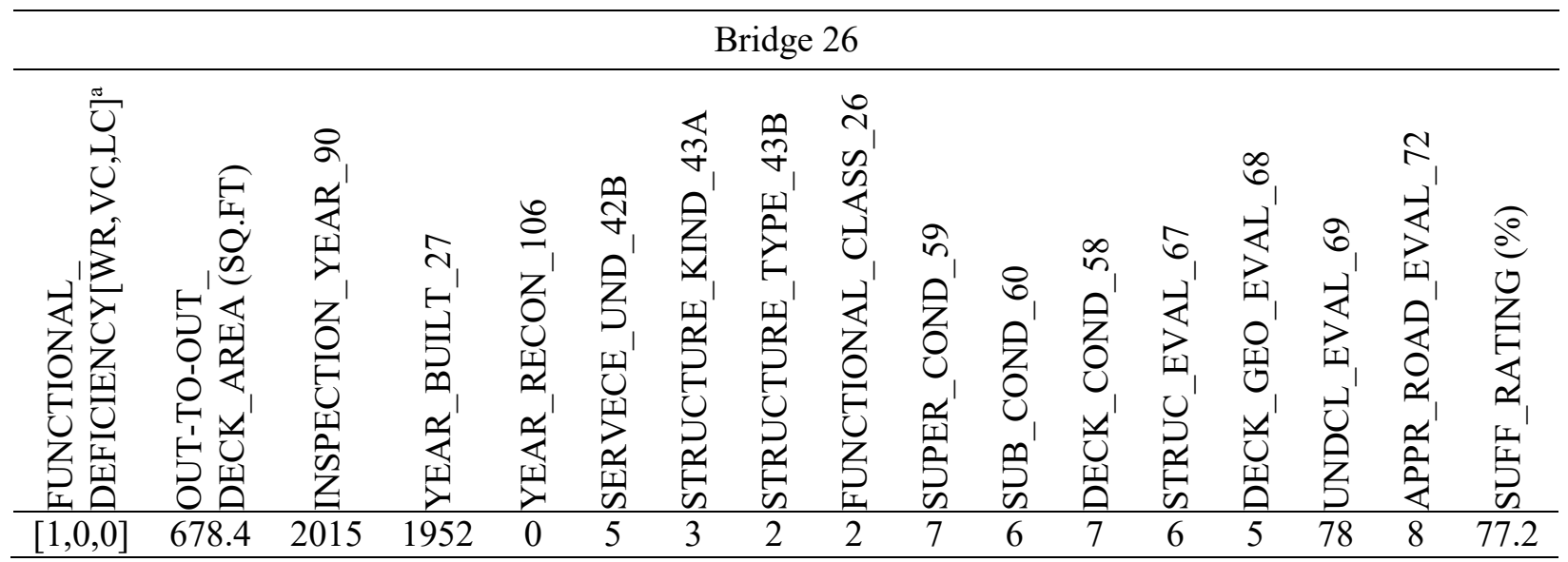

Note. $\mathrm{WR}=$ width required; $\mathrm{VC}=$ vertical clearance; $\mathrm{LC}=$ load capacity.

${ }^{a}$ The value of 1 represents to the existence of the functional deficiency, otherwise the value of 0 .

Table 6.6 Condition states of the 11 elements of Bridge 26

\begin{tabular}{lccccccccccc}
\hline & \multicolumn{10}{c}{ Elements of Bridge 26} \\
\cline { 2 - 12 } & 12 & 107 & 215 & 225 & 234 & 301 & 311 & 313 & 330 & 331 & 510 \\
\hline CS1 (\%) & 95 & 93.81 & 100 & 75 & 0 & 92.45 & 0 & 0 & 100 & 97.14 & 95 \\
CS2 (\%) & 5 & 2.50 & 0 & 0 & 93.68 & 7.55 & 100 & 100 & 0 & 2.62 & 5 \\
CS3 (\%) & 0 & 3.69 & 0 & 25 & 6.32 & 0 & 0 & 0 & 0 & 0.24 & 0 \\
CS4 (\%) & 0 & 0 & 0 & 0 & 0 & 0 & 0 & 0 & 0 & 0 & 0 \\
Qt (sq.ft) & 7303 & 840 & 72 & 20 & 174 & 318 & 24 & 24 & 420 & 420 & 7303 \\
Welm $_{\text {el\%) }}$ & 25 & 49 & 13 & 10 & 13 & 12 & 12 & 12 & 16 & 14 & 5 \\
ESL (years) & 129 & 77 & 75 & 20 & 408 & 24 & 58 & 58 & 28 & 151 & 126 \\
RSL (years) & 69 & 16 & 16 & 0 & 180 & 0 & 0 & 0 & 0 & 90 & 67 \\
RU (\$) & 54 & 2,062 & 1,591 & 38,810 & 1,186 & 72 & 9,009 & 9,009 & 296 & 212 & 21 \\
\hline
\end{tabular}

${ }^{a} \mathrm{RSL}$ is estimated from program year 1 (2020).

Table 6.7 specifies the goals and types of the different ELO problems under the unconstrained scenarios of the example. The analysis period is set for 54 years, including a 10-year program period. The discount rate is $4 \%$ over 56 years (the analysis period plus the period from current year, 2018, to program year 1, 2020). 
Table 6.7 Defining the different ELO problems of the example

\begin{tabular}{|c|c|c|c|c|}
\hline & Sample & Type & Optimization Goals & Scenario \\
\hline \multirow{3}{*}{ 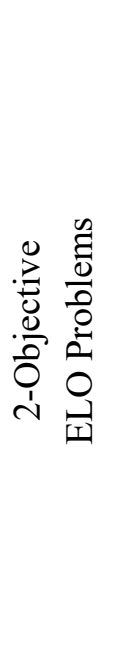 } & \multirow[t]{3}{*}{$\begin{array}{l}\text { Bridge } 26 \\
\text { with } \\
11 \\
\text { elements }\end{array}$} & \multirow[t]{3}{*}{ MCKP } & \multirow{3}{*}{$\begin{array}{l}\text { - maximize the bridge } \\
\text { health index in year } 20 \\
\text { (denoted by HI20 brg) } \\
\text { - minimize the bridge } \\
\text { LCC (denoted by } \\
\text { LCC }_{\text {brg }} \text { ) }\end{array}$} & $\begin{array}{l}\text { Scenario a: unconstrained } \\
\checkmark \quad \text { MRR, program year } 6, \\
\text { unconstrained budget and } \\
\text { performance, Figure } 6.6(\mathrm{a})\end{array}$ \\
\hline & & & & $\begin{array}{l}\text { Scenario b: unconstrained } \\
\qquad \begin{array}{l}\checkmark \\
\text { FCI, program year } 6, \\
\text { unconstrainted budget and } \\
\text { performance, Figure } 6.6(\mathrm{~b})\end{array}\end{array}$ \\
\hline & & & & $\begin{array}{l}\text { Scenario c: unconstrained } \\
\checkmark \quad \text { REP, program year } 6, \\
\text { unconstrainted budget and } \\
\text { performance, Figure } 6.6(\mathrm{c})\end{array}$ \\
\hline \multirow{3}{*}{ 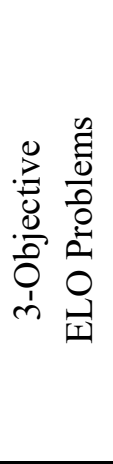 } & \multirow{3}{*}{$\begin{array}{l}\text { Bridge } 26 \\
\text { with } \\
11 \\
\text { elements }\end{array}$} & \multirow[t]{3}{*}{ MCKP } & \multirow{3}{*}{$\begin{array}{l}\text { - minimize the bridge } \\
\text { LCC (denoted by } \\
\mathrm{LCC}_{\text {brg }} \text { ) } \\
\text { - maximize the bridge } \\
\mathrm{LCC}_{\text {benefit (denoted }} \\
\text { by } \mathrm{BNT}_{\text {brg }} \text { ) } \\
\text { - maximize the bridge } \\
\text { health index in year } 20 \\
\text { (denoted by HI } 20_{\text {brg }} \text { ) }\end{array}$} & $\begin{array}{l}\text { Scenario a: unconstrained } \\
\text { Figure } 6.6(\mathrm{~d})\end{array}$ \\
\hline & & & & $\begin{array}{l}\text { Scenario b: unconstrained } \\
\text { Figure } 6.6(\mathrm{e})\end{array}$ \\
\hline & & & & $\begin{array}{l}\text { Scenario c: unconstrained } \\
\text { Figure } 6.6(\mathrm{f})\end{array}$ \\
\hline \multirow[b]{3}{*}{ 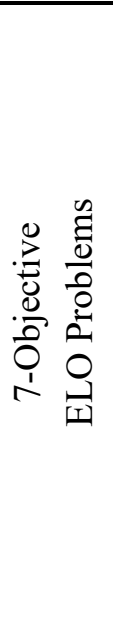 } & \multirow{3}{*}{$\begin{array}{l}\text { Bridge } 26 \\
\text { with } \\
11 \\
\text { elements }\end{array}$} & \multirow[t]{3}{*}{ MCKP } & \multirow{3}{*}{ 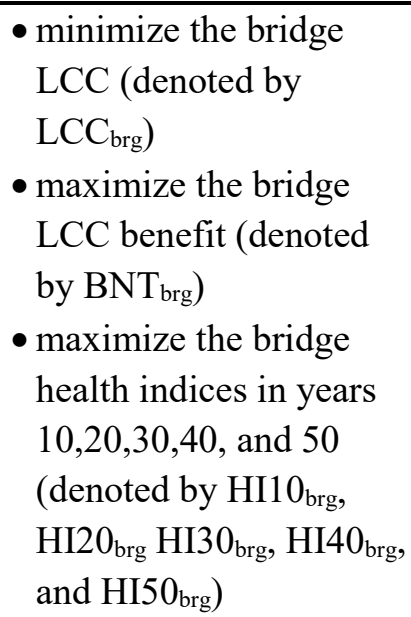 } & $\begin{array}{l}\text { Scenario a: unconstrained } \\
\text { Figure } 6.6(\mathrm{~g})\end{array}$ \\
\hline & & & & $\begin{array}{l}\text { Scenario b: unconstrained } \\
\text { Figure } 6.6(\mathrm{~h})\end{array}$ \\
\hline & & & & $\begin{array}{l}\text { Scenario c: unconstrained } \\
\text { Figure } 6.6(\mathrm{k})\end{array}$ \\
\hline
\end{tabular}


Table 6.8 shows the results associated with the 41 ELO solutions obtained for the 2-objective ELO problem, Scenario a (unconstrained budget under MRR improvement type). The optimization goal is to maximize the bridge health index in year 20 (HI20 brg $)$ and minimize the bridge LCC ( $\left.\mathrm{LCC}_{\mathrm{brg}}\right)$ for MRR improvement type with an initial intervention in program year 6 . In addition to these two main ELO objectives, the bridge initial agency cost ( $\mathrm{IAC}_{\mathrm{brg}}$ ), LCC benefit $\left(\mathrm{BNT}_{\mathrm{brg}}\right)$, and health indices at the ends of years $1,10,30,40,50$, and 54 were also determined as part of the module optimization results. 
Table 6.8 Results associated with the 41 solutions obtained for the 2-objective ELO problem, Scenario a

\begin{tabular}{|c|c|c|c|c|c|c|c|c|c|c|}
\hline Solution & $\begin{array}{c}\mathrm{IAC}_{\mathrm{brg}} \\
(\$)\end{array}$ & $\begin{array}{c}\mathrm{LCC}_{\mathrm{brg}}{ }^{\mathrm{a}} \\
(\$)\end{array}$ & $\begin{array}{c}\mathrm{BNT}_{\text {brg }} \\
(\$)\end{array}$ & $\begin{array}{l}\mathrm{HI}_{\mathrm{brg}} \\
(\%)\end{array}$ & $\begin{array}{c}\mathrm{HI} 10_{\text {brg }} \\
(\%)\end{array}$ & $\begin{array}{c}\text { HI20 } 0_{\text {brg }}{ }^{a} \\
(\%)\end{array}$ & $\begin{array}{c}\mathrm{HI} 30_{\mathrm{brg}} \\
(\%)\end{array}$ & $\begin{array}{c}\mathrm{HI} 40_{\mathrm{brg}} \\
(\%)\end{array}$ & $\begin{array}{c}\mathrm{HI} 50_{\mathrm{brg}} \\
(\%)\end{array}$ & $\begin{array}{c}\mathrm{HI}^{5} 4_{\text {brg }} \\
(\%)\end{array}$ \\
\hline 1 & $1,004,525$ & $1,298,060$ & $-1,072,572$ & 84.29 & 85.62 & 86.93 & 73.35 & 65.33 & 63.62 & 60.90 \\
\hline 2 & 331,868 & $1,128,022$ & $-902,535$ & 84.29 & 74.61 & 86.25 & 72.99 & 65.05 & 62.99 & 60.22 \\
\hline 3 & 269,591 & 812,601 & $-587,114$ & 84.29 & 74.35 & 85.66 & 72.74 & 64.90 & 63.53 & 60.79 \\
\hline 4 & 268,755 & 663,091 & $-437,603$ & 84.29 & 74.23 & 68.90 & 84.93 & 70.00 & 63.57 & 60.95 \\
\hline 5 & 347,149 & 717,100 & $-491,612$ & 84.29 & 74.74 & 69.47 & 85.19 & 70.15 & 63.03 & 60.38 \\
\hline 6 & 347,985 & $1,140,047$ & $-914,560$ & 84.29 & 74.86 & 86.25 & 72.99 & 65.05 & 62.99 & 60.22 \\
\hline 7 & $1,716,793$ & $2,085,502$ & $-1,860,015$ & 84.29 & 86.15 & 87.52 & 73.60 & 65.49 & 63.07 & 60.32 \\
\hline 8 & $1,066,802$ & $1,613,481$ & $-1,387,993$ & 84.29 & 85.88 & 87.51 & 73.61 & 65.49 & 63.07 & 60.32 \\
\hline 9 & $1,713,135$ & $2,080,609$ & $-1,855,122$ & 84.29 & 86.15 & 87.52 & 73.60 & 65.49 & 63.07 & 60.32 \\
\hline 10 & 269,591 & 816,457 & $-590,969$ & 84.29 & 74.35 & 85.77 & 72.83 & 64.98 & 63.50 & 60.76 \\
\hline 11 & 268,755 & 666,946 & $-441,459$ & 84.29 & 74.23 & 69.00 & 85.02 & 70.08 & 63.54 & 60.93 \\
\hline 12 & $1,004,525$ & $1,308,717$ & $-1,083,229$ & 84.29 & 85.62 & 87.29 & 73.48 & 65.34 & 63.03 & 60.28 \\
\hline 13 & 268,755 & 673,748 & $-448,261$ & 84.29 & 74.23 & 69.26 & 85.07 & 70.00 & 62.98 & 60.34 \\
\hline 14 & 335,526 & 851,071 & $-625,584$ & 84.29 & 74.61 & 86.21 & 73.01 & 65.05 & 62.99 & 60.22 \\
\hline 15 & $1,079,260$ & $1,620,613$ & $-1,395,125$ & 84.29 & 86.13 & 87.51 & 73.60 & 65.49 & 63.07 & 60.32 \\
\hline 16 & $1,004,525$ & $1,301,915$ & $-1,076,428$ & 84.29 & 85.62 & 87.03 & 73.44 & 65.42 & 63.59 & 60.87 \\
\hline 17 & 331,032 & 696,668 & $-471,180$ & 84.29 & 74.49 & 69.44 & 85.20 & 70.15 & 63.03 & 60.38 \\
\hline 18 & 269,591 & 817,880 & $-592,393$ & 84.29 & 74.35 & 86.01 & 72.82 & 64.97 & 63.50 & 60.76 \\
\hline 19 & 331,868 & 839,059 & $-613,571$ & 84.29 & 74.61 & 86.19 & 73.02 & 65.06 & 62.99 & 60.22 \\
\hline 20 & $1,004,525$ & $1,316,260$ & $-1,090,773$ & 84.29 & 85.62 & 87.41 & 73.56 & 65.42 & 63.00 & 60.25 \\
\hline 21 & 268,755 & 689,699 & $-464,211$ & 84.29 & 74.23 & 69.40 & 85.12 & 70.08 & 62.95 & 60.31 \\
\hline 22 & $1,082,919$ & $1,352,068$ & $-1,126,581$ & 84.29 & 86.13 & 87.50 & 73.60 & 65.48 & 63.07 & 60.32 \\
\hline 23 & $1,004,525$ & $1,303,338$ & $-1,077,851$ & 84.29 & 85.62 & 87.27 & 73.43 & 65.41 & 63.59 & 60.87 \\
\hline 24 & 347,985 & 866,610 & $-641,123$ & 84.29 & 74.86 & 86.23 & 72.99 & 65.05 & 62.99 & 60.22 \\
\hline 25 & 269,591 & 817,456 & $-591,968$ & 84.29 & 74.35 & 85.91 & 72.73 & 64.89 & 63.53 & 60.79 \\
\hline 26 & $1,066,802$ & $1,331,636$ & $-1,106,149$ & 84.29 & 85.88 & 87.47 & 73.62 & 65.49 & 63.07 & 60.32 \\
\hline 27 & 268,755 & 668,370 & $-442,882$ & 84.29 & 74.23 & 69.24 & 85.02 & 70.07 & 63.54 & 60.93 \\
\hline 28 & 268,755 & 681,292 & $-455,804$ & 84.29 & 74.23 & 69.38 & 85.14 & 70.08 & 62.95 & 60.31 \\
\hline 29 & 334,690 & 701,561 & $-476,073$ & 84.29 & 74.49 & 69.44 & 85.20 & 70.15 & 63.03 & 60.38 \\
\hline 30 & 268,755 & 663,515 & $-438,028$ & 84.29 & 74.23 & 69.00 & 85.02 & 70.08 & 63.54 & 60.93 \\
\hline 31 & 335,526 & $1,132,915$ & $-907,428$ & 84.29 & 74.61 & 86.25 & 72.99 & 65.05 & 62.99 & 60.22 \\
\hline 32 & $1,066,802$ & $1,324,517$ & $-1,099,030$ & 84.29 & 85.88 & 87.45 & 73.64 & 65.49 & 63.07 & 60.32 \\
\hline 33 & 268,755 & 667,945 & $-442,458$ & 84.29 & 74.23 & 69.14 & 84.93 & 69.99 & 63.57 & 60.96 \\
\hline 34 & $1,004,525$ & $1,309,141$ & $-1,083,654$ & 84.29 & 85.62 & 87.39 & 73.57 & 65.42 & 63.00 & 60.25 \\
\hline 35 & $1,070,461$ & $1,336,529$ & $-1,111,042$ & 84.29 & 85.88 & 87.47 & 73.62 & 65.49 & 63.07 & 60.32 \\
\hline 36 & $1,004,525$ & $1,302,914$ & $-1,077,427$ & 84.29 & 85.62 & 87.17 & 73.34 & 65.33 & 63.62 & 60.90 \\
\hline 37 & 268,755 & 674,172 & $-448,685$ & 84.29 & 74.23 & 69.36 & 85.16 & 70.08 & 62.95 & 60.31 \\
\hline 38 & $1,082,919$ & $1,625,506$ & $-1,400,018$ & 84.29 & 86.13 & 87.52 & 73.60 & 65.49 & 63.07 & 60.32 \\
\hline 39 & $1,004,525$ & $1,298,484$ & $-1,072,997$ & 84.29 & 85.62 & 87.03 & 73.44 & 65.42 & 63.59 & 60.87 \\
\hline 40 & 269,591 & 823,683 & $-598,196$ & 84.29 & 74.35 & 86.13 & 72.96 & 64.99 & 62.92 & 60.14 \\
\hline 41 & 269,591 & 813,026 & $-587,538$ & 84.29 & 74.35 & 85.77 & 72.83 & 64.98 & 63.50 & 60.76 \\
\hline
\end{tabular}

${ }^{a}$ Optimized objective. 
The recommended solutions for the ELO problems of this example are plotted in Figures 6.6(a) through (k). Figures 6.6(a), (b), and (c) display the 2-objective ELO solutions for MRR (Scenario a), FCI (Scenario b), and REP (Scenario c) improvement types, respectively. Figures 6.6(d), (e), and (f) show the 3-objective ELO solutions obtained for the three improvement types. The three radar (or spider) charts, Figures $6.6(\mathrm{~g}),(\mathrm{h})$, and $(\mathrm{k})$, relate to the 7-objective ELO problems. When dealing with more than three conflicting objectives, the relationship between them is difficult to convey visually. Thus, for the 7-objective ELO problems, radar charts are used to assist with the representation of results. The axes of these charts represent each of the objectives. The objective values are normalized to share a common scale (producing a spider-web-like appearance) and for comparing across axes.

The displayed solutions are the Pareto solutions obtained for the three improvement types (i.e., MRR, FCI, and REP) and pertain to program year 6. As emphasized previously, Pareto solutions in this research encompass the optimal or near-optimal (very close to optimal) solutions. The approach discussed earlier to verify optimally was followed for the different examples included in this dissertation. The consistency of each obtained Pareto frontier was verified by increasing the number of iterations/generations to observe any difference in shape. The obtained solutions are hosted on discontinuous Pareto frontiers (good approximation of true Pareto frontiers). 

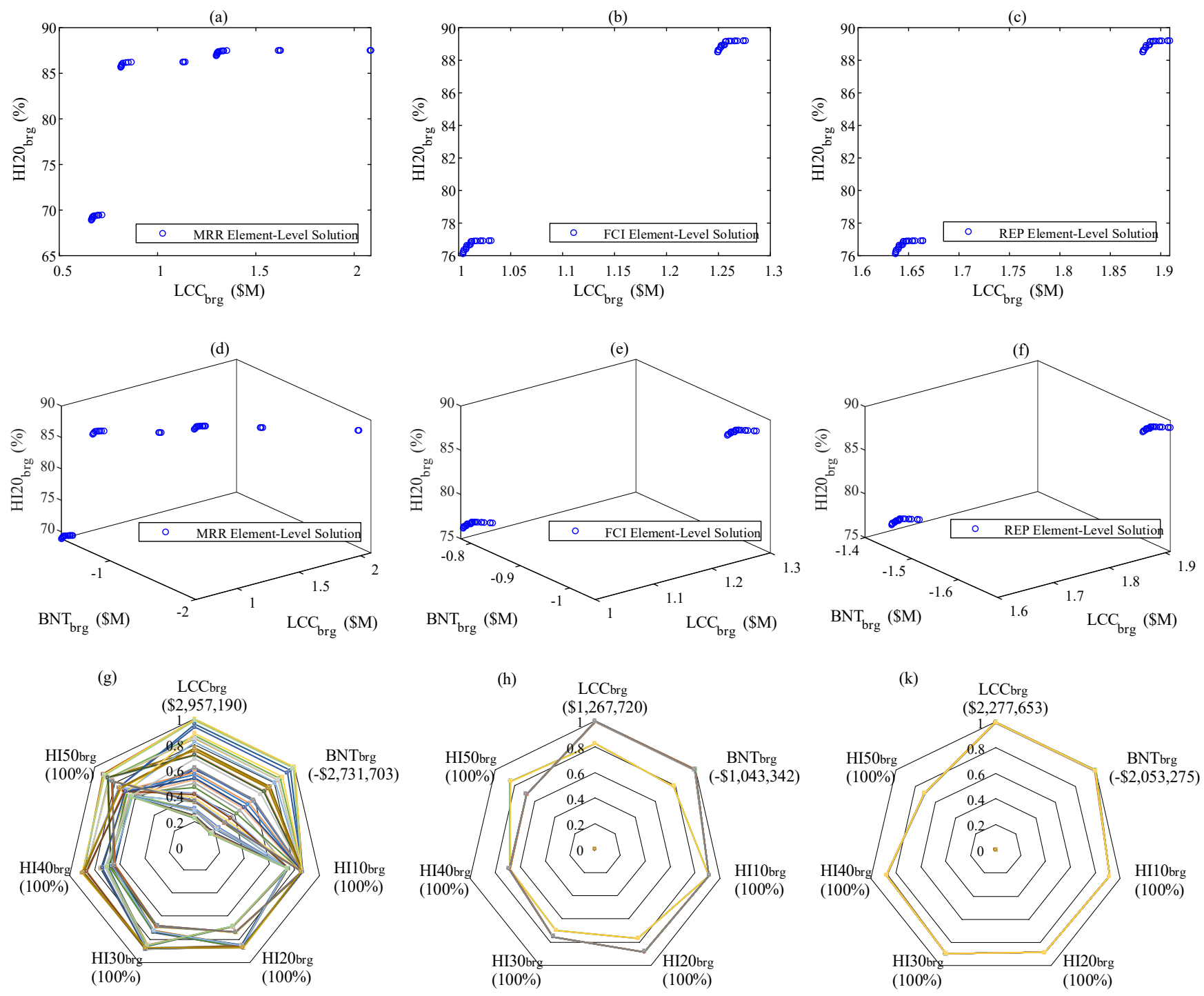

- MRR Element-Level Solutio

- FCI Element-Level Solution

$\rightarrow$ REP Element-Level Solution

Figure 6.6 Obtained solutions for the 2-objective ELO problems under (a) Scenario a, (b) Scenario b, (c) Scenario c; the 3-objective ELO problems under (d) Scenario a, (e) Scenario b, (f) Scenario c; the 7-objective ELO problems under (g) Scenario a, (h) Scenario b, (k) Scenario c 
The bridge health indices at the ends of years $1,10,20,30,40,50$, and 54 for each of these solutions are shown in Figures 6.7(a) through (k). Figures 6.7(a), (b), and (c) show the predicted bridge health indices (connected with straight lines) for the 2-objective ELO solutions. Figures 6.7(d), (e), and (f) display the predicted bridge health indices for the 3-objective ELO solutions. The bridge health indices associated with the 7-objective ELO solutions are shown in Figures 6.7(g), (h), and (k). Figures 6.7(a), (d), and (g) represent only the MRR improvement type (Scenarios a); Figures 6.7(b), (e), and (h), the FCI improvement type (Scenario b); and Figures 6.7(c), (f), and (k), the REP improvement type (Scenario c).

The obtained ELO solutions per program year produced comparable values of bridge health indices despite the differences between the LCC values. As mentioned in Chapter 4, the bridge health index is an appropriate measure to assess performance; however, it is not a complete measure of the value of the agency's investment (Chase et al., 2016). Most straight lines connecting the predicted health indices are superimposed in these figures. Changes to the overall health index are generally minuscule. Element health indices are weighted, aggregated and divided by the sum of all their weighs to constitute this overall index. Improving few element health indices (after factoring their weights) won't dramatically change the overall index. It takes substantial improvement efforts to alter the overall index.

The connected straight lines between RO LC Alternative, DN LC Alternative, or FCI/REP Alternative 1 health indices show a decline in bridge condition over time. For instance, the bridge DN health index in year $1(84.24 \%)$ is predicted to be reduced by half $(41.71 \%)$ at the end of analysis period if no improvement action is taken. Similarly, for FCI/REP Alternative 1, the scenario when no preservation actions succeed a major bridge improvement, the bridge health index is predicted to reach $47.18 \%$ by the end of analysis period. 

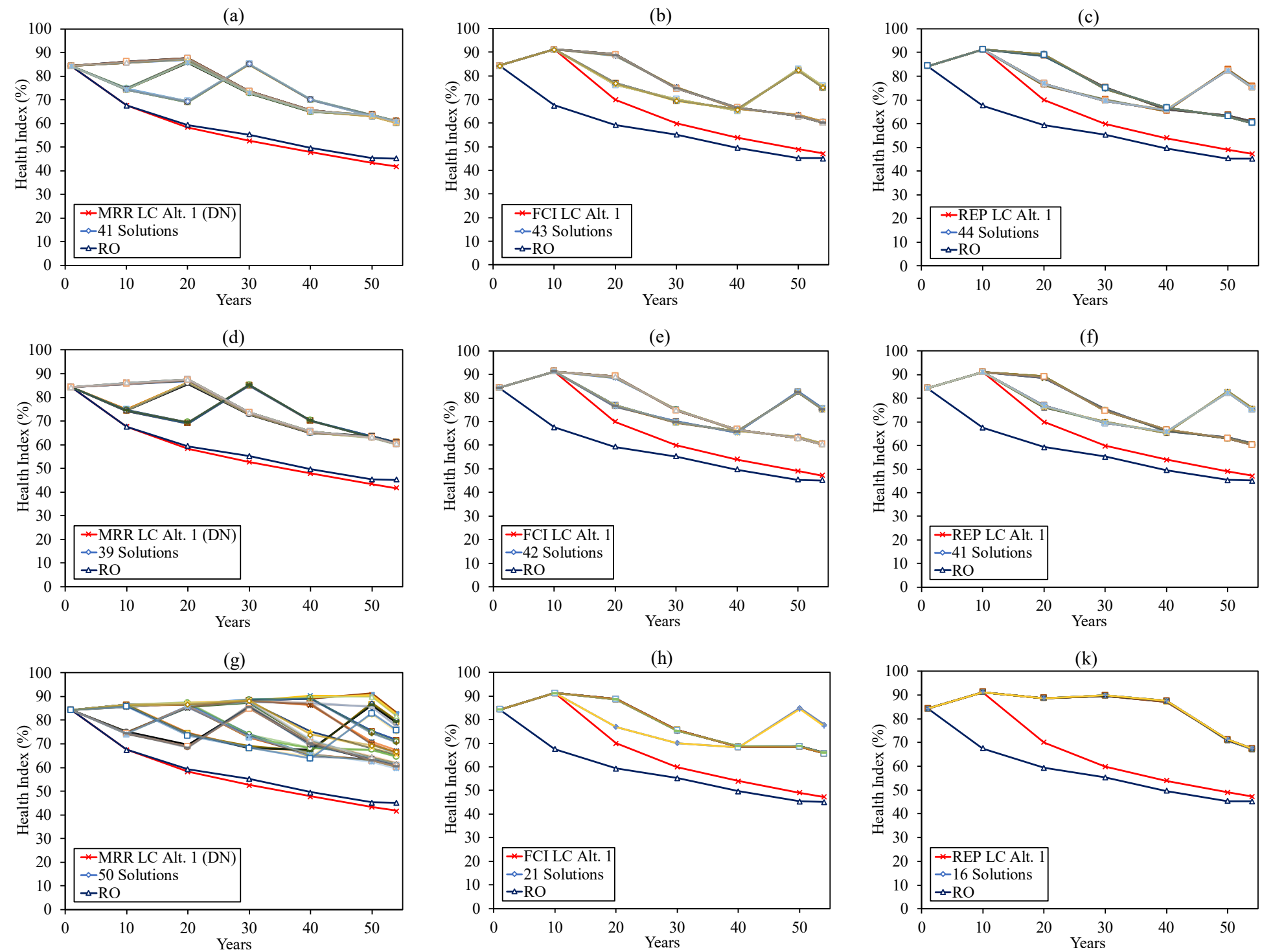

Figure 6.7 Predicted bridge health indices for the 2-objective ELO problems under (a) Scenario a, (b) Scenario b, (c) Scenario c; the 3-objective ELO problems under (d) Scenario a, (e) Scenario b, (f) Scenario c; the 7-objective ELO problems under (g) Scenario a, (h) Scenario b, (k)

Scenario c 
Table 6.9 shows the results associated with the 2-objective ELO solutions producing the least bridge LCC values for the three improvement types (Scenarios a, b, and c) with an initial intervention in program year 6 . Solutions 4,7 , and 10 produce the least bridge LCC values over the analysis period for MRR, FCI, and REP improvement types, respectively. Although the bridge is identified with a functional deficiency, Solution 4 of the preservation (MRR) improvement type produces the least bridge LCC $(\$ 663,091)$ and the highest bridge LCC benefit $(-\$ 437,603)$ —it's expected since the bridge presently has a sufficiency rating of $77.2 \%$ (representing an overall good condition). A small investment of $\$ 268,755$ ( $\mathrm{IAC}_{\mathrm{brg}}$ ) is required in program year 6 to sustain a bridge health index above $68.90 \%$ (based on HI20 brg value) over a period of 40 years.

Table 6.9 Results associated with the 2-objective ELO solutions producing the least bridge LCC values for the three improvement types

\begin{tabular}{|c|c|c|c|c|c|c|c|c|c|c|}
\hline \multicolumn{11}{|c|}{ MRR Improvement Type } \\
\hline Solution & $\begin{array}{c}\text { IACbrg } \\
(\$)\end{array}$ & $\begin{array}{c}\mathrm{LCC}_{\mathrm{brg}}{ }^{\mathrm{a}} \\
(\$)\end{array}$ & $\begin{array}{c}\mathrm{BNT}_{\mathrm{brg}} \\
(\$)\end{array}$ & $\begin{array}{c}\mathrm{HI} 1_{\text {brg }} \\
(\%)\end{array}$ & $\begin{array}{c}\mathrm{HI}_{10} 0_{\mathrm{brg}} \\
(\%)\end{array}$ & $\begin{array}{c}\mathrm{HI} 20_{\mathrm{brg}}{ }^{\mathrm{a}} \\
(\%)\end{array}$ & $\begin{array}{c}\mathrm{HI}^{3} 0_{\mathrm{brg}} \\
(\%)\end{array}$ & $\begin{array}{c}\text { HI } 40_{\mathrm{brg}} \\
(\%)\end{array}$ & $\begin{array}{c}\mathrm{HI} 50_{\mathrm{brg}} \\
(\%)\end{array}$ & $\begin{array}{c}\mathrm{HI}^{5} 4_{\text {brg }} \\
(\%)\end{array}$ \\
\hline 4 & 268,755 & 663,091 & $-437,603$ & 84.29 & 74.23 & 68.90 & 84.93 & 70.00 & 63.57 & 60.95 \\
\hline \multicolumn{11}{|c|}{ FCI Improvement Type } \\
\hline 7 & $1,453,447$ & $1,004,093$ & $-779,714$ & 84.29 & 91.20 & 76.12 & 70.08 & 65.29 & 82.90 & 75.77 \\
\hline \multicolumn{11}{|c|}{ REP Improvement Type } \\
\hline 10 & $2,319,632$ & $1,637,006$ & $-1,412,627$ & 84.29 & 91.20 & 76.12 & 70.08 & 65.29 & 82.90 & 75.77 \\
\hline
\end{tabular}

${ }^{a}$ Optimized objective.

Figures 6.8(a) through $(\mathrm{k})$ show the predicted element health indices associated with the solutions producing the least bridge LCC values for the 2-, 3-, and 7-objective ELO problems under the three improvement types with an initial intervention in program year 6. As expected, the straight lines connecting element health indices are all situated above the health index thresholds $(60 \%$ every 10 years, horizontal dashed lines in black) specified by Criteria 1 of the alternative feasibility screening process (discussed in Chapter 5). The horizontal dashed lines in red represent the health index lower-frontiers (deficiency screening thresholds, a minimum health index of $80 \%$ every 10 
years). The dashed curves above this frontier distinguish the non-deficient elements from the deficient ones. The figures representing the MRR improvement type show Elements 215 and 331 as the non-deficient elements (based on the preservation only screening covered in Chapter 5); however, the other figures representing the FCI or REP improvement type consider only Element 234 as the non-deficient element (based on the post-major improvement screening covered in Chapter 5). The thick connected lines in black represent the overall bridge health indices. Figure 6.8(1) includes the predicted DN element health indices over the analysis period-produced by DN LC Alternative. The predicted RO element health indices are shown in Figure 6.8(m) — produced by RO LC Alternative. Figure 6.8(n) shows the element health indices predicted for the scenario mimicking a major bridge improvement with no follow-up preservation actions-produced by FCI/REP LC Alternative 1. 
(a)

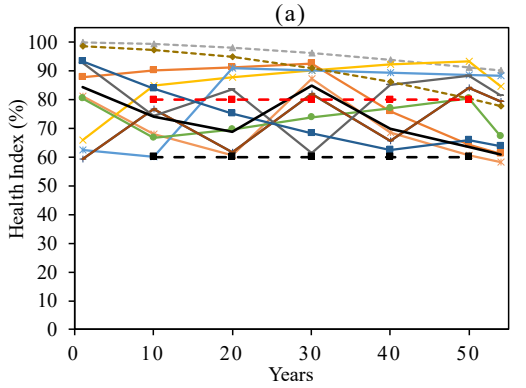

(d)

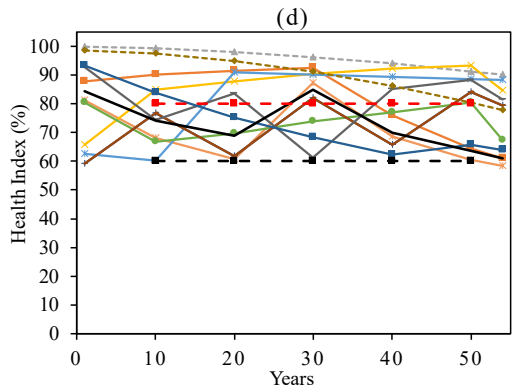

(g)

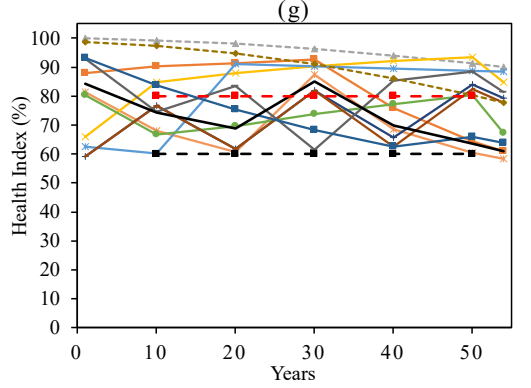

(1)

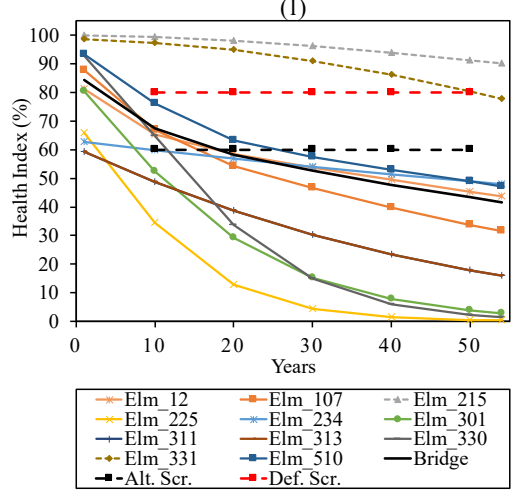

(b)

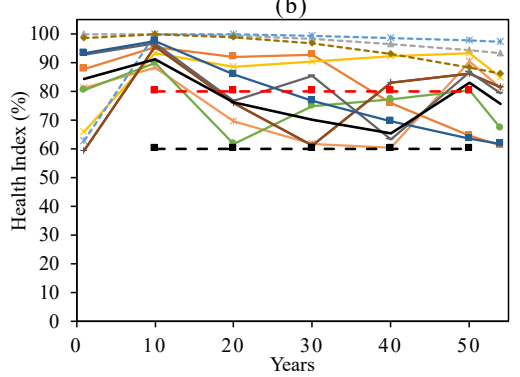

(e)

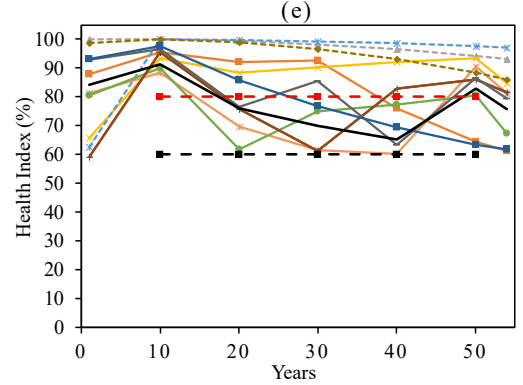

(h)

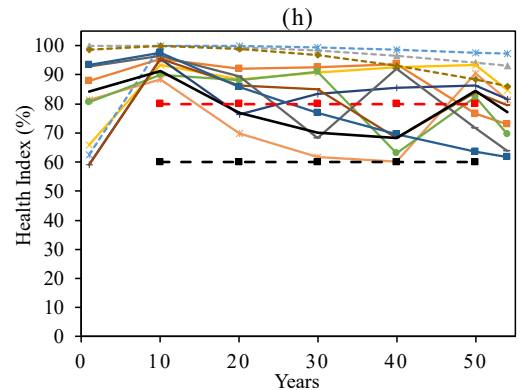

$(\mathrm{m})$

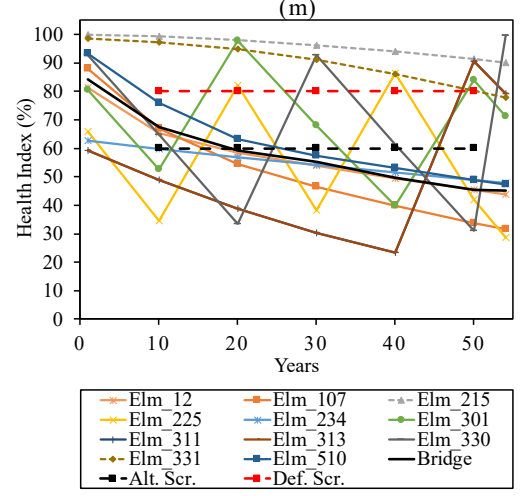

(c)

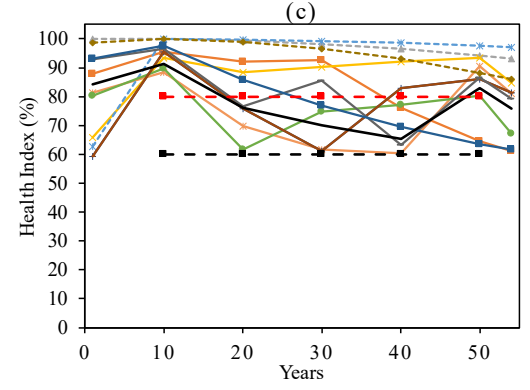

(f)

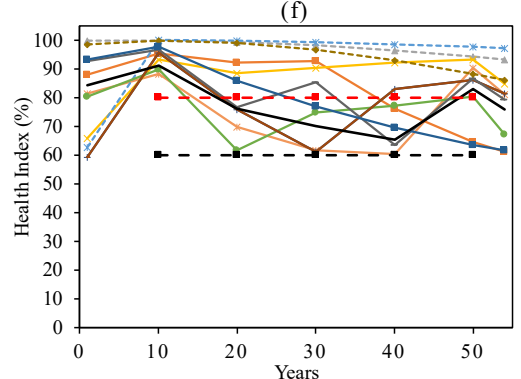

(k)

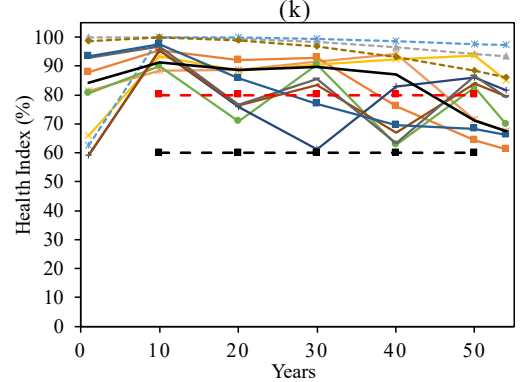

(n)

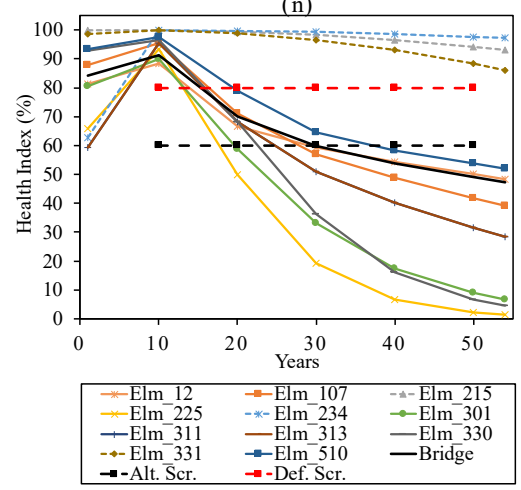

Figure 6.8 Predicted health indices associated with the solutions producing the least bridge LCC values for the 2-objective ELO problems under (a) Scenario a, (b) Scenario b, (c) Scenario c; the 3-objective ELO problems under (d) Scenario a, (e) Scenario b, (f) Scenario c; the 7-objective ELO problems under (g) Scenario a, (h) Scenario b, (k) Scenario c. Predicted health indices under (l) DN LC Alternative; (m) RO LC Alternative; (n) FCI/REP LC Alternative 1 
The objective of the NLO module is to produce a set of optimal or near-optimal solutions per improvement type and per program year for a candidate bridge. Each solution holds a set of recommended LC alternatives for the different elements of the bridge. Table 6.10 shows the recommended LC alternatives associated with Solutions 4, 7, and 10 (refer to Table 6.9). For MRR improvement type, element-level preservation actions (MRR Actions 0, 1, 2, 3, and 4) are assigned to the program year and each decision point (spaced by a 10 -year inaction period). For FCI and REP improvement types, MRR actions are assigned only to the decision points, and the bridgelevel improvement actions (FCI Action 5 and REP Action 6) are assigned only to the program year. LC Alternative 1 is always assigned to the non-deficient elements. These recommended improvement actions provide the least bridge LCCs while maintaining element health indices above the minimum acceptable limits $(60 \%$ every 10 years, set based on the alternative feasibility screening process) for program year 6 . Table 6.11 shows the feasible preservation treatments associated with these recommended improvement actions. 
Table 6.10 Element improvement actions associated with the 2-objective ELO solutions producing the least bridge LCC values for the three improvement types

\begin{tabular}{|c|c|c|c|c|c|c|c|}
\hline & $\begin{array}{l}\text { Element } \\
\text { Ref. }\end{array}$ & $\begin{array}{l}\text { LC Alt. } \\
\text { Ref. }\end{array}$ & $\begin{array}{c}\text { Pro. Year } \\
\text { (year 6) }\end{array}$ & $\begin{array}{c}\text { Dec. Point } 1 \\
\text { (year 17) }\end{array}$ & $\begin{array}{c}\text { Dec. Point } 2 \\
\text { (year 28) }\end{array}$ & $\begin{array}{c}\text { Dec. Point } 3 \\
\text { (year 39) }\end{array}$ & $\begin{array}{l}\text { Dec. Point } 4 \\
\text { (year 50) }\end{array}$ \\
\hline \multirow{11}{*}{ 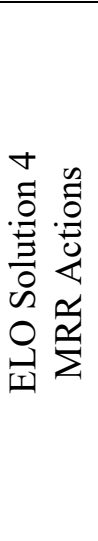 } & 12 & 2294 & 3 & 3 & 1 & 3 & 3 \\
\hline & 107 & 800 & 1 & 1 & 1 & 4 & 4 \\
\hline & $215^{\mathrm{a}}$ & 1 & 0 & 0 & 0 & 0 & 0 \\
\hline & 225 & 782 & 1 & 1 & 1 & 1 & 1 \\
\hline & 234 & 2094 & 3 & 1 & 3 & 3 & 3 \\
\hline & 301 & 1563 & 2 & 2 & 2 & 2 & 2 \\
\hline & 311 & 1042 & 1 & 3 & 1 & 3 & 1 \\
\hline & 313 & 1042 & 1 & 3 & 1 & 3 & 1 \\
\hline & 330 & 2082 & 3 & 1 & 3 & 1 & 1 \\
\hline & $331^{\mathrm{a}}$ & 1 & 0 & 0 & 0 & 0 & 0 \\
\hline & 510 & 2343 & 3 & 3 & 3 & 3 & 2 \\
\hline \multirow{11}{*}{ 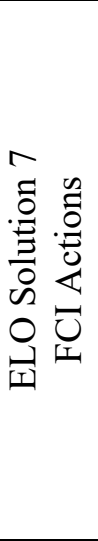 } & 12 & 462 & 5 & 3 & 3 & 2 & 1 \\
\hline & 107 & 175 & 5 & 1 & 1 & 4 & 4 \\
\hline & $215^{\mathrm{a}}$ & 1 & 5 & 0 & 0 & 0 & 0 \\
\hline & 225 & 157 & 5 & 1 & 1 & 1 & 1 \\
\hline & $234^{\mathrm{a}}$ & 1 & 5 & 0 & 0 & 0 & 0 \\
\hline & 301 & 438 & 5 & 3 & 2 & 2 & 2 \\
\hline & 311 & 457 & 5 & 3 & 3 & 1 & 1 \\
\hline & 313 & 457 & 5 & 3 & 3 & 1 & 1 \\
\hline & 330 & 417 & 5 & 3 & 1 & 3 & 1 \\
\hline & $331 \mathrm{a}$ & 1 & 5 & 0 & 0 & 0 & 0 \\
\hline & 510 & 469 & 5 & 3 & 3 & 3 & 3 \\
\hline \multirow{11}{*}{ 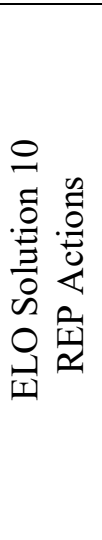 } & 12 & 462 & 6 & 3 & 3 & 2 & 1 \\
\hline & 107 & 175 & 6 & 1 & 1 & 4 & 4 \\
\hline & $215^{\mathrm{a}}$ & 1 & 6 & 0 & 0 & 0 & 0 \\
\hline & 225 & 157 & 6 & 1 & 1 & 1 & 1 \\
\hline & $234^{a}$ & 1 & 6 & 0 & 0 & 0 & 0 \\
\hline & 301 & 438 & 6 & 3 & 2 & 2 & 2 \\
\hline & 311 & 457 & 6 & 3 & 3 & 1 & 1 \\
\hline & 313 & 457 & 6 & 3 & 3 & 1 & 1 \\
\hline & 330 & 417 & 6 & 3 & 1 & 3 & 1 \\
\hline & $331^{\mathrm{a}}$ & 1 & 6 & 0 & 0 & 0 & 0 \\
\hline & 510 & 469 & 6 & 3 & 3 & 3 & 3 \\
\hline
\end{tabular}

${ }^{a}$ Non-deficient element. 
Table 6.11 Feasible preservation treatments associated with the improvement actions recommended by the 2-objective ELO solutions producing the least bridge LCC values for the three improvement types

\begin{tabular}{|c|c|c|c|c|c|c|c|c|}
\hline & $\begin{array}{c}\text { Element } \\
\text { Ref. }\end{array}$ & $\begin{array}{l}\text { Element } \\
\text { Name }\end{array}$ & $\begin{array}{c}\text { LC Alt. } \\
\text { Ref. }\end{array}$ & $\begin{array}{c}\text { Pro. } \\
\text { Year } \\
\text { (year 6) }\end{array}$ & $\begin{array}{c}\text { Dec. } \\
\text { Point } 1 \\
\text { (year 17) }\end{array}$ & $\begin{array}{c}\text { Dec. } \\
\text { Point } 2 \\
\text { (year 28) }\end{array}$ & $\begin{array}{c}\text { Dec. } \\
\text { Point } 3 \\
\text { (year 39) }\end{array}$ & $\begin{array}{c}\text { Dec. } \\
\text { Point } 4 \\
\text { (year 50) }\end{array}$ \\
\hline \multirow{11}{*}{ 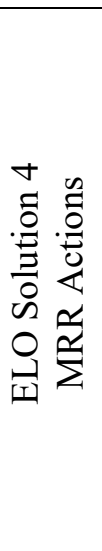 } & 12 & Re. Concrete Deck & 2294 & PM & PM & MRR & PM & PM \\
\hline & 107 & Steel Open Girder/Beam & 800 & SMMR & SMMR & SMMR & PM & PM \\
\hline & $215^{\mathrm{a}}$ & Re. Conc. Abutment & 1 & $\mathrm{DN}$ & DN & $\mathrm{DN}$ & $\mathrm{DN}$ & $\mathrm{DN}$ \\
\hline & 225 & Steel Pile & 782 & SMMR & SMMR & SMME & SMMR & SMMR \\
\hline & 234 & Re. Conc. Pier Cap & 2094 & PM & MRR & PM & PM & PM \\
\hline & 301 & Pourable Joint Seal & 1563 & RJ & RJ & RJ & RJ & RJ \\
\hline & 311 & Moveable Bearing & 1042 & MMR & PM & MMR & PM & MMR \\
\hline & 313 & Fixed Bearing & 1042 & MMR & PM & MMR & PM & MMR \\
\hline & 330 & Metal Bridge Railing & 2082 & PM & SMMR & PM & SMMR & SMMR \\
\hline & $331^{\mathrm{a}}$ & Re. Conc. Bridge Railing & 1 & $\mathrm{DN}$ & $\mathrm{DN}$ & $\mathrm{DN}$ & $\mathrm{DN}$ & $\mathrm{DN}$ \\
\hline & 510 & Wearing Surfaces & 2343 & PM & PM & PM & PM & RU \\
\hline \multirow{11}{*}{ 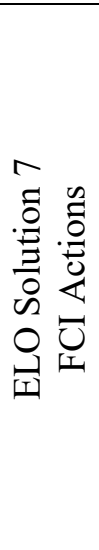 } & 12 & Re. Concrete Deck & 462 & FCI & PM & PM & $\mathrm{RD}$ & MMR \\
\hline & 107 & Steel Open Girder/Beam & 175 & FCI & SMMR & SMMR & PM & PM \\
\hline & $215^{\mathrm{a}}$ & Re. Conc. Abutment & 1 & FCI & $\mathrm{DN}$ & $\mathrm{DN}$ & $\mathrm{DN}$ & DN \\
\hline & 225 & Steel Pile & 157 & FCI & SMMR & SMMR & SMMR & SMMR \\
\hline & $234^{\mathrm{a}}$ & Re. Conc. Pier Cap & 1 & FCI & $\mathrm{DN}$ & $\mathrm{DN}$ & $\mathrm{DN}$ & $\mathrm{DN}$ \\
\hline & 301 & Pourable Joint Seal & 438 & FCI & PM & RJ & RJ & RJ \\
\hline & 311 & Moveable Bearing & 457 & FCI & PM & PM & MMR & MMR \\
\hline & 313 & Fixed Bearing & 457 & FCI & PM & PM & MMR & MMR \\
\hline & 330 & Metal Bridge Railing & 417 & FCI & PM & SMMR & PM & SMMR \\
\hline & $331^{\mathrm{a}}$ & Re. Conc. Bridge Railing & 1 & FCI & DN & $\mathrm{DN}$ & $\mathrm{DN}$ & $\mathrm{DN}$ \\
\hline & 510 & Wearing Surfaces & 469 & FCI & PM & PM & PM & PM \\
\hline \multirow{11}{*}{ 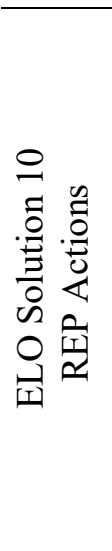 } & 12 & Re. Concrete Deck & 462 & REP & PM & PM & $\mathrm{RD}$ & MMR \\
\hline & 107 & Steel Open Girder/Beam & 175 & REP & SMMR & SMMR & PM & PM \\
\hline & $215^{\mathrm{a}}$ & Re. Conc. Abutment & 1 & REP & $\mathrm{DN}$ & $\mathrm{DN}$ & $\mathrm{DN}$ & $\mathrm{DN}$ \\
\hline & 225 & Steel Pile & 157 & REP & SMMR & SMMR & SMMR & SMMR \\
\hline & $234^{\mathrm{a}}$ & Re. Conc. Pier Cap & 1 & REP & $\mathrm{DN}$ & $\mathrm{DN}$ & $\mathrm{DN}$ & DN \\
\hline & 301 & Pourable Joint Seal & 438 & REP & PM & RJ & RJ & RJ \\
\hline & 311 & Moveable Bearing & 457 & REP & PM & PM & MMR & MMR \\
\hline & 313 & Fixed Bearing & 457 & REP & PM & PM & MMR & MMR \\
\hline & 330 & Metal Bridge Railing & 417 & REP & PM & SMMR & PM & SMMR \\
\hline & $331^{\mathrm{a}}$ & Re. Conc. Bridge Railing & 1 & REP & $\mathrm{DN}$ & $\mathrm{DN}$ & $\mathrm{DN}$ & $\mathrm{DN}$ \\
\hline & 510 & Wearing Surfaces & 469 & REP & PM & PM & PM & PM \\
\hline
\end{tabular}

Note. For more detail about these preservation treatments, refer to Implementation of the 2013 AASHTO Manual for Bridge Element Inspection (Sobanjo \& Thompson, 2016a). PM = Preventive maintenance; $\mathrm{RD}=$ Replace deck; $\mathrm{MMR}=$ Minor or major repair; $\mathrm{SMMR}=$ Spot blast and minor or major repair; $\mathrm{DN}=$ Do-nothing; RJ = Replace joint; FCI = Bridge functional improvement; REP = Bridge replacement.

${ }^{\mathrm{a}}$ Non-deficient element. 


\subsection{Summary and Conclusions}

This chapter introduced a "true" element-based MOO method that relies on results from the data processing and improvement modules. The chapter presented the module framework illustrating the different concepts and processes. It described the alternative feasibility screening process developed to reduce the ELO problem size to a manageable size and improve the computational time. The screening process recognizes the best feasible LC alternatives for each program year based on the specified criteria and optimization goals.

The ELO problem type and formulation, and the mapping approach of the problem decision variables are also discussed. The optimization problem is formulated in terms of discrete decision variables (binary values). The optimization formulation is shaped as a MCKP, involving only the selection criterion. The year-by-year optimization strategy is adapted to decompose the problem and further reduce the number decision variables. An ELO run is independently performed for each program year. The ELO problem focuses on finding a set of Pareto optimal or near-optimal solutions per program year for each improvement type.

The chapter introduced the heuristic optimization algorithm designed to solve the ELO problem. The metaheuristic NSGA-II is deployed as the main optimizer to handle the computational complexity of these large-sized optimization problems. A set of LC alternatives is derived from each obtained solution. Each recommended LC alternative represents a series of best (optimal or near-optimal) actions for a deficient element over the analysis period. Performance and LCC results associated with these $\mathrm{LC}$ alternatives (or solutions) serve as the fundamental inputs for the bridge- and network-level (top-down approach only) optimization modules. 
The chapter also included an illustrative example using the developed MATLAB-based tool prototype. The example consists of different ELO problems under unconstrained scenarios. Only one sample bridge is used in this example. Using the tool prototype, the preliminary outcomes for this sample bridge produced by the improvement module were transferred to this ELO module. Only optimization results for one program year are presented in this chapter. For the analyzed program year, under each of three improvement types, the tool produced optimal or near-optimal ELO solutions, recommended sets of intervention actions, predicted performance, and determined budget requirements. 


\section{CHAPTER 7-BRIDGE-LEVEL OPTIMIZATION MODULE}

- Task 3: Proposing an Element-Based Multi-Objective Optimization Methodology

- Task 4: Development of a Tool Prototype

- Task 5: Implementation of the Methodology through Examples of Scenarios

\subsection{Introduction}

The EB-MOO methodology consists of five modules (i.e., data processing, improvement, ELO, BLO, and NLO modules). Chapters 4 and 5 introduced the different processes and models incorporated in the data processing and improvement modules: a novel screening process to identify potential deficient bridge elements, an independent deterioration model to predict performance, a LCC model to estimate LCCs and benefits, and a simulation arrangement to generate realistic LC alternatives for three improvement types. Chapters 6 presented the ELO module framework and its different processes, laid out the problem formulation and the proposed heuristic algorithm, and included an illustrative example.

Analyzing each bridge individually to select the appropriate strategy is normally referred to as the bridge-level decision making (Abu Dabous, 2008). This chapter introduces an innovative BLO module that considers the ELO recommendations. The chapter discusses the module framework and the interaction between the element- and bridge-level modules, includes an illustrative example of results using the tool prototype, and demonstrates effectiveness and benefits. The optimization problem is either constrained or unconstrained - involving only the selection criterion for the unconstrained optimization problems. The module optimization requires a 
simultaneous optimization of multiple competing objectives - such as minimize LCC and maximize the LCC benefit and/or health indices at different points in time for a candidate bridge, seeking the best trade-off between them. The aim is to obtain a diverse set of BLO solutions for the entire program period as close as possible to the Pareto frontier; a recommended BLO solution delivers the best feasible set of LC alternatives for all deficient elements over the entire analysis period. The module can be used independently to identify the best feasible sets of improvement actions and timings for future work on a candidate bridge. It provides a systematic process to develop/assess bridge improvement or preservation programs.

Integrating bridge-level and network-level decisions and handling optimization problems of a large size is still a challenging task (Elbehairy, 2007). Bridge-level decisions complement network-level decisions. BLO results are key inputs for the bottom-up approach NLO (discussed in the subsequent section). The BLO module addresses one bridge at a time. Obtained results are stored to serve the NLO process. ELO results associated with the improvement types are grouped and expressed in summation formulas over the entire program period and all deficient elements. The grouped ELO solutions compete for minimum bridge LCCs, maximum bridge LCC benefits, and/or maximum bridge health indices.

The optimization problem is formulated in terms of binary decision variables. When an ELO solution is selected for an improvement type and a program year, the decision variable is taken as 1, otherwise 0 . The optimization problem is an NP-hard combinatorial optimization problem. Generally, near-optimal rather than optimal solutions are obtained. The BLO objectives and constraints are expressed in terms of these decision variables. The optimization formulation is shaped as a MCKP if no constraints involved other than the selection criterion—only one ELO solution (one choice) must be picked from all solutions (multiple choices). The multi-dimensional 
aspect is added to the problem when more than one constraint (e.g., budget or performance constraint) is involved. The problem is then formulated as a "Multi-Choice Multi-Dimensional Knapsack Problem" (MCMDKP). The literature review under Task 1 revealed that economic analysis, mathematical programming methods, and heuristic algorithms are the most common techniques to support bridge-level decisions. The module relies on the same metaheuristic NSGAII to solve the bridge-level NP-hard combinatorial optimization problem.

\subsection{Module Framework}

Each bridge is analyzed independently, and a set of optimal or near-optimal intervention strategies is identified for all its elements. The module has two main purposes:

(1) support the development of bridge programs, and

(2) produce bridge-level input parameters for the bottom-up approach NLO (discussed in Chapter 8).

The module helps decision makers to develop a comprehensive bridge program addressing improvement needs of the identified deficient elements - the initial implementations are always performed within the program period, accompanying with a follow-up multi-year plan. It's essential to achieve high-quality solutions at this optimization level— the bottom-up approach of the NLO module depends mainly on these BLO solutions.

In the improvement module, overviewed in Chapter 5, each bridge in the portfolio is evaluated separately for each program year-generating LC alternatives, determining LCCs and LCC benefits, and predicting performance (health indices). As discussed in Chapter 6, the ELO module focuses on finding optimal or near-optimal solutions per program year and per improvement type for each deficient element of a bridge in the portfolio. If the MRR improvement type is 
recommended, MRR LC Alternative 1 (representing the DN LC Alternative over the entire analysis period) is assigned to non-deficient elements, otherwise FCI/REP LC alternative 1 (mimicking the DN LC Alternative over the analysis years succeeding the element replacement year. The module framework exemplified in Figure 7.1 is based on the following concepts:

1. The ELO results per improvement type and per program year for each element are transferred to this module as input parameters.

2. The ELO solution results (i.e., bridge LCCs, IACs, LCC benefits, and health indices) are grouped and reorganized in single matrices as illustrated in Figure 7.1.

3. Each arranged matrix represents one type of solution results covering the entire program period and the three improvement types; this arrangement allows to unite all recommended ELO solutions to compete at the bridge level.

4. No screening process is needed to further reduce the size of these arranged matrices; the screening process is generally unnecessary for the BLO module-the total number of decision variables remains manageable even for bridges with many deficient elements (explained later in this chapter).

5. The last three matrices shown in Figure 7.1 represent the BLO solution outcomes for the bridge. The first matrix (bottom, far-left) contains the recommend LC alternatives associated with the BLO solutions (each element is assigned to an optimal or near-optimal LC alternative, an improvement type, and a program year), the second matrix, the resulted bridge initial agency costs $\left(\mathrm{IAC}_{\mathrm{brgs}}\right)$ and health indices at different points in the analysis period, and the third matrix, the values of the optimized bridge-level objectives. 


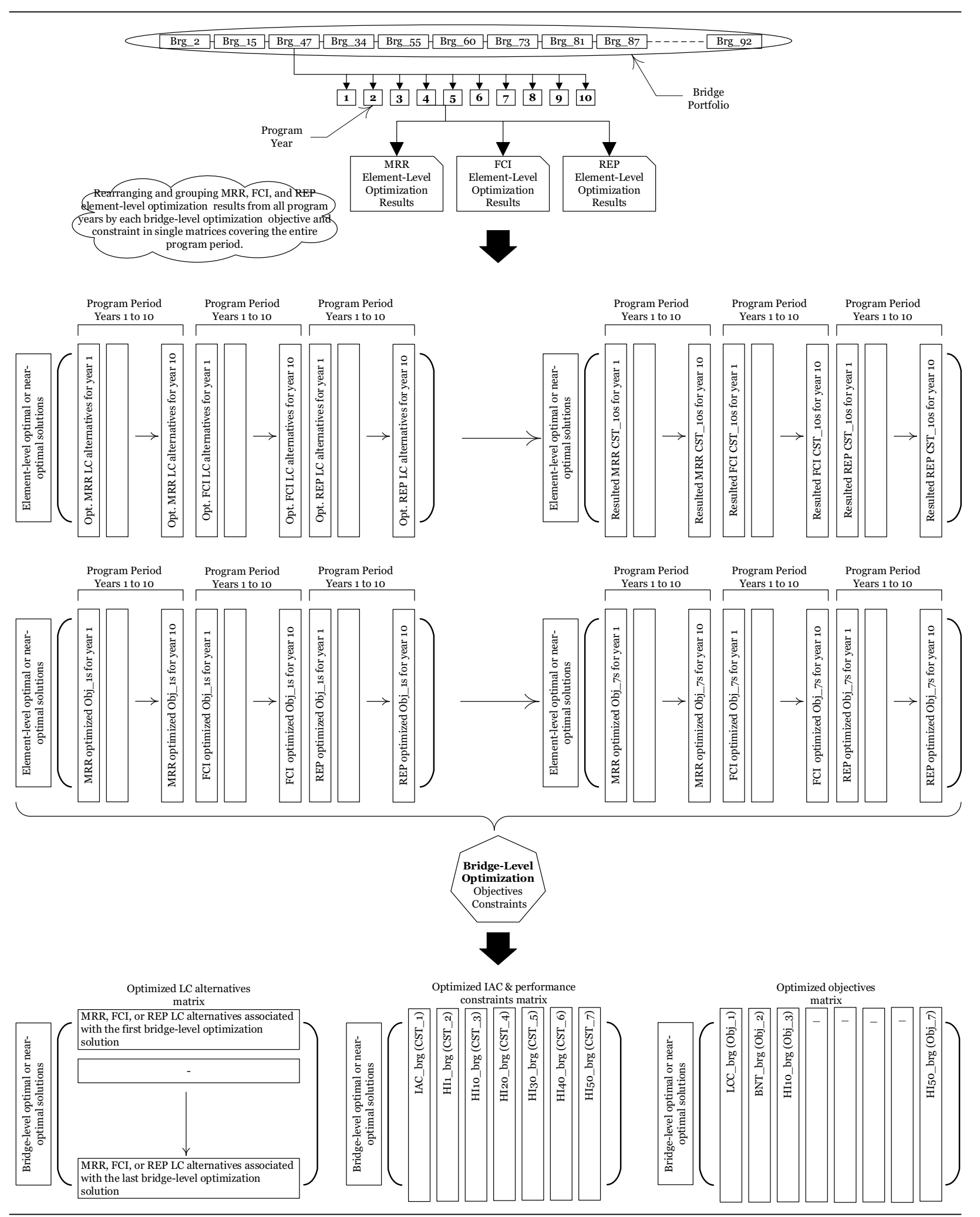

Figure 7.1 Bridge-level optimization module framework 


\subsection{Bridge-Level Optimization Problem Formulation}

As explained in Chapter 6, the product of a MOO is generally a set of non-dominated solutions. Each solution is represented by a vector of decision variables that satisfies constraints and optimizes multiple competing objectives. An optimal solution for one of the objectives is usually non-optimal for the remaining objectives. The solutions are described as Pareto optimal solutions (non-dominated solutions). Each solution on the Pareto frontier is not dominated by any other feasible solution. In this research, as mentioned in the previous chapter, Pareto solutions encompass the optimal or near-optimal (very close to optimal) solutions.

The module optimization problem requires a simultaneous optimization of multiple competing objectives, seeking the best trade-off between them. The module focuses on finding a diverse set of Pareto solutions for the entire program period. The module can be used independently to identify the optimal or near-optimal sets of improvement actions and timings for future work on a candidate bridge. The module optimization problem can be either constrained or unconstrained. BLO solutions of the unconstrainted problem serve as the input parameters for the NLO process. For a candidate bridge, the BLO goal is to simultaneously minimize the bridge LCC and maximize the bridge LCC benefit and/or health indices at different points in time subject to budget and/or performance constraints, where only one ELO solution (one choice) must be picked from all the ELO solutions (multiple choices) recommended for the three improvement types and all program years.

Bridge replacement (REP) and functional improvement (FCI) are generally recommended by a BMS due to an existing functional deficiency or other agency's rules or triggers (such as condition ratings or repair costs exceeding the replacement costs). These two REP and FCI improvement types usually are not directly incorporated in the optimization process alongside with the MRR 
improvement type. The MRR optimization is usually performed independently. In this research, these three improvement types are considered in the BLO process. Each improvement type is assessed separately in the ELO module for each program year and transferred to this bridge-level module. ELO results associated with these three improvement types are grouped and expressed in summation formulas covering the entire program period. These grouped ELO solutions compete for minimum bridge LCCs, maximum bridge LCC benefits, and/or maximum bridge health indices.

The module optimization problem falls under combinatorial category (touched on in Chapter 6). It's defined in terms of binary decision variables. The binary variables were found to be suitable for this type of combinatorial optimization problem. A decision variable is taken as 1 if an ELO solution is selected, otherwise 0. A recommended BLO solution delivers the best feasible set of LC alternatives for all deficient elements over the entire analysis period. The DN LC Alternative is assigned to each non-deficient element if the MRR improvement type is recommended, otherwise FCI/REP LC alternative 1.

A selection criterion is always considered for the constrained and unconstrained optimization problems, regardless. When no additional constraints are involved, the problem is formulated as a MCKP - Chapter 6 discusses the MCKP in greater detail. However, when multiple constraints (e.g., available budget and/or minimum acceptable performance) are considered, the multidimensional aspect is added to the problem; and therefore, it is classified as MCMDKP. It represents one of the knapsack problems identified in the literature review. Moser, Jokanovic and Shiratori (as cited in Patidar, 2006) underscored the lack of studies on attempting to develop efficient heuristics for solving this type of problems. The multi-choice aspect in the BLO stands for the fact that one ELO solution must be selected per bridge; the multi-dimensional aspect of the 
problem relates to the presence of budget and/or performance constraints (in addition to the section criterion one).

Typically, this type of optimization problems is considered an NP-hard optimization problem and generally solved using heuristics or metaheuristics to obtain approximate (near-optimal rather than optimal) solutions within a reasonable computational effort (Thompson et al., 2008). The next section covers the heuristic optimization algorithm developed for this purpose. The same metaheuristic NSGA-II is used to solve this bridge-level NP-hard optimization problem. The formulation presented herein is based on the MCMDKP. The problem can be mathematically expressed as follows:

Objectives

Minimize $L C C_{\text {brg }_{k}}$

Maximize $B N T_{b r g_{k}}$

Maximize $H I_{b r g_{k}}^{y}$ $\forall y \in H_{o b j}$

Subject to

$I A C_{\text {rrg }_{k}} \leq B G T$

$H I_{b r g_{k}}^{Z} \geq H I_{b r g_{\text {min }}}^{Z}$

$\forall z \in H_{c o n}$

$\sum_{p \in I} \sum_{j \in T} \sum_{s \in O_{k}^{p j}} X_{p j s}=1$

where

$L_{C C_{\text {brg }_{k}}}=\sum_{p \in I} \sum_{j \in T} \sum_{s \in O_{k}^{p_{j}}}\left(X_{p j s} L C C_{\text {brg }_{k}}^{p j s}\right)$
$B N T_{\text {brg }_{k}}=\sum_{p \in I} \sum_{j \in T} \sum_{s \in O_{k}^{p_{j}}}\left(X_{p j s} B N T_{b r g_{k}}^{p j s}\right)$ 


$$
\begin{aligned}
& H I_{b r g_{k}}^{y}=\sum_{p \in I} \sum_{j \in T} \sum_{s \in O_{k}^{p_{j}}}\left(X_{p j s} H I_{b r g_{k}}^{\text {ypjs }}\right) \\
& H I_{b r g_{k}}^{Z}=\sum_{p \in I} \sum_{j \in T} \sum_{s \in O_{k}^{p_{j}}}\left(X_{p j s} H I_{\text {brg }_{k}}^{z p j s}\right) \\
& I A C_{b r g_{k}}=\sum_{p \in I} \sum_{j \in T} \sum_{s \in O_{k}^{p j}}\left(X_{p j s} I A C_{b r g_{k}}^{p j s}\right) \\
& L^{L C C_{\text {brg }_{k}}}=\text { total LCC for bridge } k \text {; } \\
& B N T_{\text {brg }_{k}}=\text { total LCC benefit for bridge } k \text {; } \\
& \mathrm{HI}_{\mathrm{brg}}^{\mathrm{y}}=\text { total health index of bridge } k \text { at the end of year } y \text {; } \\
& H I_{b r g}^{Z} \quad=\text { total health index of bridge } k \text { at the end of year } z \text {; } \\
& H I_{\text {brg }}^{Z} \text { min }=\text { minimum acceptable bridge health index at the end of year } z \\
& I A C_{\operatorname{brg}_{k}}=\text { total initial agency cost incurred for bridge } k \text {; } \\
& B G T=\text { available improvement budget per bridge; }
\end{aligned}
$$

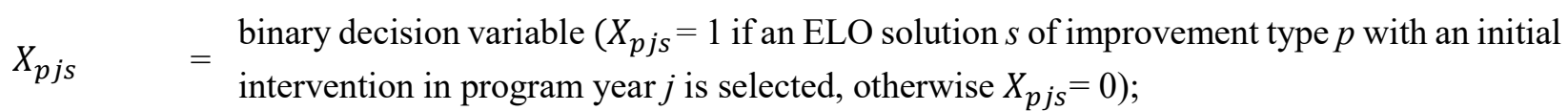

$$
\begin{aligned}
& L_{C C C_{\text {brg }}^{\text {pjs }}}=\begin{array}{l}
\text { total LCC of ELO solution } s \text { of improvement type } p \text { with an initial intervention in program } \\
\text { year } j \text { performed on bridge } k ;
\end{array} \\
& B N T_{\text {brg }_{k}}^{\text {jss }}=\begin{array}{l}
\text { total benefit of ELO solution } s \text { of improvement type } p \text { with an initial intervention in program } \\
\text { year } j \text { performed on bridge } k ;
\end{array}
\end{aligned}
$$

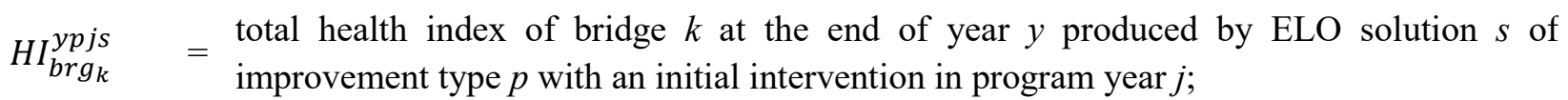

$$
\begin{aligned}
& \begin{aligned}
\mathrm{HI}_{\mathrm{brg}}^{\mathrm{zpjs}} & =\begin{array}{l}
\text { total health index of bridge } k \text { at the end of year } z \text { produced by ELO solution } s \text { of } \\
\text { improvement type } p \text { with an initial intervention in program year } j
\end{array}
\end{aligned} \\
& I A C_{\text {brg }_{k}}^{\text {pjs }}=\begin{array}{l}
\text { total initial agency cost for bridge } k \text { incurred by ELO solution } s \text { of improvement type } p \text { with } \\
\text { an initial intervention in program year } j ;
\end{array} \\
& O_{k}^{p j}=\quad \begin{array}{l}
\text { set of ELO solutions of improvement type } p \text { with an initial intervention in program year } j \\
\text { performed on bridge } k ;
\end{array}
\end{aligned}
$$


$I \quad=$ set of the three improvement types (i.e., MRR, FCI, and REP);

$T \quad=$ set of all program years (i.e., the program period);

$H_{o b j} \quad=$ set of specified years in the analysis period for health index objectives; and

$H_{\text {con }}=$ set of specified years in the analysis period for health index constraints.

Formulating the problem correctly is so essential to achieve high-quality solutions. Several formulation designs for overcoming the size of the problem were tested. The proposed formulation is flexible enough to accommodate different BLO goals with multiple constraints. The formulation accommodates ELO solutions from the three distinct improvement types (i.e., MRR, FCI, and REP) and all program years. For example, a BLO goal for a given bridge may involve minimizing the bridge LCC, Equation (7.4), maximizing the bridge LCC benefit, Equation (7.5), maximizing the bridge health indices at years 20 and 40, Equation (7.6), and maintaining a bridge health index above $70 \%$ (signifies a minimum acceptable bridge performance). In this example, four objectives subject to a performance constraint and a picking criterion, Equation (7.3), will be optimized for this bridge.

This generalized formulation calls for an optimization for the entire program period - taking into account the three improvement types and all program years to compete at this level. No screening process is needed to further reduce the size of the optimization problem. The screening process is deemed unnecessary for this level of optimization. The total number of decision variables remains manageable. A multi-year optimization strategy was found to be appropriate to accomplish this task at the bridge level. The year-by-year optimization strategy adapted in the ELO module decomposes the ELO problem, producing a set of ELO solutions per improvement type and per program year. Using all these sets as input parameters for the bridge-level optimization still generates a manageable number of decision variables with reasonable computational efforts. For 
instance, typically, a multi-year optimization strategy generates around 3,000 $(3 \times 10 \times 100)$ decision variables over a program period of 10 years for a giving bridge, where each improvement type is associated with 100 ELO solutions per program year. This number of decision variables per bridge is considered practicable even for a large network of bridges.

In this module, the BLO objectives (i.e., bridge LCC, LCC benefit, and performance) are mathematically formulated. Multiple constraints (i.e., selection criterion, available budget, and minimum desired bridge performance) are integrated in the problem formulation. A set of Pareto solutions per bridge for the entire program period is recommended. A BLO solution is the recommended ELO solution from the different sets of ELO solutions, representing a combination of the best feasible (optimal or near-optimal) LC alternatives identified for the bridge deficient elements.

A constrained BLO problem limits the decision variable space and solution diversity. Constraints can be applied at the network level. Making the optimization problem unconstrained (except for the picking criterion) at this level increases the search effort and generates unwanted bridge-level solutions - unable to satisfy either the budget or performance constraint at the network level when following the bottom-up approach. Obtaining a diverse set of BLO solutions is essential for the next level of optimization (covered in the subsequent Chapter 8). The elimination of the unwanted BLO solutions is basically delayed at this level. Nevertheless, this approach permits to increase the diversity of BLO solutions and explore enough areas of the search space.

As explain earlier, the decision in this module optimization problem is a binary choice —one of the ELO solutions is either selected or rejected. A BLO solution is represented by a vector of binary decision variables (chromosome). The encoding of decision variables is illustrated and 
discussed later in this chapter. The non-dominated solutions of the entire feasible solution space constitute the Pareto solution set. Equation (7.3) restricts the decision variable space. This constraint guarantees the selection of only one ELO solution for the analyzed bridge. Therefore, among all grouped ELO solutions, only one is recommended for the bridge. For a population of size N2, N2 BLO solutions are recommended by the optimizer. The binary decision variable is symbolized by $X_{p j s}$. The variable equals to 1 when an ELO solution $s$ of improvement type $p$ with an initial intervention in program year $j$ is assigned to bridge $k$, otherwise 0 .

LC Alternative 1 is always assigned to the non-deficient elements. For MRR improvement type, the non-deficient elements don't experience preservation actions for the entire analysis period. MRR LC Alternative 1, representing the DN LC Alternative, is assigned to each of them. For FCI and REP improvement types, the non-deficient elements still get replaced; however, no preservation actions will follow. FCI/REP LC Alternative 1 is assigned to mimic this scenario. In contrasts, the deficient elements undertake the recommended LC alternatives.

The bridge-level objectives, Equations (7.4), (7.5), and (7.6), are interdependent on the decision variable, $X_{p j s}$. These objective equations represent the bridge LCC, LCC benefit, and health indices at the ends of specified analysis years and involve only ELO solution results, considering the different sets of ELO solutions associated with the three improvement types and all program years. A set of ELO solutions, $O_{k}^{p j}$, is established for each improvement type and each program year. The contributions of the different ELO solutions within these sets are summed over the three different improvement types $(I)$ and all program years $(T)$, after applying their corresponding decision variables. 
Equation (7.1) represents the inequality of budget constraint. The initial agency cost for the entire bridge is restricted by the available budget, $B G T$. Similarly, the cost is determined by summing all initial agency costs, $I A C_{\text {brg }_{k}}$, produced by the different ELO solutions over the three different improvement types and all program years, after applying their corresponding decision variables. Equation (7.2) represents the inequality of performance constraint. The inequality permits to maintain an acceptable bridge performance ("state of good repair") or attain a higher bridge performance over a certain period — health indices at the ends of specified analysis years, ${\mathrm{HI} \mathrm{brg}_{k}}^{Z}$,

are bounded by the minimum acceptable health indices, $\mathrm{HI}_{b r g_{\text {min }}}^{Z}$. Likewise, the bridge health indices are determined by summing all bridge health indices (at the ends of the analyzed years) produced by the different ELO solutions over the three different improvement types $(I)$ and all program years $(T)$, after applying their corresponding decision variables.

\subsection{Heuristic Bridge-Level Optimization Algorithm}

The objective of the BLO module is to obtain a diverse set of LC improvement actions as close as possible to the optimal ones. Again, as explained in Chapter 6, GAs are very effective in exploring the search space and reaching global optimality. They are capable of handling large-sized NP-hard combinatorial problems and obtaining a high quality of Pareto solutions in a single run. The same metaheuristic algorithm (i.e., NSGA-II) is deployed to solve this bridge-level NP-hard combinatorial optimization problem. However, a minor integration adjustment was required to accommodate the new optimization problem features. Again, like the preceding module, users can substitute this module optimizer algorithm by any other proven metaheuristic algorithm with similar capabilities such as SPEA-2, PESA, and MOGA. 
Figure 7.2 presents the flowchart of the heuristic algorithm designed to solve the module optimization problem. NSGA-II is integrated within the algorithm to handle optimization tasks. The brute-force (or exhaustive) search technique is used in this heuristic algorithm to enumerate and examine obtained solutions. For each bridge in the portfolio, the brute-force search technique systematically assesses every single solution after certain evaluations (for NSGA-II, the product of population size and total number of generations equals to the total number of evaluations). If all solutions of this total number of evaluations satisfy the constraints, they are then deemed feasible, and the algorithm moves on to the next bridge. Otherwise, if any solution violates a constraint, the whole set of solutions is rejected, the total number of evaluations is increased by an increment, and new solutions are obtained to be assessed for the same bridge. This simple search technique controls the number of runs per bridge, efficiently manages the computational time, and guarantees the feasibility of every single produced solution.

Users set the population size, initial evaluations, evaluation increment, maximum evaluations, number of bridges to be evaluated, program period, and available budget and performance thresholds. As emphasized in Chapter 6, the initial evaluations, population size, including other optimizer default parameters (such as crossover and mutation probabilities) must be carefully chosen. They are essential to ensure a high quality of obtained solutions. The best combination of parameters, initial evaluations, and population size can be identified by trial and error or by establishing different performance metrics to assess convergence, optimality of the solutions, diversity along the Pareto frontier, computational time, computer memory use, etc. The key steps of the designed heuristic algorithm are as follows:

1. The algorithm starts with the first bridge $(i=1)$, and a total number of evaluations (Eval) equals to the total number of initial evaluations (Eval_inc). 
2. For each bridge $i$, the algorithm locates MRR, FCI, and REP ELO solution output matrices (refer to Chapter 6).

3. The ELO solution output matrices are rearranged and grouped in single matrices covering the three improvement types and entire program period.

4. A random initial population of size $N 2$ is generated following the chromosome structure (discussed in the subsequent section).

5. While the total number of evaluations $\left(E v a l+E v a l \_i n c\right)$ is less than the specified maximum value (Eval_max), the brute-force search technique is deployed.

6. The optimizer NSGA-II is called to solve the optimization problem for these evaluations.

7. The obtained BLO solution results of these evaluations are saved.

8. Feasibility of the obtained solution set is verified.

9. If all constraints are met, the algorithm exits the while loop of brute-force search technique. Otherwise, the total number of evaluations is increased by the specified increment (Eval_inc), the population is taken as the population of this number of evaluations, and steps 5,6 and 7 get repeated.

10. The algorithm advances to the bridge $(i=i+1)$, and repeats the whole process again (the above steps).

11. The algorithm terminates after all $n b$ bridges being addressed. 


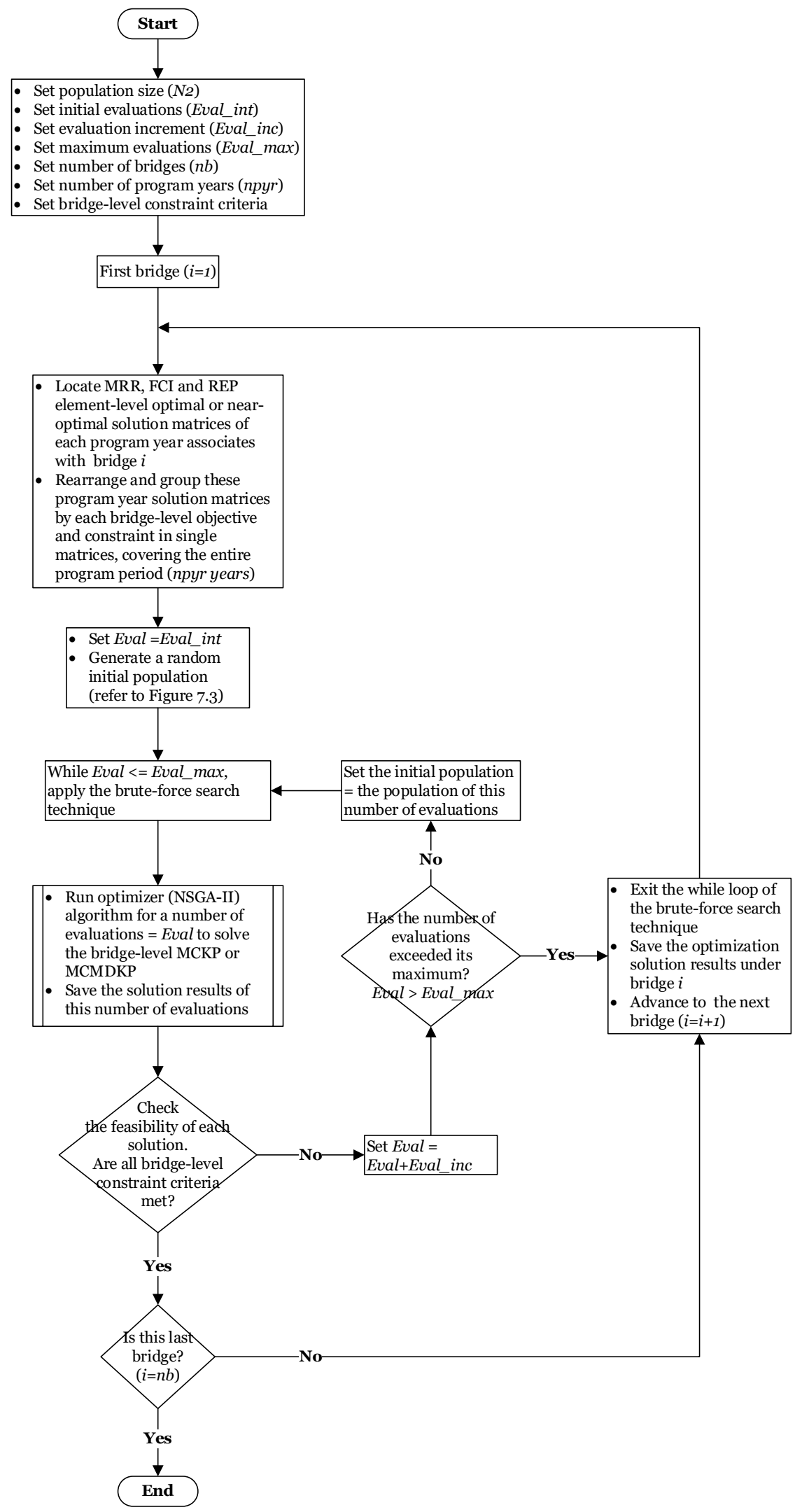

Figure 7.2 Heuristic bridge-level optimization algorithm 


\subsection{Mapping of Decision Variables}

Chapter 6 overviewed the basics process of generating a set of chromosomes (a population of solutions). The three main genetic operators (crossover, mutation, and selection/fitness) were discussed in Chapter 2. The chosen genetic optimizer, NSGA-II, relies on an elitism operator for selecting best (elite) chromosomes. Elitism operator increases the performance of GA, as it ensures the best chromosomes remain in the population. As stressed in Chapter 6, the process requires an effective encoding of the chromosome to maintain better performance and truthful representation of the problem.

GAs are capable of processing numerous chromosomes per iteration (representing one population). However, the larger the population size, the more computational efforts will take. The same is true when dealing with a large number of genes (i.e., decision variables) per chromosome. The screening process and the year-by-year optimization strategy had to be introduced in the ELO module to manage the number of decision variables and make the problem trackable with less computational efforts. For the BLO module, no screening process is required to reduce the size of the optimization problem. The screening process is deemed unnecessary for this level of optimization. The total number of decision variables remains manageable, as demonstrated earlier in this chapter. However, it's essential to control the population size in terms of number of chromosomes.

Figure 7.3 illustrates the chromosome encoding used to represent a BLO solution. A chromosome encompasses a total number of N1.npyr.nimp genes, where $N 1$ is the total number of ELO solutions per program year, npyr is the total number of program years, and nimp is the total number of improvement types. For instance, 3,000 chromosomes per bridge represents a set of 100 ELO solutions per program year, 10-year program period, and three improvement types (i.e., MRR, FCI, 
and REP). Again, a binary encoding scheme is adapted because of its simplicity and the problem allocation characteristics. Each chromosome has one binary string of genes (composed of either 0 or 1), and each gene represents a characteristic of the BLO solution.

Figure 7.3 illustrates the process of assigning decision variables to an ELO solution matrix of size $(N 1 \times 30)$ for a 10 -year program period. In this illustrative example, the first $N 1$ genes represent program year 1, the next $N 1$ genes represent program year 2, and so on until the last program year 10. All the first 10.N1 genes are assigned to MRR improvement type, and the next 10.Nlgenes to FCI improvement type, followed by another series of $10 . N 1$ genes to REP improvement type. Therefore, a total of $30 . N 1$ genes constitutes a binary chromosome. A gene with a value of 1 signifies an ELO solution is selected; in contrast, a value of 0 signifies no selection. A gene position depicts a specific problem characteristic. A gene in the fourth cell (starting from the left), for example, represents an ELO solution for MRR improvement type and program year 4. A population of size $N 2$ simply includes $N 2$ chromosomes with different random binary values. For any bridge in the portfolio, chromosomes are structured similarly. 

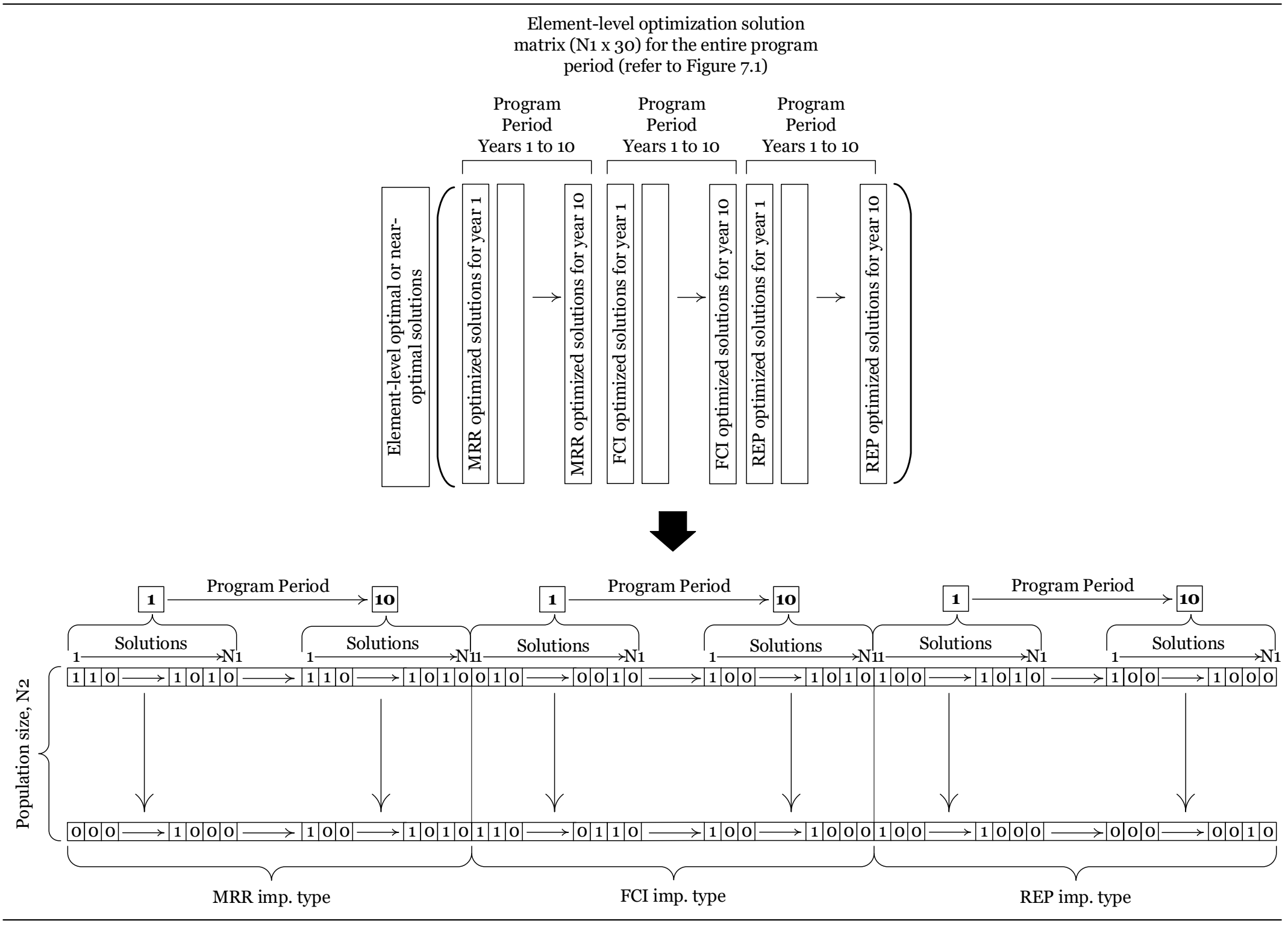

Figure 7.3 Illustration of the chromosome encoding of a BLO solution 


\subsection{Example of Module Results}

The methodology was implemented through different examples to test concepts, prove effectiveness, and demonstrate potential benefits. These examples provided an excellent opportunity to apply the different concepts of the proposed EB-MOO methodology. The examples include detailed tables and charts to communicate outcomes from the different modules. A portfolio of 40 sample bridges (introduced in Chapter 3) is used to implement the different stages of the EB-MOO methodology. These sample bridges were selected based on common features, attributes, and data completeness. Table 3.2 in Chapter 3 provides more information related to the characteristics of these sample bridges.

As discussed in Chapter 3, the MATLAB-based tool prototype, structured around the five EBMOO modules, was mainly developed to be used for the implementation. The tool prototype is considered as a "proof of concept" rather than a complete rigorous software ready for operational implementation. Using the tool prototype, the ELO results associated with these bridges were transferred to this BLO module. The tool produced a diverse set of BLO solutions, recommended set of intervention actions and timings, predicted performance, and determined budget requirements for the entire program period.

One of the sample bridges in the portfolio (i.e., Bridge 38) is used in the example presented herein. Table 7.1 presents the NBI condition ratings of this sample bridge and other related attributes. Table 7.2 shows the latest condition states of the 20 elements of this sample bridge. The bridge was built in 1959. It's a steel bascule bridge and has a low sufficiency rating of $42.5 \%$ (representing an overall poor condition). The bridge was identified with two functional deficiencies: bridge roadway width and load capacity. 
Table 7.1 NBI condition ratings of Bridge 38

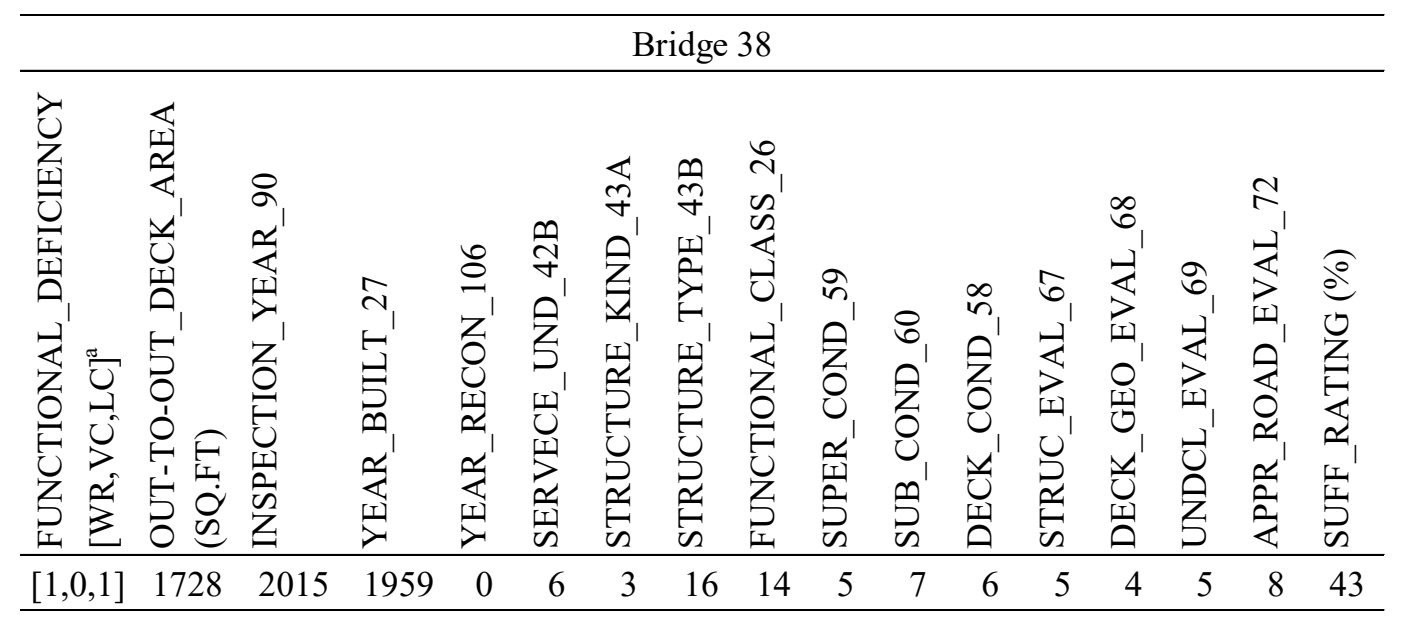

Note. $\mathrm{WR}=$ width required; $\mathrm{VC}=$ vertical clearance; $\mathrm{LC}=$ load capacity.

${ }^{a}$ The value of 1 represents to the existence of the functional deficiency, otherwise the value of 0 .

Table 7.2 Condition states of the 20 elements of Bridge 38

\begin{tabular}{|c|c|c|c|c|c|c|c|c|c|c|c|c|c|c|c|c|c|c|c|c|}
\hline & \multicolumn{20}{|c|}{ Elements of Bridge 38} \\
\hline & 12 & 28 & 29 & 107 & 109 & 113 & 152 & 210 & 215 & 220 & 226 & 227 & 231 & 234 & 301 & 310 & 311 & 313 & 330 & 331 \\
\hline CS1 (\%) & 95 & 0 & 0 & 98 & 99 & 96.92 & 98 & 0 & 100 & 100 & 16 & 100 & 84.38 & 100 & 97.32 & 0 & 100 & 100 & 100 & 99.74 \\
\hline CS2 (\%) & 5 & 100 & 100 & 2 & 1.06 & 0.00 & 1 & 100 & 0 & 0 & 84 & 0 & 15.63 & 0 & 2.68 & 100 & 0 & 0 & 0 & 0.26 \\
\hline CS3 (\%) & 0 & 0 & 0 & 0 & 0.00 & 3 & 1 & 0 & 0 & 0 & 0 & 0 & 0 & 0 & 0 & 0 & 0 & 0 & 0 & 0 \\
\hline CS4 (\%) & 0 & 0 & 0 & 0 & 0 & 0 & 0 & 0 & 0 & 0 & 0 & 0 & 0 & 0 & 0 & 0 & 0 & 0 & 0 & 0 \\
\hline Qt (sq.ft) & 13,382 & 2,834 & 882 & 780 & 1,506 & 650 & 210 & 79 & 85 & 610 & 57 & 6 & 64 & 197 & 224 & 60 & 10 & 4 & 937 & 779 \\
\hline $\mathrm{W}_{\mathrm{elm}}(\%)$ & 25 & 27 & 27 & 49 & 46 & 18 & 17 & 11 & 13 & 10 & 17 & 11 & 10 & 13 & 12 & 13 & 12 & 12 & 16 & 14 \\
\hline ESL (years) & 129 & 36 & 36 & 77 & 334 & 292 & 77 & 200 & 75 & 123 & 141 & 200 & 20 & 408 & 24 & 401 & 58 & 58 & 28 & 151 \\
\hline $\mathrm{RSL}^{\mathrm{a}}$ (years) & 69 & 0 & 0 & 18 & 273 & 228 & 17 & 96 & 16 & 64 & 67 & 141 & 0 & 349 & 0 & 235 & 0 & 0 & 0 & 92 \\
\hline $\mathrm{RU}(\$)$ & 54 & 63 & 54 & 2,062 & 848 & 481 & 1,054 & 5,822 & 1,591 & 194,049 & 38,810 & 38,810 & 1,186 & 1,186 & 72 & 9,009 & 9,009 & 9,009 & 296 & 212 \\
\hline
\end{tabular}

${ }^{a} \mathrm{RSL}$ is estimated from program year 1 (2020). 
Table 7.3 specifies the goals and types of the different BLO problems used in this example. Scenarios are defined based on imposed restrictions (by the available budget and/or minimum acceptable performance). The analysis period is set for 54 years, including a 10 -year program period. The discount rate is $4 \%$ over 56 years (the analysis period plus the period from current year, 2018, to program year 1, 2020).

Table 7.3 Defining the different BLO problems of the example

\begin{tabular}{|c|c|c|c|c|}
\hline & Sample & Optimization Goals & Type & Scenarios \\
\hline \multirow{2}{*}{ 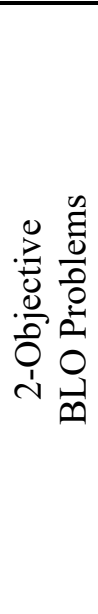 } & \multirow[t]{2}{*}{$\begin{array}{l}\text { Bridge } 38 \\
\text { with } \\
20 \\
\text { elements }\end{array}$} & \multirow[t]{2}{*}{$\begin{array}{l}\text { - maximize the bridge health } \\
\text { index in year } 20 \text { (denoted } \\
\text { by HI20 brg) } \\
\text { - minimize the bridge LCC } \\
\text { (denoted by } \text { LCC }_{\text {brg }} \text { ) }\end{array}$} & MCKP & $\begin{array}{ll}\text { Scenario a: unconstrained } \\
\checkmark & \text { unconstrained budget } \\
\checkmark & \text { unconstrained } \\
& \text { performance } \\
& \text { Figure } 7.4(\mathrm{a})\end{array}$ \\
\hline & & & MCMDKP & $\begin{array}{l}\text { Scenario b: constrained } \\
\checkmark \quad \text { budget constraint } \\
\leq \$ 2.5 \mathrm{M} \\
\checkmark \quad \text { performance constraint } \\
\geq \text { bridge health index of } \\
70 \% \text { every } 10 \text { years } \\
\text { Figure } 7.4(\mathrm{~b})\end{array}$ \\
\hline \multirow[b]{2}{*}{ 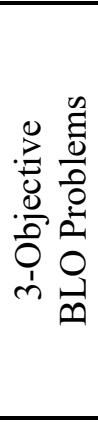 } & \multirow{2}{*}{$\begin{array}{c}\text { Bridge } 38 \\
\text { with } \\
20 \\
\text { elements }\end{array}$} & \multirow{2}{*}{$\begin{array}{l}\text { - minimize the bridge LCC } \\
\text { (denoted by } \mathrm{LCC}_{\text {brg }} \text { ) } \\
\text { - maximize the bridge LCC } \\
\text { benefit (denoted by } \\
\mathrm{BNT}_{\text {brg }} \text { ) } \\
\text { - maximize the bridge health } \\
\text { index in year } 20 \text { (denoted } \\
\text { by HI20 } 0_{\text {brg }} \text { ) }\end{array}$} & MCKP & $\begin{array}{l}\text { Scenario a: unconstrained } \\
\text { Figure } 7.4(\mathrm{c})\end{array}$ \\
\hline & & & MCMDKP & $\begin{array}{l}\text { Scenario b: constrained } \\
\text { Figure } 7.4(\mathrm{~d})\end{array}$ \\
\hline \multirow[b]{2}{*}{ 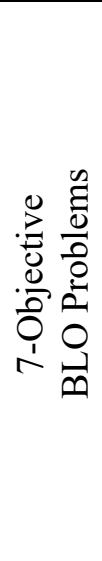 } & \multirow{2}{*}{$\begin{array}{l}\text { Bridge } 38 \\
\text { with } \\
20 \\
\text { elements }\end{array}$} & \multirow[b]{2}{*}{$\begin{array}{l}\text { - minimize the bridge LCC } \\
\text { (denoted by } \mathrm{LCC}_{\mathrm{brg}} \text { ) } \\
\text { - maximize the bridge LCC } \\
\text { benefit (denoted by } \\
\mathrm{BNT}_{\mathrm{brg}} \text { ) } \\
\text { - maximize the bridge health } \\
\text { indices in years } \\
10,20,30,40 \text {, and } 50 \\
\text { (denoted by HI10 } 0_{\mathrm{brg}} \\
\text { HI20 } 0_{\mathrm{brg}}, \mathrm{HI} 30_{\mathrm{brg}}, \mathrm{HI} 40_{\mathrm{brg}}, \\
\text { and } \mathrm{HI} 50_{\mathrm{brg}} \text { ) }\end{array}$} & MCKP & $\begin{array}{l}\text { Scenario a: unconstrained } \\
\text { Figure } 7.4(\mathrm{e})\end{array}$ \\
\hline & & & MCMDKP & $\begin{array}{l}\text { Scenario b: constrained } \\
\text { Figure } 7.4(\mathrm{f})\end{array}$ \\
\hline
\end{tabular}


Table 7.4 shows the results associated with the 35 BLO solutions recommended for the 2-objective BLO problem, Scenarios $b$ (budget constraint $\leq \$ 2.5 \mathrm{M}$; performance constraint $\geq$ bridge health index of $70 \%$ every 10 years), for the entire analysis period. The optimization goal is to maximize the bridge health index in year 20 (HI20 brg) and minimize the bridge LCC ( $\left.\mathrm{LCC}_{\mathrm{brg}}\right)$. In addition to these two main objectives, the bridge initial agency cost ( $\left.\mathrm{IAC}_{\mathrm{brg}}\right), \mathrm{LCC}$ benefit $\left(\mathrm{BNT}_{\mathrm{brg}}\right)$, and health indices at the ends of years $1,10,30,40,50$, and 54 were also determined as part of the module optimization results. 
Table 7.4 Results associated with the 35 solutions obtained for the 2-objective BLO problem, Scenario $b$

\begin{tabular}{|c|c|c|c|c|c|c|c|c|c|c|}
\hline Solution & $\begin{array}{c}\mathrm{IAC}_{\mathrm{brg}} \\
(\$)\end{array}$ & $\begin{array}{c}\mathrm{LCC}_{\mathrm{brg}}{ }^{\mathrm{a}} \\
(\$)\end{array}$ & $\begin{array}{c}\mathrm{BNT}_{\text {brg }} \\
(\$)\end{array}$ & $\begin{array}{c}\mathrm{HI}_{\mathrm{brg}} \\
(\%)\end{array}$ & $\begin{array}{c}\mathrm{HI} 10_{\text {brg }} \\
(\%)\end{array}$ & $\begin{array}{c}\text { HI } 20_{\text {brg }}{ }^{\mathrm{a}} \\
(\%)\end{array}$ & $\begin{array}{c}\mathrm{HI} 30_{\mathrm{brg}} \\
(\%)\end{array}$ & $\begin{array}{c}\mathrm{HI}_{40} \mathrm{brg}_{\mathrm{g}} \\
(\%)\end{array}$ & $\begin{array}{c}\mathrm{HI} 50_{\text {brg }} \\
(\%)\end{array}$ & $\begin{array}{c}\mathrm{HI}_{5} 4_{\text {brg }} \\
(\%)\end{array}$ \\
\hline 1 & 621,560 & $74,475,238$ & $3,818,368$ & 80.59 & 77.64 & 88.49 & 73.24 & 73.91 & 71.70 & 67.41 \\
\hline 2 & 543,639 & $74,712,273$ & $3,581,333$ & 80.59 & 78.10 & 90.72 & 74.45 & 92.93 & 72.48 & 74.21 \\
\hline 3 & 917,873 & $74,754,845$ & $3,538,760$ & 80.59 & 78.26 & 90.99 & 74.28 & 92.90 & 72.46 & 74.20 \\
\hline 4 & 803,917 & $74,552,872$ & $3,740,733$ & 80.59 & 77.78 & 88.76 & 73.41 & 73.95 & 71.70 & 67.41 \\
\hline 5 & $1,033,072$ & $75,097,015$ & $3,196,590$ & 80.59 & 78.29 & 91.03 & 74.31 & 92.91 & 72.46 & 74.21 \\
\hline 6 & $2,012,630$ & $75,392,561$ & $2,901,045$ & 80.59 & 93.37 & 96.21 & 74.59 & 70.11 & 76.87 & 78.40 \\
\hline 7 & 621,560 & $74,430,483$ & $3,863,122$ & 80.59 & 77.64 & 88.49 & 73.24 & 73.91 & 71.70 & 67.40 \\
\hline 8 & 488,898 & $74,381,069$ & $3,912,537$ & 80.59 & 77.50 & 88.28 & 73.08 & 73.87 & 72.30 & 68.04 \\
\hline 9 & 444,112 & $74,638,473$ & $3,655,133$ & 80.59 & 78.08 & 90.69 & 74.41 & 92.92 & 72.47 & 74.20 \\
\hline 10 & $1,121,511$ & $75,203,509$ & $3,090,096$ & 80.59 & 78.29 & 91.03 & 74.31 & 92.91 & 72.46 & 74.21 \\
\hline 11 & $1,121,511$ & $74,952,364$ & $3,341,241$ & 80.59 & 78.29 & 91.02 & 74.31 & 92.91 & 72.46 & 74.21 \\
\hline 12 & $2,097,390$ & $75,450,232$ & $2,843,374$ & 80.59 & 93.39 & 96.24 & 74.63 & 70.14 & 76.88 & 78.41 \\
\hline 13 & $1,893,447$ & $75,369,448$ & $2,924,158$ & 80.59 & 93.40 & 96.06 & 74.39 & 70.03 & 76.82 & 78.39 \\
\hline 14 & 317,312 & $74,596,468$ & $3,697,137$ & 80.59 & 77.95 & 90.43 & 74.25 & 92.85 & 72.40 & 74.19 \\
\hline 15 & 620,028 & $74,201,275$ & $4,092,331$ & 80.59 & 77.53 & 74.11 & 83.71 & 78.29 & 71.73 & 67.55 \\
\hline 16 & 530,689 & $74,703,174$ & $3,590,431$ & 80.59 & 78.10 & 90.72 & 74.44 & 92.93 & 72.48 & 74.21 \\
\hline 17 & 817,066 & $74,341,158$ & $3,952,447$ & 80.59 & 77.67 & 74.38 & 83.88 & 78.32 & 71.73 & 67.55 \\
\hline 18 & 487,366 & $74,107,106$ & $4,186,500$ & 80.59 & 77.40 & 73.90 & 83.54 & 78.24 & 72.34 & 68.19 \\
\hline 19 & 488,485 & $74,395,637$ & $3,897,968$ & 80.59 & 77.50 & 88.40 & 73.22 & 73.88 & 71.69 & 67.40 \\
\hline 20 & $1,004,210$ & $74,819,058$ & $3,474,547$ & 80.59 & 78.28 & 91.02 & 74.31 & 92.91 & 72.46 & 74.21 \\
\hline 21 & 317,312 & $74,590,618$ & $3,702,987$ & 80.59 & 77.95 & 89.23 & 75.62 & 92.85 & 72.40 & 74.19 \\
\hline 22 & $1,004,210$ & $75,070,203$ & $3,223,403$ & 80.59 & 78.28 & 91.02 & 74.31 & 92.91 & 72.46 & 74.21 \\
\hline 23 & 611,660 & $74,154,712$ & $4,138,894$ & 80.59 & 77.52 & 74.11 & 83.71 & 78.29 & 71.73 & 67.55 \\
\hline 24 & $1,884,393$ & $75,376,997$ & $2,916,608$ & 80.59 & 93.32 & 96.20 & 74.58 & 70.09 & 76.84 & 78.39 \\
\hline 25 & $1,033,072$ & $74,845,871$ & $3,447,735$ & 80.59 & 78.29 & 91.02 & 74.31 & 92.91 & 72.46 & 74.21 \\
\hline 26 & 794,017 & $74,277,101$ & $4,016,505$ & 80.59 & 77.66 & 74.38 & 83.88 & 78.32 & 71.73 & 67.55 \\
\hline 27 & $1,030,034$ & $74,889,040$ & $3,404,565$ & 80.59 & 78.29 & 91.02 & 74.31 & 92.91 & 72.46 & 74.21 \\
\hline 28 & 684,404 & $74,246,989$ & $4,046,616$ & 80.59 & 77.54 & 74.16 & 83.71 & 78.28 & 72.34 & 68.19 \\
\hline 29 & $1,033,072$ & $75,141,808$ & $3,151,798$ & 80.59 & 78.29 & 91.03 & 74.31 & 92.91 & 72.46 & 74.21 \\
\hline 30 & 486,953 & $74,122,202$ & $4,171,403$ & 80.59 & 77.40 & 74.02 & 83.69 & 78.25 & 71.72 & 67.54 \\
\hline 31 & 511,742 & $74,421,987$ & $3,871,619$ & 80.59 & 77.58 & 88.48 & 73.22 & 73.88 & 71.69 & 67.40 \\
\hline 32 & $1,879,254$ & $75,369,603$ & $2,924,003$ & 80.59 & 93.32 & 96.17 & 74.55 & 70.05 & 76.82 & 78.39 \\
\hline 33 & 785,854 & $74,253,677$ & $4,039,929$ & 80.59 & 77.58 & 74.17 & 83.73 & 78.31 & 72.35 & 68.20 \\
\hline 34 & 685,935 & $74,520,952$ & $3,772,653$ & 80.59 & 77.64 & 88.54 & 73.25 & 73.91 & 72.31 & 68.05 \\
\hline 35 & 326,399 & $74,595,614$ & $3,697,992$ & 80.59 & 77.96 & 89.32 & 75.55 & 92.85 & 72.39 & 74.19 \\
\hline
\end{tabular}

${ }^{\mathrm{a}}$ Optimized objective. 
The recommended solutions for the different BLO problems of the example are plotted in Figures 7.4(a) through (f). The plotted solutions are the Pareto solutions and identified for the entire analysis period. Figures 7.4(a) and (b) display the 2-objective BLO solutions for Scenarios a and b. Figures 7.4(c) and (d) present the 3-objective BLO solutions. The two radar or spider charts, Figures 7(e) and 7(f), relate to the 7-objective BLO problems. The charts show the normalized objective values (Chapter 6 discusses the reasons for using this type of charts).

All obtained solutions are considered non-dominated solutions (Pareto solutions). As emphasized previously, Pareto solutions in this research encompass the optimal or near-optimal (very close to optimal) solutions. The approach discussed in Chapter 6 to verify optimally was also followed for this example. The consistency of each obtained Pareto frontier was verified by increasing the number of iterations/generations to observe any difference in shape. The obtained solutions are hosted on discontinuous Pareto frontiers (good approximation of true Pareto frontiers). 
(a)

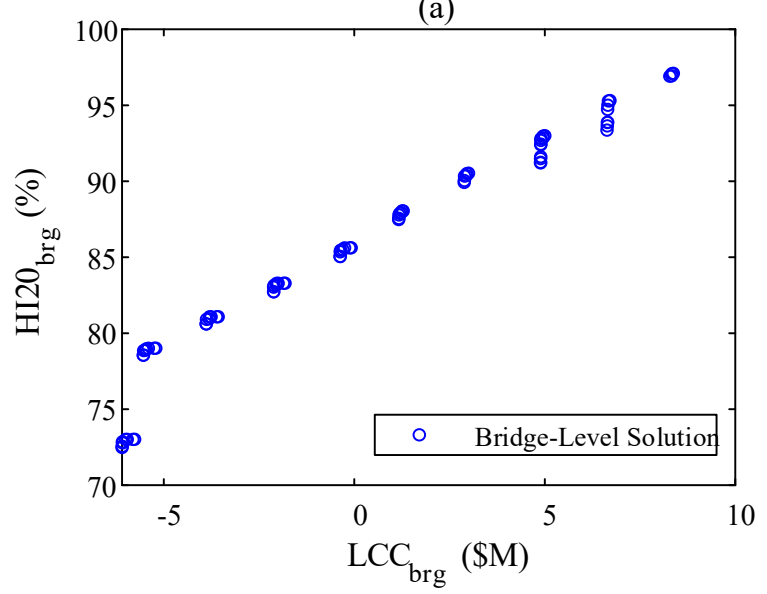

(c)
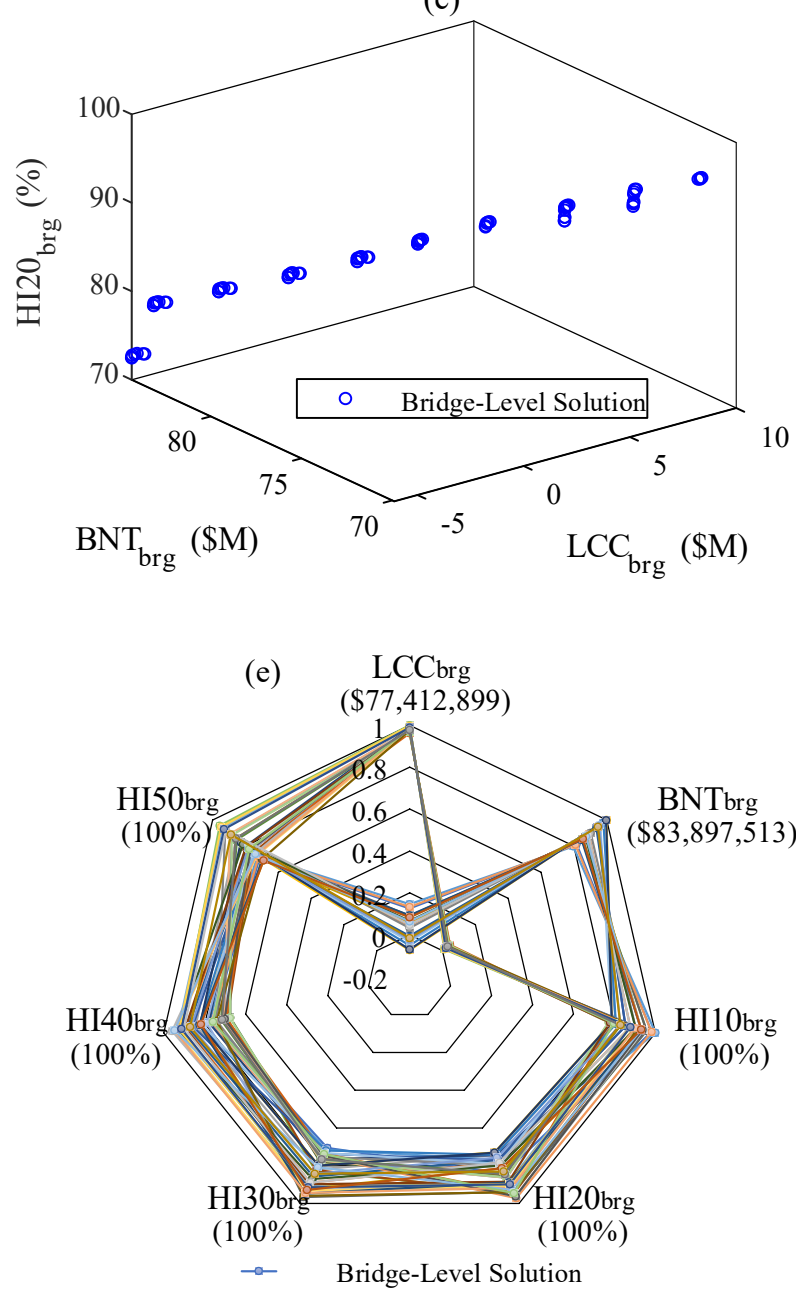

(b)

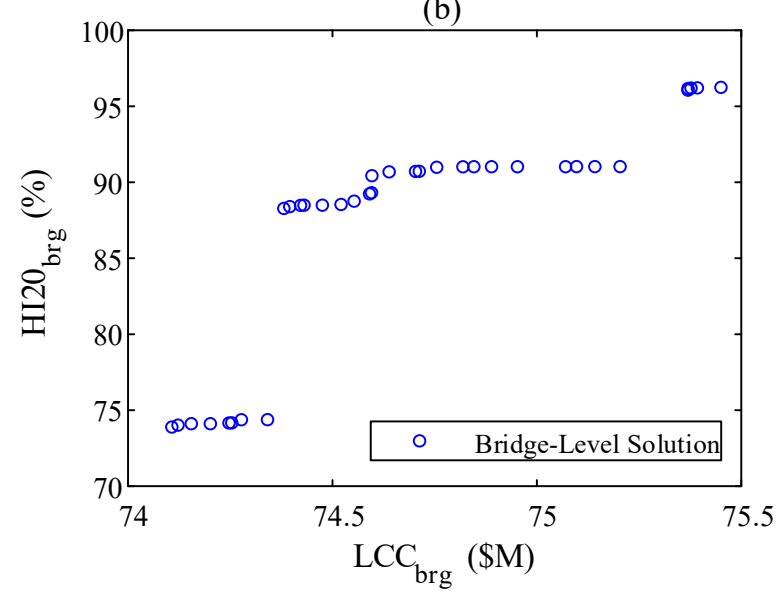

(d)
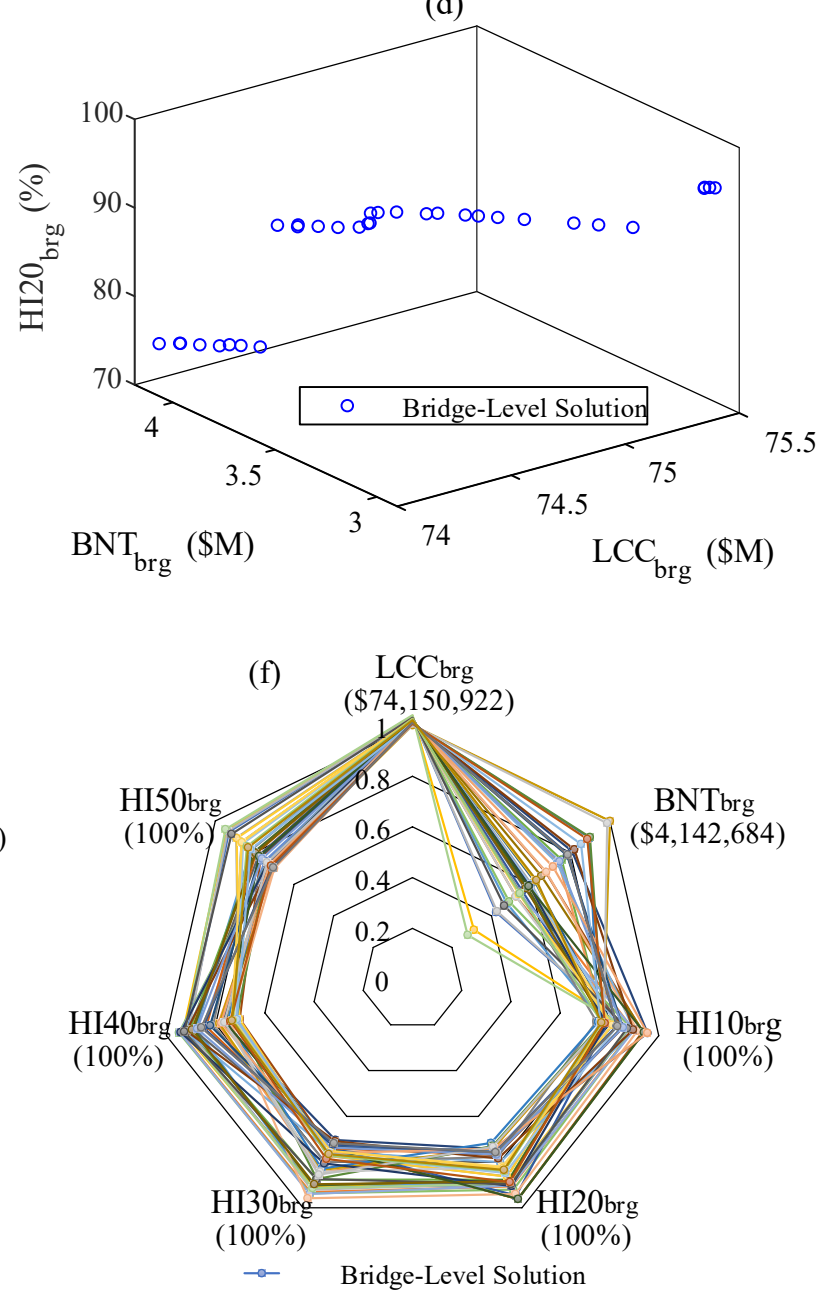

Figure 7.4 Obtained solutions for the 2-objective BLO problems under (a) Scenario a, (b) Scenario b; the 3-objective BLO problems under (c) Scenario a, (d) Scenario b; the 7-objective BLO problems under (e) Scenario a, (f) Scenario b 
The bridge health indices at the ends of years $1,10,20,30,40,50$, and 54 for each of these solutions are shown in Figures 7.5(a) through (f). Figures 7.5(a) and (b) show the predicted bridge health indices (connected with straight lines) for the 2-objective BLO solutions. Figures 7.5(c) and (d) display the predicted bridge health indices for the 3-objective BLO solutions. The bridge health indices associated with the 7-objective BLO solutions are shown in Figures 7.5(e) and (f). Figures 7.5(a), (c), and (e) represent Scenarios a; Figures 7.5(b), (d), and (f) represent Scenario b.

The RO health indices shown in these figures are slightly better than the predicted DN ones. The connected straight lines between $\mathrm{RO}$ or DN health indices illustrate a decline in bridge condition over time. The bridge DN health index is predicted to drop to $40 \%$ by year 50 . However, the bridge RO health indices are slightly better, starting from year 20. Figures 7.5 (b), (d), and (f) indicate that the recommended Pareto solutions for Scenario b satisfy the imposed performance restriction. As expected, the straight lines connecting the predicted bridge health indices are all situated above the horizontal dashed lines in black, representing the performance constraints (specified by a minimum bridge health index of $70 \%$ every 10 years).

Several Pareto solutions produced close values of bridge health indices despite the differences between the resulting LCCs. As explained in the previous chapter, changes to the overall bridge health index are generally minuscule. Element health indices are weighted, aggregated and divided by the sum of all their weighs to constitute this overall index, Equation (7.6). Preserving or improving few element health indices (after factoring their weights) won't dramatically change the overall index. It takes substantial improvement efforts to alter the overall index. 
(a)

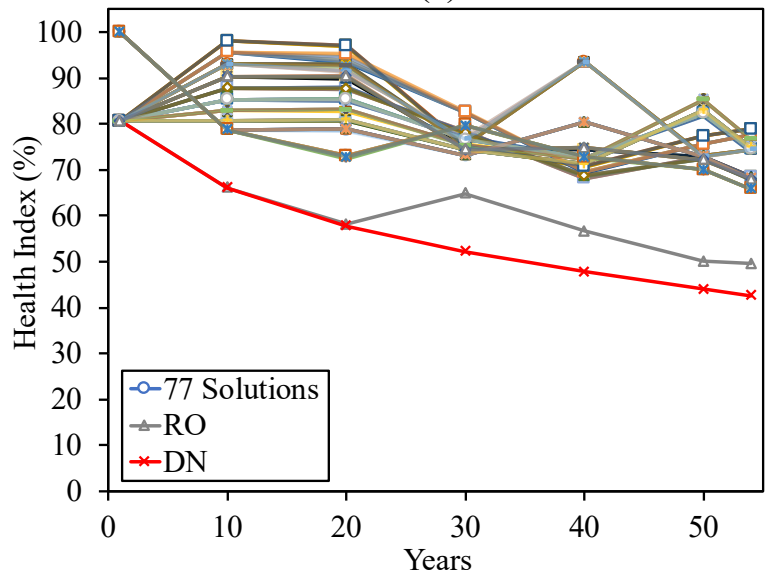

(c)

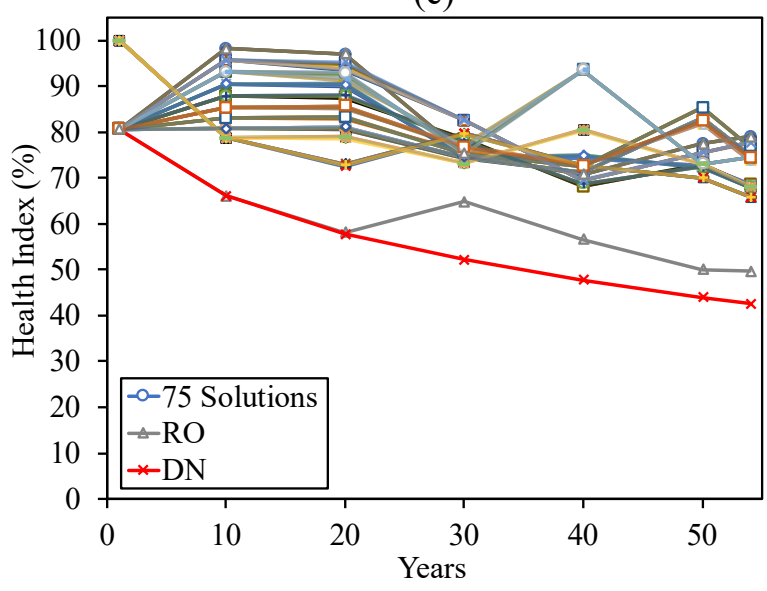

(e)

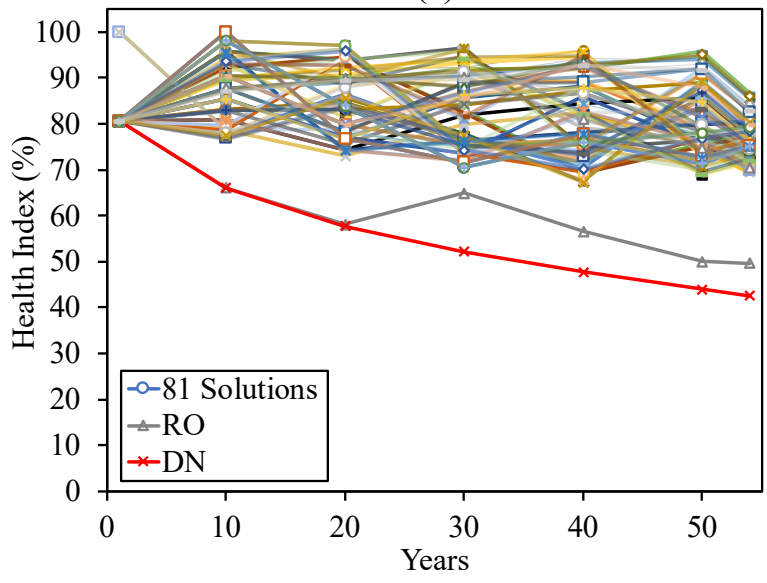

(b)

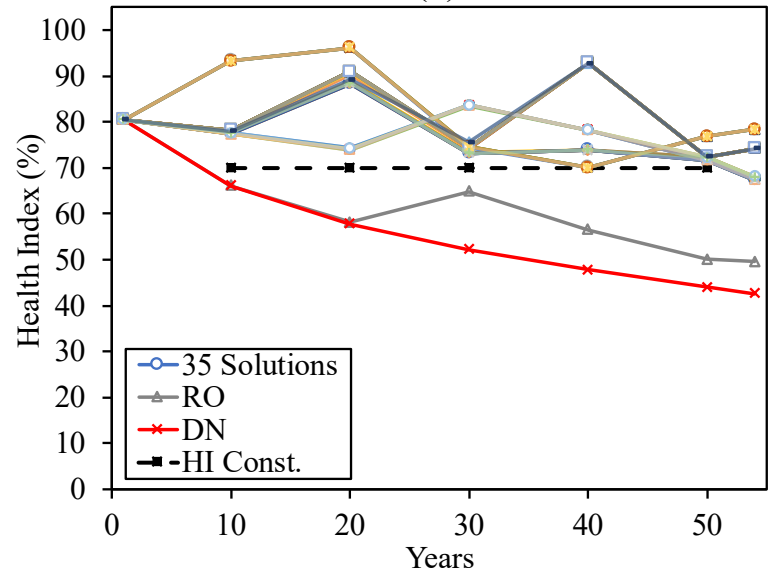

(d)

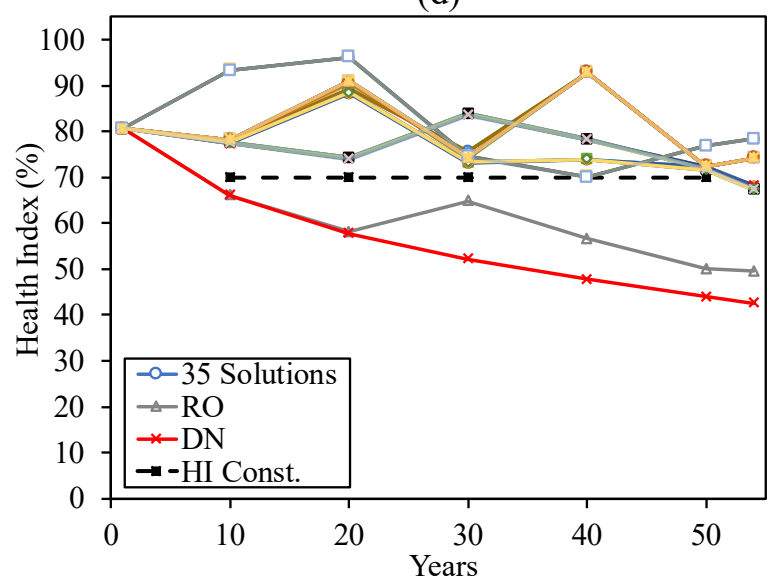

(f)

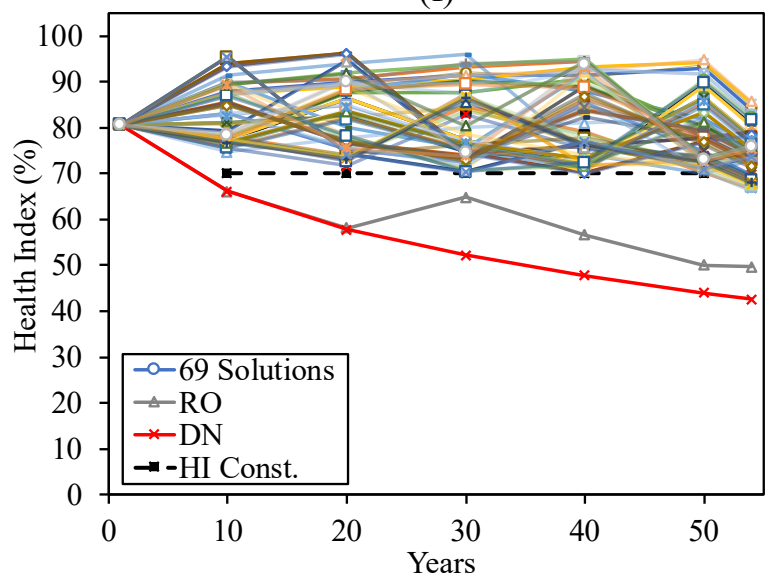

Figure 7.5 Predicted bridge health indices for the 2-objective BLO problems under (a) Scenario a, (b) Scenario b; the 3-objective BLO problems under (c) Scenario a, (d) Scenario b; the 7-objective BLO problems under (e) Scenario a, (f) Scenario b 
Table 7.5 shows the results associated with the BLO solutions producing the least bridge LCC values for the different BLO problems of the example. Although the 2- and 3-objective BLO problems have different BLO goals, it can be noticed that the presented BLO solutions (i.e., Solutions 18 and 23, respectively) produced the same results. The 3-objective BLO problems include one additional objective aimed to maximize the bridge $\mathrm{LCC}$ benefit $\left(\mathrm{BNT}_{\mathrm{brg}}\right)$. Generally, if the benefit is defined as the savings in LCC of "doing something" alternative relative to the "baseline" alternative, as defined in this research, the same alternative producing the least LCC value delivers the most LCC benefit.

Table 7.5 Results associated with the solutions producing the least bridge LCC values for the different BLO problems of the example

\begin{tabular}{|c|c|c|c|c|c|c|c|c|c|c|c|}
\hline & \multicolumn{11}{|c|}{ 2-Objective Optimization Problems } \\
\hline & Solution & $\begin{array}{c}\mathrm{IAC}_{\mathrm{brg}} \\
(\$)\end{array}$ & $\begin{array}{c}\mathrm{LCC}_{\mathrm{brg}}{ }^{\mathrm{a}} \\
(\$)\end{array}$ & $\begin{array}{c}\mathrm{BNT}_{\text {brg }} \\
(\$)\end{array}$ & $\begin{array}{c}\mathrm{HI}_{\mathrm{brg}} \\
(\%)\end{array}$ & $\begin{array}{c}\mathrm{HI} 10_{\mathrm{brg}} \\
(\%)\end{array}$ & $\begin{array}{c}\text { HI20 }{ }_{\text {brg }}^{\mathrm{a}} \\
(\%)\end{array}$ & $\begin{array}{c}\mathrm{HI}_{3} \mathrm{~b}_{\mathrm{brg}} \\
(\%)\end{array}$ & $\begin{array}{c}\mathrm{HI}_{40} \mathrm{~b}_{\mathrm{rg}} \\
(\%)\end{array}$ & 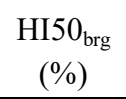 & $\begin{array}{c}\mathrm{HI}_{54}{ }_{\mathrm{brg}} \\
(\%)\end{array}$ \\
\hline Scenario a & 18 & $3,472,375$ & $-6,094,001$ & $84,352,376$ & 100.00 & 78.70 & 72.48 & 79.57 & 72.74 & 70.02 & 65.81 \\
\hline \multirow[t]{2}{*}{ Scenario b } & 18 & 487,366 & $74,107,106$ & $4,186,500$ & 80.59 & 77.40 & 73.90 & 83.54 & 78.24 & 72.34 & 68.19 \\
\hline & \multicolumn{11}{|c|}{ 3-Objective Optimization Problems } \\
\hline Scenario a & 23 & $3,472,375$ & $-6,094,001$ & $84,352,376$ & 100.00 & 78.70 & 72.48 & 79.57 & 72.74 & 70.02 & 65.81 \\
\hline \multirow[t]{2}{*}{ Scenario b } & 23 & 487,366 & $74,107,106$ & $4,186,500$ & 80.59 & 77.40 & 73.90 & 83.54 & 78.24 & 72.34 & 68.19 \\
\hline & \multicolumn{11}{|c|}{ 7-Objective Optimization Problems } \\
\hline & 4 & $3,472,375$ & $-5,639,137$ & $83,897,513$ & 100.00 & 78.70 & 73.01 & 79.80 & 82.38 & 74.30 & 69.30 \\
\hline Scenario $b$ & 22 & 586,817 & $74,150,922$ & $4,142,684$ & 80.59 & 77.47 & 73.93 & 83.50 & 78.45 & 71.94 & 67.77 \\
\hline
\end{tabular}


Figures 7.6(a) through (f) show the predicted element health indices associated with the solutions shown in Table 7.5. As expected, the straight lines connecting element health indices are all situated above the health index thresholds (60\% every 10 years, horizontal dashed lines in black) specified by Criteria 1 of the alternative feasibility screening process (discussed in Chapter 5). The horizontal dashed lines in red represent the health index lower-frontiers (deficiency screening thresholds, a minimum health index of $80 \%$ every 10 years). The dashed curves distinguish the non-deficient elements from the deficient ones. The thick connected lines in black represent the overall bridge health indices. Figure 7.6(g) includes the predicted DN element health indices over the analysis period - produced by DN LC Alternative. The predicted RO element health indices are shown in Figure 7.6(h)-produced RO LC Alternative. 
(a)

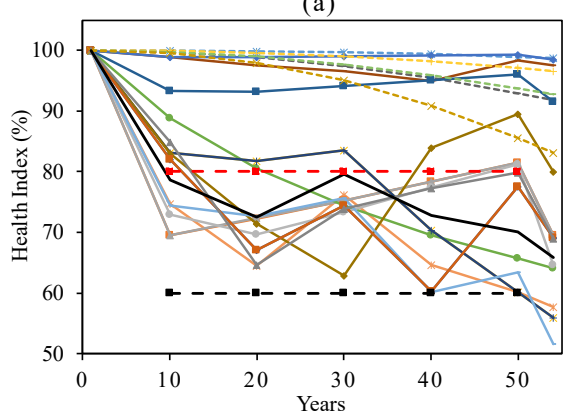

(c)

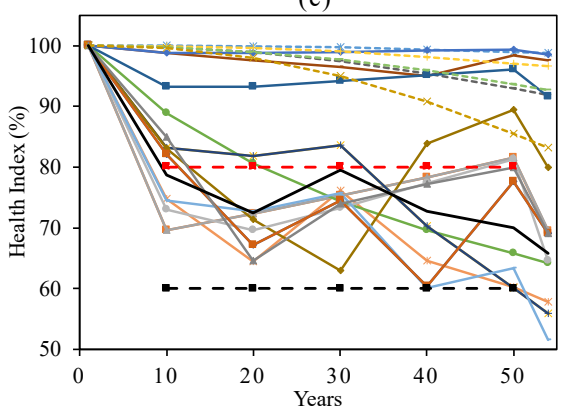

(e)

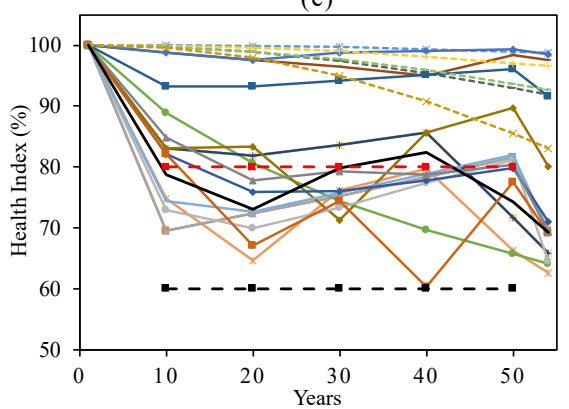

$(\mathrm{g})$

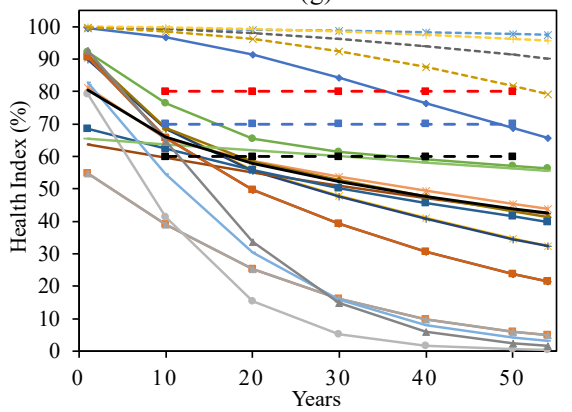

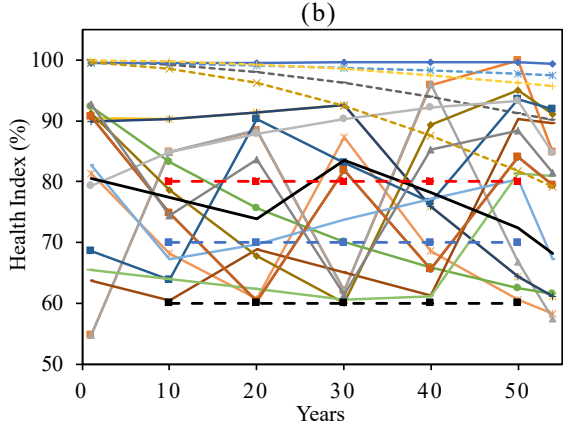

(d)

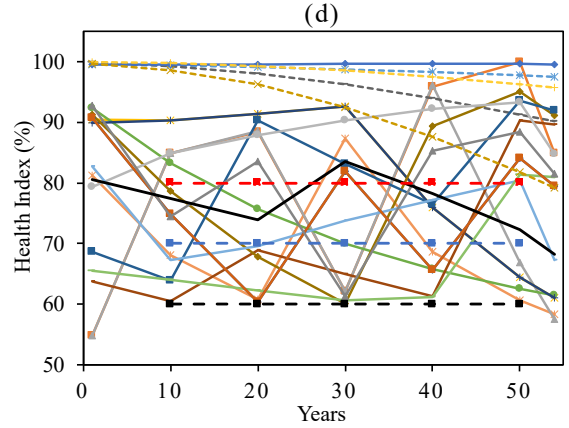

(f)

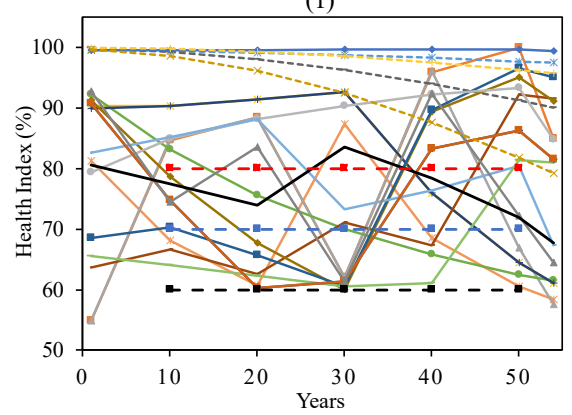

(h)

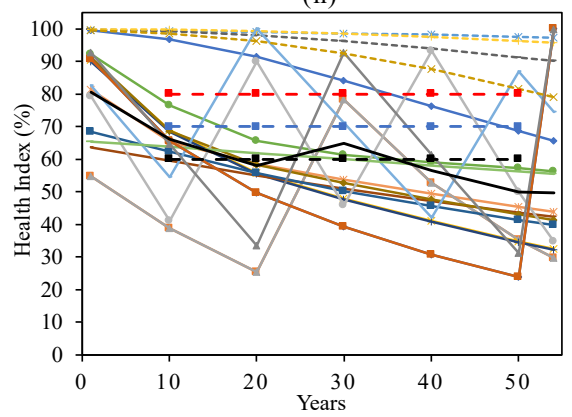

\begin{tabular}{|c|c|c|}
\hline & $=-E \ln$ & $m 29$ \\
\hline -Elm 107 & -*-Elm 109 & $\rightarrow$ Elm 113 \\
\hline -E $1{ }^{-152}$ & -Elm-210 & ---Elm² 215 \\
\hline$\rightarrow$ Elm-220 & $\rightarrow-E l m-226$ & $\rightarrow$ Elm 227 \\
\hline 231 & -Elm_234 & -Elm_- 301 \\
\hline -Elm_310 & $\rightarrow$ Elm_-311 & - Elm 313 \\
\hline$=$ Elm 330 & $-*$-Elm 331 & $\rightarrow$-Alt. $\overline{\text { Scr. }}$ \\
\hline -Bridge & $\rightarrow$-Def. Scr. & $\rightarrow-\mathrm{HI}$ Const. \\
\hline
\end{tabular}

Figure 7.6 Predicted health indices associated with the solutions producing the least bridge LCC values for the 2-objective BLO problems under (a) Scenario a, (b) Scenario b; the 3-objective BLO problems under (c) Scenario a, (d) Scenario b; the 7-objective BLO problems under (e) Scenario a, (f) Scenario b.

Predicted health indices under (g) DN LC Alternative; (h) RO LC Alternative 
The objective of the BLO module is to produce a set of optimal or near-optimal solutions for a candidate bridge. Each solution holds a recommended LC alternative for each element of the bridge. Table 7.6 shows the recommended LC alternatives associated with the solutions producing the least bridge LCC values for the 2-objective BLO problems (i.e., Solution 18, shown in Table 7.5). These recommended improvement actions provide the least bridge LCC while sustaining the desired bridge performance.

For Scenario a (unconstrained budget and performance), the major functional improvement action (FCI Action 5) is assigned to program year 1 followed by a series of element preservation actions (MRR Actions 0, 1, 2, 3, and 4) at different decision points. For an initial investment of $\$ 3,472,375$, the FCI improvement type eliminates the two functional deficiencies and incurred user costs and fully restores all bridge elements to CS1 equals $100 \%$. However, for the constrained Scenario b (limiting the budget to $\$ 2.5 \mathrm{M}$ and sustaining a minimum bridge health index of $70 \%$ every 10 years), the preservation (MRR) improvement type produced the least bridge LCC value. The recommended element preservation actions are assigned to program year 6 and each decision point (spaced by a 10 -year inaction period). LC Alternative 1 is always assigned to the non-deficient elements. Elements 109, 215, 234, 310, and 331 are the non-deficient elements (based on the postmajor improvement screening covered in Chapter 5) under the FCI improvement type; however, Element 310 is considered as a deficient element under the MRR improvement type-only Elements 109, 215, 234, and 331 are the non-deficient ones based on the preservation only screening covered in Chapter 5. The recommended improvement actions and timings represent a detailed element-level work plan. The recommended actions can be grouped into a bridge project or included under a bridge improvement program. Table 7.7 shows the feasible preservation treatments associated with these recommended improvement actions. 
Table 7.6 Element improvement actions associated with the solutions producing the least bridge LCC values for the 2-objective BLO problems

\begin{tabular}{|c|c|c|c|c|c|c|c|}
\hline & $\begin{array}{l}\text { Element } \\
\text { Ref. }\end{array}$ & $\begin{array}{c}\text { FCI } \\
\text { LC Alt. }\end{array}$ & $\begin{array}{l}\text { Pro. Year } \\
\text { (year 1) }\end{array}$ & $\begin{array}{c}\text { Dec. Point } 1 \\
\text { (year 12) }\end{array}$ & $\begin{array}{c}\text { Dec. Point } 2 \\
\text { (year 23) }\end{array}$ & $\begin{array}{c}\text { Dec. Point } 3 \\
\text { (year 34) }\end{array}$ & $\begin{array}{c}\text { Dec. Point } 4 \\
\text { (year 45) }\end{array}$ \\
\hline \multirow{21}{*}{ 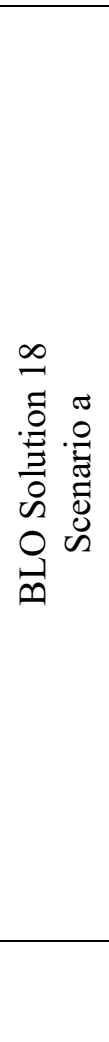 } & 12 & 418 & 5 & 3 & 1 & 3 & 2 \\
\hline & 28 & 157 & 5 & 1 & 1 & 1 & 1 \\
\hline & 29 & 157 & 5 & 1 & 1 & 1 & 1 \\
\hline & 107 & 175 & 5 & 1 & 1 & 4 & 4 \\
\hline & $109^{\mathrm{a}}$ & 1 & 5 & 0 & 0 & 0 & 0 \\
\hline & 113 & 625 & 5 & 4 & 4 & 4 & 4 \\
\hline & 152 & 175 & 5 & 1 & 1 & 4 & 4 \\
\hline & 210 & 572 & 5 & 4 & 2 & 4 & 1 \\
\hline & $215^{\mathrm{a}}$ & 1 & 5 & 0 & 0 & 0 & 0 \\
\hline & 220 & 607 & 5 & 4 & 4 & 1 & 1 \\
\hline & 226 & 157 & 5 & 1 & 1 & 1 & 1 \\
\hline & 227 & 157 & 5 & 1 & 1 & 1 & 1 \\
\hline & 231 & 157 & 5 & 1 & 1 & 1 & 1 \\
\hline & $234^{\mathrm{a}}$ & 1 & 5 & 0 & 0 & 0 & 0 \\
\hline & 301 & 163 & 5 & 1 & 1 & 2 & 2 \\
\hline & $310^{\mathrm{a}}$ & 1 & 5 & 0 & 0 & 0 & 0 \\
\hline & 311 & 417 & 5 & 3 & 1 & 3 & 1 \\
\hline & 313 & 417 & 5 & 3 & 1 & 3 & 1 \\
\hline & 330 & 407 & 5 & 3 & 1 & 1 & 1 \\
\hline & $331^{\mathrm{a}}$ & 1 & 5 & 0 & 0 & 0 & 0 \\
\hline & $\begin{array}{l}\text { Element } \\
\text { Ref. }\end{array}$ & $\begin{array}{c}\text { MRR } \\
\text { LC Alt. }\end{array}$ & $\begin{array}{l}\text { Pro. Year } \\
\text { (year 6) }\end{array}$ & $\begin{array}{c}\text { Dec. Point } 1 \\
(\text { year 17) }\end{array}$ & $\begin{array}{l}\text { Dec. Point } 2 \\
\quad(\text { year } 28)\end{array}$ & $\begin{array}{c}\text { Dec. Point } 3 \\
\text { (year 39) }\end{array}$ & $\begin{array}{c}\text { Dec. Point } 4 \\
\quad(\text { year 50) }\end{array}$ \\
\hline \multirow{20}{*}{ 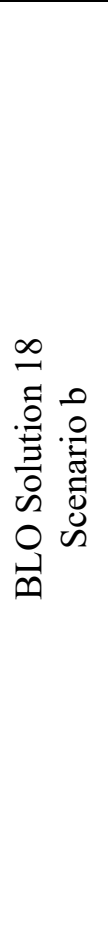 } & 12 & 2294 & 3 & 3 & 1 & 3 & 3 \\
\hline & 28 & 832 & 1 & 1 & 3 & 1 & 1 \\
\hline & 29 & 1464 & 2 & 1 & 3 & 2 & 3 \\
\hline & 107 & 800 & 1 & 1 & 1 & 4 & 4 \\
\hline & $109^{a}$ & 1 & 0 & 0 & 0 & 0 & 0 \\
\hline & 113 & 3125 & 4 & 4 & 4 & 4 & 4 \\
\hline & 152 & 800 & 1 & 1 & 1 & 4 & 4 \\
\hline & 210 & 2247 & 3 & 2 & 4 & 4 & 1 \\
\hline & $215^{\mathrm{a}}$ & 1 & 0 & 0 & 0 & 0 & 0 \\
\hline & 220 & 1857 & 2 & 4 & 4 & 1 & 1 \\
\hline & 226 & 2747 & 4 & 1 & 4 & 4 & 1 \\
\hline & 227 & 782 & 1 & 1 & 1 & 1 & 1 \\
\hline & 231 & 782 & 1 & 1 & 1 & 1 & 1 \\
\hline & $234^{\mathrm{a}}$ & 1 & 0 & 0 & 0 & 0 & 0 \\
\hline & 301 & 1563 & 2 & 2 & 2 & 2 & 2 \\
\hline & 310 & 2337 & 3 & 3 & 3 & 2 & 1 \\
\hline & 311 & 1667 & 2 & 3 & 1 & 3 & 1 \\
\hline & 313 & 1667 & 2 & 3 & 1 & 3 & 1 \\
\hline & 330 & 2082 & 3 & 1 & 3 & 1 & 1 \\
\hline & $331^{\mathrm{a}}$ & 1 & 0 & 0 & 0 & 0 & 0 \\
\hline
\end{tabular}

${ }^{\mathrm{a}}$ Non-deficient element. 
Table 7.7 Feasible preservation treatments associated with the improvement actions recommended by the solutions producing the least bridge LCC values for the 2-objective BLO problems

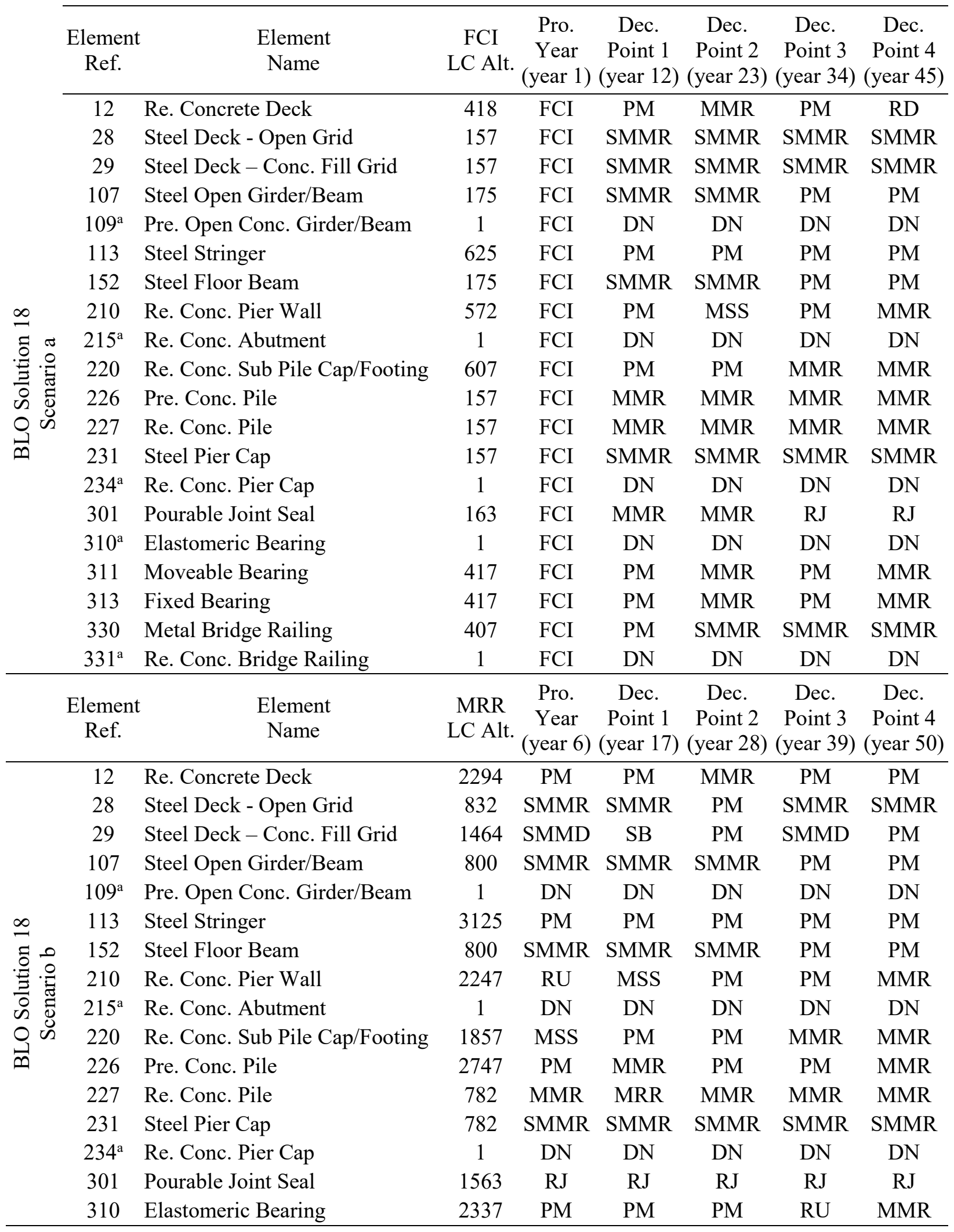


Table 7.7 Feasible preservation treatments associated with the improvement actions recommended by the solutions producing the least bridge LCC values for the 2-objective BLO problems (continued)

\begin{tabular}{clcccccc}
\hline \multicolumn{1}{c}{$\begin{array}{c}\text { BLO Solution 18 } \\
\text { Scenario b }\end{array}$} \\
$\begin{array}{c}\text { Element } \\
\text { Ref. }\end{array}$ & \multicolumn{1}{c}{$\begin{array}{c}\text { Element } \\
\text { Name }\end{array}$} & $\begin{array}{c}\text { MRR } \\
\text { LC Alt. }\end{array}$ & $\begin{array}{c}\text { Pro. } \\
\text { Year } \\
\text { (year 6) }\end{array}$ & $\begin{array}{c}\text { Dec. } \\
\text { Point 1 } \\
\text { (year 17) }\end{array}$ & $\begin{array}{c}\text { Dec. } \\
\text { Point 2 } \\
\text { (year 28) }\end{array}$ & $\begin{array}{c}\text { Dec. } \\
\text { Point 3 } \\
\text { (year 39) }\end{array}$ & $\begin{array}{c}\text { Dec. } \\
\text { Point 4 } \\
\text { (year 50) }\end{array}$ \\
\hline 311 & Moveable Bearing & 1667 & RU & PM & MMR & PM & MMR \\
313 & Fixed Bearing & 1667 & RU & PM & MMR & PM & MMR \\
330 & Metal Bridge Railing & 2082 & PM & SMMR & PM & SMMR & SMMR \\
$331^{\text {a }}$ & Re. Conc. Bridge Railing & 1 & DN & DN & DN & DN & DN \\
\hline
\end{tabular}

Note. For more detail about these preservation treatments, refer to Implementation of the 2013 AASHTO Manual for Bridge Element Inspection (Sobanjo \& Thompson, 2016a). FCI = Bridge functional improvement; $\mathrm{PM}=$ Preventive maintenance; $\mathrm{RD}=$ Replace deck; $\mathrm{MMR}=$ Minor or major repair; $\mathrm{SMMR}=$ Spot blast and minor or major repair; SMMD $=$ Spot blast and minor or major repair or replace deck; SMMU = Spot blast and minor or major repair or replace unit; SB = Spot blast; RU = Replace unit; $\mathrm{DN}=$ Do-nothing; RJ = Replace joint; MSS = Mitigate settlement or scour.

${ }^{a}$ Non-deficient element. 


\subsection{Summary and Conclusions}

This chapter introduced an innovative BLO module that considers the ELO recommendations. The chapter presented the module framework, capturing the different concepts and processes, discussed the interaction between the element- and bridge-level optimization modules, and demonstrated effectiveness and benefits. The chapter highlighted the different BLO problem types and formulations, and the mapping approach of the problem decision variables. No screening process is needed to further reduce the large size of the optimization problem. The total number of decision variables remains manageable even for a large network of bridges.

A multi-year optimization strategy was found to be appropriate for the problem formulation. The BLO problem is formulated in terms of binary decision variables. The optimization formulation is shaped as a MCKP if no constraints involved other than the selection criterion-only one ELO solution (one choice) must be picked from all solutions (multiple choices). The multi-dimensional aspect is added to the problem when more than one constraint (e.g., available budget and/or minimum desired performance) are involved; the problem is then formulated as a MCMDKP.

The chapter introduced the heuristic algorithm designed to solve the BLO problems. The module relies on the same metaheuristic algorithm (i.e., NSGA-II) discussed in Chapter 6 to solve this bridge-level NP-hard combinatorial optimization problem. The BLO process addresses one bridge at a time. The optimization process focuses on obtaining a diverse set of BLO solutions for the entire program period - a recommended BLO solution delivers an optimal or near-optimal set of LC alternatives for all the identified deficient elements over the entire analysis period. BLO results are key inputs for the bottom-up approach NLO (discussed in Chapter 8). 
The chapter included an illustrative example of module results produced by the tool prototype. Only optimization results of one sample bridge are shown in this chapter. The presented example consists of different BLO problems under constrained and unconstrained scenarios. The tool prototype produced optimal or near-optimal BLO solutions, recommended sets of intervention actions and timings, predicted performance, and determined funding requirements for the entire program period. The BLO module can be used independently to provide a systematic process to develop/assess bridge improvement/preservation programs. 


\section{CHAPTER 8-NETWORK-LEVEL OPTIMIZATION MODULE}

- Task 3: Proposing an Element-Based Multi-Objective Optimization Methodology

- Task 4: Development of a Tool Prototype

- Task 5: Implementation of the Methodology through Examples of Scenarios

\subsection{Introduction}

The main objective of the proposed EB-MOO methodology is to determine short- and long-term investment needs, and support recommending programs and implementation schedules. The EBMOO methodology depends on a quantitative process driven by bridge-related data, decision makers' preferences, and agency's preservation polices, objectives and goals. As stated throughout this dissertation, three independent optimization modules were incorporated into the EB-MOO methodology:

(1) an ELO, to identify optimal or near-optimal element intervention actions for each deficient element (in a poor condition state) of a candidate bridge;

(2) a BLO, to identify combinations of optimal or near-optimal element intervention actions for a candidate bridge; and

(3) a NLO, following either a top-down or bottom-up approach, to identify sets of optimal or near-optimal element intervention actions for a network of bridges.

The two preceding chapters introduced the ELO and BLO module frameworks, concepts and processes, laid out the problem formulations and heuristic algorithms, and included illustrative examples using the tool prototype to demonstrate effectiveness and benefits. Likewise, this chapter 
introduces the third optimization module of the methodology. The chapter discusses the NLO module frameworks, the top-down and bottom-up approaches, the screening process, and the different heuristic optimization algorithms.

A screening process referred to as "solution superiority screening" is integrated into this module to reduce the computational time by restricting the decision variable space. The screening guarantees the inclusion of superior solutions-for each network-level objective to be optimized, ELO solutions yielding the best objective values per program year are embraced. Agency's improvement rules or triggers can be established at this higher level to substitute the adapted screening process or complement it.

The optimization problem is either constrained (by budget and/or performance) or unconstrained (involving only the selection criterion). The unconstrained problem can be used to estimate the budget required to address all predicted needs, or determine whether a program is underfunded or overfunded. Usually, this information is used to justify budget requests through the legislative process. The sum of initial agency costs of all recommended LC alternatives represents the needed investment. The budget-constrained problem can be used to anticipate short- and long-term network improvement strategies under a limited available budget—supporting the development of bridge programs, TAMPs, or TIPs/STIPs. Performance-constrained problem can be used to maintain a desired network performance. Typically, performance constraints involve setting network condition targets such as a percentage of bridges in a "state of good repair," a total number of bridges classified as "good," a percentage of the structurally deficient deck areas of bridges, and an average network health index. In this research, an overall network health index (averaging health indices of all bridges in the network) is used to assess the network performance. 
The optimization formulation is shaped as a MCKP for an unconstrained network-level problem. For a budget-constrained problem, the formulation varies based on the scenario (i.e., annual budget scenario or multi-year budget scenario). Budget constraints can be imposed either annually (for each program year) or cumulatively (for all program years). The multi-year budget-constrained problem is formulated as a MCMDKP; whereas, the annual budget-constrained problem is formulated as a "Multiple Knapsack Problem" (MKP). The module optimization requires a simultaneous optimization of multiple competing objectives; for example, minimize the network LCC and maximize the network LCC benefit and/or health indices at different points in time, seeking to arrive at the best trade-off between them. The aim is to obtain a diverse set of NLO solutions for the entire program period as close as possible to the Pareto frontier-a recommended NLO solution delivers the best feasible (optimal or near-optimal) set of LC alternatives for all deficient elements of the identified high-priority bridges from the portfolio over the entire analysis period. Both BLO and ELO solution results are key inputs for the NLO process. A bottom-up approach is followed when the BLO solutions are used as input parameters. On the other hand, a top-down approach is followed when the ELO solutions are used instead.

The module focuses on finding a diverse set of Pareto solutions for a portfolio of bridges. Again, the confronted problem is an NP-hard combinatorial optimization problem. Both top-down and bottom-up optimization approaches are formulated in terms of binary decision variables. When following the top-down approach, ELO results are grouped and expressed in summation formulas over the entire program period for all deficient elements of each bridge in the portfolio-a selected ELO solution is represented by a decision variable of 1 , otherwise 0 . When following the bottomup approach, BLO results are grouped and expressed in summation formulas over all bridges in the portfolio- a selected BLO solution is represented by a decision variable of 1 , otherwise 0 . 
Generally, near-optimal rather than optimal solutions are obtained. NLO objectives and constraints are expressed in terms of these decision variables. The module relies on the same metaheuristic algorithm (i.e., NSGA-II) discussed in Chapters 6 and 7 to solve these network-level NP-hard combinatorial optimization problems.

Several examples of unconstrained and constrained (by budget and/or performance) scenarios were established for the module implementation using the MATLAB-based tool prototype. Results are presented in this chapter to demonstrate the module capabilities: generating optimal or nearoptimal solutions, predicting performance, and determining intervention strategies and funding requirements. The example results are also used to evaluate the effectiveness of the top-down and bottom-up approaches.

\subsection{Background}

Decisions are made at either the network level or bridge level. For network-level decisions, ranking procedures or established decision trees (discussed in Chapter 2) have been widely used by state DOTs and other transportation agencies, especially when dealing with a large network of bridges. Setting priorities by ranking or decision trees is usually subjective and inadequate in providing the best long-term investment strategies. LCCA, BCA, and IBCA (discussed in Chapter 5) are the common economic decision analyses used by BMSs. Typically, alternatives are prioritized by sorting their benefit-cost ratios-generally ratios equal to or greater than 1 are considered acceptable. When the budget is constrained, alternatives with the highest benefit-cost ratios are selected following a priority until the available funding is exhausted. Though, ranking alternatives based on their benefit-cost ratios usually yield misleading results. 
IBCA is considered a superior to BCA (Farid et al., 1988). Robert (2017) described how IBCA is used in NBIAS to provide a near-optimal solution for a budget-constrained problem rather than exact optimization methods - due to their limitations for large-sized problems. NBIAS relies on an IBC heuristic called "MINBEN," described in (Robert et al., 2009), for sorting improvement alternatives. The heuristic provides a near-optimal solution within acceptable running time. Improvement alternatives that do not follow the Law of Diminishing Marginal Return are eliminated from consideration. Under a limited available budget, improvement alternatives that produce the highest benefits per additional cost are selected.

As stated in Chapter 5, BrM uses a multi-criteria decision analysis based on the utility theory. Each criterion for a bridge is associated with a utility value determined through weighting, scaling and amalgamation techniques. BrM uses two types of improvement actions: bridge- and network-level actions. A bridge-level action represents a single or small group of bridge work item(s). A networklevel action represents an entire set of actions for a network of bridges. The benefit of a networklevel action is calculated from the incremental increase in the overall utility value of the bridge (referred to as $\Delta$ Utility). A $\Delta$ Utility ratio is determined for each network-level action. The system selects network-level actions with the highest $\Delta$ Utility ratios. A cutoff is reached when all performance and budget constraints are met (Johnson \& Boyle, 2017).

Agency's rules and/or triggers are used by most BMSs to limit the number of possible improvement actions per year. At the network level, these improvement actions are often described in broad terms and applied to the entire bridge or its major components. Thus, much of the elementspecific information is lost. These high-level actions reduce the total number of possible combinations of actions and eventually the execution time. These actions lack of details and 
typically are not meant to produce a comprehensive bridge program. Obtained network-level investment strategies are generally used for high-level planning and programming purposes.

There are two NLO approaches among BMSs: (1) top-down approach, where the optimization determines the network-level goals, and then the improvement needs for individual bridges; and (2) bottom-up approach, where the bridge improvement needs are determined first. The top-down approach emphasizes on planning and strategy management at the network level. The bottom-up approach focuses on bridge-level analysis which dictates the network strategy (Lake \& Seskis, 2013). The top-down approach optimization produces high-level strategies (generally based on bridge component- or network-level actions) to meet the network goals and objectives. The bottom-up approach optimization uses the identified component- or element-level improvement strategies for each bridge as input parameters for the optimization process. The latter preserves bridge information and subsequently produces more refined network-level improvement strategies. The drawback of this approach is that a separate analysis for each bridge is required; and therefore, increasing the problem complexity, simulation, and computational time. For large networks, the top-down approach is generally the preferred approach due to the less computational requirements. Bridge improvement recommendations are made generally in terms of network-level improvement actions. Work refinements at the component or element level are generally left to the bridge manager judgments to compensate for the loss of bridge-specific information in the aggregation step (Yeo et al., 2013). 


\subsection{Module Framework}

Typically, decision makers are faced to simultaneously evaluate several differing preferences. In most cases, other non-economic preferences, targets and restrictions contribute in the decision making at the network level. True MOO methodologies are effective in optimizing multiple competing network-level objectives (such as minimizing the network LCC, and maximizing the network LCC benefit and performance) subject to constraints (such as limited available budget and minimum acceptable network performance). This research introduces a true network-level MOO procedure to overcome the limitations of existing approaches discussed in the background section. The proposed NLO module attempts to guarantee a diverse set of optimal or near-optimal solutions with a reasonable computational effort - the economically unattractive element LC alternatives are eliminated at the element level to produce screened sets of input parameters for this higher level of optimization.

The top-down and bottom-up approaches are defined differently in this research. The NLO of this module follows a novel concept but keeping the same terminology. Basically, a bottom-up approach is followed when BLO solutions (input parameters) are used in determining networklevel needs and recommending investment strategies; however, when ELO solutions (input parameters) are used instead, then the top-down approach is followed. In either approach, both levels of solutions originate from an unconstrained optimization to increase diversity and ultimately the search space. Recommended network-level investment strategies (by either approach) are detailed at the element level.

Figure 8.1 presents the framework of the top-down approach optimization-ELO solutions, associated with the three improvement types and all program years, for each bridge in the portfolio compete at this level. Only one ELO solution per bridge is selected. As discussed in Chapter 6, the 
ELO module focuses on finding Pareto solutions per program year and per improvement type for each deficient element of a bridge in the portfolio. A set of $\mathrm{LC}$ alternatives is derived from each solution. Each recommended LC alternative represents a series of best (optimal or near-optimal) actions for a deficient element over the analysis period. Results associated with these ELO solutions serve as the key inputs for the top-down approach optimization.

Figure 8.2 shows the framework adapted for the bottom-up approach optimization-BLO solutions for each bridge in the portfolio compete at this higher level. No more than one BLO solution per bridge is selected. The BLO module (covered in Chapter 7) independently produces a diverse set of Pareto solutions per program period for each bridge in the portfolio- $\mathrm{a}$ recommended BLO solution delivers an optimal or near-optimal set of LC alternatives for all deficient elements over the entire analysis period. Results associated with these BLO solutions serve as the key inputs for the bottom-up approach optimization. As mentioned throughout the preceding modules, if the MRR improvement type is recommended, MRR LC Alternative 1 (to mimic the DN LC Alternative over the entire analysis period) is assigned to the non-deficient elements, otherwise FCI/REP LC alternative 1 (to mimic the DN LC Alternative over the analysis years succeeding the element replacement year). 


\subsubsection{Top-Down Approach}

The module framework exemplified in Figure 8.1 is based on the following concepts:

1. ELO solution results (from an unconstrained optimization) per improvement type and per program year for each element of each bridge in the portfolio are transferred to this module as input parameters.

2. A screening process referred to as "solution superiority screening," discussed in the subsequent section, is deployed to reduce the NLO problem size, guarantee the inclusion of the best ELO solution results (input parameters) in the optimization process, and improve the computational time. Solution results are sorted based on the preferred screening criteria and/or optimization goals. However, this screening can be bypassed for small networks of bridges.

3. The screened ELO solution results (serving as the input parameters) are grouped and reorganized in single matrices as illustrated in Figure 8.1.

4. Single matrices representing each type of solution results are arranged to cover the entire program period, the three improvement types, and the entire portfolio.

5. The last three matrices shown in Figure 8.1 represent the NLO solution outcomes for the entire portfolio. The first matrix (bottom, far-left) contains the recommended LC alternatives associated with the NLO solutions (each bridge element is assigned to an optimal or near-optimal LC alternative, an improvement type, and a program year), the second matrix includes the resulting network initial agency costs and health indices at different points in the analysis period, and the third matrix contains the values of optimized network-level objectives. 


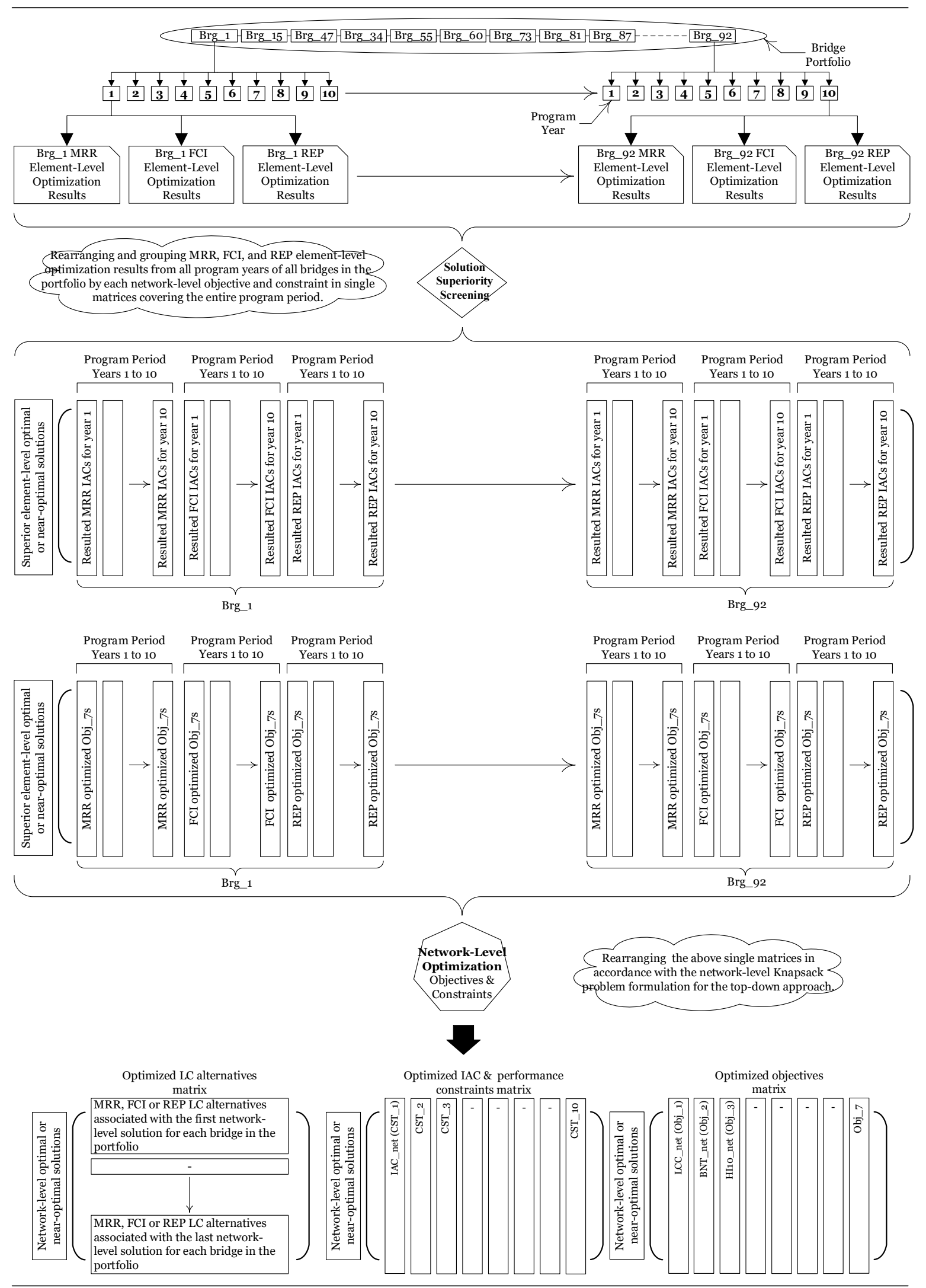

Figure 8.1 Network-level optimization module framework for the top-down approach 


\subsubsection{Bottom-Up Approach}

The module framework presented in Figure 8.2 is based on the following concepts:

1. BLO solution results (from an unconstrained optimization) per improvement type and per program year for each element of a bridge in the portfolio are transferred to this module as input parameters. A screening process is generally unnecessary for this bottom-up approach optimization. No need to further reduce the size of the optimization problem. The total number of decision variables remains manageable, even for a large network of bridges.

2. These BLO solution results are grouped and reorganized in single matrices as illustrated in Figure 8.2.

3. Single matrices representing each type of solution results are arranged to cover all bridges in the portfolio.

4. The last three matrices shown in Figure 8.2 represent the NLO outcomes for the entire portfolio. The first matrix (bottom, far-left) contains the recommend LC alternatives associated with the NLO solutions (each bridge element is assigned to an optimal or nearoptimal LC alternative, an improvement type, and a program year), the second matrix includes the resulting network initial agency costs and health indices at different points in the analysis period, and the third matrix contains the values of optimized network-level objectives. 

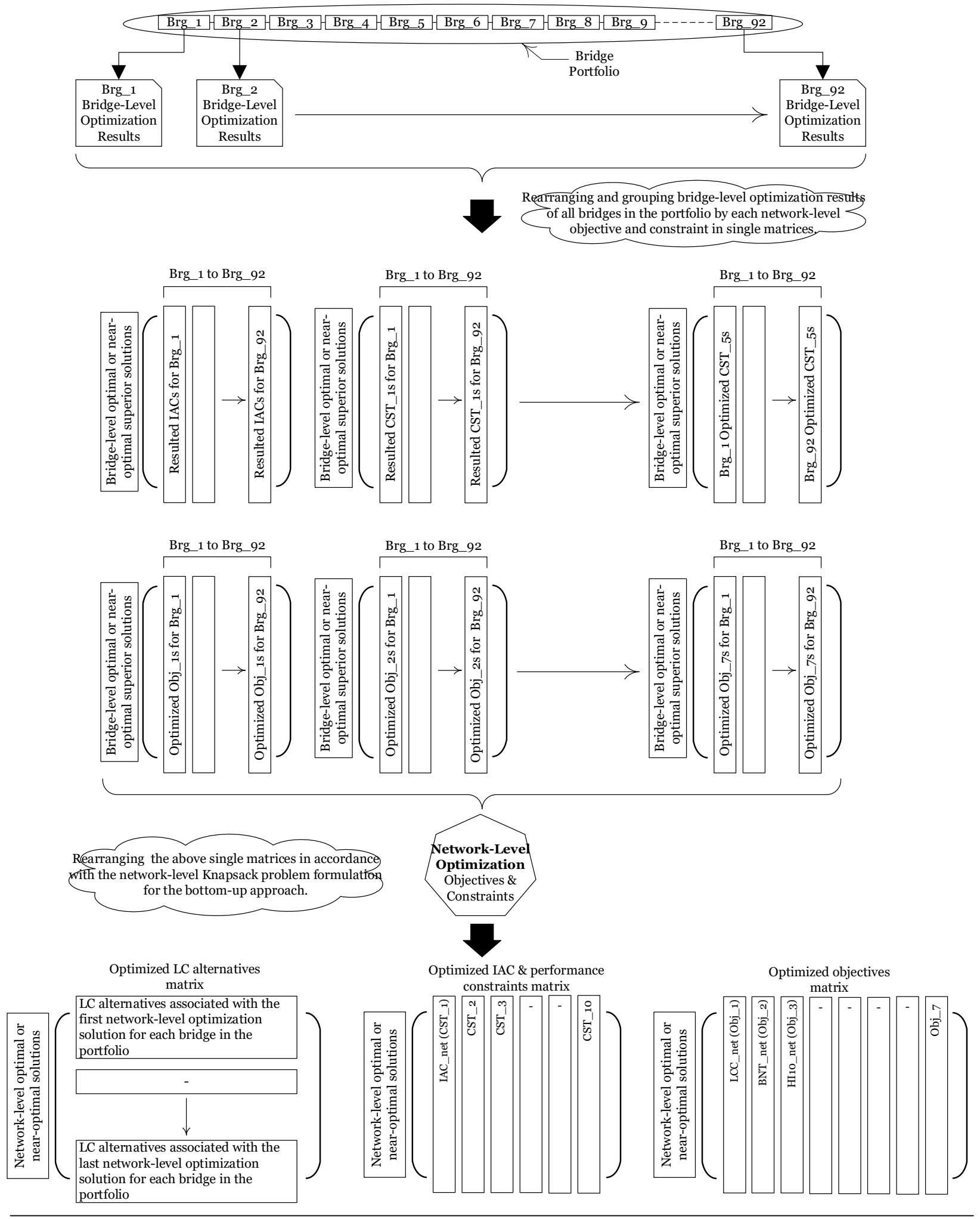

Brg 1 to $\mathrm{Brg} 92$

Figure 8.2 Network-level optimization module framework for the bottom-up approach 


\subsection{Solution Superiority Screening}

Although the improvement and ELO modules deploy several screening processes to reduce the problem size and improve the computational time, the problem size still represents a challenge at this higher level of optimization. Incorporating element- and bridge-level details into this module complicates the NLO process. The complexity of the problem substantially increases when the number of involved bridges increases, and consequently the solution space. Therefore, deploying a strategy to reduce the number of possible solutions is essential (Elbehairy, 2007). The module top-down approach could generate thousands or even millions of decision variables. For instance, 600,000 decision variables represent a top-down approach optimization problem for a network of 200 bridges (considering 100 ELO solutions per program year for each bridge). This large-scale problem becomes extremely difficult to manage, especially with common computers. Achieving heuristic solutions as close as to the Pareto frontier requires tremendous computational efforts and computer memory.

Chapter 6 introduced a 2-stage screening process (i.e., alternative feasibility screening process) to guide the ELO search toward global optimality within a reasonable computational time. A similar concept is endorsed at this higher level of optimization. An arrangement process referred to as "solution superiority screening" is integrated into this module to make the problem tractable with a reasonable computational effort—by restricting the decision variable space without affecting the quality of solutions. However, the process can be avoided if the running time is not a concern. This process reduces the optimization problem size and guarantees the inclusion of the superior (best) solution results in the NLO process. The process identifies ELO solutions (input parameters) producing the best results in alignment with the NLO goal. For each network-level objective to be optimized, the ELO solutions yielding the best element-level objective values per program year 
are embraced-generally, a cutoff value is assigned to control the number of these superior ELO solutions. Different cutoff values can be specified based on relative importance of network-level objectives, permitting acceptance of additional ELO solutions for consideration. A cutoff value of 1 produces only the maximum or minimum values, depending on the NLO goal. Let's reconsider the earlier network example for an optimization problem consisting of three network-level objectives. If 1 is taken as the cutoff value, the 100 ELO solutions are reduced to 3 ELO solutions per program year, and consequently the 600,000 decision variables are reduced to 18,000 . The screening process is generally unnecessary for the bottom-up approach optimization. The total number of decision variables remains manageable even for a large network of bridges. For the same network of 200 bridges, considering 100 BLO solutions per bridge, the bottom-up approach produces 20,000 decision variables.

Figure 8.3 illustrates an example of the solution superiority screening process. Three networklevel objectives to be optimized with a user-specified cutoff value of 10 . The example optimization goal is to simultaneously minimizing the network LCC and maximizing the network LCC benefit and health index at the end of year 20. For each program year and each improvement type, the corresponding ELO solution results are sorted in descendant or ascendant order, aligning with the NLO objectives. In this example, bridge LCCs, LCC benefits, and health indices at the end of year 20 (HI20s) are sorted independently. The 10 smallest bridge LCCs, the 10 highest bridge LCC benefits, and the 10 highest bridge HI20s are identified. Associated ELO solutions with these sorted results are classified as the superior ones and grouped in single matrices for consideration. These superior ELO solutions are compatible with the NLO goal. Thus, NLO solutions producing maximum or minimum network-level objective values are guaranteed - extending the search space exploration. 


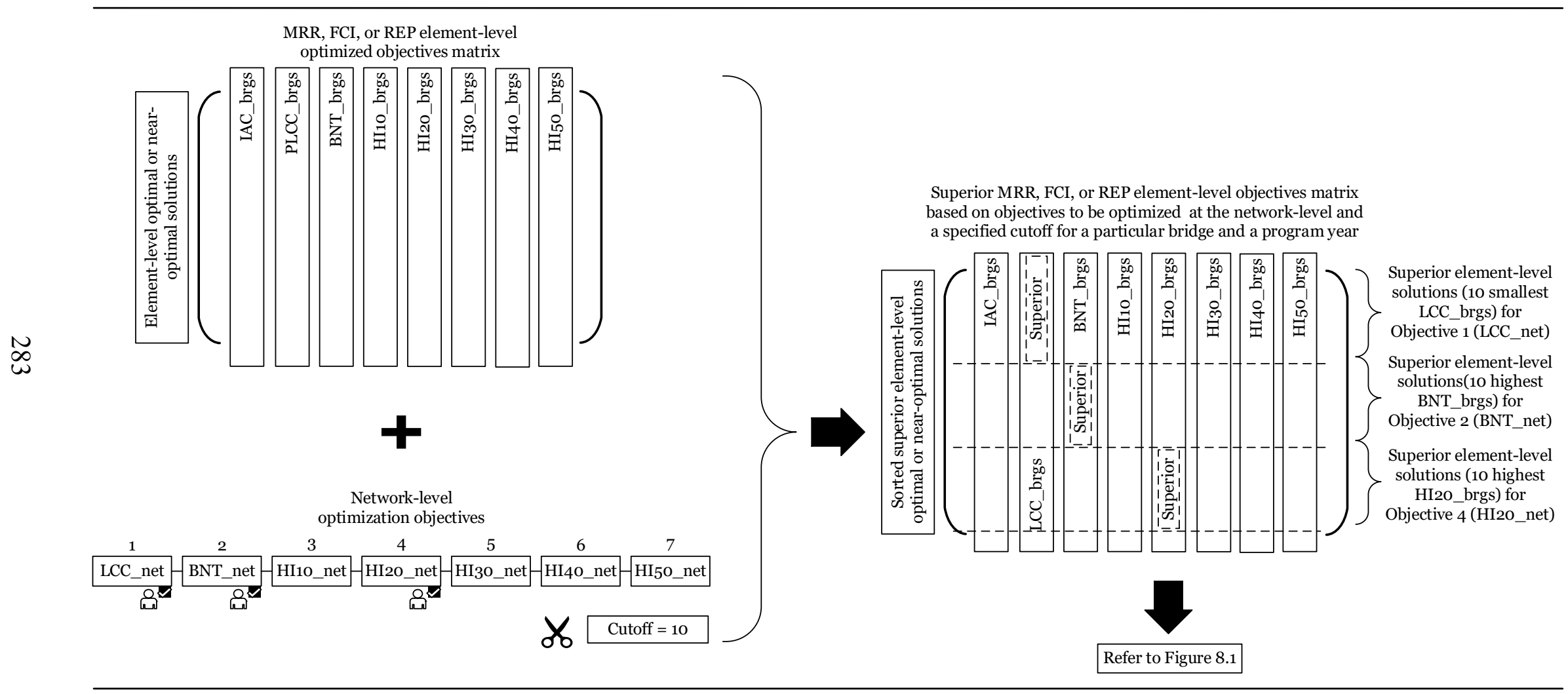

Figure 8.3 Illustration of the solution superiority screening process 


\subsection{Network-Level Optimization Problem Formulations}

Constructing a trade-off frontier composed of NLO solutions requires a simultaneous optimization of multiple network-level objectives. The NLO goal is to minimize network LCCs, maximize network LCC benefits, and/or maximize network health indices at different points in time (averaging health indices of all bridges in the network or portfolio) subject to network budget and/or performance constraints. The module focuses on finding a diverse set of Pareto solutions for a portfolio (network) of bridges while meeting the network budget and performance constraints. Each NLO solution identifies a set of high-priority bridges from the portfolio for improvement work. Each recommended bridge is allied with the appropriate improvement type, program year, and LC alternatives for all its elements. The non-selected bridges (considered as less-priority bridges) due to a budget restriction automatically constitute the backlog. If a backlog is not an option, the optimization is forced to consider every single bridge in the portfolio while relaxing certain constraints.

As mentioned earlier, the module top-down and bottom-up approaches are defined differently in this research. Basically, a bottom-up approach is followed when BLO solutions (input parameters) are used in determining network-level needs, and a top-down approach is followed when ELO solutions (input parameters) are used instead. In either approach, both levels of solutions originate from unconstrained optimization problems, increasing diversity and ultimately the search space. The solution superiority screening process identifies superior ELO solutions as input parameters for the top-down approach. As emphasized in the previous section, this screening process is deemed essential for large-sized optimization problems. The process makes the problem tractable with a reasonable computational effort-it reduces the problem size by focusing mainly on superior ELO solutions. The screening process is generally unnecessary for the bottom-up 
approach optimization. The total number of decision variables remains manageable even for a large network of bridges.

The top-down and bottom-up approaches in this research are formulated differently. Their formulations are defined in terms of binary decision variables. NLO objectives are functions of these decision variables. The binary variables were found to be suitable for these types of combinatorial optimization problems. A decision variable is 1 if an ELO (for the top-down approach) or BLO (for the bottom-up approach) solution is selected, otherwise 0. Their formulations are covered in the subsequent two sections. Budget and performance scenarios examined in this module are categorized as follows:

- $\quad$ Case $\mathrm{A}$ - all bridges in the portfolio must be selected

- Case B-not necessarily all bridges in the portfolio must be selected

For Case A's scenarios, the specified selection criterion restricts the decision variable space to one recommendation per bridge. Each bridge in the portfolio must be associated with only one set of LC alternatives for all its elements. This case is used when a backlog is not an option; every single bridge in the portfolio is considered - certain constraints can be relaxed to make the problem trackable, if necessary. For Case B's scenarios, no more than one set of LC alternatives per bridge can be recommended-reducing the budget causes improvement work on some bridges to be postponed. Not all bridges will be programmed under a restricted budget. Case B relaxes the selection criterion (the selection of each bridge is no fully enforced) to focus on bridges of urgent improvement needs (generally in a poor condition state) and satisfy the available budget. The nonselected bridges (the less urgent) are added to the backlog. Those low-priority bridges still require work to be performed, but most likely improvement needs change as they continue to deteriorate. 
The NLO problem can be either constrained (e.g., available budget and/or minimum desired performance) or unconstrained. The selection criterion of Case A or Case B is always considered, regardless. When no additional constraints are involved (i.e., the unconstrained problem for this research), the problem is formulated as a MCKP (Chapter 6 discusses the MCKP in greater detail). However, when multiple constraints are considered (i.e., the constrained problem for this research), the multi-dimensional aspect is added, and the problem is classified as a MCMDKP (Chapter 7 covers the MCMDKP). Budget constraints can be imposed either annually (for each program year) or cumulatively (for the entire program period). A multi-year budget scenario is formulated as a MCMDKP. An annual budget scenario is formulated as a MKP. The MKP focuses on satisfying the capacity constraints of the multiple knapsacks. It is used in several applications such as bin packing, cutting stock, and financial management (Ramasamy, 2013). MumfordValenzuela (2005) explained the MKP concept using the following example.

For a simple knapsack problem, a set of $n$ objects $O=\left\{O_{1}, O_{2}, O_{3}, \ldots O_{n}\right\}$ and a knapsack of capacity $C$ are given. Each object $O_{i}$ has an associated profit $P_{i}$ and weight $W_{i}$. The objective is to find a subset $S$ from $O$ such the sum of weights over the objects in $S$ does not exceed the knapsack capacity $C$ and yields a maximum profit. The MKP may involves $m$ knapsacks of different capacities, $C_{1}, C_{2}, C_{3}, \ldots C_{m}$. Selected objects must be placed in all $m$ knapsacks - neither the weight of an object $O_{i}$ nor its profit $P_{i}$ is fixed. For a demonstration, a small MKP with 10 objects and two knapsacks is defined in Table 8.1. A Pareto solution set for this small problem is presented in Table 8.2. 
Table 8.1 Example of MKP with 10 objects and two knapsacks

\begin{tabular}{|c|c|c|c|c|}
\hline \multirow{2}{*}{$\begin{array}{l}\text { Object } \\
\text { Number }\end{array}$} & \multicolumn{2}{|c|}{$\begin{array}{c}\text { Knapsack } 1 \\
\text { Capacity }=38\end{array}$} & \multicolumn{2}{|c|}{$\begin{array}{c}\text { Knapsack } 2 \\
\text { Capacity }=35\end{array}$} \\
\hline & Weight & Profit & Weight & Profit \\
\hline 1 & 9 & 2 & 3 & 3 \\
\hline 2 & 8 & 7 & 4 & 9 \\
\hline 3 & 2 & 4 & 2 & 1 \\
\hline 4 & 7 & 5 & 4 & 5 \\
\hline 5 & 3 & 6 & 9 & 3 \\
\hline 6 & 6 & 2 & 5 & 8 \\
\hline 7 & 1 & 7 & 4 & 2 \\
\hline 8 & 3 & 3 & 8 & 6 \\
\hline 9 & 9 & 7 & 3 & 1 \\
\hline 10 & 3 & 1 & 7 & 3 \\
\hline
\end{tabular}

Table 8.2 Obtained Pareto solution set for the MKP example defined in Table 8.1

\begin{tabular}{ccc}
\hline $\begin{array}{c}\text { Knapsack 1 } \\
\text { Profit }\end{array}$ & $\begin{array}{c}\text { Knapsack 2 } \\
\text { Profit }\end{array}$ & $\begin{array}{c}\text { Objects in } \\
\text { Knapsacks }\end{array}$ \\
\hline 39 & 27 & $\{2,3,4,5,7,8,9\}$ \\
38 & 29 & $\{2,3,4,5,6,7,9\}$ \\
36 & 30 & $\{2,3,5,6,7,8,9\}$ \\
35 & 32 & $\{2,3,4,6,7,8,9\}$ \\
34 & 33 & $\{2,3,4,5,6,8,9\}$ \\
32 & 34 & $\{2,4,6,7,8,9,10\}$ \\
29 & 35 & $\{1,2,3,4,5,6,8\}$ \\
27 & 36 & $\{1,2,4,6,7,8,10\}$ \\
\hline
\end{tabular}

The module MKP formulation uses the same analogy. Each program year is assigned to a different knapsack to accept the maximum number of bridges until the allocated annual budget is exhausted. Each bridge is assigned to only one knapsack and affiliated with only one ELO solution. Each knapsack must at least accommodate one bridge. The MKP formulation requires input parameters per program year to meet the multiple knapsack capacities. Only ELO solutions produce results per program year. Therefore, the module MKP is always shaped as a top-down approach optimization problem. The MKP is an NP-hard combinatorial optimization problem. As emphasized in Chapters 6 and 7, this type of problems is solved using heuristics or metaheuristics 
to achieve approximate (near-optimal rather than optimal) solutions within a reasonable computational effort (Thompson et al., 2008). The next section introduces the heuristic algorithms developed to solve the module NLO problems.

\subsubsection{Top-Down Approach}

The formulation presented in this section is based on the MCMDKP and MKP and limited only to the top-down approach. The problem can be mathematically expressed as follows:

Objectives

Minimize $L C C_{\text {net }}$

Maximize $B N T_{\text {net }}$

Maximize $H I_{\text {net }}^{y}$

$\forall y \in H_{o b j}$

Subject to

$I A C_{\text {net }}^{\text {total }} \leq B G T_{\text {net }}^{\text {total }} \quad$ Only for total budget scenarios

$I A C_{n e t}^{j} \leq B G T_{n e t}^{j}$

Only for annual budget scenarios

$\forall j \in T$

$H I_{\text {net }}^{Z} \geq H I_{\text {net }}^{Z}{ }_{\text {min }}$

$\forall z \in H_{\text {con }}$

$\sum_{p \in I} \sum_{j \in T} \sum_{s \in O_{k}^{p j}} X_{k p j s}=1$

Only for scenarios of Case A

$\forall k \in P$

$\sum_{p \in I} \sum_{j \in T} \sum_{s \in O_{k}^{p_{j}}} X_{k p j s} \leq 1$

Only for scenarios of Case B

$\forall k \in P$

where

$L C C_{n e t}=\sum_{k \in P} L C C_{b r g_{k}}^{D N}+\sum_{k \in P} \sum_{p \in I} \sum_{j \in T} \sum_{s \in O_{k}^{p j}}\left[X_{k p j s}\left(L C C_{b r g_{k}}^{p j s}-L C C_{b r g_{k}}^{D N}\right)\right]$ 


$$
\begin{aligned}
& B N T_{n e t}=\sum_{k \in P} B N T_{b r g_{k}}^{D N}+\sum_{k \in P} \sum_{p \in I} \sum_{j \in T} \sum_{s \in O_{k}^{p j}}\left[X_{k p j s}\left(B N T_{b r g_{k}}^{p j s}-B N T_{b r g_{k}}^{D N}\right)\right] \\
& H I_{\text {net }}^{y}=\frac{1}{n b} \sum_{k \in P} H I_{\text {brg }_{k}}^{y D N}+\frac{1}{n b} \sum_{k \in P} \sum_{p \in I} \sum_{j \in T} \sum_{s \in O_{k}^{p j}}\left[X_{k p j s}\left(H I_{b r g_{k}}^{\text {ypjs }}-H I_{\text {brg }_{k}}^{y D N}\right)\right] \\
& H I_{\text {net }}^{Z}=\frac{1}{n b} \sum_{k \in P} H I_{\text {brg }_{k}}^{Z D N}+\frac{1}{n b} \sum_{k \in P} \sum_{p \in I} \sum_{j \in T} \sum_{s \in O_{k}^{p j}}\left[X_{k p j s}\left(H I_{\text {brg }_{k}}^{z p j s}-H I_{\text {brg }_{k}}^{Z D N}\right)\right] \\
& I A C_{\text {net }}^{\text {total }}=\sum_{k \in P} \sum_{p \in I} \sum_{j \in T} \sum_{s \in O_{k}^{p j}}\left(X_{k p j s} I A C_{b r g_{k}}^{p j s}\right) \\
& I A C_{n e t}^{j}=\sum_{k \in P} \sum_{p \in I} \sum_{s \in O_{k}^{p j}}\left(X_{k p j s} I A C_{b r g_{k}}^{p j s}\right) \\
& L C C_{n e t} \quad=\text { total network LCC; } \\
& B N T_{\text {net }}=\text { total network LCC benefit; } \\
& H I_{\text {net }}^{y} \quad=\text { network health index at the end of year } y \\
& H I_{\text {net }}^{Z} \quad=\text { network health index at the end of year } z \\
& H I_{\text {net }}^{Z}=\text { minimum acceptable network health index at the end of year } z \\
& I A C_{\text {net }}^{\text {total }}=\text { network initial agency cost for the entire program period; } \\
& B G T_{\text {net }}^{\text {total }}=\text { available total improvement budget for the entire program period; } \\
& I A C_{\text {net }}^{j} \quad=\text { network initial agency cost incurred in program year } j \text {; } \\
& B G T_{\text {net }}^{j} \quad=\text { available improvement budget for program year } j \\
& X_{k p j s}=\begin{array}{l}
\text { binary decision variable }\left(X_{k p j s}=1 \text { if bridge } k \text { ELO solution } s \text { of improvement type } p\right. \text { with } \\
\text { an initial intervention in program year } \left.j \text { is selected, otherwise } X_{k p j s}=0\right) ;
\end{array} \\
& L_{C C} C_{b r g}^{D N} \quad=\text { total DN LCC for bridge } k \\
& L_{C C}^{\text {pjs }} \text { brg }_{k}=\begin{array}{l}
\text { total LCC of ELO solution } s \text { of improvement type } p \text { with an initial intervention in program } \\
\text { year } j \text { performed on bridge } k ;
\end{array} \\
& B N T_{b r g}^{D N} \quad \text { total DN LCC benefit for bridge } k \text {; }
\end{aligned}
$$




$$
\begin{aligned}
& B N T_{\text {brg }_{k}}^{\text {pjs }}=\begin{array}{l}
\text { total LCC benefit of ELO solution } s \text { of improvement type } p \text { with an initial intervention in } \\
\text { program year } j \text { performed on bridge } k ;
\end{array} \\
& \begin{array}{l}
\mathrm{HI}_{\mathrm{brg}}^{\mathrm{ypjs}} \\
=
\end{array} \quad \begin{array}{l}
\text { total health index of bridge } k \text { at the end of year } y \text { produced by ELO solution } s \text { of } \\
\text { improvement type } p \text { with an initial intervention in program year } j ;
\end{array} \\
& H I_{b r g}^{y D N}=\text { total DN health index of bridge } k \text { at the end of year } y \text {; }
\end{aligned}
$$

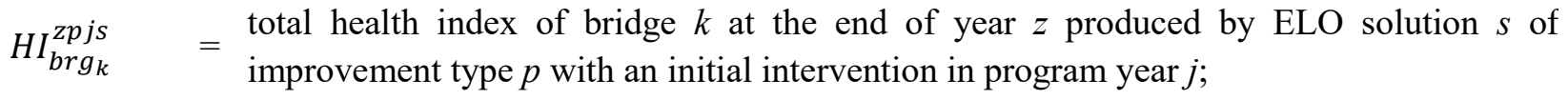

$$
\begin{aligned}
& \mathrm{HI}_{\mathrm{brg}_{k}}^{Z D N}=\text { total DN health index of bridge } k \text { at the end of year } z \text {; } \\
& I A C_{\text {brg }_{k}}^{\text {pjs }}=\quad \begin{array}{l}
\text { total initial agency cost for bridge } k \text { incurred by ELO solution } s \text { of improvement type } p \\
\text { with an initial intervention in program year } j ;
\end{array} \\
& O_{k}^{p j} \quad=\begin{array}{l}
\text { set of ELO solutions of improvement type } p \text { with an initial intervention in program year } j \\
\text { performed on bridge } k ;
\end{array} \\
& I \quad=\text { set of the three improvement types (i.e., MRR, FCI, and REP); } \\
& T \quad=\text { set of all program years (i.e., the program period); } \\
& H_{o b j} \quad=\text { set of specified years in the analysis period for health index objectives; } \\
& H_{\text {con }} \quad=\text { set of specified years in the analysis period for health index constraints; } \\
& P \quad=\text { portfolio of bridges; and } \\
& n b \quad=\text { total number of bridge in portfolio } P \text {. }
\end{aligned}
$$

Formulating the problem correctly is so essential to achieve high-quality solutions. Several formulation designs for overcoming the size of the problem were tested. The proposed formulation is flexible enough to accommodate different NLO goals with multiple constraints. The formulation accommodates ELO solutions from the three distinct improvement types (i.e., MRR, FCI, and REP) and all program years. The different module optimization objectives (i.e., network LCC, LCC benefit, and performance) and constraints (i.e., selection criterion, available network budget, and minimum desired network performance) are mathematically represented. For example, an 
optimization goal for a network may involve minimizing the total network LCC, Equation (8.6), maximizing the total network LCC benefit, Equation (8.7), maximizing the network health indices at years 20 and 40, Equation (8.8), and maintaining the network health index above 70\% (signifies a minimum acceptable network performance). In this example, four network-level objectives subject to two network performance constraints, Equation (8.4), and a picking criterion, Equation (8.5), will be optimized for this network-level problem.

The multi-year optimization strategy in the formulation was found to be appropriate for this level of assessment under the top-down approach. As emphasized earlier, the solution superiority screening process is critical for the top-down approach optimization. It identifies the superior ELO solutions to serve as input parameters. Without this screening process, this multi-year strategy encounters a huge number of decision variables, and the computational time to reach convergence is substantial, especially when dealing with a large network of bridges - most likely, global optimality cannot be attained.

A set of Pareto solutions is recommended for the entire portfolio. A NLO solution is a set of ELO solutions, where each represents a combination of optimal or near-optimal LC alternatives recommended for the deficient elements of one of the bridges in the portfolio. LC Alternative 1 (representing the DN LC Alternative) is always assigned to the non-deficient elements. For MRR improvement type, a non-deficient element doesn't experience preservation actions for the entire analysis period-MRR LC Alternative 1 is assigned. For FCI and REP improvement types, a nondeficient element still get replaced; however, no preservation actions will follow-FCI/REP LC Alternative 1 is assigned. 
As explain earlier, the decision in this module optimization problem is a binary choice- one of the ELO solutions is either selected or rejected. A NLO solution is represented by a vector of binary decision variables (chromosome). The encoding of decision variables is illustrated and discussed later in this chapter. The non-dominated solutions of the entire feasible solution space constitute the Pareto solution set. For Case A's scenarios, Equation (8.4) is added to restrict the decision variable space. This constraint equation guarantees the selection of only one ELO solution per bridge. Therefore, among all grouped ELO solutions, only one must be recommended for each bridge. Equation (8.5) is used instead for Case B's scenarios; no more than one ELO solution per bridge is recommended by this inequality constraint. The binary decision variable is symbolized by $X_{k p j s}$. The variable equals to 1 if bridge $k$ ELO solution $s$ of improvement type $p$ with an initial intervention in program year $j$ is selected, otherwise 0 .

The network-level objectives, Equations (8.6), (8.7), and (8.8), are interdependent on the decision variable $\left(X_{k p j s}\right)$. These equations, respectively, represent the network LCC, LCC benefit, and performance at the ends of specified analysis years $\left(H_{o b j}\right)$, involving only ELO solution results associated with the bridges in the portfolio. A unique set of ELO solutions, $O_{k}^{p j}$, is established for each bridge $k$ and performed improvement type $p$ with an initial intervention in program year $j$. The contributions of the different ELO solutions within these sets are summed over all bridges in the portfolio $(P)$, the three different improvement types $(I)$, and all program years $(T)$, after applying their corresponding decision variables.

Equation (8.9) represents the inequality of performance constraints. This equation permits to maintain an acceptable network performance ("state of good repair") or attain a higher network performance over a certain period; the network health indices at the ends of specified analysis 
years, $H I_{\text {net }}^{Z}$, are bounded by the network health index thresholds, $H I_{\text {net }}^{Z}$ min (minimum acceptable network health indices). Likewise, these network health indices are determined by summing and averaging health indices at the ends of specified years $\left(H_{c o n}\right)$ of all bridges in the portfolio $(P)$ produced by the ELO solutions of all sets $\left(O_{k}^{p j}\right)$, after applying their corresponding decision variables.

Equation (8.1) represents the inequality of multi-year budget constraint. The total network initial agency cost for the entire program period, $I A C_{\text {net }}^{\text {total }}$, is restricted by the available total budget, $B G T_{\text {net }}^{\text {total }}$. The network initial cost for the entire program period is determined by summing all incurred bridge initial agency costs—-produced by the different ELO solutions of all sets $\left(O_{k}^{p j}\right)$ over the three different improvement types $(I)$ and all program years $(T)$, after applying their corresponding decision variables.

Equation (8.2) represents the inequality of annual budget constraint. The optimization process aims to meet the available budget for each program year. The network initial agency cost for program year $j, I A C_{n e t}^{j}$, is restricted by the available budget for the same program year $j, B G T_{n e t}^{j}$. The network initial cost for a program year is determined by summing only the bridge initial agency costs incurred in the same program year-produced by the different ELO solutions of only the sets $\left(O_{k}^{p j}\right)$ with the same program year $(j)$ over the three different improvement types $(I)$, after applying their corresponding decision variables. 


\subsubsection{Bottom-Up Approach}

The formulation presented in this section is based on the MCMDKP and limited only to the bottomup approach. The problem can be mathematically expressed as follows:

Objectives

Minimize $L C C_{\text {net }}$

Maximize $B N T_{\text {net }}$

Maximize $H I_{\text {net }}^{y}$

$\forall y \in H_{o b j}$

Subject to

$$
\begin{array}{ll}
I A C_{\text {net }}^{\text {total }} \leq B G T_{\text {net }}^{\text {total }} & \text { Only for total budget scenarios } \\
H I_{\text {net }}^{Z} \geq H I_{\text {net }}^{Z} \text { min } & \forall z \in H_{\text {con }} \\
\sum_{w \in S_{k}} X_{k w}=1 & \text { Only for scenarios of Case A } \\
\sum_{w \in S_{k}} X_{k w} \leq 1 & \forall k \in P
\end{array}
$$

where

$$
\begin{aligned}
& L C C_{n e t}=\sum_{k \in P} L C C_{b r g_{k}}^{D N}+\sum_{k \in P} \sum_{w \in S_{k}}\left[X_{k w}\left(L C C_{b r g_{k}}^{w}-L C C_{b r g_{k}}^{D N}\right)\right] \\
& B N T_{n e t}=\sum_{k \in P} B N T_{b r g_{k}}^{D N}+\sum_{k \in P} \sum_{w \in S_{k}}\left[X_{k w}\left(B N T_{b r g_{k}}^{w}-B N T_{b r g_{k}}^{D N}\right)\right] \\
& H I_{n e t}^{y}=\frac{1}{n b} \sum_{k \in P} H I_{b r g_{k}}^{y D N}+\frac{1}{n b} \sum_{k \in P} \sum_{w \in S_{k}}\left[X_{k w}\left(H I_{b r g_{k}}^{y w}-H I_{b r g_{k}}^{y D N}\right)\right] \\
& H I_{n e t}^{Z}=\frac{1}{n b} \sum_{k \in P} H I_{b r g_{k}}^{z D N}+\frac{1}{n b} \sum_{k \in P} \sum_{w \in S_{k}}\left[X_{k w}\left(H I_{b r g_{k}}^{y w}-H I_{b r g_{k}}^{z D N}\right)\right] \\
& I A C_{\text {net }}^{\text {total }}=\sum_{k \in P} \sum_{w \in S_{k}}\left(X_{k w} I A C_{b r g_{k}}^{w}\right)
\end{aligned}
$$




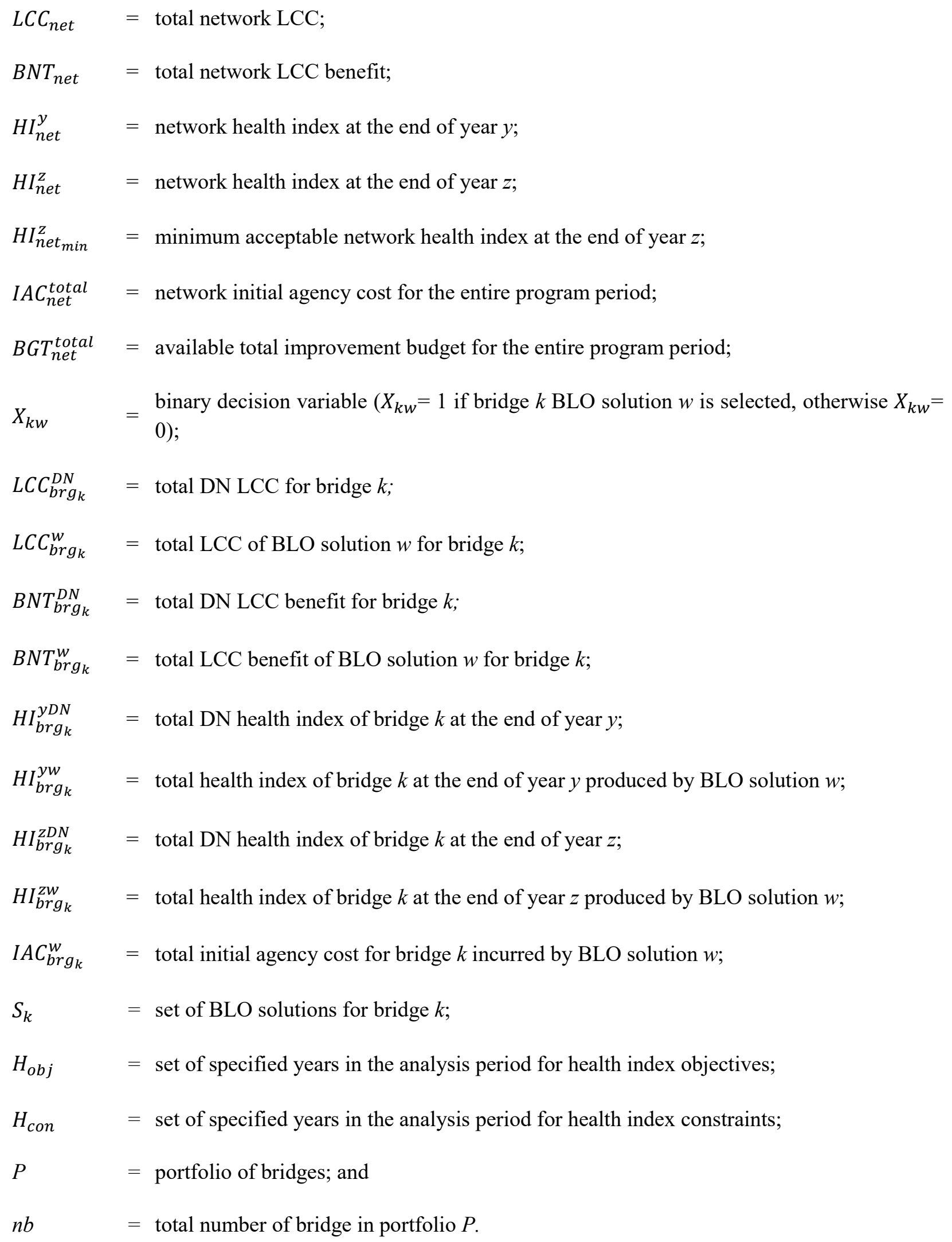


The bottom-up approach optimization formulation is flexible enough to accommodate different NLO goals with multiple constraints. The formulation accommodates BLO solutions. The different network-level objectives (i.e., network LCC, LCC benefit, and performance) and constraints (i.e., selection criterion, available network budget, and minimum desired network performance) are mathematically represented. For example, an optimization goal for a given network may involve minimizing the total network LCC, Equation (8.16), maximizing the total network LCC benefit, Equation (8.17), maximizing the network health indices at years 20 and 40, Equation (8.18), and maintaining the network health index above $70 \%$ (signifies a minimum acceptable network performance). In this example, four network-level objectives subject to two network performance constraints, Equation (8.14), and a picking criterion, Equation (8.15), will be optimized for this network-level problem.

As demonstrated in the previous section, the screening process is generally unnecessary for the bottom-up approach optimization. The total number of decision variables remains manageable even for a large network of bridges. A set of Pareto solutions is recommended for the entire portfolio. A NLO solution is a set of BLO solutions, where each represents a combination of optimal ort near-optimal LC alternatives identified for the deficient elements of one of the bridges in the portfolio. LC Alternative 1 (representing the DN LC Alternative) is always assigned to the non-deficient elements. For MRR improvement type, a non-deficient element doesn't experience preservation actions for the entire analysis period-MRR LC Alternative 1 is assigned. For FCI and REP improvement types, a non-deficient element still get replaced; however, no preservation actions will follow-FCI/REP LC Alternative 1 is assigned. 
The multi-year optimization strategy in the formulation was also found to be appropriate for this level of assessment under the bottom-up approach. Again, the decision in this optimization problem is a binary choice - one of the BLO solutions is either selected or rejected. A NLO solution is represented by a vector of binary decision variables (chromosome). The encoding of decision variables is illustrated and discussed later in this chapter. The non-dominated solutions of the entire feasible solution space constitute the Pareto solution set. For Case A's scenarios, Equation (8.14) restricts the decision variable space. This constraint guarantees the selection of only one BLO solution per bridge. Therefore, among all grouped BLO solutions, only one must be recommended for each bridge. For Case B's scenarios, Equation (8.15) is used instead; no more than one BLO solution per bridge is recommended by this inequality constraint. The binary decision variable is symbolized by $X_{k w}$. The variable equals to 1 if bridge $k$ BLO solution $w$ is selected, otherwise 0 .

The network-level objectives, Equations (8.16), (8.17), and (8.18), are interdependent on the decision variable $\left(X_{k w}\right)$. These equations, respectively, represent the network LCC, LCC benefit, and performance at the ends of specified analysis years $\left(H_{o b j}\right)$, involving only BLO solution results associated with the bridges in the portfolio. A unique set of BLO solutions $\left(S_{k}\right)$ is established for each bridge in the portfolio $(P)$. The contributions of these different BLO solutions within these sets are summed over all bridges in the portfolio $(P)$, after applying their corresponding decision variables.

Equation (8.13) represents the inequality of performance constraints. This equation permits to maintain an acceptable network performance ("state of good repair") or attain a higher performance over a certain period; the network health indices at the ends of specified analysis years, $H I_{\text {net }}^{Z}$, are bounded by the network health index thresholds, $H I_{\text {net }_{\text {min }}}^{Z}$ (minimum acceptable 
network health indices). Likewise, these network health indices are determined by summing and averaging health indices at the ends of specified years $\left(H_{c o n}\right)$ of all bridges in the portfolio $(P)$ produced by the BLO solutions of all sets $\left(S_{k}\right)$, after applying their corresponding decision variables. Equation (8.12) represents the inequality of multi-year budget constraint. The total network initial agency cost for the entire program period, $I A C_{\text {net }}^{\text {total }}$, is restricted by the available total budget, $B G T_{\text {net }}^{\text {total }}$. The network initial cost for the entire program period is determined by summing all incurred bridge initial agency costs — produced by the different BLO solutions of all sets $\left(S_{k}\right)$, after applying their corresponding decision variables.

\subsection{Heuristic Network-Level Optimization Algorithms}

A Trade-off between competing objectives requires several independent runs by varying certain parameters; and yet, optimal or near-optimal solutions are not guaranteed. MOO methodologies are effective in optimizing multiple competing objectives subject to all kinds of constraints (e.g., budget and/or performance constraints). They help providing a complete knowledge of the problem (Talbi, 2009). MOO methodologies guarantee a diverse set of optimal or near-optimal solutions - constituting a frontier of trade-offs. The objective of the NLO module is to obtain a diverse set of optimal or near-optimal LC alternatives (Pareto solutions) for the entire networkto support decision making through a trade-off or "what-if" scenario analysis between the obtained Pareto solutions. Again, the same metaheuristic algorithm (i.e., NSGA-II) is deployed at this higher level of optimization. NSGA-II is integrated as the main optimizer into the heuristic optimization algorithms proposed for the top-down and bottom-up approaches. A minor integration adjustment was required to accommodate the NLO problem features. As stated previously, the optimizer algorithm can be substituted by other metaheuristics with similar capabilities such as SPEA-2, PESA, and MOGA. 
The brute-force search technique is also used in these heuristic NLO algorithms to enumerate and examine obtained NLO solutions. Every single NLO solution after certain evaluations (for NSGAII, the product of population size and total number of generations equals to the total number of evaluations) is assessed. NLO solutions for a certain number of evaluations are accepted if they all satisfy the problem constraints. Otherwise, if any of them violates any constraint, the whole set of solutions is rejected, the total number of evaluations is increased by an increment, and new solutions are obtained to be assessed for the same network. This simple search technique controls the number of runs, efficiently manages the computational time, and guarantees the feasibility of every single produced solution.

Users set the population size, initial evaluations, evaluation increment, maximum evaluations, number of bridges to be evaluated, program period, and available budget (annual or multi-year budget) and performance thresholds (minimum desired network performance). Once more, the initial evaluations, population size, including other optimizer default parameters must be carefully selected to ensure a high quality of obtained solutions. As stated in Chapter 6, the best combination of parameters, initial evaluations, and population size can be identified by trial and error or by establishing different performance metrics to assess convergence, optimality of the solutions, diversity along the Pareto frontier, computational time, computer memory use, etc. 


\subsubsection{Top-Down Approach}

Figure 8.4 presents the flowchart of the heuristic algorithm developed to solve the module topdown approach NLO problem. The key steps of the developed heuristic algorithm are as follows:

1. The algorithm starts with a total number of evaluations (Eval) equals to the total number of initial evaluations (Eval_inc).

2. The algorithm locates superior MRR, FCI, and REP ELO solution matrices of each program year for each bridge in the portfolio.

3. The algorithm rearranges and groups these program year solution matrices of each bridge by network-level objectives and constraints in single matrices covering the entire program period.

4. The algorithm rearranges and combines all these individual bridge solution matrices into a single matrix consistent with the NLO problem formulation for the top-down approach.

5. A random initial population of size $N 3$ is generated following the chromosome structure for the top-down approach (discussed later).

6. While the total number of evaluations $\left(E v a l+E v a l \_i n c\right)$ is less than the specified maximum value (Eval_max), the brute-force search technique is deployed.

7. The optimizer NSGA-II is called to solve the optimization problem for these evaluations.

8. The obtained NLO solution results of these evaluations are saved.

9. Feasibility of the obtained solution set is verified.

10. If all constraints are met, the algorithm exits the while loop of brute-force search technique, saves the solutions, and terminates. Otherwise, if any solution fails the feasibility check, the total number of evaluations is increased by the specified increment (Eval_inc), the population is taken as the population of this number of evaluations, and steps $6,7,8$ and 9 get repeated. 


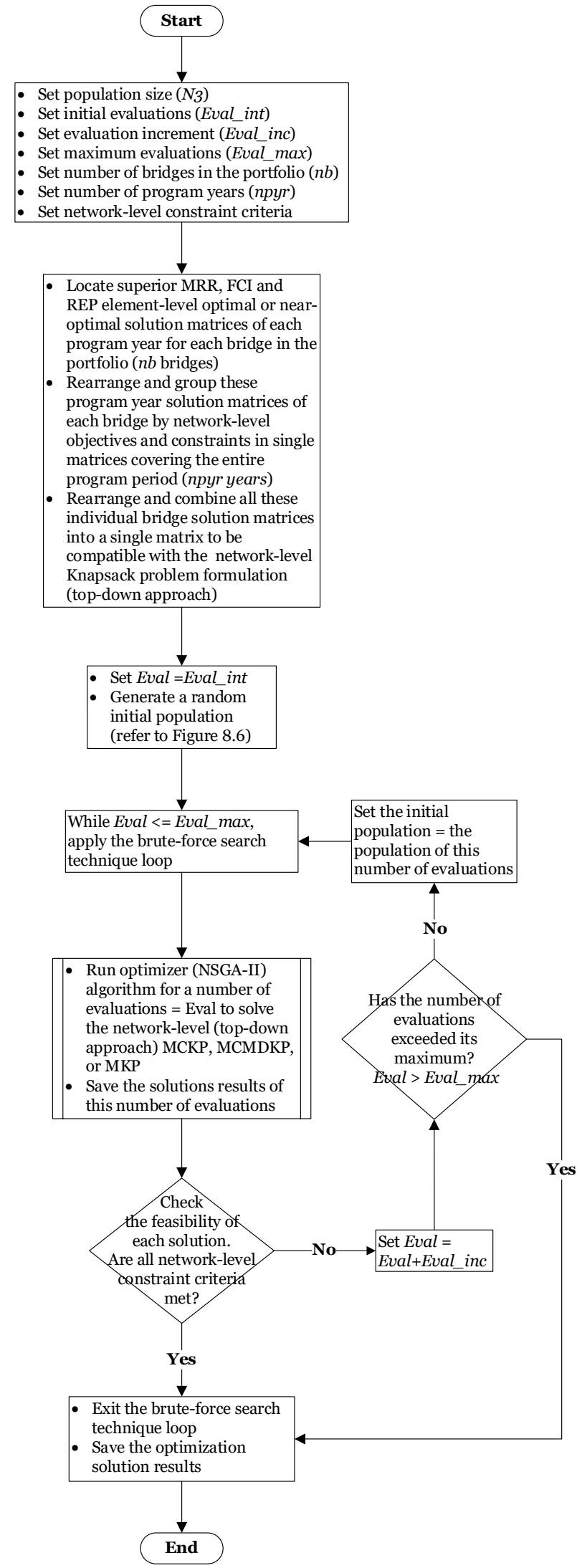

Figure 8.4 Heuristic network-level optimization algorithm for the top-down approach 


\subsubsection{Bottom-up Approach}

Figure 8.5 presents the flowchart of the heuristic algorithm developed to solve the module bottomup approach NLO problem. The key steps of the developed heuristic algorithm are as follows:

1. The algorithm starts with a total number of evaluations (Eval) equals to the total number initial evaluations (Eval_inc).

2. The algorithm locates BLO solution matrices for each bridge in the portfolio.

3. The algorithm rearranges and groups these program year solution matrices of each bridge by network-level objectives and constraints in single matrices covering the entire program period.

4. The algorithm rearranges all these grouped individual BLO solution matrices to be compatible with the NLO problem formulation for the bottom-up approach.

5. A random initial population of size $N 3$ is generated following the chromosome structure for the bottom-up approach (discussed in the subsequent section).

6. While the total number of evaluations $($ Eval + Eval_inc $)$ is less than the specified maximum value (Eval_max), the brute-force technique search technique is deployed.

7. The optimizer NSGA-II is called to solve the optimization problem for these evaluations.

8. The obtained NLO solution results of these evaluations are saved.

9. Feasibility of the obtained solution set is verified.

10. If all constraints are met, the algorithm exits the while loop of brute-force search technique, saves the solutions, and terminates. Otherwise, if any solution fails the feasibility check, the total number of evaluations is increase by the specified increment (Eval_inc), the population is taken as the population of this number of evaluations, and steps $6,7,8$ and 9 get repeated. 


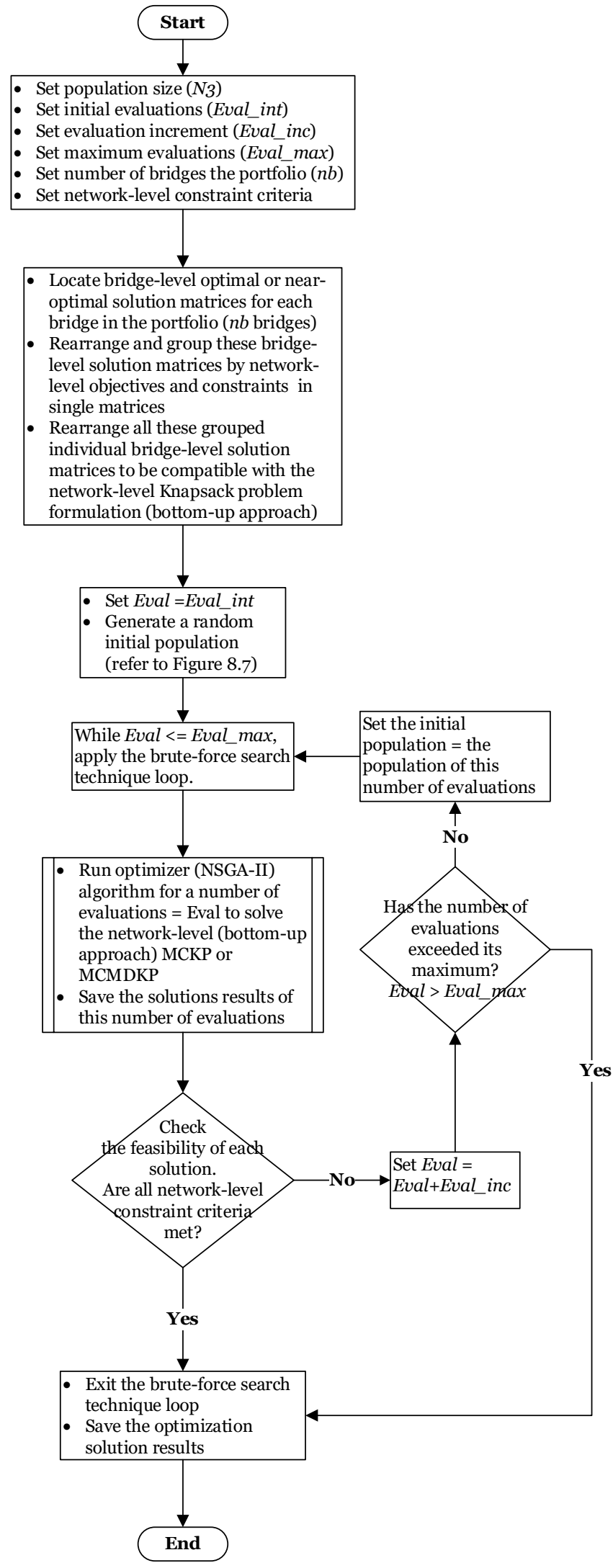

Figure 8.5 Heuristic network-level optimization algorithm for the bottom-up approach 


\subsection{Mapping of Decision Variables}

Chapter 6 discussed the basics process of generating a set of chromosomes (a population of solutions). The three main genetic operators (i.e., crossover, mutation, and selection/fitness) were explained in Chapter 2. The optimizer algorithm, NSGA-II, relies on an elitism operator for selecting best (elite) chromosomes. Elitism operator increases the performance of GA, as it ensures the best chromosomes remain in the population. Again, effectively encoding the chromosome for each approach is crucial to achieve a better GA performance and a truthful representation of the problem. Both approaches have two different chromosome structures. The following sections introduce these two structures.

\subsubsection{Top-down Approach}

As discussed earlier, the formulation encompasses the three improvement types and all program years. ELO solutions per bridge compete for selection at this level of optimization. This multi-year optimization strategy requires a vast number of chromosomes to process. This large number of chromosomes impacts the computer memory and computational time, especially when dealing with a large network of bridges-more likely, local rather than global optimality is attained. Similarly, the number of genes (i.e., decision variables) per chromosome affects the GA performance. Therefore, it's essential to control the number of chromosomes per population.

As emphasized earlier, the solution superiority screening process had to be introduced for the topdown approach optimization to manage the number of decision variables and make the problem trackable with less computational efforts. The process reduces the number of decision variables per chromosome. It identifies the superior ELO solutions to serve as input parameters. As demonstrated in the previous section, typically, a given bridge is associated with $3,000(3 \times 10 \times$ 100) decision variables over a program period of 10 years and the three improvement types, 
considering 100 ELO solutions per program year. So, for a network of 200 bridges, 600,000 (3,000 $\times 200$ ) decision variables are involved. When using the screening process, the 100 ELO solutions are reduced to 3 ELO solutions per program year, and consequently the 600,000 decision variables are reduced to 18,000 .

Figure 8.6 illustrates the chromosome encoding used to represent a top-down approach NLO solution. A chromosome encompasses a total number of $c f . n p y r . n i m p . n b$ genes, where $c f$ is the total number of superior ELO solution per program year, npyr is the total number of program years, nimp is the total number of improvement types, and $n b$ is the total number of bridges in the portfolio. For instance, a total of 60,000 chromosomes represents a set of 10 superior ELO solutions per program year, a 10-year program period, three improvement types (i.e., MRR, FCI, and REP), and a portfolio of 200 bridges. Again, a binary encoding scheme is adapted because of its simplicity and the problem allocation characteristics. Each chromosome has one binary string of genes (composed of either 0 or 1 ), and each gene represents a characteristic of the NLO solution.

Figure 8.6 shows an example of assigning decision variables to an ELO solution matrix of size ( $c f$ $\times 30$ ) for the entire portfolio. In this illustrative example, the first $c f$ genes represent program year 1 , the next $c f$ genes represent program year 2, and so on until the last program year 10 . The first 10.cf genes are assigned to MRR improvement type, and the next 10.cf genes to FCI improvement type, followed by another series of 10.cf genes to REP improvement type. These first 30.cf genes are dedicated to the first ranked bridge in the portfolio, Brg_1, the next 30.cf genes are arranged in the same manner and dedicated to the second ranked bridge, Brg_2, and so on until the last bridge in the portfolio, Brg_92. A gene with a value of 1 signifies an ELO solution is selected; in contrast, a value of 0 signifies no selection. A gene position depicts a specific problem characteristic. A gene in the fourth cell (starting from the left), for example, represents the fourth 
superior ELO solution (i.e., Solution 4) associated with the first bridge (Brg_1) for MRR improvement type and program year 4 . A population of size $N 3$ simply includes $N 3$ chromosomes with different random binary values. 


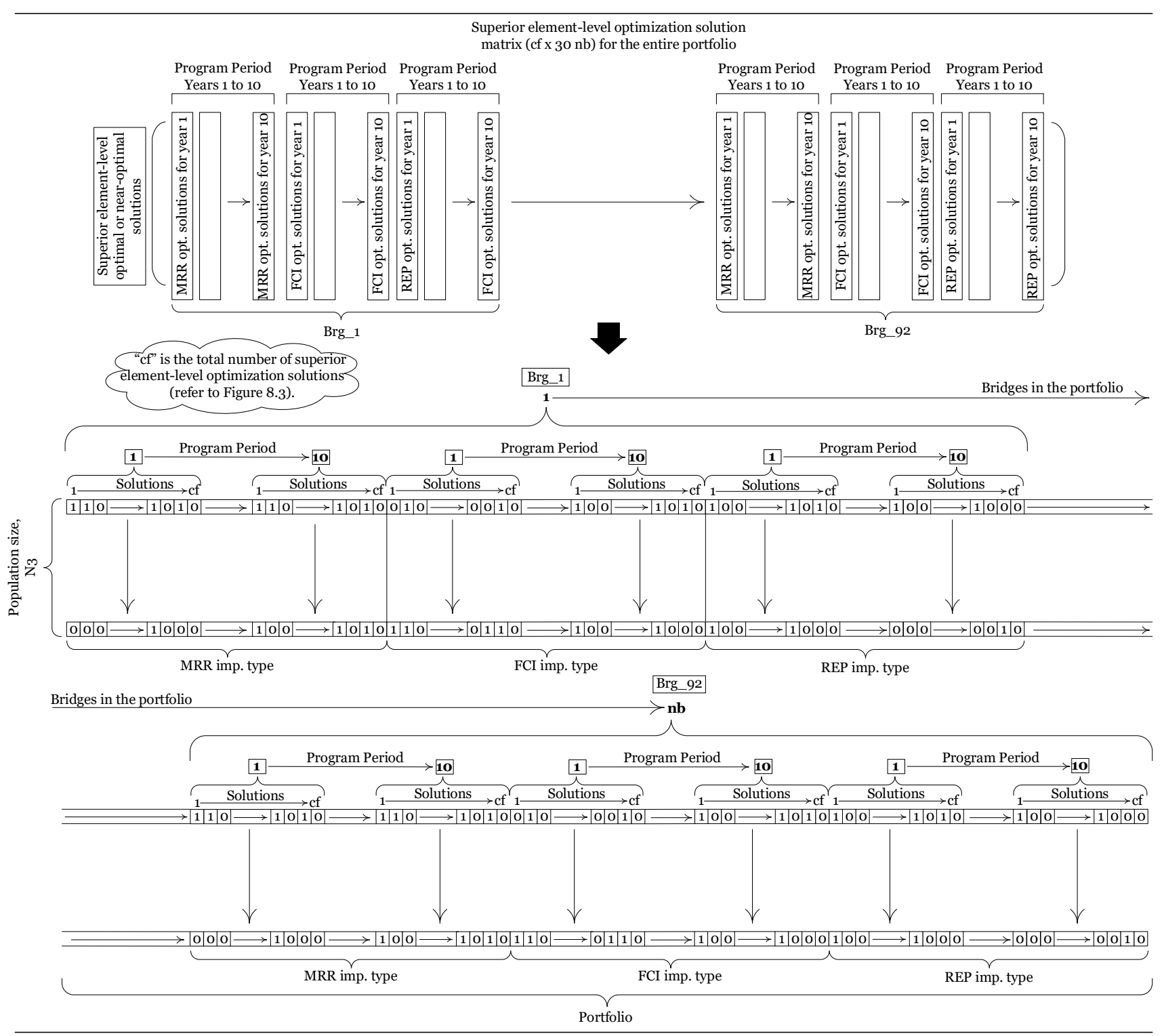

Figure 8.6 Illustration of the chromosome encoding of a top-down approach NLO solution 


\subsubsection{Bottom-Up Approach}

When following the bottom-up approach, BLO solutions per bridge produced by the BLO module compete for selection. As demonstrated earlier in the previous section, the screening process is generally unnecessary for the bottom-up approach optimization. The total number of decision variables remains manageable even for a large network of bridges. However, the larger the population size, the more chromosomes are generated. A large number of chromosomes impacts the computer memory and computational time. Therefore, it's important to carefully choose the population size to achieve a better GA performance and a high quality of solutions (refer to Chapters 2 and 6 for more discussions).

Figure 8.7 illustrates the chromosome encoding used to represent a bottom-up approach NLO solution. A chromosome encompasses a total number of $N 2 . n b$ genes, where $N 2$ is the total number of BLO solutions per bridge, and $n b$ is the total number of bridges in the portfolio. For instance, a total of 20,000 chromosomes represents a set of 100 BLO solutions per bridge, and a portfolio of 200 bridges. Each chromosome has one binary string of genes (composed of either 0 or 1), and each gene represents a characteristic of the NLO solution.

Figure 8-7 shows an example of assigning decision variables to a BLO solution matrix of size (N2 $\times n b)$ for the entire portfolio. In this illustrative example, the first $N 2$ genes represent the first bridge, Brg_1, the next N2 genes represent the second ranked bridge, Brg_2, and so on until the last bridge in the portfolio, Brg_92. A gene with a value of 1 signifies a BLO solution is selected; in contrast, a value of 0 signifies no selection. A gene position depicts a specific problem characteristic. A gene in the fourth cell (starting from the left), for example, represents the fourth BLO solution (i.e., Solution 4) of the first bridge, Brg_1. A population of size N3 simply includes $N 3$ chromosomes with different random binary values. 


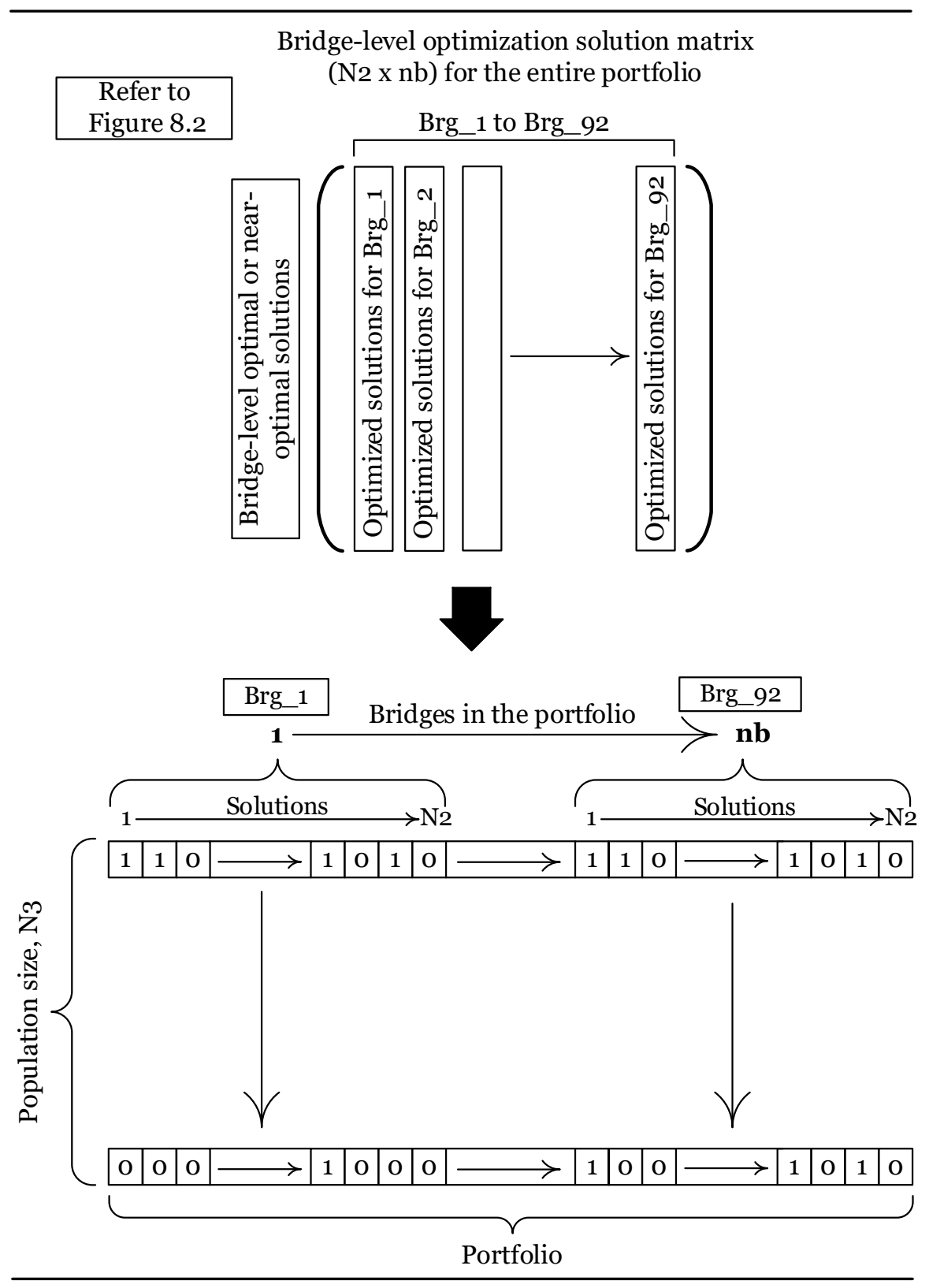

Figure 8.7 Illustration of the chromosome encoding of a bottom-up approach NLO solution 


\subsection{Examples of Module Results}

Chapter 3 introduced the MATLAB-based tool prototype developed for the implementation of the five EB-MOO modules. The tool prototype is considered a "proof of concept" rather than a complete rigorous software ready for operational implementation. The methodology was implemented through several examples using the tool prototype to test concepts, prove effectiveness, and demonstrate potential benefits. The examples include detailed tables and charts to communicate outcomes from the different modules. The NLO module can handle a large portfolio with hundreds of bridges. In these examples, a portfolio of 40 sample bridges (introduced in Chapter 3) is used to demonstrate the module produced NLO results. These sample bridges were selected based on common features, attributes, and data completeness. Table 3.2 in Chapter 3 provides information related to the different characteristics of these sample bridges. These examples provided an excellent opportunity to apply the proposed EB-MOO methodology and assess the effectiveness of the tool prototype in terms of the following areas:

- ability of finding Pareto solutions, identifying optimal or near-optimal intervention actions, predicting performance, and determining funding requirements with less computational efforts;

- interaction between the element-, bridge-, and network-level optimization modules, along with the different screening processes;

- diversity and quality of Pareto solutions obtained by either the top-down or bottom-up approach optimization;

- $\quad$ quality of predicted investment needs and performance; and

- $\quad$ ease of conducting trade-offs between funding levels and performance. 
A priori articulation of preference approach is often followed when multiple objectives are aggregated into one, as in the weighted sum or utility function method. Decision makers provide preferences (relative weights) prior to the optimization process. As explained in Chapter 3 , the methodology follows a different approach referred to as posteriori articulation of preference approach-Pareto solutions are first determined, and then presented to the decision makers to select the best ones based on preferences. This approach helps providing a complete knowledge of the problem and exploring the whole set of Pareto solutions (Talbi, 2009). The adapted posteriori articulation approach is tested through the examples presented herein. Different constrained (by budget and/or performance) and unconstrained scenarios were established to facilitate decision making by comparing the recommended investment strategies. Trade-offs between the NLO solutions obtained for these different scenarios support identifying the best investment strategies that address short- and long-term goals and objective priorities. The examples constitute of different optimization goals and problem types (defined in Tables 8.3 and 8.4). As discussed earlier in this chapter. Two cases of budget and performance scenarios can be analyzed throughout this module:

- Case A-all bridge in the portfolio must be selected

- Case B - not necessarily all bridges in the portfolio must be selected

Examples 1, 2, 3 and 4 include the scenarios under Case A (defined in Table 8.3). Examples 5, 6, 7, and 8 constitute of the scenarios under Case B (defined in Table 8.4). For the scenarios under Case A, the selection criterion restricts the decision variable space to one recommendation per bridge. Each bridge in the portfolio must be associated with one set of LC alternatives for all its elements. This case is used when a backlog is not an option; every single bridge in the portfolio must be considered — if Pareto solutions can't be obtained, certain constraints can be relaxed to 
make the problem trackable. For the scenarios under Case B, the selection criterion is relaxed such as the mandated recommendation per bridge is not fully enforced — not necessary a set of LC alternatives must be identified for each bridge in the portfolio. By relaxing the selection criterion, Case B's scenarios direct the focus mainly toward the high-priority bridges (of urgent improvement needs), at the same time satisfying available budgets. The non-selected bridges (the less urgent) automatically constitute the backlog.

The analysis period is set for 54 years, including a 10 -year program period. The discount rate is $4 \%$ over 56 years (the analysis period plus the period from current year, 2018, to program year 1, 2020). Both the top-down and bottom-up approaches were examined and compared under these different scenarios. All obtained solutions are considered non-dominated solutions (Pareto solutions). As emphasized throughout this dissertation, Pareto solutions in this research encompass the optimal or near-optimal (very close to optimal) solutions. To verify optimally, the consistency of each obtained Pareto frontier was verified by increasing the number of iterations/generations and observing the difference between shapes. The obtained solutions are hosted on concave Pareto frontiers (good approximation of true Pareto frontiers). 
Table 8.3 Defining the different NLO problems of the examples under Case A

\begin{tabular}{|c|c|c|c|c|c|c|}
\hline & & Optimization Goals & App. & Type & Scenarios & $\begin{array}{c}\text { Pareto } \\
\text { Obtained? }\end{array}$ \\
\hline \multirow{10}{*}{ 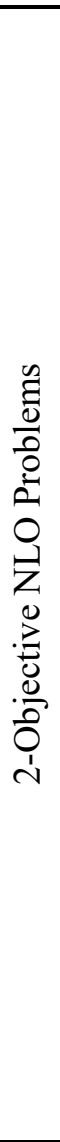 } & \multirow{8}{*}{ 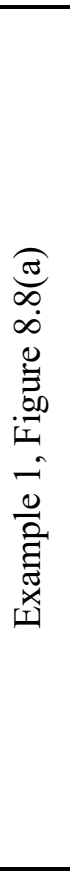 } & \multirow{8}{*}{$\begin{array}{l}\text { - maximize the network } \\
\text { health index in year } 20 \\
\text { (denoted by HI20 } 0_{\text {net }} \\
\text { - minimize the network } \\
\text { LCC (denoted by } \\
\text { LCC }_{\text {net }} \text { ) }\end{array}$} & \multirow[t]{4}{*}{$\begin{array}{l}\text { Top- } \\
\text { Down }\end{array}$} & MCKP & $\begin{array}{l}\text { Scenario 1a: } \\
\text { unconstrained } \\
\checkmark \text { unconstrained budget } \\
\checkmark \text { unconstrained } \\
\quad \text { performance }\end{array}$ & Yes \\
\hline & & & & \multirow[t]{3}{*}{ MCMDKP } & $\begin{array}{l}\text { Scenario 2a: constrained } \\
\checkmark \quad \text { total budget } \leq \$ 80 \mathrm{M} \\
\checkmark \quad \text { network health index } \\
\quad \geq 70 \% \text { every } 10 \text { years }\end{array}$ & Yes \\
\hline & & & & & $\begin{array}{l}\text { Scenario 3a: constrained } \\
\checkmark \quad \text { total budget } \leq \$ 30 \mathrm{M}\end{array}$ & No \\
\hline & & & & & $\begin{array}{ll}\text { Scenario 4a: constraint } \\
\checkmark \quad \text { total budget } \leq \$ 50 \mathrm{M} \\
\checkmark \quad \text { network health index } \\
\geq 70 \% \text { every } 10 \text { years } \\
\quad\end{array}$ & Yes \\
\hline & & & \multirow{4}{*}{$\begin{array}{l}\text { Bottom } \\
\text {-Up }\end{array}$} & MCKP & Scenario 1a & Yes \\
\hline & & & & \multirow[t]{3}{*}{ MCMDKP } & Scenario $2 \mathrm{a}$ & No \\
\hline & & & & & Scenario $3 \mathrm{a}$ & No \\
\hline & & & & & Scenario $4 a$ & $\mathrm{No}$ \\
\hline & \multirow{2}{*}{ 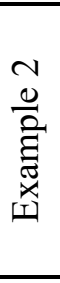 } & \multirow{2}{*}{$\begin{array}{l}\text { - maximize the network } \\
\text { health index in year } 20 \\
\text { (denoted by HI } 20_{\text {net }} \text { ) } \\
\text { - minimize the network } \\
\text { LCC (denoted by } \\
\text { LCC }_{\text {net }} \text { ) }\end{array}$} & \multirow[t]{2}{*}{$\begin{array}{l}\text { Top- } \\
\text { Down }\end{array}$} & \multirow[t]{2}{*}{ MKP } & $\begin{array}{l}\text { Scenario 5a: constrained } \\
\checkmark \quad \text { annual budget } \leq \$ 3 \mathrm{M}\end{array}$ & No \\
\hline & & & & & $\begin{array}{ll}\text { Scenario 6a: constrained } \\
\checkmark \quad \text { annual budget } \leq \$ 8 \mathrm{M} \\
\checkmark \quad \text { network health index } \\
\geq 70 \% \text { every } 10 \text { years }\end{array}$ & No \\
\hline \multirow{10}{*}{$\begin{array}{l}0 \\
0 \\
0 \\
0 \\
0 \\
0 \\
0 \\
0 \\
Z \\
0 \\
0 \\
: 0 \\
0 \\
0 \\
0 \\
0 \\
1 \\
n\end{array}$} & \multirow{8}{*}{ 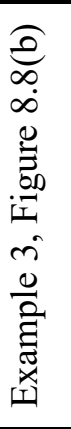 } & \multirow{8}{*}{$\begin{array}{l}\text { - maximize the network } \\
\text { health index in year } 20 \\
\left(\text { denoted by HI20 } 0_{\text {net }} \text { ) }\right. \\
\text { - maximize the network } \\
\text { LCC benefit (denoted } \text { by BNT }_{\text {net }} \text { ) } \\
\text { - minimize the network } \\
\text { LCC (denoted by } \text { LCC }_{\text {net }} \text { ) }\end{array}$} & \multirow{4}{*}{$\begin{array}{l}\text { Top- } \\
\text { Down }\end{array}$} & MCKP & Scenario 1a & Yes \\
\hline & & & & MCMDKP & Scenario $2 \mathrm{a}$ & Yes \\
\hline & & & & & Scenario $3 \mathrm{a}$ & No \\
\hline & & & & & Scenario $4 \mathrm{a}$ & Yes \\
\hline & & & \multirow{4}{*}{$\begin{array}{c}\text { Bottom } \\
\text {-Up }\end{array}$} & MCKP & Scenario 1a & Yes \\
\hline & & & & \multirow[t]{3}{*}{ MCMDKP } & Scenario $2 \mathrm{a}$ & No \\
\hline & & & & & Scenario $3 \mathrm{a}$ & No \\
\hline & & & & & Scenario $4 \mathrm{a}$ & No \\
\hline & & \multirow[b]{2}{*}{$\begin{array}{l}\text { - maximize the network } \\
\text { health index in year } 20 \\
\text { (denoted by HI } 20_{\text {net }} \text { ) } \\
\text { - maximize the network } \\
\text { LCC benefit (denoted } \\
\text { by BNT } \text { net }_{\text {) }} \\
\text { - minimize the network } \\
\text { LCC (denoted by } \\
\text { LCC }_{\text {net }} \text { ) }\end{array}$} & \multirow{2}{*}{$\begin{array}{l}\text { Top- } \\
\text { Down }\end{array}$} & \multirow[t]{2}{*}{ MKP } & Scenario 5a & No \\
\hline & 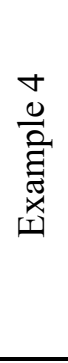 & & & & Scenario 6a & No \\
\hline
\end{tabular}


Table 8.4 Defining the different NLO problems of the examples under Case B

\begin{tabular}{|c|c|c|c|c|c|c|}
\hline & & Optimization Goals & App. & $\begin{array}{l}\text { Problem } \\
\text { Type }\end{array}$ & Scenarios & $\begin{array}{c}\text { Pareto } \\
\text { Obtained? }\end{array}$ \\
\hline \multirow{6}{*}{ 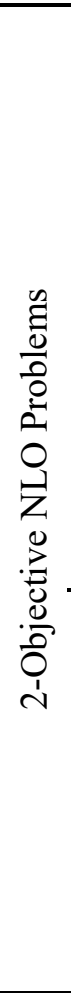 } & \multirow{4}{*}{ 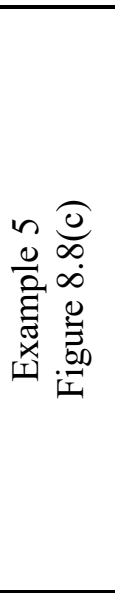 } & \multirow{4}{*}{$\begin{array}{l}\text { - maximize the network } \\
\text { health index in year } 20 \\
\text { (denoted by HI20 } 0_{\text {net }} \text { ) } \\
\text { - minimize the network } \\
\text { LCC (denoted by } \\
\text { LCC }_{\text {net }} \text { ) }\end{array}$} & $\begin{array}{l}\text { Top- } \\
\text { Down }\end{array}$ & MCMDKP & $\begin{array}{l}\text { Scenario 3b: constrained } \\
\checkmark \quad \text { total budget } \leq \$ 30 \mathrm{M}\end{array}$ & Yes \\
\hline & & & \multirow[t]{3}{*}{$\begin{array}{c}\text { Bottom } \\
\text {-Up }\end{array}$} & \multirow[t]{3}{*}{ MCMDKP } & $\begin{array}{ll}\text { Scenario } 2 \text { b: constraint } \\
\checkmark & \text { total budget } \leq \$ 80 \mathrm{M} \\
\checkmark & \text { network health } \\
\text { index } \geq 70 \% \text { every } \\
10 \text { years }\end{array}$ & Yes \\
\hline & & & & & Scenario $3 b$ & Yes \\
\hline & & & & & $\begin{array}{ll}\text { Scenario } 4 \text { b: constrained } \\
\checkmark & \text { total budget } \leq \$ 50 \mathrm{M} \\
\checkmark & \text { network health } \\
& \text { index } \geq 70 \% \text { every } \\
& 10 \text { years }\end{array}$ & Yes \\
\hline & \multirow[b]{2}{*}{ 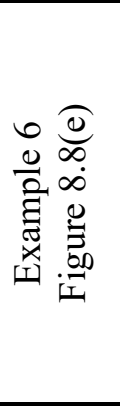 } & \multirow{2}{*}{$\begin{array}{l}\text { - maximize the network } \\
\text { health index in year } 20 \\
\left.\text { (denoted by HI } 20_{\text {net }}\right) \\
\text { - minimize the network } \\
\text { LCC (denoted by } \\
\text { LCC }_{\text {net }} \text { ) }\end{array}$} & \multirow[t]{2}{*}{$\begin{array}{l}\text { Top- } \\
\text { Down }\end{array}$} & \multirow[t]{2}{*}{ MKP } & $\begin{array}{l}\text { Scenario 5b: constrained } \\
\checkmark \quad \text { annual budget } \leq \\
\$ 3 \mathrm{M}\end{array}$ & Yes \\
\hline & & & & & $\begin{array}{ll}\text { Scenario 6b: constrained } \\
\checkmark & \text { annual budget } \leq \\
& \$ 8 \mathrm{M} \\
\checkmark & \text { network health } \\
& \text { index } \geq 70 \% \text { every } \\
& 10 \text { years }\end{array}$ & Yes \\
\hline \multirow{6}{*}{ 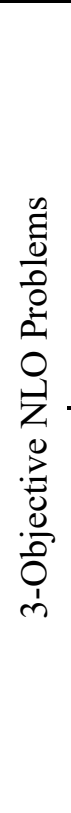 } & \multirow{4}{*}{ 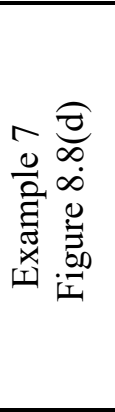 } & \multirow{4}{*}{ 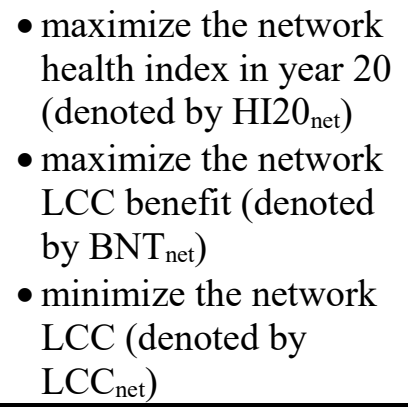 } & $\begin{array}{l}\text { Top- } \\
\text { Down }\end{array}$ & MCMDKP & Scenario $3 b$ & Yes \\
\hline & & & \multirow{3}{*}{$\begin{array}{c}\text { Bottom } \\
\text {-Up }\end{array}$} & \multirow[t]{3}{*}{ MCMDKP } & Scenario $2 b$ & Yes \\
\hline & & & & & Scenario 3b & Yes \\
\hline & & & & & Scenario $4 b$ & Yes \\
\hline & \multirow[b]{2}{*}{ 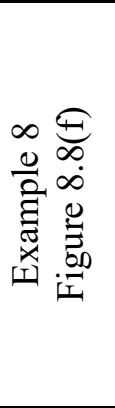 } & \multirow[b]{2}{*}{$\begin{array}{l}\text { - maximize the network } \\
\text { health index in year } 20 \\
\text { (denoted by HI } 20_{\text {net }} \text { ) } \\
\text { - maximize the network } \\
\text { LCC benefit (denoted } \\
\text { by BNT } \text { net }_{\text {) }} \\
\text { - minimize the network } \\
\text { LCC (denoted by } \\
\text { LCC }_{\text {net }} \text { ) }\end{array}$} & \multirow{2}{*}{$\begin{array}{l}\text { Top- } \\
\text { Down }\end{array}$} & \multirow[t]{2}{*}{ MKP } & Scenario $5 \mathrm{~b}$ & Yes \\
\hline & & & & & Scenario $6 \mathrm{~b}$ & Yes \\
\hline
\end{tabular}




\section{Obtained Pareto Frontiers for the Different Scenarios}

The objective of this NLO module is to obtain a diverse set of optimal or near-optimal NLO solutions for the entire network, constituting a frontier of trade-offs (Pareto frontier). The recommended NLO solutions for the different examples are plotted in Figures 8.8(a) through (f). The plotted solutions represent the Pareto solutions (constituting the Pareto frontiers for the different scenarios) for the entire analysis period. Scenarios under Case A were initially analyzed; however, Pareto solutions were obtained only for the top-down approach Scenarios 1a, 2a and 4a, and the bottom-up approach Scenario 1a. On the other hand, Pareto solutions were obtained for all scenarios under Case B. The scenarios which Pareto frontiers couldn't be obtained under Case A were used in the examples of Case B.

Most BMSs start with the MRR optimization at the bridge level and use the produced results at the network level. To a certain extent, the module bottom-up approach resembles this common BMS optimization approach. For Case A, scenario results demonstrate that the top-down approach NLO problems are more trackable than the bottom-up approach ones. The bottom-up approach optimization failed to deliver Pareto solutions for the constrained scenarios under Case A (requiring the selection of every single bridge in the portfolio). The top-down approach optimization benefits from the diversity of the input parameters (ELO solutions per program year) along with a superiority screening process, guiding the search toward global optimality. The bottom-up approach optimization lacks this level of diversity as it relies mainly on the BLO solutions obtained for the entire program period. It's worth mentioning that the convergence time increases almost exponentially with the size of the portfolio when bypassing the screening process for the top-down approach optimization. However, this issue wasn't noticed for the bottom-up approach optimization. The benefit of the bottom-up approach optimization can be recognized for 
most of the unconstrained scenarios or constrained scenarios under Case B: Pareto solutions are obtained in less computational time than the top-down approach.

Examples 1 and 3 include the MCKPs (unconstrained scenarios) and MCMDKPs (constrained scenarios). Examples 2 and 4 include only the MKPs (constrained scenarios). Figure 8.8(a) displays the 2-objective NLO solutions for the top-down approach Scenarios 1a, 2a and 4a, and the bottom-up approach Scenario 1a. Figure 8.8(b) presents the 3-objective NLO solutions for these same scenarios. Pareto frontiers for the unconstrained scenarios of Examples 1 and 3 are practically superimposed—not necessarily derived from the same recommended LC alternatives.

The displayed NLO solutions of the unconstrained scenarios in Figures 8.8(a) and (b) indicate that both the top-down and bottom-up approaches converge to the same Pareto frontier-though, more studies are needed to confirm this observation. These figures also include the Pareto frontiers for two constrained scenarios of Examples 1 and 3 (i.e., top-down approach Scenarios 2a and 4a). The figures demonstrate that Pareto frontiers for the constrained scenarios are bounded by the ones for the unconstrained scenarios - this finding is expected because a Pareto frontier of an unconstrained scenario is viewed as the ultimate optimal boundary. For Examples 1 and 3, Pareto solutions couldn't be obtained for the top-down approach scenarios restricting the total budget to $\$ 30$ million or less, and for the bottom-up approach constrained scenarios - the 40 bridges together could not be addressed. Similarly, for Examples 2 and 4, Pareto solutions couldn't be obtained for the topdown approach scenarios restricting the annual budget to $\$ 8$ million or less and the network performance to a minimum network health index of $70 \%$ every 10 years.

Examples 5 and 7 include the MCMDKPs (constrained scenarios). Examples 6 and 8 include only the MKPs (constrained scenarios). The top-down approach Scenarios $3 b, 5 b$ and $6 b$, and the 
bottom-up approach Scenarios 2b, $3 \mathrm{~b}$ and $4 \mathrm{~b}$ under Case B are the scenarios of the examples under Case A which Pareto frontiers could not be obtained. By relaxing the selection criterion of Case B, Pareto solutions were obtained for all these scenarios. Figures 8.8(c) and (e) display the 2objective NLO solutions for these scenarios. Figures 8.8(d) and (f) present the 3-objective NLO solutions for these same scenarios. Pareto frontiers for the top-down approach Scenario 3b and the bottom-up approach Scenario 3b in Figures 8.8(c) and (d) of Examples 5 and 7 are in line to some extent. This observation suggests that the top-down and bottom-up approaches lead to a similar Pareto frontier when considering the same constrained scenario under Case B. Figures 8.8(c) and (d) include also the Pareto frontiers for two other scenarios of Examples 5 and 7 (i.e., bottom-up approach Scenarios $2 \mathrm{~b}$ and $4 \mathrm{~b}$ ). Figures 8.8(e) and 8.8(f) show the Pareto frontiers obtained for the annual budget scenarios of Examples 6 and 8, i.e., Scenario 5a (restricted by an annual budget of $\$ 3$ million) and Scenario 6a (restricted by an annual budget of $\$ 8$ million and a minimum network health index of $70 \%$ every 10 years). 
(a)

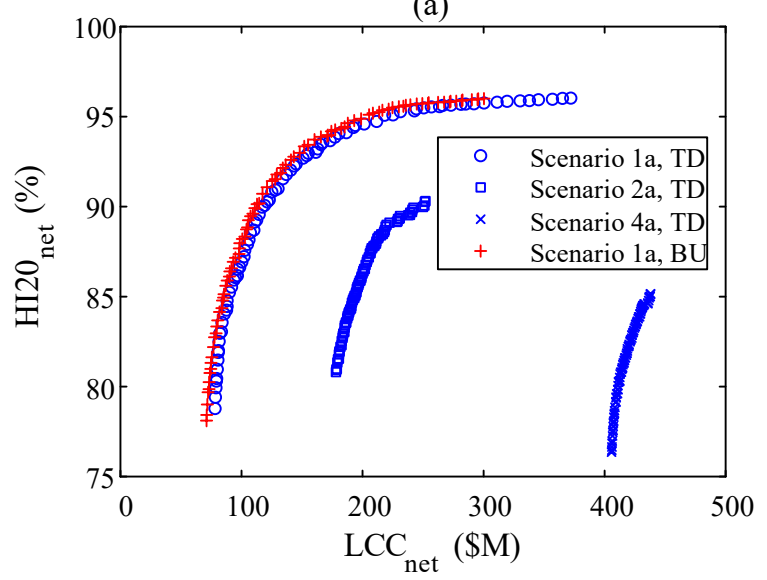

(c)

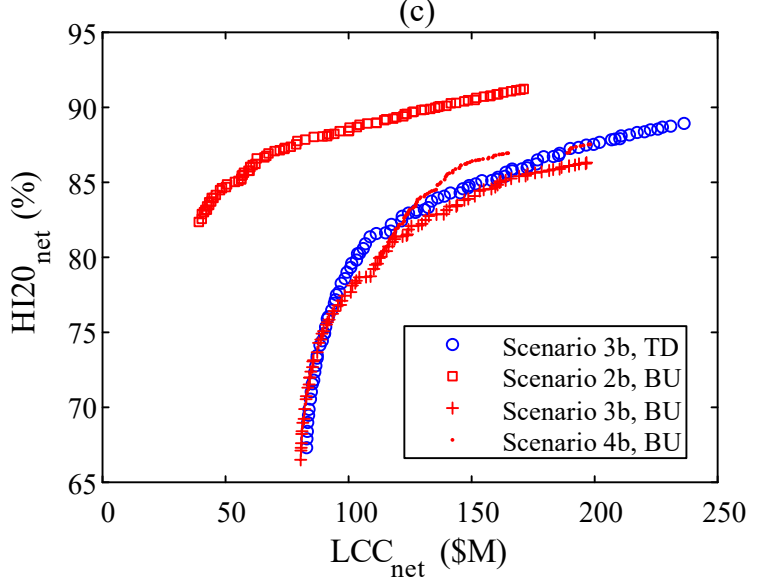

(e)

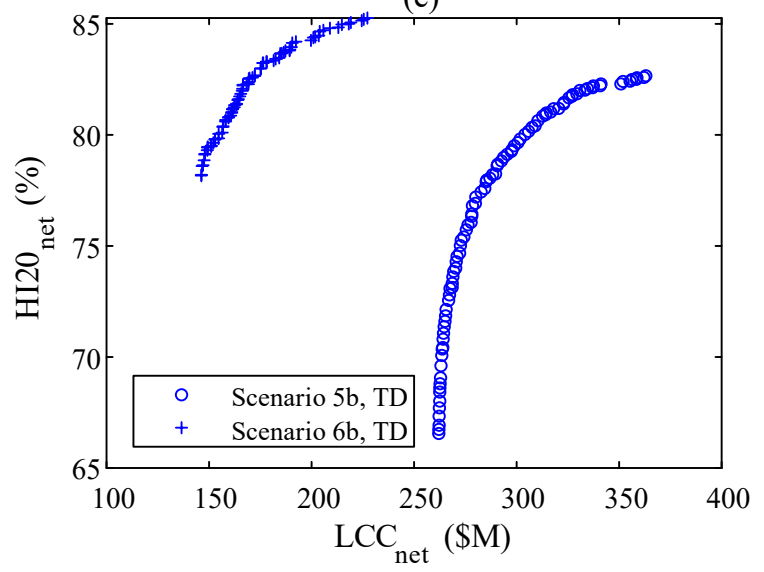

(b)

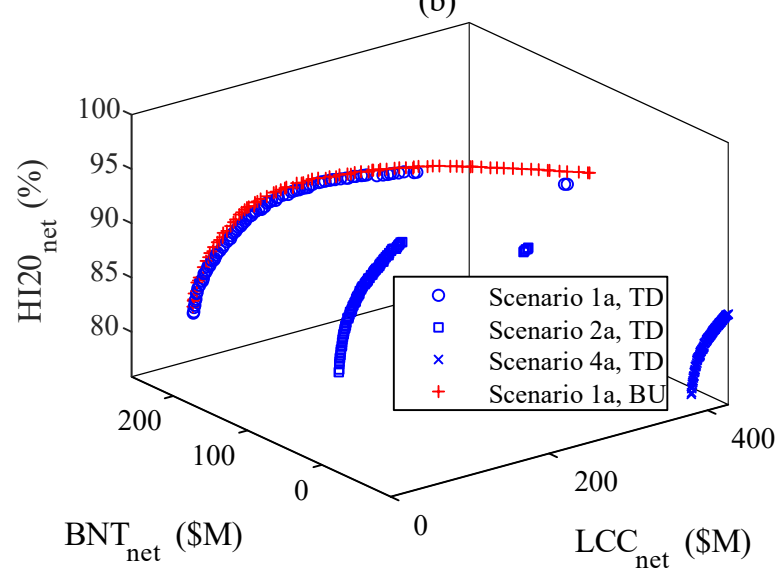

(d)

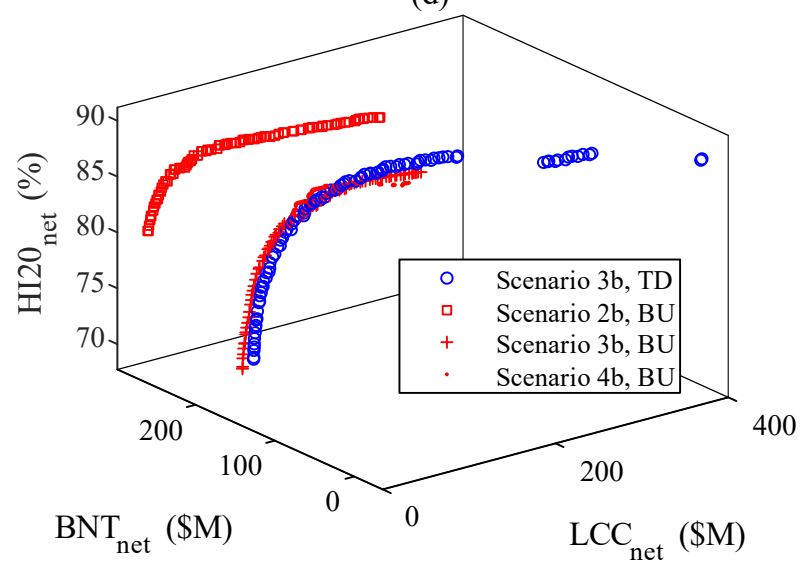

(f)

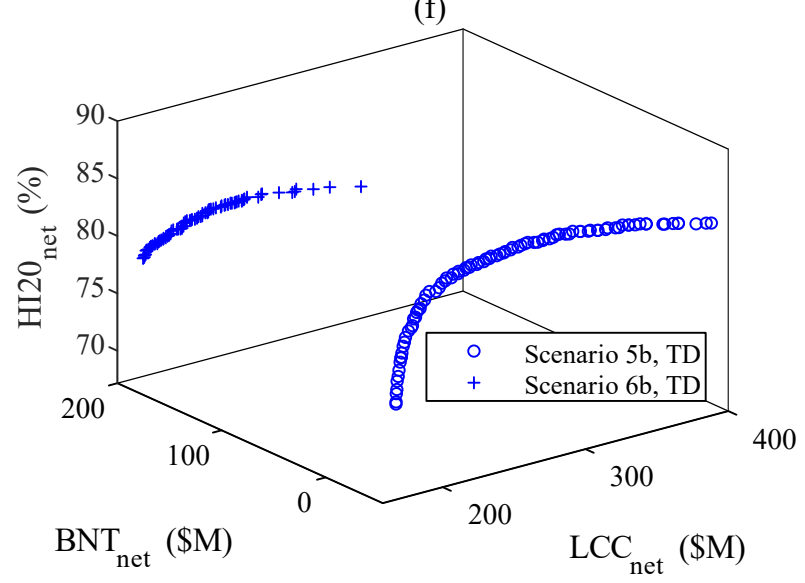

Figure 8.8 Obtained Pareto frontiers for the 2-objective NLO problems of (a) Example 1, (c) Example 5, (e) Example 6; the 3-objective NLO problems of (b) Example 3, (d) Example 7, (f) Example 8 


\section{Solutions with the Maximum Network LCC Benefits for the 3-Objective NLO Problems}

Table 8.5 shows the results associated with the NLO solutions producing the highest network LCC benefit ( $\mathrm{BNT}_{\text {net }}$ ) values for the different scenarios of Examples 3, 4, 7, and 8 (3-objective NLO problems). The unconstrained Scenario 1a (bottom-up approach) provides the highest network LCC benefit of $\$ 255,943,701$ for an investment of $\$ 123,431,339$ (Total IAC $_{\text {net). For an investment }}$ of less than $\$ 50$ million (exactly $\$ 49,798,578$ ), the top-down approach Scenario 4a unveils that all 40 bridges can be selected for improvement work, at the same time delivering the desired network performance (above the health index threshold of $70 \%$ every 10 years); however, this same scenario produces the least network LCC benefit $(-\$ 68,526,776)$. For an investment of $\$ 44,765,461$, the annual budget scenario (i.e., Scenario $6 \mathrm{~b}$ ) provides a better network LCC benefit $(\$ 175,351,664)$, improves 35 bridges, and maintains the desired network performance.

The results of Table 8.5 are visually presented in Figures 8.9 and 8.10 . Figure 8.9 shows the network total initial agency costs (Total IAC $\mathrm{Int}_{\text {net }}$, LCCs ( $\left.\mathrm{LCC}_{\mathrm{net}}\right)$, and LCC benefits $\left(\mathrm{BNT}_{\mathrm{net}}\right)$ associated with each scenario, and Figure 8.9 illustrates the resulting performance (network health indices). These visual presentations clearly assist to quickly grasp the main differences between these scenarios. For example, it's easily noticed from the bar charts that the bottom-up approach constrained Scenario $2 \mathrm{~b}$ has the highest network benefit and the least LCC for an initial investment of $\$ 79$ million. However, this scenario addresses only 35 bridges versus the 40 bridges addressed by the bottom-up approach Scenario 2a for the same level of investment.

The network health indices at the ends of years $1,10,20,30,40,50$, and 54 associated with the solutions listed in Table 8.5 are plotted (connected with straight lines) in Figure 8.9. As expected, the straight lines connecting the predicted network health indices for the performance-constrained scenarios are situated above the horizontal dashed lines in black representing the network 
performance constraints (specified by a minimum network health index of $70 \%$ every 10 years). The NLO solutions associated with these performance-constrained scenarios produce close values of network health indices despite the differences between the investment levels. As explained in the previous chapter, at the bridge level, the change to the bridge health index is generally minuscule. Element health indices are weighted, aggregated and divided by the sum of all their weighs to constitute this index. Preserving or improving few element health indices (after factoring their weights) won't dramatically change this index. It takes substantial improvement efforts to alter this bridge health index, and consequently the overall network health index-by aggregating all these bridge health indices and averaging them in Equation (8.8). The budget-constrained scenarios (i.e., Scenarios $3 \mathrm{~b}, 3 \mathrm{a}$, and $5 \mathrm{~b}$ in Table 8.5 ) produced the worst performance. Starting from program year 10, the predicted network health indices continue declining below the network performance threshold (horizontal dashed lines in black). The connected straight lines between DN health indices illustrate a decline of network condition over time; the network health index is predicted to drop to $42 \%$ by year 50 . 
Table 8.5 Results produced by the solutions with the maximum network LCC benefits for the 3-objective NLO problems

\begin{tabular}{|c|c|c|c|c|c|c|c|c|c|c|c|c|c|}
\hline & 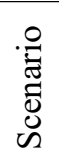 & 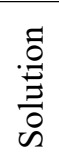 & 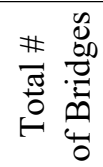 & $\begin{array}{c}\text { Total IAC } \text { Inet } \\
(\$)\end{array}$ & $\begin{array}{c}\mathrm{LCC}_{\mathrm{net}}^{\mathrm{a}} \\
(\$)\end{array}$ & $\begin{array}{c}\mathrm{BNT}_{\text {net }}^{\mathrm{a}} \\
(\$)\end{array}$ & $\begin{array}{l}\mathrm{HI}_{1 \mathrm{net}} \\
(\%)\end{array}$ & $\begin{array}{c}\text { HI10 } \\
(\%)\end{array}$ & $\begin{array}{c}\mathrm{HI} 20_{\mathrm{net}}^{\mathrm{a}} \\
(\%)\end{array}$ & $\begin{array}{c}\mathrm{HI} 30_{\text {net }} \\
(\%)\end{array}$ & $\begin{array}{c}\text { HI } 40_{\text {net }} \\
(\%)\end{array}$ & $\begin{array}{c}\text { HI50 }{ }_{\text {net }} \\
(\%)\end{array}$ & $\begin{array}{c}\text { HI54 } \\
\text { (\%) }\end{array}$ \\
\hline \multirow{3}{*}{$\begin{array}{l}\text { Top-Down } \\
\text { Approach }\end{array}$} & $1 \mathrm{a}$ & 1 & 40 & $122,797,455$ & $77,501,223$ & $255,518,628$ & 81.76 & 81.41 & 80.19 & 73.09 & 72.18 & 71.05 & 69.27 \\
\hline & $2 \mathrm{a}$ & 3 & 40 & $78,952,662$ & $172,304,296$ & $160,937,323$ & 82.18 & 79.21 & 75.86 & 73.64 & 73.54 & 70.28 & 68.33 \\
\hline & $4 a$ & 2 & 40 & $49,798,578$ & $401,920,691$ & $-68,526,776$ & 80.32 & 74.56 & 76.53 & 72.58 & 74.34 & 70.11 & 67.98 \\
\hline Bottom-Up App. & $1 \mathrm{a}$ & 1 & 40 & $123,431,339$ & $77,288,472$ & $255,943,701$ & 81.80 & 80.46 & 80.78 & 71.06 & 73.35 & 71.60 & 69.74 \\
\hline Top-Down App. & $3 b$ & 12 & 19 & $25,927,292$ & $98,935,710$ & $234,387,746$ & 80.81 & 72.90 & 68.67 & 62.58 & 60.30 & 57.60 & 55.39 \\
\hline \multirow{3}{*}{$\begin{array}{l}\text { Bottom-Up } \\
\text { Approach }\end{array}$} & $2 b$ & 4 & 35 & $79,419,639$ & $34,945,002$ & $298,350,021$ & 80.87 & 78.62 & 79.41 & 70.65 & 70.16 & 70.23 & 68.17 \\
\hline & $3 b$ & 3 & 20 & $28,927,215$ & $92,152,135$ & $241,150,613$ & 81.76 & 73.36 & 67.74 & 62.70 & 61.39 & 58.32 & 56.17 \\
\hline & $4 \mathrm{~b}$ & 20 & 35 & $47,602,938$ & $122,174,527$ & $211,127,112$ & 79.97 & 78.82 & 81.82 & 70.22 & 70.04 & 70.10 & 68.44 \\
\hline Top-Down & $5 b$ & 10 & 19 & $15,084,891$ & $260,378,040$ & $72,953,649$ & 80.94 & 72.59 & 67.18 & 64.34 & 61.24 & 56.68 & 54.59 \\
\hline Approach & $6 \mathrm{~b}$ & 22 & 35 & $44,765,461$ & $157,935,436$ & $175,351,664$ & 79.98 & 78.61 & 79.06 & 70.32 & 70.33 & 70.01 & 67.36 \\
\hline
\end{tabular}

a Optimized objective. 


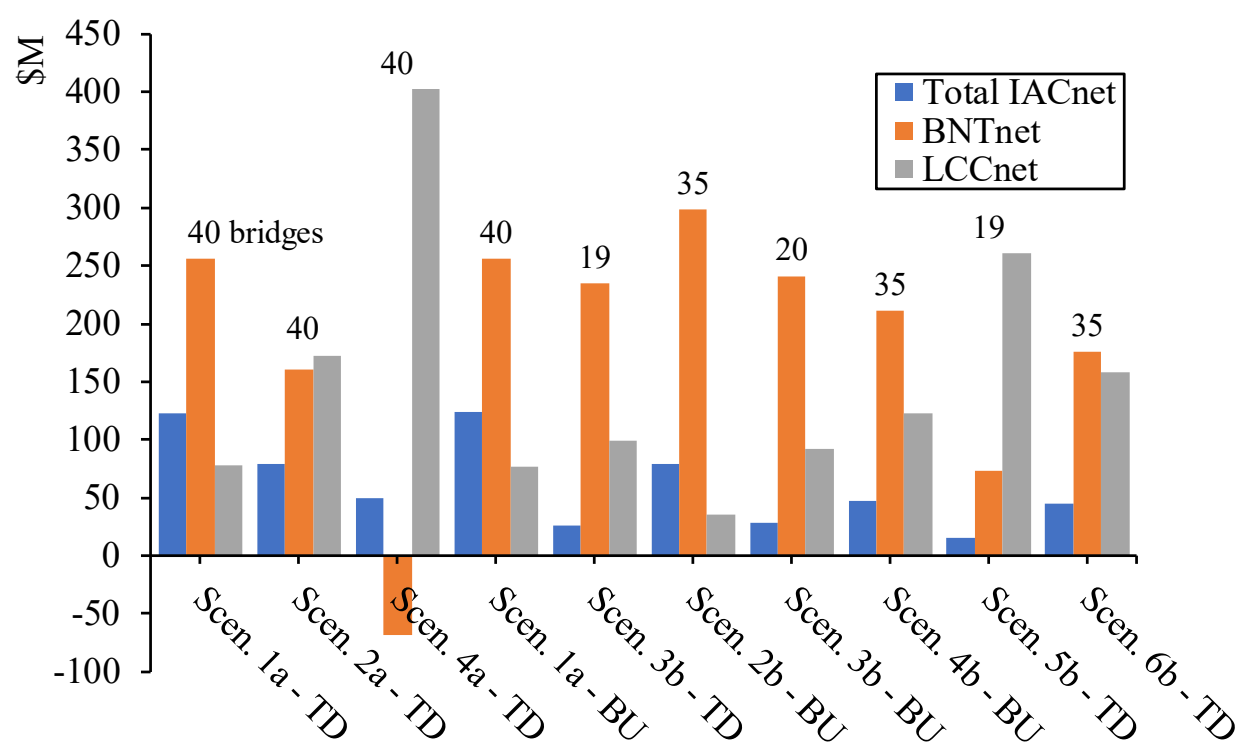

Figure 8.9 Network costs produced by the solutions with the maximum network LCC benefits for the 3objective NLO problems (Table 8.5)

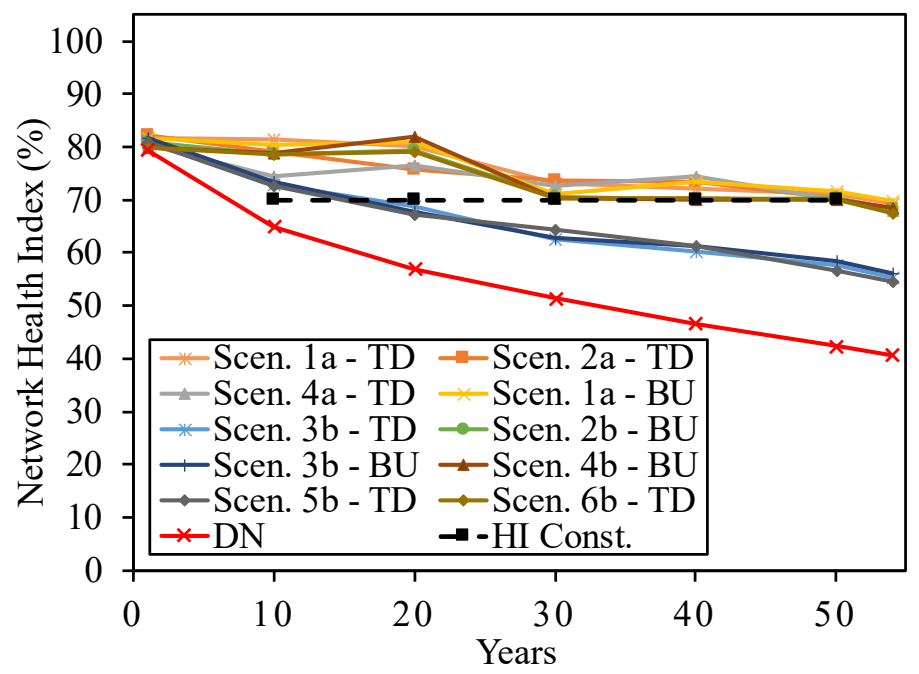

Figure 8.10 Network health indices produced by the solutions with the maximum network LCC benefits for the 3-objective NLO problems (Table 8.5)

Table 8.6 lays out the initial investment distributions over the 10 -year program period provided by the same NLO solutions listed in Table 8.5. These annual investment levels produce the highest network LCC benefit values for the scenarios in Table 8.5. For each program year, the table shows the number of selected bridges and the estimated initial agency cost (Ann. IAC $\mathrm{net}_{\text {). }}$. 
Table 8.6 Initial investment distributions over the 10-year program period for the 3-objective NLO problems

\begin{tabular}{|c|c|c|c|c|c|c|c|c|c|c|c|c|c|}
\hline & \multirow{2}{*}{\multicolumn{2}{|c|}{ Scenario Solution }} & & \multicolumn{10}{|c|}{ Program Years } \\
\hline & & & & 1 & 2 & 3 & 4 & 5 & 6 & 7 & 8 & 9 & 10 \\
\hline \multirow{6}{*}{$\begin{array}{l}\text { Top-Down } \\
\text { Approach }\end{array}$} & \multirow{2}{*}{$1 \mathrm{a}$} & \multirow{2}{*}{1} & \# of Bridges & 5 & 3 & 0 & 0 & 1 & 7 & 5 & 2 & 16 & 1 \\
\hline & & & Ann. IACnet (\$) & $8,647,578$ & $41,861,524$ & 0 & 0 & $3,072,814$ & $34,583,466$ & $1,205,503$ & $11,790,365$ & $17,141,875$ & $4,494,330$ \\
\hline & \multirow{2}{*}{$2 \mathrm{a}$} & \multirow{2}{*}{3} & \# of Bridges & 6 & 3 & 2 & 0 & 0 & 6 & 8 & 4 & 8 & 3 \\
\hline & & & Ann. IACnet (\$) & $9,651,365$ & $18,540,169$ & $16,585,072$ & 0 & 0 & $7,944,124$ & $2,597,060$ & $8,385,497$ & $4,803,601$ & $10,445,775$ \\
\hline & \multirow{2}{*}{$4 a$} & \multirow{2}{*}{2} & \# Brg & 5 & 2 & 3 & 1 & 2 & 7 & 9 & 0 & 11 & 0 \\
\hline & & & Ann. IACnet (\$) & $12,791,904$ & $5,391,934$ & $14,405,961$ & $1,187,519$ & $1,252,097$ & $5,969,196$ & $2,671,247$ & 0 & $6,128,721$ & 0 \\
\hline \multirow{2}{*}{$\begin{array}{c}\text { Bottom-Up } \\
\text { Approach }\end{array}$} & \multirow{2}{*}{ 1a } & \multirow{2}{*}{1} & \# of Bridges & 5 & 3 & 0 & 0 & 0 & 8 & 7 & 2 & 14 & 1 \\
\hline & & & Ann. IACnet (\$) & $8,661,204$ & $41,155,240$ & 0 & 0 & 0 & $37,898,355$ & $6,034,831$ & $11,790,365$ & $16,197,170$ & $1,694,174$ \\
\hline \multirow{2}{*}{$\begin{array}{l}\text { Top-Down } \\
\text { Approach }\end{array}$} & \multirow{2}{*}{$3 b$} & \multirow{2}{*}{12} & \# of bridges & 3 & 2 & 2 & 1 & 1 & 4 & 2 & 0 & 4 & 0 \\
\hline & & & Ann. IACnet (\$) & $3,974,277$ & $4,673,301$ & $1,314,915$ & $1,003,787$ & 289,739 & $8,528,996$ & $1,391,556$ & 0 & $4,750,721$ & 0 \\
\hline \multirow{6}{*}{$\begin{array}{c}\text { Bottom-Up } \\
\text { Approach }\end{array}$} & \multirow{2}{*}{$2 b$} & \multirow{2}{*}{4} & \# of Bridges & 3 & 1 & 1 & 1 & 0 & 4 & 8 & 4 & 13 & 0 \\
\hline & & & Ann. IAC & $7,242,253$ & $1,200,926$ & $1,003,787$ & 204,399 & 0 & $45,395,540$ & $9,178,742$ & $3,044,707$ & $12,149,285$ & 0 \\
\hline & \multirow{2}{*}{$3 b$} & \multirow{2}{*}{3} & \# of Bridges & 5 & 2 & 1 & 1 & 0 & 4 & 3 & 1 & 3 & 0 \\
\hline & & & Ann. IACnet (\$) & $8,647,578$ & $1,293,525$ & $1,003,787$ & 311,128 & 0 & $7,488,315$ & $2,088,280$ & 334,558 & $7,760,043$ & 0 \\
\hline & \multirow{2}{*}{$4 b$} & \multirow{2}{*}{20} & \# of Bridges & 1 & 1 & 1 & 2 & 0 & 3 & 7 & 5 & 15 & 0 \\
\hline & & & Ann. IACnet (\$) & $3,472,375$ & $3,472,375$ & 289,739 & $1,405,326$ & 0 & $11,574,863$ & $6,295,066$ & $3,165,749$ & $17,927,446$ & 0 \\
\hline \multirow{4}{*}{$\begin{array}{c}\text { Top-Down } \\
\text { Approach }\end{array}$} & \multirow{2}{*}{$5 b$} & \multirow{2}{*}{10} & \# of Bridges & 3 & 1 & 1 & 1 & 1 & 3 & 4 & 2 & 2 & 1 \\
\hline & & & Ann. IACnet (\$) & $1,505,689$ & 726,609 & $1,200,926$ & 311,128 & 845,716 & $2,365,095$ & $2,687,943$ & $2,998,005$ & 749,607 & $1,694,174$ \\
\hline & \multirow{2}{*}{$6 b$} & \multirow{2}{*}{22} & \# of Bridges & 1 & 1 & 3 & 1 & 3 & 4 & 7 & 4 & 10 & 1 \\
\hline & & & Ann. IACnet (\$) & $3,894,783$ & $4,494,330$ & $7,242,253$ & 204,399 & $3,592,249$ & $6,385,036$ & $5,010,814$ & $4,581,903$ & $4,979,787$ & $4,379,906$ \\
\hline
\end{tabular}


Results shown in Table 8.6 are visually presented in Figures 8.11(a) and (b). The three-dimensional bar charts represent the distributions of selected bridges and initial agency spending over the 10year program period for the scenarios in Table 8.5. The distributions reveal that years 2 and 6 receive the largest initial investment levels (mainly for the unconstrained scenarios and the bottomup approach constrained Scenario 2b). Most bridges are recommended for improvement work between years 6 and 9; a minor improvement work is assigned to either year 4 or 5 .

(a)

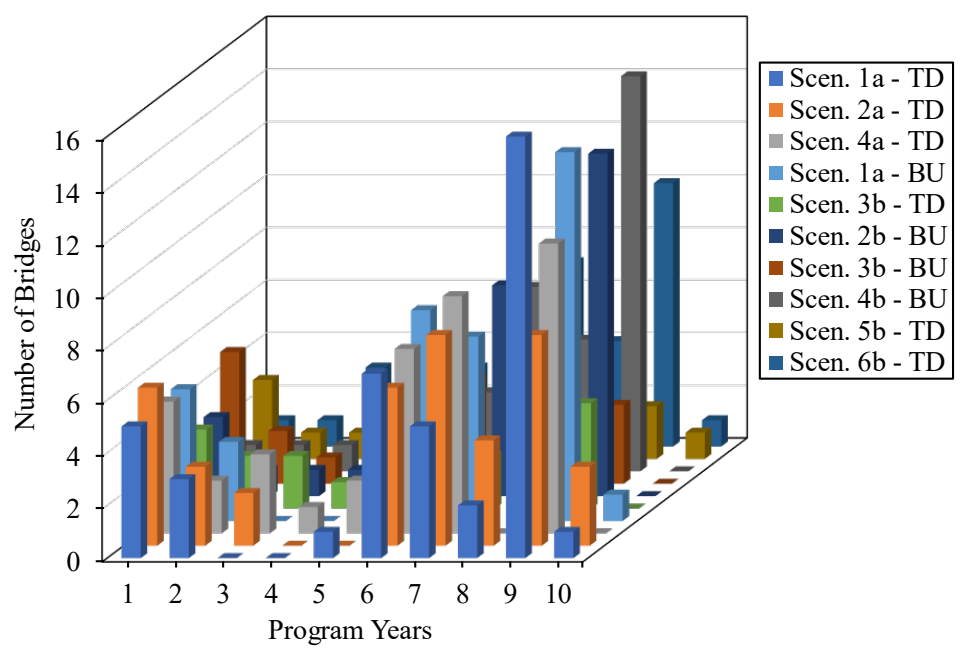

(b)

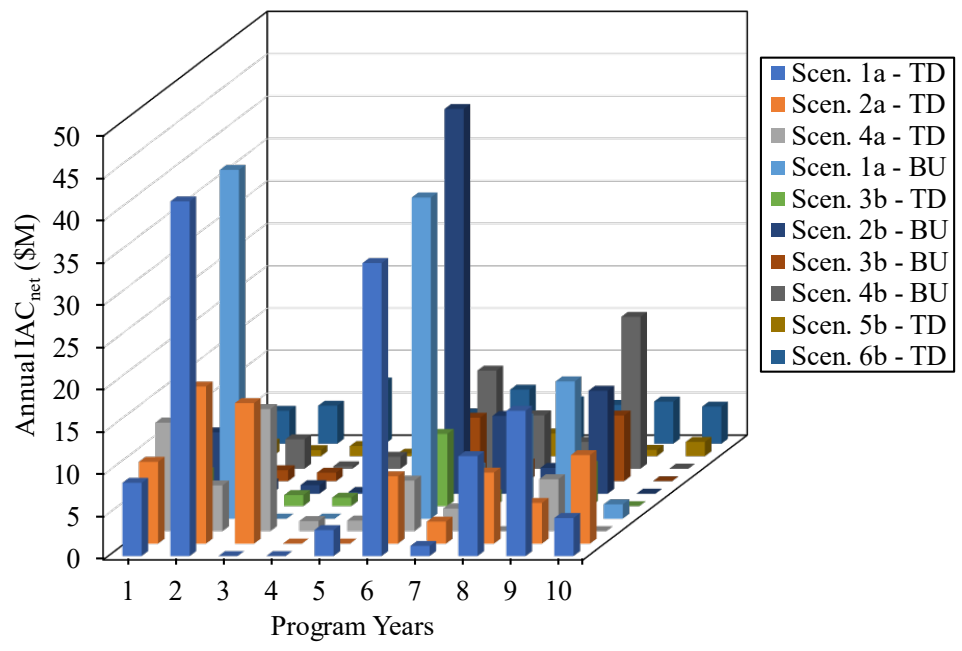

Figure 8.11 (a) Selected bridges; (b) Initial agency spending distribution over the 10-year program period for the 3-objective NLO problems (Table 8.6) 


\section{NLO Solutions Recommended for Example 3, Scenario 2a, Top-Down Approach}

Table 8.7 shows the results associated with the 86 NLO solutions recommended for the top-down approach Scenario 2a of Example 3 (3-objective NLO problem constrained by a budget of $\$ 80$ million, and a minimum network health index of $70 \%$ every 10 years). The optimization goal is to maximize the network health index in year 20 (HI20 $0_{\text {net }}$, maximize the network LCC benefit $\left(\mathrm{BNT}_{\text {net }}\right)$, and minimize the network $\mathrm{LCC}\left(\mathrm{LCC}_{\mathrm{net}}\right)$. In addition to these three main optimization objectives, the network initial agency cost (IAC $\mathrm{brg}$ ), and health indices at the ends of years 1, 10, $30,40,50$, and 54 were also determined as part of the module optimization results. Solution 3 produces the highest network LCC benefit of $\$ 160,937,323$ for an investment of $\$ 78,952,662$ (less than the available budget of $\$ 80$ million) and provides the desired network performance. 
Table 8.7 Results produced by the NLO solutions recommended for Example 3, Scenario 2a, Top-Down Approach

\begin{tabular}{|c|c|c|c|c|c|c|c|c|c|c|}
\hline Solution & $\begin{array}{c}\mathrm{IAC}_{\text {net }} \\
(\$)\end{array}$ & $\begin{array}{c}\mathrm{LCC}_{\text {net }}^{\mathrm{a}} \\
(\$)\end{array}$ & $\begin{array}{c}\mathrm{BNT}_{\text {net }}^{\mathrm{a}} \\
(\$)\end{array}$ & $\begin{array}{l}\mathrm{HI} 1_{\text {net }} \\
(\%)\end{array}$ & $\begin{array}{c}\text { HI10 } 0_{\text {net }} \\
(\%)\end{array}$ & $\begin{array}{c}\mathrm{HI} 20_{\text {net }}^{\mathrm{a}} \\
(\%)\end{array}$ & $\begin{array}{c}\text { HI30 } 0_{\text {net }} \\
(\%)\end{array}$ & $\begin{array}{c}\text { HI } 40_{\text {net }} \\
(\%)\end{array}$ & $\begin{array}{c}\mathrm{HI} 50_{\text {net }} \\
(\%)\end{array}$ & $\begin{array}{c}\text { HI54 }{ }_{\text {net }} \\
(\%)\end{array}$ \\
\hline 1 & $79,404,905$ & $295,708,356$ & $37,615,082$ & 79.97 & 82.12 & 88.78 & 73.02 & 70.06 & 72.11 & 71.07 \\
\hline 2 & $79,404,905$ & $292,665,355$ & $40,658,083$ & 79.97 & 81.86 & 88.34 & 72.90 & 70.66 & 71.89 & 70.93 \\
\hline 3 & $78,952,662$ & $172,304,296$ & $160,937,323$ & 82.18 & 79.21 & 75.86 & 73.64 & 73.54 & 70.28 & 68.33 \\
\hline 4 & 900,081 & $209,661,114$ & $123,627,095$ & 80.46 & 1.23 & 87.67 & 72.76 & 70.01 & 71.87 & 70.67 \\
\hline 5 & $79,404,905$ & $295,175,228$ & $38,148,211$ & 79.97 & 81.96 & 88.66 & 73.00 & 70.69 & 72.03 & 71.03 \\
\hline 6 & $78,952,662$ & $172,789,426$ & $160,498,783$ & 82.64 & 79.06 & 76.83 & 73.41 & 73.59 & 70.01 & 68.14 \\
\hline 7 & 79,900 & 211,63 & $121,648,398$ & 80.46 & 81.58 & 88.12 & 73.11 & 70.08 & 71.93 & 70.79 \\
\hline 8 & $79,900,081$ & $211,042,315$ & $122,245,894$ & 80.46 & 81.58 & 88.01 & 72.85 & 70.34 & 71.90 & 70.78 \\
\hline 9 & 79,468 & $186,832,114$ & $146,456,095$ & 80.87 & 80.90 & 83.66 & 72.70 & 71.98 & 71.06 & 69.98 \\
\hline 10 & $79,356,772$ & $184,997,733$ & $148,290,475$ & 80.87 & 81.25 & 83.09 & 72.94 & 72.67 & 71.10 & 70.01 \\
\hline 11 & $79,165,614$ & $178,557,898$ & $154,730,310$ & 81.34 & 80.35 & 80.90 & 72.66 & 73.02 & 71.03 & 69.59 \\
\hline 12 & $78,952,469$ & 172,58 & $160,671,981$ & 82.18 & 79.18 & 76.12 & 73.59 & & 70.27 & \\
\hline 13 & 79,73 & 206,33 & 126,948 & 80.46 & 9 & 87.45 & 73.56 & 70.23 & 71.67 & 70.62 \\
\hline 14 & 34 & 204,8 & $128,470,808$ & 80.46 & 1.69 & 87.02 & 73.32 & 71.07 & 71.69 & 70.62 \\
\hline 15 & 46 & 173 & 159 & 82.22 & 79.16 & 77.46 & 72.89 & 73.78 & 70.05 & 68.38 \\
\hline 16 & & 779 & 140 & 80.46 & .81 & .78 & 73.01 & 72.01 & 71.35 & 70.32 \\
\hline 17 & & 175 & 157 & 81.34 & .41 & 79.64 & 72.63 & 73.50 & 70.61 & 69.27 \\
\hline 18 & & 176 & 156 & 81.76 & & & 72.89 & & 71.45 & 69.70 \\
\hline 19 & & 176 & 156 & 81.34 & 80.38 & 80.17 & 72.53 & 73.51 & 71.16 & 69.84 \\
\hline 20 & $79,716,170$ & $202,867,395$ & $130,420,813$ & 80.46 & 81.78 & 86.82 & 73.39 & 70.25 & 71.65 & 70.59 \\
\hline 21 & $79,185,057$ & $175,538,077$ & $157,730,783$ & 81.76 & 0.37 & 44 & 72.79 & 73.18 & 70.85 & 69.38 \\
\hline 22 & $79,165,614$ & 176,95 & $156,336,896$ & 81.34 & 80.15 & 80.23 & 72.72 & 72.80 & 71.21 & 69.57 \\
\hline 23 & $79,468,854$ & $183,394,142$ & $149,894,067$ & 81.34 & & & 72.61 & 71.97 & 71.19 & \\
\hline 24 & 79,65 & 762,436 & 135 & 80.46 & & 79 & 73.09 & 70.98 & 71.45 & 70.46 \\
\hline 25 & 159 & 199 & 5,668 & 80.46 & 8 & 86.19 & 73.30 & 70.80 & 71.55 & 70.50 \\
\hline 26 & & 0 & 152 & 81.2 & 0 & 81.77 & 73.01 & 72.59 & 71.37 & 69.99 \\
\hline 27 & & 18 & 145 & 80.87 & 1 & 6 & 72.97 & 71.49 & 71.10 & 70.08 \\
\hline 28 & 79 & 184 & 148 & 81.34 & 57 & 82.97 & 72.94 & 71.81 & 70.96 & 69.88 \\
\hline 29 & $79,4 \varepsilon$ & 195 & 137 & 80.46 & & & 73.20 & & 71.45 & 70.40 \\
\hline 30 & $79,165,614$ & $180,352,998$ & $152,935,211$ & 81.34 & 80.58 & 81.50 & 72.84 & 73.05 & 70.87 & 69.50 \\
\hline 31 & $79,695,875$ & $198,646,966$ & $134,641,243$ & 80.46 & 81.81 & 85.95 & 72.93 & 71.17 & 71.52 & 70.52 \\
\hline 32 & $79,468,854$ & $185,533,685$ & $147,754,523$ & 80.87 & 81.02 & 83.23 & 72.78 & 72.09 & 71.19 & 70.13 \\
\hline 33 & $79,244,963$ & $179,273,837$ & $154,014,372$ & 81.76 & & 81.25 & 73.09 & 72.61 & 71.34 & 69.96 \\
\hline 34 & & & & & & & 72.64 & & 71.17 & 70.15 \\
\hline 35 & & & 138 & 80. & 8 & 85.31 & 72.95 & 71.15 & 71.45 & 70.33 \\
\hline 36 & 79,601 & 190,7 & 142,4 & 80.8 & 81 & 84.48 & 73.05 & 71.56 & 71.12 & 70.13 \\
\hline 37 & 79,90 & 213 & 119, & 80.46 & 81.68 & 88.32 & 72.96 & 70.37 & 72.04 & 70.88 \\
\hline 38 & 79,7 & 203,7 & $129,536,283$ & 80.46 & 81.78 & 86.98 & 73.23 & 70.45 & 71.72 & 70.65 \\
\hline 39 & $79,489,149$ & $195,374,216$ & $137,913,993$ & 80.46 & 81.78 & 85.43 & 72.95 & 71.62 & 71.43 & 70.40 \\
\hline 40 & $79,716,170$ & $201,982,865$ & $131,305,343$ & 80.46 & 81.78 & 86.66 & 73.54 & 70.06 & 71.58 & 70.54 \\
\hline 41 & $79,356,772$ & $181,821,433$ & $151,466,775$ & 80.87 & 80.68 & 82.15 & 72.67 & 72.30 & 71.23 & 69.99 \\
\hline 42 & $79,900,081$ & $212,524,341$ & $120,763,868$ & 80.46 & 81.58 & 88.29 & 72.96 & 70.27 & 72.00 & 70.85 \\
\hline 43 & $79,468,854$ & $189,982,223$ & $143,305,986$ & 80.46 & 81.81 & 84.35 & 73.07 & 71.89 & 71.18 & 70.20 \\
\hline 44 & & $177,288,478$ & 155,999 & 81.76 & 30 & 80.50 & 72.99 & 72.54 & 71.19 & 69.67 \\
\hline 45 & & $175,281,979$ & $158,006,229$ & 81.34 & 80.16 & 79.36 & 72.47 & 73.47 & 70.85 & 69.29 \\
\hline 46 & $79,244,963$ & $178,748,158$ & $154,540,051$ & 81.34 & 80.36 & 81.05 & 72.59 & 73.05 & 71.34 & 69.98 \\
\hline
\end{tabular}


Table 8.7 Results produced by the NLO solutions recommended for Example 3, Scenario 2a, Top-Down Approach (continued)

\begin{tabular}{|c|c|c|c|c|c|c|c|c|c|c|}
\hline Solution & $\begin{array}{c}\text { IAC }_{\text {net }} \\
(\$)\end{array}$ & $\begin{array}{c}\mathrm{LCC}_{\text {net }}^{\mathrm{a}} \\
(\$)\end{array}$ & $\begin{array}{c}\mathrm{BNT}_{\text {net }}^{\mathrm{a}} \\
(\$)\end{array}$ & $\begin{array}{c}\text { HI } 1_{\text {net }} \\
(\%)\end{array}$ & $\begin{array}{c}\text { HI10 } \\
(\%)\end{array}$ & $\begin{array}{c}\text { HI } 20_{\text {net }}{ }^{\mathrm{a}} \\
(\%)\end{array}$ & $\begin{array}{c}\mathrm{HI} 30_{\text {net }} \\
(\%)\end{array}$ & $\begin{array}{c}\mathrm{HI} 40_{\text {net }} \\
(\%)\end{array}$ & $\begin{array}{c}\mathrm{HI} 50_{\text {net }} \\
(\%)\end{array}$ & $\begin{array}{c}\text { HI54 } 4_{\text {net }} \\
(\%)\end{array}$ \\
\hline 47 & $9,468,854$ & $184,152,485$ & $149,135,724$ & 80.87 & 80.67 & 82.89 & 72.69 & 71.76 & 71.15 & 70.02 \\
\hline 48 & & & & & & & 4 & & & \\
\hline 49 & & 35 & 924 & 64 & 04 & 7.96 & 2.63 & & & 3.82 \\
\hline 50 & & & & .64 & & .13 & 3.10 & & & \\
\hline 51 & & 27 & 153,629, & & & & & & & \\
\hline 52 & 244,9 & $7,863,628$ & 155,424 & & & 89 & & & & 9.92 \\
\hline 53 & & $175,099,100$ & 158,169 & 1.34 & & .14 & 2.53 & & & \\
\hline 54 & & & 515 & 1.29 & & 3.25 & & 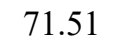 & & .05 \\
\hline 55 & & 5 & 123 & 0.46 & & .85 & 01 & & & 0.73 \\
\hline 56 & & & & & & & 0 & & & \\
\hline 57 & & & & & & & & & & \\
\hline 58 & & & & & & & & & & \\
\hline 59 & & & & & & & & & & \\
\hline 60 & & & & & & & & & & \\
\hline 61 & & & 157 & 4 & & & & & & \\
\hline 62 & & & & 7 & & 7 & 1 & & & 98 \\
\hline & & & & & & & & & & \\
\hline & & & & & & & & & & \\
\hline 6 & & & & 2 & & & & & & \\
\hline 6 & & & & & & & & & & \\
\hline 6 & & & & 6 & & & & & & \\
\hline 6 & & & & & & & & & & \\
\hline 69 & & & & 80.46 & & & & & & \\
\hline & & & & & & & & & & \\
\hline 7 & & & & 4 & & & & & & \\
\hline 7 & & & & & & & & & & \\
\hline 7 & & & & & & & & & & \\
\hline 7 & & & & & & & & & & \\
\hline 75 & & 044 & 3,164 & 80.46 & & & & & & 70.49 \\
\hline 76 & & & & & & & & & & \\
\hline 7 & & & & & & & & & & \\
\hline & & & & & & & & & & \\
\hline & & & & & & & & & & \\
\hline 80 & & & & & & & & & & \\
\hline 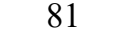 & & & & 80.87 & & & & & & 70.27 \\
\hline 8 & & & & 80.46 & & & & & & 0.53 \\
\hline 8 & & & & & & & & & & 37 \\
\hline & & & & & & & & & & 69.92 \\
\hline & & & & & & & & & & \\
\hline$\theta$ & $79,468,854$ & $187,328,785$ & $145,959,424$ & 80.87 & 81.25 & 83.83 & 72.96 & 72.12 & 71.02 & 70.03 \\
\hline
\end{tabular}

${ }^{a}$ Optimized objective. 


\section{Bridge-Level Results of the NLO Solution with the Maximum Network LCC Benefit for Example 3, Scenario 2a, Top-Down Approach}

Table 8.8 shows the bridge-level results associated with the NLO solution delivering the highest network LCC benefit for the top-down approach Scenario 2a of Example 3 (i.e., Solution 3 in Table 8.7). For each bridge in the portfolio, the table displays the sufficiency rating, number of years since the last construction or reconstruction, existing functional deficiencies, recommended program year and improvement type, estimated bridge initial agency cost, bridge LCC and bridge LCC benefit, and predicted bridge health indices at the end of years 1, 10, 20, 30, 40, 50, and 54 . The table reveals that the MRR improvement type is assigned to the bridges with high sufficiency ratings - for bridges in "good" condition (high sufficiency ratings), the preservation treatments are generally cost-effective (high LCC benefits) for maintaining the desired performance. The highest initial investment $(\$ 17,032,021)$ is allocated to Bridge 25 for a major functional improvement in year 2 . 
Table 8.8 Bridge-level results of the NLO solution with the maximum network LCC benefit for Example 3, Scenario 2a, Top-Down Approach

\begin{tabular}{|c|c|c|c|c|c|c|c|c|c|c|c|c|c|c|c|}
\hline Bridge & $\begin{array}{c}\text { Functional } \\
\text { Deficiency } \\
{[\mathrm{WR}, \mathrm{VC}, \mathrm{LC}]^{\mathrm{a}}}\end{array}$ & $\begin{array}{c}\text { Suff. } \\
\text { Rating }\end{array}$ & $\begin{array}{c}\text { Years Since } \\
\text { Con. or } \\
\text { Recon. }\end{array}$ & $\begin{array}{l}\text { Pro. } \\
\text { Year }\end{array}$ & $\begin{array}{l}\text { Imp. } \\
\text { Type }\end{array}$ & $\begin{array}{c}\mathrm{IAC}_{\mathrm{brg}} \\
(\$)\end{array}$ & $\begin{array}{c}\mathrm{LCC}_{\mathrm{brg}} \\
\quad(\$)\end{array}$ & $\begin{array}{c}\mathrm{BNT}_{\text {brg }} \\
(\$)\end{array}$ & $\begin{array}{c}\mathrm{HI}_{\text {brg }} \\
(\%)\end{array}$ & $\begin{array}{c}\mathrm{HI} 10_{\text {brg }} \\
(\%)\end{array}$ & $\begin{array}{c}\mathrm{HI} 20_{\text {brg }} \\
(\%)\end{array}$ & $\begin{array}{c}\mathrm{HI} 30_{\text {brg }} \\
(\%)\end{array}$ & $\begin{array}{c}\mathrm{HI} 40_{\mathrm{brg}} \\
(\%)\end{array}$ & $\begin{array}{c}\mathrm{HI} 50_{\text {brg }} \\
(\%)\end{array}$ & $\begin{array}{c}\mathrm{HI}_{54}{ }_{\mathrm{brg}} \\
(\%)\end{array}$ \\
\hline 1 & {$[0,0,0]$} & 92.1 & 63 & 6 & 1 & 183,905 & 329,401 & $-523,953$ & 85.12 & 75.21 & 69.47 & 85.94 & 69.76 & 62.74 & 60.26 \\
\hline 2 & {$[0,0,0]$} & 82.2 & 20 & 7 & 1 & 580,189 & 268,682 & $-1,797,320$ & 83.22 & 76.39 & 69.17 & 71.35 & 91.86 & 73.39 & 72.84 \\
\hline 3 & {$[0,0,0]$} & 83.2 & 8 & 7 & 1 & 543,158 & 332,442 & $-1,771,832$ & 83.32 & 75.90 & 68.55 & 70.78 & 91.68 & 72.82 & 72.26 \\
\hline 4 & {$[0,0,1]$} & 93.1 & 32 & 6 & 2 & $2,708,267$ & $-3,788,041$ & $5,588,272$ & 83.15 & 88.74 & 72.56 & 66.94 & 65.88 & 82.43 & 76.20 \\
\hline 5 & {$[1,0,0]$} & 52.5 & 71 & 9 & 2 & $1,694,174$ & $-4,630,465$ & $2,806,724$ & 77.00 & 97.96 & 97.24 & 74.32 & 69.85 & 76.03 & 78.38 \\
\hline 6 & {$[0,0,1]$} & 91.7 & 33 & 7 & 1 & 0 & 278,942 & $-537,903$ & 86.56 & 72.49 & 65.14 & 63.87 & 91.07 & 71.37 & 68.94 \\
\hline 7 & {$[0,0,1]$} & 96 & 6 & 10 & 2 & $4,379,906$ & $10,408,658$ & $-11,078,065$ & 83.60 & 100.00 & 74.31 & 65.11 & 75.19 & 64.91 & 64.14 \\
\hline 8 & {$[0,0,0]$} & 98.9 & 15 & 7 & 1 & 1,029 & $1,688,043$ & $-5,910,849$ & 89.16 & 72.81 & 62.80 & 63.45 & 89.31 & 69.10 & 66.59 \\
\hline 9 & {$[0,1,0]$} & 83.9 & 15 & 6 & 1 & 36,880 & 632,368 & $-1,195,086$ & 72.06 & 68.93 & 87.56 & 74.29 & 68.26 & 68.20 & 66.10 \\
\hline 10 & {$[0,1,0]$} & 72.4 & 55 & 9 & 1 & 351,974 & $1,651,068$ & $-1,958,288$ & 69.91 & 66.71 & 92.39 & 74.19 & 67.56 & 77.37 & 74.52 \\
\hline 11 & {$[1,0,0]$} & 74.3 & 62 & 6 & 1 & 254,561 & $-1,689,337$ & 711,877 & 78.14 & 71.39 & 68.07 & 88.21 & 70.55 & 64.79 & 61.92 \\
\hline 12 & {$[1,0,0]$} & 74.3 & 14 & 6 & 1 & 266,182 & $-1,936,558$ & 708,615 & 73.09 & 68.81 & 85.95 & 74.37 & 64.67 & 63.02 & 60.19 \\
\hline 13 & {$[1,0,0]$} & 59.9 & 92 & 10 & 2 & $3,894,783$ & $-2,877,501$ & $3,503,879$ & 80.33 & 100.00 & 75.69 & 71.38 & 73.60 & 69.21 & 74.09 \\
\hline 14 & {$[0,0,1]$} & 66 & 64 & 8 & 1 & $1,665,872$ & $36,266,635$ & $-6,551,301$ & 80.30 & 70.60 & 67.45 & 90.00 & 70.15 & 63.73 & 65.24 \\
\hline 15 & {$[0,0,1]$} & 43.8 & 52 & 8 & 1 & $3,902,297$ & $92,289,673$ & $-12,316,264$ & 82.87 & 70.68 & 66.29 & 90.34 & 70.39 & 63.45 & 64.39 \\
\hline 16 & {$[0,1,0]$} & 61 & 66 & 1 & 2 & $1,200,926$ & $1,777,238$ & $7,634,634$ & 100.00 & 77.14 & 68.83 & 78.04 & 66.50 & 61.14 & 58.12 \\
\hline 17 & {$[1,0,1]$} & 44.1 & 66 & 3 & 1 & $11,714,650$ & $44,284,498$ & $-44,675,334$ & 74.67 & 64.75 & 77.36 & 70.08 & 66.12 & 82.46 & 73.48 \\
\hline 18 & {$[1,0,1]$} & 46.5 & 8 & 2 & 1 & $1,197,019$ & $-4,079,567$ & $-1,909,360$ & 81.59 & 73.16 & 69.55 & 78.51 & 70.02 & 66.59 & 61.98 \\
\hline 19 & {$[1,0,0]$} & 68.5 & 56 & 10 & 2 & $2,171,086$ & $-4,654,115$ & $2,156,118$ & 74.18 & 100.00 & 73.92 & 70.37 & 75.66 & 72.47 & 79.26 \\
\hline 20 & {$[1,1,0]$} & 74.9 & 61 & 1 & 2 & $1,003,787$ & $1,136,764$ & $3,156,973$ & 100.00 & 78.09 & 70.46 & 78.96 & 67.44 & 61.82 & 58.91 \\
\hline 21 & {$[1,1,0]$} & 74.9 & 61 & 8 & 2 & $1,003,787$ & $1,793,613$ & $2,800,360$ & 75.39 & 96.07 & 94.67 & 78.47 & 67.33 & 74.63 & 75.40 \\
\hline 22 & {$[1,0,1]$} & 62.8 & 21 & 9 & 2 & 289,739 & $1,217,166$ & $2,550,120$ & 62.44 & 97.03 & 98.85 & 70.39 & 71.05 & 71.32 & 64.82 \\
\hline 23 & {$[1,0,1]$} & 57.7 & 81 & 3 & 1 & $4,870,422$ & $13,102,974$ & $-13,839,523$ & 79.26 & 71.09 & 67.31 & 81.65 & 68.16 & 62.43 & 59.60 \\
\hline 24 & {$[0,0,0]$} & 99.7 & 26 & 8 & 1 & $1,813,541$ & $-2,215,366$ & $-2,959,958$ & 83.66 & 72.68 & 66.99 & 92.14 & 73.27 & 66.77 & 67.62 \\
\hline 25 & {$[0,0,1]$} & 65.5 & 54 & 2 & 2 & $17,032,021$ & $12,933,994$ & $38,362,535$ & 78.64 & 82.15 & 73.17 & 69.11 & 83.95 & 73.10 & 70.19 \\
\hline 26 & {$[1,0,0]$} & 77.2 & 67 & 9 & 1 & 356,850 & 941,936 & $-716,448$ & 84.29 & 73.29 & 90.22 & 70.78 & 65.21 & 73.42 & 72.93 \\
\hline 27 & {$[1,0,0]$} & 80 & 71 & 9 & 2 & 520,061 & 497,529 & $-162,977$ & 82.87 & 97.66 & 94.60 & 70.53 & 64.42 & 70.30 & 67.54 \\
\hline 28 & {$[0,1,0]$} & 77.9 & 61 & 9 & 2 & 833,033 & $1,055,137$ & $-581,272$ & 77.95 & 97.17 & 95.82 & 68.55 & 62.24 & 70.56 & 67.28 \\
\hline 29 & {$[0,0,0]$} & 83.6 & 16 & 7 & 1 & 695,013 & $-4,973,625$ & $1,130,403$ & 74.52 & 68.95 & 63.57 & 66.77 & 91.26 & 66.32 & 66.16 \\
\hline 30 & {$[0,0,0]$} & 83.6 & 69 & 7 & 1 & 696,543 & $-4,930,835$ & $1,104,251$ & 74.52 & 68.97 & 63.57 & 67.23 & 91.13 & 66.23 & 66.07 \\
\hline 31 & {$[1,0,0]$} & 79.7 & 63 & 2 & 2 & 311,128 & 492,026 & $2,859,277$ & 81.58 & 77.20 & 68.16 & 75.38 & 65.28 & 77.31 & 68.84 \\
\hline 32 & {$[0,0,0]$} & 98.1 & 63 & 7 & 1 & 40,212 & $4,163,530$ & $-4,467,331$ & 86.18 & 71.18 & 62.98 & 63.48 & 89.27 & 68.85 & 66.29 \\
\hline 33 & {$[0,0,0]$} & 96.2 & 63 & 7 & 1 & 40,916 & $4,292,234$ & $-4,628,631$ & 86.10 & 71.08 & 62.93 & 63.13 & 89.44 & 68.91 & 66.34 \\
\hline 34 & {$[0,0,0]$} & 56.4 & 24 & 9 & 1 & 236,353 & 227,239 & $-309,853$ & 77.36 & 79.29 & 93.96 & 70.67 & 67.66 & 75.25 & 81.26 \\
\hline 35 & {$[1,0,0]$} & 55 & 53 & 9 & 1 & 521,417 & $-4,765,372$ & $1,002,583$ & 78.49 & 77.45 & 93.65 & 70.06 & 65.51 & 73.33 & 77.20 \\
\hline 36 & {$[1,0,1]$} & 60.1 & 66 & 6 & 2 & $4,494,330$ & $-2,606,195$ & $2,766,468$ & 65.62 & 90.04 & 75.98 & 69.13 & 66.56 & 85.86 & 77.79 \\
\hline 37 & {$[1,0,1]$} & 42.5 & 60 & 1 & 2 & $3,472,375$ & $-6,094,001$ & $84,352,376$ & 100.00 & 78.70 & 72.48 & 79.57 & 72.74 & 70.02 & 65.81 \\
\hline 38 & {$[1,0,1]$} & 42.5 & 62 & 1 & 2 & $3,472,375$ & $-6,094,001$ & $84,352,376$ & 100.00 & 78.70 & 72.48 & 79.57 & 72.74 & 70.02 & 65.81 \\
\hline 39 & {$[0,0,1]$} & 69.5 & 65 & 1 & 2 & 204,399 & $-5,141,769$ & $16,579,971$ & 100.00 & 75.19 & 66.57 & 72.79 & 65.03 & 75.47 & 67.68 \\
\hline 40 & {$[1,0,1]$} & 59.6 & 78 & 1 & 2 & 297,502 & 719,254 & $14,701,061$ & 100.00 & 74.95 & 73.82 & 65.82 & 73.63 & 64.19 & 60.87 \\
\hline \multirow[t]{2}{*}{ Network } & & & & & & $\begin{array}{c}\mathrm{IAC}_{\text {net }} \\
(\$)\end{array}$ & $\begin{array}{c}\mathrm{LCC}_{\text {net }}^{\mathrm{b}} \\
(\$)\end{array}$ & $\begin{array}{c}\mathrm{BNT}_{\text {net }}^{\mathrm{b}} \\
(\$)\end{array}$ & $\begin{array}{c}\mathrm{HI} 1_{\text {net }} \\
(\%)\end{array}$ & $\begin{array}{c}\mathrm{HI} 10_{\text {net }} \\
(\%)\end{array}$ & $\begin{array}{c}\mathrm{HI} 20_{\text {net }}^{\mathrm{b}} \\
(\%)\end{array}$ & $\begin{array}{c}\mathrm{HI} 30_{\mathrm{net}} \\
(\%) \\
\end{array}$ & $\begin{array}{c}\mathrm{HI}_{4} 40_{\text {net }} \\
(\%)\end{array}$ & $\begin{array}{c}\mathrm{HI} 50_{\text {net }} \\
(\%)\end{array}$ & $\begin{array}{c}\mathrm{HI} 54_{\text {net }} \\
(\%)\end{array}$ \\
\hline & & & & & & $78,952,662$ & $172,304,296$ & $160,937,323$ & 82.18 & 79.21 & 75.86 & 73.64 & 73.54 & 70.28 & 68.33 \\
\hline
\end{tabular}

${ }^{a}$ The value of 1 represents to the existence of the functional deficiency, otherwise the value of $0 .{ }^{b}$ Optimized objective.
To 
The investment levels shown in Table 8.8 are visually presented in Figure 8.12 -a series of bar charts representing the annual initial agency costs by improvement type. The figure clearly assists in visualizing the allocation of budget between program years and improvement types specified by NLO Solution 3, delivering the maximum network LCC benefit for the top-down approach Scenario 2a of Example 3. For instance, the bar charts show that most of the budget is distributed among 11 bridges in the first three program years. No allocations in program years 4 and 5 . The bar charts also show that most of the budget is allocated for functional improvement work.

The network health indices at the ends of years $1,10,20,30,40,50$, and 54 for each of the 86 NLO solutions (refer to Table 8.7) recommended for the top-down approach Scenario 2a of Example 3 are shown in Figure 8.13. As expected, the straight lines connecting these predicted network health indices are all situated above the horizontal dashed lines in black, representing the network performance constraints (a minimum network health index of $70 \%$ every 10 years). The thick connected lines in black represents the network performance produced by NLO Solution 3. These 86 NLO solutions produced close values of network health indices despite the differences between the resulting LCCs. As explained earlier, it takes substantial improvement efforts to alter the overall network health index — determined by aggregating all the bridge health indices and averaging them in Equation (8.8). The connected straight lines in red represent the network declining condition over time under the DN LC Alternative; the DN network health index is predicted to drop to $42 \%$ by year 50 . 


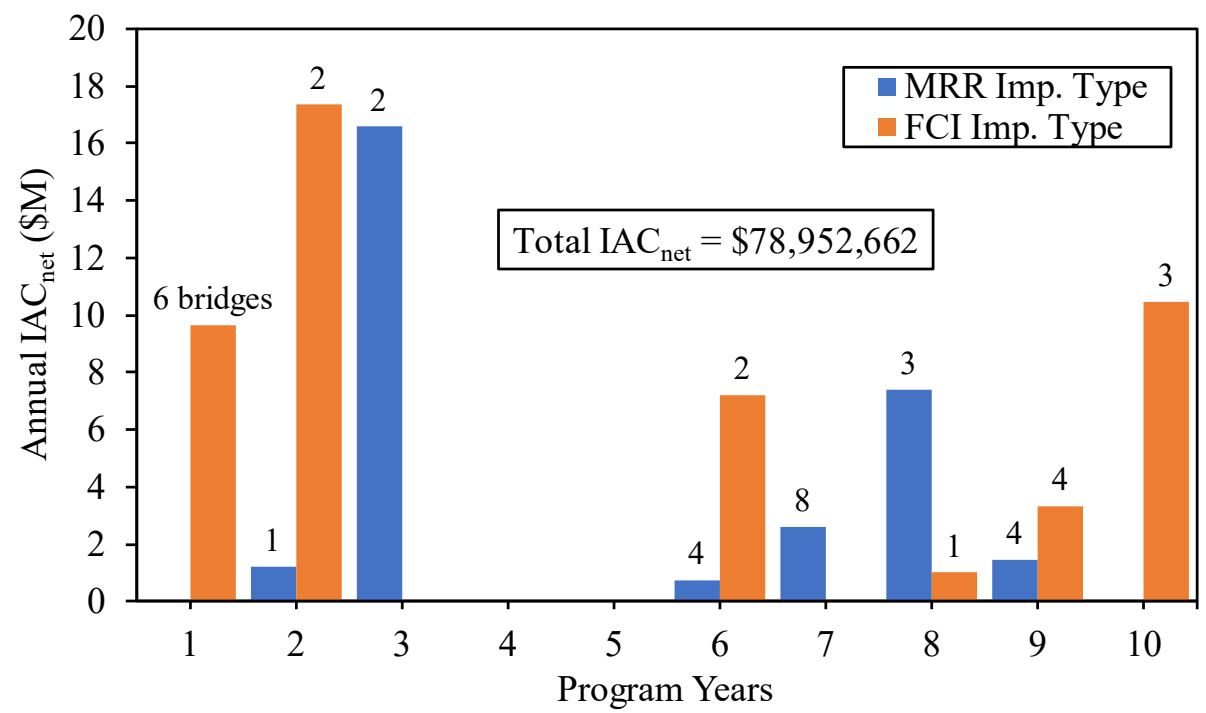

Figure 8.12 Annual initial agency costs per improvement type produced by the NLO solution with the maximum network LCC benefit for Example 3, Scenario 2a, Top-Down Approach

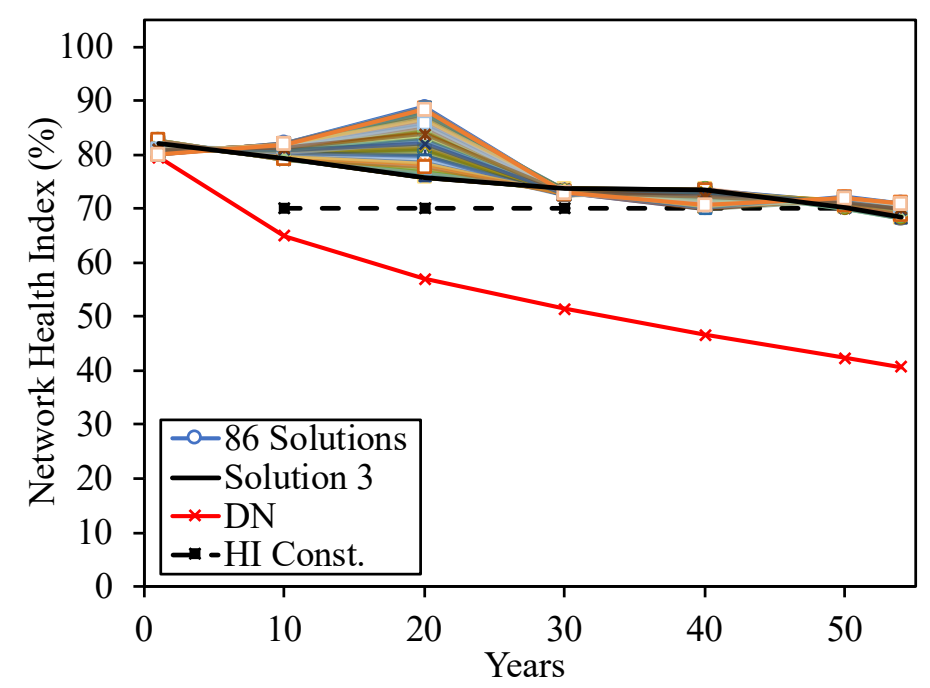

Figure 8.13 Network health indices produced by the NLO solutions recommended for Example 3, Scenario 2a, Top-Down Approach 


\section{LC Alternatives Recommended by the NLO Solution with the Maximum Network LCC Benefit for Example 3, Scenario 2a, Top-Down Approach}

Table 8.9 shows the LC alternatives recommended by NLO Solution 3, delivering the maximum network LCC benefit for the top-down approach Scenario 2a of Example 3, for each sample bridge in the portfolio. MRR and FCI improvement types are the only ones recommended by NLO Solution 3 for this portfolio. The module assumes the replacement option (REP improvement type) is always a feasible and eligible to compete. However, preservation (MRR) and functional (FCI) improvement types are generally the least expensive. Moreover, both FCI and REP improvement types fully restore all elements providing the same level of performance. For each bridge, the table discloses the recommended improvement type (third column), program year (second column), and LC alternative for each of its elements (starting from the fourth column). LC Alternative 1 is always assigned to the non-deficient elements. The recommended LC alternatives collectively yield the maximum network benefit while maintaining the network performance above the minimum acceptable limits for the top-down approach Scenario 2a of Example 3.

Bridge 17 is selected to further explore these recommended LC alternatives by NLO Solution 3. Table 8.10 presents the NBI condition ratings of this sample bridge and other related attributes. Table 8.11 shows the latest condition states of the 19 elements of this sample bridge. The bridge was built in 1953. It's a large steel truss bridge and has a low sufficiency rating of $44.1 \%$ (representing an overall poor condition). The bridge was identified with two functional deficiencies: bridge roadway width and load capacity. 
Table 8.9 LC alternatives recommended by NLO Solution 3 with the maximum network LCC benefit for Example 3, Scenario 2a, Top-Down Approach

\begin{tabular}{|c|c|c|c|c|c|c|c|c|c|c|c|c|c|c|c|c|c|c|c|c|c|c|c|c|}
\hline \multirow{3}{*}{$\frac{\text { Bridge }}{1}$} & \multirow{3}{*}{$\begin{array}{c}\text { Pro. } \\
\text { Year } \\
6\end{array}$} & \multirow{3}{*}{$\begin{array}{c}\text { Imp. } \\
\text { Type } \\
\text { MRR }\end{array}$} & \multicolumn{22}{|c|}{$\begin{array}{c}\text { Elements } \\
\text { LC Alternatives }\end{array}$} \\
\hline & & & 12 & 107 & 215 & 227 & 234 & 301 & 311 & 313 & 331 & 510 & & & & & & & & & & & & \\
\hline & & & 2294 & 800 & 1 & 782 & 1 & 1563 & 2167 & 1667 & 1 & 2343 & & & & & & & & & & & & \\
\hline \multirow{2}{*}{2} & \multirow{2}{*}{7} & \multirow{2}{*}{ MRR } & 12 & 28 & 29 & 38 & 107 & 109 & 113 & 152 & 210 & 215 & 226 & 227 & 234 & 300 & 301 & 304 & 310 & 313 & 330 & 331 & 333 & \\
\hline & & & 2309 & 1032 & 1684 & 2184 & 800 & 1 & 3125 & 800 & 2497 & 1 & 3122 & 782 & 1 & 1558 & 1558 & 2338 & 1 & 2182 & 2082 & 1 & 2342 & \\
\hline \multirow{2}{*}{3} & \multirow{2}{*}{7} & \multirow{2}{*}{ MRR } & 12 & 28 & 29 & 38 & 107 & 109 & 113 & 152 & 210 & 215 & 226 & 227 & 234 & 300 & 301 & 304 & 310 & 313 & 330 & 331 & 333 & \\
\hline & & & 2309 & 1032 & 1059 & 2184 & 800 & 1 & 3125 & 800 & 3122 & 1 & 3122 & 782 & 1 & 2183 & 1558 & 2338 & 1 & 2182 & 2082 & 1 & 2342 & \\
\hline \multirow[b]{2}{*}{4} & \multirow{2}{*}{6} & \multirow{2}{*}{ FCI } & 12 & 38 & 109 & 215 & 220 & 226 & 227 & 234 & 301 & 310 & 331 & & & & & & & & & & & \\
\hline & & & 462 & 438 & 1 & 1 & 607 & 157 & 157 & 1 & 438 & 1 & 1 & & & & & & & & & & & \\
\hline \multirow[b]{2}{*}{5} & \multirow{2}{*}{9} & \multirow{2}{*}{ FCI } & 12 & 28 & 29 & 107 & 110 & 113 & 152 & 210 & 215 & 220 & 225 & 227 & 231 & 234 & 301 & 313 & 330 & 331 & & & & \\
\hline & & & 209 & 157 & 159 & 157 & 194 & 625 & 300 & 247 & 1 & 232 & 282 & 157 & 282 & 1 & 158 & 182 & 282 & 1 & & & & \\
\hline & & & 12 & 104 & 215 & 227 & 234 & 301 & 303 & 311 & 313 & 331 & & & & & & & & & & & & \\
\hline 6 & 7 & MRR & 2309 & 1 & 1 & 3107 & 1 & 2183 & 2184 & 2307 & 2307 & 1 & & & & & & & & & & & & \\
\hline & & & 12 & 102 & 215 & 227 & 231 & 234 & 303 & 314 & 331 & & & & & & & & & & & & & \\
\hline 7 & 10 & $\mathrm{FCl}$ & 418 & 550 & 1 & 1 & 157 & 1 & 438 & 467 & 1 & & & & & & & & & & & & & \\
\hline & & & 12 & 102 & 104 & 215 & 220 & 227 & 234 & 300 & 301 & 303 & 310 & 314 & 331 & & & & & & & & & \\
\hline 8 & 7 & MRR & 1934 & 3050 & 1 & 1 & 3107 & 782 & 1 & 2183 & 1558 & 2184 & 1 & 2332 & 2344 & & & & & & & & & \\
\hline & & & 12 & 109 & 215 & 227 & 234 & 301 & 310 & 311 & 313 & 331 & & & & & & & & & & & & \\
\hline 9 & 6 & MRR & 218 & 1 & 1 & 782 & 1 & 938 & 1 & 2292 & 2292 & 1 & & & & & & & & & & & & \\
\hline & & & 12 & 105 & 109 & 215 & 227 & 234 & 301 & 310 & 311 & 331 & & & & & & & & & & & & \\
\hline 10 & 9 & MRR & 208 & 1093 & 1 & 1 & 782 & 1 & 2033 & 1 & 2037 & 1469 & & & & & & & & & & & & \\
\hline & & & 12 & 107 & 205 & 215 & 220 & 226 & 234 & 301 & 311 & 313 & 330 & 331 & & & & & & & & & & \\
\hline 11 & 6 & MRR & 2168 & 800 & 782 & 1 & 3032 & 1247 & 1 & 2188 & 1042 & 1042 & 2292 & 1 & & & & & & & & & & \\
\hline & & & 12 & 107 & 205 & 215 & 220 & 226 & 234 & 301 & 311 & 313 & 330 & 331 & & & & & & & & & & \\
\hline 12 & 6 & MRR & 218 & 800 & 782 & 1 & 3032 & 1247 & 1 & 2188 & 1042 & 1042 & 2292 & 1 & & & & & & & & & & \\
\hline & & & 12 & 28 & 29 & 110 & 113 & 120 & 152 & 210 & 215 & 220 & 227 & 234 & 301 & 304 & 311 & 313 & 330 & 331 & 510 & & & \\
\hline 13 & 10 & FCI & 418 & 157 & 159 & 469 & 625 & 250 & 175 & 1 & 1 & 607 & 1 & 1 & 158 & 443 & 412 & 412 & 407 & 1 & 468 & & & \\
\hline & & & 12 & 107 & 113 & 141 & 152 & 215 & 227 & 234 & 301 & 304 & 311 & 313 & 331 & & & & & & & & & \\
\hline 14 & 8 & MRR & 2168 & 782 & 3125 & 2735 & 800 & 1 & 3122 & 1 & 2158 & 2313 & 2157 & 1657 & 1 & & & & & & & & & \\
\hline & & & 12 & 107 & 109 & 113 & 120 & 147 & 152 & 161 & 215 & 220 & 227 & 234 & 301 & 304 & 311 & 313 & 331 & 333 & & & & \\
\hline 15 & 8 & MRR & 2293 & 782 & 1 & 3125 & 2675 & 794 & 800 & 1042 & 1 & 3032 & 1232 & 1 & 1533 & 2314 & 2157 & 2157 & 1094 & 2342 & & & & \\
\hline 16 & 1 & & 12 & 107 & 215 & 227 & 234 & 301 & 311 & 313 & 330 & 331 & & & & & & & & & & & & \\
\hline 16 & 1 & $\mathrm{FCl}$ & 418 & 175 & 1 & 157 & 1 & 163 & 417 & 417 & 407 & 1 & & & & & & & & & & & & \\
\hline & & & 12 & 30 & 107 & 113 & 120 & 152 & 161 & 215 & 220 & 225 & 227 & 234 & 301 & 304 & 311 & 313 & 330 & 331 & 510 & & & \\
\hline 17 & 3 & MRR & 1462 & 2188 & 800 & 3122 & 2675 & 800 & 1592 & 1 & 3107 & 782 & 1247 & 2341 & 2188 & 2339 & 912 & 1457 & 1032 & 2344 & 1719 & & & \\
\hline & & & 12 & 28 & 29 & 107 & 113 & 120 & 152 & 210 & 215 & 220 & 225 & 226 & 227 & 234 & 301 & 302 & 304 & 311 & 312 & 313 & 330 & 333 \\
\hline 18 & 2 & MRR & 2293 & 782 & 2032 & 800 & 3125 & 2675 & 800 & 2862 & 1 & 3107 & 782 & 3122 & 1247 & 1 & 813 & 782 & 2188 & 2292 & 1068 & 2082 & 1032 & 2344 \\
\hline & & & 12 & 28 & 29 & 107 & 109 & 113 & 152 & 210 & 215 & 220 & 226 & 234 & 301 & 304 & 310 & 311 & 313 & 330 & 331 & 333 & & \\
\hline 19 & 10 & $\mathrm{FCl}$ & 418 & 157 & 159 & 157 & 1 & 625 & 175 & 1 & 1 & 607 & 157 & 1 & 158 & 443 & 1 & 412 & 412 & 407 & 1 & 467 & & \\
\hline & & & 12 & 107 & 215 & 227 & 234 & 301 & 311 & 313 & 331 & 333 & & & & & & & & & & & & \\
\hline 20 & 1 & FCI & 418 & 175 & 1 & 157 & 1 & 163 & 417 & 417 & 1 & 469 & & & & & & & & & & & & \\
\hline
\end{tabular}


Table 8.9 LC alternatives recommended by NLO Solution 3 with the maximum network LCC benefit for Example 3, Scenario 2a, Top-Down Approach (continued)

\begin{tabular}{|c|c|c|c|c|c|c|c|c|c|c|c|c|c|c|c|c|c|c|c|c|c|c|}
\hline \multirow{3}{*}{$\frac{\text { Bridge }}{21}$} & \multirow{3}{*}{$\begin{array}{c}\text { Pro. } \\
\text { Year } \\
8\end{array}$} & \multirow{3}{*}{$\begin{array}{c}\text { Imp. } \\
\text { Type }\end{array}$} & \multicolumn{20}{|c|}{$\begin{array}{c}\text { Elements } \\
\text { LC Alternatives }\end{array}$} \\
\hline & & & 12 & 107 & 215 & 227 & 234 & 301 & 311 & 313 & 331 & 333 & & & & & & & & & & \\
\hline & & & 209 & 157 & 1 & 157 & 1 & 158 & 282 & 282 & 1 & 219 & & & & & & & & & & \\
\hline \multirow{2}{*}{22} & \multirow{2}{*}{9} & \multirow{2}{*}{$\mathrm{FCI}$} & 30 & 102 & 113 & 152 & 215 & 311 & & & & & & & & & & & & & & \\
\hline & & & 189 & 350 & 250 & 300 & 1 & 282 & & & & & & & & & & & & & & \\
\hline \multirow{2}{*}{23} & \multirow{2}{*}{3} & \multirow{2}{*}{ MRR } & 12 & 107 & 113 & 120 & 152 & 161 & 210 & 215 & 225 & 227 & 231 & 234 & 303 & 311 & 313 & 331 & & & & \\
\hline & & & 2168 & 800 & 3125 & 2675 & 800 & 2292 & 3122 & 1 & 782 & 1247 & 782 & 1 & 2188 & 917 & 2292 & 1 & & & & \\
\hline \multirow{2}{*}{24} & \multirow{2}{*}{8} & \multirow{2}{*}{ MRR } & 12 & 38 & 107 & 109 & 210 & 215 & 220 & 226 & 234 & 300 & 302 & 304 & 310 & 331 & 333 & & & & & \\
\hline & & & 2293 & 283 & 782 & 1 & 3122 & 1 & 3032 & 3122 & 1 & 2169 & 1559 & 1688 & 1 & 1 & 2342 & & & & & \\
\hline \multirow{2}{*}{25} & \multirow{2}{*}{2} & \multirow{2}{*}{ FCI } & 12 & 109 & 215 & 220 & 225 & 234 & 301 & 311 & 313 & 331 & 0 & & & & & & & & & \\
\hline & & & 434 & 1 & 1 & 607 & 157 & 1 & 188 & 434 & 432 & 1 & 0 & & & & & & & & & \\
\hline \multirow{2}{*}{26} & 0 & & 12 & 107 & 215 & 225 & 234 & 301 & 311 & 313 & 330 & 331 & 510 & & & & & & & & & \\
\hline & 9 & MRR & 2083 & 782 & 1 & 782 & 2093 & 1408 & 787 & 787 & 2032 & 1 & 2339 & & & & & & & & & \\
\hline & & & 38 & 215 & 225 & 234 & 301 & 330 & 331 & 510 & & & & & & & & & & & & \\
\hline 27 & 9 & $\mathrm{FCl}$ & 184 & 1 & 282 & 1 & 158 & 282 & 1 & 344 & & & & & & & & & & & & \\
\hline 28 & 0 & ECI & 38 & 215 & 226 & 234 & 301 & 330 & 331 & & & & & & & & & & & & & \\
\hline 28 & 9 & FCI & 184 & 1 & 157 & 1 & 158 & 282 & 1 & & & & & & & & & & & & & \\
\hline 20 & 7 & MPR & 28 & 29 & 38 & 107 & 113 & 152 & 210 & 215 & 220 & 227 & 231 & 234 & 301 & 310 & 311 & 330 & 331 & 333 & & \\
\hline 29 & 1 & MIRR & 1032 & 1684 & 2184 & 800 & 3110 & 800 & 3122 & 1 & 3032 & 782 & 782 & 1 & 1558 & 1 & 2182 & 832 & 1 & 2342 & & \\
\hline 30 & 7 & MPR & 28 & 29 & 38 & 107 & 113 & 152 & 210 & 215 & 220 & 227 & 231 & 234 & 301 & 310 & 311 & 330 & 331 & 333 & & \\
\hline 30 & 7 & MRR & 1032 & 1684 & 2184 & 800 & 3050 & 800 & 3122 & 1 & 3032 & 782 & 782 & 1 & 1558 & 1 & 2182 & 832 & 1 & 2342 & & \\
\hline & & & 38 & 210 & 215 & 225 & 234 & 301 & 331 & 510 & & & & & & & & & & & & \\
\hline 31 & 2 & $\mathrm{FCl}$ & 417 & 572 & 1 & 157 & 1 & 188 & 1 & 469 & & & & & & & & & & & & \\
\hline & & & 12 & 102 & 210 & 215 & 231 & 300 & 301 & 310 & 314 & 331 & & & & & & & & & & \\
\hline 32 & 7 & MRR & 2309 & 3050 & 3122 & 1 & 782 & 2308 & 1558 & 1 & 2332 & 1 & & & & & & & & & & \\
\hline & & & 12 & 102 & 210 & 215 & 231 & 300 & 301 & 310 & 314 & 331 & & & & & & & & & & \\
\hline 33 & 7 & MRR & 2309 & 3050 & 3122 & 1 & 782 & 2308 & 2183 & 1 & 2332 & 1 & & & & & & & & & & \\
\hline & & & 12 & 28 & 107 & 113 & 152 & 215 & 225 & 227 & 234 & 301 & 302 & 311 & 313 & 330 & & & & & & \\
\hline 34 & 9 & MRR & 2083 & 782 & 782 & 3110 & 800 & 1 & 782 & 782 & 1 & 1408 & 1533 & 2037 & 1412 & 2032 & & & & & & \\
\hline & & & 12 & 28 & 107 & 113 & 144 & 152 & 210 & 215 & 220 & 227 & 300 & 330 & 331 & 0 & & & & & & \\
\hline 35 & 9 & MRR & 2083 & 782 & 782 & 3125 & 2342 & 800 & 1872 & 3125 & 2732 & 782 & 919 & 2032 & 2344 & 0 & & & & & & \\
\hline & & & 12 & 28 & 107 & 215 & 220 & 225 & 226 & 227 & 231 & 234 & 301 & 304 & 310 & 311 & 313 & 330 & 331 & & & \\
\hline 36 & 6 & FCI & 462 & 207 & 175 & 1 & 607 & 157 & 157 & 157 & 157 & 1 & 438 & 468 & 1 & 457 & 457 & 417 & 1 & & & \\
\hline & & & 12 & 28 & 29 & 107 & 109 & 113 & 152 & 210 & 215 & 220 & 226 & 227 & 231 & 234 & 301 & 310 & 311 & 313 & 330 & 331 \\
\hline 37 & 1 & FCI & 418 & 157 & 157 & 175 & 1 & 625 & 175 & 572 & 1 & 607 & 157 & 157 & 157 & 1 & 163 & 1 & 417 & 417 & 407 & 1 \\
\hline & & & 12 & 28 & 29 & 107 & 109 & 113 & 152 & 210 & 215 & 220 & 226 & 227 & 231 & 234 & 301 & 310 & 311 & 313 & 330 & 331 \\
\hline 38 & 1 & FCI & 418 & $\begin{array}{c}20 \\
157\end{array}$ & 157 & 175 & 1 & 625 & 175 & 572 & 1 & 607 & 157 & 157 & 157 & $\begin{array}{c}254 \\
1\end{array}$ & 163 & 1 & 417 & 417 & 407 & 1 \\
\hline & & & 38 & 210 & 215 & 220 & 226 & 234 & 301 & 330 & 510 & & & & & & & & & & & \\
\hline 39 & 1 & FCI & $\begin{array}{c}50 \\
417\end{array}$ & 572 & 1 & 607 & 157 & $\begin{array}{c}254 \\
1\end{array}$ & 163 & 407 & 469 & & & & & & & & & & & \\
\hline & & & 38 & 210 & 215 & 234 & 301 & 330 & 510 & & & & & & & & & & & & & \\
\hline 40 & 1 & FCI & 209 & 247 & 1 & 1 & 163 & 282 & 344 & & & & & & & & & & & & & \\
\hline
\end{tabular}


Table 8.10 NBI condition ratings of Bridge 17

\begin{tabular}{|c|c|c|c|c|c|c|c|c|c|c|c|c|c|c|c|c|}
\hline \multicolumn{17}{|c|}{ Bridge 17} \\
\hline 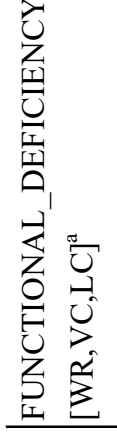 & 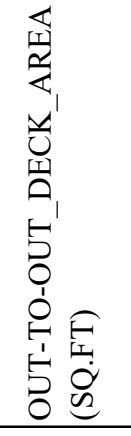 & 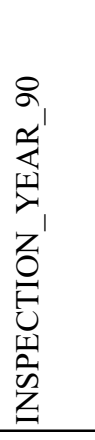 & 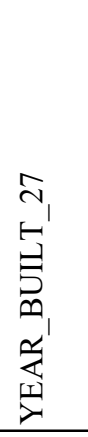 & 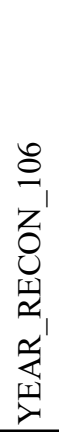 & 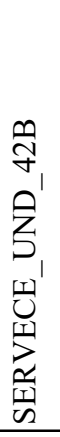 & 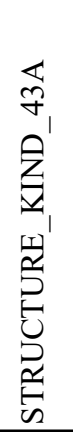 & 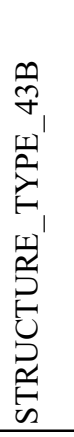 & 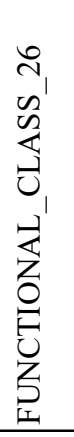 & 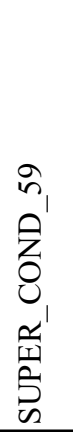 & $\begin{array}{l}8 \\
8_{1} \\
2_{1} \\
0 \\
0_{1} \\
n^{\prime} \\
\overbrace{2}\end{array}$ & 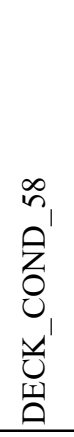 & 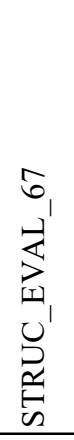 & 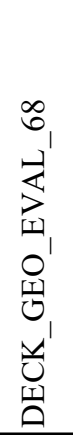 & 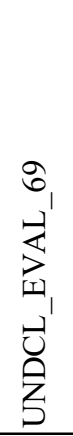 & 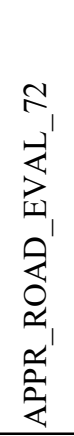 & 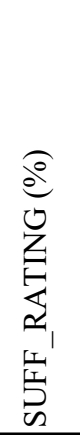 \\
\hline$[1,0,1]$ & 39791.4 & 2016 & 1953 & 0 & 6 & 4 & 10 & 12 & 5 & 6 & 5 & 5 & 2 & 3 & 8 & 44.1 \\
\hline
\end{tabular}

Table 8.11 Condition states of the 19 elements of Bridge 17

\begin{tabular}{|c|c|c|c|c|c|c|c|c|c|c|c|c|c|c|c|c|c|c|c|}
\hline & \multicolumn{19}{|c|}{ Elements of Bridge 17} \\
\hline & 12 & 30 & 107 & 113 & 120 & 152 & 161 & 215 & 220 & 225 & 227 & 234 & 301 & 304 & 311 & 313 & 330 & 331 & 510 \\
\hline CS1 (\%) & 69 & 100 & 54 & 81 & 98 & 71.61 & 83 & 100 & 100 & 0 & 0 & 63.89 & 99.93 & 93.00 & 2.19 & 22.32 & 98.37 & 81.97 & 100 \\
\hline CS2 (\%) & 4 & 0 & 35 & 6 & 2.28 & 21.46 & 17 & 0 & 0 & 0 & 25 & 35.65 & 0 & 7.00 & 97.81 & 70.64 & 1.63 & 14.41 & 0 \\
\hline CS3 (\%) & 27 & 0 & 11 & 13 & 0 & 7 & 0 & 0 & 0 & 100 & 75 & 0.46 & 0 & 0 & 0 & 0 & 0 & 3.62 & 0 \\
\hline CS4 (\%) & 0 & 0 & 0 & 0.17 & 0 & 0 & 0 & 0 & O & 促 & 0 & 0 & 0.07 & 0 & 0 & 7.03 & 0 & 0 & 0 \\
\hline Qt (sq.ft) & 381,290 & 47,038 & 16,085 & 50,664 & 10,436 & 20,189 & 6 & 118 & 305 & 100 & 283 & 2,805 & 2,786 & 1,742 & 183 & 327 & 6,028 & 16,910 & 47,038 \\
\hline $\mathrm{W}_{\text {elm }}(\%)$ & 25 & 49 & 49 & 18 & 50 & 17 & 70 & 13 & 10 & 10 & 11 & 13 & 12 & 12 & 12 & 12 & 16 & 14 & 5 \\
\hline ESL (years) & 129 & 36 & 77 & 292 & 168 & 77 & 77 & 75 & 123 & 20 & 200 & 408 & 24 & 61 & 58 & 58 & 28 & 151 & 126 \\
\hline RSL $^{\text {a }}$ (years) & 49 & 0 & 10 & 210 & 109 & 13 & 17 & 17 & 65 & 0 & 44 & 296 & 0 & 1 & 0 & 0 & 0 & 81 & 68 \\
\hline RU (\$) & 54 & 54 & 2,062 & 481 & 1,380 & 1,054 & 9,009 & 1,591 & 194,049 & 38,810 & 38,810 & 1,186 & 72 & 178 & 9,009 & 9,009 & 296 & 212 & 21 \\
\hline
\end{tabular}

${ }^{\mathrm{a}} \mathrm{RSL}$ is estimated from program year 1 (2020). 
Table 8.12 shows the LC alternatives recommended by NLO Solution 3 for Bridge 17. Different element preservation actions (MRR Actions $0,1,2,3$, and 4) are assigned to the program year (i.e., program year 3) and each decision point (spaced by a 10-year inaction period). LC Alternative 1 is assigned to the non-deficient element (i.e., Element 215). The recommended improvement actions and timings represent a detailed element-level work plan. The recommended actions can be grouped into a bridge project or included under a bridge preservation program. Table 8.13 shows the feasible preservation treatments associated with these recommended improvement actions.

Table 8.12 LC alternatives recommended by NLO Solution 3 for Bridge 17

\begin{tabular}{|c|c|c|c|c|c|c|c|}
\hline & $\begin{array}{l}\text { Element } \\
\text { Ref. }\end{array}$ & $\begin{array}{c}\text { MRR } \\
\text { LC Alt. }\end{array}$ & $\begin{array}{c}\text { Pro. Year } \\
\text { (year 3) }\end{array}$ & $\begin{array}{c}\text { Dec. Point } 1 \\
\text { (year 14) }\end{array}$ & $\begin{array}{l}\text { Dec. Point } 2 \\
\text { (year 25) }\end{array}$ & $\begin{array}{c}\text { Dec. Point } 3 \\
\text { (year 36) }\end{array}$ & $\begin{array}{c}\text { Dec. Point } 4 \\
\text { (year 47) }\end{array}$ \\
\hline \multirow{19}{*}{ 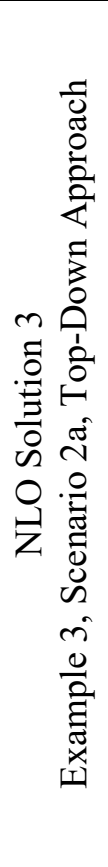 } & 12 & 1462 & 2 & 1 & 3 & 2 & 1 \\
\hline & 30 & 2188 & 3 & 2 & 2 & 2 & 2 \\
\hline & 107 & 800 & 1 & 1 & 1 & 4 & 4 \\
\hline & 113 & 3122 & 4 & 4 & 4 & 4 & 1 \\
\hline & 120 & 2675 & 4 & 1 & 1 & 4 & 4 \\
\hline & 152 & 800 & 1 & 1 & 1 & 4 & 4 \\
\hline & 161 & 1592 & 2 & 2 & 3 & 3 & 1 \\
\hline & $215^{\mathrm{a}}$ & 1 & 0 & 0 & 0 & 0 & 0 \\
\hline & 220 & 3107 & 4 & 4 & 4 & 1 & 1 \\
\hline & 225 & 782 & 1 & 1 & 1 & 1 & 1 \\
\hline & 227 & 1247 & 1 & 4 & 4 & 4 & 1 \\
\hline & 234 & 2341 & 3 & 3 & 3 & 3 & 0 \\
\hline & 301 & 2188 & 3 & 2 & 2 & 2 & 2 \\
\hline & 304 & 2339 & 3 & 3 & 3 & 2 & 3 \\
\hline & 311 & 912 & 1 & 2 & 1 & 2 & 1 \\
\hline & 313 & 1457 & 2 & 1 & 3 & 1 & 1 \\
\hline & 330 & 1032 & 1 & 3 & 1 & 1 & 1 \\
\hline & 331 & 2344 & 3 & 3 & 3 & 3 & 3 \\
\hline & 510 & 1719 & 2 & 3 & 3 & 3 & 3 \\
\hline
\end{tabular}

${ }^{\mathrm{a}}$ Non-deficient element. 
Table 8.13 Feasible preservation treatments associated with the improvement actions recommended by NLO Solution 3 for Bridge 17

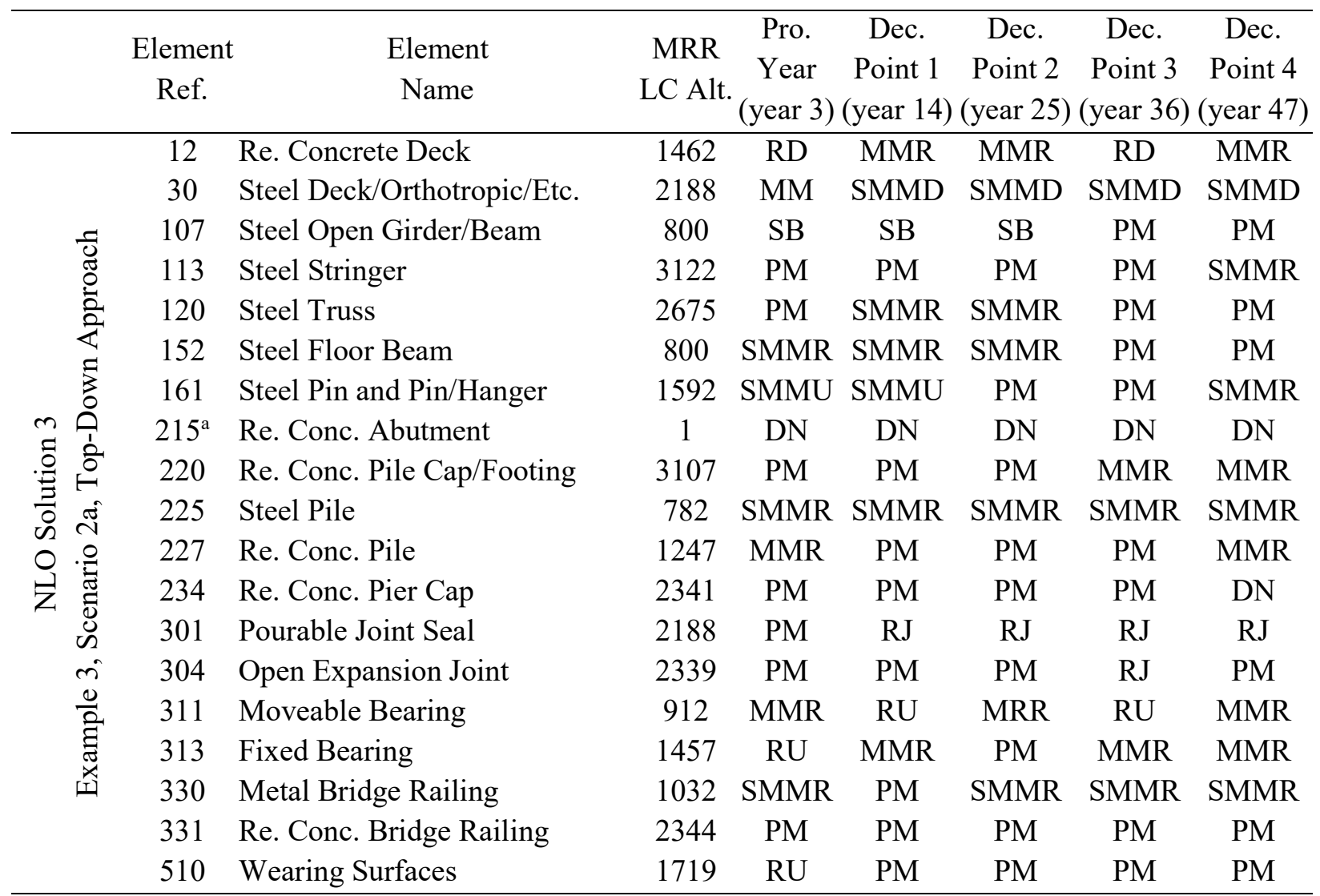

Note. For more detail about these preservation treatments, refer to Implementation of the 2013 AASHTO Manual for Bridge Element Inspection (Sobanjo \& Thompson, 2016a). PM = Preventive maintenance; RD $=$ Replace deck; MMR $=$ Minor or major repair; SMMR $=$ Spot blast and minor or major repair; SMMD $=$ Spot blast and minor or major repair or replace deck; SMMU = Spot blast and minor or major repair or replace unit; $\mathrm{SB}=$ Spot blast; $\mathrm{RU}=$ Replace unit; $\mathrm{DN}=$ Do-nothing; $\mathrm{RJ}=$ Replace joint.

${ }^{a}$ Non-deficient element.

Figure 8.14(a) shows the predicted element health indices under NLO Solution 3 for Bridge 17. As expected, the straight lines connecting element health indices are all situated above the health index thresholds (60\% every 10 years, horizontal dashed lines in black) specified by Criteria 1 of the alternative feasibility screening process (discussed in Chapter 5). The horizontal dashed lines in red represent the health index lower-frontiers (deficiency screening thresholds, a minimum health index of $80 \%$ every 10 years). The dashed curves distinguish the non-deficient elements from the deficient ones. The thick connected lines in black represent the overall bridge health 
indices. Figure 8.14(b) includes the predicted DN element health indices over the analysis periodproduced by DN LC Alternative. The predicted RO element health indices are shown in Figure 8.14(c) — produced by RO LC Alternative.

(a)

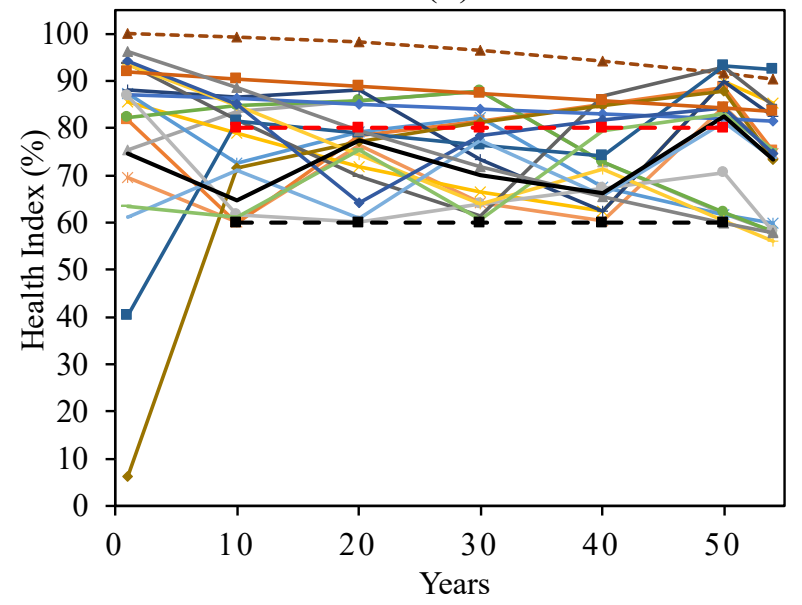

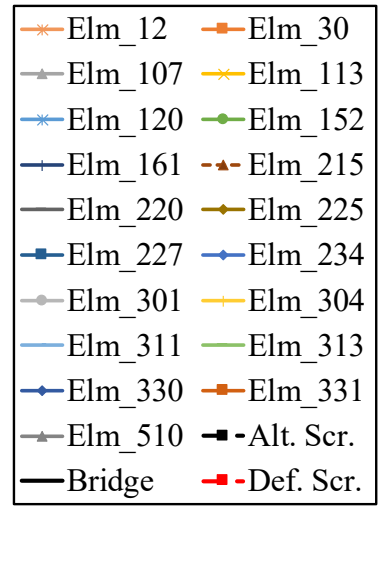

(b)

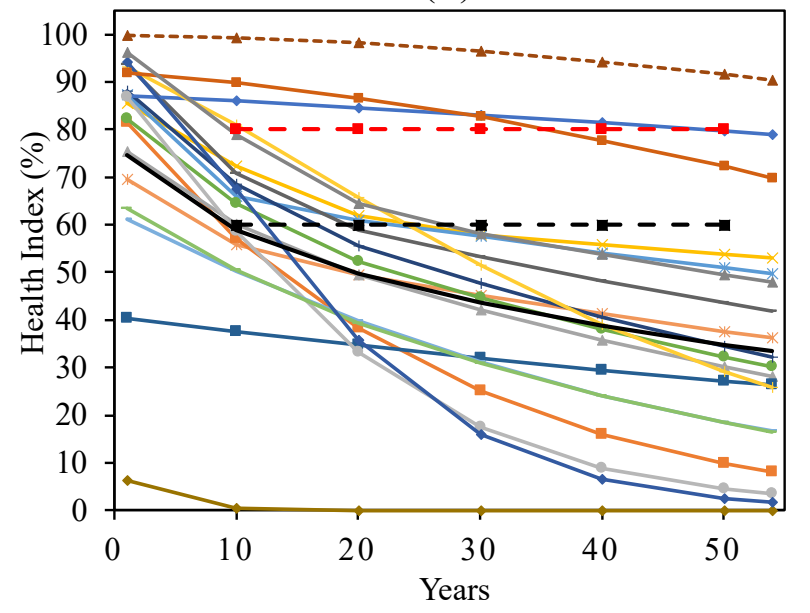

(c)

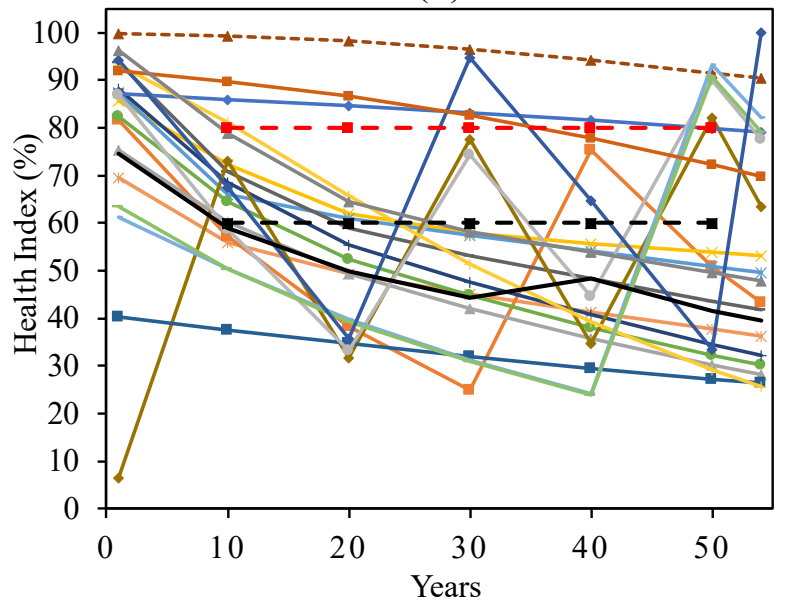

Figure 8.14 Predicted health indices for Bridge 17 and all its elements under (a) LC alternatives recommended by NLO Solution 3; (b) DN LC Alternative; (c) RO LC Alternative 


\subsection{Summary and Conclusions}

This chapter introduced the NLO module of the methodology. The chapter defined the top-down and bottom-up approaches followed at this higher level of optimization. A bottom-up approach is followed when BLO results are used as input parameters. Whereas, the top-down approach is followed when ELO results are used instead. For each approach, the chapter laid out the optimization framework, the optimization problem types and formulations, and the mapping approaches of decision variables. The multi-year optimization strategy was found to be appropriate for the problem formulations. The NLO problems are formulated in terms of binary decision variables.

A solution superiority screening process is used with the top-down approach to restrict the decision variable space and guide the optimization search toward global optimality within a reasonable computational time. The optimization formulation is shaped as a MCKP for an unconstrained NLO problem. For a constrained NLO problem, the formulation varies based on the budget constraint type. Budget constraints can be imposed either annually (for each program year) or cumulatively (for all program years). The multi-year budget-constrained problem is formulated as a MCMDKP. Whereas, the annual budget-constrained problem is formulated as a MKP.

The chapter also introduced the heuristic NLO algorithms for top-down and bottom-up approaches. The module relies on the same metaheuristic algorithm (i.e., NSGA-II) discussed in Chapters 6 and 7 to solve these NP-hard combinatorial NLO problems. The aim is to obtain a diverse set of optimal or near-optimal solutions as close as possible to the Pareto frontier-a recommended NLO solution delivers an optimal or near-optimal LC alternatives for the deficient elements of each selected bridge over the entire analysis period. The chapter included several examples of constrained (by budget and/or performance) or unconstrained scenarios for the module 
implementation using the tool prototype. Two cases of budget- and performance-constrained scenarios can be analyzed throughout this module: Case A-all bridge in the portfolio must be selected, and Case B — not necessarily all bridges must be selected. The examples constitute of different optimization goals and problem types. A portfolio of 40 sample bridges (introduced in Chapter 3) is used in these examples. Example results were visually presented in this chapter to demonstrate the ability of this module in generating Pareto frontiers, predicting network performance, determining investment needs, and facilitating trade-off analyses. The diversity and quality of NLO solutions obtained by either the top-down or bottom-up approach, and the intervention strategies recommended to maintain the desired network performance within the available budget were examined and discussed in this chapter.

The displayed Pareto frontiers for the unconstrained scenarios of these examples are practically superimposed. This observation suggests that both the top-down and bottom-up approaches converge to the same Pareto frontier for the same unconstrained scenario - though, more studies are needed to confirm this observation. Pareto frontiers for the constrained scenarios are bounded by the ones for the unconstrained scenarios - this finding is expected because a Pareto frontier of an unconstrained scenario is viewed as the ultimate optimal boundary.

Under Case A, example results demonstrate that the top-down approach NLO problems are more trackable than the bottom-up approach ones. The top-down approach benefits from the diversity of the input parameters (ELO solutions per program year) along with a superiority screening process, guiding the search toward global optimality. The bottom-up approach lacks this level of diversity as it relies mainly on the BLO solutions obtained for the entire program period. The convergence time increases almost exponentially with the size of the portfolio when bypassing the screening process for the top-down approach optimization. However, this issue wasn't noticed in 
the bottom-up approach optimization. The benefit of the bottom-up approach optimization can be recognized for the unconstrained scenarios or the constrained scenarios under Case B: Pareto solutions are obtained in less computational time than the top-down approach optimization. The module can be used to determine funding requirements and short- and long-term investment strategies for a network or portfolio of bridges, and facilitate trade-offs among funding levels and performance to help decision makers sort through the recommended investment strategies. 


\section{CHAPTER 9-CONCLUSIONS, CONTRIBUTIONS AND RECOMMENDATIONS}

\subsection{Summary and Conclusions}

MAP-21 mandates the development of a risk-based TAMP and use of a performance-based approach in transportation planning and programming. This research introduced a systematic EBMOO methodology integrated into a goal-driven TAM framework to

(1) improve bridge management,

(2) support state DOTs with their transition efforts to comply with the MAP-21 requirements,

(3) determine short- and long-term intervention strategies and funding requirements, and

(4) facilitate trade-offs between funding levels and performance.

The proposed methodology focuses on one transportation asset class (i.e., bridge) and is structured around the following five modules:

1. Data Processing Module

2. Improvement Module

3. Element-level Optimization Module

4. Bridge-level Optimization Module

5. Network-level Optimization Module 
Table 1.1 in Chapter 1 lists the activities associated with the different research tasks. The research approach was structured around the following five main tasks:

$\checkmark$ Task 1: Conducting a Literature Review

$\checkmark$ Task 2: Proposing a Goal-Driven Transportation Asset Management Framework

$\checkmark$ Task 3: Proposing an Element-Based Multi-Objective Methodology

$\checkmark$ Task 4: Development of a Tool Prototype

$\checkmark$ Task 5: Implementation of the Methodology through Examples of Scenarios

The literature review (under Task 1, Chapter 2) revealed that empirical and non-empirical ranking methods, IBCA, and multiple criteria analyses are widely applied for selecting bridge projects. A large variety of optimization techniques such as mathematical programming methods and metaheuristics have been applied to solve bridge optimization problems. The use of one technique versus another depends on the characteristics of the optimization problem. EAs produce a high quality of solutions (generally optimal or near-optimal) in a reasonable time for non-linear largesized optimization problems. The literature review was devoted to investigate this family of population-based search metaheuristics, specifically focusing on GAs to identify the most appropriate for integration. It was determined that the metaheuristic NSGA-II is well-suited for solving the different complex non-linear optimization problems (i.e., NP-hard combinatorial optimization problems) of this research in less computational efforts - it guarantees solution diversity and convergence to a near true Pareto frontier (front hosting optimal solutions). NSGAII is deployed as the main optimizer for the three optimization modules of the proposed EB-MOO methodology. 
The intent of Task 2 is to transfer previous work on TAM and best practices, identified throughout the literature review under Task 1, to refine the conceptual TAM framework introduced in the research proposal. Chapter 3 presented the refined goal-driven TAM framework, applying the principles of the of the Transportation Asset Management Guide: Prepared for NCHRP Project, 20-24(11) (Cambridge Systematics, Inc. et al., 2002) and discussed the different framework steps and interactions with the TAMP and the long-range planning and programming process. A comprehensive overview of the EB-MOO methodology (developed under Task 3) integrated into the refined goal-driven TAM framework is included in this chapter. The chapter elaborated on the posteriori articulation of preference approach followed by methodology — Pareto solutions are first determined, and then presented to the decision makers to select the best based on preferences. The chapter touched on the different types of analyses (i.e., sensitivity, "what-if" scenario, and tradeoff analyses) that can be performed to explore the whole set of Pareto solutions and communicate resulting impacts of limited resources and needs to achieve performance goals. The same chapter discussed the developed MATLAB-based tool prototype (structured around the proposed five modules of the EB-MOO methodology as part of Task 4). Several examples of unconstrained and constrained scenarios were established as part of Task 5 to test/validate concepts, prove effectiveness, and demonstrate and communicate potential benefits using the tool prototype. A sample set of existing bridges (portfolio) chosen for all these examples is introduced in this chapter.

Chapter 4 introduced the data processing module framework and described its different underlying concepts. The module relies on decision makers' preferences and inputs, quality data, information provided in the TAMP and TIP/STIP, leading-edge forecasting, and up-to-date cost models. The chapter discussed the two common types of bridge inspection data (i.e., NBI and NBE inspection data), the Weibull/Markov deterioration model used to predict the performance of an 
element over an analysis period and determine its ESL and RSL, the Florida DOT's AE models adapted to forecast element conditions when action are taken, and the performance measures (i.e., Caltrans' bridge and element health indices) used to assess the extent of deterioration and effectiveness of actions. The module assumes that a preservation policy is followed throughout the LC of the bridge and a major improvement work on the bridge fully restores all its elements. The chapter defined the preservation, functional improvement and replacement actions (i.e., MRR Actions 0, 1, 2, 3 and 4, FCI Action 5, and REP Action 6). It laid out the process of assessing function improvement needs (i.e., widening, raising, and strengthening improvement needs) and revealed the user cost models used to estimate incurred user costs. The three types of element LC profiles representing the "base" and "improvement" scenarios (i.e., DN, RO, and AE LC profiles) are introduced in this chapter with illustrative examples.

Chapter 5 presented a basic framework of the improvement module to visualize the modeling approach followed to generate LC alternatives (series of element improvement actions) and estimate LCCs and LCC benefits. The chapter discussed the novel screening process developed to focus on potential deficient elements and the new simulation arrangement to generate realistic ("real-life") LC alternatives for the three improvement types (i.e., MRR, FCI, and REP). The module relies on two independent models (i.e., deterioration and LCC models) to predict conditions and estimate LCCs and LCC benefits. The LCC and benefit modeling approaches are expressed in this chapter. An illustrative example of module results using the MATLAB-based tool prototype is also included. The implementation proved the capability of the module in producing reliable LC alternative results. The tool successfully identified potential deficient elements, predicted performance, generated LC alternatives, constructed LC profiles, and determined all incurred LCCs and LCC benefits. The module results can be used independently to 
determine bridge investment needs for bridge programming and planning. $\mathrm{BCA}$, IBCA, or optimization heuristics can be deployed to identify bridge combinations of LC alternatives representing the proper intervention strategies. The module LC alternative results are transferred to the ELO module to be used in the optimization process.

Chapter 6 presented the ELO module framework illustrating the different concepts and processes. The chapter described the alternative feasibility screening process developed to reduce the ELO problem size to a manageable size and improve the computational time. The screening process recognizes the best feasible LC alternatives for each program year based on the agency-specified criteria and optimization goals. The optimization problem is formulated in terms of binary decision variables. The optimization formulation is shaped as a MCKP, involving only the selection criterion. The year-by-year optimization strategy is adapted to decompose the optimization problem and further reduce the number decision variables. An ELO run is independently performed for each program year. The ELO problems focus on finding a set of Pareto solutions per program year for each improvement type-Pareto solutions in this research encompass the optimal or near-optimal (very close to optimal) solutions. A set of LC alternatives is derived from each obtained solution. Each recommended LC alternative represents a series of best (optimal or near-optimal) actions for a deficient element over the analysis period. Performance and LCC results associated with these LC alternatives (or solutions) serve as the fundamental inputs for the bridge- and network-level (top-down approach only) optimization modules. An illustrative example using the developed tool prototype is included in this chapter. The example consists of different ELO problems under unconstrained scenarios. Only one sample bridge was used in this example. For the analyzed program year, under each of three improvement types, the tool 
successfully produced optimal or near-optimal ELO solutions, recommended sets of intervention actions, predicted performance, and determined budget requirements.

Chapter 7 introduced an innovative BLO module that considers the ELO recommendations. No screening process is needed to further reduce the large size of the optimization problem. The total number of decision variables remains manageable even for a large network of bridges. A multiyear optimization strategy was found to be appropriate for the problem formulation. The problem is formulated in terms of binary decision variables. The optimization formulation is shaped as a MCKP if no constraints involved other than the selection criterion-only one ELO solution (one choice) must be picked from all solutions (multiple choices). The multi-dimensional aspect is added to the problem when more than one constraint (e.g., budget and/or performance) are involved; the problem is then formulated as a MCMDKP. The BLO process addresses one bridge at a time. The optimization process focuses on obtaining a diverse set of BLO solutions for the entire program period — a recommended BLO solution delivers an optimal or near-optimal set of LC alternatives for all the identified deficient elements over the entire analysis period. The presented example in this chapter consists of different BLO problems under constrained and unconstrained scenarios. Only optimization results of one sample bridge are shown in this chapter. The tool prototype successfully produced optimal or near optimal BLO solutions, recommended sets of intervention actions and timings, predicted performance, and determined funding requirements for the entire program period. The BLO module can be used independently to provide a systematic process to develop/assess bridge improvement/preservation programs.

Chapter 8 introduced the NLO module and defined the top-down and bottom-up approaches followed at this higher level of optimization. A bottom-up approach is followed when BLO results are used as input parameters; whereas, the top-down approach is followed when ELO results are 
used instead. The multi-year optimization strategy was found to be appropriate for the problem formulations. The NLO problems are formulated in terms of binary decision variables. A solution superiority screening process is used with the top-down approach to restrict the decision variable space and guide the optimization search toward global optimality within a reasonable computational time. The optimization formulation is shaped as a MCKP for an unconstrained NLO problem. For a constrained NLO problem, the formulation varies based on the budget constraint type. Budget constraints can be imposed either annually (for each program year) or cumulatively (for all program years). The multi-year budget-constrained problem is formulated as a MCMDKP. Whereas, the annual budget-constrained problem is formulated as a MKP. The aim is to obtain a diverse set of NLO solutions as close as possible to the Pareto frontier - a recommended NLO solution delivers an optimal or near-optimal LC alternatives for the deficient elements of each selected bridge in the portfolio over the entire analysis period. To verify optimally, the consistency of each obtained Pareto frontier was verified by increasing the number of iterations/generations and observing the difference between shapes.

The chapter included several examples of constrained (by available budget and/or minimum acceptable performance) or unconstrained scenarios for the module implementation using the tool prototype. Two cases of budget- and performance-constrained scenarios can be analyzed throughout this module: Case A-all bridge in the portfolio must be selected, and Case B-not necessarily all bridges must be selected. The examples constitute of different optimization goals and problem types. A portfolio of 40 sample bridges (introduced in Chapter 3 ) is used in these examples. The displayed Pareto frontiers for the unconstrained scenarios of these examples are practically superimposed. This observation suggests that both the top-down and bottom-up approaches converge to the same Pareto frontier for the same unconstrained scenario - though, 
more studies are needed to confirm this observation. Pareto frontiers for the constrained scenarios are bounded by the ones for the unconstrained scenarios - this finding is expected because a Pareto frontier of an unconstrained scenario is viewed as the ultimate optimal boundary.

Under Case A, example results demonstrate that the top-down approach NLO problems are more trackable than the bottom-up approach ones. The top-down approach benefits from the diversity of the input parameters (ELO solutions per program year) along with a superiority screening process, guiding the search toward global optimality. The bottom-up approach lacks this level of diversity as it relies mainly on the BLO solutions obtained for the entire program period. The convergence time increases almost exponentially with the size of the portfolio when bypassing the screening process for the top-down approach optimization. However, this issue wasn't noticed in the bottom-up approach optimization. The benefit of the bottom-up approach optimization can be recognized for the unconstrained scenarios or the constrained scenarios under Case B: Pareto solutions are obtained in less computational time than the top-down approach optimization. The module can be used to determine funding requirements, and short- and long-term investment strategies for a network or portfolio of bridges.

The different examples presented in this dissertation demonstrated the capability of the EB-MOO methodology to generate a high quality of solutions (generally optimal or near-optimal), predict performance, and determine proper intervention actions and funding requirements. The five modules of the methodology collectively provide a systematic process to (1) support developing/evaluating improvement programs/transportation plans, and (2) facilitate trade-offs among funding levels and performance to help decision makers sort through the recommended investment strategies. 


\subsection{Lessons Learned}

Lessons learned from the development process and the implementation of the EB-MOO methodology can be summarized as follows:

- The state of the practice among transportation agencies using performance data to support decision making for asset management, planning and programming varies considerably.

- Economic and performance measures drive investment decisions. Appropriate optimization objectives based on agency's goals, and policies and customer expectations are keys to the success of a $\mathrm{MOO}$ methodology for transportation asset planning and programming.

- A MOO methodology provides a systematic process with more transparency, addresses public needs, supports the development and evaluation of improvement programs and transportation plans, and facilitates trade-offs among funding levels and performance.

- A MOO methodology serves as a balanced decision support tool for asset managers to sort through the recommended investment strategies, test more realistic decision scenarios, and ultimately improve asset management.

- Investment strategies detailed at the element level provide a defensible approach to justify recommendations.

- Sufficient and high-quality/reliable element-level data are essential to ensure an effective element-based MOO process.

- Cost, action effectiveness, and deterioration models may not be mature for certain transportation agencies. However, the deployment of a MOO methodology to support decision making should not be delayed due to this limitation; a MOO methodology allows 
the flexibility to accommodate agency's existing models, preferences, and preservation policies.

- Considering a preservation strategy approach in the simulation process of LC alternatives extents the bridge service life and provides the most cost-effective improvement strategies.

- Efficient optimization formulations based on either the year-by-year or multi-year optimization strategy along with appropriate alternative screening processes at different levels of the optimization are effective in making the optimization problem trackable with less computational efforts.

- An alternative screening process is generally unnecessary for the BLO. The total number of decision variables remains manageable even for bridges with many deficient elements.

- The top-down and bottom-up approaches followed by the NLO often converge to the same Pareto frontier under the same unconstrained scenario. Though, Pareto frontiers for the constrained scenarios are bounded by the ones for the unconstrained scenarios.

- An alternative screening process is essential for top-down approach to guide the NLO search toward global optimality within a reasonable computational effort.

- A MOO methodology provides maximum return on investment, simulates investment and performance scenarios, and facilitates trade-off analyses-to identify appropriate strategies/scenarios, understand relationships between them, and communicate any impacts.

- Optimization using a robust metaheuristic optimizer provides the level of efficiency needed to solve complex (non-linear and combinatorial) optimization problems and achieve solution diversity and convergence to a near true Pareto frontier. 
- Successful deployment of a MOO methodology relies on key players and top-level agency commitments. Preferences should be provided by asset managers or program planners involved in asset management, planning and programming, development of policy objectives and performance measures, or resource allocations. Asset managers can validate the recommended priorities, funding requirements, and produced infographic depictions.

- A MOO methodology provides a quantitative process driven by decision makers' preferences, agency's preservation policy and objectives, and data inputs. The methodology follows a posteriori articulation of preference approach—Pareto solutions are first determined, and then presented to the decision makers to be explored and select the best based on preferences. Multiple criteria analyses facilitate the selection of the best solution.

- A trade-off or "what-if" scenario analysis allows decision makers to trade-off between sets of LC alternatives or optimization objectives. This type of analysis is essential to identify the appropriate course of actions, adjust preferences and funding levels, and communicate resulting impacts. Pareto solutions are indispensable for this type of analysis. Pareto solutions obtained for different scenarios, in alignment with the long-term goals, should be evaluated for possible implications on resource allocations and performance.

- It's important to periodically revisit optimization objectives (measures) and constraints (available budget and performance targets) to ensure their effectiveness in the actual decision making. Asset managers should perform this kind of assessments and recommend their adjustments over time.

- Considering risk in the decision-making process supports achieving a reasonable informed decision. Risk events can have impacts on system levels in various terms-impacting 
performance and ability to deliver the recommended investment strategies or effectively manage assets.

- Experts are encouraged to identify risks that could impact the serviceability and achievement of performance targets. The optimized timing of interventions over the analysis period should be explored considering any identified relevant risks — usually based on bridge or network attributes such as location, environmental, traffic volume, etc.

- Visualization plays a major role in communicating with clarity results obtained from the complex optimization analysis to the public and stakeholders. Simplicity in presenting optimization results helps create buy-in. These results should be presented in simple formats - charts and graphs can be used to simplify results in ways that are easy to comprehend and clearly convey the message. 


\subsection{Contributions}

This research contributed to the body knowledge in the areas of TAM and BMSs. The most salient contributions of this research can be summarized as follows:

\section{- Better understanding of transportation asset management needs}

Task 1 of this research consists of conducting a comprehensive review of literature across multiple resources to explore the availability of research work and findings related to the research areas and objectives, identify best practices, and ensure no duplication of efforts but rather build on previous research. The literature review identified significant references relevant to the research objectives-reflecting the current state-of-the-art in TAM, transportation planning and programming, risk assessment, MCDM, and MOO. The gathered information and best practices from these references provided the background to shape the TAM framework and the EB-MOO methodology. Table 2.1 in Chapter 2 lists these significant references. Each of them is accompanied with a brief description.

The literature review revealed that empirical and non-empirical ranking methods, IBCA, multiple criteria analyses are widely applied for selecting bridge projects. A large variety of optimization techniques such as mathematical programming methods and metaheuristics have been applied to solve bridge optimization problems. The use of one technique versus another depends on the characteristics of the optimization problem. Various investment analysis tools and systems using optimization techniques for bridge investment decision making either by state DOTs, FHWA, or

researchers were discoursed in this dissertation. Different MOO approaches, methods and techniques, and analytical tools commonly used to support investment decision making involving multiple criteria (or objectives) were identified. The importance of carrying out a trade-off analysis in TAM was highlighted. Obtained set of optimal solutions can be further examined through trade- 
off analyses to identify the appropriate strategies/scenarios, understand relationships between them, and communicate any impacts. EAs produce a high quality of solutions (optimal or nearoptimal) in a reasonable time for non-linear large-sized problems. The literature review was devoted to investigate this family of population-based search metaheuristics, specifically focusing on GAs to identify the most appropriate for integration. NSGA-II was identified as the appropriate metaheuristic algorithm for solving complex (non-linear and combinatorial) optimization problems in less computational efforts - it guarantees solution diversity and convergence to a near true Pareto frontier.

\section{- Proposing a goal-driven transportation asset management framework}

A goal-driven framework applying the principles of the Transportation Asset Management Guide: Prepared for NCHRP Project, 20-24(11) (Cambridge Systematics, Inc. et al., 2002) is proposed to support state DOTs with their transition efforts to performance management and performancebased planning and required by MAP-21. The framework focuses on one transportation asset class (i.e., bridge) and relies on quality data and agency established policies, goals, performance measures and targets, anticipated funding levels, and customer expectations to guide the management process of assets. The framework can be expanded to accommodate other asset classes or modes. The framework is designed to be integrated into the long-range planning and programming process - to provide more transparency, address public needs, and support the development and evaluation of the LRTP, TIP/STIP, and TAMP. The framework is structured around a continuing performance monitoring to assess effectiveness, identify gaps, and adjust as needed. This research was also undertaken to develop a novel MOO methodology integrated into this proposed framework, serving as a decision support tool to

(1) identify candidate bridge projects for inclusion in the LRTP or TIP/STIP; 
(2) set project/program priorities, revaluate funding allocations, or assess impacts of programmed types of bridge work (i.e., preservation, rehabilitation, and replacement) in the TIP/STIP on system performance; and

(3) evaluate different investment strategies and set targets through scenario analyses.

\section{- Developing a "true" multi-objective optimization methodology and a tool prototype for implementation}

Typically, decision makers are faced to simultaneously evaluate several differing preferences. In most cases, other non-economic preferences (objectives), targets and restrictions (constraints) contribute in the decision making. Most BMSs transfer this type of MOO problem to a singleobjective problem by scaling, weighting, and aggregating all competing objectives that could be easily solved by mathematical programming algorithms/methods. Although the approach is straightforward and guarantees global optimality, it's sensitive to the selected weights, requires advance knowledge of relative importance of each objective, and limits solution diversity. It requires several independent runs by varying weights to achieve the desired diversity. Decisions are made at either the network level or bridge level. For network-level decisions, ranking procedures or established decision trees (discussed in Chapter 2) have been widely used by state DOTs and other transportation agencies, especially when dealing with a large network of bridges. LCCA, BCA, and IBCA (discussed in Chapter 5) are the common economic decision analyses used by BMSs. When dealing with a budget-constrained scenario, alternatives with the highest benefit-cost ratios are selected in descending order until the available funding is exhausted. Though, at this higher level of assessment, setting priorities by ranking based on benefit-cost ratios results or decision trees is usually subjective and inadequate in providing the best long-term investment strategies. 
True MOO methodologies consist of a simultaneous optimization of multiple competing objectives subject to constraints. They guarantee a diverse set of optimal or near-optimal solutions, constituting a frontier of trade-offs between objectives. This research introduced a systematic true MOO methodology for the three levels of assessment (i.e., element, bridge, and network levels), laid out its five module frameworks, and presented the different optimization problem types and formulations, the adapted mapping approaches of decision variables, and the designed heuristic optimization algorithms. A MATLAB-based tool prototype structured around the proposed five modules of the EB-MOO methodology was developed. Several examples of unconstrained and constrained scenarios were established to test/validate concepts, prove effectiveness, and demonstrate and communicate potential benefits using the tool prototype. The proposed MOO methodology overcomes the discussed limitations, produces detailed element-level improvement strategies within a reasonable computational effort, provides maximum return on investment, simulates investment and performance scenarios, and permits trade-offs among competing objectives.

\section{- Developing a methodology based on a posteriori articulation of preference approach}

Due to the large variation among bridge management and maintenance practices, preservation policies, and performance measures adapted by state DOTs, the methodology relies on inputs and preferences from experts and decision makers, familiar with the state DOT's internal procedures and practices, to support the implemented processes and different analyses (i.e., LCCA, optimization process, sensitivity analysis, trade-off analysis, and adjustment of measures and targets). A priori articulation of preference approach (discussed in Chapter 2) is often followed when multiple objectives are aggregated into one, as in the weighted sum or utility function method. Decision makers provide preferences (relative weights) prior the optimization process. 
The methodology follows a different approach referred to as posteriori articulation of preference approach-Pareto solutions are first determined, and then presented to the decision makers to select the best one based on preferences. This approach helps providing a complete knowledge of the problem and exploring the whole set of Pareto solutions (Talbi, 2009).

The multiple criteria analyses discussed in Chapter 2 facilitate the selection of the best solution. Asset managers can validate optimized priorities, funding requirements, and produced infographic depictions. They can also run sensitivity/scenario analysis for funding uncertainty by manipulating budget constraints, discount rates, model parameters, or other variables. Optimization results can be further explored by a trade-off or "what-if" scenario analysis (covered in Chapter 2) between obtained Pareto solutions. The analysis allows decision makers to trade-off between sets of LC alternatives or optimization objectives. This type of analysis is essential for identifying the appropriate course of actions, adjusting preferences and funding levels, and communicating resulting impacts. Pareto solutions are indispensable for this type of analysis. Pareto solutions from different scenarios, in alignment with the long-term goal, are evaluated for possible implications on resource allocations and performance. It's important to periodically revisit measures and targets to ensure their effectiveness in the actual decision making and the development of long-term investment strategies. Asset managers through the proposed EB-MOO modules will be able to perform this kind of assessments and recommend adjustments to targets or measures over timefor example, based on trends of actual investments and separate analyses, measures that were proven to be irrelevant or ineffective, or targets believed to be unachievable (set too high). 


\section{- Developing a methodology producing detailed element-level improvement strategies}

Little research work has been focused on systematic element-based optimization methodologies for bridge project selection. This research proposes a novel MOO methodology to assess bridge element improvement needs for an effective management of bridge activities in both short- and long-term planning horizons. Agency's rules or triggers are used by most BMSs to limit the number of possible improvement actions per year. At the network level, these improvement actions are often described in broad terms and applied to the entire bridge or its major components. Thus, much of the element-specific information is lost. These high-level actions are generally used in the top-down approach assessment, reducing possible combinations of actions and eventually the execution time. These actions lack of details and typically are not meant to produce a comprehensive bridge improvement program. The proposed methodology depends on a quantitative process driven by decision makers' preferences (derived from the agency's preservation policy, objectives, and constraints), element and bridge data inputs, and defined feasible element and bridge improvement actions. Three independent optimization levels are incorporated into the methodology:

(1) an ELO, to identify optimal or near-optimal element intervention actions for each deficient element (in a poor condition state) of a candidate bridge;

(2) a BLO, to identify combinations of optimal or near-optimal element intervention actions for a candidate bridge; and

(3) a NLO, following either a top-down or bottom-up approach, to identify sets of optimal or near-optimal element intervention actions for a network of bridges.

Heuristic optimization algorithms using a robust genetic optimizer (i.e., NSGA-II) for each optimization level were developed to efficiently solve the complex (non-linear and combinatorial) 
optimization problems and achieve solution diversity and convergence to near true Pareto frontiers. The recommended element improvement actions and timings represent a detailed element-level improvement strategy for bridge planning and programming.

\section{- Overcoming the limitations of existing top-down and bottom-up approaches followed by the network-level optimization}

The literature review revealed two common approaches followed by the NLO among BMSs: (1) top-down approach, optimization determines the network-level goals, and then the improvement needs for individual bridges; and (2) bottom-up approach, where the bridge improvement needs are determined first. The top-down approach optimization produces high-level strategies (generally based on component-level or network-level actions) to meet the network-level goals and objectives. The bottom-up approach optimization uses the identified component- or elementlevel improvement strategies for each bridge as input parameters for the NLO process. The latter preserves bridge information and subsequently produces more refined network-level improvement strategies. The drawback of this approach is that a separate analysis for each bridge is required; and therefore, increasing the problem complexity, simulation, and eventually computational time. For a large network of bridges, the top-down approach is generally the preferred approach due to the less computational requirements. Bridge improvement recommendations are made generally in terms of network-level improvement actions. Work refinements at the component level or element level are generally left to the bridge manager judgments to compensate for the loss of bridge-specific information in the aggregation step (Yeo et al., 2013).

The presented research attempts to overcome the limitations of existing approaches. The top-down and bottom-up approaches are defined differently in this research. The NLO module bottom-up approach is followed when BLO solutions (input parameters) are used in determining network- 
level needs and recommending investment strategies; however, when superior ELO solutions (identified through the solution superiority screening process) are used as input parameters, then the top-down approach is followed. In either approach, both levels of solutions originate from an unconstrained optimization to increase diversity and ultimately the search space-recommended network-level investment strategies (by either approach) are detailed at the element level and obtained with reasonable computational efforts.

\section{- Developing a novel simulation arrangement to generate realistic ("real-life") LC alternatives for three improvement types}

Not all possible combinations of improvement actions and timings over an extended analysis period get considered in LC economic analyses - capturing incurred costs due to each possible alternative and any effect on performance leads to tremendous computational efforts and processing time. Thus, the recommended alternatives do not necessarily guarantee the optimal allocation of resources (Kachua, 2012). The methodology is designed to overcome most of these limitations. The methodology deploys an independent deterioration model (i.e., Weibull/Markov model), to predict performance, and a LCC model, to estimate LCCs and LCC benefits.

Three types of LC profiles (i.e., DN, RO, and AE LC profiles) are constructed for each element to predict bridge and element health indices at different points in time, and estimate RSLs and LCCs. The DN LC profile represents a "base" scenario of predicting condition of an untreated element This scenario simulates the element declining condition when no action is ever taken until reaching its end-of-life threshold. The RO LC profile represents another "base" scenario of predicting condition of an element that experiences only replacement actions. The schematic mimics the "worst-first" strategy: the element gets entirely replaced as it deteriorates to a poor condition without experiencing any treatments (no actions). The AE LC profile represents an "improvement" 
scenario of predicting condition of a treated element. An AE profile of an element is represented by a series of improvement actions. When an action is taken, an immediate change in condition happens per the $\mathrm{AE}$ model, while subsequent forecasting up to the next action is based on the hybrid (Weibull/Markov) deterioration model.

The module assumes that a preservation policy is followed throughout the LC of the bridge, and a major improvement work on the bridge fully restores all elements. Preservation actions are considered the most cost-effective actions for the long term. Thus, they always subsequent a major improvement work on the bridge for the remaining analysis period. Preservation actions account for the large portion of the AE LC profile. To generate LC alternatives for each element, all possible AE LC profiles must first be constructed. A LC alternative is defined by a path of actions and an improvement type. Each AE LC profile is laid out in a cash-flow diagram following a LC alternative action path. It's possible to generate all possible LC alternatives; however, the number will be unmanageable. The methodology relies on a simulation arrangement to generate manageable number of realistic ("real-life") LC alternatives for MRR, FCI and REP improvement types based on the following rules:

- Action effectiveness profile consists of 5 cycles

- First cycle falls always after a program year

- Preservation action selection is made at the end of each inaction period (referred to as a decision point)

- Ten years of inaction period between decision points

- Action is implemented in one year 
Based on these rules, $3125 \mathrm{MRR}, 625 \mathrm{FCI}$, and $625 \mathrm{REP}$ LC alternatives per program year are generated for each deficient element. A given bridge with just few elements can be associated with an enormous number of possible combinations of LC alternatives. The bridge LCCs and LCC benefits are determined for each of these feasible combinations of LC alternatives to be compared. The proposed EB-MOO methodology deviates from the common approaches (i.e., BCA and IBCA) used by most BMSs for selecting alternatives. LC alternative results are processed by the optimization modules to obtain the optimal or near-optimal combinations of LC alternatives for each analyzed bridge.

\section{- Developing efficient optimization problem formulations and introducing three novel screening processes to overcome computer memory and processing time limitations}

To overcome computer memory and processing time limitations, efficient optimization problem formulations were developed based on the appropriate optimization strategy (either year-by-year or multi-year strategy), considering multiple competing objectives and performance and (annual and multi-year) budget constraints. In addition, the methodology relies on the following screening processes:

Element Deficiency Screening Process-The proposed improvement module depends on a screening process that evaluates the extent of element deterioration. Some elements may have deteriorated to a level where major repairs or proactive preservation efforts are necessary, and others may show no sign of deterioration. Each bridge is screened for candidacy. This process is referred to as "element deficiency screening." A bridge with or expected to acquire deficient elements is considered a candidate for improvement. The focus is to ensure vulnerable bridges are being elevated in the programming process. A bridge identified with no potential deficient

elements is excluded from consideration — the "state of good repair" is assumed to be maintained 
in the entire analysis period. Users set minimum element health index limits at different points in the analysis period. Element DN health indices at the end of analysis years 10, 20, 30, 40, and 50 are predicted for each element. A health index lower-frontier is constructed for each element (joining minimum limits). Elements with health indices falling under this lower-frontier are classified as "deficient." For MRR improvement type, the identified set of potential deficient elements is considered for all program years. For FCI and REP improvement types, preservation needs are assessed for the period succeeding the program year. All elements are replaced (restoring CS1 to $100 \%$ ) in the program year because of a major improvement performed on the entire bridge. A separate screening is performed for each of the other program years.

Alternative Feasibility Screening Process-A given bridge can be associated with many possible combinations of LC alternatives. This huge number makes the optimization problem very challenging and costly to solve in terms of computational time and computer memory. This largescale optimization problem becomes extremely difficult to manage. Achieving heuristic solutions as close as to the Pareto frontier requires tremendous computational efforts. The need for a strategy that guides the optimization search toward global optimality within a reasonable computational time became indispensable. A screening process referred to as "alternative feasibility screening" was introduced in the ELO module to a make the problem more tractable without affecting the quality of solutions - attaining a manageable problem size dramatically improves the optimization computational time. To achieve a reasonable problem size and guarantee inclusion of most suitable LC alternatives, the process relies on two distinct stages of screening:

(1) an initial screening stage-feasible LC alternatives are identified after eliminating the economically unattractive ones, and 
(2) a final screening stage - feasible LC alternatives producing results in alignment with the ELO goal are further identified and classified as the best feasible LC alternatives for consideration.

The initial stage of the screening process focuses on recognizing feasible LC alternatives for each deficient element. The final stage improves the computational time by imposing additional screening criteria. The approach reduces the dimensionality of the optimization search space (i.e., the space of all feasible solutions) by emphasizing on the best feasible LC alternatives. It allows to efficiently explore the search space toward the optimal frontier without affecting the solution quality. This additional screening assures inclusion of the best feasible LC alternative results to serve as input parameters in the ELO process. These best LC alternatives are compatible with the ELO goal. Thus, solutions producing maximum or minimum objective values, depending on the optimization goal, are guaranteed. Obtained solutions are considered superior to all other solutions in the search space.

Solution Superiority Screening Process-Incorporating element- and bridge-level details into the NLO module complicates the NLO process. The complexity of the problem substantially increases when the number of involved bridges increases, and consequently the solution space. Therefore, deploying a strategy to reduce the number of possible solutions is essential (Elbehairy, 2007). Although the improvement or ELO modules deploy two different screening processes to reduce the problem size and improve the computational time, the problem size still represents a challenge at the network level. The module top-down approach could generate thousands or even millions of decision variables. This large-scale problem becomes extremely difficult to manage, especially with common computers. Achieving heuristic solutions as close as to the Pareto frontier requires a tremendous computational effort and computer memory. 
An arrangement process referred to as "solution superiority screening" is integrated into the NLO module to make the problem tractable with reasonable computational efforts - by restricting the decision variable space without affecting the quality of solutions. However, the process can be avoided if the running time is not a concern. This process reduces the optimization problem size and guarantees the inclusion of the superior (best) ELO solution results in the NLO process. The process identifies ELO solutions (input parameters) producing the best results in alignment with the NLO goal. For each network-level objective to be optimized, ELO solutions yielding the best objective values per program year are embraced — generally, a cutoff value is assigned to control the number of these superior ELO solutions. Thus, NLO solutions producing maximum or minimum objective values are guaranteed—extending the search space exploration. The screening process is generally unnecessary for the bottom-up approach optimization. The total number of decision variables remains manageable even for a large network of bridges. 


\subsection{Recommendations}

Several uncertainties and limitations were identified while pursuing this research. To further prove robustness and advance capabilities of the proposed EB-MOO methodology, the following recommendations could be explored in future research or studies:

\section{- Comparison study between the proposed EB-MOO methodology and the IBC heuristic}

IBCA is considered a superior to BCA and other empirical and non-empirical ranking methods (Farid et al., 1988). The IBCA is used by most BMSs for sorting alternatives at the network level. To further assess robustness/effectiveness and communicate benefits of the proposed methodology, a comparison study is recommended between the intervention action priorities determined by the tool prototype and the IBC heuristic used by BrM, NBIAS, or another state DOT's BMS (covered in Chapter 5) — assessing differences and impacts in terms of performance, funding requirements, and achievement of desired targets under different budget- and performance-constrained scenarios for a hypothetical network of bridges.

\section{- Examine other performance measures or health indices to substitute the adapted Caltrans' bridge and element health indices}

The obtained optimization solutions produce comparable values of bridge health indices despite the differences between the LCC values. As mentioned in Chapter 4, the bridge health index is an appropriate measure to assess performance; however, it is not a complete measure of the value of the agency's investment (Chase et al., 2016). Changes to the overall health index are generally minuscule. Element health indices are weighted, aggregated and divided by the sum of all their weighs to constitute this overall index. Improving few element health indices (after factoring their weights) won't dramatically change the overall index. It takes substantial improvement efforts to alter the overall index. Other performance measures or indices to assess the structural or functional 
health of a bridge can be examined to substitute the adapted Caltrans' bridge and element health indices.

\section{- Further investigate the solution convergence following either the top-down or bottom-up approach optimization under an unconstrained scenario}

The solutions produced by the NLO module following either the top-down or bottom-up approach appear to converge to the same Pareto frontier under the same an unconstrained scenario. Though, more studies are needed to confirm this observation.

\section{- Incorporate deterioration refinements quantifying effects of different protections or environments}

Elements deteriorate at different rates in different natural environments. The concept of bridge environment is incorporated in many deterioration models. A bridge is assigned to an environment based on the climate zone definitions of HPMS. Likewise, the different effects of protective elements (such as coatings, wearing surfaces, cathodic protections, joints, and drainage systems) are considered in deterioration models by introducing the concept of deterioration refinements. These concepts are ignored in the deterioration modeling of the proposed methodology. The inclusion of any deterioration refinements is beyond the scope of this research. Though, the data processing module is well-suited to accommodate any deterioration refinements that quantify effects of different protections or environments.

\section{- Factoring risk in the life-cycle cost modeling}

A broad range of risk events could impact achievement of bridge-related performance targets and the ability to deliver planned investments or manage assets effectively, and the performance of a network of assets or a single asset. Considering risk in the decision-making process supports achieving a reasonable informed decision. As emphasized in Chapter 2, efforts have been made to 
assess the threat of natural and man-made hazards in BMSs. Vulnerability or risk cost models quantify consequences resulting from natural hazards (such as earthquake, scour, and flooding). The NCHRP Project 20-07, Task 378, (Thompson, 2018) developed a risk assessment guideline for the LCCA in BMSs based on likelihood probability models for sixteen different hazards and a process for monetizing risk. The guideline can be considered in the LCCA to account for risk. The improvement module LCC model doesn't consider risk costs. Only agency and user costs are considered in the LCC model. Adding the risk aspect in the recommended investment strategies is beyond the scope of this research. Nevertheless, the module is well-suited to admit the recommended guideline concepts or other risk models.

\section{- Investigate the possibility of evolving the proposed methodology to a cross-asset multi- objective optimization methodology}

MAP-21 (23 CFR 515.9) requires state DOTs to incorporate a LCP process into their TAMPs, at a minimum, for pavements and bridges on the NHS and recommends similar process for other transportation assets. FHWA (2017a) recommends developing a strategy for managing each asset class or asset sub-group by minimizing the LCCs, while achieving the state DOT's targets for asset condition. The proposed goal-driven framework focuses on one transportation asset class (i.e., bridge). The framework can be expanded to accommodate other asset classes to facilitate crossasset resource allocation decisions through trade-off analyses between asset classes, and support the LCP process. This effort requires evolving the integrated EB-MOO methodology to a crossasset MOO methodology that considers information from the different asset classes for cross-asset management, programming and planning. 


\section{- Investigate sensitivity of the produced improvement strategies to default variables}

Sensitivity analysis as defined by Tarquin and Blank is "a study to see how the economic decision will be altered if certain factors are varied" (as cited in Farid et al., 1988). The accuracy of the proposed EB-MOO methodology results depends on the accuracy of the input data, the model parameters, assumptions, and other factors. Variables that most likely to impact results should be determined first - if a small variation of the variable changes the decision, the results are sensitive to that variable; otherwise, it's considered not sensitive (Farid et al., 1988). Sensitivity of the produced improvement strategies (i.e., optimization solutions) to the following default variables (believed sensitive) under different budget- and performance-constrained scenarios should be carried out through a series of tests to determine their appropriate values, evaluate impacts on funding requirements and performance, and assess the robustness of produced results.

- discount rate (default, $4 \%$ ),

- end-of-life threshold (defined as CS4=50\%),

- deficiency screening thresholds (default, minimum health index of $80 \%$ every 10 years)

- feasibility screening thresholds (default, minimum health index of $60 \%$ every 10 years)

- feasibility screening cutoff value (default, 50)

- $\quad$ superiority screening cutoff value (default, 2)

- inaction period (default, 10 years between decision points) 


\section{- Implementation through a case study involving a pilot state DOT using a user-friendly version of the tool prototype}

The deterioration, AE, and user cost models considered in the development of the EB-MOO methodology are based on studies (mainly for Florida DOT, TRB, AASHTO, and FHWA) presented in the literature. It is highly recommended that the proposed methodology to be customized for a pilot state DOT (using the pilot state DOT's AE, and user cost, deterioration models and preservation policy and/or triggers), and implemented through a case study to prove effectiveness and demonstrate/communicate potential benefits. The case study will provide the pilot state DOT with an excellent opportunity to apply the EB-MOO methodology and compare results. The case study should consist of bridges with sufficient data and from the same network (e.g., bridges that share the same decision-making entity, geographical area, vicinity, or other characteristics) — to ensure a high reliability of outcomes.

To achieve a successful implementation of the case study, the MATLAB-based tool prototype should be first migrated to a standalone or web-based product for ease of use by the pilot state DOT or other transportation agencies. The new user-friendly version of the tool (practical and ready-to-use) should include a front-end interface that provides most of the controls (user-specified performance measures, model parameters, inputs and preferences) and outputs in one convenient layout while reserving all complex mathematical calculations and programming languages for back-end processing. Tabulations, graphs, and dashboards should be used for summarizing, presenting, and displaying data and results.

A decision-making group consisting of key personnel from the pilot state DOT with various backgrounds and diverse expertise should be assembled to support the case study implementation. The group should include asset managers or program planners who make decisions about project 
priority setting, budgeting and programming, and experts who are responsible for overseeing bridge-related activities (i.e., inspection, preservation, maintenance, rehabilitation, and replacement) and familiar with the performance history of bridges and the state internal bridge preservation and maintenance practices. Coordination with the decision-making group should proceed throughout the case study implementation. Members of the decision-making group should be asked for feedback at different stages of the case study implementation and should be able to

- input preferences into the new user-friendly version of the tool;

- manipulate budget constraints and adjust performance measures and targets for the tradeoff analysis; and

- validate the recommended priorities, funding requirements, and infographic depictions produced by the tool. 


\section{LIST OF REFERENCES}

Avni, A., Burley, P., Casey, P., Cherney, J., Christiansen, L., Daly, J. S., ... \& Minotti, J. (2015). Literature searches and literature reviews for transportation research projects. How to search, where to search, and how to put it all together: Current practices. Transportation Research Circular, (E-C194).

AASHTO. (2013a). AASHTO Manual for Bridge Element Inspection. Washington, DC: Author.

AASHTO. (2013b). Transportation asset management guide: A focus on implementation. Washington D.C.: Author.

Adams, T. M., Kang, M., \& Pincheira, J. A. (2009). Sensitivity analysis of Bridge Health Index to element failure costs and conditions. Midwest Regional University Transportation Center.

Abu Dabous, S. (2008). A decision support methodology for rehabilitation management of concrete bridges (Doctoral dissertation, Concordia University).

AASHTO. (1986). Guide for design of pavement structures. Washington DC: Author.

Boyle, Z. (2017, April 26). AASHTOWare bridge management 5.2.3.: conducting trade-off analysis [PowerPoint slides]. Training session presented at the Eleventh International Bridge and Structures Management Conference.

Bai, Q. (2012). Trade-off analysis in multi-objective optimization for transportation asset management (Doctoral dissertation). Retrieved from

https://docs.lib.purdue.edu/dissertations/AAI3543353/

Chase, S., Adu-Gyamfi, Y., Aktan, A., \& Minaie, E. (2016). Synthesis of national and international methodologies used for bridge health indices. (Report No. FHWA-HRT-15081). Retrieved from https://www.fhwa.dot.gov/publications/research/infrastructure/structures/bridge/15081/index .$c f m$

Caltrans. (2016). SHOPP project prioritization phase 2: Application of a project prioritization framework to the 2016 SHOPP. Retrieved from:

http://www.dot.ca.gov/hq/transprog/shopp.htm

Caltrans. (2015). SHOPP pilot project phase 1: A framework for project prioritization. Retrieved from http://www.dot.ca.gov/hq/transprog/shopp.htm

Cambridge Systematics, Inc. (2013). Colorado DOT's risk-based asset management plan.

Retrieved from

http://www.tamptemplate.org/wpcontent/uploads/tamps/022_coloradodot.pdf 
Cambridge Systematics, Inc. (2011a). NCHRP Report 706: Uses of risk management and data Management to support target-setting for performance-based resource allocation by transportation agencies. Washington, DC: Transportation Research Board of the National Academies.

Cambridge Systematics, Inc. (2011b). National bridge investment analysis system 4.0 technical manual. Washington, DC: FHWA.

Cambridge Systematics, Inc. (2010). NCHRP Report 666: Target-setting methods and data management to support performance-based resource allocation by transportation agencies. Washington, DC: Transportation Research Board of the National Academies.

Cambridge Systematics, Inc. (2009). NCHRP Report 632: An Asset management framework for the interstate highway system. Washington, DC: Transportation Research Board of the National Academies.

Cambridge Systematics, Inc., PB Consult, \& Texas Transportation Institute. (2006). NCHRP Report 551: Performance measures and targets for transportation asset management. Washington, DC: Transportation Research Board of the National Academies.

Chinneck, J. W. (2006). Practical optimization: a gentle introduction. Retrieved from http://www. sce. carleton. ca/faculty/chinneck/po. html.

Cambridge Systematics, Inc., PB Consult, \& System Metrics Group, Inc. (2005) NCHRP Report 545: Analytical tools for asset management. Washington, DC: Transportation Research Board of the National Academies.

Cambridge Systematics, Inc., Parsons Brinckerhoff Quade \& Douglas, Inc., Ray Jorgenson Associates, Inc., \& Thompson, P. D. (2002). Transportation asset management guide: prepared for NCHRP Project, 20-24(11). Washington, DC: AASHTO.

Corne, D. W., Knowles, J. D., \& Oates, M. J. (2000, September). The Pareto envelope-based selection algorithm for multi-objective optimization. In Proceedings of the Six International Conference on Parallel Problem Solving from Nature (pp. 839-848).

Day, R. O. (2005). Explicit building block multi-objective evolutionary computation: methods and applications (Doctoral dissertation). Retrieved from https://apps.dtic.mil/docs/citations/ADA437215

Deb, K., Pratap, A., Agarwal, S., \& Meyarivan, T. A. M. T. (2002). A fast and elitist multiobjective genetic algorithm: NSGA-II. IEEE transactions on evolutionary computation, 6(2), 182-197.

Deb, K. (2001). Multi-objective optimization using evolutionary algorithms (Vol. 16). John Wiley \& Sons. 
Elbehairy, H. (2007). Bridge management system with integrated life cycle cost optimization. (Doctoral dissertation, University of Waterloo).

Erickson, M., Mayer, A., \& Horn, J. (2001, March). The niched Pareto genetic algorithm 2 applied to the design of groundwater remediation systems. In Proceedings of the First International Conference on Evolutionary Multi-Criterion Optimization (pp. 681-695).

FHWA. (2018). Bridge preservation guide (Report. No. FHWA-HIF-11042). Retrieved from https://www.fhwa.dot.gov/bridge/preservation/guide/guide.pdf

FHWA. (2017a). Using a life-cycle planning process to support asset management. Retrieved from https://www.fhwa.dot.gov/asset/pubs/life_cycle_planning.pdf

FHWA. (2017b). Incorporating risk management into transportation asset management plans. Retrieved from https://www.fhwa.dot.gov/asset/pubs/incorporating_rm.pdf

Flannery, A., Manns, J., \& Venner, M. (2016). NCHRP Synthesis of Highway Practice 494: Lifecycle cost analysis for management of highway assets. Washington, DC: Transportation Research Board of the National Academies.

FHWA. (2015). Introduction to element-level bridge inspection course. Washington D.C.: Author.

Frangopol, D. M., \& Liu, M. (2007). Maintenance and management of civil infrastructure based on condition, safety, optimization, and life-cycle cost. Structure and infrastructure engineering, 3(1), 29-41.

FHWA. (2002). Life-cycle cost analysis primer. Washington, DC: Author.

FHWA. (1999). Asset management primer. Washington, DC: Author.

FHWA. (1995). Recording and coding guide for the structure inventory and appraisal of the nation's bridges (Report No. No. FHWA-PD-96-001). Washington, DC: Author.

Farid, F., Johnston, D. W., Laverde, M.A., Chen, C. J. \& Rihani, B. S. (1988). Feasibility of incremental benefit-cost analysis for optimal allocation of limited budgets to maintenance, rehabilitation and replacement of bridges (Report No. FHWA-DP-71-02). Washington, DC: FHWA.

Grant, M., D'Ignazio, J., Bond, A., \& McKeeman, A. (2013). Performance-based planning and programming guidebook. Retrieved from https://www.fhwa.dot.gov/planning/performance_based_planning/pbpp_guidebook/

Hugh, H. (2003). NCHRP Report 483: Bridge life cycle cost analysis (BLCCA). Washington DC: Transportation Research Board of the National Academies. 
Hwang, C. L., \& Masud, A. S. M. (1979). Multiple objectives decision making-methods and applications. Berlin, Germany: Springer.

Holland, J. H. (1975). Adaptation in natural and artificial systems. Ann Arbor, MI: The University of Michigan press.

Johnson, J., \& Boyle, Z. (2017, April). Implementation of AASHTOWare Bridge Management 5.2.3 to meet agency policies and objectives for bridge management and address FHWA requirements. In Proceedings of the Eleventh International Bridge and Structures Management Conference, (pp. 188-212).

Jiang, Y., \& Sinha, K. C. (1989). The development of optimal strategies for maintenance, rehabilitation, and replacement of highway bridges volume 6: performance analysis and optimization. Indianapolis, IN: Indiana Department of Transportation

Kachua, S. G. (2012). A large-scale optimization algorithm to support cross-assets long-term planning in transportation asset management (Doctoral dissertation). Available from ProQuest Dissertations and Theses database. (NR98397)

Kachua, S. G. (2011). A large-scale optimization algorithm to support cross-assets long-term planning in transportation asset management (Doctoral dissertation, University of New Brunswick).

Krugler, P. E., Chang-Albitres, C. M., Pickett, K. W., Smith, R. E., Hicks, I. V., Feldman, R. M., ... Guikema, S. D. (2007). Asset management literature review and potential applications of simulation, optimization and decision analysis techniques for right-of-way and transportation planning and programming. Austin, Texas: Texas Department of Transportation.

Konak, A., Coit, D. W., \& Smith, A. E. (2006). Multi-objective optimization using genetic algorithms: A tutorial. Reliability Engineering \& System Safety, 91(9), 992-1007.

Kunkle, D. (2005). A summary and comparison of MOEA algorithms. Retrieved from http://www.ccs.neu.edu/home/kunkle/papers/techreports/moeaComparison.pdf

Knowles, J. D., \& Corne, D.W. (2000). Approximating the nondominated front using the Pareto archived evolution strategy. Evolutionary Computation, 8(2),149-172

Keeney, R. L., \& Raiffa, H. (1993). Decisions with multiple objectives: preferences and value trade-offs. Cambridge university press.

Labi, S. (2014). Introduction to civil engineering systems: A systems perspective to the development of civil engineering facilities. Hoboken, NJ: John Wiley \& Sons.

Lake, N., \& Seskis, J. (2013). Bridge management using performance models (No. AP-T258/13). 
Maggiore, M., \& Ford, K. (2016). NCHRP Report 806: Cross-asset resource allocation and the impact on transportation system performance. Washington, DC: Transportation Research Board of the National Academies.

MnDOT. (2015). Bridge preservation and improvement guidelines. Retrieved from http://www.dot.state.mn.us/bridge/pdf/bridge-preservation-and-improvement-guidelines2016-2020.pdf

Markow, M. J., \& Hyman, W. A. (2009). NCHRP Synthesis of Highway Practice 397: Bridge management systems for transportation agency decision making. Washington, DC: Transportation Research Board of the National Academies.

Mumford-Valenzuela, C. L. (2005). A simple approach to evolutionary multiobjective optimization. In Evolutionary Multiobjective Optimization (pp. 55-79). Springer, London.

Miettinen, K. M. (1999). Nonlinear multi-objective optimization. Norwell, MA: Kluwer Academic Publishers.

Moser, M., Jokanovic, D. P., \& Shiratori, N. (1997). An algorithm for the multidimensional multiple-choice knapsack problem. IEICE transactions on fundamentals of electronics, communications and computer sciences, 80(3), 582-589.

Mohamed, H. A. H. (1995). Development of optimal strategies for Bridge Management Systems (Doctoral dissertation, Carleton University).

NYSDOT. (2014). Transportation asset management plan draft v 05-02-14. Retrieved from: http://www.tamptemplate.org/wp-content/uploads/tamps/023_newyorkstatedot.pdf

Neumann, L. A. (1997). NCHRP Synthesis of Highway Practice 243: Methods for capital programming and project selection. Washington, DC: Transportation Research Board of the National Academies.

O’ Connor, D., \& Hyman, W. A. (1989). Bridge management systems (Report No. FHWA-DP71-01R). Washington, DC: FHWA.

Okabe, T., Jin, Y., \& Sendhoff, B. (2003, December). A critical survey of performance indices for multi-objective optimization. In The 2003 Congress on Evolutionary Computation, 2003. CEC'03. (Vol. 2, pp. 878-885). IEEE.

Patidar, V., Labi, S., Sinha, K. C., \& Thompson, P. D. (2007). NCHRP Report 590: Multiobjective optimization for bridge management systems. Washington, DC: Transportation Research Board of the National Academies.

Patidar V. (2006). Multi-criteria optimization in bridge management (Doctoral dissertation). Retrieved from https://docs.lib.purdue.edu/dissertations/AAI3260025/ 
Robert, W. (2017, April). Modeling of life-cycle alternatives in the National Bridge Investment Analysis System. In Proceedings of the Eleventh International Bridge and Structures Management Conference, (pp. 100-113).

Riquelme, N., Von Lücken, C., \& Baran, B. (2015, October). Performance metrics in multiobjective optimization. In The 2015 Latin American Computing Conference (CLEI) (pp. 111). IEEE.

Ramasamy, H. (2013). Toward a natural genetic/evolutionary algorithm for multiobjective optimization (Doctoral dissertation, Illinois Institute of Technology).

Robert, W. E., Gurenich, D. I., \& Thompson, R. E. (2009). Multiperiod bridge investment optimization utilizing Pontis results and budget constraints by work type (No. 09-2853).

Sobanjo, J. O., \& Thompson, P. D. (2016a). Implementation of the 2013 AASHTO manual for bridge element inspection. Retrieved from https://rosap.ntl.bts.gov/view/dot/37491

Sobanjo, J. O., \& Thompson, P. D. (2016b). Florida DOT's project level analysis tool user manual. Tallahassee, FL: Florida Department of Transportation.

Sobanjo, J. O., \& Thompson, P. D. (2016c). Florida DOT's network analysis tool user manual. Tallahassee, FL: Florida Department of Transportation.

Shoghli, O. (2014). A Decison Support System for Multi-Objective Multi-Asset Roadway Asset Management (Doctoral dissertation, Virginia Tech).

Sobanjo, J. O., \& Thompson, P. D. (2011). Enhancement of the FDOT's project level and network level bridge management analysis tools. (Contract. No. BDK83, 977-01). Retrieved from https://rosap.ntl.bts.gov/view/dot/18692

Sobanjo, J. O., \& Thompson, P. D. (2007). Decision Support for Bridge Programming and budgeting. Retrieved from https://trid.trb.org/view/805606

Sobanjo, J. O., \& Thompson, P. D. (2004). Project planning models for Florida's bridge management system (Contract No. BC 352-9). Retrieved from https://trid.trb.org/view/697924

Saito, M., \& Sinha, K. C. (1989). The development of optimal strategies for maintenance, rehabilitation, and replacement of highway bridges volume 5: priority ranking method. Indianapolis, IN: Indiana Department of Transportation

Schaffer, J. D. (1985). Multiple objective optimization with vector evaluated genetic algorithms. In Proceedings of the First International Conference on Genetic Algorithms and their Applications (pp. 93-100). 
Thompson, P. D., Bye, P., Western, J., \& Valeo, M. (2018, July). Risk assessment for bridge management systems. In Maintenance, Safety, Risk, Management and Life-Cycle Performance of Bridges. In Proceedings of the 8 Ninth International Conference on Bridge Maintenance, Safety and Management, (p. 246).

Thompson, P. D., Ford, K. M., Armin, M. H.R., Labi, S., Sinha, K. C., \& Shirole, A. (2012). NCHRP Report 713: Estimating life expectancies of highway assets. Washington, DC: Transportation Research Board of the National Academies.

Talbi, E. G. (2009). Metaheuristics: from design to implementation (Vol. 74). Hoboken, NJ: John Wiley \& Sons.

Thompson, P., Sinha, K. C., Labi, S., \& Patidar, V. (2008). Multi-objective optimization for bridge management systems. International Bridge and Structure Management, 195.

Taboada, H. (2007). Multi-objective optimization algorithms considering objective preferences and solution clusters (Doctoral dissertation, Rutgers University).

Turner, A., \& Richardson, J. (1994). Bridge management system data needs and data collection. Transportation Research Circular, (423), (pp. 5-15).

Tarquin, A. J., \& Blank, L. T. (1976). Engineering economy: a behavioral approach. McGrawHill.

U.S. DOT. (2015). Status of the nation's highways, bridges, and transit: Conditions \& performance. Washington, DC: The U.S. Department of Transportation.

Walls, J. \& Smith, M. R., (1998). Life-cycle cost analysis in pavement design-interim technical bulletin (Report No. FHWA-SA-98-079). Retrieved from

https://www.fhwa.dot.gov/infrastructure/asstmgmt/013017.pdf

Yeo, H., Yoon, Y., \& Madanat, S. (2013). Algorithms for bottom-up maintenance optimization for heterogeneous infrastructure systems. Structure and Infrastructure Engineering, 9(4), 317-328.

Zitzler, E., Laumanns, M., \& Thiele, L. (2001). SPEA2: Improving the strength Pareto evolutionary algorithm. TIK-report, 103.

Zitzler, E., Deb, K., \& Thiele, L. (2000). Comparison of multi-objective evolutionary algorithms: Empirical results. Evolutionary computation, 8(2), 173-195. 